\title{
Ecology of New Zealand Deep-sea Chondrichthyans
}

\author{
Brittany Finucci
}

A thesis submitted to the Victoria University of Wellington in fulfilment of the requirements for the degree of Doctor of Philosophy in Marine Biology

Victoria University of Wellington

Te Whare Wānanga o te Ūpoko o te Ika a Māui

2017 
This thesis was conducted under the supervision of:

Dr. Matthew R. Dunn (primary supervisor)

National Institute of Water and Atmospheric Research Wellington, New Zealand

Dr. Emma Jones (secondary supervisor)

National Institute of Water and Atmospheric Research Auckland, New Zealand 


\section{Abstract}

Deep-sea chondrichthyans represent nearly half of the known species of sharks, rays, and chimaeras. Most are poorly known, largely due to their historically low economic value, and thus, low prioritization for research efforts and targeted sampling. Globally, many deep-sea fisheries have proven to be unsustainable, as deep-sea species are generally characterised with life history traits, resulting in low biological productivity. Although generally not targeted, there is a lack of data on New Zealand deep-sea chondrichthyans, despite regularly occurring as bycatch, with no mitigation in place to limit catches.

This thesis described aspects of life histories for data deficient deep-sea chondrichthyans caught as bycatch in New Zealand deep-sea fisheries. In Chapter II, research trawl survey data were used to describe and evaluate length-weight relationships, which were found to greatly differ from parameters reported by FishBase. This was followed by the application of a set of models to detect changes in weight at length relationships, and assess if these changes correspond to biological or ecological events, such as length-at-maturity or ontogenetic changes in diet.

Chapter III evaluates deep-sea chondrichthyan aggregations and social associations. Not all species were found to engage in aggregative behaviour, but those that did suggested patterns of sex- and size-specific associations which varied with catch density. Adult females were caught most frequently in low densities and were highly associated with other adult females, adult males consistently highly associated with each other, and the highest density catches were dominated by juvenile individuals. These trends may be driven by factors such as foraging, predator avoidance or sexual conflict avoidance.

Chapters IV, V, and VI examine, respectively, details of the reproduction, life history, and diet of prickly dogfish (Oxynotus bruniensis), longnose spookfish (Harriotta raleighana) and Pacific spookfish (Rhinochimaera pacifica), and brown chimaera (Chimaera carophila) and black ghost shark (Hydrolagus homonycteris). All species were found to have life histories characteristic of low productivity, including reaching maturation at a large proportion of their maximum length, and having low fecundity. Additional novel biological results included: DNA identification of prey revealed that $O$. 
bruniensis preyed exclusively on the egg capsules of holocephalans, potentially making it the only known elasmobranch with a diet reliant solely upon other chondrichthyans; sperm storage was confirmed in female H. raleighana, $R$. pacifica, and C. carophila; and sexual dimorphism in snout length was found in $H$. raleighana, where male relative snout size increased at sexual maturity, suggesting that the snout is a secondary sexual characteristic.

The depth range of most New Zealand deep-sea chondrichthyans may provide some refuge from current fishing activity. However, results from this thesis have suggested that the species examined here have life histories characteristic of low productivity, and engage in behaviours that will have implications for selective mortality by spatially or temporally stratified fishing. Oxynotus bruniensis, in particular, is likely at higher risk from the impact of fishing than currently estimated, given its reproductive characteristics, highly specialised diet, and distribution overlap with deep-sea fisheries. Continued monitoring and a greater collection of biological data from additional and alternative sources (e.g. fisheries observer program, local fishers, underwater vehicles and video) is recommended to fully understand and negate mortality from human activities. 


\section{Acknowledgements}

Matt Dunn - I can't thank you enough. You truly made this $\mathrm{PhD}$ experience one I have thoroughly enjoyed. Thanks for the always entertaining and informative conversations that went off on the most random of tangents; for the initiation (a.k.a. sending me off to sea), for spending countless hours reviewing $\mathrm{R}$ with me, and driving me to the zoo with my dead sharks. Thank you for always being available and supporting all my findings, regardless how weird they were. A big thank you to Emma Jones as well, for all your assistance with my $\mathrm{PhD}$, despite the long distance. I know it wasn't easy being in another city, but I greatly appreciate all your valuable insight, hosting me in Auckland, and making the time for Wellington visits.

Thank you to everyone at NIWA who I've had the opportunity to meet and work with over the course of my thesis: to Darren Stevens and Neil Bagley for overseeing sample collection on surveys; thanks again to Darren for encouragement with my lab work and assisting me with the diet analysis; to Jeff Forman for allowing me to invade and make a mess of the feeding lab; to Dean Stotter for the prickly dogfish; Sadie Mills for advice on the chimaera diets; Warrick Lyon, Kath Large, Di Tracey for their encouragement and support. An extra special thanks to the staff and crew of TAN1401 for helping me collect the bulk of the specimens needed for my thesis. I also really appreciate resisting any urge to throw me overboard during the trip. I promise next time I won't be so sick.

Thank you to Victoria University of Wellington, as well as the Hutton Fund for providing the funding for this research. Thanks to the Ministry for Primary Industries (MPI) for providing access to the research trawl survey database and to the MPI Observer Program for collecting additional samples for me. These specimens were incredibly valuable and filled in many of the gaps for my sampling (gravid prickly dogs!) Thanks to the Museum of New Zealand Te Papa Tongarewa for access to the collections, and to Andrew Stewart and Carl Struthers in particular, for their advice, support, coffee, and willingness to examine the fish I could not identify; to Bruce Marshall for advice on the chimaera diet analysis. Thanks to Jane Anderson and St John Wakefield of Massey University for assisting with the histological work; and to Deepblue Seafoods for kindly 
retaining shark catches for me and packaging them up in scooter friendly boxes, as well as supporting my attendance at international conferences.

A special thank you to the NZ shark community for their overwhelming support throughout the years; to Malcolm Francis for first suggesting the idea of working on the deep-sea chondrichthyans, for the additional data, invaluable support, generous nature, and the fantastic shark nerd shindigs; to Clinton Duffy for your infectious enthusiasm for shark research and for the incredibly useful advice and inspiration; and to Kat Goddard thank you for welcoming me into the NZ science community. This all started here from a chance meeting with you!

To the wider shark community for all their wisdom, support, and encouragement along the way: the anonymous and not so anonymous reviewers for the extremely helpful comments for my publications; Carlos Bustamante; Chris Bird; Simon Pierce and the Marine Megafauna Foundation; South African Shark Conservancy; Jeff and Carol Carrier; Cassie Rigby; the Oceania Chondrichthyan Society, the American Elasmobranch Society, and FSBI for welcoming me into the global communities, hosting fantastic conferences, and supporting my attendance at conferences. Thank you to the Natural History Museum, Will White and CSIRO Hobart, and MAGNT Darwin for allowing me to poke and prod through museum collections. And of course, a big thank you to Pete Kyne (and the all-knowing Sant Mat) for proving my worth as bug bait, and all the incredibly helpful support during the final stretch of this work.

Thank you to the Wellington Muay Thai community and all my training partners that kept the stress levels down and supported me through four fights during the course of this thesis. Thank you to Angus for always finding me food, and to my fellow PhD-ers, Fran, Steph, and Katie, for the always thought-provoking yarns - I'd be a lot richer now if it wasn't for all those coffee breaks; and to Annie - thank you.... for being you?

And finally, thank you to my parents, Vito and Sharon, for always supporting my decisions and encouraging me to pursue my passions, even if it did mean moving some 15 $000 \mathrm{~km}$ away from home to spend all my time with creepy-looking monsters of the deep.

Instead of ending with a meaningful quote, I would like to use this space to formally apologise to everyone in the Allen building at NIWA for that time I stunk up the building with rotten chimaeras. 


\section{Table of Contents}

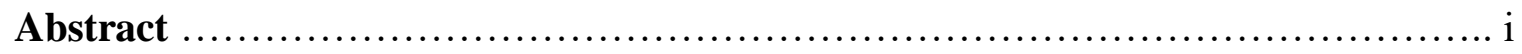

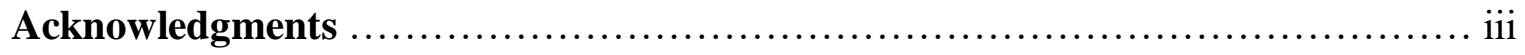

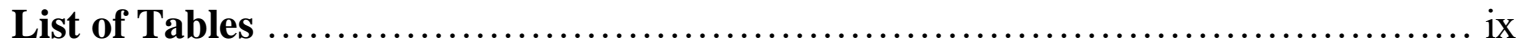

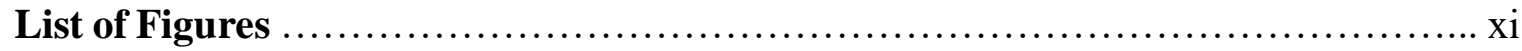

Contributions and Publication Status of Dissertation Chapters .................. xvii

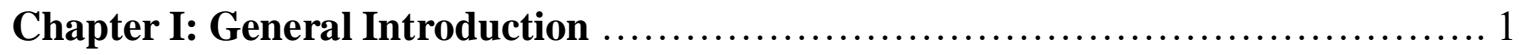

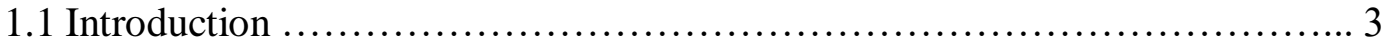

1.2 Deep-Sea Fisheries and Chondrichthyan Catches in New Zealand ............ 4

1.3 Shark Fisheries and Bycatch Management in New Zealand .................. 6

1.4 Ecological Risk Assessment for the Effects of Fishing (ERAEF) ............. 8

1.5 The Importance of Life History ......................................... 10

1.6 Study Species ....................................................... 13

1.6.1 Chimaeras (Chondrichthyes: Holocephali) ..................... 13

1.6.2 Longnose spookfish Harriotta raleighana Goode \& Bean, 1895 ..... 14

1.6.3 Pacific spookfish, Rhinochimaera pacifica (Mitsukuri, 1895) ...... 15

1.6.4 Brown chimaera Chimaera carophila Kemper, Ebert, Naylor \&

Didier, 2014 ................................................ 16

1.6.5 Black ghost shark Hydrolagus homonycteris Didier, 2008 ......... 17

1.6.6 Sharks (Chondrichthyes: Elasmobranchii) ........................ 18

1.6.7 Prickly dogfish Oxynotus bruniensis (Ogilby, 1893) .............. 19

1.7 Overview \& Objectives ................................................... 22

Chapter II: Length-weight relationships of deep-sea chondrichthyans .............. 24

2.1 Abstract ................................................................. 25

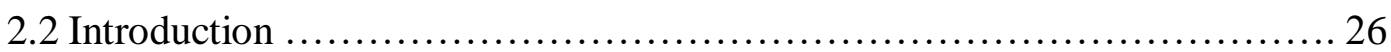

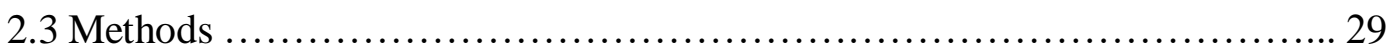

2.3.1 Research trawl data .............................................. 29

2.3.2 Species studied ......................................... 31

2.3.3 Part I: Estimating the length-weight relationship (LWR) ......... 31

2.3.4 Part II: Fitting a more complex length-weight model .............. 32

2.4 Results .......................................................... 37

2.4.1 Length-weight relationships ................................ 37

2.4.2 FishBase LWR parameter estimates ........................... 37

2.4.3 Inflection points in the LWR ................................ 38

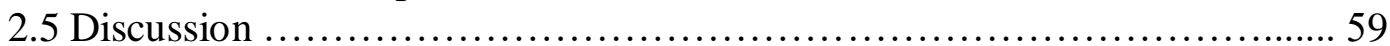

2.5.1 FishBase parameter estimates ................................. 60

2.5.2 Form factor and depth ......................................... 62

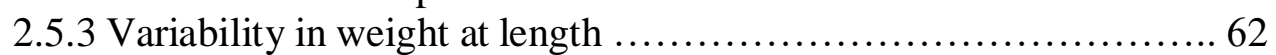

2.5.4 Early length $L W R$ variation detections ........................6 63

2.5.5 Mid-length $L W R$ variation detections ........................ 64

2.5.6 Late length LWR variation detections ......................... 66

Chapter III: Social associations in deep-sea chondrichthyans .................... 70 


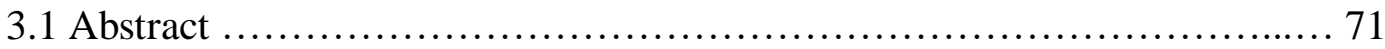

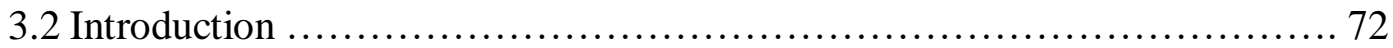

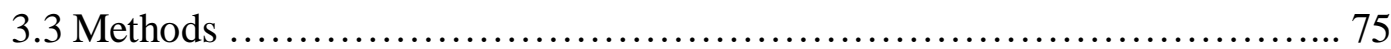

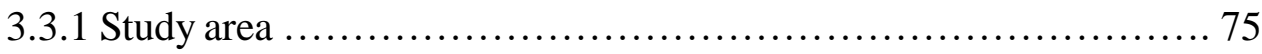

3.3.2 Research trawl catch data ................................. 75

3.3.3 Social association analysis .................................. 76

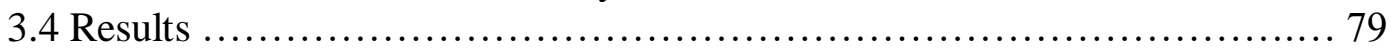

3.4.1 Catch densities ............................................... 79

3.4.2 Hydrolagus novaezealandiae ................................. 80

3.4 .3 Squalus acanthias ........................................ 82

3.4.4 Etmopterus lucifer ....................................... 84

3.4.5 Deania calcea ............................................... 86

3.4.6 Centroselachus crepidater ................................... 88

3.4.7 Etmopterus granulosus ..................................... 90

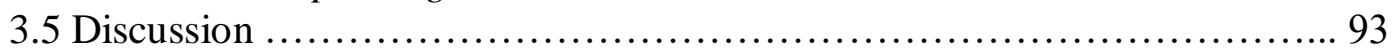

3.5.1 Reclusive females ........................................ 95

3.5.2 Adult males associated with other adult males .................. 96

3.5.3 Highest densities dominated by juveniles ....................... 98

3.5.4 Individual species trends ..................................... 99

Chapter IV: Reproductive biology and feeding habits of the prickly dogfish Oxynotus bruniensis (Ogilby 1893) .................................................. 101

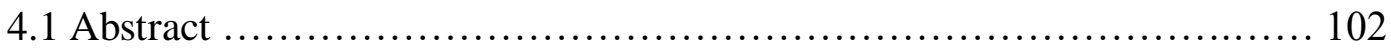

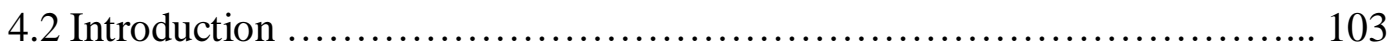

4.3 Methods ............................................................ 105

4.3.1 Study area and specimen collection ......................... 105

4.3.2 Biological data ........................................... 105

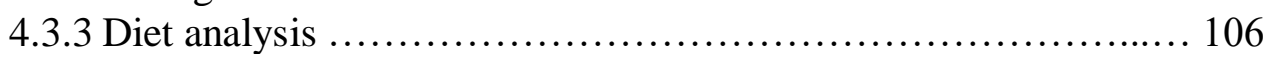

4.4 Results ............................................................. 110

4.4.1 Specimen collection and length-mass relationship ................ 110

4.4.2 Male reproduction ......................................... 115

4.4.3 Female reproduction ...................................... 116

4.4.4 Diet analysis .............................................. 118

4.5 Discussion ......................................................... 122

4.5.1 Reproductive characteristics .............................. 122

4.5.2 Oxynotus bruniensis $\operatorname{diet}$.................................... 123

Chapter V: Reproductive biology of two deep-sea chimaeras, longnose spookfish (Harriotta raleighana), and Pacific spookfish (Rhinochimaera pacifica) .............. 126

5.1 Abstract ............................................................. 127

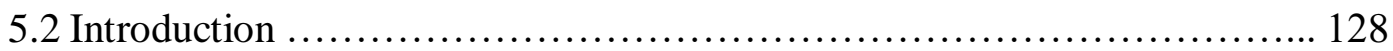

5.3 Methods ....................................................... 130

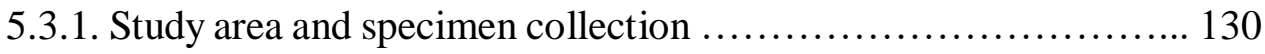

5.3.2 Biological data .......................................... 130

5.3.3 Light microscopy and transmission electron microscopy .......... 134

5.4 Results ........................................................... 135

5.4.1 Harriotta raleighana ........................................ 135

5.4 .2 Size-at-maturity .......................................... 136

5.4.3 Female reproductive characteristics ........................ 137

5.4.4 Male reproductive characteristics ........................... 139 
5.4.5 Rhinochimaera pacifica ................................... 141

5.4.6 Size-at-maturity .......................................... 142

5.4.7 Female reproductive characteristics ........................ 142

5.4.8 Male reproductive characteristics ........................... 143

5.4 .9 Sperm storage .......................................... 146

5.4.10 Preorbital snout length .................................... 147

5.5 Discussion ......................................................... 148

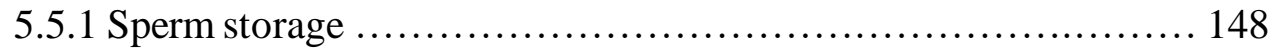

5.5.2 Secondary sexual characteristics .......................... 149

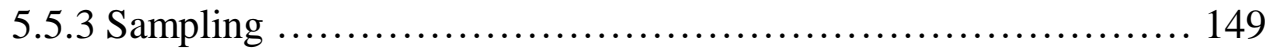

5.5.4 Review of chimaeroid reproductive biology ................... 151

Chapter VI: First observations of the biology of two rarely observed deep-sea chimaeras, Chimaera carophila and Hydrolagus homonycteris .................... 156

6.1 Abstract ...................................................... 157

6.2 Introduction .......................................................... 158

6.3 Methods ....................................................... 160

6.3.1 Specimen collection and biological data ..................... 160

6.3.2 Sperm storage .......................................... 162

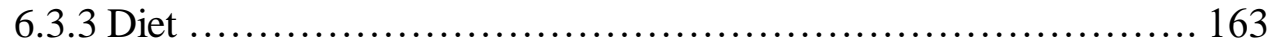

6.4 Results ............................................................ 164

6.4.1 Chimaera carophila distribution............................. 164

6.4.2 Hydrolagus homonycteris distribution........................ 164

6.4.3 Chimaera carophila specimen size range and length-mass relationship 167

6.4.4 Hydrolagus homonycteris specimen size range and length-mass relationship .................................................... 168

6.4.5 Size-at-maturity ........................................ 170

6.4.6 Chimaera carophila reproductive characteristics ................ 171

6.4.7 Hydrolagus homonycteris reproductive characteristics ............ 171

6.4.8 Diet ....................................................... 174

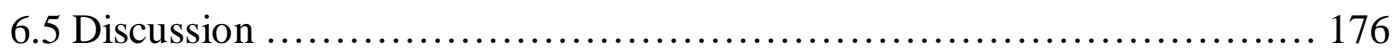

6.5.1 Biological sampling .................................... 176

6.5.2 Diet ........................................................... 178

6.5.3 Rare, uncommon, or under-sampled? ..................... 178

Chapter VII: General Discussion ...................................... 182

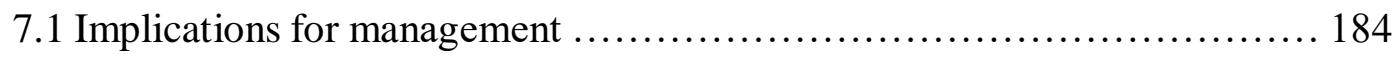

7.2 Continued data collection ............................................ 185

7.3 Future research ........................................................ 187

7.3.1 Ageing ................................................ 187

7.3.2 Identification of regions of importance for life history stages ...... 188

7.3.3 Taxonomy ................................................. 189

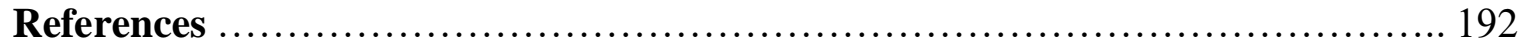

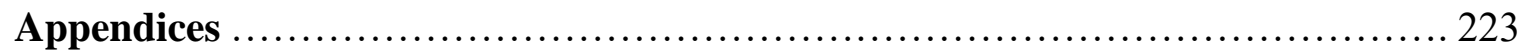




\section{List of Tables}

Table 1. Additional chondrichthyan fishes mentioned in thesis and their status on the IUCN Red List of Threatened Species (IUCN 2016) ........................................ 21

Table 2.1. Summary of mid-water and bottom trawls from Ministry for Primary Industry (MPI) research trawl survey datasets, carried out in different marine regions of New Zealand from 1972 - 2013. Trawls were divided into one of five regions: Southland and Sub-Antarctic (trawls at latitude of $46^{\circ} \mathrm{S}$ or lower, Bagley \& O'Driscoll, 2012; Bagley et al., 2013); Chatham Rise and East Coast South Island (ESCI); West Coast of North and South Island; North Island and Cook Strait; outside the EEZ on Lord Howe Rise and Norfolk Ridge 34

Table 2.2. Summary of mid-water and bottom trawl dataset with the number of trawls, the minimum and maximum mean depth, the number of tows by depth range, and the number of tows by month 35

Table 2.3. Length-at-maturity $(\mathrm{cm})$ by sex and length-at-birth $(\mathrm{cm})$ for selected chondrichthyans (total length, $L_{\mathrm{T}}$, for squaloids; chimaera length, $L_{\mathrm{C}}$, for chimaeroids) .. 36

Table 2.4. Length-weight relationships $[\log (W)=\log (a)+b \times \log (L)]$ for selected deepsea chondrichthyans. * Significant difference between males and females

Table 2.5. For species where a significant difference of LWR was found between males and females, weight estimates (g) for lengths of a 30,50, 70, and $100 \mathrm{~cm}$ fish (total length, $L_{\mathrm{T}}$, for squaloids; chimaera length, $L_{\mathrm{C}}$, for chimaeroids), as well as weight estimates (g) for each sex at mean observed length $(\mathrm{cm})$. Weight estimates for 70 and $100 \mathrm{~cm}$ lengths only given for species that reach that length or greater

Table 2.6. Comparison of weight (g) estimates using LWR parameters from FishBase and this study at mean species length $(\mathrm{cm})$ (total length, $L_{\mathrm{T}}$, for squaloids; chimaera length, $L_{\mathrm{C}}$, for chimaeroids). FishBase LWR estimate accuracy was described as under- ( $\downarrow$ ) or overestimating $(\uparrow)$ fish size relative to estimates in this study, or providing similar results $(<10 \%$ difference) $(-)$. * Significant difference $(P<0 \cdot 05)$. Refer to Table 2.3 for sample sizes 47

Table 2.7. Relative AIC values for the four models (Linear mean \& Constant variance, $\mathrm{L}+\mathrm{CV}$; Broken stick mean \& Constant variance, BS+CV; Linear mean \& Variance shelf, $\mathrm{L}+\mathrm{VS}$; Broken stick mean \& Variance shelf, BS+VS) 51

Table 2.8. Estimates of inflection points, confidence intervals, and significance of the variance $(\alpha)$ and mean $(\beta)$ for each species by the best fitting model as determined by AIC. Additional results in Appendix II

Table 3.1. Length at first maturity $\left(L_{50}\right)$ by sex for selected chondrichthyans from New Zealand literature (total length, $L_{\mathrm{T}}$, for elasmobranchs; chimaera length, $L_{\mathrm{C}}$, for chimaeras) 
Table 3.2. Density of catches ( $n$ per $\mathrm{km}$ ) as a proportion of the total catch recorded, with mean depth $(\mathrm{m})$ across all densities and maximum density (number of individuals $/ \mathrm{km}$ ) .. 92

Table 4.1. Primers used for amplification of $\operatorname{cox} 1$ and $n a d h 2$ mitochondrial genes 109

Table 5.1. Macroscopic criteria used to determine maturity in male and female chimaeroids

Table 5.2. Published and unpublished studies on holocephalan reproduction by species, location, depth, sample size by sex (M: male; F: female), length measurement (SL: standard length; TL: total length; FL: fork length; CL: chimaera length; PCL: precaudal length; BDL: body length; SVL: snout to vent length), length range by sex, measurements to assess maturity by sex $(\mathrm{Cl}$ : clasper length; G: gonad mass; OV: oviducal gland width; U: uterus width; H: histology; S: steroid hormone), length-at-maturity $\left(L_{50}\right), L_{50}$ as proportion of maximum length, age at maturity $\left(A_{50}\right)$, and ovarian fecundity. * refers to those studies that have included more than one length measurement 154

Table 6.1. Digestive tract contents composition from Chimaera carophila and Hydrolagus homonycteris by absolute number $(N)$, frequency of occurrence $(F)$, and weight $(W)$ for prey grouped at taxonomic level. 175

Table 6.2. Research bottom trawl catch records of chimaera species on Chatham Rise from 1978 to 2013 by depth (m) 


\section{List of Figures}

Figure 1.1 Adult female Harriotta raleighana $\left(89 \mathrm{~cm} L_{\mathrm{C}}\right.$, Chatham Rise) ............. 15

Figure 1.2 Adult male Rhinochimaera pacifica $\left(106 \mathrm{~cm} L_{\mathrm{C}}\right.$, Chatham Rise) ............ 16

Figure 1.3 Adult male Chimaera carophila $\left(78 \mathrm{~cm} L_{\mathrm{C}}\right.$, Chatham Rise) ................. 17

Figure 1.4 Adult female Hydrolagus homonycteris ( $86 \mathrm{~cm} L_{\mathrm{C}}$, Chatham Rise) ........... 18

Figure 1.5 Juvenile male Oxynotus bruniensis (44 $\mathrm{cm} L_{\mathrm{T}}$, Chatham Rise) .............. 20

Figure 2.1 Location of research trawl points around New Zealand from 1972-2013, with inclusion of Exclusive Economic Zone (EEZ) and isobaths (400 m, 1000 m, 1500 m) ... 30

Figure 2.2 Variation in form factor $\left(a_{3.0}\right)$ with mean depth $(\mathrm{m})$ by family for (A) chimaeroids (Callorhinchidae, + ; Chimaeridae, $\times$; Rhinochimaeridae, $\circ$ ) and (B) squaloids

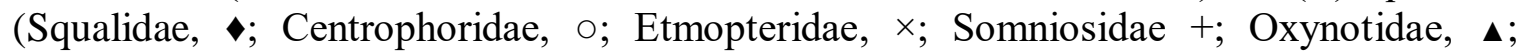
Dalatiidae, $\bullet$ ) ............................................................... 46

Figure 2.3 LWR $\left(W=a L^{b}\right)$ (left panel) and log-transformed LWR $[\log (W)=\log (a)+b \times$ $\log (L)]$ (right panel) for (A) Rhinochimaera pacifica (overestimation from FishBase parameters) (B) Oxynotus bruniensis (underestimation from FishBase parameters) and (C) Hydrolagus trolli (under- and overestimation from FishBase parameters) with fitted LWR from this study (red) and FishBase LWR estimate (blue). See Appendix I for additional plots 48

Figure 2.4 LWR $\left(W=a L^{b}\right)$ (left panel), log-transformed LWR $[\log (W)=\log (a)+b \times \log$ $(L)$ ] (middle panel), and in logspace, the frequency of distribution of weight at mean observed length (right panel), for (A) Hydrolagus bemisi, (B) Squalus acanthias, (C) Centrophorus squamosus, (D) Deania calcea, and (E) Etmopterus granulosus, with fitted LWR from this study (red) and FishBase LWR estimate (blue). Dashed line indicates \pm one standard deviation (S.D.) of weight at mean observed length 49

Figure 2.5 An example of suspected increase in variance of weight at length with increasing length. LWR $\left(W=a L^{b}\right)$ and $\log$-transformed LWR $[\log (W)=\log (a)+b \times \log$ $(L)]$ for combined sex Etmopterus granulosus ( $\uparrow$, indication of variability in weight at length) 50

Figure 2.6 Correlation between length-at-maturity $(\mathrm{cm})$ and variance estimate $(\mathrm{cm})$ by $(A)$ sex (O, male; + , female) and (B) order $(\times$, chimaeroids, $\nabla$, squaloids); and correlation between length-at-maturity $(\mathrm{cm})$ and mean estimate $(\mathrm{cm})$ by $(\mathrm{C})$ sex $(\circ$, male; + , female) and (D) order ( $\times$, chimaeroids, $\nabla$, squaloids). $\vdots, 10 \%$ and $20 \%$ margins of length estimates. Most estimates $(c .75 \%)$ of the variance inflection point were within $20 \%$ of reported length-at-maturity (A, B), and were more accurate for length-at-maturity for females (over males, A) and squaloids (over chimaeroids, B). Approximately half of the estimates of the mean inflection point were beyond a margin of $20 \%$ of reported length-at-maturity (C,D), 
and were more accurate for length-at-maturity for males (over females, C) and chimaeroids (over squaloids, D). .............................................................. 54

Figure 2.7 Linear and best fitting model for female Centroscymnus owstonii (CYO). Top left panel: linear model with constant variance; top right panel: residuals for the best fitting model from a linear model with constant variance (horizontal lines show the mean with \pm 1 standard deviations); middle two panels: same data summarised by boxplots; bottom left panel: the same as top left, with residuals from the linear model, but with the mean and \pm 1 standard deviation superimposed; bottom right: fitted model plotted on top of the original data on the original linear scale. !, inflection point of the variance shelf $(\alpha)$; |, inflection point of the broken stick $(\beta)$

Figure 2.8 Linear and best fitting model for male Squalus acanthias (SPD). Top left panel: linear model with constant variance; top right panel: residuals for the best fitting model from a linear model with constant variance (horizontal lines show the mean with \pm 1 standard deviations); middle two panels: same data summarised by boxplots; bottom left panel: the same as top left, with residuals from the linear model, but with the mean and \pm 1 standard deviation superimposed; bottom right: fitted model plotted on top of the original data on the original linear scale. !, inflection point of the variance shelf $(\alpha)$; |, inflection point of the broken stick $(\beta)$

Figure 2.9 Linear and best fitting model for male Deania calcea (SND). Top left panel: linear model with constant variance; top right panel: residuals for the best fitting model from a linear model with constant variance (horizontal lines show the mean with \pm 1 standard deviations); middle two panels: same data summarised by boxplots; bottom left panel: the same as top left, with residuals from the linear model, but with the mean and \pm 1 standard deviation superimposed; bottom right: fitted model plotted on top of the original data on the original linear scale. |, inflection point of the broken stick ( $\beta$ )

Figure 2.10 Linear and best fitting model for male Etmopterus granulosus (ETB). Top left panel: linear model with constant variance; top right panel: residuals for the best fitting model from a linear model with constant variance (horizontal lines show the mean with \pm 1 standard deviations); middle two panels: same data summarised by boxplots; bottom left panel: the same as top left, with residuals from the linear model, but with the mean and \pm 1 standard deviation superimposed; bottom right: fitted model plotted on top of the original data on the original linear scale. $\vdots$, inflection point of the variance shelf $(\alpha)$

Figure 3.1 Hydrolagus novaezealandiae, by catch density, the proportional class composition ( $\square$ ) and proportional social associations (--- juvenile male; - - - juvenile female; - - - adult male; - - - adult female) of (A) juvenile male, (B) juvenile female, (C) adult male, and (D) adult female. Densities at which social associations were significantly different from that expected by overall catch composition indicated with *. OA $=$ overall proportion of sample for each class 81

Figure 3.2 Squalus acanthias, by catch density, the proportional class composition ( $\square$ ) and proportional social associations (-- juvenile male; - - juvenile female; - - - adult male; - - - adult female) of (A) juvenile male, (B) juvenile female, (C) adult male, and (D) adult female. Densities at which social associations were significantly different from that 
expected by overall catch composition indicated with *. OA $=$ overall proportion of sample for each class

Figure 3.3 Etmopterus lucifer, by catch density, the proportional class composition ( $\square$ ) and proportional social associations (--- juvenile male; -- juvenile female; - - - adult male; - - - adult female) of (A) juvenile male, (B) juvenile female, (C) adult male, and (D) adult female. Densities at which social associations were significantly different from that expected by overall catch composition indicated with *. OA $=$ overall proportion of sample for each class 85

Figure 3.4 Deania calcea, by catch density, the proportional class composition ( $\square$ ) and proportional social associations (--- juvenile male; --- juvenile female; - - - adult male; - - - adult female) of (A) juvenile male, (B) juvenile female, (C) adult male, and (D) adult female. Densities at which social associations were significantly different from that expected by overall catch composition indicated with *. OA $=$ overall proportion of sample for each class

Figure 3.5 Centroselachus crepidater, by catch density, the proportional class composition ( $\square$ ) and proportional social associations (--- juvenile male; --- juvenile female; - - - adult male; - - - adult female) of (A) juvenile male, (B) juvenile female, (C) adult male, and (D) adult female. Densities at which social associations were significantly different from that expected by overall catch composition indicated with $*$. OA = overall proportion of sample for each class

Figure 3.6 Etmopterus granulosus, by catch density, the proportional class composition ( $\square$ ) and proportional social associations (-- juvenile male; --- juvenile female; - - - adult male; - - - adult female) of (A) juvenile male, (B) juvenile female, (C) adult male, and (D) adult female. Densities at which social associations were significantly different from that expected by overall catch composition indicated with $*$. OA $=$ overall proportion of sample for each class 91

Figure 4.1 Distribution of all survey trawls stations ( $\square, n=41644$ ) and catch records of Oxynotus bruniensis $(+, n=464)$ within New Zealand waters ....

Figure 4.2 Depth distribution of Oxynotus bruniensis by (A) catch-record frequency of $O$. bruniensis capture $(\square, n=464)$ and frequency of trawl-survey stations $(\cdots, n=41644)$ and by (B) sex and maturity stage: IM, immature male; MM, mature male; IF, immature female; MF, mature female $(n=112)$. Most catch occurred around the $400 \mathrm{~m}$ depth range

Figure 4.3 Total length $\left(L_{\mathrm{T}}\right)$-frequency distribution for ( $\square$ ) male and ( $\square$ ) female Oxynotus bruniensis $(n=112)$

Figure 4.4 Figure 4.4 Total length $\left(L_{\mathrm{T}}\right)$-mass $\left(M_{\mathrm{T}}\right)$ relationship for Oxynotus bruniensis males $(\circ ; n=48)$ and females $(+; n=64)$ with best fit exponential relationship plotted. ANCOVA results reported below 113

Figure 4.5 Logistic model fitted total length $\left(L_{\mathrm{T}}\right)$-maturity ogive for Oxynotus bruniensis, with the observed proportion of mature $(\circ)$ males and $(+)$ females in each size interval. $\vdots, L_{\mathrm{T}}$ at $50 \%$ mature $\left(L_{50}\right)$ for males $\left(L_{50}=54.7 \mathrm{~cm}\right)$ and females $\left(L_{50}=64 \mathrm{~cm}\right)$. 114 
Figure 4.6 Relationship between total length $\left(L_{\mathrm{T}}\right)$ and (A) gonadosomatic index $\left(I_{\mathrm{G}}\right)$, and (B) proportional inner clasper length $\left(L_{\mathrm{C}}\right)$, for Oxynotus bruniensis based on macroscopic maturity stages: $\circ$, juvenile; + , maturing; $\times$, mature $)$ ! , estimated $L_{\mathrm{T}}$ at $50 \%$ mature $\left(L_{50}=\right.$ $54 \cdot 7 \mathrm{~cm})$

Figure 4.7 Relationship between total length $\left(L_{\mathrm{T}}\right)$ and $(\mathrm{A})$ gonadosomatic index $\left(I_{\mathrm{G}}\right),(\mathrm{B})$ proportional width of oviducal gland, (C) uterus and (D) diameter of largest ovarian follicle for female Oxynotus bruniensis, based on macroscopic maturity stages: ०, juvenile; +, maturing; $\times$, mature; $\bullet$, gravid I; $\bullet$, gravid II; $\diamond$, post-partum. $\vdots$, estimated $L_{\mathrm{T}}$ at $50 \%$ mature $\left(L_{50}=64 \cdot 0 \mathrm{~cm}\right)$ 117

Figure 4.8 Examples of stomach contents from Oxynotus bruniensis including (A) egg case fragments and (B) Harriotta raleighana embryo

Figure 4.9 Neighbour-joining distance (NJ) and maximum-likelihood (ML) tree of cytochrome oxidase subunit 1 ( coxl) haplotypes found from stomach contents $(*)$ and 18 holocephalan species (R., Rhinochimaera spp.; H., Hydrolagus spp.; C., Chimaera spp.), including the Oxynotidae clade (O., Oxynotus spp.) as the out group, based on the K2P model. Estimation of the nodes stability is expressed as a percentage (1000 bootstrap

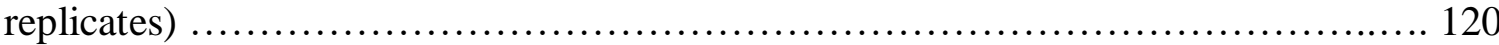

Figure 4.10 Neighbour-joining distance (NJ) and maximum-likelihood (ML) tree of nicotinamide adenine dinucleotide subunit 2 (nadh2) haplotypes found from stomach contents (*) and eight holocephalan species (R., Rhinochimaera spp.; H., Hydrolagus spp.; $C$., Chimaera spp.), including the Oxynotidae clade (O., Oxynotus spp.) as the out group, based on the K2P model. Estimation of the nodes stability is expressed as a percentage (1000 bootstrap replicates) 121

Figure 5.1 (A) Adult female Harriotta raleighana $\left(89 \mathrm{~cm} L_{\mathrm{C}}\right)$ and (B) adult male

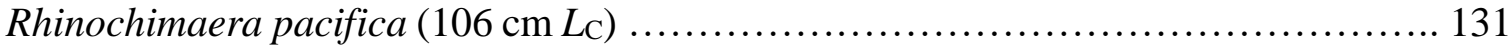

Figure 5.2 Chimaera length $\left(L_{C}\right)$-frequency distribution for ( $\square$ ) female and ( $\square$ )

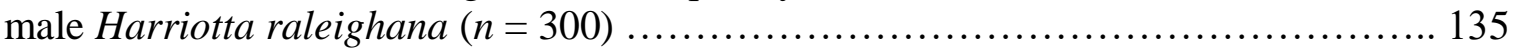

Figure 5.3 Logistic model fitted chimaera length (Lc)-maturity ogive for Harriotta raleighana, with the observed proportion of mature (A) females and (B) males in each size interval. $\vdots, L c$ at $5 \%, 50 \%$ [ $\left(L_{50}\right)$ for females $\left(L_{50}=75.8 \mathrm{~cm}\right)$ and males $\left.\left(L_{50}=62.8 \mathrm{~cm}\right)\right]$, and $95 \%$ maturity determined from fitted logistic curves 136

Figure 5.4 Relationship between chimaera length $(L c)$ and $(\mathrm{A})$ gonadosomatic index $\left(I_{\mathrm{G}}\right)$, (B) proportional width of oviducal gland, (C) uterus and (D) diameter of largest ovarian follicle for female Harriotta raleighana, based on macroscopic maturity stages: $\circ$, juvenile; +, maturing; $\times$, mature; $\bullet$, gravid; $\bullet$, post-laying. $\vdots$, estimated $L_{\mathrm{T}}$ at $50 \%$ mature $\left(L_{50}=75.8 \mathrm{~cm}\right)$ with $5-95 \%$ mature as grey bar ................................. 138

Figure 5.5 Relationship between chimaera length $(L c)$ and $(\mathrm{A})$ gonadosomatic index $\left(I_{\mathrm{G}}\right)$, (B) proportional inner clasper length $\left(L_{\mathrm{CL}}\right),(\mathrm{C})$ testes length, and (D) seminal vesicle width for male Harriotta raleighana based on macroscopic maturity stages: $\odot$, juvenile; +, 
maturing; $\times$, mature). !, estimated $L_{\mathrm{T}}$ at $50 \%$ mature $\left(L_{50}=62.8 \mathrm{~cm}\right)$ with $5-95 \%$ mature as grey bar

Figure 5.6 Chimaera length $\left(L_{C}\right)$-frequency distribution for ( $\square$ ) female and $(\square)$ male Rhinochimaera pacifica $(n=168)$

Figure 5.7 Logistic model fitted chimaera length $(L c)$-maturity ogive for Rhinochimaera pacifica, with the observed proportion of mature (A) females and (B) males in each size interval. $\vdots, L c$ at $5 \%, 50 \%$ [ $\left(L_{50}\right)$ for females $\left(L_{50}=125 \cdot 0 \mathrm{~cm}\right)$ and males $\left.\left(L_{50}=105 \cdot 3 \mathrm{~cm}\right)\right]$, and $95 \%$ maturity determined from fitted logistic curves 142

Figure 5.8 Relationship between chimaera length $(L c)$ and $(\mathrm{A})$ gonadosomatic index $\left(I_{\mathrm{G}}\right)$, (B) proportional width of oviducal gland, (C) uterus and (D) diameter of largest ovarian follicle for female Rhinochimaera pacifica, based on macroscopic maturity stages: $\circ$, juvenile; +, maturing; $\times$, mature; $\bullet$ gravid; $\bullet$, post-laying. !, estimated $L_{\mathrm{T}}$ at $50 \%$ mature $\left(L_{50}=125 \cdot 0 \mathrm{~cm}\right)$ with $5-95 \%$ mature as grey bar

Figure 5.9 Relationship between chimaera length $(L c)$ and $(\mathrm{A})$ gonadosomatic index $\left(I_{\mathrm{G}}\right)$, (B) proportional inner clasper length $\left(L_{\mathrm{CL}}\right),(\mathrm{C})$ testes length, and (D) seminal vesicle width for male Rhinochimaera pacifica based on macroscopic maturity stages: $\odot$, juvenile; + , maturing; $\times$, mature). $\vdots$, estimated $L_{\mathrm{T}}$ at $50 \%$ mature $\left(L_{50}=105.3 \mathrm{~cm}\right)$ with $5-95 \%$ mature as grey bar....

Figure 5.10 (A) Light microscopy image of sperm storage tubules from terminal zone of the oviducal gland of Harriotta raleighana (* indicates sperm bundle), and (B) transmission electron microscopy of a sperm bundles $(*)$.......................... 146

Figure 5.11 Preorbital length $\left(L_{\mathrm{PB}}\right)$ as a function of chimaera length $(L \mathrm{c})$ for $(\mathrm{A})$ Harriotta raleighana and (B) Rhinochimaera pacifica ( $\circ$, female; $\times$, male). The vertical dashed line and bar indicates the macroscopic $L_{50}$ (line) and $95 \%$ C.I. (bar) for male $H$. raleighana. A LOESS (moving average) smoother is plotted for male and female samples (solid line)

Figure 6.1 Adult (A) male Chimaera carophila $(78 \mathrm{~cm} \mathrm{Lc)}$ and (B) female Hydrolagus homonycteris $(86 \mathrm{~cm} \mathrm{Lc})$. Patchy colouration of both specimens due to skin damage from trawl

Figure 6.2 Distribution of deep-sea trawl-survey stations $(\square, n=23245)$ and catch-records of (A) Chimaera carophila $(+, n=781)$ and (B) Hydrolagus homonycteris $(+, n=415)$ within New Zealand waters 165

Figure 6.3 Proportion of survey trawls conducted by depth class $(\cdots, n=23245)$, and proportion of survey trawls that captured (A) Chimaera carophila and (B) Hydrolagus homonycteris by depth class $(\square, n=781$ and $n=415)$; and frequency of trawl survey captures of (C) Chimaera carophila and (D) Hydrolagus homonycteris by sex and maturity stage: $\square$, immature male; $\square$, mature male; $\square$, immature female; $\square$, mature female 166 
Figure 6.4 Chimaera length $(L c)$-frequency distributions by sex $[(\square)$ male and ( $\square$ ) female] for trawl survey specimens of (A) Chimaera carophila $(n=183)$ and (B) Hydrolagus homonycteris $(n=79)$

Figure 6.5 Logistic model fitted total length (Lc)-maturity ogive for (A) Chimaera carophila and (B) Hydrolagus homonycteris, with the observed proportion of mature (O) males and $(\times)$ females in each size interval. $\vdots, L c$ at $50 \%\left(L_{50}\right)$ determined from fitted logistic curves

Figure 6.6 Relationship between chimaera length $(L c)$ and proportional inner clasper length $\left(L_{\mathrm{CL}}\right)$, for $(\mathrm{A})$ Chimaera carophila and (B) Hydrolagus homonycteris, based on macroscopic maturity stages: $\bigcirc$, juvenile; +, maturing; $\times$, mature). !, estimated $L c$ at $50 \%$ mature $\left(L_{50}=72.5 \mathrm{~cm}[\mathrm{~A}]\right.$ and $\left.79 \cdot 1 \mathrm{~cm}[\mathrm{~B}]\right)$

Figure 6.7 Relationship between chimaera length $(L c)$ and $(A)$ proportional width of oviducal gland, (B) uterus for female Chimaera carophila, and relationship between chimaera length $(L c)$ and $(C)$ proportional width of oviducal gland, (D) uterus for female Hydrolagus homonycteris based on macroscopic maturity stages: $\odot$, juvenile; + , maturing; $\times$, mature; $\diamond$, gravid I; $\bullet$, gravid II; $\diamond$, post-partum. $\vdots$, estimated $L c$ at $50 \%$ mature $\left(L_{50}=\right.$ $82.7 \mathrm{~cm} L \mathrm{c}$ and $80 \cdot 1 \mathrm{~cm} L \mathrm{c}$ ) 173

Figure 6.8 Light microscopy image of sperm storage tubules (SST) from terminal zone of the oviducal gland of Chimaera carophila $(*$ indicates sperm bundle) 


\section{Contributions and Publication Status of Dissertation Chapters}

\section{Chapter I: General Introduction}

Editing by M. Dunn and E. Jones.

Chapter II: Predicting size-at-maturity in fishes from their weight-at-length

Model described in Section 2.3.4 created by R.A. Arnold (Victoria University) and M.R. Dunn (NIWA). Study design and data analysis by B. Finucci and M. Dunn. Editing by M. Dunn and E. Jones.

Chapter III: Social associations in deep-sea chondrichthyans

Study design and data analysis by B. Finucci and M. Dunn. Editing by M. Dunn and E. Jones.

Chapter IV: Reproductive biology and feeding habits of the prickly dogfish Oxynotus bruniensis (Ogilby 1893)

Study design by B. Finucci. Analysis of DNA from diet samples (Section 4.3.3) by C. Bustamante (University of Queensland). Editing by M. Dunn and E. Jones.

Published as: Finucci, B., Bustamante, C., Jones, E.G. \& Dunn, M.R. (2016). Reproductive biology and feeding habits of the prickly dogfish Oxynotus bruniensis. Journal of Fish Biology 89, 2326-2344. DOI: 10.1111/jfb.13116.

Chapter V: Reproductive biology of two deep-sea chimaeras, longnose spookfish (Harriotta raleighana), and Pacific spookfish (Rhinochimaera pacifica)

Study design by B. Finucci. Preparation of histology slides for light microscopy and transmission electron microscopy (Section 5.3.3) by J. Anderson (Massey University). Editing by M. Dunn and E. Jones.

Published as: Finucci, B., Dunn, M.R., Jones, E.G. \& Anderson, J. (2016). Reproductive biology of the two deep-sea chimaerids, longnose spookfish (Harriotta raleighana) and Pacific spookfish (Rhinochimaera pacifica). Deep Sea Research Part I: Oceanographic Research Papers. Deep Sea Research Part I, DOI: 10.1016/j.dsr.2016.11.008.

Chapter VI: First observations of the biology of two rarely observed deep-sea chimaeras, Chimaera carophila and Hydrolagus homonycteris

Study design by B. Finucci. Diet analysis (Section 6.3.3) completed with D. Stevens (NIWA). Editing by M. Dunn and E. Jones.

Published as: Finucci, B., Stevens, D.W., Jones, E.G., \& Dunn, M.R. (2017). Some observations on the biology of two rarely seen deep-sea chimaerids, Chimaera carophila and Hydrolagus homonycteris. Journal of Fish Biology 90, 2020-2040. DOI: 10.1111/jfb.13284.

\section{Chapter VII: General Discussion}

Editing by M. Dunn and E. Jones. 



\section{CHAPTER I}

General Introduction 


\subsection{Introduction}

The class Chondrichthyes (cartilaginous fishes) includes around 1200 species of sharks, rays, and chimaeras. Presently, there are over 500 described species of true sharks (orders Carcharhiniformes, Heterodontiformes, Echinorhiniformes, Hexanchiformes, Lamniformes, Orectolobiformes, Pristiophoriformes, Squaliformes, Squatiniformes), 630 batoids (orders Myliobatiformes, Torpediniformes, Rajiformes, and Rhinopristiformes), and 49 chimaeroids (order Chimaeriformes) (Weigmann, 2016; Last et al., 2016). Of all known chondrichthyans, nearly half are classified as deep-sea, in which the majority of their lifecycle is spent at depths beyond $200 \mathrm{~m}$ (Kyne \& Simpfendorfer, 2007).

In New Zealand (NZ), currently 117 species of chondrichthyans (Roberts et al., 2015), representing 31 families, have been identified from within the Economic Exclusive Zone (EEZ), many of which are deep-sea. This large diversity of cartilaginous fish species ( $10 \%$ of the global fauna) continues to increase with improved species identification, and taxonomic resolution. Between 2008 and 2011 alone, four new species were described from NZ waters, all of which were considered deep-sea and rare (Francis \& Lyon, 2012), and included one species of chimaerid, the black ghost shark Hydrolagus homonycteris Didier $2008^{1}$, the roughskin catshark Apristurus ampliceps Sasahara, Sato \& Nakaya 2008, the sapphire skate Notoraja sapphira Séret \& Last 2009, and the blueeye lantern shark Etmopterus viator Straube 2011. The latest addition was the description of the brown chimaera Chimaera carophila Kemper, Ebert, Naylor \& Didier 2014 in November 2014 (Kemper et al., 2014).

There is growing global conservation concern as many chondrichthyan populations continue to decline (Dulvy et al., 2014). Chondrichthyans have been well documented for life history traits which result in low productivity (slow growth, high longevity, late maturation, low fecundity) which increases their vulnerability to fishing pressure and population depletion (Cortés, 2000; Dulvy et al., 2014). These traits have been found to be more pronounced in deep-sea species (García et al., 2008; Simpfendorfer \& Kyne, 2009; Rigby \& Simpfendorfer, 2015). Many deep-sea chondrichthyans are poorly known taxonomically and their populations remain data deficient, largely due to their historically low economic value, and thus, low prioritization

\footnotetext{
${ }^{1}$ Species nomenclature and discription authority follows Eschmeyer et al. (2016).
} 
for research efforts and targeted sampling (Francis, 1998; Kyne \& Simpfendorfer, 2010; Holt et al., 2013; Cotton \& Grubbs, 2015).

The inaccessibility of deep-sea chondrichthyans to most fisheries has allowed these species to generally be considered less threatened than their shallow water relatives (Dulvy et al., 2014). Notwithstanding, as inshore fisheries become overexploited, the expansion and commercial importance of deep-sea fisheries have increased on a global scale (Morato et al., 2006b; Watson \& Morato, 2013; Clark, 2016). Deep-sea fisheries already reach the maximum depths attained by most chondrichthyans (Priede et al., 2006; Norse et al., 2012), resulting in significant chondrichthyan bycatch (Musick et al., 2000; Graham \& Daley, 2011; Moore et al., 2013). Some deep-sea chondrichthyans have already shown significant population declines, which in turn have resulted in the implementation of zero allowable landed catch regulations for species in certain regions (Graham et al., 2001; ICES, 2009; Neat et al., 2015).

\subsection{Deep-Sea Fisheries and Chondrichthyan Catches in New Zealand}

Deep-sea fisheries were not established in New Zealand until the creation of the Exclusive Economic Zone (EEZ) in 1978 (Mace et al., 2013). Pre-1980s, most New Zealand fisheries were restricted to small inshore domestic fleets, and fishing beyond the 12 nautical mile Territorial Sea was dominated by foreign fleets from Japan, Korea, and the Soviet Union (Clark et al., 1988). In 1986, the Quota Management System (QMS) was introduced, designed as a single-species management system with the objective to maximize economic value of New Zealand fisheries, while ensuring sustainability (Clark et al., 1988). Individual transferable quotas (ITQs) were established to represent shares of Total Allowable Commercial Catches (TACCs) for each stock to incentivize accurate catch reporting and decrease discarding and under-reporting (Clark et al., 1988; Mace et al., 2013). Three chondrichthyans, rig Mustelus lenticulatus Phillipps, 1932, elephantfish Callorhinchus milii, Bory de St Vincent, 1823, and school shark Galeorhinus galeus (L. 1758), were included from the introduction of the QMS (Francis, 1998). The first deepsea chondrichthyan, dark ghost shark Hydrolagus novaezealandiae (Fowler, 1911), was not introduced until 1998-99 (MacGibbon, 2016), with pale ghost shark Hydrolagus bemisi Didier, 2002, following in the subsequent year (MacGibbon \& Fu, 2013).

TACCs make up a large proportion of the overall Total Allowable Catch (TAC), which also includes allowances for recreational fisheries, customary fisheries, and other 
fishing-related mortality (Mace et al., 2013). Recreational and customary catches are considered to be negligible for most QMS species (e.g. MacGibbon, 2016), while other fishing-related mortality, specifically post-release mortality, is largely unknown (Clarke et al., 2013). TACCs were originally determined by the basis of catch histories (Clark et $a l ., 1988)$, and are routinely re-evaluated with the objective of ensuring catches allow stocks to support utilization at the maximum sustainable level. To date, only one chondrichthyan stock (M. lenticulatus) has had a full quantitative stock assessment, which are dependent on specific input data (relative abundance index, catch histories, catch composition, biological parameters such as growth, natural mortality, and fecundity) unavailable for most chondrichthyans (Francis \& Lyon, 2012). Without this information, TACCs have been based on absolute biomass estimates derived from trawl surveys, or as a proportion of recent landings (Francis, 1988).

At present, there are approximately 80 species of chondrichthyans caught by commercial vessels in New Zealand (Francis, 2015). In 2011-12 (from 1 October to 30 September), the deep-sea fisheries were responsible for a third of total annual shark catches (MPI, 2013). There is very little, if any, targeted fishing for deep-sea chondrichthyans in New Zealand waters (Ford et al., 2015). Most deep-sea chondrichthyan catch occurs in the trawl fisheries targeting hoki Macruronus novaezelandiae Hector, 1871, orange roughy Hoplostethus atlanticus Collett, 1889, and oreos (Family Oreosomatidae). Smaller catches are also recorded in fisheries for arrow squid (Nototodarus spp), southern blue whiting Micromesistius australis Norman, 1937, jack mackerel (Trachurus spp), scampi Metanephrops challengeri (Balss, 1914), and ling Genypterus blacodes (Forster, 1801) (Blackwell, 2010; Ballara, 2015). Additional catch is recorded from commercial surface and bottom longlining (Francis, 2015).

Chondrichthyans are generally dead when hauled on deck, and are either processed for fins, liver, or fishmeal, or discarded altogether (Ministry of Fisheries, 2008). Approximately $45 \%$ of non-QMS deep-sea chondrichthyan catch is fully utilized (MPI, 2013).

Deep-sea chondrichthyan catches within the New Zealand EEZ have been primarily comprised of a handful of species. Hydrolagus novaezealandiae and $H$. bemisi have been caught, and continue to be caught, in such large numbers as bycatch that they were added into the QMS in 1998-99 (MacGibbon \& Fu, 2013). The longnose spookfish Harriotta raleighana Goode \& Bean, 1895, is one of the most common bycatch species not managed by the QMS (MPI, 2015). Eight sharks not managed by the QMS are also 
commonly observed: shovelnose dogfish Deania calcea (Lowe, 1839), longnose velvet dogfish Centroscymnus crepidater (Barbosa du Bocage \& de Brito Capello, 1864), leafscale gulper shark Centrophorus squamosus (Bonnaterre, 1788), Baxter's dogfish Etmopterus granulosus (Günther, 1880), Owston's dogfish Centroscymnus owstonii Garman, 1906, Lucifer's dogfish Etmopterus lucifer Jordan \& Snyder, 1902, Plunket's shark Centroscymnus plunketi (Waite, 1909) and seal shark Dalatias licha (Bonnaterre, 1788) (Blackwell, 2010).

Catches of deep-sea chondrichthyans increased in the early 1990s, peaked in the 2000s, and have since declined (Francis, 1998; Ministry of Fisheries, 2008). Over 1000 tonnes of non-QMS chondrichthyan bycatch was landed by the deep-sea fishing fleet in 2014/15 (MPI, 2016b), although this amount is probably underestimated due to discarding and misreporting (Parker \& Francis, 2012). Worldwide, shark catches are often unregulated, misidentified, unrecorded, aggregated, or discarded at sea, resulting in a lack of species-specific landings information (Bornatowski et al., 2013; Dulvy et al., 2014). In New Zealand, the inability to distinguish deep-sea species has been a prolonged problem, resulting in the use of aggregate species ("generic") codes for catch records by the commercial industry and the Ministry for Primary Industries Observer Program (Francis, 1998; Francis \& Lyon, 2012). Data lumped into generic codes cannot be used to determine trends in species biomass (Francis, 1998; Ford et al., 2015). A total of 15 generic chondrichthyan codes have been used for catch that cannot be identified to the species level: two for chimaerids (Chimaeras, CHI and Ghost sharks, HYD), seven for sharks (Deepwater Dogfish, DWD; Other Sharks \& Dogfish, OSD; Deepsea Sharks, CEN; Sharks, SHA; Lantern sharks, ETM; Catsharks, CSH; Whaler sharks, RSH), and six for batoids (Deepsea skates, BTH; Other skates, OSK; Rays, RAY; Skates, SKA; Stingrays, STR; and Blind electric rays, BER) (Francis, 2015). Both DWD and OSD have seen a substantial decline in use in recent years, coinciding with the release of updated species identification guidebooks (Francis \& Lyon, 2012; MPI, 2016b).

\subsection{Shark Fisheries and Bycatch Management in New Zealand}

All commercial and non-commercial fishing in New Zealand is managed under the Fisheries Act 1996, encompassing all chondrichthyan catches, both targeted and bycatch (Clark et al., 1988; Ministry of Fisheries, 2008). Specific chondrichthyan conservation and management is guided under the National Plan of Action for Sharks 
(NPOA-Sharks) (Ministry of Fisheries, 2008). Developed by the Ministry of Fisheries (now Ministry for Primary Industries, MPI), the New Zealand NPOA-Sharks was first released in 2008 and reviewed in 2013 (MPI, 2013). In accordance with the objectives of the Food and Agriculture (FAO) International Plan of Action-Sharks (IPOA-Sharks), the NPOA-Sharks was created with the goal of ensuring "the conservation and management of sharks and their long-term sustainable use" (FAO, http://www.fao.org/ipoasharks/background/about-ipoa-sharks/en/). Similarly, the purpose of the New Zealand Fisheries Act 1996 is to "provide for the utilisation of fisheries resources while ensuring sustainability" (Fisheries Act 1996, http://www.legislation.govt.nz/act/public/1996/0088/152.0/whole.html\#whole).

There are three categories of management for New Zealand chondrichthyans (MPI, 2013). The first is "protected species", which includes chondrichthyans that are not managed under the QMS and have been identified as unable to withstand any utilization (Ministry of Fisheries, 2008; MPI, 2013). These species are protected under the Wildlife Act 1953, which applies to the New Zealand EEZ, or the Fisheries Act 1996, applying to New Zealand flagged fishing vessels and nationals on the High Seas (outside the EEZ), or both. Catches of these species cannot be retained by law, and must be reported. New Zealand has seven protected species, white shark Carcharodon carcharias (L. 1758), basking shark Cetorhinus maximus (Gunnerus, 1765), whale shark Rhincodon typus Smith, 1828, oceanic whitetip Carcharhinus longimanus (Poey, 1861), deep-water nurse shark Odontaspis ferox (Risso, 1810), giant manta ray Manta birostris (Walbaum, 1792), and spine-tailed devil ray Mobula japanica (Müller \& Henle, 1841) (MPI, 2013). Two additional species, smooth hammerhead Sphyrna zygaena (L. 1758) and sharpnose sevengill shark Heptranchias perlo (Bonnaterre, 1788), are protected under Schedule 4C of the Fisheries Act 1996, in which there are no permits to target these species and they may only be taken as bycatch (Ministry of Fisheries, 2008).

The majority (90\%, 11 species) of commercial chondrichthyan catches in New Zealand are in the second category, which are species managed under the Quota Management System (QMS) (MPI, 2013). These include spiny dogfish Squalus acanthias L. 1758, G. galeus, C. milii, M. lenticulatus, shortfin mako Isurus oxyrinchus Rafinesque 1810, porbeagle Lamna nasus (Bonnaterre, 1788), blue shark Prionace glauca (L. 1758), H. novaezealandiae, H. bemisi, smooth skate Dipturus innominatus (Garrick \& Paul, 1974), and rough skate Zearaja nasuta (Müller \& Henle, 1841). All QMS species must be landed, with the exception of those species listed under Schedule 6 of the Fisheries Act 
(S. acanthias, P. glauca, I. oxyrinchus, L. nasus, M. lenticulatus, G. galeus, D.

innominatus, Z. nasuta). Under the provisions of Schedule 6, species may be returned to the sea if they are likely to survive upon return, and that the return takes place as soon as possible. Exceptions to this condition under Schedule 6 apply to S. acanthias and highly migratory species (P. glauca, I. oxyrinchus, L. nasus), which allows fishers to discard these animals alive, dead, or near-dead.

The remaining chondrichthyan species are listed in a third category, as "open access". These are species, including most deep-sea chondrichthyans, in which there is negligible economic value, and little mitigation in place to protect these species or to reduce catches (MPI, 2013). There are no TACCs for species in this category and no requirement to report catches (MPI, 2016a). With limited budgets for fisheries research, priority is given to monitor and assess high value fish stocks, while species deemed to have little or no commercial value, receive very little, if any, research attention (Mace $e t$ $a l ., 2013)$. Even the need for basic biological information is often overlooked (Francis \& Lyon, 2012; Ford et al., 2015; Simpfendorfer \& Kyne, 2015). A review of published and unpublished literature on research and monitoring studies on cartilaginous fishes in New Zealand since the release of the NPOA in 2008 found that $30 \%$ of known species failed to be mentioned in any study (Francis \& Lyon, 2012). With limited recorded data on demographics, abundance, distribution, and catch history, it is not known how fishing pressures are affecting these populations (Ministry of Fisheries, 2008; Francis \& Lyon, 2012; Ford et al., 2015).

\subsection{Ecological Risk Assessment for the Effects of Fishing (ERAEF)}

In the absence of this information, it can be very difficult to manage category 3 (open access) species and determine where research efforts should be directed. Ecological risk assessments have been used to help inform the management of these species. In recent years, the use of Ecological Risk Assessments for the Effects of Fishing (ERAEF; also referred to as Ecological Risk Assessment, ERA) have become an increasingly popular scientific tool in the developing approach of ecosystem-based fisheries management (EBFM) (see Hobday et al., 2007; Gallagher et al., 2012; Ford et al., 2015) as a means of understanding the wider ecological impacts of fishing (Cortés et al., 2010). ERAs are often applied for assisting management bodies with the identification of stocks that are most vulnerable to overfishing (Cortés et al., 2010). Additionally, ERAs can be 
used to prioritize research efforts by focusing on data poor species with high susceptibility (Braccini et al., 2006). The flexible nature of an ERA, which can be undertaken at different levels depending on data availability, has been commonly applied to bycatch species where biological and fishery information are often sparse (Stobutzki et al., 2002; Ford et al., 2015). ERAs have been widely adopted within federally managed fisheries in Australia (Hobday et al., 2007), and adapted for international application, including institutions such as the Marine Stewardship Council (MSC) (Ponte, 2012), the International Commission for the Conservation of Atlantic Tuna (ICCAT) (ICCAT, 2008; Cortés et al., 2015), and the Western Central Pacific Fisheries Commission (WCPFC) (Kirby \& Molony, 2006).

In 2014, a qualitative (Level 1) risk assessment was conducted for all New Zealand chondrichthyans to assess risk from commercial fishing, with the intention to inform management and assist prioritizing action (Ford et al., 2015). The objective of a Level 1 risk assessment is to identify which risks can lead to significant impact on a species, habitat, or community, as well as identify knowledge gaps (Hobday et al., 2007). Non-fishing threats (e.g. noise pollution, electromagnetic fields, loss of habitat, eutrophication, alteration of behaviour by ecotourism) were not included in the risk assessment, as a review concluded information on non-fishing threats were scarce and a less imminent threat to shark populations than commercial fishing (Francis \& Lyon, 2013). A modified Scale Intensity Consequence Analysis (SICA, Smith et al., 2007) approach was used for the risk assessment because it was considered most relevant for commercial fishing and outlined clear descriptions of fishing intensity parameters (Ford et al., 2015). SICA is generally used as a rapid screening tool, applying a "plausible worst case" approach to risk evaluation to measure the total level of impact from activity (in this case, intensity of fishing activity) and its ecological consequence. SICA requires available information on the activity (spatial and temporal scale of fishing), and the biology of species evaluated (population size, geographic range, genetic structure, age/size/sex structure, reproductive capacity, behaviour/movement) (Smith et al., 2007; Hobday et al., 2007). Risk was considered on a national (EEZ-wide) scale, and an expert panel scored risk to each taxon from commercial fishing based on biological productivity and distribution overlap with fisheries. Intensity and consequence of the fishery to the shark taxa was scored on a scale of 1 to 6 ( 1 being low, and 6 being high), and then multiplied together for a total risk score (maximum possible score of 36; a high score 
denoting high risk). Species that were very infrequently seen, or poorly taxonomically described, were not assessed at this time.

Of the 84 taxa evaluated, only 14 species were judged to have existing data that were sound for risk assessment purposes. Most species (88\%) were labelled with data that "exist but poor" (37) or "few data" (37), resulting in low confidence in the risk scores for 43 taxa, many of which were deep-sea. The deep-sea sharks were often given a consequence score of four (actual, or potential for, unsustainable impact), which was based on either their known, or assumed, low productivity (Simpfendorfer \& Kyne, 2009). The highest consequence scores (all 4.5) were all applied to deep-sea non-QMS species and included Centroscymnus crepidater, Centroscymnus plunketi, and Dawson's catshark Bythaelurus dawsoni (Springer, 1971). Several species were given the highest intensity score (6); half of these species were deep-sea, and with the exception of carpet shark Cephaloscyllium isabellum (Bonnaterre, 1788), were all managed under the QMS. Five species, all of which were deep-sea, were scored with a total risk of 20 or higher (21, C. isabellum; 21, Zearaja nasuta; 20, Dipturus innominatus; 20, Etmopterus granulosus; 20, Dalatias licha).

The outcomes of this risk assessment suggested that, despite fisheries frequently catching sharks across a wide proportion of their range, commercial fishing was not currently causing substantial unsustainable impacts to New Zealand chondrichthyans (Ford et al., 2015). However, from this exercise, it was evident that most New Zealand chondrichthyans were in need of greater data collection, and more research was recommended for species identification (taxonomy), updated distribution ranges, and species biology for estimates of population parameters and abundance indices.

\subsection{The Importance of Life History}

As of 2016, of those species which have been assessed, $30 \%$ of chondrichthyans in New Zealand waters are classified as data deficient on an international level because of a lack of biological knowledge (www.iucnredlist.org/). Studies on lesser known or noncommercial species contribute to our overall knowledge of chondrichthyan ecology (Simpfendorfer \& Wetherbee, 2015). In recent years, however, chondrichthyan life history research has declined (Simpfendorfer et al., 2011). Heightened species threat levels (Dulvy et al., 2014) and public concern for shark conservation has increased 
pressure for more favourable non-lethal scientific sampling, which can impede some life history studies (Heupel \& Simpfendorfer, 2010; Simpfendorfer et al., 2011).

Understanding life history parameters, including age, growth, and reproduction, is essential for providing baseline information on population dynamics, and assessing a species' susceptibility to population depletion (Cortés, 2004). These parameters have been used to provide trait comparisons among taxonomic groups, evaluating interspecific, intraspecific and intrapopulation variation, and have since established many fundamental correlations between the effect of body size and life history traits in sharks (Cortés, 2000; Frisk et al., 2001). For example, across taxonomic groups, both males and females reach maturity at an average of $75 \%$ of their maximum size (Cortés, 2000), with females generally mature at a larger size and older age than males (e.g. Hancett, 1988; Horn, 1997; Parker \& Francis, 2012). This has been attributed to the large female size required to carry pups, and decreased energy partitioning allotted for somatic growth in favour of reproduction, thus delaying the onset of sexual maturity in females (Springer, 1967; Hoenig \& Gruber, 1990; Cortés, 2000). Additionally, a strong positive correlation between size-at-maturity and maximum size in both sexes has been noted (Cortés, 2000; Frisk et al., 2001), while interspecific maternal length has been found to be positively correlated with litter size and offspring size, which may increase pup survival through greater maternal investment (e.g. Hussey et al., 2010). Such findings form the context for interpretation of many studies pertaining to genetics and behaviour (Simpfendorfer \& Wetherbee, 2015), as well as extinction risk (e.g. Dulvy et al., 2008; Rigby \& Simpfendorfer, 2015).

For marine fishes, including elasmobranchs, body size can indicate a species' vulnerability to exploitation (Reynolds et al., 2005). Larger species have been characterised with low productivity (slow somatic growth, low reproductive output, low maximum population growth rate), and even small levels of fishing mortality can drive local extinctions (e.g. Pardo et al., 2016). In the absence of information, declines in susceptible chondrichthyan populations can occur very rapidly, and can often go undetected until the damage is irreversible. The rapid expansion and collapse of several shark fisheries have been documented in many regions of the world, including the Maldives (Kyne \& Simpendorfer, 2007), India (Akhilesh, 2014), the Northeast Atlantic (ICES, 2009), and Australia (Graham et al. 2001). After only 20 years of targeted fishing, the gulper shark (Centrophorus spp.) stock collapsed off the Maldives in the early 2000s, while over a 20 year period (1976/77-1996/97), substantial declines (up to 90\%) of 
relative abundance of deepwater chondrichthyans in the Australian South East Fishery trawl grounds were reported (Graham et al. 2001). Knowledge of life history dynamics is required for understanding a species' ability to withstand exploitation, recover following overfishing, and is used in stock assessments and ecological risk assessments (Cortés, 2007; Francis \& Lyon, 2012), all of which are essential for effective conservation and ecosystem-based management (Cotton \& Grubbs, 2015). 


\subsection{Study Species}

The following species, available from research trawl survey catches, were selected for this thesis because they are considered data deficient. There is a lack of available data on length-weight relationships, growth, age, longevity, maturity, fecundity, reproductive periodicity and seasonality, spatial areas of importance for specific ages/size classes (e.g. egg-laying grounds, breeding grounds, nursery grounds), diet, behaviour, migration patterns, stocks, recruitment, and population size, structure, and connectivity.

\subsubsection{Chimaeras (Chondrichthyes: Holocephali)}

Extant holocephalans and their closest fossil relatives belong to the Order Chimaeriformes. Commonly referred to as chimaeroid fishes, chimaeras, ghost sharks, rabbitfish, ratfish, or spookfish, holocephalans differ from other chondrichthyan fishes on account of their unique form of holostyly (upper jaw is fused to the neurocranium), as well as a reduced number of tooth elements in exchange for non-replaceable, hypermineralized tooth plates (Didier et al., 2012).

Chimaeriformes is comprised of three families, distinguishable by their snout morphology. The first, Callorhinchidae (plow-nosed chimaeroids), consists of three species in one genera (Callorhinchus), all of which are recorded from the southern hemisphere. One species, Callorhinchus milii, is found in New Zealand waters. The second family, Rhinochimaeridae (long-nosed chimaeroids), is globally distributed, consisting of eight species across three genera (Harriotta, Neoharriotta, and Rhinochimaera), and are distinguished from other chimaeroids by having long tapered snouts. Three species are known from New Zealand waters, the small spine spookfish Harriotta haeckeli Karrer, 1972, Harriotta raleighana, and Rhinochimaera pacifica. Chimaeridae (short-nosed chimaeroids) is the third and most specious family, with 38 species across two genera (Chimaera and Hydrolagus). There are eight recognized species in New Zealand, giant black ghost shark Hydrolagus $c f$. affinis (de Brito Capello, 1868), leopard chimaera Chimaera panthera Didier, 1998, Chimaera carophila, giant chimaera Chimaera lignaria Didier, 2002, Hydrolagus novaezealandiae, Hydrolagus homonycteris, Hydrolagus bemisi, and pointynose blue ghost shark Hydrolagus trolli Didier \& Séret, 2002 (Roberts et al., 2015). 
With the exception of members of Callorhinchidae, all known holocephalans are classified as benthic, deep-sea species (Kyne \& Simpfendorfer, 2007; Cotton \& Grubbs, 2015), and include some of the deepest recorded chondrichthyans (Preide et al., 2006). Chimaeroids are widespread geographically, but most species are known to have a rather restricted range and a high degree of endemism (Didier et al., 2012). At least three chimaeroids (C. carophila, $H$. novaezealandiae, $H$. bemisi) are endemic to the New Zealand region (Roberts et al., 2015).

1.6.2 Longnose spookfish Harriotta raleighana Goode \& Bean, 1895

Harriotta raleighana (Fig. 1.1) was confirmed in New Zealand waters in 1967, becoming the third holocephalan to be reported in New Zealand (after C. milii and $H$. novaezealandiae), and the first recording of a rhinochimaerid (Garrick, 1971). First described from the North Atlantic in 1895, H. raleighana is a globally reported species with a patchy distribution on the continental slope and the ocean floor across the Atlantic, the north Pacific, and in the south Pacific off Australia and New Zealand (Last \& Stevens, 2009). It is the only chimaeroid species with a known global distribution (Didier et al. 2012). Within New Zealand, H. raleighana is widespread, from the Norfolk Ridge to the Campbell Plateau, at depths as shallow as $200 \mathrm{~m}$ (Last \& Stevens, 2009), but is most commonly found at depths of 500-1500 m (Roberts et al., 2015).

Harriotta raleighana can grow to a length of $120 \mathrm{~cm}$ chimaera length $\left(L_{\mathrm{C}}\right.$, tip of the snout to the posterior edge of the supracaudal fin, excluding the caudal filament), although it has not been documented larger than $90 \mathrm{~cm} L_{\mathrm{C}}$ in New Zealand (Roberts et al., 2015). Like all other chimaeroids, it is oviparous. Cox \& Francis (1999) report estimated size-at-birth between 10 and $13 \mathrm{~cm} L_{\mathrm{C}}$. Size-at-maturity is thought to occur between 25 and $30 \mathrm{~cm}$ body length ( $L_{\mathrm{BD}}$, dorsal edge of gill opening to origin of upper caudal fin) for males and $35 \mathrm{~cm} L_{\mathrm{BD}}$ for females (D.D. Didier, Millersville University, pers. comm.). In the North Atlantic, size and sexual segregation has been noted in sub-adults and adults, with juveniles being more common in shallower waters and females in deeper water (Holt et al., 2013). The diet of $H$. raleighana has been examined in both the Pacific and Atlantic populations, and consisted primarily of benthic invertebrates such as polychaetes, crustaceans, gastropods and amphipods (Mauchline \& Gordon, 1983; Dunn et al., 2010a). The elongated snout of $H$. raleighana is thought to be used as a sensory probe to locate prey in the sediment (Mauchline \& Gordon, 1983). 
There are no target fisheries for H. raleighana, however, it is regularly caught as bycatch in many trawl and line fisheries (Jacob et al., 1998; Allain et al., 2003; Ballara, 2015). Over 100 tonnes were landed in New Zealand by deep-sea fleets in the 2014/15 fishing season (MPI, 2016b). Historically, the majority of catches were landed and processed as fish meal (Ministry of Fisheries, 2008). Its widespread circumglobal distribution and apparent abundance where it is known to occur has warranted $H$. raleighana to be listed as Least Concern on the International Union for Conservation of Nature (IUCN) Red List of Threatened Species (Dagit et al., 2016).

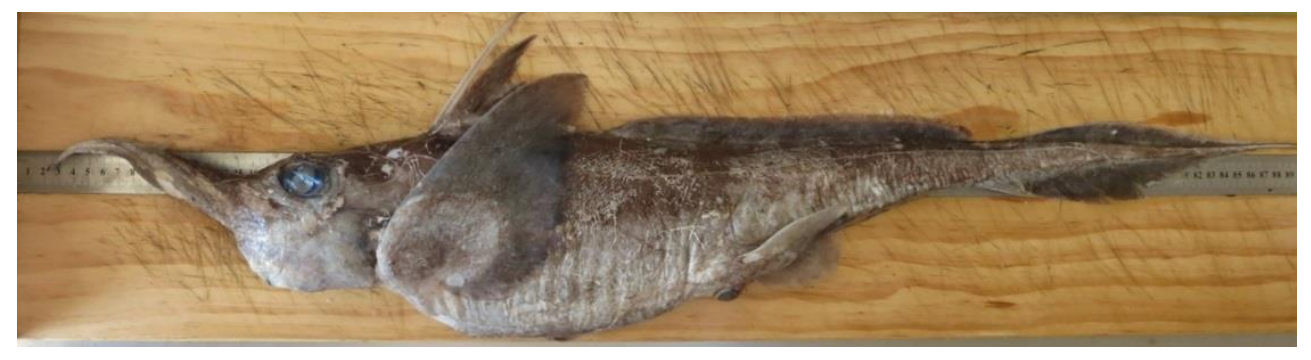

Figure 1.1 Adult female Harriotta raleighana ( $89 \mathrm{~cm} L_{\mathrm{C}}$, Chatham Rise).

\subsubsection{Pacific spookfish Rhinochimaera pacifica (Mitsukuri, 1895)}

Rhinochimaera pacifica (Fig. 1.2), first described in Japan in 1895 as Harriotta pacifica Mitsukuri, 1895, was later moved to the genus Rhinochimaera because of its smooth dental plates (Garman, 1901). Rhinochimaera pacifica has a patchy distribution in the Pacific and Indian Oceans (Didier \& Nakaya, 1999), recorded across the Japanese Archipelago to the South China Sea (Shao \& Hwang, 1997; Shinohara et al., 2009), Australia (Last \& Stevens, 2009), and eastern Pacific off Peru (Chirichigno, 1974). It was first recorded in New Zealand in 1968, when two specimens were found on separate trawls on Chatham Rise by RV Kaiyo Maru (Inada \& Garrick, 1979). Since then, $R$. pacifica has been found more widely distributed along the continental shelf (Francis, 1979; Didier \& Nakaya, 1999). In Southland and the sub-Antarctic region of New Zealand, it is caught in hoki (Macruronus novaezelandiae) and other middle-depth (200$800 \mathrm{~m}$ ) species trawl surveys (Bagley et al., 2013). Rhinochimaera pacifica occurs sympatrically with $H$. raleighana, and is most commonly at depths below $700 \mathrm{~m}$ (1911290 m) (Roberts et al., 2015). 
Very little is known about $R$. pacifica. It is defined by its prominent conical snout that can measure anywhere from $50 \%$ to $87 \%$ of its body length (Didier \& Nakaya, 1999). Rhinochimaera pacifica can reach a maximum length of at least $130 \mathrm{~cm} L_{\mathrm{C}}(\mathrm{Cox} \&$ Francis, 1999), is oviparous (Last \& Stevens, 2009), and thought to reach sexual maturity at $50 \mathrm{~cm} L_{\mathrm{BD}}$ (Dagit \& Kyne, 2015). All other aspects of its biology and population structure are unknown.

In New Zealand, there are no target fisheries for $R$. pacifica and it is only occasionally caught as bycatch in deep-sea fisheries (approximately 1 tonne annually; MPI, 2016b). Bycatch between 1990-91 and 2010-11 was increasing (MPI, 2013), although since little trawling occurs $>1200 \mathrm{~m}$ depth around New Zealand, this species would likely find some refuge at depth. In Australia, closure of most areas below $700 \mathrm{~m}$ depth provides substantial refuge for R. pacifica (Patterson et al., 2014) and its southern Australian range overlaps with some marine protected areas in the Commonwealth Marine Reserve network. At this time, it is listed as Least Concern on the IUCN Red List (Dagit \& Kyne, 2015).

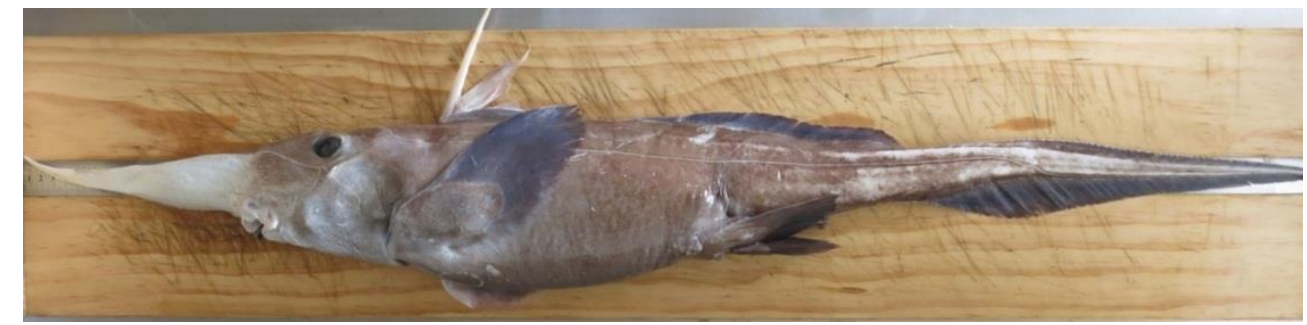

Figure 1.2 Adult male Rhinochimaera pacifica (106 $\mathrm{cm} L_{\mathrm{C}}$, Chatham Rise).

\subsubsection{Brown chimaera Chimaera carophila Kemper, Ebert, Naylor \& Didier, 2014}

The brown chimaera, Chimaera carophila (Fig. 1.3), was previously known as Chimaera sp C., and thought to be the same species, or a conspecific, of the longspine chimaera, Chimaera macrospina Didier, Last \& White, 2008 (McMillian et al., 2011), until it was described in 2014 as a species endemic to the New Zealand region. It is part of the Australasian species-complex which also includes the southern chimaera Chimaera fulva Last \& White, 2008, C. macrospina, and the shortspine chimaera Chimaera obscura Didier, Last \& White, 2008 (Kemper et al., 2014). Chimaera carophila is known from central and southern New Zealand waters, including Chatham Rise and slope, Challenger 
Plateau, Campbell Plateau, Hikurangi Trough, and Bounty Platform, at depths between 846 and $1350 \mathrm{~m}$ (most common beyond $1000 \mathrm{~m}$ ) (Roberts et al., 2015).

Maximum length has been recorded at $103 \mathrm{~cm} L_{\mathrm{C}}$ (Roberts et al., 2015), but there are no age, reproductive data, or abundance indices for $C$. carophila. There are also few data on biology, population structure, or fisheries impacts. Based on on the number of records in commercial, observer and research trawl database, population size has been classified by expert opinion as relatively small for $C$. carophila (Ford et al., 2015), and bycatch in deep-sea trawl fisheries to be low (less than 5 tonnes reported in the 2008-09 to 2012-13 fishing years). Chimaera carophila has yet to be assessed on the IUCN Red List.

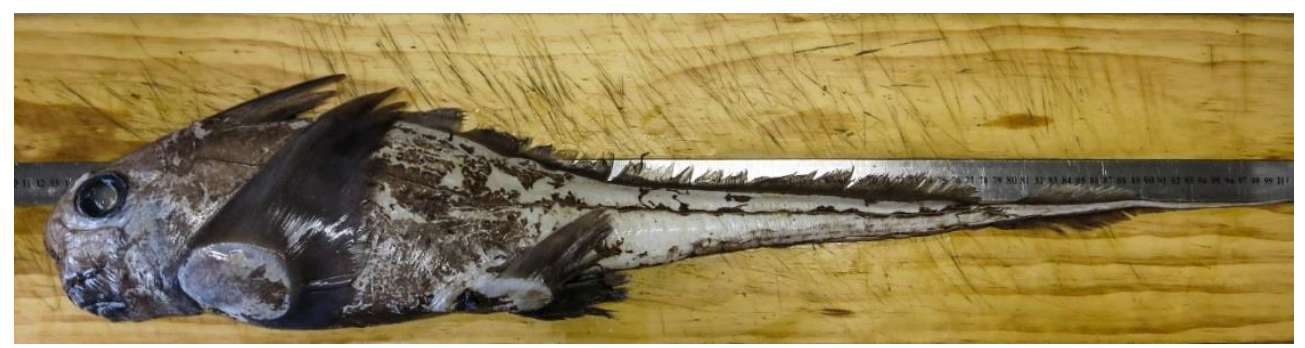

Figure 1.3 Adult male Chimaera carophila $\left(78 \mathrm{~cm} L_{\mathrm{C}}\right.$, Chatham Rise).

\subsubsection{Black ghost shark Hydrolagus homonycteris Didier, 2008}

Hydrolagus homonycteris (Fig. 1.4) is known from the continental slope and seamounts of southeastern Australia, Tasmania, and New Zealand (Last \& Stevens, 2009). It is thought to have a widespread distribution around New Zealand, and is occasionally caught in deep-sea fishing grounds around Chatham Rise and Lord Howe Rise at depths of 886 m to 1447 m, and possibly deeper (Didier, 2008; Roberts et al., 2015).

No age, reproductive data, or abundance indices are available for $H$. homonycteris. It has been measured to lengths of about $105 \mathrm{~cm} L_{\mathrm{C}}$ and, like all chimaeroids, is presumed to be oviparous (Last \& Stevens, 2009). Didier (2008) speculated that sexual maturity is reached at lengths greater than $60 \mathrm{~cm}$ (males) and 85 $\mathrm{cm}$ (females) based upon preserved specimens. Size-at-birth is unknown (Roberts et al., 2015). 
Estimated catch for H. homonycteris in New Zealand is low (less than 5 tonnes reported from the 2008-09 to 2012-13 fishing years), which is caught solely as bycatch in deep-sea trawl and longline fisheries (Francis, 2015). Very few records exist from the fisheries observer program, suggesting this species may be misidentified with other chimaerids (Ford et al., 2015). Hydrolagus homonycteris was once commonly caught as bycatch in the orange roughy (Hoplostethus atlanticus) fishery off Tasmania (Last \& Stevens 2009), but this fishery has since closed (Patterson et al., 2016). It is unknown if H. homonycteris is caught in orange roughy fisheries around New Zealand. Population sizes in New Zealand waters have been classified by expert opinion as moderate for $H$. homonycteris based on the scale of catches (Ford et al., 2015). On the basis of minimal distributional overlap with fishing at this time, a recent review of its IUCN Red List status moved H. homonycteris from Data Deficient to Least Concern (Theiss et al., 2015).

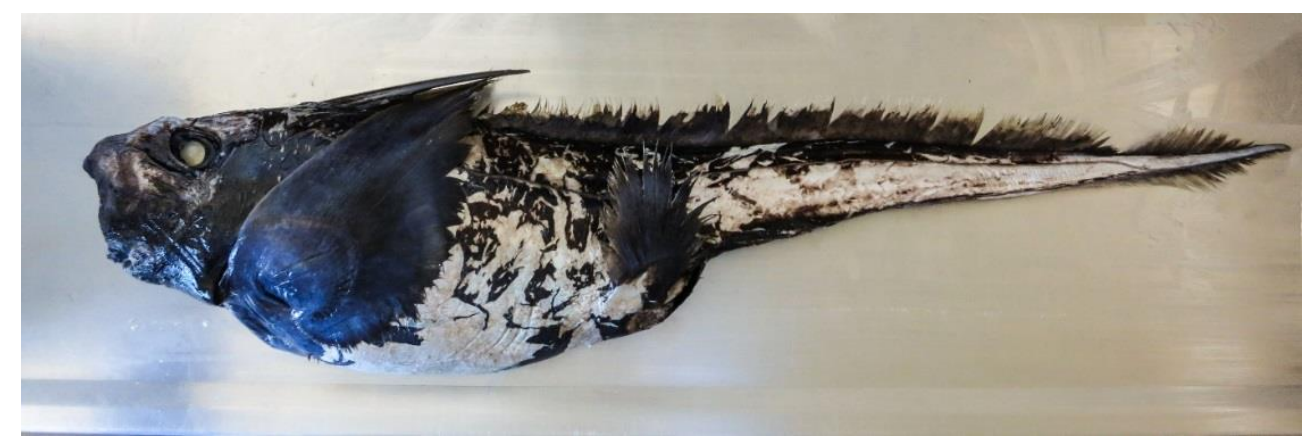

Figure 1.4 Adult female Hydrolagus homonycteris ( $86 \mathrm{~cm} L_{\mathrm{C}}$, Chatham Rise).

\subsubsection{Sharks (Chondrichthyes: Elasmobranchii)}

The larger of the two subclass of Chondrichthyes, Elasmobranchii includes the true sharks (Selachii) and the rays (Batoidea), spread across thirteen extant Orders. The Order Squaliformes, also known as the dogfish sharks, are recorded nearly worldwide and account for nearly half of all deep-sea chondrichthyan fishes (Kyne \& Simpfendorfer, 2010). The Squaliformes is the second most specious shark order, with 119 species across six families (Centrophoridae, Dalatiidae, Etmopteridae, Oxynotidae, Somniosidae, Squalidae) (Weigmann, 2016). Species from all families are known to occur in New Zealand, with a total of 32 species identified to date (Roberts et al., 2015), and additional species yet to be described. 
Oxynotus bruniensis (Fig. 1.5) is one of five species of the family Oxynotidae, known as angular or rough sharks (Yano \& Matsuura, 2002). Oxynotus bruniensis has a limited distribution, inhabiting temperate waters of the outer continental and insular shelves and slopes of southern Australia and New Zealand (Garrick, 1960; Last \& Stevens, 2009). In New Zealand waters, O. bruniensis have been reported most frequently off the east coast of the South Island from depths of 200-1000m (Beentjes et al., 2002; Francis et al., 2002; Tracey et al., 2004). Records from Rumble V seamount in the southern part of the Kermadec volcanic arc (Gordon, 2013) have since speculated that $O$. bruniensis may be more abundant on rough ground (Ford et al., 2015).

Literature regarding the biology and ecology of $O$. bruniensis is scarce. A maximum total length $\left(L_{\mathrm{T}}\right)$ has been reported at $75 \mathrm{~cm} L_{\mathrm{T}}$ in New Zealand but specimens up to $91 \mathrm{~cm} L_{\mathrm{T}}$ have been reported from Australia (Last \& Stevens, 2009). Females are lecithotrophic, viviparous, and are thought to have low fecundity (Compagno, 1984). Length-at-maturity is estimated at $67 \mathrm{~cm} L_{\mathrm{T}}$ for females, and between 55 and $60 \mathrm{~cm} L_{\mathrm{T}}$ for males (Roberts et al., 2015). Size-at-birth is estimated at $24 \mathrm{~cm} L_{\mathrm{T}}$ and females are thought to produce at least 7 pups per litter (Francis, 2003). The diet is unknown (Dunn et $a l ., 2013)$, although a relatively small mouth suggests that $O$. bruniensis may feed on benthic invertebrates and fishes (Last \& Stevens, 2009).

Oxynotus bruniensis has little (to no) current value to New Zealand fisheries (Ministry of Fisheries, 2008), and is caught as bycatch predominately in deep-sea trawl fisheries targeting hoki (Macruronus novaezelandiae) (MPI, 2015). Most catch is discarded, although nearly 25 tonnes were landed between the 2010-11 and 2014-15 fishing seasons in New Zealand (MPI, 2016b). No clear abundance indices trends exist for $O$. bruniensis, but risk from fishing was judged by expert opinion to be moderate (Ford et al., 2015). With a lack of information, O. bruniensis is listed as Data Deficient on the IUCN Red List (Francis, 2003). 


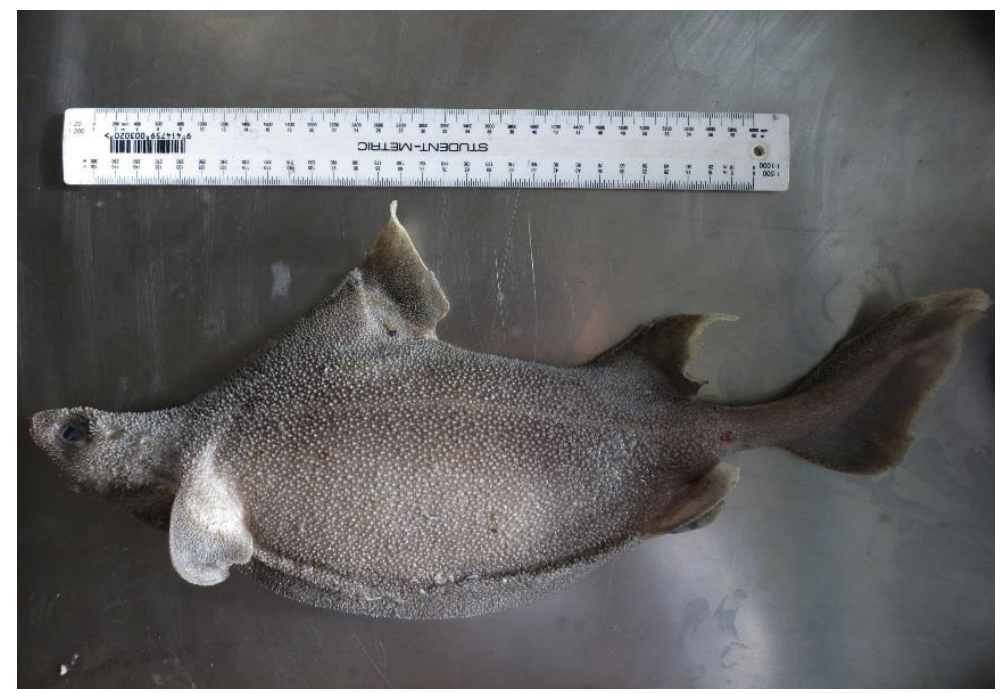

Figure 1.5 Juvenile male Oxynotus bruniensis ( $44 \mathrm{~cm} L_{\mathrm{T}}$, Chatham Rise). 
Table 1. Additional chondrichthyan fishes mentioned in thesis and their status on the IUCN Red List of Threatened Species (IUCN 2016).

\begin{tabular}{|c|c|c|}
\hline Family & Species & $\begin{array}{l}\text { IUCN Red List } \\
\text { Status }\end{array}$ \\
\hline \multirow[t]{6}{*}{ Chimaeridae } & $\begin{array}{l}\text { Smallspine spookfish } \\
\text { Harriotta haeckeli Karrer, } 1972\end{array}$ & Least Concern \\
\hline & $\begin{array}{l}\text { Pale ghost shark } \\
\text { Hydrolagus bemisi (Didier, 2002) }\end{array}$ & Least Concern \\
\hline & $\begin{array}{l}\text { Dark ghost shark } \\
\text { Hydrolagus novaezealandiae (Fowler, 1911) }\end{array}$ & Least Concern \\
\hline & $\begin{array}{l}\text { Pointy-nose blue chimaera } \\
\text { Hydrolagus trolli Didier \& Séret } 2002\end{array}$ & Least Concern \\
\hline & $\begin{array}{l}\text { Giant chimaera } \\
\text { Chimaera lignaria Didier, } 2002\end{array}$ & Least Concern \\
\hline & $\begin{array}{l}\text { Leopard chimaera } \\
\text { Chimaera panthera Didier, } 1998\end{array}$ & Data Deficient \\
\hline \multirow[t]{2}{*}{ Centrophoridae } & $\begin{array}{l}\text { Leafscale gulper shark } \\
\text { Centrophorus squamosus (Bonnaterre, 1788) }\end{array}$ & $\begin{array}{l}\text { Vulnerable; } \\
\text { Data Deficient } \\
\text { Australasia region }\end{array}$ \\
\hline & $\begin{array}{l}\text { Shovelnose dogfish, Birdbeak dogfish } \\
\text { Deania calcea (Lowe, 1839) }\end{array}$ & $\begin{array}{l}\text { Least Concern; } \\
\text { Endangered Europe }\end{array}$ \\
\hline \multirow[t]{2}{*}{ Etmopteridae } & $\begin{array}{l}\text { Baxter's dogfish, New Zealand lanternshark } \\
\text { Etmopterus granulosus (Gunther 1880) }\end{array}$ & Least Concern \\
\hline & $\begin{array}{l}\text { Lucifer dogfish, Blackbelly lanternshark } \\
\text { Etmopterus lucifer Jordan \& Snyder, } 1902\end{array}$ & Least Concern \\
\hline \multirow[t]{4}{*}{ Somniosidae } & $\begin{array}{l}\text { Longnose velvet dogfish } \\
\text { Centroscymnus crepidater (Barbosa du Bocage \& } \\
\text { de Brito Capello, 1864) }\end{array}$ & Least Concern \\
\hline & $\begin{array}{l}\text { Portuguese dogfish } \\
\text { Centroscymnus coelolepis Barbosa du Bocage \& } \\
\text { de Brito Capello, } 1864\end{array}$ & Near Threatened \\
\hline & $\begin{array}{l}\text { Owston's dogfish } \\
\text { Centroscymnus owstonii Garman, } 1906\end{array}$ & Least Concern \\
\hline & $\begin{array}{l}\text { Plunket shark } \\
\text { Centroscymnus plunketi (Waite, 1910) }\end{array}$ & Near Threatened \\
\hline Dalatiidae & $\begin{array}{l}\text { Seal shark, Kitefin shark } \\
\text { Dalatias licha (Bonnaterre, 1788) }\end{array}$ & Near Threatened \\
\hline Squalidae & $\begin{array}{l}\text { Spiny dogfish or piked dogfish } \\
\text { Squalus acanthias L. } 1758\end{array}$ & $\begin{array}{l}\text { Vulnerable; } \\
\text { Least Concern in } \\
\text { Australasia region }\end{array}$ \\
\hline
\end{tabular}




\subsection{Overview \& Objectives}

This thesis presents new information of several data poor chondrichthyan species found in New Zealand waters, which can be utilized in future management and conservation decisions.

Chapter II describes and evaluates the length-weight relationships (LWRs) for New Zealand deep-sea chondrichthyans, as well as assesses changes in length-weight data to estimate life history events, such as length-at-maturity. Maturity is often difficult to determine because of a lack of seasonality in the deep ocean.

Chapter III examines aggregation and social association behaviour of deep-sea chondrichthyans. If particular size or sex classes aggregate for developmental, environmental, or social reasons, these characteristics could increase species' vulnerability to fishing.

Chapters IV, V, and VI are a series of chapters describing the biology and ecology of several poorly known chondrichthyans: Chapter IV on the diet and reproductive biology of prickly dogfish (Oxynotus bruniensis); Chapter V on the reproductive biology of the two deep-sea longnose chimaeroids, long-nose spookfish (Harriotta raleighana) and Pacific spookfish (Rhinochimaera pacifica); and Chapter VI on the first observations of the biology of two rarely observed deep-sea chimaeroids, black ghost shark (Hydrolagus homonycteris) and brown chimaera (Chimaera carophila).

The discussion chapter (VII) draws conclusions on data collected from the previous chapters, evaluating species vulnerability to overexploitation, how this information can be incorporated into future management decisions, and where resources can be best allocated to future research.

Chapters III-VI have been prepared as stand-alone manuscripts to facilitate publication, and therefore each chapter has its own, more detailed and specific introduction. 


\section{CHAPTER II}

\section{Length-weight relationships of deep-sea chondrichthyans}




\subsection{Abstract}

Body size is one of the most important traits determining individual performance and life history, and can indicate individual and species' vulnerability to exploitation. Length-weight relationships (LWRs) are a necessary component to stock assessment models and fisheries management, and despite forming the basis of many important fisheries science applications, well described LWR for many species, including many deep-sea chondrichthyans, do not exist. The first objective of this study was to describe and evaluate LWRs for New Zealand deep-sea chondrichthyans and compare these parameters to FishBase estimates. The second objective was to examine any changes in the LWRs that may be a result of biological or ecological factors, such as maturity. A significant difference of the LWR between the sexes was found for most species, with females growing larger in length, and weighing more at a given length than males. LWR parameter estimates provided by the online database, FishBase, were found to differ significantly from those calculated in this study. Variability in weight at length was observed across most species, and appeared to increase with increasing length. A flexible model using permutations of a broken stick and variance shift was applied to LWRs and detected several trends in weight at length, discussed in further detail in this chapter. The model presented here could be applied to any marine fish where length and weight data are available, and may be particularly useful to provide insight into biological and ecological factors for species where macroscopic evaluations are unavailable or difficult to estimate, or when it may take years to collect such data. 


\subsection{Introduction}

Basic information on abundance and population trends, taxonomy, life history, and the ecological roles of deep-sea chondrichthyans are often lacking (Simpfendorfer \& Kyne, 2009; Simpfendorfer \& Wetherbee, 2015). Life history characteristics (e.g. age structure, reproduction, diet) are important for providing basic information on population dynamics, which are required for understanding a species' ability to withstand exploitation and recover from decline (Cortés, 2007; Francis \& Lyon, 2012). Morphological traits influence growth, development, reproduction and mortality, which in turn affect individual fitness as well as population stability and extinction risk (De Roos et al., 2003; DeAngelis \& Huston, 1987; Ebenman, 1988; Ohlberger et al., 2013). Body size is one of the most important traits determining individual performance and life history, such as energy metabolism, lifespan, and offspring size (Peters, 1983, Calder, 1984), and can indicate individual and species' vulnerability to exploitation (Reynolds et al., 2005). Maximum body size can be predictive of extinction threat for many marine fish (Olden et al., 2007). Large body size and slow population growth rates have been found to be associated with species decline when mortality from fishing is the most significant threat (e.g. Dulvy et al., 2003).

In most fish, body size and growth is described as allometric (changes in weight are relatively greater than changes in length), as opposed to isometric (growth rate is approximately the same for all body parts) (Fulton, 1904). Thus, increase in weight is a more pronounced aspect of growth than is increase in length (Ricker, 1958). The relationship between length and weight is expressed by the power equation, $W=a L^{b}$, where $W$ is weight $(\mathrm{g}), L$ is length $(\mathrm{cm})$, and $a$ and $b$ are constants (Le Cren, 1951; Froese, 2006). Most values of parameter $a$ range between $0 \cdot 001$ and $0 \cdot 05$, and are used to describe body shape (eel-like, $a=0 \cdot 001$; fusiform, $a=0 \cdot 01$; spherical $a=0 \cdot 1$ ), while parameter $b$ describes the body form or condition, and generally has a value of approximately 3 (Froese, 2006). Deviations from $b=3$ indicate a decrease in condition or elongated body form (negative allometric growth, $b<3$ ), or, increase in condition or increased width with increased length (positive allometric growth, $b>3$ ) (Hile, 1936). If $b=3$, the body of an individual is increasing in all dimensions in the same proportions over time, and thus, small individuals would have the same form and condition as large individuals (isometric growth) (Jobling, 2002; Froese, 2006). 
Length-weight relationships (LWRs), while often overlooked, are an important component of stock assessment models and fisheries management (Froese, 2006). These relationships can be used to index condition and to convert lengths to weights (Froese, 2006; Kulbicki et al., 2005), and can be used to compare regional life history strategies and morphological differences (Froese, 2006; Karachle \& Stergiou, 2012; Froese et al., 2014). Parameters from LWRs can also be extracted to produce an index of the body shape of a species or given population (form factor, $a_{3.0}$ ), where low form values are designated to an elongated fish shape, while high values to a more spherical shape (Froese, 2006). Form factor has been correlated with environmental factors, such as depth, where a more elongated body form is favoured with increasing depth, and may increase swimming performance in the deep-sea (Neat \& Campbell, 2013; Farre et al., 2016). Despite forming the basis of many important fisheries science applications, well described LWR for many species, including many chondrichthyans, do not exist (Barría et al., 2015; Froese et al., 2014).

FishBase, an online global comprehensive database of fish species (http://www.fishbase.org/), provides information on a wide range of biological and ecological characteristics for over 30000 fish species. Included among the biological information are LWR estimates produced from a Bayesian hierarchical approach, where generalized LWR parameters were modelled on existing LWR studies and four assigned body shape groups (eel-like, elongated, fusiform, and short \& deep) (Froese et al., 2014). For data limited species ( $<5$ studies with LWR data), information was derived from related species (by genus, subfamily or family and with the same body shape). The final project has resulted in LWR parameter estimates for nearly all known fishes, which have since been applied to fisheries science (e.g. Apostolidis et al., 2015; Simmons et al., 2016; Wiedenmann et al., 2016). Estimates of length-weight relationships, however, can show large variations from true estimates of population parameters, which may be accounted for by inadequate sampling (Morato et al., 2001), or a lack of indication of taxonomic relationship beyond the species level (e.g. genus, family) when estimates are inferred from related species (Kulbicki et al., 2005). Although these parameters are provided, they may not be useful beyond general length-weight conversions.

Most teleosts demonstrate indeterminate growth patterns, with the highest rates of growth observed in early stages of life, followed by a subsequent decline with increasing age (Le Gros Clark \& Medawar, 1945; Ricker, 1958). Variability in weight between fish of the same length has been found to exist both within, and between, populations 
(Jobling, 2002), and may be correlated to environmental conditions (e.g. water temperature, Hile, 1936; Jobling, 1997), ontogenetic shifts in diet (Stergiou \& Fourtouni, 1991), body condition (Jobling, 2002), variations in behaviour (Ricker, 1979), and habitat (Dunn \& Forman, 2011). Changes in weight are also observed as maturity, and a subsequent reproductive cycle, is reached (Ricker, 1979; Wootton, 1990). In chondrichthyans, which are generally characterized with low reproductive output, large energetic expenditure is often allocated into reproduction, particularly for maternal reproductive investment (Hoenig \& Gruber, 1990). As the onset of sexual maturity is reached, weight of an individual may increase due to changes in gonad (Francis et al., 2000) or offspring (Baremore \& Passerotti, 2013) weight, and decrease as a result of intensive breeding behaviour or parturition (Pratt \& Carrier, 2001).

Fisheries-independent research trawls within the New Zealand Exclusive Economic Zone (EEZ) have been in operation since the 1970s (O'Driscoll et al., 2011), compiling a large database of biological and environmental information. The main objective of these surveys is to estimate biomass of important commercial species, although length and weight measurements of many bycatch species, including deep-sea chondrichthyans, have also been recorded. This information is readily available to researchers, but is often greatly underutilized. For bycatch species, this data could hold a wealth of information regarding species' life history for which we know little about, and would otherwise not have the opportunity to sample. The first objective of this study aims to describe and evaluate LWRs for deep-sea chondrichthyans caught in these surveys, and compare these parameters to FishBase estimates. The second objective is to assess the feasibility of fulling exploiting the database for indicators of life history characteristics by examining more complex patterns in the LWRs, which may be expressed as changes in the mean or variability of weight at length. These changes may be a result of biological or ecological factors, such as maturity, and will be examined and discussed in further detail. 


\subsection{Methods}

\subsubsection{Research trawl data}

Data from fisheries-independent research trawls within the New Zealand Exclusive Economic Zone (EEZ) were obtained from the New Zealand Ministry for Primary Industries (MPI). Biological sample data were selected where the average bottom depth was $>200 \mathrm{~m}$, the gear used was any form of bottom or mid-water trawl, gear performance was rated as at least satisfactory (catch unlikely to be reduced by gear performance, as per NIWA criteria), latitude was between 25 and $55^{\circ} \mathrm{S}$, and longitude between $160^{\circ} \mathrm{E}$ and $170^{\circ} \mathrm{W}$. The resulting dataset consisted of 358 trips and 18505 trawls, completed by 33 vessels, dating from December 1976 to January 2013. Of these, 9258 trawls were completed by the RV Tangaroa. Over half of all trawls occurred on the Chatham Rise or East Coast of the South Island (ECSI) (Fig. 2.1, Table 2.1). Gear depth of mid-water trawls was between 375-538 $\mathrm{m}$ and bottom trawls between 386-882 m (Table 2.2). Most tows occurred at depths between 400-1200 m, with most recorded in the second half of the year (July to December) (Table 2.2). For each tow, the details of the fishing operation were recorded, and the catch was sorted by species and sampled for biological statistics (species, sex, length, weight). Data were groomed to remove any implausible outliers (e.g. a Harriotta raleighana measured at a length of $6 \mathrm{~m}$ and no weight recorded). 


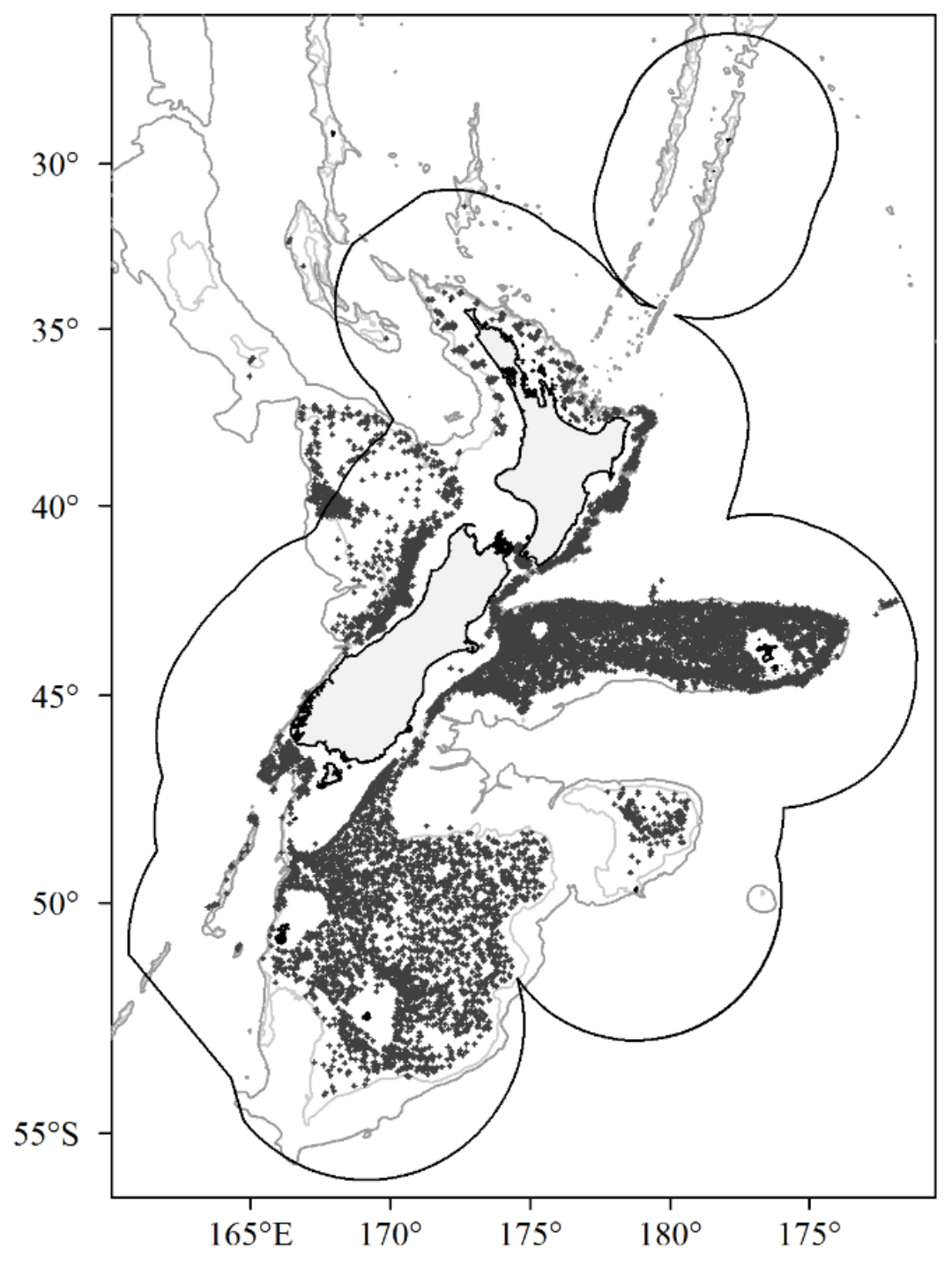

Figure 2.1 Location of research trawl points around New Zealand from 1972-2013, with inclusion of Exclusive Economic Zone (EEZ) and isobaths (400 m, 1000 m, 1500 m). 


\subsubsection{Species studied}

Selected species for analysis included common and infrequently caught deep-sea species from offshore New Zealand waters. These were nine chimaeroids: smallspine spookfish (Harriotta haeckeli), longnose spookfish (Harriotta raleighana), Pacific spookfish (Rhinochimaera pacifica), brown chimaera (Chimaera carophila), giant chimaera (Chimaera lignaria), pale ghost shark (Hydrolagus bemisi), black ghost shark (Hydrolagus homonycteris), dark ghost shark (Hydrolagus novaezealandiae), and pointynose blue ghost shark (Hydrolagus trolli); and eleven squaloids: spiny dogfish (Squalus acanthias), leafscale gulper shark (Centrophorus squamosus), shovelnose dogfish (Deania calcea), Baxter's dogfish (Etmopterus granulosus), Lucifer dogfish (Etmopterus lucifer), Portuguese dogfish (Centroscymnus coelolepis), Owston's dogfish (Centroscymnus owstonii), longnose velvet dogfish (Centroscymnus crepidater), Plunket's shark (Scymnodon plunketi), prickly dogfish (Oxynotus bruniensis), and seal shark (Dalatias licha).

\subsubsection{Part I: Estimating the length-weight relationship (LWR)}

Length and weight measurements were extracted for all selected species. Lengths (total length, $L_{\mathrm{T}}$ : tip of the snout to posterior end of caudal fin, or chimaera length, $L_{\mathrm{C}}$ : tip of the snout to the posterior edge of the supracaudal fin, excluding the caudal filament) were rounded down to the nearest centimetre and weights to the nearest gram. The lengthweight relationship (LWR) for male, female, and combined sexes was estimated using the $\log$ form of the equation, $W=a L^{b}$ :

$$
\log (W)=\log (a)+b \times \log (L)
$$

where $W$ is weight, $L$ is length, and $a$ and $b$ are parameters (Le Cren, 1951). Parameters and their confidence intervals were estimated using nonlinear least squares $(n l s)$ in R. The LWR from FishBase was also plotted for comparison. FishBase LWR parameter estimates were obtained for each species from http://www.fishbase.org/ (accessed 30 October 2014), with the exception of C. carophila, as it is a recently described species and is not yet on the database, and LWR parameters for Callorhinchus milli were extracted from Gorman (1963). 
To test for deviations from isometry $(b=3)$, a Student $t$-test following Pauly (1984) was used, and ANCOVA was used to compare LWR between sexes (Froese, 2006). A $t$-test was also used to evaluate differences between the Fishbase LWR parameter estimates and the LWR estimated in this study (Zar, 1996). Significance in all tests was accepted at $P<0 \cdot 05$. The form factor $\left(a_{3.0}\right)$ was calculated for each species using the equation, $a_{3.0}=10^{\log (a)-S(b-3)}$, where $a$ and $b$ are the parameters of the LWR, and $S$ is the slope of the regression of $\log (a)$ vs $b$. A mean slope $S=-1.358$ was used as calculated by Froese (2006). Form factor was plotted against mean depth by family.

\subsubsection{Part II: Fitting a more complex length-weight model}

Length and weight data were selected for species with a combined sexed dataset of > 50 observations, removing $H$. haeckeli, C. lignaria, $H$. trolli, and $C$. coelolepis from this analysis. Data were separated by sex. In this section of the study, model parameters were estimated using Maximum Likelihood, assuming normally distributed errors. Unlike $n l s$, Maximum Likelihood estimates the variance $(\sigma)$, which was required to allow $\sigma$ changes over length.

A linear model was fitted to log-weight and log-length data, with an alternative specification of the mean incorporating a broken stick (allows the mean to take two different gradients, $\beta_{1}$ and $\beta_{3}$ ) at $x=\beta_{2}$ :

$$
\mu(\beta, x)=\beta_{0}+\beta_{1} x+\beta_{3} \min \left(x-\beta_{2}, 0\right)
$$

such that the final term in this model was included only when fish length, $x$, exceeded $\beta_{2}$. The model was then further extended to allow a simple level shift in the variance $\left(\sigma^{2}\right)$ at $x$ $=\alpha_{1}$ :

$$
\log \sigma^{2}=\tau(\alpha, x)=\alpha_{0}+\alpha_{2} I\left(x>\alpha_{1}\right)
$$

where $\tau=\log \sigma^{2}=\alpha_{0}$ and $I(\cdot)$ is an indicator function (taking a value of 1 or 0 in the presence/absence of the event). A basic step function, $I\left(x>\alpha_{1}\right)$ is not differentiable, and so, to ensure differentiability and convergence, this model was fitted as:

$$
\log \sigma^{2}=\tau(\alpha, x)=\alpha_{0}+\alpha_{2} /\left(1+\exp \left(-\left(x-\alpha_{1}\right) / e^{\alpha 3}\right)\right)
$$


where $e^{\alpha 3}=0 \cdot 01$ was held fixed. The second variance term, $\alpha_{2}$, is therefore added into the model depending on the value of the indicator function $I(x)$ :

$$
I(x)=1 /\left(1+\exp \left(-\left(x-\alpha_{1}\right) / e^{\alpha 3}\right)\right)
$$

which has a value either zero (or very close to zero) when $x<\alpha_{1}$, or one (or very close to one) when $x>\alpha_{1}$. The fixed term $e^{\alpha 3}$ gives a smooth transition between 0 and 1 , with the width of the transition determined by $\alpha_{3}$. The value of $\mathrm{e}^{\alpha 3}$ was selected and fixed at 0.01 in this study to give a transition appropriate to the observed data.

Four versions of this model were fitted to each of the 16 datasets: permutations of broken stick absent (L) or present (BS), and the variance shelf absent (CV) or present (VS). Akaike information criterion (AIC) was used to select the best fitting model, since AIC includes a complexity term penalising overly complex models (Akaike, 1987). In the presentation of results, the minimum AIC was subtracted so that the model where the AIC is zero is the best fitting model for that dataset. Due to bimodality, which distorted the behaviour of the LWR, an additional two datasets of $C$. crepidater were included, where each sex was truncated at a length of $90 \mathrm{~cm} L_{\mathrm{T}}$.

The inflection point of the variance $(\alpha)$ and mean $(\beta)$, if estimated by the best fitting model, were compared to previously reported estimates for mean length-atmaturity and size-at-birth, using New Zealand based literature, when available (Table 2.3). All statistical analyses were carried out using R (R Core Team, 2012). 
Table 2.1. Summary of mid-water and bottom trawls from Ministry for Primary Industry (MPI) research trawl survey datasets, carried out in different marine regions of New Zealand from 1972 - 2013. Trawls were divided into one of five regions: Southland and Sub-Antarctic (trawls at latitude of $46^{\circ} \mathrm{S}$ or lower, Bagley \& O'Driscoll, 2012; Bagley et al., 2013); Chatham Rise and East Coast South Island (ESCI); West Coast of North and South Island; North Island and Cook Strait; outside the EEZ on Lord Howe Rise and Norfolk Ridge.

\begin{tabular}{|c|c|c|c|c|c|c|c|}
\hline \multirow[t]{2}{*}{ Year } & \multirow[t]{2}{*}{ No. of trawls } & \multirow{2}{*}{$\begin{array}{c}\text { Mean no. of } \\
\text { trawls per } \\
\text { year }\end{array}$} & \multicolumn{5}{|c|}{ No. of trawls by area } \\
\hline & & & $\begin{array}{c}\text { Southland, } \\
\text { Sub-Antarctic }\end{array}$ & $\begin{array}{c}\text { Chatham } \\
\text { Rise, ECSI }\end{array}$ & West Coast & $\begin{array}{c}\text { North Island, } \\
\text { Cook Strait }\end{array}$ & Outside EEZ \\
\hline 1972-76 & 2 & $<1$ & 2 & 0 & 0 & 0 & 0 \\
\hline 1977-81 & 917 & 183 & 316 & 351 & 223 & 27 & 0 \\
\hline 1982-86 & 2258 & 451 & 356 & 1081 & 318 & 499 & 4 \\
\hline 1987-91 & 4066 & 813 & 703 & 1951 & 990 & 417 & 5 \\
\hline $1992-96$ & 4738 & 947 & 1266 & 2706 & 44 & 722 & 0 \\
\hline 1997-2001 & 2116 & 423 & 383 & 1264 & 232 & 237 & 1 \\
\hline $2002-2006$ & 2089 & 417 & 553 & 1202 & 236 & 98 & 0 \\
\hline 2007-2011 & 1823 & 364 & 420 & 966 & 271 & 166 & 0 \\
\hline 2012-2013 & 496 & 248 & 91 & 313 & 84 & 8 & 0 \\
\hline Total & 18505 & & 4090 & 9834 & 2398 & 2174 & 10 \\
\hline
\end{tabular}


Table 2.2. Summary of mid-water and bottom trawl dataset with the number of trawls, the minimum and maximum mean depth, the number of tows by depth range, and the number of tows by month.

\begin{tabular}{|c|c|c|c|c|c|c|c|c|c|c|c|c|c|}
\hline \multirow{3}{*}{ year } & \multirow{3}{*}{$\begin{array}{l}\text { no. of } \\
\text { trawls }\end{array}$} & \multicolumn{4}{|c|}{ mean depth of trawl (m) } & \multirow{2}{*}{\multicolumn{4}{|c|}{ no. of trawls by mean depth (m) }} & \multirow{2}{*}{\multicolumn{4}{|c|}{ no. of trawls by month }} \\
\hline & & mid-wat & trawl & bottom $\mathbf{t}$ & & & & & & & & & \\
\hline & & $\min$ & $\max$ & $\min$ & $\max$ & $0-400$ & $\begin{array}{l}400- \\
800\end{array}$ & $\begin{array}{l}800- \\
1200\end{array}$ & $1200+$ & $\begin{array}{l}\text { January } \\
\text { - March }\end{array}$ & $\begin{array}{l}\text { April - } \\
\text { June }\end{array}$ & $\begin{array}{c}\text { July - } \\
\text { September }\end{array}$ & $\begin{array}{l}\text { October - } \\
\text { December }\end{array}$ \\
\hline 1972-76 & 2 & - & - & 386 & 401 & 1 & 1 & 0 & 0 & 0 & 0 & 0 & 2 \\
\hline 1977-81 & 917 & 461 & 507 & 497 & 523 & 327 & 451 & 137 & 0 & 258 & 144 & 314 & 201 \\
\hline 1982-86 & 2258 & 430 & 439 & 770 & 791 & 236 & 741 & 1259 & 20 & 232 & 474 & 911 & 641 \\
\hline 1987-91 & 4066 & 422 & 462 & 851 & 882 & 223 & 1051 & 2595 & 197 & 82 & 407 & 2087 & 1490 \\
\hline $1992-96$ & 4738 & 483 & 538 & 788 & 841 & 508 & 1474 & 2460 & 295 & 1371 & 1130 & 1215 & 1022 \\
\hline 1997-2001 & 2116 & 375 & 409 & 654 & 694 & 432 & 926 & 748 & 10 & 597 & 308 & 608 & 603 \\
\hline 2002-2006 & 2089 & 393 & 450 & 691 & 716 & 379 & 917 & 762 & 31 & 489 & 248 & 666 & 686 \\
\hline 2007-2011 & 1823 & 382 & 441 & 707 & 741 & 266 & 729 & 763 & 64 & 607 & 339 & 331 & 546 \\
\hline 2012-2013 & 496 & - & - & 624 & 642 & 98 & 276 & 108 & 14 & 256 & 57 & 92 & 91 \\
\hline Total & 18506 & & & & & 2470 & 6566 & 8832 & 631 & 3892 & 3107 & 6224 & 5282 \\
\hline
\end{tabular}


Table 2.3. Length-at-maturity $(\mathrm{cm})$ by sex and length-at-birth $(\mathrm{cm})$ for selected chondrichthyans (total length, $L_{\mathrm{T}}$, for squaloids; chimaera length, $L_{\mathrm{C}}$, for chimaeroids).

\begin{tabular}{|c|c|c|c|c|c|c|c|}
\hline \multicolumn{2}{|r|}{ Species } & \multirow{2}{*}{$\begin{array}{c}\text { Length } \\
\text { range }(\mathrm{cm})\end{array}$} & \multicolumn{2}{|c|}{ Maturity (cm) } & \multirow[t]{2}{*}{ Source } & \multirow{2}{*}{$\begin{array}{l}\text { Length- } \\
\text { at-birth } \\
\text { (cm) }\end{array}$} & \multirow[t]{2}{*}{ Source } \\
\hline & & & Female & Male & & & \\
\hline \multicolumn{8}{|l|}{ Chimaeriformes } \\
\hline & Harriotta raleighana & $19 \cdot 9-97 \cdot 9$ & 76 & 63 & Finucci et al. (2016) & $10-13$ & Cox \& Francis (1999) \\
\hline & Rhinochimaera pacifica & $28 \cdot 6-165 \cdot 2$ & 125 & 105 & Finucci et al. (2016) & - & \\
\hline & Chimaera carophila & $35 \cdot 4-103 \cdot 9$ & 83 & 75 & Finucci et al. (2017) & - & \\
\hline & Hydrolagus bemisi & $13 \cdot 9-89 \cdot 1$ & 69 & 59 & Horn (1997) & $9-12$ & Cox \& Francis (1999) \\
\hline & Hydrolagus homonycteris & $50 \cdot 6-99 \cdot 8$ & 83 & 79 & Finucci et al. (2017) & - & \\
\hline & Hydrolagus novaezealandiae & $18 \cdot 9-81 \cdot 0$ & 62 & 52 & Horn (1997) & 5 & Horn (1997) \\
\hline \multicolumn{8}{|l|}{ Squaliformes } \\
\hline & Squalus acanthias & $24 \cdot 2-107 \cdot 0$ & 73 & 58 & Hanchet (1988) & 22 & Last \& Stevens (2009) \\
\hline & Centrophorus squamosus & $37 \cdot 9-145 \cdot 0$ & 119 & 99 & Parker \& Francis (2012) & $35-40$ & Last \& Stevens (2009) \\
\hline & Deania calcea & $20 \cdot 4-126 \cdot 5$ & 106 & 78 & Parker \& Francis (2012) & 30 & Last \& Stevens (2009) \\
\hline & Etmopterus granulosus & $17 \cdot 3-85 \cdot 8$ & 64 & 55 & Wetherbee (1996) & $21-23$ & Last \& Stevens (2009) \\
\hline & Etmopterus lucifer & $16 \cdot 3-54 \cdot 8$ & 34 & 29 & Galland (2015) & 15 & Last \& Stevens (2009) \\
\hline & Centroscymnus owstonii & $28 \cdot 2-148 \cdot 0$ & 98 & 71 & King \& Clark (1987) & 30 & Last \& Stevens (2009) \\
\hline & Centroselachus crepidater & $28 \cdot 0-109 \cdot 8$ & 81 & 62 & King \& Clark (1987) & $30-35$ & Last \& Stevens (2009) \\
\hline & Scymnodon plunketi & $29 \cdot 0-150 \cdot 2$ & 129 & 100 & Garrick (1959) & $32-36$ & Last \& Stevens (2009) \\
\hline & Oxynotus bruniensis & $41 \cdot 4-73 \cdot 7$ & 64 & 55 & Finucci et al. (2016) & $25-27$ & Finucci et al. (2016) \\
\hline & Dalatias licha & $35 \cdot 7-153 \cdot 6$ & 120 & 95 & King \& Clark (1987) & $30-40$ & Last \& Stevens (2009) \\
\hline
\end{tabular}




\subsection{Results}

\subsubsection{Length-weight relationships}

Parameter $a$ of length-weight relationships (LWRs) for combined sexes ranged from $a=0.0008$ (Harriotta haeckeli) to $a=0.0525$ (Hydrolagus homonycteris), while the parameter $b$ ranged from 2.44 (H. homonycteris) to 3.48 (Oxynotus bruniensis) (Table 2.4). The LWR coefficient of determination $\left(r^{2}\right)$ ranged from 0.852 to $0 \cdot 997$. Most species (70\%) had significant positive allometric growth with the exception of H. homonycteris, Hydrolagus trolli, Centroscymnus coelolepis, and O. bruniensis. Two species, Chimaera carophila $(b=2 \cdot 52)$ and Hydrolagus bemisi $(b=2 \cdot 89)$, had significant negative allometric growth. LWRs were significantly different between the sexes for most species (75\%), but were not significantly different for $H$. bemisi, $H$. trolli, Chimaera lignaria, $C$. coelolepis, and $O$. bruniensis. With the exception of Hydrolagus novaezealandiae and $H$. trolli, females had larger $b$ values than males (Table 2.4).

Where significant LWR differences were found between the sexes, males were usually heavier in the smallest length classes, and females heavier in the largest length classes and at the mean observed length (Table 2.5). Male $H$. novaezealandiae were found to weigh more than females across all length classes, while female Etmopterus lucifer weighed more than male E. lucifer across the length range. Male Scymnodon plunketi weighed more than female S. plunketi at the mean observed length. No significant LWR differences were found between the sexes of $C$. lignaria, H. bemisi, $H$. trolli, C. coelolepis, and $O$. bruniensis (Table 2.5). In both the chimaeroid and squaloid families, no pattern was observed between form factor $\left(a_{3.0}\right)$ and mean depth (m) (Fig. 2.2), however, members of the Rhinochimaeridae appeared to have lower $a_{3.0}$ than other chimaeroids in relation to their depth range.

\subsubsection{FishBase LWR parameter estimates}

Most LWR parameter estimates from FishBase were significantly different from those calculated in this study (Fig. 2.3, Table 2.6). At the mean observed length of each species, FishBase LWR parameters significantly underestimated weights for H. haeckeli, C. lignaria, $H$. novaezealandiae, and $O$. bruniensis, and significantly overestimated weights for $H$. raleighana, $R$. pacifica, $H$. homonycteris, E. lucifer, $C$. coelolepis, 
Centroscymnus owstonii, Centroselachus crepidater, S. plunketi, and D. licha.

Hydrolagus trolli was the only species where FishBase parameters were found not to be significantly different (Table 2.6). FishBase and the present study had similar weight at length estimates (< 10\% difference) for H. bemisi, S. acanthias, C. squamosus, Deania calcea, and Etmopterus granulosus. These weight estimates did not fall within the mean \pm one standard deviation (S.D.) of weight at mean observed length for these species, however, they were still found to be significantly different (Fig. 2.4, Appendix I).

\subsubsection{Inflection points in the LWR}

Variability in weight at length was observed across all species, and often appeared to increase with increasing length (Fig. 2.5). Of the four models, the Broken stick mean \& Variance shelf (BS+VS) model was most often selected (47\%), followed by Broken stick mean \& Constant variance $(\mathrm{BS}+\mathrm{CV})(31 \%)$, Linear mean \& Constant variance (L+CV) (16\%), and Linear mean \& Variance shelf (L+VS) (6\%) (Table 2.7).

Both variance $(\alpha)$ and mean $(\beta)$ were selected for $38 \%$ of the samples (Fig. 2.7, Fig. 2.8), with only mean ( $\beta$ ) inflection points detected for $59 \%$ of samples (Fig. 2.9), and only variance $(\alpha)$ inflection points were detected for $88 \%$ of samples (Fig. 2.10). Location $(\mathrm{cm})$ of the inflection points in either variance $(\alpha)$ or mean $(\beta)$, or both, from the best fitting model were credible for all species, with the exception of Oxynotus bruniensis, where no credible estimates were found (Table 2.8, Appendix II). Full results are reported in Appendix III.

Most estimates (c. 75\%) of the variance inflection point were within $20 \%$ of reported length-at-maturity [Fig. 2.6(A), Fig. 2.6(B)]. The inflection point of the variance generally underestimated length-at-maturity for females more than males, and $83 \%$ of female length estimates and $57 \%$ of male length estimates from the variance were accurate within $20 \%$ [Fig. 2.6(A)]. The inflection point of the variance underestimated most length-at-maturity for both squaloids and chimaeroids, and $83 \%$ of squaloid and $57 \%$ of chimaeroid length estimates from the variance were accurate within $20 \%$ [Fig. 2.6(B)].

Half of the estimates of the mean inflection point were beyond a margin of $20 \%$ of reported length-at-maturity [Fig. 2.6(C), Fig. 2.6(D)]. The inflection point of the mean mostly underestimated length-at-maturity for females, and 38\% of female length estimates and $62 \%$ of male length estimates from the mean were accurate within $20 \%$ 
[Fig. 2.6(C)]. The inflection point of the mean underestimated most length-at-maturity for most squaloids, and $41 \%$ of squaloid and $67 \%$ of chimaeroid length estimates from the mean were accurate within $20 \%$ [Fig. 2.6(D)].

Estimates of the variance inflection point were closest to the published length-atmaturity ( $<5 \%$ difference) for male $C$. carophila, female $C$. owstonii, female $C$. crepidater, and female $S$. plunketi, while estimates of the mean inflection point were closest to published length-at-maturity values (<5\% difference) for female $\mathrm{H}$. bemisi, both sexes $H$. homonycteris, male $H$. novaezealandiae, male $D$. calcea, and female $C$. crepidater (Table 2.9, Fig. 2.6).

Examples of the model fits are shown in Figures $2.7-2.10$, with details of the model estimates reported in Table 2.8 and Appendix II. In some cases, a decrease in the mean (Fig. 2.7, Fig. 2.8) or decline in the variance (Fig. 2.10) was provided early in the observed length range, while in other species, the mean increased (Fig. 2.9) or the variance increased (Fig. 2.8) in larger fish. In a few cases, the inflection point of the variance or mean was estimated to occur outside of the majority of the data (Fig. 2.9), and these were therefore not considered credible. 
Table 2.4. Length-weight relationships $[\log (W)=\log (a)+b \times \log (L)]$ for selected deep-sea chondrichthyans. * Significant difference between males and females.

\begin{tabular}{|c|c|c|c|c|c|c|c|c|c|c|c|c|c|c|c|c|}
\hline \multirow[t]{2}{*}{ Family } & \multirow[t]{2}{*}{ Species } & \multirow[t]{2}{*}{ Sex } & \multicolumn{6}{|c|}{ Length (cm) } & \multicolumn{2}{|c|}{ Weight (g) } & \multirow[b]{2}{*}{ SD } & \multirow[b]{2}{*}{$a$} & \multirow[b]{2}{*}{$\begin{array}{r}95 \% \\
\text { C.I. }\end{array}$} & \multirow[b]{2}{*}{$b$} & \multirow[b]{2}{*}{$\begin{array}{c}95 \% \\
\text { C.I. }\end{array}$} & \multirow[b]{2}{*}{$r^{2}$} \\
\hline & & & $n$ & Min & Max & Mean & SD & Min & Max & Mean & & & & & & \\
\hline \multirow[t]{9}{*}{ Rhinochimaeridae } & \multirow[t]{3}{*}{$\begin{array}{l}\text { Harriotta } \\
\text { haeckeli }\end{array}$} & Combined* & 23 & 46 & 73 & 63 & 10 & 325 & 1625 & 1023 & 483 & 0.0008 & $\begin{array}{r}0.0003- \\
0.0021\end{array}$ & 3.37 & $\begin{array}{r}3.14- \\
3.59\end{array}$ & 0.977 \\
\hline & & Male & 8 & 46 & 54 & 50 & 3 & 325 & 515 & 423 & 59 & 0.1487 & $\begin{array}{r}0.0096- \\
2.2986\end{array}$ & 2.03 & $\begin{array}{r}1.33- \\
2.74\end{array}$ & 0.876 \\
\hline & & Female & 15 & 62 & 73 & 70 & 3 & 755 & 1625 & 1343 & 220 & 0.0001 & $\begin{array}{r}0.000001- \\
0.0097\end{array}$ & 3.81 & $\begin{array}{r}2.79- \\
4.84\end{array}$ & 0.820 \\
\hline & \multirow[t]{3}{*}{$\begin{array}{l}\text { Harriotta } \\
\text { raleighana }\end{array}$} & Combined* & 4429 & 20 & 98 & 69 & 14 & 10 & 3195 & 1142 & 620 & 0.0033 & $\begin{array}{r}0.0031- \\
0.0036\end{array}$ & 2.99 & $\begin{array}{r}2.97- \\
3.00\end{array}$ & 0.966 \\
\hline & & Male & 2210 & 20 & 92 & 65 & 11 & 10 & 2830 & 876 & 355 & 0.0075 & $\begin{array}{r}0.0060- \\
0.0083\end{array}$ & 2.78 & $\begin{array}{r}2.75- \\
2.80\end{array}$ & 0.957 \\
\hline & & Female & 2219 & 23 & 98 & 72 & 15 & 40 & 3195 & 1406 & 709 & 0.0028 & $\begin{array}{r}0.0025- \\
0.0030\end{array}$ & 3.04 & $\begin{array}{r}3.02- \\
3.06\end{array}$ & 0.978 \\
\hline & \multirow[t]{3}{*}{$\begin{array}{l}\text { Rhinochimaera } \\
\text { pacifica }\end{array}$} & Combined* & 1269 & 29 & 159 & 117 & 21 & 36 & 7550 & 3205 & 1557 & 0.0010 & $\begin{array}{r}0.0009- \\
0.0012\end{array}$ & 3.12 & $\begin{array}{r}3.08- \\
3.15\end{array}$ & 0.964 \\
\hline & & Male & 741 & 29 & 146 & 115 & 16 & 36 & 6360 & 2731 & 801 & 0.0022 & $\begin{array}{r}0.0017- \\
0.0027\end{array}$ & 2.95 & $\begin{array}{r}2.90- \\
2.99\end{array}$ & 0.952 \\
\hline & & Female & 528 & 36 & 159 & 121 & 26 & 90 & 7550 & 3872 & 2042 & 0.0007 & $\begin{array}{r}0.0005- \\
0.0008\end{array}$ & 3.21 & $\begin{array}{r}3.17- \\
3.25\end{array}$ & 0.976 \\
\hline \multirow[t]{3}{*}{ Chimaeridae } & \multirow[t]{3}{*}{$\begin{array}{l}\text { Chimaera } \\
\text { carophila }\end{array}$} & Combined* & 159 & 35 & 103 & 77 & 13 & 300 & 4805 & 2264 & 863 & 0.0374 & $\begin{array}{r}0.0220- \\
0.0635\end{array}$ & 2.52 & $\begin{array}{r}2.40- \\
2.64\end{array}$ & 0.913 \\
\hline & & Male & 104 & 35 & 103 & 78 & 11 & 300 & 4805 & 2193 & 674 & 0.0657 & $\begin{array}{r}0.0331- \\
0.1306\end{array}$ & 2.38 & $\begin{array}{r}2.23- \\
2.54\end{array}$ & 0.897 \\
\hline & & Female & 55 & 43 & 99 & 77 & 15 & 430 & 4490 & 2397 & 1134 & 0.0173 & $\begin{array}{r}0.0079- \\
0.0377\end{array}$ & 2.71 & $\begin{array}{r}2.53- \\
2.89\end{array}$ & 0.944 \\
\hline
\end{tabular}




\begin{tabular}{|c|c|c|c|c|c|c|c|c|c|c|c|c|c|c|c|c|}
\hline \multirow[t]{2}{*}{ Family } & \multirow[t]{2}{*}{ Species } & \multirow[t]{2}{*}{ Sex } & \multicolumn{5}{|c|}{ Length (cm) } & \multicolumn{4}{|c|}{ Weight (g) } & \multirow[b]{2}{*}{$a$} & \multirow[b]{2}{*}{$\begin{array}{r}95 \% \\
\text { C.I. } \\
\end{array}$} & \multirow[b]{2}{*}{$b$} & \multirow[b]{2}{*}{$\begin{array}{r}95 \% \\
\text { C.I. }\end{array}$} & \multirow[b]{2}{*}{$r^{2}$} \\
\hline & & & $n$ & Min & $\operatorname{Max}$ & Mean & SD & Min & Max & Mean & SD & & & & & \\
\hline & \multirow[t]{3}{*}{$\begin{array}{l}\text { Chimaera } \\
\text { lignaria }\end{array}$} & Combined & 44 & 78 & 131 & 106 & 15 & 3300 & 17400 & 8623 & 3987 & 0.0018 & $\begin{array}{r}0.0005- \\
0.0067\end{array}$ & 3.28 & $\begin{array}{r}3.00- \\
3.56\end{array}$ & 0.931 \\
\hline & & Male & 15 & 84 & 119 & 100 & 12 & 3300 & 11900 & 6762 & 2572 & 0.0047 & $\begin{array}{r}0.0003- \\
0.0617\end{array}$ & 3.07 & $\begin{array}{r}2.51- \\
2.63\end{array}$ & 0.908 \\
\hline & & Female & 29 & 78 & 131 & 109 & 15 & 3460 & 17400 & 9657 & 4289 & 0.0018 & $\begin{array}{r}0.0003- \\
0.0095\end{array}$ & 3.29 & $\begin{array}{r}2.93- \\
3.65\end{array}$ & 0.932 \\
\hline & \multirow[t]{3}{*}{$\begin{array}{l}\text { Hydrolagus } \\
\text { bemisi }\end{array}$} & Combined & 26168 & 14 & 94 & 65 & 11 & 15 & 4450 & 1680 & 703 & 0.0093 & $\begin{array}{r}0.0090- \\
0.0095\end{array}$ & 2.89 & $\begin{array}{r}2.88- \\
2.89\end{array}$ & 0.968 \\
\hline & & Male & 13002 & 14 & 89 & 64 & 9 & 15 & 3930 & 1588 & 569 & 0.0093 & $\begin{array}{r}0.0090- \\
0.0097\end{array}$ & 2.88 & $\begin{array}{r}2.87- \\
2.89\end{array}$ & 0.960 \\
\hline & & Female & 13166 & 18 & 94 & 66 & 12 & 32 & 4450 & 1771 & 804 & 0.0092 & $\begin{array}{r}0.0089- \\
0.0096\end{array}$ & 2.89 & $\begin{array}{r}2.88- \\
2.89\end{array}$ & 0.973 \\
\hline & \multirow[t]{3}{*}{$\begin{array}{l}\text { Hydrolagus } \\
\text { homonycteris }\end{array}$} & Combined* & 68 & 51 & 100 & 87 & 8 & 652 & 4310 & 2879 & 658 & 0.0525 & $\begin{array}{r}0.0178- \\
0.1549\end{array}$ & 2.44 & $\begin{array}{r}2.20- \\
2.68\end{array}$ & 0.857 \\
\hline & & Male & 51 & 65 & 96 & 86 & 6 & 1320 & 3330 & 2708 & 391 & 0.5173 & $\begin{array}{r}0.1533- \\
1.7461\end{array}$ & 1.92 & $\begin{array}{r}1.65- \\
2.19\end{array}$ & 0.799 \\
\hline & & Female & 17 & 51 & 100 & 88 & 12 & 652 & 4310 & 3392 & 982 & 0.0149 & $\begin{array}{r}0.0036- \\
0.0025\end{array}$ & 2.74 & $\begin{array}{r}2.43- \\
3.06\end{array}$ & 0.955 \\
\hline & \multirow[t]{3}{*}{$\begin{array}{l}\text { Hydrolagus } \\
\text { novaezealandiae }\end{array}$} & Combined* & 27878 & 19 & 81 & 53 & 10 & 20 & 3200 & 1023 & 509 & 0.0022 & $\begin{array}{r}0.0021- \\
0.0022\end{array}$ & 3.25 & $\begin{array}{r}3.25- \\
3.26\end{array}$ & 0.979 \\
\hline & & Male & 13238 & 19 & 81 & 55 & 11 & 20 & 3200 & 1144 & 590 & 0.0020 & $\begin{array}{r}0.0019- \\
0.0021\end{array}$ & 3.27 & $\begin{array}{r}3.26- \\
3.28\end{array}$ & 0.982 \\
\hline & & Female & 14640 & 19 & 73 & 52 & 8 & 25 & 2540 & 887 & 353 & 0.0024 & $\begin{array}{r}0.0023- \\
0.0025\end{array}$ & 3.22 & $\begin{array}{r}3.21- \\
3.23\end{array}$ & 0.972 \\
\hline & \multirow[t]{3}{*}{ Hydrolagus trolli } & Combined & 33 & 53 & 119 & 91 & 20 & 700 & 8800 & 4124 & 2245 & 0.0086 & $\begin{array}{r}0.0033- \\
0.0222\end{array}$ & 2.87 & $\begin{array}{r}2.66- \\
3.08\end{array}$ & 0.960 \\
\hline & & Male & 22 & 68 & 114 & 98 & 12 & 1405 & 7220 & 4549 & 1660 & 0.0039 & $\begin{array}{r}0.0004- \\
0.0334\end{array}$ & 3.04 & $\begin{array}{r}2.57- \\
3.51\end{array}$ & 0.896 \\
\hline & & Female & 11 & 53 & 119 & 78 & 25 & 700 & 8800 & 3274 & 3025 & 0.0058 & $\begin{array}{r}0.0017- \\
0.0198\end{array}$ & 2.98 & $\begin{array}{r}2.69- \\
3.26\end{array}$ & 0.982 \\
\hline
\end{tabular}




\begin{tabular}{|c|c|c|c|c|c|c|c|c|c|c|c|c|c|c|c|c|}
\hline \multirow[t]{2}{*}{ Family } & \multirow[t]{2}{*}{ Species } & \multirow[t]{2}{*}{ Sex } & \multicolumn{5}{|c|}{ Length (cm) } & \multicolumn{4}{|c|}{ Weight (g) } & \multirow[b]{2}{*}{$a$} & \multirow[b]{2}{*}{$\begin{array}{r}95 \% \\
\text { C.I. }\end{array}$} & \multirow[b]{2}{*}{$b$} & \multirow[b]{2}{*}{$\begin{array}{r}95 \% \\
\text { C.I. }\end{array}$} & \multirow[b]{2}{*}{$r^{2}$} \\
\hline & & & $n$ & Min & $\operatorname{Max}$ & Mean & SD & Min & Max & Mean & SD & & & & & \\
\hline \multirow[t]{3}{*}{ Squalidae } & \multirow[t]{3}{*}{$\begin{array}{l}\text { Squalus } \\
\text { acanthias }\end{array}$} & Combined* & 32402 & 25 & 107 & 70 & 12 & 50 & 5995 & 1604 & 962 & 0.0009 & $\begin{array}{r}0.0009- \\
0.0010\end{array}$ & 3.36 & $\begin{array}{r}3.35- \\
3.36\end{array}$ & 0.956 \\
\hline & & Male & 12584 & 25 & 92 & 64 & 8 & 65 & 4625 & 1039 & 359 & 0.0031 & $\begin{array}{r}0.0029- \\
0.0033\end{array}$ & 3.04 & $\begin{array}{r}3.03- \\
3.06\end{array}$ & 0.934 \\
\hline & & Female & 19818 & 26 & 107 & 73 & 13 & 50 & 5995 & 1962 & 1049 & 0.0012 & $\begin{array}{r}0.0011- \\
0.0012\end{array}$ & 3.31 & $\begin{array}{r}3.30- \\
3.32\end{array}$ & 0.965 \\
\hline \multirow[t]{6}{*}{ Centrophoridae } & \multirow[t]{3}{*}{$\begin{array}{l}\text { Centrophorus } \\
\text { squamosus }\end{array}$} & Combined* & 1690 & 38 & 145 & 88 & 33 & 220 & 23600 & 6082 & 6069 & 0.0011 & $\begin{array}{r}0.0011- \\
0.0012\end{array}$ & 3.35 & $\begin{array}{r}3.34- \\
3.37\end{array}$ & 0.991 \\
\hline & & Male & 623 & 38 & 131 & 82 & 24 & 240 & 19300 & 3920 & 3189 & 0.0019 & $\begin{array}{r}0.0016- \\
0.0020\end{array}$ & 3.23 & $\begin{array}{r}3.20- \\
3.25\end{array}$ & 0.991 \\
\hline & & Female & 1067 & 38 & 145 & 91 & 36 & 220 & 23600 & 7368 & 6948 & 0.0010 & $\begin{array}{r}0.0008- \\
0.0010\end{array}$ & 3.39 & $\begin{array}{r}3.38- \\
3.41\end{array}$ & 0.992 \\
\hline & Deania calcea & Combined* & 23841 & 20 & 127 & 79 & 19 & 70 & 8700 & 2279 & 1332 & 0.0016 & $\begin{array}{r}0.0015- \\
0.0016\end{array}$ & 3.19 & $\begin{array}{r}3.18- \\
3.20\end{array}$ & 0.997 \\
\hline & & Male & 11142 & 26 & 107 & 77 & 14 & 70 & 6500 & 1920 & 595 & 0.0029 & $\begin{array}{r}0.0027- \\
0.0030\end{array}$ & 3.05 & $\begin{array}{r}3.03- \\
3.06\end{array}$ & 0.968 \\
\hline & & Female & 12699 & 20 & 127 & 81 & 22 & 75 & 8700 & 2604 & 1683 & 0.0013 & $\begin{array}{r}0.0012- \\
0.0013\end{array}$ & 3.24 & $\begin{array}{r}3.23- \\
3.25\end{array}$ & 0.983 \\
\hline \multirow[t]{6}{*}{ Etmopteridae } & \multirow[t]{3}{*}{$\begin{array}{l}\text { Etmopterus } \\
\text { granulosus }\end{array}$} & Combined* & 14892 & 17 & 86 & 60 & 12 & 25 & 3620 & 1279 & 643 & 0.0032 & $\begin{array}{r}0.0031- \\
0.0034\end{array}$ & 3.11 & $\begin{array}{r}3.11- \\
3.12\end{array}$ & 0.975 \\
\hline & & Male & 6179 & 18 & 82 & 56 & 10 & 30 & 2695 & 948 & 375 & 0.0047 & $\begin{array}{r}0.0044- \\
0.0049\end{array}$ & 3.02 & $\begin{array}{r}3.01- \\
3.03\end{array}$ & 0.972 \\
\hline & & Female & 8713 & 17 & 86 & 63 & 13 & 25 & 3620 & 1475 & 706 & 0.0030 & $\begin{array}{r}0.0028- \\
0.0031\end{array}$ & 3.14 & $\begin{array}{r}3.12- \\
3.15\end{array}$ & 0.975 \\
\hline & \multirow[t]{3}{*}{$\begin{array}{l}\text { Etmopterus } \\
\text { lucifer }\end{array}$} & Combined* & 3881 & 16 & 55 & 39 & 6 & 10 & 775 & 211 & 103 & 0.0016 & $\begin{array}{r}0.0015- \\
0.0018\end{array}$ & 3.19 & $\begin{array}{r}3.16- \\
3.21\end{array}$ & 0.945 \\
\hline & & Male & 2113 & 17 & 55 & 39 & 5 & 15 & 480 & 186 & 55 & 0.0034 & $\begin{array}{r}0.0029- \\
0.0038\end{array}$ & 2.98 & $\begin{array}{r}2.95- \\
3.02\end{array}$ & 0.932 \\
\hline & & Female & 1768 & 16 & 54 & 39 & 8 & 10 & 775 & 242 & 134 & 0.0012 & $\begin{array}{r}0.0011- \\
0.0014\end{array}$ & 3.28 & $\begin{array}{r}3.25- \\
3.31\end{array}$ & 0.959 \\
\hline
\end{tabular}




\begin{tabular}{|c|c|c|c|c|c|c|c|c|c|c|c|c|c|c|c|c|}
\hline \multirow[t]{2}{*}{ Family } & \multirow[t]{2}{*}{ Species } & \multirow[t]{2}{*}{ Sex } & \multicolumn{5}{|c|}{ Length (cm) } & \multicolumn{4}{|c|}{ Weight (g) } & \multirow[b]{2}{*}{$a$} & \multirow[b]{2}{*}{$\begin{array}{r}95 \% \\
\text { C.I. } \\
\end{array}$} & \multirow[b]{2}{*}{$b$} & \multirow[b]{2}{*}{$\begin{array}{r}95 \% \\
\text { C.I. }\end{array}$} & \multirow[b]{2}{*}{$r^{2}$} \\
\hline & & & $n$ & Min & $\operatorname{Max}$ & Mean & SD & Min & Max & Mean & SD & & & & & \\
\hline \multirow[t]{12}{*}{ Somniosidae } & \multirow[t]{3}{*}{$\begin{array}{l}\text { Centroscymпиs } \\
\text { coelolepis }\end{array}$} & Combined & 46 & 29 & 121 & 46 & 19 & 150 & 10100 & 957 & 2109 & 0.0031 & $\begin{array}{r}0.0019- \\
0.0053\end{array}$ & 3.14 & $\begin{array}{r}3.00- \\
3.27\end{array}$ & 0.979 \\
\hline & & Male & 27 & 31 & 121 & 44 & 18 & 150 & 10100 & 785 & 1932 & 0.0037 & $\begin{array}{r}0.0024- \\
0.0057\end{array}$ & 3.08 & $\begin{array}{r}2.96- \\
3.19\end{array}$ & 0.992 \\
\hline & & Female & 19 & 29 & 106 & 48 & 20 & 180 & 9100 & 1201 & 2372 & 0.0031 & $\begin{array}{r}0.0011- \\
0.0086\end{array}$ & 3.16 & $\begin{array}{r}2.89- \\
3.42\end{array}$ & 0.972 \\
\hline & \multirow[t]{3}{*}{$\begin{array}{l}\text { Centroscymnus } \\
\text { owstonii }\end{array}$} & Combined* & 1778 & 28 & 148 & 79 & 20 & 120 & 21300 & 3552 & 2730 & 0.0028 & $\begin{array}{r}0.0026- \\
0.0031\end{array}$ & 3.17 & $\begin{array}{r}3.15- \\
3.19\end{array}$ & 0.982 \\
\hline & & Male & 997 & 31 & 125 & 76 & 13 & 140 & 10600 & 2621 & 1051 & 0.0055 & $\begin{array}{r}0.0048- \\
0.0061\end{array}$ & 3.00 & $\begin{array}{r}2.97- \\
3.03\end{array}$ & 0.978 \\
\hline & & Female & 781 & 28 & 148 & 82 & 26 & 120 & 21300 & 4747 & 3615 & 0.0023 & $\begin{array}{r}0.0020- \\
0.0026\end{array}$ & 3.22 & $\begin{array}{r}3.20- \\
3.25\end{array}$ & 0.988 \\
\hline & \multirow[t]{3}{*}{$\begin{array}{l}\text { Centroselachus } \\
\text { crepidater }\end{array}$} & Combined* & 8469 & 28 & 110 & 68 & 19 & 100 & 6490 & 1821 & 1380 & 0.0021 & $\begin{array}{r}0.0020- \\
0.0022\end{array}$ & 3.18 & $\begin{array}{r}3.17- \\
3.19\end{array}$ & 0.983 \\
\hline & & Male & 3133 & 28 & 98 & 60 & 13 & 100 & 3890 & 1047 & 552 & 0.0043 & $\begin{array}{r}0.0040- \\
0.0045\end{array}$ & 3.00 & $\begin{array}{r}2.98- \\
3.01\end{array}$ & 0.980 \\
\hline & & Female & 5336 & 29 & 110 & 73 & 20 & 100 & 6490 & 2282 & 1513 & 0.0019 & $\begin{array}{r}0.0018- \\
0.0020\end{array}$ & 3.21 & $\begin{array}{r}3.20- \\
3.22\end{array}$ & 0.983 \\
\hline & \multirow[t]{3}{*}{$\begin{array}{l}\text { Scymnodon } \\
\text { plunketi }\end{array}$} & Combined* & 411 & 29 & 150 & 88 & 29 & 130 & 27100 & 5497 & 5048 & 0.0036 & $\begin{array}{r}0.0031- \\
0.0042\end{array}$ & 3.11 & $\begin{array}{r}3.08- \\
3.15\end{array}$ & 0.987 \\
\hline & & Male & 156 & 29 & 128 & 90 & 26 & 140 & 12200 & 5365 & 3553 & 0.0046 & $\begin{array}{r}0.0037- \\
0.0057\end{array}$ & 3.05 & $\begin{array}{r}3.00- \\
3.10\end{array}$ & 0.990 \\
\hline & & Female & 255 & 30 & 150 & 87 & 30 & 130 & 27100 & 5578 & 5788 & 0.0031 & $\begin{array}{r}0.0025- \\
0.0038\end{array}$ & 3.15 & $\begin{array}{r}3.10- \\
3.19\end{array}$ & 0.987 \\
\hline \multirow[t]{3}{*}{ Oxynotidae } & \multirow[t]{3}{*}{$\begin{array}{l}\text { Oxynotus } \\
\text { bruniensis }\end{array}$} & Combined & 59 & 41 & 74 & 61 & 6 & 845 & 6560 & 2720 & 1159 & 0.0015 & $\begin{array}{r}0.0003- \\
0.0073\end{array}$ & 3.48 & $\begin{array}{r}3.10- \\
3.87\end{array}$ & 0.852 \\
\hline & & Male & 27 & 41 & 65 & 57 & 5 & 845 & 2940 & 2002 & 516 & 0.0183 & $\begin{array}{r}0.0024- \\
0.1351\end{array}$ & 2.86 & $\begin{array}{r}2.36- \\
3.35\end{array}$ & 0.844 \\
\hline & & Female & 32 & 49 & 74 & 64 & 6 & 1235 & 6560 & 3327 & 1209 & 0.0015 & $\begin{array}{r}0.0001- \\
0.0230\end{array}$ & 3.50 & $\begin{array}{r}2.84- \\
4.16\end{array}$ & 0.791 \\
\hline
\end{tabular}




\begin{tabular}{|c|c|c|c|c|c|c|c|c|c|c|c|c|c|c|c|c|}
\hline \multirow[t]{2}{*}{ Family } & \multirow[t]{2}{*}{ Species } & \multirow[t]{2}{*}{ Sex } & \multicolumn{7}{|c|}{ Length (cm) } & \multicolumn{2}{|c|}{ Weight (g) } & \multirow[b]{2}{*}{$a$} & \multirow[b]{2}{*}{$\begin{array}{r}95 \% \\
\text { C.I. }\end{array}$} & \multirow[b]{2}{*}{$b$} & \multirow[b]{2}{*}{$\begin{array}{r}95 \% \\
\text { C.I. }\end{array}$} & \multirow[b]{2}{*}{$r^{2}$} \\
\hline & & & $n$ & Min & Max & Mean & SD & Min & Max & Mean & SD & & & & & \\
\hline \multirow[t]{3}{*}{ Dalatiidae } & Dalatias licha & Combined* & 896 & 36 & 154 & 66 & 29 & 195 & 31500 & 2797 & 5149 & 0.0014 & $\begin{array}{r}0.0013- \\
0.0015\end{array}$ & 3.30 & $\begin{array}{r}3.28- \\
3.32\end{array}$ & 0.991 \\
\hline & & Male & 351 & 36 & 143 & 61 & 23 & 200 & 15700 & 1716 & 2558 & 0.0018 & $\begin{array}{r}0.0015- \\
0.0021\end{array}$ & 3.23 & $\begin{array}{r}3.19- \\
3.27\end{array}$ & 0.987 \\
\hline & & Female & 545 & 37 & 154 & 69 & 32 & 195 & 31500 & 3505 & 6194 & 0.0012 & $\begin{array}{r}0.0011- \\
0.0013 \\
\end{array}$ & 3.33 & $\begin{array}{r}3.31- \\
3.36 \\
\end{array}$ & 0.992 \\
\hline
\end{tabular}


Table 2.5. For species where a significant difference of LWR was found between males and females, weight estimates (g) for lengths of a 30, 50, 70, and $100 \mathrm{~cm}$ fish (total length, $L_{\mathrm{T}}$, for squaloids; chimaera length, $L_{\mathrm{C}}$, for chimaeroids), as well as weight estimates $(\mathrm{g})$ for each sex at mean observed length $(\mathrm{cm})$. Weight estimates for 70 and 100 $\mathrm{cm}$ lengths only given for species that reach that length or greater.

\begin{tabular}{|c|c|c|c|c|c|c|c|}
\hline \multirow[t]{2}{*}{ Family } & \multirow[t]{2}{*}{ Species } & \multicolumn{4}{|c|}{$\begin{array}{l}\text { Weight (g) at given length } \\
\text { measurement }(\mathrm{cm})\end{array}$} & \multirow{2}{*}{$\begin{array}{c}\text { Mean } \\
\text { length } \\
(\mathrm{cm})\end{array}$} & \multirow{2}{*}{$\begin{array}{c}\text { Weight } \\
\text { (g) at } \\
\text { mean } \\
\text { length }\end{array}$} \\
\hline & & 30 & 50 & 70 & 100 & & \\
\hline \multirow[t]{9}{*}{ Rhinochimaeridae } & Harriotta haeckeli & & & & & & \\
\hline & Male & 148 & 418 & 828 & - & 49.7 & 413 \\
\hline & Female & 42 & 297 & 1071 & - & 69.6 & 1046 \\
\hline & Harriotta raleighana & & & & & & \\
\hline & Male & 96 & 395 & 1007 & 2712 & 64.8 & 812 \\
\hline & Female & 86 & 409 & 1137 & 3365 & 71.8 & 1228 \\
\hline & Rhinochimaera pacifica & & & & & & \\
\hline & Male & 50 & 224 & 605 & 1730 & 114.4 & 2573 \\
\hline & Female & 37 & 193 & 569 & 1788 & 120.9 & 3288 \\
\hline \multirow[t]{9}{*}{ Chimaeridae } & Chimaera carophila & & & & & & \\
\hline & Male & 215 & 726 & 1618 & 3781 & 77.8 & 2079 \\
\hline & Female & 174 & 695 & 1731 & 4550 & 76.7 & 2219 \\
\hline & Hydrolagus homonycteris & & & & & & \\
\hline & Male & 353 & 942 & 1797 & 3562 & 84.4 & 2572 \\
\hline & Female & 168 & 681 & 1715 & 4560 & 88.4 & 3252 \\
\hline & Hydrolagus novaezealandiae & & & & & & \\
\hline & Male & 137 & 730 & 2193 & - & 54.7 & 979 \\
\hline & Female & 140 & 728 & 2154 & - & 51.5 & 801 \\
\hline \multirow[t]{3}{*}{ Squalidae } & Squalus acanthias & & & & & & \\
\hline & Male & 98 & 465 & 1295 & - & 64.0 & 987 \\
\hline & Female & 91 & 492 & 1497 & - & 73.3 & 1747 \\
\hline \multirow[t]{6}{*}{ Centrophoridae } & Centrophorus squamosus & & & & & & \\
\hline & Male & 109 & 568 & 1683 & 5322 & 82.0 & 2803 \\
\hline & Female & 100 & 564 & 1769 & 5936 & 91.3 & 4362 \\
\hline & Deania calcea & & & & & & \\
\hline & Male & 91 & 434 & 1209 & 3583 & 77.3 & 1635 \\
\hline & Female & 81 & 421 & 1252 & 3973 & 81.3 & 2033 \\
\hline \multirow[t]{6}{*}{ Etmopteridae } & Etmopterus granulosus & & & & & & \\
\hline & Male & 134 & 626 & 1729 & - & 55.9 & 879 \\
\hline & Female & 129 & 641 & 1843 & - & 63.0 & 1324 \\
\hline & Etmopterus lucifer & & & & & & \\
\hline & Male & 85 & 390 & - & - & 38.5 & 179 \\
\hline & Female & 86 & 460 & - & - & 39.0 & 203 \\
\hline \multirow[t]{9}{*}{ Somniosidae } & Centroscymnus owstonii & & & & & & \\
\hline & Male & 148 & 688 & 1888 & 5509 & 76.1 & 2429 \\
\hline & Female & 134 & 695 & 2056 & 6486 & 82.4 & 3471 \\
\hline & Centroselachus crepidater & & & & & & \\
\hline & Male & 115 & 533 & 1461 & 4259 & 60.0 & 919 \\
\hline & Female & 105 & 540 & 1589 & 4988 & 72.5 & 1780 \\
\hline & Scymnodon plunketi & & & & & & \\
\hline & Male & 148 & 704 & 1965 & 5833 & 90.2 & 4252 \\
\hline & Female & 139 & 695 & 2003 & 6151 & 86.9 & 3949 \\
\hline \multirow[t]{3}{*}{ Dalatiidae } & Dalatias licha & & & & & & \\
\hline & Male & 109 & 568 & 1684 & 5334 & 60.8 & 1067 \\
\hline & Female & 102 & 562 & 1723 & 5651 & 69.0 & 1641 \\
\hline
\end{tabular}




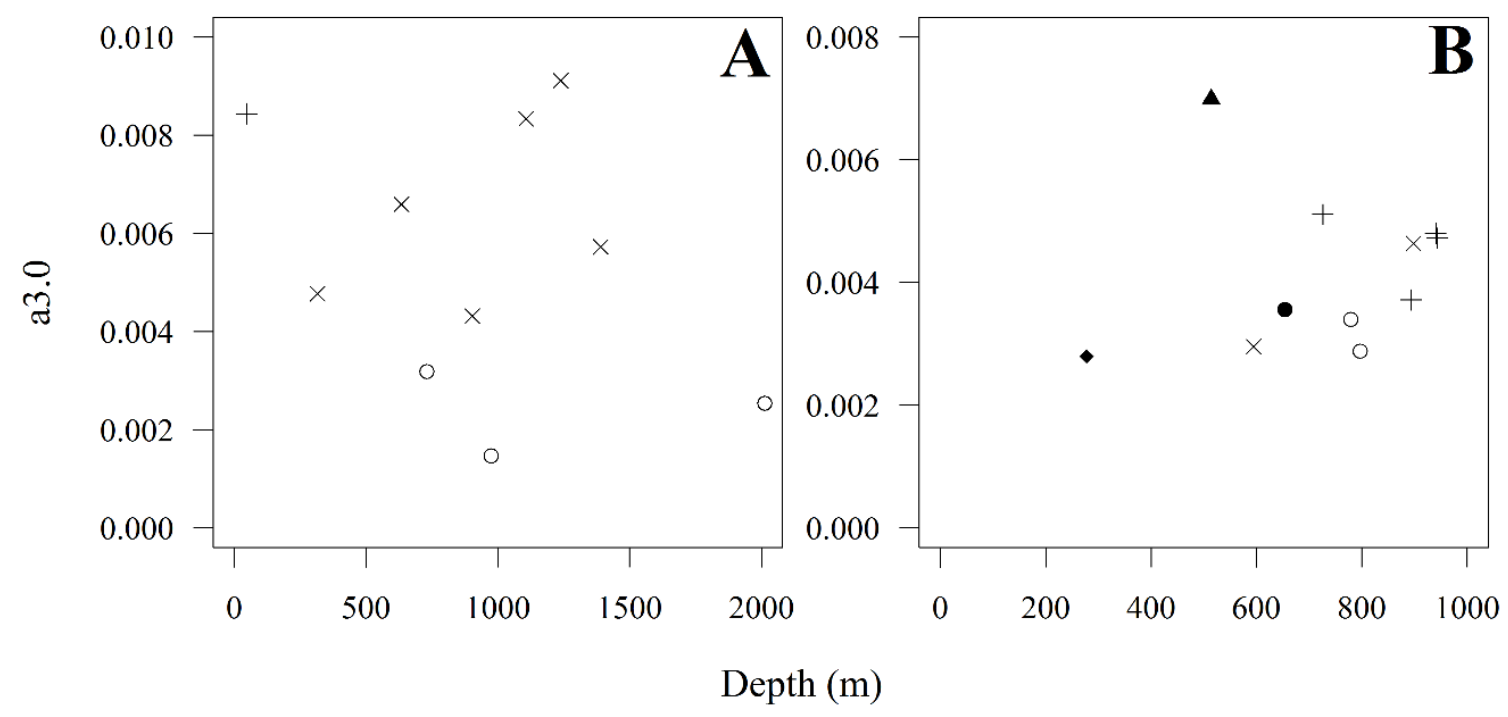

Figure 2.2 Variation in form factor $\left(a_{3.0}\right)$ with mean depth $(\mathrm{m})$ by family for $(\mathrm{A})$ chimaeroids (Callorhinchidae, +; Chimaeridae, $\times$; Rhinochimaeridae, $\circ$ ) and (B) squaloids (Squalidae, $\$$; Centrophoridae, ०; Etmopteridae, $\times$; Somniosidae +; Oxynotidae, ^; Dalatiidae, •). 
Table 2.6. Comparison of weight (g) estimates using LWR parameters from FishBase and this study at mean species length $(\mathrm{cm})$ (total length, $L_{\mathrm{T}}$, for squaloids; chimaera length, $L_{\mathrm{C}}$, for chimaeroids). FishBase LWR estimate accuracy was described as under- $(\downarrow)$ or overestimating $(\uparrow)$ fish size relative to estimates in this study, or providing similar results $(<10 \%$ difference) $(-)$. $*$ Significant difference $(P<0 \cdot 05)$. Refer to Table 2.3 for sample sizes.

\begin{tabular}{|c|c|c|c|c|c|}
\hline Family & Species & $\begin{array}{l}\text { Mean } \\
\text { length } \\
(\mathrm{cm})\end{array}$ & $\begin{array}{c}\text { FishBase } \\
\text { (g) }\end{array}$ & $\begin{array}{c}\text { This } \\
\text { study } \\
(\mathrm{g})\end{array}$ & $\begin{array}{l}\text { FishBase } \\
\text { estimate } \\
\text { accuracy }\end{array}$ \\
\hline \multirow[t]{3}{*}{ Rhinochimaeridae } & Harriotta haeckeli & 62.7 & 1575 & 911 & $\downarrow^{*}$ \\
\hline & Harriotta raleighana & 68.5 & 2076 & 1010 & $\uparrow^{*}$ \\
\hline & Rhinochimaera pacifica & 117.1 & 11062 & 2859 & $\uparrow^{*}$ \\
\hline \multirow[t]{5}{*}{ Chimaeridae } & Chimaera lignaria & 102.9 & 5602 & 7173 & $\downarrow^{*}$ \\
\hline & Hydrolagus bemisi & 64.7 & 1604 & 1591 & $-*$ \\
\hline & Hydrolagus homonycteris & 85.6 & 3165 & 2728 & $\uparrow^{*}$ \\
\hline & Hydrolagus novaezealandiae & 53.2 & 670 & 891 & $\downarrow^{*}$ \\
\hline & Hydrolagus trolli & 90.3 & 3735 & 3527 & - \\
\hline Squalidae & Squalus acanthias & 69.7 & 1266 & 1412 & $-*$ \\
\hline \multirow{2}{*}{ Centrophoridae } & Centrophorus squamosus & 87.9 & 3825 & 3724 & $-*$ \\
\hline & Deania calcea & 79.4 & 2024 & 1832 & $-*$ \\
\hline \multirow[t]{2}{*}{ Etmopteridae } & Etmopterus granulosus & 60.1 & 1155 & 1125 & $-*$ \\
\hline & Etmopterus lucifer & 38.7 & 294 & 189 & $\uparrow^{*}$ \\
\hline \multirow[t]{4}{*}{ Somniosidae } & Centroscymnus coelolepis & 55.9 & 1600 & 950 & $\uparrow^{*}$ \\
\hline & Centroscymnus owstonii & 78.9 & 4010 & 2850 & $\uparrow^{*}$ \\
\hline & Centroselachus crepidater & 67.9 & 2533 & 1414 & $\uparrow^{*}$ \\
\hline & Scymnodon plunketi & 88.1 & 5865 & 4060 & $\uparrow^{*}$ \\
\hline Oxynotidae & Oxynotus bruniensis & 61.0 & 1445 & 2551 & $\downarrow^{*}$ \\
\hline Dalatiidae & Dalatias licha & 65.8 & 1707 & 1395 & $\uparrow^{*}$ \\
\hline
\end{tabular}


Figure 2.3 LWR $\left(W=a L^{b}\right)$ (left panel) and $\log$-transformed LWR $[\log (W)=\log (a)+b$ $\times \log (L)]$ (right panel) for (A) Rhinochimaera pacifica (overestimation from FishBase parameters) (B) Oxynotus bruniensis (underestimation from FishBase parameters) and (C) Hydrolagus trolli (under- and overestimation from FishBase parameters) with fitted LWR from this study (red) and FishBase LWR estimate (blue). See Appendix I for additional plots.
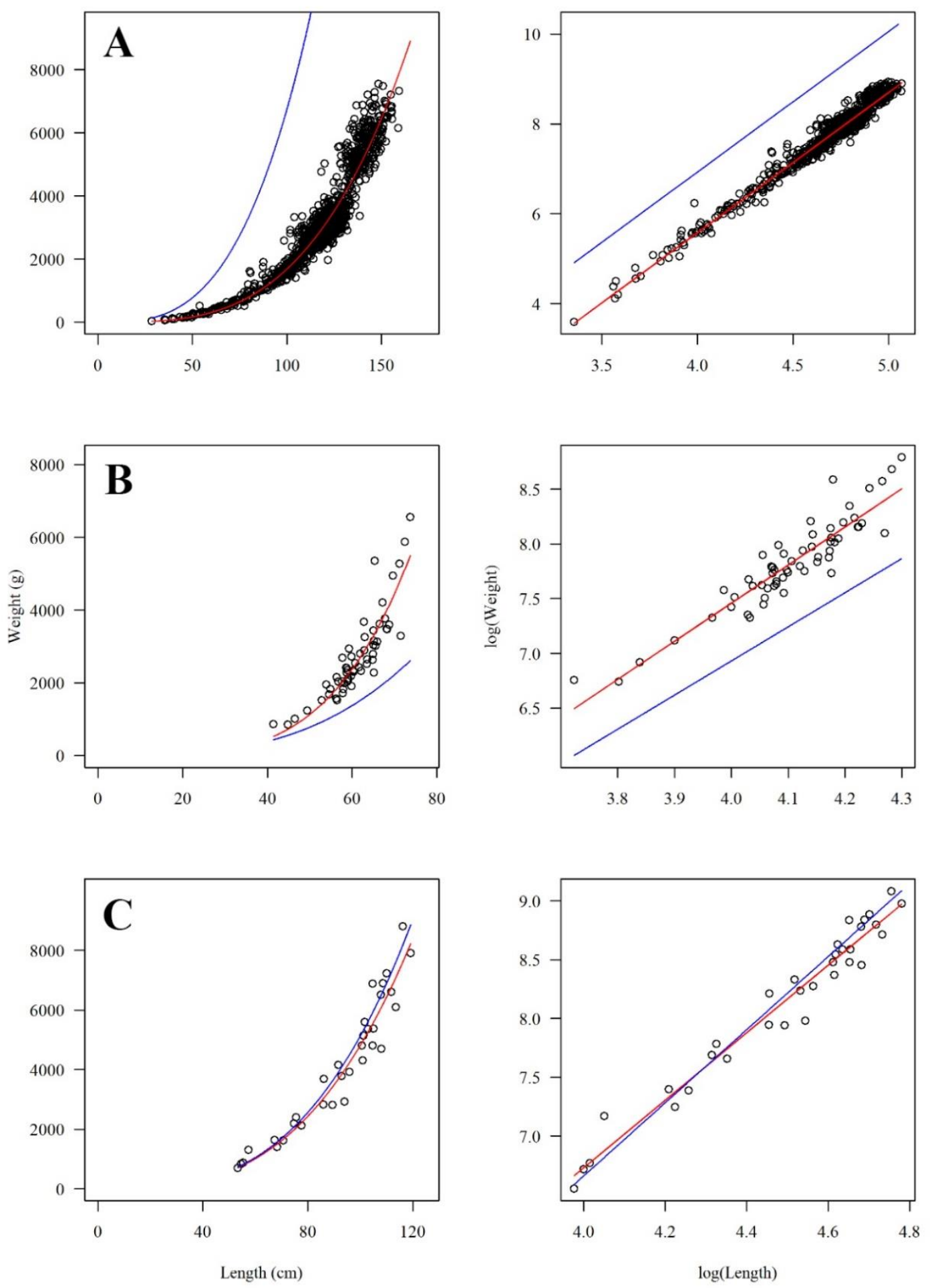
Figure 2.4 LWR $\left(W=a L^{b}\right)$ (left panel), $\log$-transformed LWR $[\log (W)=\log (a)+b \times$ $\log (L)]$ (middle panel), and in logspace, the frequency of distribution of weight at mean observed length (right panel), for (A) Hydrolagus bemisi, (B) Squalus acanthias, (C) Centrophorus squamosus, (D) Deania calcea, and (E) Etmopterus granulosus, with fitted LWR from this study (red) and FishBase LWR estimate (blue). Dashed line indicates \pm one standard deviation (S.D.) of weight at mean observed length.
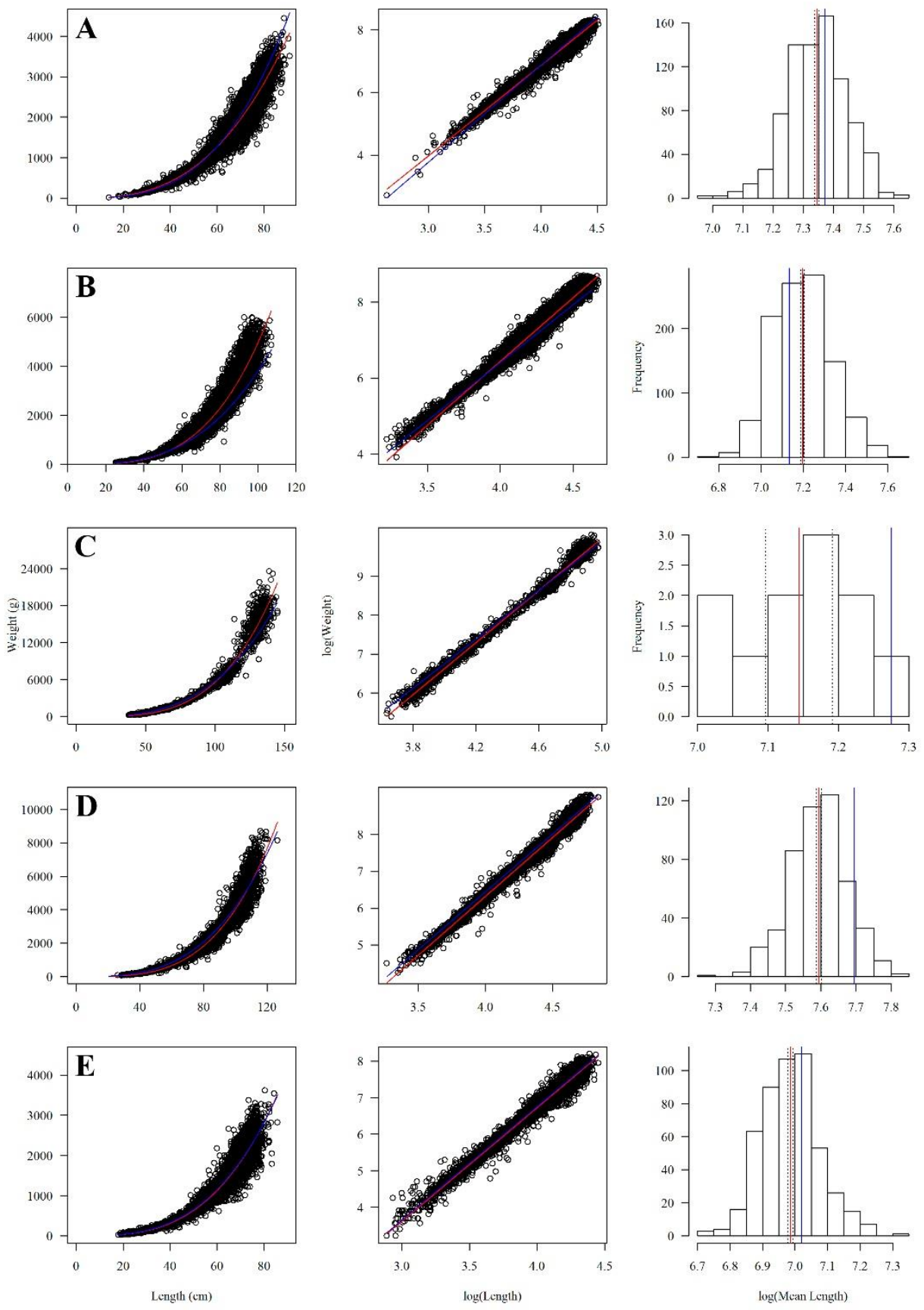

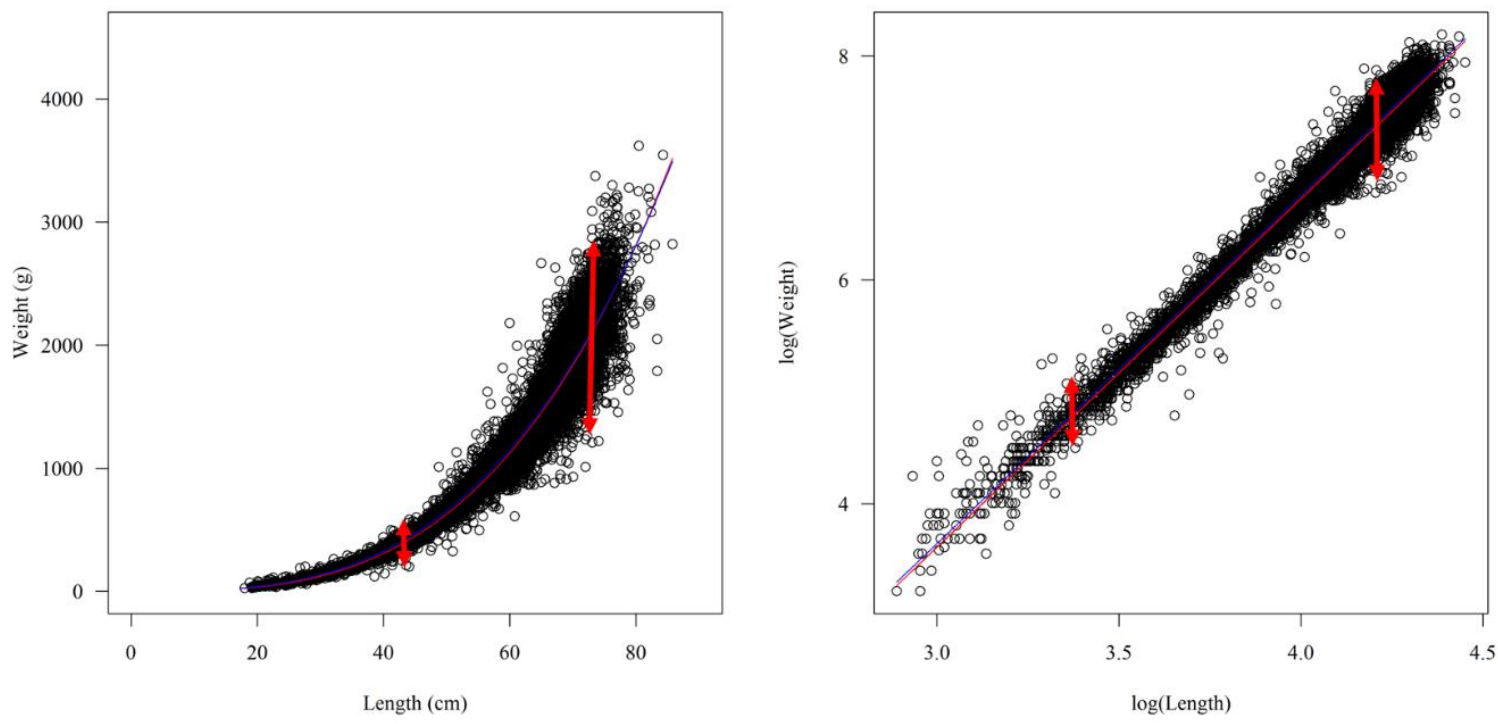

Figure 2.5 An example of suspected increase in variance of weight at length with increasing length. LWR $\left(W=a L^{b}\right)$ and $\log$-transformed LWR $[\log (W)=\log (a)+b \times \log$ $(L)]$ for combined sex Etmopterus granulosus ( $\uparrow$, indication of variability in weight at length). 
Table 2.7. Relative AIC values for the four models (Linear mean \& Constant variance, $\mathrm{L}+\mathrm{CV}$; Broken stick mean \& Constant variance, BS+CV; Linear mean \& Variance shelf, L+VS; Broken stick mean \& Variance shelf, BS+VS).

\begin{tabular}{|c|c|c|c|c|c|c|}
\hline \multirow[b]{2}{*}{ Species } & \multirow[b]{2}{*}{ Sex } & \multirow[b]{2}{*}{$n$} & \multicolumn{4}{|c|}{ Model selection } \\
\hline & & & $\mathrm{L}+\mathrm{CV}$ & $\mathrm{BS}+\mathrm{CV}$ & $\mathbf{L}+\mathbf{V S}$ & BS+VS \\
\hline \multicolumn{7}{|l|}{ Chimaeriformes } \\
\hline \multirow[t]{2}{*}{ Harriotta raleighana } & M & 2210 & 135.2 & 72.7 & 10.0 & 0.0 \\
\hline & $\mathrm{F}$ & 2219 & 20.2 & 6.6 & 19.9 & 0.0 \\
\hline \multirow[t]{2}{*}{ Rhinochimaera pacifica } & M & 741 & 120.6 & 0.0 & 31.7 & 35.5 \\
\hline & $\mathrm{F}$ & 528 & 37.3 & 19.4 & 0.0 & 4.0 \\
\hline \multirow[t]{2}{*}{ Chimaera carophila } & M & 104 & 33.9 & 24.9 & 37.9 & 0.0 \\
\hline & $\mathrm{F}$ & 55 & 4.1 & 6.9 & 0.0 & 12.1 \\
\hline \multirow[t]{2}{*}{ Hydrolagus bemisi } & M & 12715 & 150.8 & 0.0 & 90.7 & 114.2 \\
\hline & $\mathrm{F}$ & 12657 & 62.7 & 0.0 & 64.8 & 46.8 \\
\hline \multirow[t]{2}{*}{ Hydrolagus homonycteris } & $\mathrm{M}$ & 51 & 43.6 & 19.1 & 32.2 & 0.0 \\
\hline & $\mathrm{F}$ & 17 & 323.4 & 0.0 & 272.1 & 4.0 \\
\hline \multirow[t]{2}{*}{ Hydrolagus novaezealandiae } & $\mathrm{M}$ & 10959 & 625.3 & 199.7 & 452.5 & 0.0 \\
\hline & $\mathrm{F}$ & 12193 & 74.4 & 27.7 & 0.0 & 31.7 \\
\hline \multicolumn{7}{|l|}{ Squaliformes } \\
\hline \multirow[t]{2}{*}{ Squalus acanthias } & M & 19818 & 43.6 & 19.1 & 32.2 & 0.0 \\
\hline & $\mathrm{F}$ & 12584 & 323.4 & 0.0 & 272.1 & 4.0 \\
\hline \multirow[t]{2}{*}{ Centrophorus squamosus } & M & 614 & 7.1 & 0.0 & 9.2 & 9.4 \\
\hline & $\mathrm{F}$ & 1033 & 86.3 & 22.2 & 74.3 & 0.0 \\
\hline \multirow[t]{2}{*}{ Deania calcea } & M & 7019 & 333.3 & 0.0 & 320.4 & 291.8 \\
\hline & $\mathrm{F}$ & 7771 & 628.2 & 233.2 & 136.8 & 0.0 \\
\hline \multirow[t]{2}{*}{ Etmopterus granulosus } & M & 5439 & 200.0 & 71.2 & 0.0 & 114.4 \\
\hline & $\mathrm{F}$ & 7241 & 129.4 & 63.3 & 49.7 & 0.0 \\
\hline \multirow[t]{2}{*}{ Etmopterus lucifer } & M & 2026 & 83.0 & 0.0 & 87.0 & 4.0 \\
\hline & $\mathrm{F}$ & 1675 & 99.6 & 92.2 & 91.6 & 0.0 \\
\hline \multirow[t]{2}{*}{ Centroscymnus owstonii } & M & 972 & 52.0 & 38.2 & 37.4 & 0.0 \\
\hline & $\mathrm{F}$ & 757 & 92.8 & 39.6 & 62.6 & 0.0 \\
\hline \multirow[t]{2}{*}{ Centroselachus crepidater } & M & 445 & 81.9 & 0.0 & 31.4 & 48.4 \\
\hline & $\mathrm{F}$ & 1284 & 332.5 & 13.8 & 143.2 & 0.0 \\
\hline \multirow[t]{2}{*}{ Centroselachus crepidater 2} & M & 357 & 26.2 & 11.6 & 9.3 & 0.0 \\
\hline & $\mathrm{F}$ & 978 & 31.2 & 0.0 & 22.7 & 19.3 \\
\hline \multirow[t]{2}{*}{ Scymnodon plunketi } & $\mathrm{M}$ & 154 & 1.7 & 4.5 & 0.0 & 9.0 \\
\hline & $\mathrm{F}$ & 249 & 28.0 & 8.7 & 11.2 & 0.0 \\
\hline \multirow[t]{2}{*}{ Oxynotus bruniensis } & $\mathrm{M}$ & 27 & 0.0 & 2.3 & 3.4 & 6.5 \\
\hline & $\mathrm{F}$ & 32 & 0.0 & 1.8 & 0.9 & 5.6 \\
\hline \multirow[t]{2}{*}{ Dalatias licha } & $\mathrm{M}$ & 344 & 0.4 & 0.0 & 1.4 & 1.6 \\
\hline & $\mathrm{F}$ & 525 & 28.4 & 5.5 & 14.5 & 0.0 \\
\hline
\end{tabular}


Table 2.8. Estimates of inflection points, confidence intervals, and significance of the variance $(\alpha)$ and mean $(\beta)$ for each species by the best fitting model as determined by AIC. Additional results in Appendix II.

\begin{tabular}{|c|c|c|c|c|c|c|c|c|c|c|c|}
\hline \multirow{2}{*}{$\begin{array}{l}\text { Species } \\
\text { Chimaeriformes }\end{array}$} & \multirow[t]{2}{*}{ Sex } & \multirow[t]{2}{*}{$\begin{array}{l}\text { Inflection point } \\
\text { of variance }(\alpha)\end{array}$} & \multicolumn{2}{|c|}{ Confidence Interval } & \multirow[t]{2}{*}{ p-value } & \multirow[t]{2}{*}{ Variance $(\mathbf{c m})$} & \multirow[t]{2}{*}{$\begin{array}{c}\text { Inflection point } \\
\text { of mean }(\boldsymbol{\beta}) \\
\end{array}$} & \multicolumn{2}{|c|}{ Confidence Interval } & \multirow[t]{2}{*}{ p-value } & \multirow[t]{2}{*}{ Mean $(\mathrm{cm})$} \\
\hline & & & & & & & & & & & \\
\hline \multirow[t]{2}{*}{ Harriotta raleighana } & M & 4.33 & 4.32 & 4.35 & $<0.001$ & 76.1 & 3.69 & 3.65 & 3.74 & $<0.001$ & 40.1 \\
\hline & $\mathrm{F}$ & 3.58 & 3.54 & 3.61 & $<0.001$ & 35.8 & 3.89 & 3.83 & 3.95 & $<0.001$ & 48.9 \\
\hline \multirow[t]{2}{*}{ Rhinochimaera pacifica } & M & - & - & - & - & - & 4.74 & 4.73 & 4.75 & $<0.001$ & 114.1 \\
\hline & $\mathrm{F}$ & 4.91 & 4.89 & 4.93 & $<0.001$ & 135.7 & 3.20 & 3.16 & 3.25 & $<0.001$ & 24.6 \\
\hline \multirow[t]{2}{*}{ Chimaera carophila } & M & 4.26 & 4.24 & 4.28 & & 70.8 & - & - & - & - & - \\
\hline & $\mathrm{F}$ & 4.26 & 4.20 & 4.31 & $<0.001$ & 70.5 & - & - & - & - & - \\
\hline \multirow[t]{2}{*}{ Hydrolagus bemisi } & M & - & - & - & - & - & 4.26 & 4.25 & 4.26 & $<0.001$ & 70.6 \\
\hline & $\mathrm{F}$ & - & - & - & - & - & 4.23 & 4.21 & 4.25 & $<0.001$ & 68.8 \\
\hline \multirow[t]{2}{*}{ Hydrolagus homonycteris } & M & - & - & - & - & - & 4.41 & 4.39 & 4.42 & $<0.001$ & 82.0 \\
\hline & $\mathrm{F}$ & - & - & - & - & - & 4.45 & 4.33 & 4.58 & $<0.001$ & 85.8 \\
\hline \multirow[t]{2}{*}{ Hydrolagus novaezealandiae } & M & 3.70 & 3.69 & 3.71 & $<0.001$ & 40.4 & 4.01 & 4.00 & 4.01 & $<0.001$ & 55.0 \\
\hline & $\mathrm{F}$ & 3.58 & 3.53 & 3.63 & $<0.001$ & 35.8 & - & - & - & - & - \\
\hline \multicolumn{12}{|l|}{ Squaliformes } \\
\hline \multirow[t]{2}{*}{ Squalus acanthias } & M & 4.46 & 4.43 & 4.49 & $<0.001$ & 86.8 & 3.85 & 3.81 & 3.89 & $<0.001$ & 47.1 \\
\hline & $\mathrm{F}$ & - & - & - & - & - & 3.84 & 3.81 & 3.87 & $<0.001$ & 46.6 \\
\hline \multirow[t]{2}{*}{ Centrophorus squamosus } & M & - & - & - & - & - & 3.80 & 3.77 & 3.84 & $<0.001$ & 44.8 \\
\hline & $\mathrm{F}$ & 4.63 & 4.58 & 4.69 & $<0.001$ & 102.8 & 4.45 & 4.41 & 4.49 & $<0.001$ & 85.7 \\
\hline \multirow[t]{2}{*}{ Deania calcea } & M & - & - & - & - & - & 4.38 & 4.37 & 4.40 & $<0.001$ & 80.1 \\
\hline & $\mathrm{F}$ & 4.60 & 4.59 & 4.61 & $<0.001$ & 99.4 & 3.95 & 3.92 & 3.97 & $<0.001$ & 42.7 \\
\hline \multirow[t]{2}{*}{ Etmopterus granulosus } & M & 3.79 & 3.77 & 3.81 & $<0.001$ & 44.2 & - & - & - & - & - \\
\hline & $\mathrm{F}$ & 4.34 & 4.33 & 4.35 & $<0.001$ & 77.0 & 3.60 & 3.54 & 3.65 & $<0.001$ & 36.5 \\
\hline \multirow[t]{2}{*}{ Etmopterus lucifer } & M & - & - & - & - & - & 3.61 & 3.60 & 3.63 & $<0.001$ & 37.0 \\
\hline & $\mathrm{F}$ & 3.33 & 3.27 & 3.38 & $<0.001$ & 27.8 & - & - & - & - & - \\
\hline
\end{tabular}




\begin{tabular}{|c|c|c|c|c|c|c|c|c|c|c|c|}
\hline \multirow{2}{*}{$\begin{array}{l}\text { Species } \\
\text { Centroscymnus owstonii }\end{array}$} & \multirow{2}{*}{$\begin{array}{r}\text { Sex } \\
\mathrm{M}\end{array}$} & \multirow{2}{*}{$\begin{array}{c}\begin{array}{c}\text { Inflection point } \\
\text { of variance }(\boldsymbol{\alpha})\end{array} \\
4.48\end{array}$} & \multicolumn{2}{|c|}{ Confidence Interval } & \multirow{2}{*}{$\begin{array}{c}\text { p-value } \\
<0.001\end{array}$} & \multirow{2}{*}{$\begin{array}{c}\text { Variance (cm) } \\
88.3\end{array}$} & \multirow{2}{*}{$\begin{array}{c}\begin{array}{c}\text { Inflection point } \\
\text { of mean }(\boldsymbol{\beta})\end{array} \\
4.03\end{array}$} & \multicolumn{2}{|c|}{ Confidence Interval } & \multirow{2}{*}{$\begin{array}{l}\text { p-value } \\
<0.001\end{array}$} & \multirow{2}{*}{$\begin{array}{c}\text { Mean }(\mathrm{cm}) \\
56.3\end{array}$} \\
\hline & & & 4.46 & 4.50 & & & & 3.98 & 4.08 & & \\
\hline & $\mathrm{F}$ & 4.59 & 4.56 & 4.62 & $<0.001$ & 98.5 & 4.41 & 4.37 & 4.44 & $<0.001$ & 82.1 \\
\hline \multirow[t]{2}{*}{ Centroselachus crepidater } & M & - & - & - & - & - & 4.45 & 4.41 & 4.49 & $<0.001$ & 85.4 \\
\hline & $\mathrm{F}$ & 4.46 & 4.45 & 4.48 & $<0.001$ & 86.8 & 4.40 & 4.38 & 4.42 & $<0.001$ & 81.6 \\
\hline \multirow[t]{2}{*}{ Centroselachus crepidater 2} & M & - & - & - & - & - & 3.72 & 3.68 & 3.75 & $<0.001$ & 41.2 \\
\hline & $\mathrm{F}$ & - & - & - & - & - & 4.31 & 4.28 & 4.35 & $<0.001$ & 74.7 \\
\hline \multirow[t]{2}{*}{ Scymnodon plunketi } & M & 4.32 & 4.24 & 4.39 & $<0.001$ & 74.9 & - & - & - & - & - \\
\hline & $\mathrm{F}$ & 4.83 & 4.79 & 4.86 & $<0.001$ & 125.2 & 4.12 & 4.04 & 4.21 & $<0.001$ & 61.8 \\
\hline \multirow[t]{2}{*}{ Oxynotus bruniensis } & M & - & - & - & - & - & - & - & - & - & - \\
\hline & $\mathrm{F}$ & - & - & - & - & - & - & - & - & - & - \\
\hline \multirow[t]{2}{*}{ Dalatias licha } & M & - & - & - & - & - & 3.74 & 3.70 & 3.78 & $<0.001$ & 42.1 \\
\hline & $\mathrm{F}$ & 4.61 & 4.55 & 4.67 & $<0.001$ & 100.8 & 3.77 & 3.75 & 3.79 & $<0.001$ & 43.3 \\
\hline
\end{tabular}




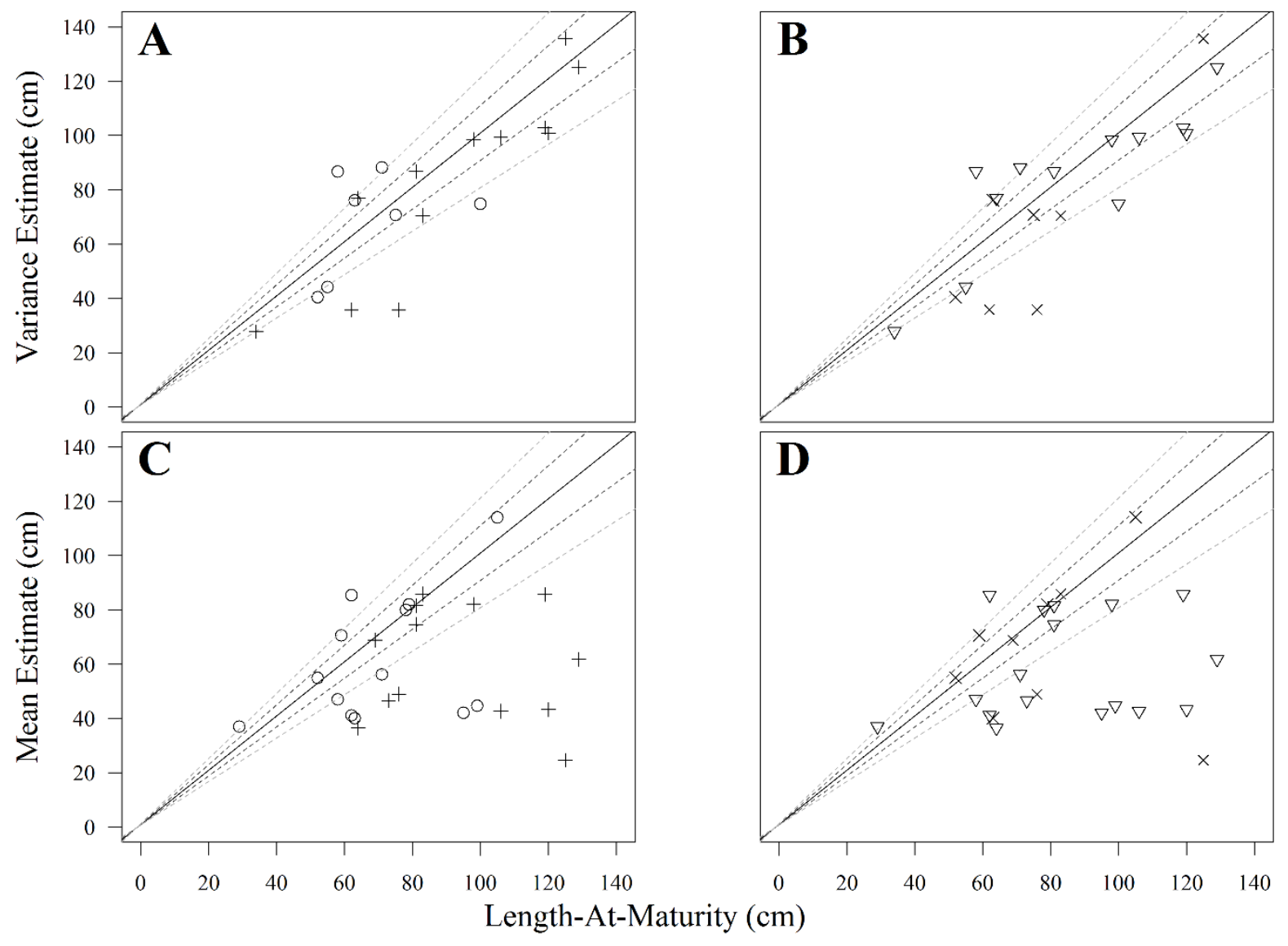

Figure 2.6 Correlation between length-at-maturity $(\mathrm{cm})$ and variance estimate $(\mathrm{cm})$ by (A) $\operatorname{sex}(\circ$, male; + , female) and (B) order ( $\times$, chimaeroids, $\nabla$, squaloids); and correlation between length-at-maturity $(\mathrm{cm})$ and mean estimate $(\mathrm{cm})$ by $(\mathrm{C})$ sex $(\circ$, male; + , female) and (D) order ( $\times$, chimaeroids, $\nabla$, squaloids). $\vdots, 10 \%$ and $20 \%$ margins of length estimates. Most estimates (c. 75\%) of the variance inflection point were within $20 \%$ of reported length-at-maturity (A, B), and were more accurate for length-at-maturity for females (over males, A) and squaloids (over chimaeroids, B). Approximately half of the estimates of the mean inflection point were beyond a margin of $20 \%$ of reported lengthat-maturity $(\mathrm{C}, \mathrm{D})$, and were more accurate for length-at-maturity for males (over females, C) and chimaeroids (over squaloids, D). 

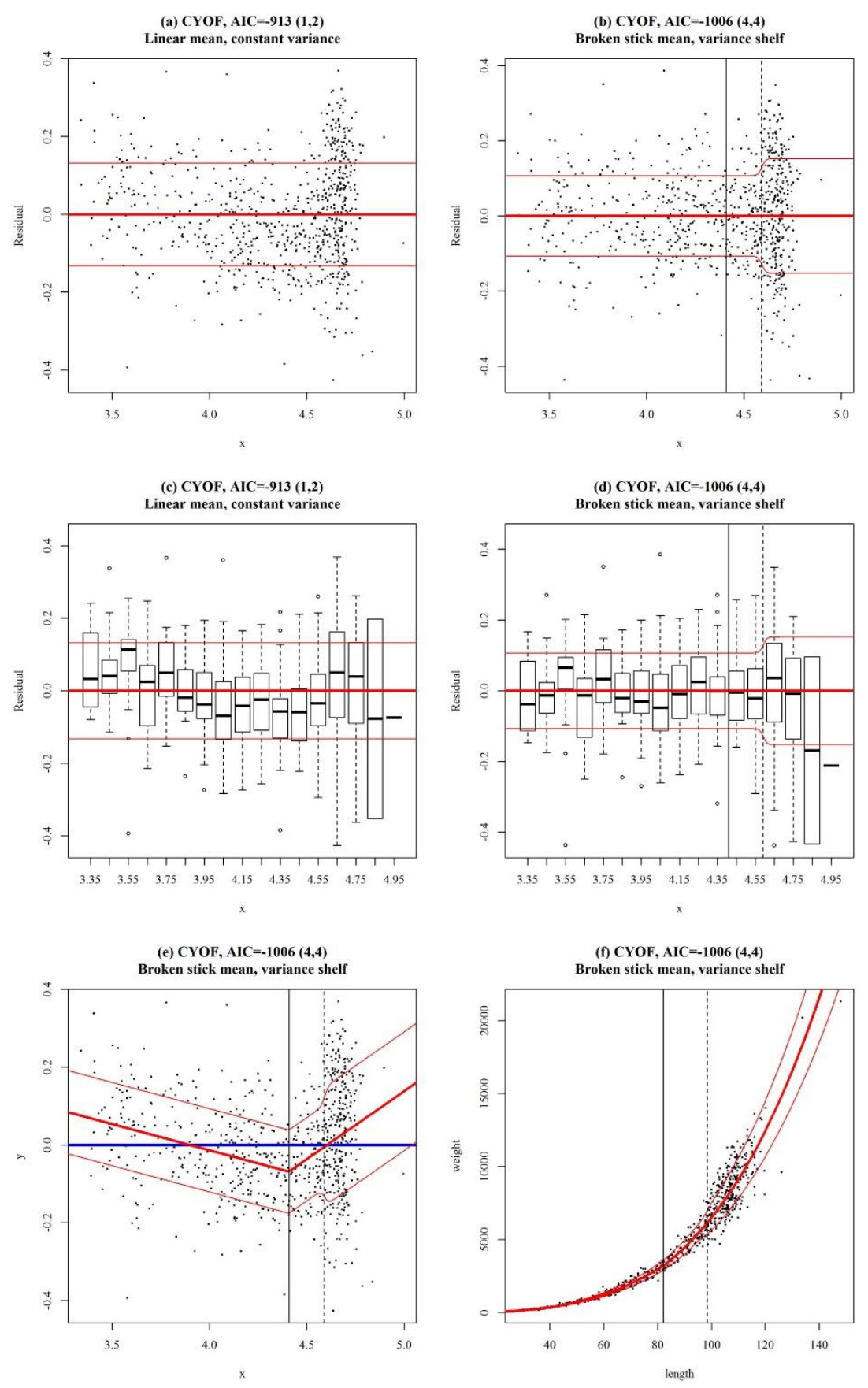

Figure 2.7 Linear and best fitting model for female Centroscymnus owstonii (CYO). Top left panel: linear model with constant variance; top right panel: residuals for the best fitting model from a linear model with constant variance (horizontal lines show the mean with \pm 1 standard deviations); middle two panels: same data summarised by boxplots; bottom left panel: the same as top left, with residuals from the linear model, but with the mean and \pm 1 standard deviation superimposed; bottom right: fitted model plotted on top of the original data on the original linear scale. $\vdots$, inflection point of the variance shelf $(\alpha)$; I, inflection point of the broken stick $(\beta)$. 

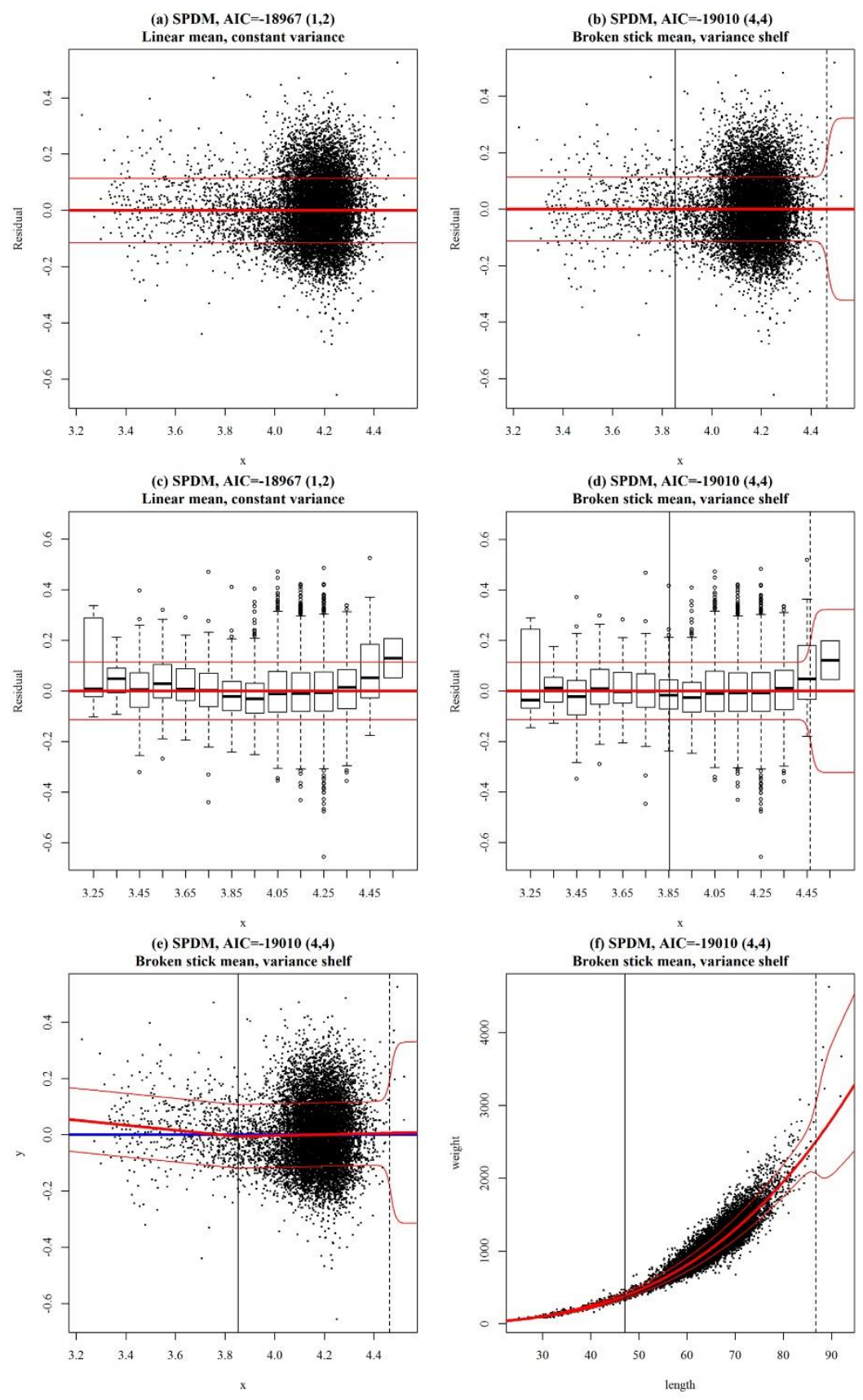

Figure 2.8 Linear and best fitting model for male Squalus acanthias (SPD). Top left panel: linear model with constant variance; top right panel: residuals for the best fitting model from a linear model with constant variance (horizontal lines show the mean with \pm 1 standard deviations); middle two panels: same data summarised by boxplots; bottom left panel: the same as top left, with residuals from the linear model, but with the mean and \pm 1 standard deviation superimposed; bottom right: fitted model plotted on top of the original data on the original linear scale. inflection point of the broken stick $(\beta)$. 

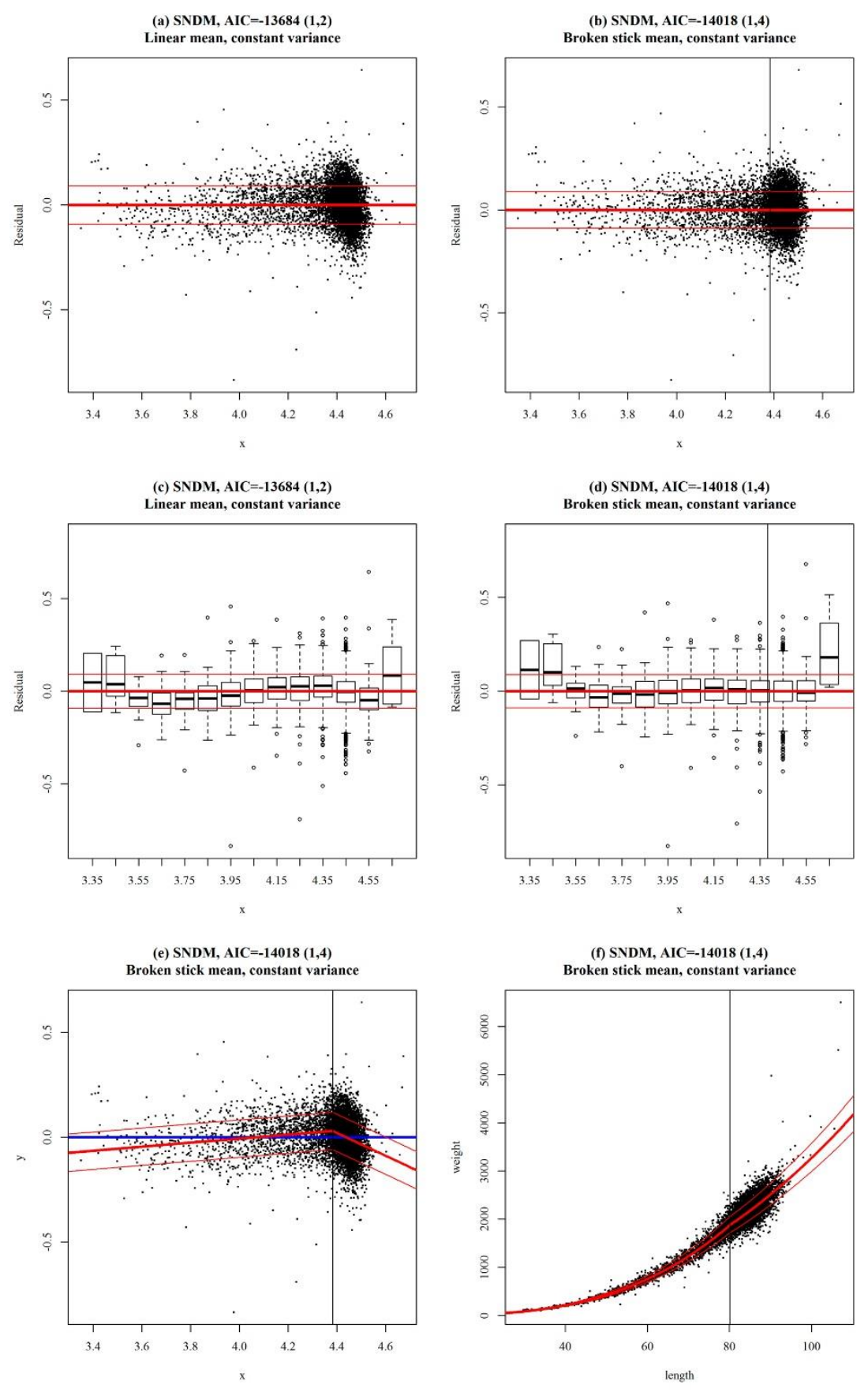

Figure 2.9 Linear and best fitting model for male Deania calcea (SND). Top left panel: linear model with constant variance; top right panel: residuals for the best fitting model from a linear model with constant variance (horizontal lines show the mean with \pm 1 standard deviations); middle two panels: same data summarised by boxplots; bottom left panel: the same as top left, with residuals from the linear model, but with the mean and \pm 1 standard deviation superimposed; bottom right: fitted model plotted on top of the original data on the original linear scale. |, inflection point of the broken stick $(\beta)$. 

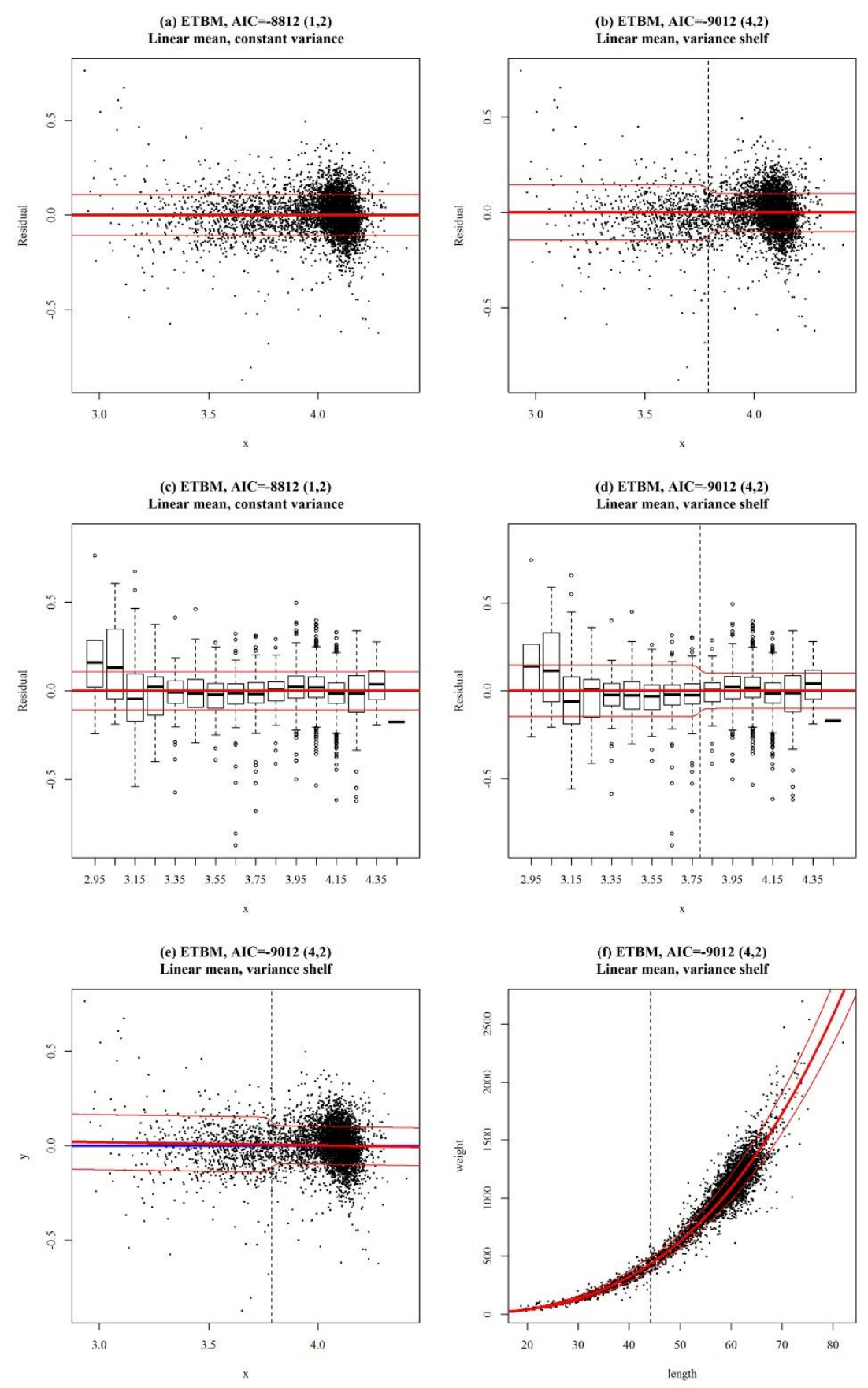

Figure 2.10 Linear and best fitting model for male Etmopterus granulosus (ETB). Top left panel: linear model with constant variance; top right panel: residuals for the best fitting model from a linear model with constant variance (horizontal lines show the mean with \pm 1 standard deviations); middle two panels: same data summarised by boxplots; bottom left panel: the same as top left, with residuals from the linear model, but with the mean and \pm 1 standard deviation superimposed; bottom right: fitted model plotted on top of the original data on the original linear scale. $\vdots$, inflection point of the variance shelf $(\alpha)$. 


\subsection{Discussion}

The first part of this study estimated length-weight relationships (LWRs) for deepsea chondrichthyans caught around New Zealand, and according to FishBase, are the first reporting of LWR parameters for Harriotta haeckeli, Harriotta raleighana, Rhinochimaera pacifica, Chimaera carophila, Chimaera lignaria, Hydrolagus homonycteris, Hydrolagus trolli, Etmopterus granulosus, Etmopterus lucifer, Scymnodon plunketi, Oxynotus bruniensis, and Dalatias licha anywhere across their range. These are also the first LWR reporting for Deania calcea, Centroscymnus coelolepis, Centroscymnus owstonii and Centroselachus crepidater from the New Zealand region. Most species, with the exception of Chimaera carophila and Hydrolagus bemisi, had positive allometric growth that significantly deviated from isometry, signifying that these species grow relatively faster in weight than length.

A significant difference of the LWR between the sexes was found for most species, with females not only growing larger in length than males, but also weighing more at a given length (larger $b$ values). Female chondrichthyans generally mature at a larger size and older age than males, which is most likely a requirement for significant reproductive maternal investment (Springer, 1967; Hoenig \& Gruber, 1990; Cortés, 2000). With the exception of Hydrolagus novaezealandiae and $H$. trolli, females had larger $b$ values than males. Mature females made up a small proportion of $H$. novaezealandiae samples (MacGibbon, 2016), and this lack of large, reproductively active females may explain why males had the larger $b$ value. For $H$. trolli, and for those species where no difference in LWRs between the sexes was found, this might be accounted for by small sample sizes ( $<50$ individuals of each sex). Differences in the $b$ parameters between the sexes also appears to be greater in squaloids than chimaeroids, which is expected given that maternal investment is more pronounced in species that exhibit viviparity over oviparity (Pethybridge et al., 2011b).

Parameters $a$ and $b$ of $H$. bemisi (unsexed, $a=0 \cdot 00936, b=2 \cdot 89$, O'Driscoll $\&$ Bagley, 2004), Squalus acanthias (male, $a=0 \cdot 00275, b=3 \cdot 05$; female, $a=0 \cdot 00139, b=$ 3·25, Annala $\&$ Sullivan, 1997) and Centrophorus squamosus (male, $a=0 \cdot 0019, b=$ 3.23; female, $a=0 \cdot 0010, b=3 \cdot 38$, Parker $\&$ Francis, 2012) had similar values to those previously reported from New Zealand waters, but differed for Hydrolagus novaezealandiae ( $a=0 \cdot 00120, b=3 \cdot 39$, O’Driscoll $\&$ Bagley, 2004). This may be due to regional variation, as the estimates of O’Driscoll \& Bagley (2004) were from Sub- 
Antarctic specimens only. Chondrichthyans have also been shown to exhibit regional variation in growth rates (Lucifora et al., 1999; Driggers et al., 2004; Neer \& Thompson, 2005), and for stock assessments, it would be best to develop regional LWRs. At this time, however, little is known about population connectivity of deep-sea chondrichthyans in New Zealand and thus data were treated as being from one population, thereby ignoring any geographic, seasonal, and inter-annual variation (Froese, 2006). LWR parameters for $S$. acanthias from this study were found to differ from those in the Mediterranean (e.g. Ismen et al., 2009; Barría et al., 2015) and Northeast Atlantic (Coull et al., 1989). Barría et al. (2015) reported similar parameters for Dalatias licha in the north-western Mediterranean Sea, where $C$. coelolepis had negative allometric growth ( $b$ $=2 \cdot 29)$, as opposed to positive allometric growth in New Zealand $(b=3 \cdot 14)$.

Temporal variation in size can vary from year to year, and may also play a critical factor in LWRs. Changes in weight at length can vary with reproductive seasonality. Ripening of gonads can account for a considerable proportion of the total weight of a fish, and following a breeding period, the decreased body condition of a spent individual will reduce total body weight (Le Cren, 1951). Seasonal fluctuations in environmental conditions can also influence body weight in fish: in largemouth bass Micropterus salmoides (Lacepède, 1802), growth was highest with increased water temperature (Niimi \& Beamish, 1974); and a lack of available prey for smaller M. salmoides was determined to cause growth disparity of fish in an initial year class (Shelton et al., 1979). Sizes of the study species could have been confounded by the regional and temporal scale of sampling, however, sampling across all available time and space was used for this analysis in an effort to reduce any potential bias.

\subsubsection{FishBase parameter estimates}

We have shown that LWR parameter estimates provided by the online database, FishBase, differed significantly from most of those calculated in this study. Although FishBase has a method to estimate LWR parameters that do not exist for many species, these estimates should be used with caution. The FishBase estimates could greatly overor underestimate fish size (>10\%). A Rhinochimaera pacifica, for example, would weigh an estimated $2859 \mathrm{~g}$ at a length of $c .120 \mathrm{~cm} L_{\mathrm{C}}$ (Table 2.5), corresponding well to measurements by Finucci et al. (2016b). Using FishBase estimates for this species, the same specimen would have an estimated weight of $11062 \mathrm{~g}$, a roughly four-fold 
overestimation (Table 2.5). The variations in parameter estimates can have important implications for fisheries applications, such as biomass estimates, and have the potential to greatly overestimate population size, acceptable catch limits, and thus, the overall sustainability of the fisheries. It is also likely that this discrepancy has been applied across most fish populations. Gerritsen \& McGrath (2007) found a bias of up to $10 \%$ in weight could occur in biomass estimates if the LWR from one stock of whiting Merlanguis merlangus (L. 1758) was applied to neighbouring stocks of whiting from the same region. The importance of LWRs in stock assessments may be underestimated, at least in New Zealand, where LWRs are generally assumed to be constant over time and space (MPI, 2014).

Small sample sizes might be sufficient to establish a LWR, although it is recommended to include roughly equal numbers of randomly selected small, medium, and large-size specimens to sufficiently evaluate the relationship of parameter $b$ (Froese, 2006). For some species, the smallest and largest size classes were missing or limited in the dataset. In this study, there were no small individuals $(<35 \mathrm{~cm})$ available for $H$. homonycteris and C. carophila, as well as O. bruniensis, (size at birth c. $25-27 \mathrm{~cm} L_{\mathrm{T}}$ for O. bruniensis, Finucci et al. (2016a); unknown but presumed $<35 \mathrm{~cm} L_{\mathrm{C}}$ for $H$. homonycteris and C. carophila). For several of the larger species, such as $C$. owstonii, $S$. plunketi, and $D$. licha, all of which can grow to lengths of $160 \mathrm{~cm} L_{\mathrm{T}}$, data were few for the largest of size classes ( $>120 \mathrm{~cm} L_{\mathrm{T}}$ ). These missing size classes may explain some of the LWR differences observed in these species, and further collection of length and weight measurements across all size classes is recommended for accurate LWR estimation.

In the analysis undertaken of FishBase data to estimate LWR parameters, only a dozen of the 119 recognized species of the order Squaliformes (Weigmann, 2016) were included, all of which were categorized with an elongated or fusiform body shape. Only two species in this study were reported in that analysis (S. acanthias, C. owstonii). While the analysis included species representative of five of the seven the families of the order Squaliformes (Squalidae, Etmopteridae, Centrophoridae, Dalatiidae, Somniosidae), squaloids are a diverse group of species with a range of morphological characteristics and life history differences (Kyne \& Simpfendorfer, 2010). Many of these are species are data deficient, and LWR would have been estimated using related species and/or similar body shape (Froese et al., 2014). Averaging LWR parameters for related species has previously been proven to be statistically flawed as most LWR studies are given at the species level 
with no indication of relationships at a higher taxonomic level (genus or family) (Kulbicki et al., 2005).

From the over- and underestimation of weight at length relationships produced for many species, it appears that it may not be best to generalize parameters based on relatedness and body type alone, and perhaps, life history strategies should also be taken into account. For example, despite being in the same genus, E. granulosus and E. lucifer have different life history strategies: demersal, maximum size of $85 \mathrm{~cm} L_{\mathrm{T}}$ vs. mesopelagic, maximum size of $45 \mathrm{~cm} L_{\mathrm{T}}$, respectively (Last \& Stevens, 2009), and mean depths of occurrence ( $898 \mathrm{~m}$ vs. $595 \mathrm{~m}$, respectively). However, both species were given the same FishBase parameter estimates derived from estimates for smooth lanternshark Etmopterus pusillus (Lowe, 1839) and velvetbelly lanternshark Etmopterus spinax (L. 1758) from the Western Mediterranean (Froese, 2006). Weights calculated using LWR parameters from this study for E. granulosus were similar to those in FishBase (but still significantly different), while the weights calculated for E. lucifer were quite different.

\subsubsection{Form factor and depth}

Depth of occurrence should also be taken into consideration, as it explains a lot of variability in deep-sea life histories, including growth (Drazen \& Haedrich, 2012). Fluctuations in pressure, light levels, temperature, oxygen, and food availability (Gage \& Tyler, 1991), reduced metabolic rate with increasing depth (Drazen \& Seibel, 2007), as well as a decline in predation pressure due to reduced megafaunal density (Haedrich \& Rowe, 1977), may all contribute to changes in growth across species. Depth can also influence body form $\left(a_{3.0}\right)$ of fishes, where a more elongated body form is favoured with increasing depth, thought to be linked to more efficient swimming performance in the deep-sea (Neat \& Campbell, 2013; Farre et al., 2016). No correlation between chimaeroid or squaloid body form and depth was observed in this study, which was also reported previously from deep-sea species caught on Rockall Trough, and the lack of change in body shape may act as a constraint to deep-sea elasmobranch depth distributions beyond 2000 m (Neat \& Campbell, 2013). 


\subsubsection{Variability in weight at length}

Variability in weight at length was observed across most species, and appeared to increase with increasing length. This observed variability deviates from the expected LWR $\left(W=a L^{b}\right)$, which may affect the parameters estimates. In addition to subdividing species data by sex, it seemed more appropriate to model data sets with multiple LWRs for different size classes, as a simple LWR would lead to bias over some length ranges. This study has used a flexible model to detect changes in the inflection point of the mean, variance, or both, in weight at a given length. An estimate for the inflection point of the variance was reported for most species (c. 90\%), whereas an estimate for the inflection point of the mean was only reported approximately $60 \%$ of the time. The inflection point of the variance also found length estimates that more consistently corresponded with previously reported length-at-maturity values. This may indicate that the variance inflection point in LWRs could be correlated with the onset of maturity, while the mean inflection point may be attributed to a different ecological factor. Three general changes in the LWRs were detected by the model for deep-sea chondrichthyans: one occurring shortly after birth, one occurring mid-size, and one occurring around sizes which have been reported as length-at-maturity estimates.

\subsubsection{Early length LWR variation detections}

In many species, the inflection point of the mean or variance of weight at length was detected shortly after length-at-birth or at a small length $(<50 \mathrm{~cm})$. In a cohort, fish are expected to initially vary in size, which may be the result of environmental or congenital physiological differences, or variability in the time of hatching (Ricker, 1958). Environmental heterogeneity (e.g. changes in oxygen levels, salinity) may influence feeding ability and metabolism, and thus, overall growth patterns (Wootton, 1990; Pfister \& Peacor, 2002; Jobling, 2002). Temperature is the most prevalent environmental factor that influences aquatic organisms (Wootton, 1990; Jobling, 1997), and has been found to play a pivotal role in determining development rates and timing of hatching in chimaeroids and other oviparous chondrichthyans (Ballard et al., 1993; Lyon et al., 2009). As ectotherms, variations in external conditions have been correlated with sitespecific spawning rates, with warmer waters increasing embryonic physiological activity, and thus, varying the rate of development and hatching periods across spawning areas 
(Hoff, 2008; Lyon et al., 2009). The variability around the LWR was observed at a small length for several chimaeroids, but these length estimates were much larger than reported length-at-birth (e.g. H. novaezealandiae: length-at-birth $c .5 \mathrm{~cm} L_{\mathrm{C}}$, model change at 35.8 $\mathrm{cm}$ ), and thus, must be influenced by some factor beyond time of hatching.

In many of the early length LWR variation detections, there was an initial decrease in the mean or decline in the variance, which would suggest that the average individual decreased in weight at length, or that there was less variability in weight at length of individuals in these size classes. Population density can influence growth rates through increased competition for resources (e.g. food), and has been reported to increase individual size variability and decrease the growth rate of the average fish in a population (Rubenstein, 1981). High population densities are characteristic of chondrichthyan nursery areas, and while presumed to decrease mortality by providing optimal growth conditions, aggregations of juvenile individuals can increase mortality from food limitations or increased predation from larger fish (Heupel et al., 2007). While little is known about nursery or pupping grounds for the study species, some chondrichthyans, particularly oviparous species, engage in congregative nursery behaviour (Etnoyer \& Warrenchuk, 2007). Large clusters of egg cases from oviparous species have been located near cold seeps (Truede et al., 2011), cold-water coral reef habitat (Henry et al., 2013), and various discreet locations of the outer and middle shelf and upper slope of canyons (Hoff, 2016), and it has been suspected the New Zealand chimaeroids engage in the same behaviour (McCutcheon, 1980). The estimates provided by the mean and variance may indicate the size at which these individuals move away from high density areas, potentially reducing competition for resources, and thus, reducing variability in weight at length.

\subsubsection{Mid-length LWR variation detections}

Inflection points that were detected well beyond length-at-birth but before reported length-at-maturity were generally characterised by changes in the mean, shifting from a decline to an increase or stabilization after the inflection point. These changes may indicate shifts in diet with ontogeny. Ontogenetic diet shifts can have important effects on growth, affecting an individual's ability to exploit different resources as it changes in body size (Werner \& Gilliam, 1984). For chimaeroids, some broad diet patterns have been observed in a number of species, regardless of differences in location and depth 
range (Dunn et al., 2010). In several of the deep-sea species, including Harriotta raleighana, Hydrolagus bemisi, and $H$. novaezealandiae, smaller individuals were found to feed predominately on polychaetes, small crustaceans, and gastropods, while larger fish relied more on decapod crustaceans (Macpherson, 1980, Mauchline \& Gordon 1983, Moura et al., 2005; Dunn et al., 2010). This shift in diet may be a reflection of the change in growth of the jaw structure. Huber et al. (2008) reported that in spotted ratfish Hydrolagus colliei (Lay \& Bennett, 1839), as fish length doubled, its ability to exert crushing force increased by six-fold. This mechanism may allow chimaeroids to feed on a larger or stronger variety of prey as body size increases. Greater net energetic payoff are thought to be associated with larger prey items (Lowe et al., 1996), which may increase growth rates (Werner \& Gilliam, 1984).

Ontogenetic changes in diet have been reported in a number of deep-sea elasmobranchs (e.g. Albo-Puigserver et al., 2015). Increased cephalopod consumption was found to be positively correlated with increasing length for velvet belly lanternshark E. spinax (MacPherson, 1979), as well as Dalatias licha (Matallanas, 1982). With the use of a combination of stomach content, stable isotope, and signature fatty acid profile analysis, ontogenetic diet shifts were confirmed for Centroselachus crepidater (Pethybridge et al., 2011a; Pethybridge et al., 2012). A significant positive correlation between total length and $\delta 15 \mathrm{~N}$ values, an indication of ontogenetic shifts (Araújo et al., 2011), were observed for Deania calcea (Iitembu \& Richoux, 2015). In E. granulosus, the importance of teleost prey tended to increase with shark length (Hallet \& Daley, 2010), while crustaceans appeared to be important in the diet of smaller individuals $(<55$ $\mathrm{cm} L_{\mathrm{T}}$ ) and absent from larger fish (> $75 \mathrm{~cm} L_{\mathrm{T}}$ ) (Hallet \& Daley, 2010).

In most cases, however, any analysis of ontogenetic changes in diet are often constricted by small sample sizes, or missing length classes, particularly smaller individuals (Matallanas, 1982; Preciado et al., 2009; Iitembu \& Richoux, 2015), making it difficult to pinpoint at what length these changes are occurring. Macroscopic observations may also provide biased results if study species regurgitate upon capture, scavenge from fishing vessels, or forage in trawl nets upon capture (Dunn et al., 2010; Dunn et al., 2013). For example, S. acanthias is known for its opportunistic foraging behaviour, which is supported by widespread spatial and temporal variations in diet (Belleggia et al., 2012). While it has been reported that smaller fish fed more on small crustaceans and salps, and the diet of larger individuals was comprised more of large and scavenged fishes, suspected offal scavenging from fishing vessels may mask macroscopic 
detection of diet changes with ontogeny in S. acanthias (Dunn et al., 2013). Applying the models presented here to datasets may provide better insight to when these diet shifts occur. With the use of the Broken Stick model, the inflection point of the mean for both sexes of $S$. acanthias was estimated at $c .47 \mathrm{~cm} L_{\mathrm{T}}$. This length estimate is well beyond reported size at birth $\left(c .22 \mathrm{~cm} L_{\mathrm{T}}\right)$, and underestimates length-at-maturity $\left(58 \mathrm{~cm} L_{\mathrm{T}}\right.$ for males, $73 \mathrm{~cm} L_{\mathrm{T}}$ for females, Table 2.3). Previous studies of $S$. acanthias from Patagonia waters found a diet shift at approximately $62 \mathrm{~cm} L_{\mathrm{T}}$, correlating with the onset of maturity (García de La Rosa \& Sánchez, 1997). However, this estimated shift was based purely on aggregated sex and size-based analysis. Further division of classes found considerable difference in diet between immature and mature individuals, which may be associated with sex and size segregation, or shift of habitat utilization (from pelagic to demersal) (Alonso et al., 2002). Given that this change was detected at approximately the same length in both sexes, it may instead pinpoint the previously suspected shift in diet for $S$. acanthias from New Zealand.

\subsubsection{Late length LWR variation detections}

Most estimates (c.75\%) of the variance inflection point and approximately half of the mean inflection point estimates were accurate within $20 \%$ of reported length-atmaturity. These were generally characterised by an increase in the variance inflection point for females, and a decline in mean after the point of inflection for males. The use of the variance to estimate length-at-maturity for females seems appropriate given that in most chondrichthyans, females attain a larger overall size (length and weight) than males, most likely a requirement for significant reproductive maternal investment (Hoenig \& Gruber, 1990; Cortés, 2000). Female chondrichthyans can undergo large fluctuations in weight at length during their reproductive cycle; increased growth has been observed with the gonad development and the production of large yolky oocytes required to nourish offspring (Wourms, 1977), and female weight can also increase with offspring size (Baremore \& Passerotti, 2013), or large litters (Yano, 1995). These increases in weight would then be followed by large declines in weight after parturition, and decreased body condition during any subsequent resting or regenerating period (Girard \& du Boit, 1999). The model also generally underestimated length-at-maturity for females, which may suggest that females begin relocating energy from somatic to reproductive growth (Roff, 1983) at length classes earlier than what is detected from macroscopic observation. 
For males, an observed decline of the mean after the point of inflection may represent a reduction in body condition associated with reproductive costs. Liver condition, used as an index of overall body condition, has been found to decrease in sexually mature males (Springer, 1967). It has been hypothesised that, during breeding periods, males may engage in prolonged fasting (Springer, 1967), increased activity or aggressive behaviour to acquire females, or may have to produce large quantities of sperm to engage in sperm competition (Pratt \& Carrier, 2001). All of these behaviours would involve significant energetic requirements, and as observed in the model, would likely reduce weight at length in reproductively active males.

Estimates of the variance appear to be more appropriate for estimating length-atmaturity for squaloids than chimaeroids. In squaloids, embryos develop within the mother, gestation can last up to 24 months (Ketchen, 1972; Guallart \& Vincent 2001), and litter sizes have been reported > 10 (Wetherbee, 1999; Daley et al., 2002), and up to 40 pups (Yano, 1995). Size and experience of the female can also influence reproductive output (maximum litter size, offspring size) (Cortes, 2000; Braccini et al., 2007), and it would be expected that more variability in weight at length would be observed in female squaloids. In comparison, chimaeroids produce egg capsules that encapsulate individual embryos and are deposited on the seafloor (Dean, 1906; Didier et al., 2012). In captive $H$. colliei, pairs of egg capsules were laid every 10 to 14 days over a period of several months (Didier \& Rosenberger, 2002). Given the relatively reduced maternal reproductive investment in chimaeroids, it would be expected that less variability in weight at length would be observed, and changes in mean may be better suited to estimate length-at-maturity for this group.

Estimates by the model may also provide some indication of reproductive seasonality. Deep-sea chondrichthyans are thought to engage in asynchronous reproductive cycles (Yano, 1995; Wetherbee, 1996; Daley et al., 2002), which allows for parturition to occur when individual or environmental conditions are optimal. This would result in individuals at different stages of their reproductive cycle (gravid, post-partum, regenerating, resting), and thus, it would be expected that individual variability of weight at length would be high among mature individuals. In the squaloids, most late length LWR variation detections were changes in the variance, possibly indicating asynchronous breeding cycles, which has been previously suspected for some of the study species, including D. calcea, E. granulosus, C. crepidater, S. plunketi, and D. licha (Daley et al., 2002). In contrast, species with a synchronous breeding cycle would be expected to have 
low variability in weight at length, and rather, a change in the mean size, as all mature individuals should change in weight around the same time. Nothing is known about reproductive seasonality in New Zealand chimaeroids, however, estimates of the mean inflection point from the model were most observed amongst the chimaeroids. For the ghost sharks ( $H$. bemisi and H. novaezealandiae), it has been proposed that these species engage in either synchronous (Horn, 1997), or year-round breeding (McCutcheon, 1980); results from the model would suggest the former. Through macroscopic observations, a discrete reproductive cycle in $H$. colliei was reported off the west coast of North America (Barnett et al., 2009).

Many deep-sea chondrichthyans are data deficient, with little data available for even the most basic of biology. However, large data sets of basic measurements (length, weight, sex) collected by research trawl surveys often exist, spanning over many years, and are often underutilized. The model presented here could be applied to any marine fish where length and weight data are routinely collected from research surveys, and may be particularly useful to provide insight into biological and ecological factors for species where macroscopic evaluations are unavailable or difficult to estimate, or when it may take years to collect such data. 


\section{CHAPTER III}

Social associations in deep-sea

chondrichthyans 


\subsection{Abstract}

Group living has been recorded across a diverse range of chondrichthyans. Understanding these interactions is necessary to address a broad set of biological questions, but due to a variety of factors, any inferences of social associations in deep-sea cartilaginous fishes have yet to be described. In this study, social associations in deep-sea chondrichthyans were evaluated across class and group size. Common and infrequently caught deep-sea chondrichthyans from offshore New Zealand waters (6 holocephalans, 10 elasmobranchs) were selected using fisheries-independent research trawl surveys on Chatham Rise dating back to the 1970s. Results indicated that not all selected species engaged in aggregative behaviour, but those that do suggested patterns of sex- and sizespecific socially driven associations which varied with catch density. Several trends were observed in the social association analysis: 1) adult females were caught most frequently in low densities and were highly associated with other adult females; 2) adult males were consistently highly associated with each other; and 3) the highest densities were dominated by juvenile individuals. The nature of these gatherings will have implications for selective mortality of populations by spatial or temporally stratified, or aggregationtargeting, fishing. 


\subsection{Introduction}

Group living has been recorded across a diverse range of coastal, pelagic, and deep-sea chondrichthyans (the cartilaginous fishes: sharks, rays and chimaeras) (Springer, 1967). Congregations can be single- (Economakis \& Lobel, 1998) or multi-species (Speed et al., 2011), and correlated with environmental factors such as depth, temperature, or benthos (Klimley, 1987; Speed et al., 2012; Nosal et al., 2013), or biological factors, such as prey abundance (Sims et al., 2006), and have been attributed to foraging (Klimley, 1987), reproduction (Speed et al., 2011), energy conservation (refuging) (Economakis \& Lobel, 1998), and social preference (Guttridge et al., 2009; Mourier et al., 2012).

Chondrichthyans may form assemblages dominated by particular sizes, sexes, or developmental stages (Girard \& Du Boit, 1999; Mucientes et al., 2009). Sexual segregation is characteristic of many chondrichthyan populations, including deep-sea species (Yano \& Tanaka, 1988). Females may migrate away from males to habitats which are thought to provide the energy-rich prey needed to reach the larger body size required to support large, well developed embryos (Klimley, 1987), or where optimal environmental conditions (e.g. warmer water) may increase the rate of embryonic development (Economakis \& Lobel, 1998). Spatial segregation may also provide refuge for females from energetically expensive and potentially damaging multiple mating events due to harassing males (Pratt \& Carrier, 2001). Nursery grounds, where relatively high densities of juveniles occur, may be characterised by increased rates of growth (e.g. higher food abundance, optimal temperature), and segregation and refuge from larger predatory species (Heupel et al., 2007).

Cartilaginous fishes have well-developed cognitive abilities, and are capable of complex social behaviours (Schluessel, 2015). Lemon sharks Negaprion brevirostris (Poey, 1868) have been documented to engage in active partner preference through nonrandom associations between specific individuals (Guttridge et al., 2009). The spotted wobbegong Orectobolobus maculatus (Bonnaterre, 1788) and blacktip reef sharks Carcharhinus melanopterus (Quoy \& Gaimard, 1824) have the capacity to form shortterm (Armansin et al., 2016), and long-term social bonds (Mourier et al., 2012), showing that chondrichthyans do not just aggregate, but may form social groups.

Measuring social interactions in most chondrichthyan species is difficult, due to their highly mobile nature, and the logistical constraints of tracking individuals in the 
open sea (Mourier et al., 2012). Consequently, social behaviour studies on wild chondrichthyans have been limited to inshore species that display some degree of site fidelity (e.g. Guttridge et al., 2009). Network approaches are increasingly popular tools for analysing chondrichthyan organization (Jacoby et al., 2012), and are useful for understanding social complexity at the individual, community, and population level (Croft et al., 2008). However, these approaches require individuals to be uniquely identified (Whitehead, 2008), which can be achieved by direct field observations (Economakis \& Lobel, 1998), acoustic telemetry (Espinoza et al., 2011), and photoidentification (Mourier et al., 2012). Applying these techniques to the deep-sea has been extremely difficult, because to the difficulty of making observations, the high equipment development and deployment costs, unknown survivorship of tagged individuals with extreme hydrostatic pressure change (Daley et al., 2015), and the relatively low probability of recovering individuals to retrieve data from electronic tagging (RodriguezCabello \& Sanchez, 2016). Some deep-sea species also appear to engage in movements over extensive open habitat at depths beyond the working capacity of most tagging technology (Rodríguez-Cabello et al., 2016), while monitoring methods (e.g. baited remote underwater video systems, BRUVs) can be intrusive to the deep-sea environment and result in biased observations (McLean et al., 2015). Some of the earliest insights into the structure of chondrichthyan groupings were gained from relatively simple analyses, using the catch composition of commercial trawl catches (Wearmouth \& Sims, 2008). Analysis of both commercial and research trawl survey catches have been used to infer that many deep-sea cartilaginous fishes are spatially segregated by size class and/or sex (Holt et al., 2013; Moura et al., 2014). However, inferences about aggregations and social grouping in deep-sea species have yet to be made.

The objective of this study was to characterize the catch composition of deep-sea chondrichthyans, using extensive research trawl catch data, and following the companion preference analysis methods of Underwood (1981). Underwood's (1981) method uses simple statistics to describe companions for each class of animal (a class being defined by e.g. sex, maturity) and was originally used to describe the pattern of social groupings in Common Eland Tragelaphus oryx (Pallas, 1766). Eland were free ranging, making it difficult to trace individuals over time. However, the use of class of animal allowed for general inferences of companion preference to be made from repeated measurements of individual group (herd) structures. In this study, eland are replaced by deep-sea chondrichthyans, and a group is replaced by an individual trawl catch. We apply the 
Underwood (1981) method across several chondrichthyan species, allowing us to identify common patterns, and develop hypotheses for why these patterns repeatedly occur. 


\subsection{Materials and methods}

\subsubsection{Study area}

Chatham Rise, located to the east of New Zealand, is a region known for its complex bathymetry and high biodiversity (McClatchie et al., 1997). The high biological productivity is influenced by the subtropical front, which provides a mixing of warm, nutrient-poor northern subtropical waters and cold, nutrient-rich waters of the Subantarctic (Heath, 1985). It is one of New Zealand's most important fishing grounds, and as a result, has been a focus for research efforts, resulting in the most comprehensive time series of marine species observations within the New Zealand Exclusive Economic Zone (EEZ) (O’Driscoll et al., 2011).

\subsubsection{Research trawl catch data}

Fisheries-independent research trawl survey catch and effort data on Chatham Rise, to the east of New Zealand, were obtained from the New Zealand Ministry for Primary Industries (MPI) trawl database. Data were selected where fishing gear was any form of bottom or mid-water trawl, gear performance was rated as at least satisfactory, latitude was between $42.5^{\circ}$ and $45^{\circ} \mathrm{S}$, and longitude between $172^{\circ} \mathrm{E}$ and $173^{\circ} \mathrm{W}$. The resulting data set consisted of 185 voyages, with 9305 bottom trawls and 182 midwater trawls, by 23 vessels, between 1978 and 2013. Individual trawl depth ranged between 202-2218 $\mathrm{m}($ median $=841 \mathrm{~m})$, distance between the trawl net wings $2.6-126.9 \mathrm{~m}$ $($ median $=19 \mathrm{~m})$, and distance between the trawl doors 6-237 $\mathrm{m}($ median $=116 \mathrm{~m})$. Most trawls were completed by RV Tangaroa (58 surveys and 5349 trawls), following stratified random survey designs, using a full-wing bottom trawl with a $60 \mathrm{~mm}$ codend mesh, towed on flat seabed (O'Driscoll et al., 2011). Additional surveys had a variety of objectives, largely targeting orange roughy Hoplostethus atlanticus, Collett, 1889 and oreos (Oreosomatidae), as summarised by Dunn et al. 2009. For each tow, the details of the fishing operation were recorded, and the catch was sorted by species and sampled for biological statistics (for chondrichthyans, sex, mass, total length (TL, cm): tip of the snout to posterior end of caudal fin, or chimaera length, $(\mathrm{CL}, \mathrm{cm})$ : tip of the snout to the posterior edge of the supracaudal fin, excluding the caudal filament). For this study, the 
species for analysis included both common and infrequently caught deep-sea chondrichthyans (Table 3.1).

Species density for each trawl was estimated as the total number of individuals in the catch divided by the total distance $(\mathrm{km})$ towed (Appendix IV). Depth of each tow was estimated as the average of the depth at the start and end positions. Individuals were classified by species, sex, and then maturity class as, (i) juvenile/immature or (ii) adult/mature, using New Zealand estimates of mean length at maturity ( $\left.L_{50}\right)$ (Table 3.1 ).

\subsubsection{Social association analysis}

Social associations were examined for data sets where the catch densities suggested aggregations may occur (densities $>50 \mathrm{fish} / \mathrm{km}$ ) (Table 3.2). Etmopterus lucifer, an unusually small species (Table 3.1), was also included in the analysis to allow a comparison across species size, for a total of one chimaeroid and five squaloids. Following Underwood (1981), if $q$ groups (in this case, each group is one class in one trawl) are sighted, the $j$ th group having $x_{j}$ members, each member of that group has $x_{j}-1$ companions. The total number of animals sighted is the sum of all group sizes,

$$
\sum_{j=1}^{q} x_{j}
$$

The average number of companions for the average animal sighted (C) is then,

$$
C=\sum_{j=1}^{q} x_{j}\left(x_{j}-1\right) / \sum_{j=1}^{q} x_{j}=\left(\sum_{j=1}^{q} x_{j}^{2} / \sum_{j=1}^{q} x_{j}\right)-1
$$

The group containing the average animal has $C_{1}$ members of a given class, $y$, where $y_{j}$ is the number of animals of that class in the jth group. The mean number of companions of a particular class is then,

$$
C_{1}=\sum_{j=1}^{q}\left(x_{j} y_{j}\right) / \sum_{j=1}^{q} x_{j}
$$

The average member of a particular class, the "subject" class, has $C_{2}$ or $C_{3}$ companions of a given associating class. If $p$ groups containing the subject class are sighted, the $k$ th group having $y_{k}$ members of the subject class and $z_{k}$ members of the associating class, 
then the intra-class gregariousness $\left(C_{2}\right.$, the mean number of companions of the same class) is given by,

$$
C_{2}=\left(\sum_{k=1}^{p} y^{2} k / \sum_{k=1}^{p} y_{k}\right)-1
$$

And the inter-class gregariousness $\left(C_{3}\right.$, the mean number of companions of a different class) is,

$$
C_{3}=\sum_{k=1}^{p}\left(y_{k .} z_{k}\right) / \sum_{k=1}^{p} y_{k}
$$

Statistics describing social associations were calculated for tows where two or more fish were caught, in fish density bins: 1 fish/ $\mathrm{km}$ or less, $>1-5$ fish $\mathrm{km}^{-1},>5-10$ fish $\mathrm{km}^{-1},>10-20$ fish km $\mathrm{km}^{-1}>20-50$ fish $\mathrm{km}^{-1},>50-100$ fish $\mathrm{km}^{-1},>100-200$ fish $\mathrm{km}^{-1}, 200$ 1000 fish $\mathrm{km}^{-1}$, and $>1000$ fish $\mathrm{km}^{-1}$. To test whether class compositions for each density bin were significantly different to that expected from the total catch composition, a Chisquare $\left(\chi^{2}\right)$ test was used, where for each class the number of companions $\left(\mathrm{n} \mathrm{km}^{-1}\right)$ by class (from Eqns. 4 and 5) was the observed, and the total catch composition by class ( $\mathrm{n}$ $\mathrm{km}^{-1}$ ) was he expected. The null hypothesis was that, at each density, the companions for each class would be the same as that seen in the overall catch. A significant difference was concluded at $P<0 \cdot 05$. Across all species, the minimum number of individuals in any density bin was 249 , with no less than 11 in any class, therefore the potential bias caused by subtracting the focal individual from the observed catch composition (Eqn. 4) was negligible and ignored. All analyses were carried out using R (R Core Team, 2012). 
Table 3.1. Length at maturity $\left(L_{50}\right)$ by sex for selected chondrichthyans from New Zealand literature (total length, $L_{\mathrm{T}}$, for elasmobranchs; chimaera length, $L_{\mathrm{C}}$, for chimaeras).

\begin{tabular}{|c|c|c|c|c|}
\hline \multirow[t]{2}{*}{ Species } & \multirow{2}{*}{$\begin{array}{c}\text { Length } \\
\text { Range }(\mathrm{cm})\end{array}$} & \multicolumn{2}{|c|}{ Maturity $(\mathrm{cm})$} & \multirow[t]{2}{*}{ Source } \\
\hline & & Female & Male & \\
\hline \multicolumn{5}{|l|}{ Chimaeriformes } \\
\hline Harriotta raleighana & $19 \cdot 9-97 \cdot 9$ & 76 & 63 & $\begin{array}{l}\text { Finucci et al., } \\
\quad(2016 \mathrm{~b})\end{array}$ \\
\hline Rhinochimaera pacifica & $28 \cdot 6-165 \cdot 2$ & 125 & 105 & $\begin{array}{l}\text { Finucci et al., } \\
\quad(2016 \mathrm{~b})\end{array}$ \\
\hline Chimaera carophila & $35 \cdot 4-103 \cdot 9$ & 83 & 75 & $\begin{array}{l}\text { Finucci } \text { et al. } \\
\text { (2017) }\end{array}$ \\
\hline Hydrolagus bemisi & $13 \cdot 9-89 \cdot 1$ & 69 & 59 & Horn (1997) \\
\hline Hydrolagus homonycteris & $50 \cdot 6-99 \cdot 8$ & 83 & 79 & $\begin{array}{l}\text { Finucci et al., } \\
\text { (2017) }\end{array}$ \\
\hline Hydrolagus novaezealandiae & $18 \cdot 9-81 \cdot 0$ & 62 & 52 & Horn (1997) \\
\hline \multicolumn{5}{|l|}{ Squaliformes } \\
\hline Squalus acanthias & $24 \cdot 2-107 \cdot 0$ & 73 & 58 & $\begin{array}{l}\text { Hanchet } \\
\text { (1988) }\end{array}$ \\
\hline Centrophorus squamosus & $37 \cdot 9-145 \cdot 0$ & 119 & 99 & $\begin{array}{c}\text { Parker \& } \\
\text { Francis (2012) }\end{array}$ \\
\hline Deania calcea & $20 \cdot 4-126 \cdot 5$ & 106 & 78 & $\begin{array}{c}\text { Parker \& } \\
\text { Francis (2012) }\end{array}$ \\
\hline Etmopterus granulosus & $17 \cdot 3-85 \cdot 8$ & 64 & 55 & $\begin{array}{l}\text { Wetherbee } \\
\text { (1996) }\end{array}$ \\
\hline Etmopterus lucifer & $16 \cdot 3-54 \cdot 8$ & 40 & 34 & $\begin{array}{l}\text { Galland } \\
(2015)\end{array}$ \\
\hline Centroscymnus owstonii & $28 \cdot 2-148 \cdot 0$ & 98 & 71 & $\begin{array}{c}\text { King \& Clark } \\
\text { (1987) }\end{array}$ \\
\hline Centroselachus crepidater & $28 \cdot 0-109 \cdot 8$ & 81 & 62 & $\begin{array}{c}\text { King \& Clark } \\
\text { (1987) }\end{array}$ \\
\hline Scymnodon plunketi & $29 \cdot 0-150 \cdot 2$ & 129 & 100 & Garrick (1959) \\
\hline Oxynotus bruniensis & $41 \cdot 4-73 \cdot 7$ & 64 & 55 & $\begin{array}{l}\text { Finucci et al., } \\
\quad \text { (2016a) }\end{array}$ \\
\hline Dalatias licha & $35 \cdot 7-153 \cdot 6$ & 120 & 95 & $\begin{array}{l}\text { King \& Clark } \\
\quad \text { (1987) }\end{array}$ \\
\hline
\end{tabular}




\subsection{Results}

\subsubsection{Catch densities}

When converted to density (number of individuals/km), Hydrolagus bemisi was the most frequently recorded chimaeroid in the trawl database with 2039 records, followed by Hydrolagus novaezealandiae $(n=1553)$, Harriotta raleighana $(n=$ 843), Rhinochimaera pacifica $(n=386)$, Chimaera carophila $(n=100)$, and Hydrolagus homonycteris $(n=10)$. Hydrolagus novaezealandiae had the highest density catch at 1629.1 individuals/km, followed by $H$. bemisi (44.7), $H$. raleighana (26.6), $R$. pacifica (18.8), C. carophila (7.9), and H. homonycteris (2.9). For squaloids, Squalus acanthias was the most recorded $(n=2312)$, then Deania calcea $(n=2302)$, Etmopterus granulosus $(n=1070)$, Centroselachus crepidater $(n=742)$, Centroscymnus owstonii $(n=445)$, Etmopterus lucifer $(n=436)$, Centrophorus squamosus $(n=$ 365), Dalatias licha $(n=349)$, Scymnodon plunketi $(n=162)$, and Oxynotus bruniensis $(n=41)$. The highest density catches (individuals $/ \mathrm{km}$ ) recorded for squaloids were (in decreasing order): S. acanthias (16523) E. granulosus (10047), D. calcea (119.6), C. crepidater (71.4), C. squamosus, C. owstonii, and S. plunketi (all 33·3), E. lucifer (17·1), D. licha (7·1), and O. bruniensis (2·6) (Table 3.2, Appendix IV).

Table 3.2 gives the proportions of the total catch records calculated to represent different densities for each species. For chimaeroids, all species except $H$. bemisi, were most frequently recorded at a density estimated from a trawl sample of $\leq 1$ individual/km: C. carophila (0.74), H. homonycteris (0·70), R. pacifica (0.63), H. raleighana $(0 \cdot 58)$, and $H$. novaezealandiae $(0 \cdot 22)$. The most common density category for $H$. bemisi was 1 - 5 individuals $/ \mathrm{km}$. Only $H$. novaezealandiae was estimated to have catch densities $\geq 100$ individuals $/ \mathrm{km}$ or greater (proportion of occurrence $=0 \cdot 11$ ) and therefore, was the only chimaeroid included in the social association analysis. For six of the elasmobranchs, most of the catches were recorded at a densities of $\leq 1$ individual/km: O. bruniensis (0.98), D. licha (0.95), S. plunketi (0.80), C. squamosus (0.77), C. owstonii (0.58), and E. lucifer (0.52). In four species, the highest proportion of catches were at densities of $>1-5$ individual $/ \mathrm{km}$ : $C$. crepidater $(0 \cdot 50), D$. calcea (0.36), E. granulosus $(0 \cdot 44)$, and S. acanthias $(0 \cdot 33)$. Proportion of occurrence of catch densities $\geq 100$ individuals $/ \mathrm{km}$ or greater for these species were: $C$. 
crepidater (0·001), D. calcea (0·007), E. granulosus (0·08), and S. acanthias (0·12). These four species, as well as E. lucifer, were included in the social association analysis.

\subsubsection{Hydrolagus novaezealandiae}

Hydrolagus novaezealandiae catches were equally distributed by sex $\left(\chi^{2}=104 \cdot 78, P\right.$ $>0.05$ ), and comprised predominately of juvenile individuals. At the lowest densities, individuals in all classes were significantly associated with other individuals of the same class (Fig. 3.1).

Juveniles were most prevalent in the highest density catches. Juvenile males increased in catch proportion with increasing density, were significantly associated with each other, and showed low association patterns with adults at high densities [Fig. 3.1(A)]. Juvenile females were significantly associated with other juvenile females at low densities [Fig. 3.1(B)].

Adult males were significantly associated with other adult males only at the lowest and highest densities [Fig. 3.1(C)]. Adult females made up a large proportion of the catch at low densities. Adult females were significantly associated with each other across all densities, and with adult males at high densities, while showing little association with juveniles at low density [Fig. 3.1(D)]. 

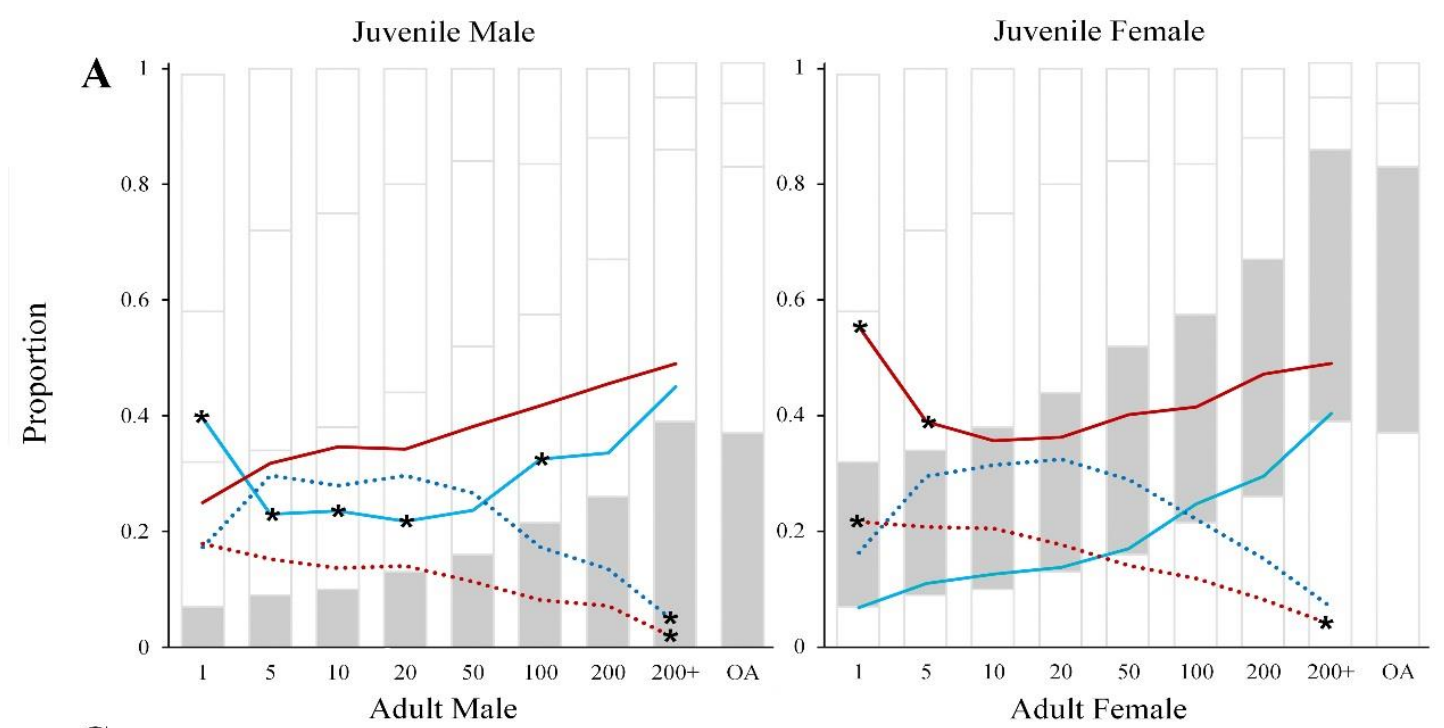

B

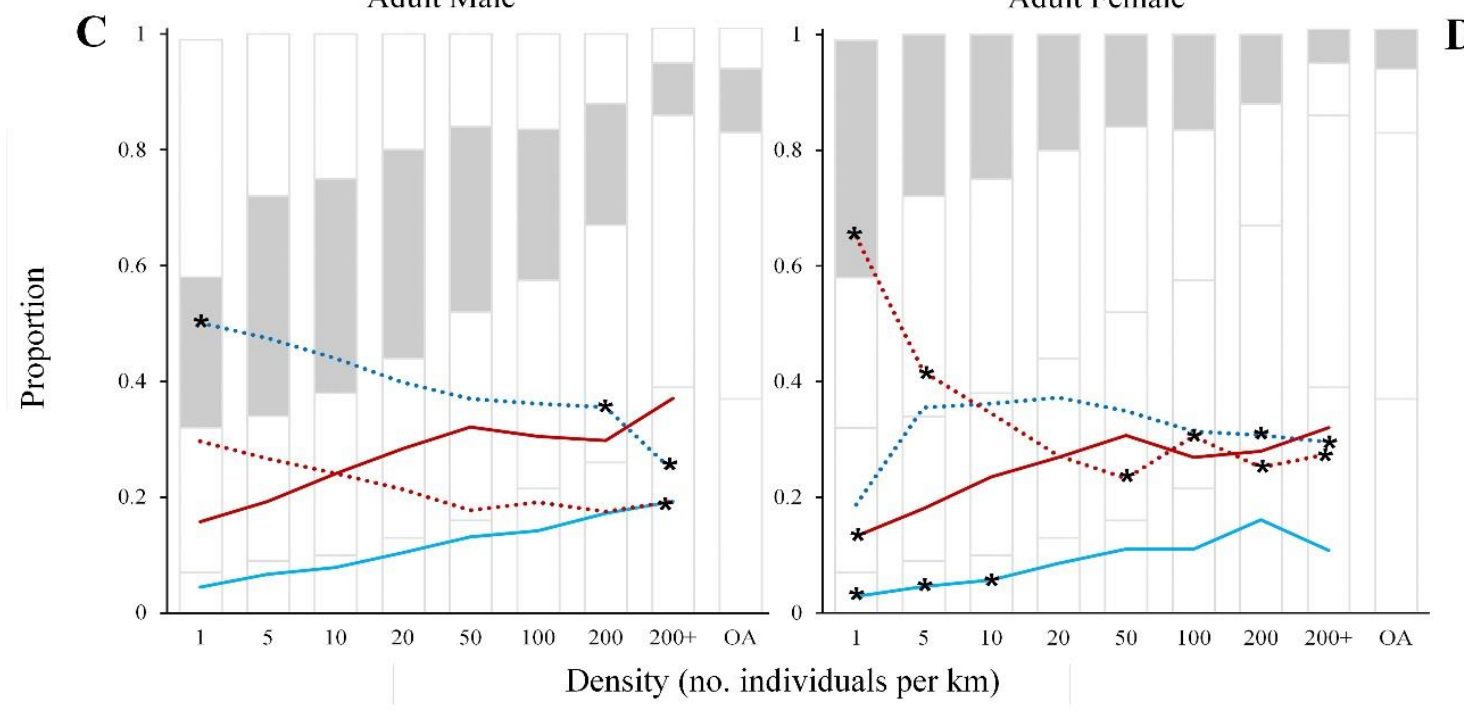

Figure 3.1 Hydrolagus novaezealandiae, by catch density, the proportional class composition ( $\square$ ) and proportional social associations (--- juvenile male; --- juvenile female; - - - adult male; - - - adult female) of (A) juvenile male, (B) juvenile female, (C) adult male, and (D) adult female. Densities at which social associations were significantly different from that expected by overall catch composition indicated with *. OA $=$ overall proportion of sample for each class. 


\subsubsection{Squalus acanthias}

Squalus acanthias catches were composed predominately of juvenile individuals, with a male to female ratio of 1.5:1 $\left(\chi^{2}=9252 \cdot 08, P<0 \cdot 05\right)$. At the lowest densities, individuals in all classes were significantly associated with other individuals of the same class (Fig. 3.2).

Juveniles were prevalent in high density catches. Juvenile males were significantly associated with other juvenile males across all densities, and showed low association patterns with adult females [Fig. 3.2(A)]. Juvenile females were significantly associated with other juvenile females, regardless of density [Fig. 3.2(B)].

Adult males were significantly associated with other adult males, and were associated with adult females at low densities and juvenile females at high densities [Fig. 3.2(C)]. The proportion of the catch that included males, both juvenile and adult, increased with increasing density. Adult females made up a large proportion of the catch at low densities. Adult females were significantly associated with other adult females across all densities, while maintaining low associations with males, regardless of class [Fig. 3.2(D)]. 

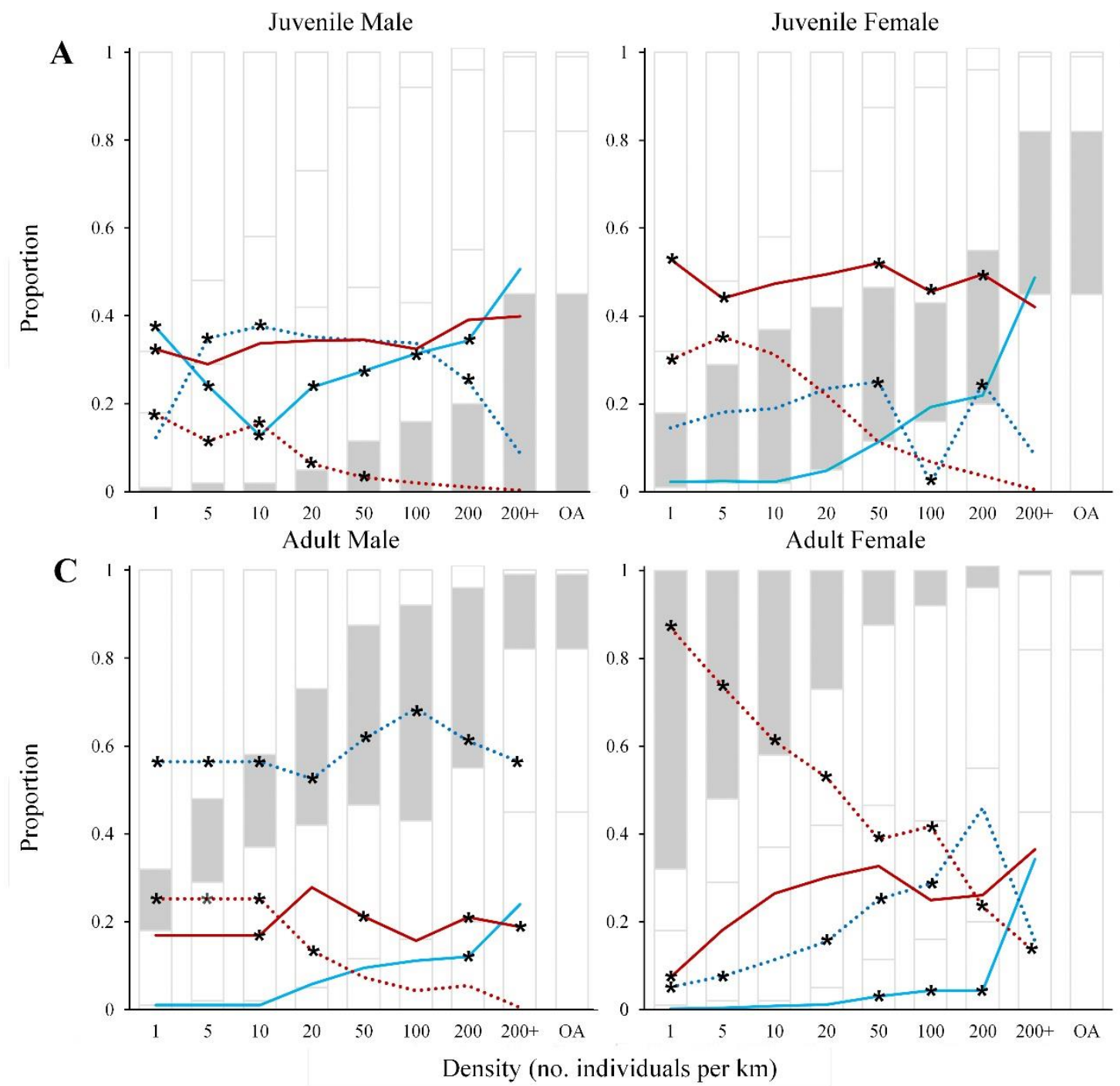

D

Figure 3.2 Squalus acanthias, by catch density, the proportional class composition ( $\square$ ) and proportional social associations (--- juvenile male; --- juvenile female; - - - adult male; - - - adult female) of (A) juvenile male, (B) juvenile female, (C) adult male, and (D) adult female. Densities at which social associations were significantly different from that expected by overall catch composition indicated with *. OA $=$ overall proportion of sample for each class. 


\subsubsection{Etmopterus lucifer}

Etmopterus lucifer catches were composed mostly of adult males and juvenile females, and had a 1:1 sex ratio $\left(\chi^{2}=5.53, P>0 \cdot 05\right)$. At the lowest densities, individuals in all classes were significantly associated with other individuals of the same class (Fig. $3.3)$.

Juvenile males and females increased in numbers with increasing density. Across densities, juveniles of both sexes were significantly associated with other juveniles and showed low association patterns with adults [Fig. 3.3(A, B)].

Lowest densities were comprised largely of adult males and adult females, and a large proportion of the catches included adult males (c. 40\%). Adult males significantly associated with other adult males at lower densities [Fig. 3.3(C)]. Adult females formed the highest proportion of catch at the lowest densities, and were significantly associated with other adult females across all densities. [Fig. 3.3(D)]. 

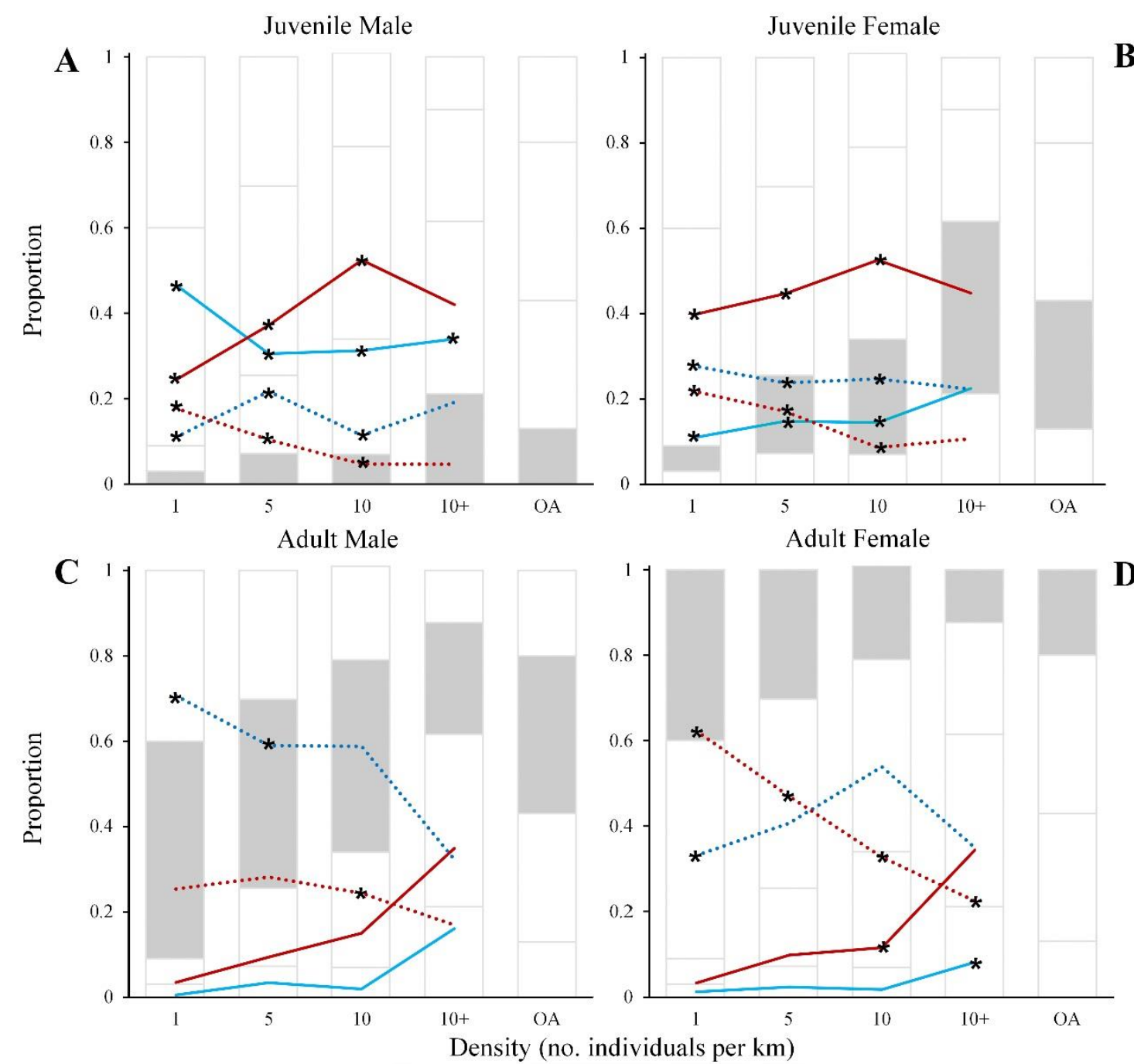

Figure 3.3 Etmopterus lucifer, by catch density, the proportional class composition ( $\square$ ) and proportional social associations (--- juvenile male; -- - juvenile female; - - - adult male; - - - adult female) of (A) juvenile male, (B) juvenile female, (C) adult male, and (D) adult female. Densities at which social associations were significantly different from that expected by overall catch composition indicated with $* . \mathrm{OA}=$ overall proportion of sample for each class. 


\subsubsection{Deania calcea}

The sex ratio of Deania calcea catches was approximately 1:1.5 in favour of females $\left(\chi^{2}=166 \cdot 53, P<0 \cdot 05\right)$. Catches were comprised mostly of juvenile females. At the lowest densities, individuals in all classes were significantly associated with other individuals of the same class (Fig. 3.4).

Juvenile males were significantly associated with other juvenile males across all densities [Fig. 3.4(A)]. Social associations for juvenile females suggested high association with other juvenile females across all densities, but these trends were not significant [Fig. 3.4(B)].

Adult males displayed some significant association patterns with other adult males at low densities [Fig. 3.4(C)], while adult females were significantly associated with other adult females across all densities [Fig. 3.4(D)]. 

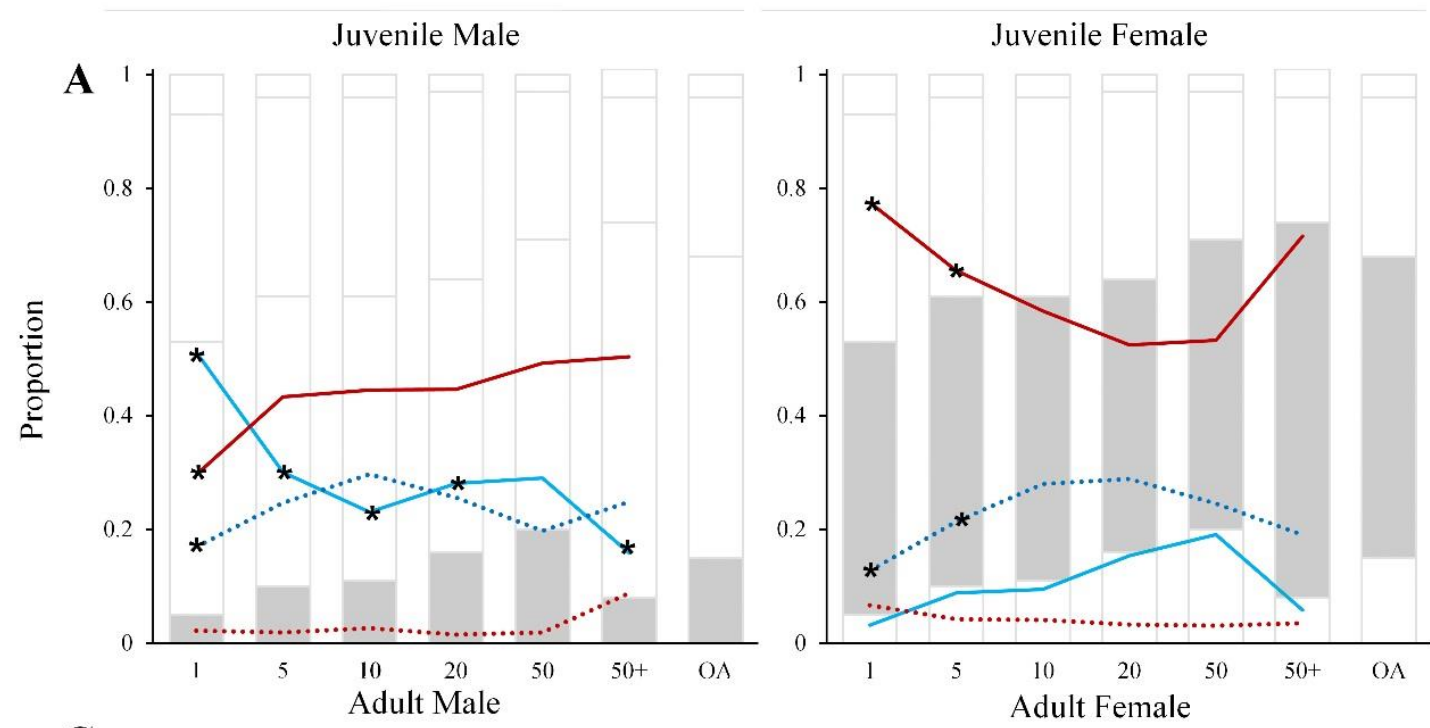

B
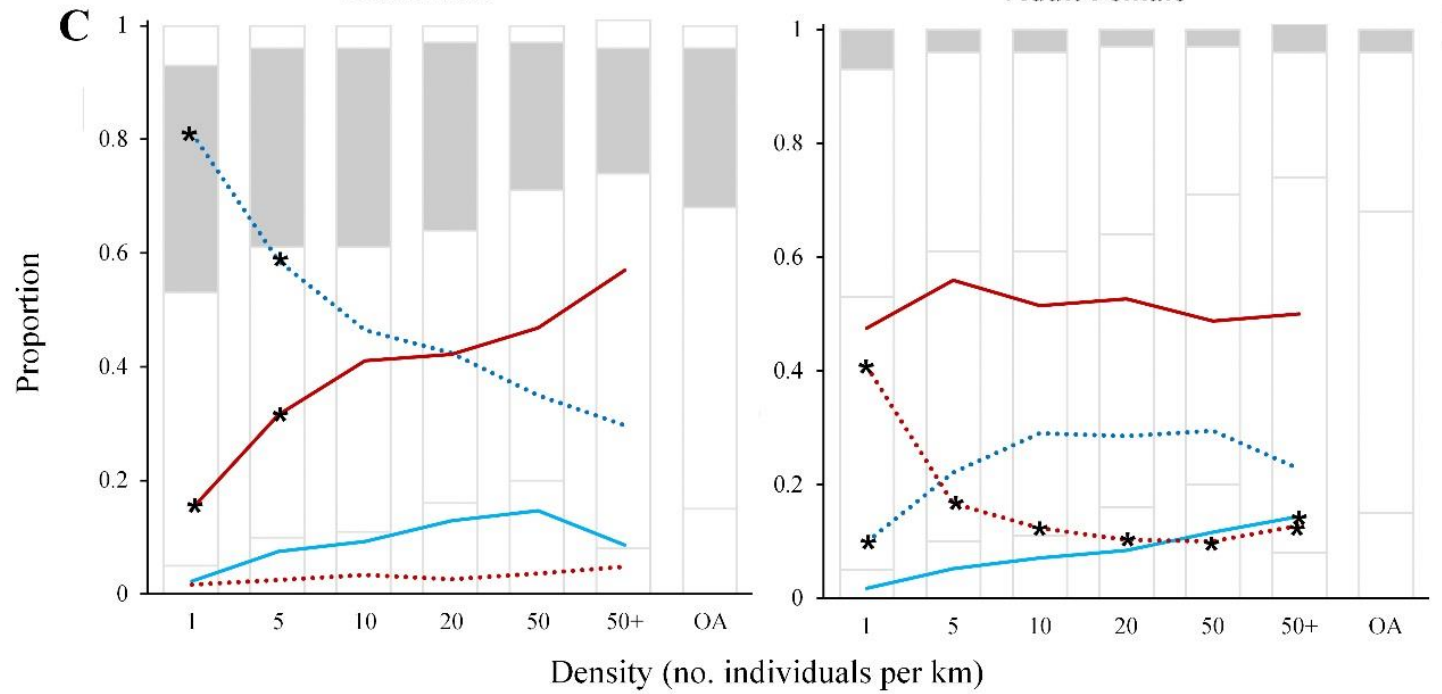

D

Figure 3.4 Deania calcea, by catch density, the proportional class composition ( $\square$ ) and proportional social associations (- - juvenile male; --- juvenile female; - - - adult male; - - - adult female) of (A) juvenile male, (B) juvenile female, (C) adult male, and (D) adult female. Densities at which social associations were significantly different from that expected by overall catch composition indicated with *. OA = overall proportion of sample for each class. 
Centroselachus crepidater catches were equally distributed in $\operatorname{sex}\left(\chi^{2}=127 \cdot 17, P\right.$ $>0.05)$ and maturity stage. At the lowest densities, individuals in all classes were significantly associated with other individuals of the same class (Fig. 3.5).

Juvenile males increased in catch proportion with increasing density, were significantly associated with each other, and showed significantly low association patterns for adult females [Fig. 3.5(A)]. Juvenile female proportions were consistent across density and showed no association with any class [Fig. 3.5(B)].

Adult males were significantly associated with other adult males across all densities [Fig. 3.5(C)]. Adult females were significantly associated with other adult females across all densities. [Fig. 3.5(D)]. 

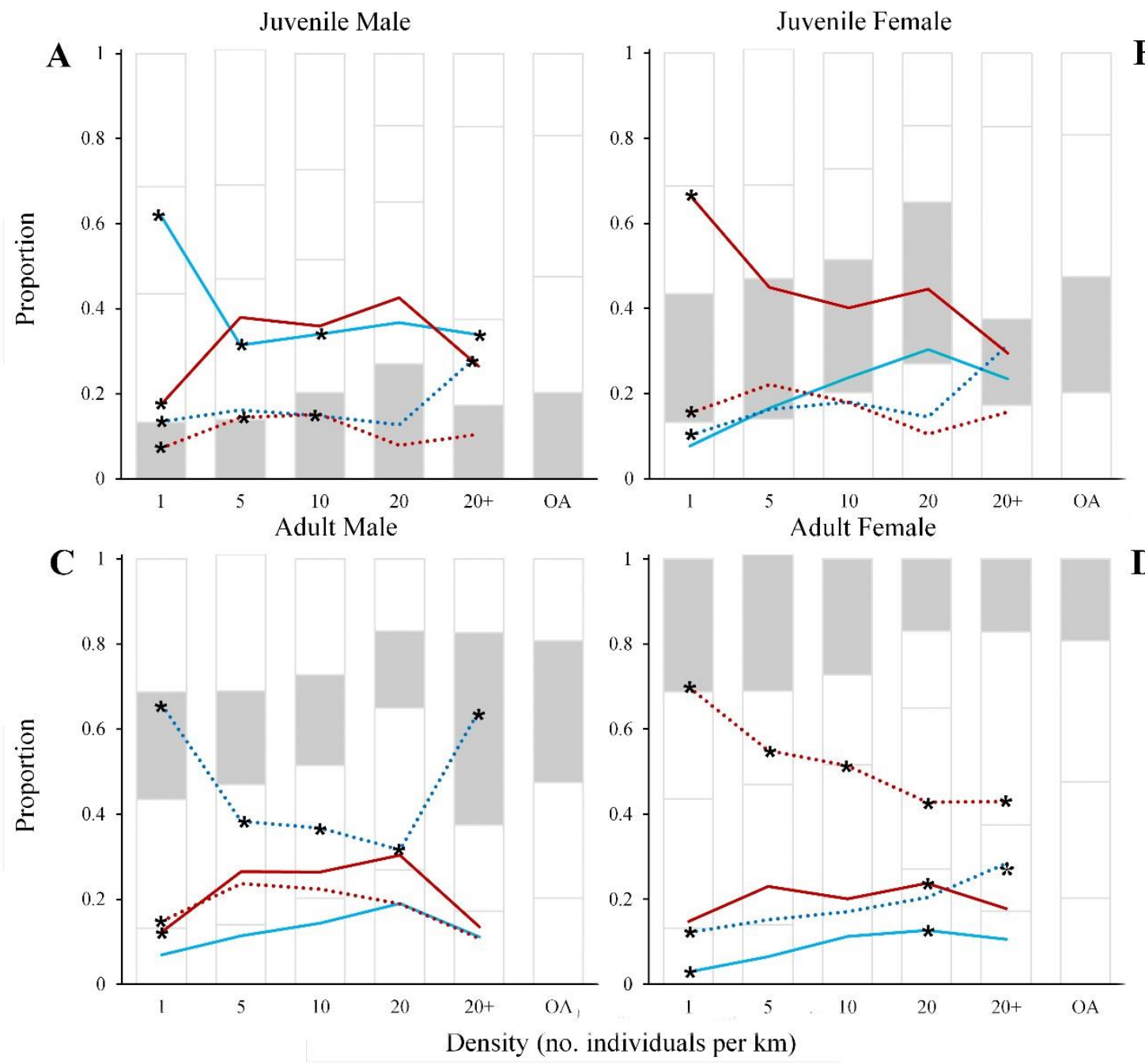

D

Figure 3.5 Centroselachus crepidater, by catch density, the proportional class composition ( $\square$ ) and proportional social associations (--- juvenile male; --- juvenile female; - - - adult male; - - - adult female) of (A) juvenile male, (B) juvenile female, (C) adult male, and (D) adult female. Densities at which social associations were significantly different from that expected by overall catch composition indicated with $*$. OA $=$ overall proportion of sample for each class. 


\subsubsection{Etmopterus granulosus}

Catches of Etmopterus granulosus were composed primarily of mature females, with an approximate $4: 1$ ratio in favour of females $\left(\chi^{2}=49700 \cdot 83, P<0 \cdot 05\right)$. At the lowest densities, individuals in all classes were significantly associated with other individuals of the same class (Fig. 3.6).

Juveniles of both sexes, particularly males, made up a small proportion of the overall catches and decreased in proportion of catch as densities increased. Juvenile males were significantly associated with other juvenile males, and showed low association with adult females at low densities [Fig. 3.6(A)]. Juveniles were most prevalent in high density catches. Juvenile females were significantly associated with other juvenile females at low densities [Fig. 3.6(B)].

The proportion of catch that included adult males was highest at densities of 100 individuals per kilometre. Adult males were significantly associated with other adult males across densities [Fig. 3.6(C)]. Adult females made up a large proportion of all densities, and made up the largest proportion of large density catches. Adult were significantly associated with other adult males across most densities [Fig. 3.6(D)]. 

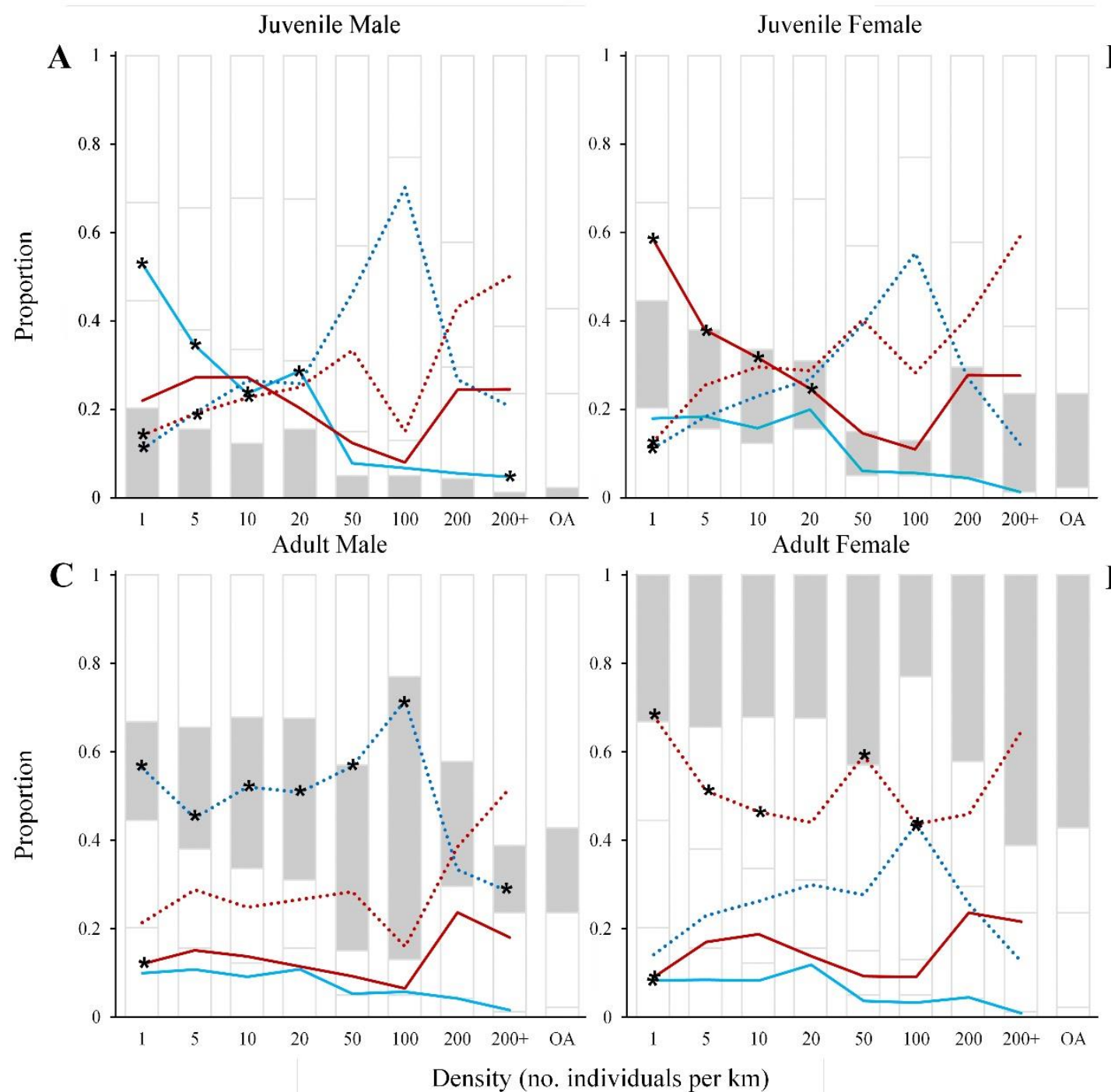

D

Figure 3.6 Etmopterus granulosus, by catch density, the proportional class composition (口) and proportional social associations (-- juvenile male; --- juvenile female; - - adult male; - - - adult female) of (A) juvenile male, (B) juvenile female, (C) adult male, and (D) adult female. Densities at which social associations were significantly different from that expected by overall catch composition indicated with $*$. OA $=$ overall proportion of sample for each class. 
Table 3.2. Density of catches ( $n$ per $\mathrm{km}$ ) as a proportion of the total catch recorded, with mean depth (m) across all densities and maximum density (number of individuals/km).

\begin{tabular}{|c|c|c|c|c|c|c|c|c|c|c|c|c|}
\hline \multirow[t]{2}{*}{ species } & \multirow{2}{*}{$\begin{array}{l}\text { catch } \\
(n)\end{array}$} & \multicolumn{9}{|c|}{ density $(n$ per $\mathrm{km}$ ) as proportion of catch } & \multirow{2}{*}{$\begin{array}{c}\text { Mean } \\
\text { Depth } \\
(\mathbf{m})\end{array}$} & \multirow{2}{*}{$\begin{array}{c}\text { Max Density } \\
\text { (individuals/km) }\end{array}$} \\
\hline & & $n \leq 1$ & $>1-5$ & $>5-10$ & $\begin{array}{c}>10- \\
20\end{array}$ & $\begin{array}{c}>20- \\
50\end{array}$ & $\begin{array}{c}>50- \\
100\end{array}$ & $\begin{array}{l}>100 \\
-200\end{array}$ & $\begin{array}{c}>200- \\
1000\end{array}$ & $\begin{array}{c}1000 \\
+\end{array}$ & & \\
\hline \multicolumn{13}{|l|}{ Chimaeriformes } \\
\hline Chimaera carophila & 100 & 0.740 & 0.250 & 0.010 & 0 & 0 & 0 & 0 & 0 & 0 & 1124 & 7.9 \\
\hline Hydrolagus homonycteris & 10 & 0.700 & 0.300 & 0 & 0 & 0 & 0 & 0 & 0 & 0 & 1186 & 2.9 \\
\hline Rhinochimaera pacifica & 386 & 0.627 & 0.368 & 0.003 & 0.003 & 0 & 0 & 0 & 0 & 0 & 990 & 18.8 \\
\hline Harriotta raleighana & 843 & 0.577 & 0.382 & 0.037 & 0.004 & 0.001 & 0 & 0 & 0 & 0 & 733 & 26.6 \\
\hline Hydrolagus bemisi & 2039 & 0.368 & 0.487 & 0.122 & 0.020 & 0.003 & 0 & 0 & 0 & 0 & 616 & 44.7 \\
\hline Hydrolagus novaezealandiae & 1553 & 0.218 & 0.158 & 0.127 & 0.166 & 0.219 & 0.057 & 0.029 & 0.024 & 0.002 & 335 & 1629.1 \\
\hline \multicolumn{13}{|l|}{ Squaliformes } \\
\hline Oxynotus bruniensis & 41 & 0.976 & 0.024 & 0 & 0 & 0 & 0 & 0 & 0 & 0 & 501 & 2.6 \\
\hline Dalatias licha & 349 & 0.946 & 0.049 & 0.003 & 0 & 0 & 0 & 0 & 0 & 0 & 634 & 7.1 \\
\hline Scymnodon plunketi & 162 & 0.796 & 0.117 & 0.031 & 0.025 & 0.031 & 0 & 0 & 0 & 0 & 712 & 33.3 \\
\hline Centrophorus squamosus & 365 & 0.770 & 0.208 & 0.014 & 0.005 & 0.003 & 0 & 0 & 0 & 0 & 833 & 33.3 \\
\hline Centroscymnus owstonii & 445 & 0.580 & 0.393 & 0.013 & 0.009 & 0.004 & 0 & 0 & 0 & 0 & 947 & 33.3 \\
\hline Etmopterus lucifer & 436 & 0.523 & 0.413 & 0.048 & 0.014 & 0 & 0 & 0 & 0 & 0 & 553 & 17.1 \\
\hline Deania calcea & 2302 & 0.334 & 0.363 & 0.142 & 0.100 & 0.054 & 0.005 & 0.002 & 0 & 0 & 780 & 119.6 \\
\hline Centroselachus crepidater & 742 & 0.307 & 0.503 & 0.105 & 0.058 & 0.024 & 0.001 & 0 & 0 & 0 & 909 & 71.4 \\
\hline Squalus acanthias & 2312 & 0.192 & 0.330 & 0.152 & 0.109 & 0.096 & 0.050 & 0.032 & 0.032 & 0.007 & 300 & 16523.0 \\
\hline Etmopterus granulosus & 1070 & 0.186 & 0.443 & 0.130 & 0.096 & 0.062 & 0.020 & 0.019 & 0.032 & 0.011 & 922 & 10047.0 \\
\hline
\end{tabular}




\subsection{Discussion}

This chapter has examined aggregation behaviour and social associations amongst deep-sea chondrichthyans. All species displayed some social grouping when classified using size and maturity classes (juvenile male, juvenile female, adult male, adult female). Several consistent trends were observed in the social association analysis: adult females were caught most frequently in low densities and were highly associated with other adult females; 2) adult males were consistently highly associated with each other; and, 3) the highest densities were usually dominated by juveniles. Two species, Deania calcea and Etmopterus granulosus, had trends that differed from these observations, with $D$. calcea catches being comprised of mainly juvenile females and few social association trends were noted, and E. granulosus catches consisting mainly of adult females. Catches for some species were most frequently recorded as individuals (Oxynotus bruniensis (0.98), Dalatias licha (0.95), and Scymnodon plunketi (0.80), suggesting that these are largely solitary species. The low density catches of these species may be correlated to a reduction in biomass from fishing (Dunn et al., 2013), or as high trophic level predators, may be reflected by their diets that rely on other chondrichthyans (Dunn et al., 2013; Navarro et al., 2014; Finucci et al., 2016a).

In chondrichthyan research, the term aggregation is often used loosely and may not be explicitly defined. Speed et al. (2011) defined an aggregation as five or more individuals being present in the same area at the same time, which was based on mean nearest neighbour distance calculations by Heupel \& Simpfendorfer (2005). Klimley (1987) considered scalloped hammerhead (Sphyrna lewini) individuals separated by no more than four body lengths to be within the bounds of the study group, while other studies have suggested individuals were capable of interactions at distances $4-5$ metres apart (Smith et al., 2015, Armansin et al., 2016). In teleosts, shoaling fish have been described as those grouping within one body length, although larger distances may designate more loosely organised shoals (Pitcher \& Partridge, 1979). General consensus considers associating fish within four body lengths of each other (Hensor et al., 2005), which would greatly vary in distance across the species studied here given the size differences (Etmopterus lucifer: maximum length of $55 \mathrm{~cm} L_{\mathrm{T}}$ vs. Dalatias licha: maximum length of $153 \mathrm{~cm} L_{\mathrm{T}}$ ). For species with catch densities of $1000+$ individuals $/ \mathrm{km}$ (equivalent to $>1$ individual $/ \mathrm{m}$ ), these high density catches equate to distances between individuals that would realistically interact with each other. Thus, 
species were chosen for the social association analysis based on their likeliness to group in large numbers (largest catch densities).

For the purpose of this study, species were presumed to be equally distributed in the area swept by the trawl, as the species occurrence within the trawl path is unknown. Multiple trawl capture methods were included in the analysis to maximize coverage across Chatham Rise and maximize representation of all size and maturity stages. Trawls can be selective as larger sharks may avoid capture, and part of a population may be beyond range of trawling (Clarke et al., 2005). A previous investigation of deep-sea shark catches from Chatham Rise found no significant difference in the abundance or mean diversity of species captured by different trawl surveys using multiple trawl methods, suggesting that any differences in shark catch between surveys was related to sampling location, rather than temporal or methodological differences (Wetherbee, 2000). Future analysis could be coupled with video footage using towed vehicles or net-mounted cameras (Gerlotto et al., 2010) to observe fish behaviour, and examine the average distance between individuals within a group. Dividing trawls into monthly or seasonal sets may provide changes within group structure throughout the year, possibly giving some indication of reproductive seasonality (e.g. changes in adult male and female associations over time). Seasonal trends were not available for this analysis as a large proportion of surveys were completed in summer months (O'Driscoll et al., 2011).

We recognize that large densities may be influenced by non-social behaviour, such as habitat or food availability. Deep-sea chondrichthyans have been associated with particular sea features (Tracey et al., 2012). However, most deep-sea chondrichthyans are widely distributed across Chatham Rise (Wetherbee, 2000; MacGibbon, 2016), which may be attributed to the highly productive nature of Chatham Rise (McClatchie et al., 1997). In a study by Wetherbee (2000), Etmopterus granulosus has been found to be the most ubiquitous within the squaloids examined, being present in nearly all depths and locations on Chatham Rise. The feeding habitats of most of the study species have been described, with varying degree of depth and location preferences between species, consistent with niche separation to reduce interspecific competition (Dunn et al., 2010a; Dunn et al., 2013). With the exception of O. bruniensis, which appears to rely solely on chondrichthyan egg cases (Finucci et al., 2016a), it was shown that prey items were not exclusive, and species showed some adaptive foraging, scavenging from fishing vessels, and fairly ubiquitous distributions (Dunn et al., 2013). While we cannot completely rule out that these affiliations may be influenced by a non-social behaviour or site preference, 
given the productivity of Chatham Rise, widespread occurrence and adaptable feeding habits of the study species, as well as strong association trends produced here across multiple species at varying densities, particularly in adult individuals, we believe social selection within these groupings has been recognized.

\subsubsection{Reclusive females}

Regardless of density, adult females showed strong associations for other adult females, and little association with both juvenile and adult males. Sexual segregation has been frequently recorded in deep-sea elasmobranchs (Yano \& Tanaka, 1988; Coelho \& Erzini, 2010; Moura et al., 2014) and more recently, in chimaeroids (Holt et al., 2013). Segregation of the sexes is thought to have evolved in response to the male reproductive strategy, which promotes relatively low reproductive investment and is orientated towards frequent mating with multiple receptive partners (Wearmouth \& Sims, 2008). Mating can come at high costs to female chondrichthyans as multiple copulations can leave potentially serious inflictions to the body and pectoral fins caused by males (Carrier et al., 1994; Pratt \& Carrier, 2001), and harassment can reduce feeding efficiency (Magurran \& Sehers, 1994) and increase risk of predation (Wearmouth \& Sims, 2008), all of which may negatively impact female fitness. These factors create a benefit for females to avoid multiple mating events (Economakis \& Lobel, 1998). There is an adaptive benefit to females that invest in delayed fertilization, sperm storage, or refuge en masse (Wearmouth \& Sims, 2008). Under captive conditions, female small-spotted catsharks Scyliorhinus canicula (L. 1758) were shown to spend more time in socially stable aggregations with other females, correlating with greater resilience to male disturbance (Jacoby et al., 2010). Single-sex female groups were also found to have increased foraging activity than those in mixed-sex groups, which was attributed to factors associated with sexual conflict (e.g. male harassment and female avoidance of males) (Kimber et al., 2009).

Adult females were most frequently captured in low densities. If mature female chondrichthyans are naturally occurring at low densities, this observation is consistent with an apparent lack of large, reproductively active females (e.g. the presence of vitellogenic activity or egg capsules) in catches, as observed for many deep-sea chondrichthyan species (Girard \& Du Buit, 1999; Moura et al., 2014; Finucci et al., 2016b). The apparent lack of reproductively active females has been attributed to 
individuals inhabiting depths beyond fishing efforts, or in areas inaccessible to, or outside of, trawl surveys (Parker \& Francis, 2012). Gear selectivity is a factor that can explain size and sex catch composition, and whilst catchability is poorly understood for these species, it has been suggested that large specimens might be able to avoid nets (Clarke et $a l .$, 2005). In this study, however, adult males often represented large proportions of the samples across most densities, so we would expect females of similar size to be captured as well.

Deep-sea chondrichthyans are thought to engage in extensive migrations (Rodríguez-Cabello \& Sánchez, 2014), which may be associated with reproductive activity (Clark \& King, 1989; Moura et al., 2014). In the shallow water lemon shark Negaprion brevirostris (Poey, 1868), gravid females have been found to undertake longdistance migrations to give birth in sheltered areas far from adult conspecifics (Feldheim et al., 2002). It seems more plausible that temporal segregation, rather than gear selectivity, explains the missing females for some species. However, previous studies which have included sites in New Zealand beyond Chatham Rise have still seldom recorded mature females (Clark \& King, 1989; Parker \& Francis, 2012; Moura et al., 2014). Given this general pattern of low mature female density across all species (with the exception of Etmopterus granulosus), it appears that mature female chondrichthyans on Chatham Rise may be found naturally in lower densities than other classes.

\subsubsection{Adult males associated with other adult males}

In all species, adult males were consistently highly associated with other adult males across all densities. Male affiliation is known across many vertebrate taxa, and has been attributed to cooperative foraging (Mitani \& Watts, 1999), reproduction (e.g. courtship, copulation) (Taborsky, 2009), and territorial defence (Grinnell et al., 1995); the latter of which has not been demonstrated in elasmobranchs (Gruber et al., 1988). While large groups of elasmobranchs have been recorded feeding on the same resource, evidence of cooperative hunting through social facilitation in chondrichthyans is often speculative, as it is unclear if sharks are actually cooperating or aggregating for prey items (Motta \& Wilga, 2001). Some lantern sharks (Etmopterus spp.) have been suggested to forage in groups based on the identification of large prey items in stomach contents (Springer, 1967; MacPherson, 1980), however, it is possible that these species were simply scavenging (Neiva et al., 2006). 
Cooperative behaviour in males for reproductive purposes has been documented in a several elasmobranchs, including whitetip reef sharks Triaenodon obesus (Rüppell, 1837) (Whitney et al., 2004) and nurse sharks Ginglymostoma cirratum (Bonnaterre, 1788) (Carrier et al., 1994). Termed 'group courtship', this behaviour refers to multiple males simultaneously grasping the same female within a mating aggregation (Whitney et al., 2004). Group courtship, while competitively motivated, is thought to be functionally cooperative by increasing the probability of copulation for one male if another has also taken hold of the female (Carrier et al., 1994). Size dimorphism in chondrichthyans favours larger-bodied females, which may allow females to avoid mating attempts (DalyEngel et al., 2010). With cooperative behaviour (e.g. mobbing or herding), however, males could overcome size disadvantages to mate with unwilling females (Pratt \& Carrier, 2001; Whitney et al., 2004). If adult females are found naturally at low densities, as shown in this analysis, it may be advantageous for males to work in groups to mate with all encountered females (Taborsky, 2009).

The male for male associations may suggest for a lek mating system in the deepsea chondrichthyans analysed here. Lekking has evolved independently across many vertebrate taxa, including teleosts (e.g. Nordeide \& Folstad, 2000), and consists of an aggregation of males that females visit with the sole purpose of mating (Höglund \& Alatalo, 1995). It requires no male paternal care, the absence of any unique resources to females other than the males, and involves female mate choice (Bradbury, 1981). In the deep-sea, where resources may be limited, lekking may reduce the cost of a female searching for mates because the congregation of males would make mate selection easier (Wickman \& Jansson, 1997). Most lekking species are sexually dimorphic (Höglund \& Alatalo, 1995). As well as overall size, sexual dimorphism in chondrichthyans has been reported in some other characteristics, including the electric organ of some skates (e.g. Morson \& Morrissey, 2007) and the snout size of Harriotta raleighana (Finucci et al., 2016b). A previous proposal for a chondrichthyan lek system was challenged on account of a variety of factors, including confirmation of multiple paternity in a single litter (eliminating female mate choice) (Domeier \& Nasby-Lucas, 2013). Multiple paternity has been documented in five of the eight extant orders of sharks, including the Squaliformes (Fitzpatrick et al., 2012), but has yet to be identified in chimaeroids. In addition, it appears more likely that females would refuge from, rather than actively seek out male partners, given the high fitness costs to females from aggressive mating events as outlined previously, at least in elasmobranchs. For chimaeroids, it is difficult to make similar 
conclusions at this time with such little data available on their reproductive behaviour. However, some observations have been made from captive individuals, particularly egglaying in Hydrolagus colliei (Tozer \& Didier, 2004), and these individuals could be useful for future reproductive behavioural studies.

\subsubsection{Highest densities dominated by juveniles}

The highest density catches of several species were dominated by juvenile individuals, and were found to be a relatively equal mix of the sexes. Areas with high densities of juveniles are characteristic of nursery grounds, along with juvenile site fidelity for extended periods of time and repeated use of the designated area across years (Heupel et al., 2007). Grouping at an early life history stage is thought to reduce mortality via antipredator behaviour (Krause \& Ruxton, 2002). Chondrichthyans have been found to make up a large proportions of the diet of other chondrichthyans, particularly in the deep-sea (Navarro et al., 2014; Finucci et al., 2016a). Smaller-bodied lantern sharks Etmopterus spp. have been reported in the diet of a number of larger-bodied species, including Dalatias licha (Navarro et al., 2014) and Scymnodon plunketi (Dunn et al., 2010b). Intra-specific predation of juveniles by larger individuals has also been documented across a range of elasmobranchs (Guttridge et al., 2012), as well as in $H$. colliei (Johnson, 1967). Egg cases of oviparous chondrichthyans also fall prey to a selection of species, including gastropods, elasmobranchs, teleosts, and mammals (Bor \& Santos, 2003; Finucci et al., 2016a). The identification of deep-sea nursery or egg laying grounds which meet the criteria outlined by Heupel et al. (2007) is difficult to validate, but large clusters of egg cases from oviparous species have been located near cold seeps (Truede et al., 2011), cold-water coral reef habitat (Henry et al., 2013), and various discreet locations of the outer and middle shelf and upper slope of canyons (Hoff, 2016). Mernoo Bank has been suspected as an area of importance for juvenile $H$. bemisi and $H$. novaezealandiae based on observations of large numbers of juveniles, but further investigation into this region is required (Horn, 1997).

In size assortment preference experiments, juvenile sharks preferred to spend more time with other individuals of the same size class, suggesting group composition was influenced by social preference (Guttridge et al., 2009). Our study has demonstrated that juveniles were associated with other juveniles, particularly at low densities. Associating with individuals of similar body size has been thought to increase 
information sharing and foraging efficiency, while reducing vulnerability to predation (Ranta et al., 1994). It is unknown if social preference is maintained through familiarity and/or individual recognition in juvenile sharks (Guttridge et al., 2009), but our study has indicated that for some deep-sea chondrichthyans, sex and size-specific associations, which may be influenced by maturity, may be established at a small size and then continue into adulthood.

\subsubsection{Individual species trends}

Squalus acanthias showed the strongest trends for social associations across all densities and class. The trends observed may be explained by its high abundance (O’Driscoll et al., 2011) and strong synchronous breeding seasons (Hanchet, 1998). Species that did not exhibit patterns of social association, or form high densities which may account for breeding aggregations, may engage in asynchronous reproductive cycles (Daly-Engel et al., 2010). A lack of defined breeding cycles would be advantageous for species in habitats where resources, breeding partners, or breeding opportunities are limited (Veríssimo et al., 2010). Asynchronous breeding cycles have been noted in some deep-sea elasmobranchs (Yano \& Tanaka, 1988; Girard \& du Boit, 1999), and have been suspected for E. granulosus (Wetherbee, 1999; Daley et al., 2002), D. calcea, and C. crepidater (Daley et al., 2002). Reports are conflicting in the chimaeroids, and for $H$. bemisi and $H$. novaezealandiae, it has been suggested that spawning may occur on a seasonal basis (Horn, 1997) or year-round (McCutcheon, 1980). Since neither H. bemisi nor $H$. novaezealandiae showed strong association patterns similar to S. acanthias, this may imply that these species engage in asynchronous breeding cycles.

The aggregations of Etmopterus granulosus were different from the other chondrichthyans in this study, with the sample dominated by mature females and the largest catch densities comprised of mature individuals. Wetherbee (1999) reported nearly twice as many females as males from Chatham Rise, with the majority of the sample (76 $-80 \%$ ) composed of mature individuals. The high tendency for adults to prefer associating with fish of the same sex and size, in conjunction with a strong association with features such as seamounts (Hallett \& Daley, 2010; Tracey et al., 2004), suggest male or female dominated seamount aggregations. Many seamounts are intensively fished for orange roughy (Hoplostethus atlanticus), and E. granulosus makes up a significant component of bycatch in orange roughy fisheries (Clark et al., 2000; Daley et al., 2002). 
Spatially focused fishing pressures on seamounts could result in differential mortality of the sexes (Mucientes et al., 2009).

Information on juvenile E. granulosus is limited on Chatham Rise, and gravid females with embryos have not been recorded (Wetherbee, 1999). With an estimated sizeat-birth of c. $20 \mathrm{~cm}$ total length (Wetherbee, 1999), it is unlikely that small individuals were not retained by research trawls, and might therefore be at depths outside most bottom trawl fishing efforts on Chatham Rise. It is also plausible that this species may be pupping elsewhere, as gravid females have been captured from Cook Strait off Wellington, New Zealand (Finucci, unpublished data). Etmopterus granulosus was listed as the second highest at-risk species not managed under New Zealand's Quota Management System (QMS) by a recent Level 1 Qualitative Risk Assessment, on account of low productivity (e.g. late age of maturity, moderate fecundity) and high habitat overlap with fishing effort (Ford et al., 2015). Potential high unintentional sex-specific capture of adult E. granulosus supports this high risk ranking, and continual monitoring of this species is needed.

This study has found evidence of a general pattern of sex- and size-class structuring in deep-sea chondrichthyans. Social associations were inferred in multiple species, which may be driven by a variety of factors, including foraging and predator avoidance, as well as avoidance of sexual conflict. The nature of these associations may result in selective mortality of components of populations by spatial or temporally stratified, or aggregation-targeting, fishing, which may increase population vulnerability. The recognition of social interactions in deep-sea chondrichthyans, and the resulting segregation, not only provides an insight into the behaviour of these species, but is required for effective monitoring and management of the effect of human activities on their populations. 


\section{CHAPTER IV}

Reproductive biology and feeding habits of the prickly dogfish Oxynotus bruniensis (Ogilby 1893) 


\subsection{Abstract}

The reproductive biology and diet of prickly dogfish Oxynotus bruniensis, a deepsea elasmobranch, endemic to the outer continental and insular shelves of southern Australia and New Zealand, and caught as bycatch in demersal fisheries, are described from specimens caught in New Zealand waters. A total of 53 specimens were obtained from research surveys and commercial fisheries, including juveniles and adults ranging in size from $33 \cdot 5-75 \cdot 6 \mathrm{~cm}$ total length $\left(L_{\mathrm{T}}\right)$. Estimated size-at-maturity was $54.7 \mathrm{~cm} L_{\mathrm{T}}$ in males and $64.0 \mathrm{~cm} L_{\mathrm{T}}$ in females. Three gravid females $\left(65 \cdot 0,67 \cdot 5\right.$ and $\left.71 \cdot 2 \mathrm{~cm} L_{\mathrm{T}}\right)$ were observed, all with eight embryos. Size-at-birth was estimated to be $25-27 \mathrm{~cm} L_{\mathrm{T}}$. Vitellogenesis was not concurrent with embryo development. Analysis of diet from stomach contents, including DNA identification of prey using the mitochondrial genes coxl and nadh2, revealed that $O$. bruniensis preys exclusively on the egg capsules of holocephalans, potentially making it the only known elasmobranch with a diet reliant solely upon other chondrichthyans. Based on spatial overlap with deep-sea fisheries, a highly specialized diet, and reproductive characteristics representative of a low productivity fish, the commercial fisheries bycatch of $O$. bruniensis may put this species at relatively high risk of overfishing. 


\subsection{Introduction}

The prickly dogfish Oxynotus bruniensis (Ogilby 1893), is one of five species of the globally distributed monotypic sharks of the family Oxynotidae, known as angular or rough sharks (Yano \& Matsuura, 2002). Extant species comprise the angular roughshark Oxynotus centrina (L. 1758) and the sailfin roughshark Oxynotus paradoxus Frade 1929 in the eastern Atlantic Ocean, and Caribbean roughshark Oxynotus caribbaeus Cervigón 1961, Japanese roughshark Oxynotus japonicus Yano \& Murofushi 1985 and prickly dogfish O. bruniensis, endemic to the Caribbean, Japanese waters and the Tasman Sea region, respectively (Yano \& Matsuura, 2002; Yano, 2004; Ebert et al., 2013). Oxynotus bruniensis has a limited distribution, inhabiting temperate waters of the outer continental and insular shelves of southern Australia and New Zealand (Garrick, 1960; Last \& Stevens, 2009). In New Zealand waters, O. bruniensis have been reported most frequently off the east coast of the South Island from depths of 200-1000 m (Beentjes et al., 2002; Francis et al., 2002; Tracey et al., 2004).

With a unique triangular shaped body, sail-like dorsal fins and distinctive enlarged dermal denticles, members of the family Oxynotidae are easily identifiable, but sightings seem to be uncommon and their life history is virtually unknown (Capapé et al.,1999; Yano et al., 2002; Messing et al., 2013). Literature regarding the biology and ecology of $O$. bruniensis is scarce, which is reflected in the current conservation assessment of Data Deficient made by the IUCN Red List (Francis, 2003). Maximum total length $\left(L_{\mathrm{T}}\right)$ of $O$. bruniensis has been reported at $75 \mathrm{~cm}$ (Roberts et al., 2015). Females are lecithotrophic, viviparous and thought to have low fecundity (Compagno, 1984). Lengthat-maturity is estimated at $67 \mathrm{~cm} L_{\mathrm{T}}$ for females, and between 55 and $60 \mathrm{~cm} L_{\mathrm{T}}$ for males, and size-at-birth is $c .24 \mathrm{~cm} L_{\mathrm{T}}$ (Francis, 2003). The diet is unknown (Dunn et al., 2013), although a relatively small mouth suggests that $O$. bruniensis may feed on benthic invertebrates and fishes (Last \& Stevens, 2009).

Oxynotus bruniensis has little (to no) value to New Zealand fisheries (Ministry of Fisheries, 2008) and is caught as bycatch predominately in deep-sea trawl fisheries targeting hoki Macruronus novaezelandiae (MPI, 2015). Most catch is discarded, although nearly $25 \mathrm{t}$ were landed between the 2009-2010 and 2013-2014 fishing seasons in New Zealand (MPI, 2015). Mortality rate of any discards, as well as the value or use of landed fish, are unknown. The potential effects of commercial fishing on O. bruniensis in New Zealand waters are unknown, but risk to fishing is considered moderate based on 
this species having some distribution beyond the range of fishing effort (Ford et al., 2015). Basic biological information, including length- and age-at-maturity, growth rates and reproductive output are important for fisheries biology (Ricker, 1958; Cailliet \& Goldmann, 2004), and in assessing a species' susceptibility to fishing (Stevens et al., 2000; Dulvy et al., 2014). This study examines life history traits of $O$. bruniensis for the first time, focusing on reproduction and diet, in order to provide baseline data for future conservation assessments. 


\subsection{Methods}

\subsubsection{Study area and specimen collection}

Specimens were primarily obtained during fisheries independent research trawl surveys conducted by the National Institute of Water and Atmospheric Research (NIWA), on board RV Tangaroa in Chatham Rise and sub-Antarctic waters of New Zealand during 2014 and 2016, and RV Kaharoa around the North Island of New Zealand in 2015. Trawl surveys were stratified-random with a primary objective to provide relative abundance indices of important commercial middle-depth fishes (O'Driscoll et al., 2011; Bagley et al., 2013). Sampling strata were defined by location and depth, and fishing occurred on trawlable fishing grounds. For RV Tangaroa, a full-wing bottom trawl net with a 60-mm codend mesh was towed for $5.6 \mathrm{~km}$ (3 nautical miles), at a speed of $6 \cdot 5 \mathrm{~km} \mathrm{~h}^{-1}(3 \cdot 5$ knots) at depths from 300 to $1300 \mathrm{~m}$. Trawls completed by RV Kaharoa used a two-panel trawl net with a $60 \mathrm{~mm}$ codend mesh, towed for $2.8 \mathrm{~km}(1.5$ nautical miles $)$ at a speed of $6.5 \mathrm{~km} \mathrm{~h}^{-1}$ (3.5 knots), at depths up to $400 \mathrm{~m}$. All fish caught were frozen whole at sea and brought back to the laboratory for analyses. Samples were also obtained from within the New Zealand Exclusive Economic Zone (EEZ) by Ministry for Primary Industries (MPI) scientific observers aboard commercial fishing vessels during 2015.

Catch records and biological information (length, mass, sex and maturity stage) on $O$. bruniensis caught during previous survey trawls were accessed from the New Zealand MPI research trawl database to increase sample size. Bottom trawl catches dating back to 1998 and made between $34 \cdot 90^{\circ}-53 \cdot 37^{\circ} \mathrm{S}$ and $166 \cdot 01^{\circ}-175 \cdot 44^{\circ} \mathrm{W}$ provided 464 catch records of $O$. bruniensis, of which 59 fish were also sampled for biological information.

\subsubsection{Biological data}

For all specimens dissected, total mass $\left(M_{\mathrm{T}}\right), L_{\mathrm{T}}$, snout to vent length $\left(L_{\mathrm{SV}}\right)$ and inner clasper length $\left(L_{C}\right)$ were recorded following Francis (2006a). Sex was determined externally by the presence (males) or absence (females) of claspers. The mass of the liver and gonads were recorded, and the oviducal gland and uterus (in females) and testes (in males) were measured for length and width. The degree of calcification of claspers and development of the epididymis were also noted. The number of vitellogenic follicles and 
the largest ovarian follicle diameter were recorded for each ovary. Lengths were measured to the nearest $0 \cdot 1 \mathrm{~cm}$, and mass to the nearest $0 \cdot 1 \mathrm{~g}$. Gonadosomatic index $\left(I_{\mathrm{G}}\right)$ was calculated as the gonad mass divided by $M_{\mathrm{T}}$. ANCOVA was used to compare relationships between $L_{\mathrm{T}}$ and $M_{\mathrm{T}}$, and $L_{\mathrm{T}}$ and $L_{\mathrm{SV}}$ between sexes, and paired-sample $t$-tests were used to evaluate differences between measurements of the right and left reproductive tracts of males and females (Zar, 1996), with significance for all tests accepted at $P<0.05$.

Specimens were classified into one of six maturity stages as used by the New Zealand on-board scientific observers (M.P. Francis \& W.S. Lyon, NIWA, pers. comm.). Males were identified as immature (claspers shorter than pelvic fins, soft and uncalcified, unable or difficult to splay open), maturing (claspers longer than pelvic fins, soft and calcified, unable or difficult to splay open or rotate forwards) or mature (clasper longer than pelvic fins, hard and calcified, able to splay open and rotate forwards to expose clasper spine). Six maturity stages were used in females: from immature (ovaries small and undeveloped, and ovarian follicles small or not visible, translucent whitish colour), maturing (some ovarian follicles enlarged, up to pea-sized or larger, coloured white to cream), mature (large ovarian follicles, bright yellow in colour), gravid I (uteri contain eggs or egg cases but embryos are not visible), gravid II (uteri contain visible embryos) and post-partum (distended and vascularized uteri indicating recent birth). Ovarian fecundity (number of large, yolky follicles) and uterine fecundity (number of embryos) were determined from female stages mature, gravid I and gravid II.

Size-at-maturity $\left(L_{50}\right)$ was calculated for males and females separately by fitting a logistic model to the proportion of mature individuals at length using non-linear least squares. Maturity data (immature 0 , mature 1) were aggregated by $5 \mathrm{~cm} L_{\mathrm{T}}$ size class intervals for each sex. The logistic function had the form $Y_{a}=\left[1+19^{\left(L_{50}\right.}{ }^{-a) / L} \text { to95 }\right]^{-1}$, where $Y_{a}$ is the proportion of mature individuals in size class $a$, and $L_{50}$ and $L_{\mathrm{to} 95}$ are parameters. All statistical analyses were carried out using R (R Core Team, 2012).

\subsubsection{Diet analysis}

Stomachs were dissected from all specimens, and stomach contents were rinsed with distilled water on a 500- $\mu \mathrm{m}$ steel sieve. Recognizable prey items were identified to the lowest possible taxon using reference guides. A tissue sample was taken from all prey items that could not be identified with reference guides, and preserved in $90 \%$ ethanol. 
Total genomic DNA was extracted from each tissue sample using the salting out procedure of Miller et al. (1988). A small piece of the tissue (c. $100 \mathrm{mg}$ ) was digested overnight at $56^{\circ} \mathrm{C}$ in a $1.5 \mathrm{ml}$ microcentrifuge tube containing $600 \mu \mathrm{l}$ of lysis buffer [0.05 M Tris-HCl, pH 8; 0. 1 M EDTA; $5 \mathrm{M} \mathrm{NaCl}$ and $5 \mathrm{M}$ sodium dodecyl sulphate (SDS)] and $20 \mu \mathrm{l}$ of proteinase $\mathrm{K}\left(10 \mathrm{mg} \mathrm{ml}^{-1}\right)$. Proteins were precipitated using $\mathrm{NaCl}$ and discarded. Nucleic acid was precipitated by isopropyl alcohol and ethanol before being dried and resuspended in Tris-EDTA buffer. Two separate sets of universal primers developed for DNA barcoding and phylogenetic studies of fishes were used for amplification of the mitochondrial genes $\operatorname{cox} 1$ and nadh2 (Table 4.1). Polymerase chain reaction (PCR) amplification was performed in a $10 \mu 1$ solution containing 20-50 ng of template DNA, $1 \mu 1$ of $10 \times$ reaction buffer Taq DNA polymerase buffer (Applied Biosystems; www.appliedbiosystems.com), $1 \mu 1$ of deoxynucleotide triphosphates (dNTPs) $(10 \mathrm{mM}), 1 \mu \mathrm{M}$ of each primer and 0.5 units of Taq DNA polymerase (Applied Biosystems). Negative controls in all PCR runs were completed to confirm the absence of cross-contamination. The thermal cycle conditions were $95^{\circ} \mathrm{C}$ for $1 \mathrm{~min}$ (initial denaturation), followed by 35 cycles of $95^{\circ} \mathrm{C}$ for $1 \mathrm{~min}$ (denaturation), $54-57^{\circ} \mathrm{C}$ for $1 \mathrm{~min}$ (annealing) and $72^{\circ} \mathrm{C}$ for $1 \mathrm{~min}$ (extension). Double-stranded DNA products were purified using $1 \mathrm{U}$ of ExoSAP-it (GE Healthcare Bio-Sciences, Australia) at $37^{\circ} \mathrm{C}$ for 45 min, followed by an inactivation step at $80^{\circ} \mathrm{C}$ for $15 \mathrm{~min}$. Purified PCR products were bidirectional sequenced using the BigDye Kit v3.1 (Applied Biosystems) on an ABI Prism 3130xl Genetic Analyser (Applied Biosystems). The same mitochondrial genes were also amplified and sequenced from ethanol-preserved muscle tissue of $O$. bruniensis.

Nucleotide sequences were edited and aligned using Geneious R8 (Biomatters Ltd; http://www.biomatters.com/). Because visual identification of prey suggested holocephalans were the main dietary component, sequences from holocephalans, as well as Oxynotus, were obtained from Barcode of Life Data (BOLD) (Ratnasingham \& Hebert, 2007) and GenBank (Geer et al., 2010) for phylogenetic comparisons. Using Kimura's two-parameter (K2P) distance model, Neighbour Joining (NJ) and Maximum Likelihood (ML) algorithms were calculated for sequence divergences (Kimura, 1980). Uncorrected genetic ( $p$ ) distances with 1000 bootstrap replicates (Nei \& Kumar, 2000) were carried out in PhyML (Guindon \& Gascuel, 2003). Base pair differences and nucleotide diversity among the haplotypes were determined using DnaSP version $5 \cdot 10$ 
(Librado \& Rozas, 2009). Novel haplotype sequences were submitted to global databases (e.g. GenBank and BOLD) under the accession numbers listed in Appendix V. 
Table 4.1. Primers used for amplification of $\operatorname{cox} 1$ and nadh 2 mitochondrial genes.

\begin{tabular}{|c|c|c|}
\hline Name & Sequence $\left(5^{\prime}-3^{\prime}\right)$ & Reference \\
\hline Fish_F2 & TCG ACT AAT CAT AAA GAT ATC GGC AC & Ward et al. (2005) \\
\hline Fish_R2 & ACT TCA GGG TGA CCG AAG AAT CAG AA & Ward et al. (2005) \\
\hline Elasmo_F1 & CAT ACC CCA AAC ACG TTG GTT AA & Naylor et al. (2005) \\
\hline Elasmo_R2 & AAG ATC RAT GCT TAC TCA CCT AG & Naylor et al. 2005) \\
\hline ILEM & AAG GAG CAG TTT GAT AGA GT & Naylor et al. (2005) \\
\hline ASNM & AAC GCT TAG CTG TTA ATT AA & Naylor et al. (2005) \\
\hline
\end{tabular}




\subsection{Results}

\subsubsection{Specimen collection and length-mass relationship}

Combining all available information, most catch records of $O$. bruniensis were from Chatham Rise, with additional records from the continental shelf of the South Island (Bounty Trough) and Campbell Plateau (Fig. 4.1). The northernmost record was from the western tip of the North Island $\left(34.9^{\circ} \mathrm{S}, 172 \cdot 0^{\circ} \mathrm{E}\right)$ at a depth of $869 \mathrm{~m}$. Depth of captures ranged from 127 to $1071 \mathrm{~m}$, with individuals most frequently captured between 400 and $500 \mathrm{~m}($ median $=471 \mathrm{~m})$ [Fig. 4.2(A)]. Mature males predominated in shallower waters and females in deeper waters [Fig. 4.2(B)].

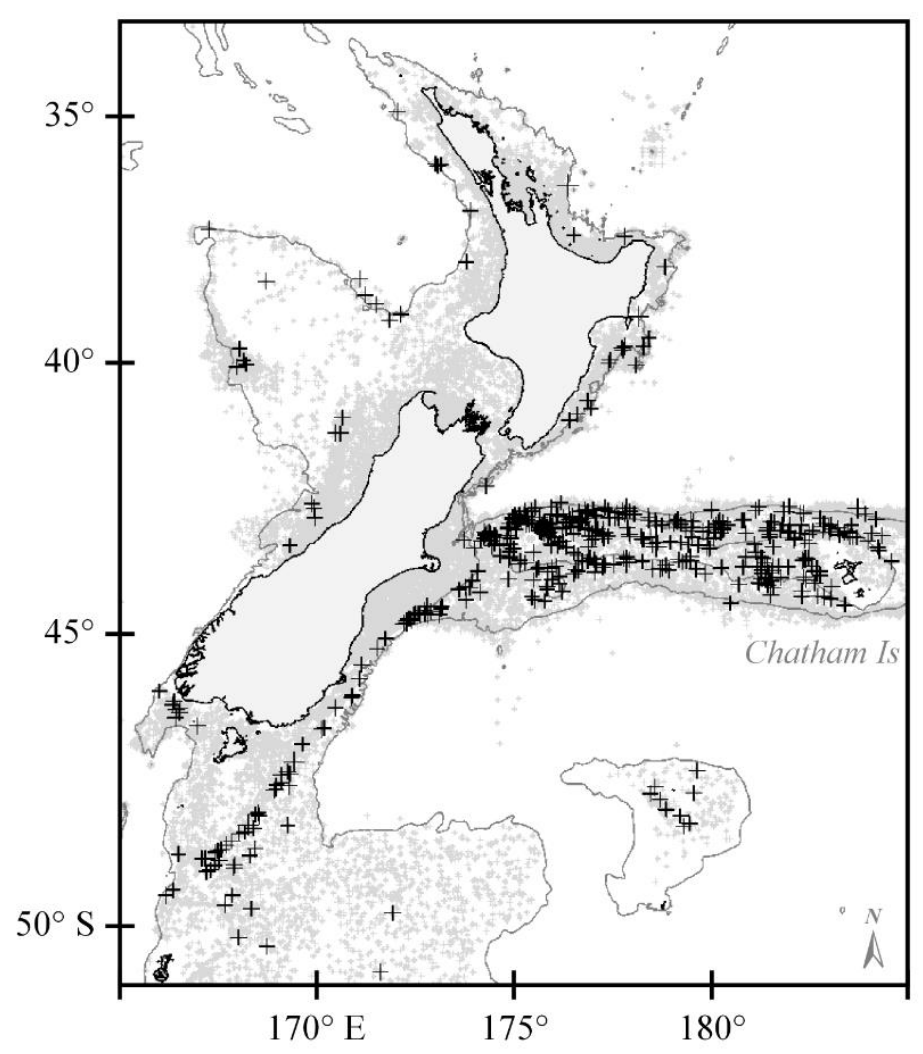

Figure 4.1 Distribution of all survey trawls stations $(\square, n=41644)$ and catch records of Oxynotus bruniensis $(+, n=464)$ within New Zealand waters. 


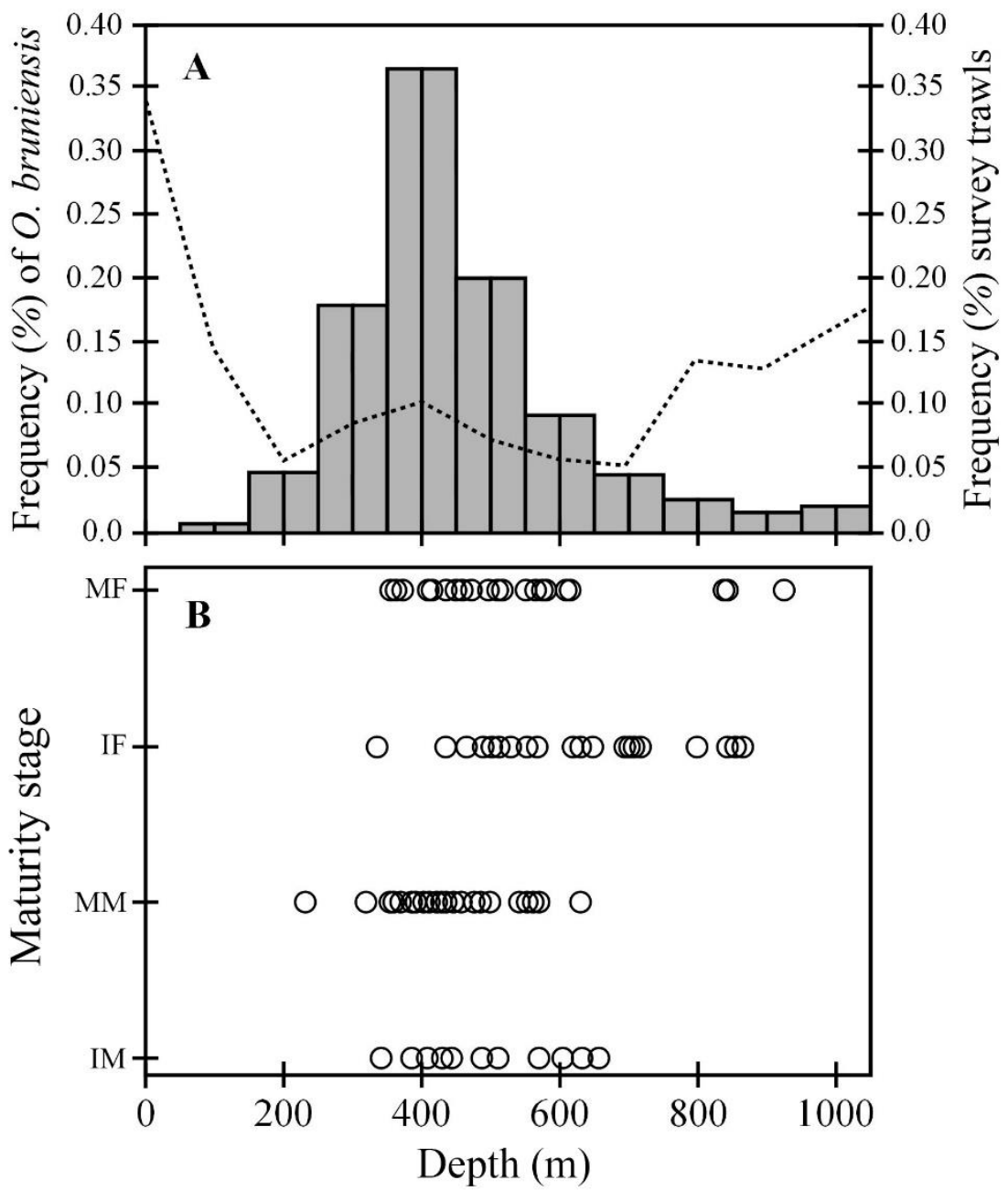

Figure 4.2 Depth distribution of Oxynotus bruniensis by (A) catch-record frequency of $O$. bruniensis capture ( $\square, n=464)$ and frequency of trawl-survey stations $(\cdots, n=41644)$ and by (B) sex and maturity stage: IM, immature male; MM, mature male; IF, immature female; MF, mature female $(n=112)$. Most catch occurred around the $400 \mathrm{~m}$ depth range. 
During this study, a total of 53 specimens of $O$. bruniensis were collected and dissected between 2014 and 2016: of these, 34 (16 males, 18 females) were obtained from 370 survey trawls, and 19 ( 5 male, 14 females) were obtained by the MPI observer programme. Additional data on 59 specimens from previous survey trawls included 27 males and 32 females, giving a total data set of 112 individuals (48 males and 64 females). Males ranged from 33.5 to $65.1 \mathrm{~cm} L_{\mathrm{T}}\left(\right.$ median $\left.=58.4 \mathrm{~cm} L_{\mathrm{T}}\right)$ and females from 49.4 to $75.6 \mathrm{~cm} L_{\mathrm{T}}\left(\right.$ median $=65.4 \mathrm{~cm} L_{\mathrm{T}}$ ) (Fig. 4.3). $M_{\mathrm{T}}$ for males and females varied from 145 to $2940 \mathrm{~g}$ and 1235 to $6560 \mathrm{~g}$, respectively. A significant difference between sexes was found in the relationship between $L_{\mathrm{T}}$ and $M_{\mathrm{T}}\left(\mathrm{ANCOVA}, F_{1,108}=\right.$ $21 \cdot 01, P<0 \cdot 05$; Fig. 4.4). The $L_{\mathrm{SV}}$ to $L_{\mathrm{T}}$ relationship $\left(L_{\mathrm{SV}}=0.608 L_{\mathrm{T}}-2.479\right.$ in females and $L_{\mathrm{SV}}=0.654 L_{\mathrm{T}}-2.068$ in males) was not significantly different by sex (ANCOVA, $\left.F_{1,47}=0 \cdot 025, P>0 \cdot 05\right)$.

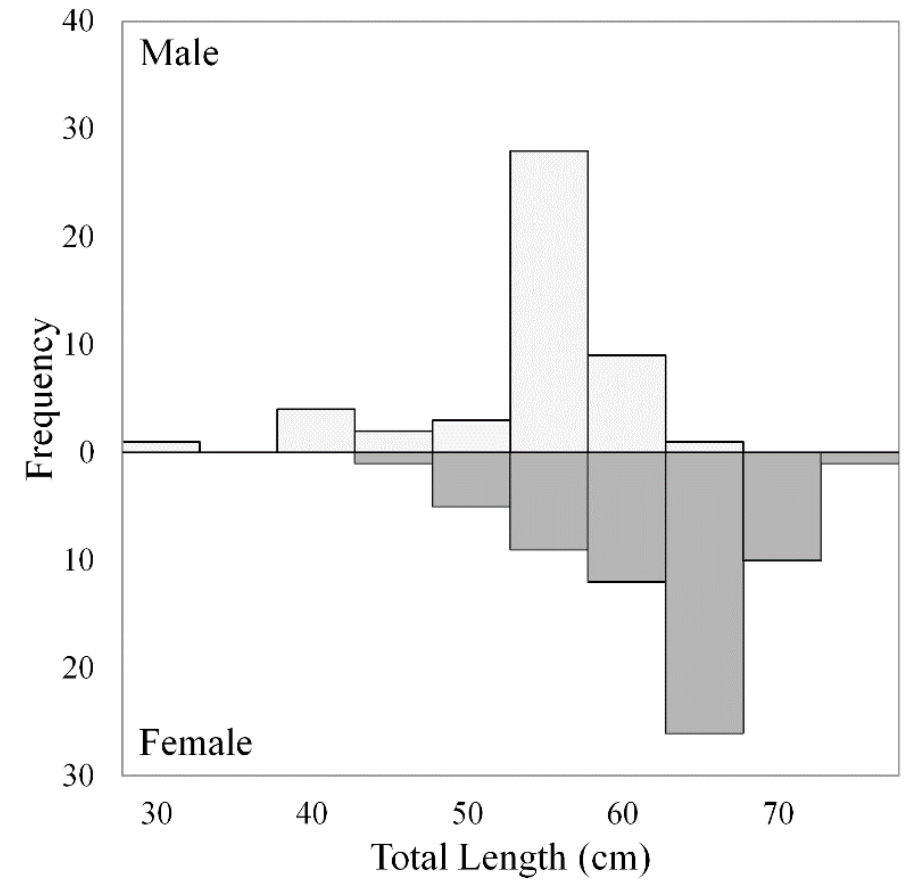

Figure 4.3 Total length $\left(L_{\mathrm{T}}\right)$-frequency distribution for ( $\square$ ) male and ( $\square$ ) female Oxynotus bruniensis $(n=112)$. 


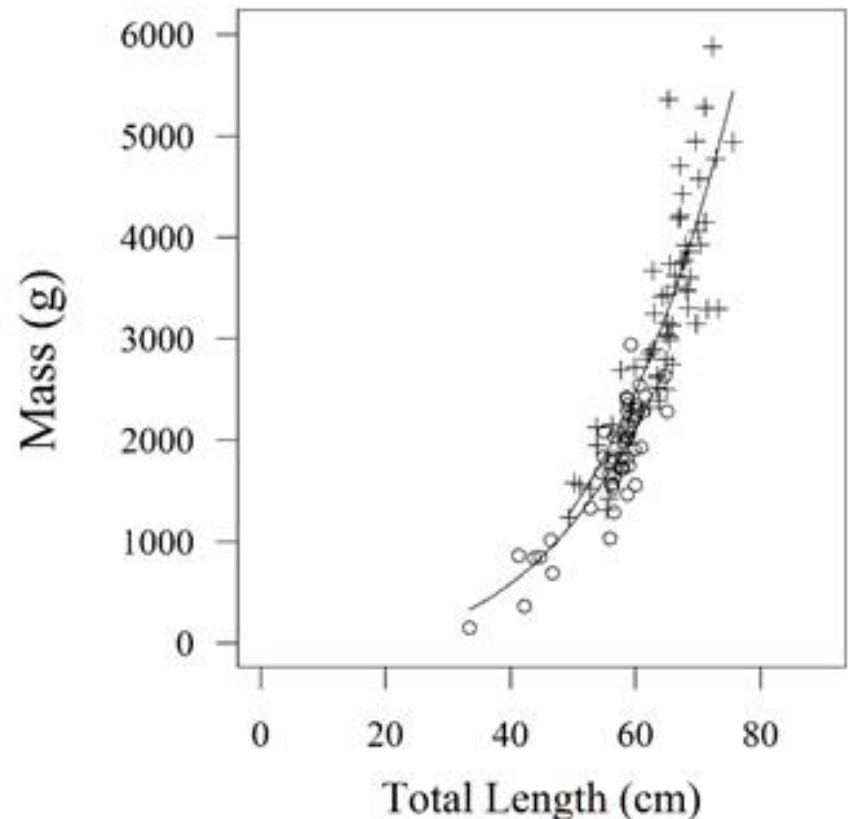

\begin{tabular}{|c|c|c|c|c|c|c|}
\hline & df. & Sum Sq & Mean Sq & $F$ value & $\operatorname{Pr}(P F)$ & \\
\hline length & 1 & 119476673 & 119476673 & 445.88 & $<0.005$ & $* * *$ \\
\hline sex & 1 & 4014009 & 4014009 & 14.98 & $<0.005$ & $* * *$ \\
\hline length:sex & 1 & 5629144 & 5629144 & 21.01 & $<0.005$ & $* * *$ \\
\hline Residuals & 108 & 28939098 & 267955 & & & \\
\hline - & & & & & & \\
\hline gnif. code & & $001^{\text {‘**' } 0.0}$ & '*' 0.05 ' & 1 & & \\
\hline
\end{tabular}

Figure 4.4 Total length $\left(L_{\mathrm{T}}\right)$-mass $\left(M_{\mathrm{T}}\right)$ relationship for Oxynotus bruniensis males $(\circ ; n=48)$ and females $(+; n=64)$ with best fit exponential relationship plotted. ANCOVA results reported below. 


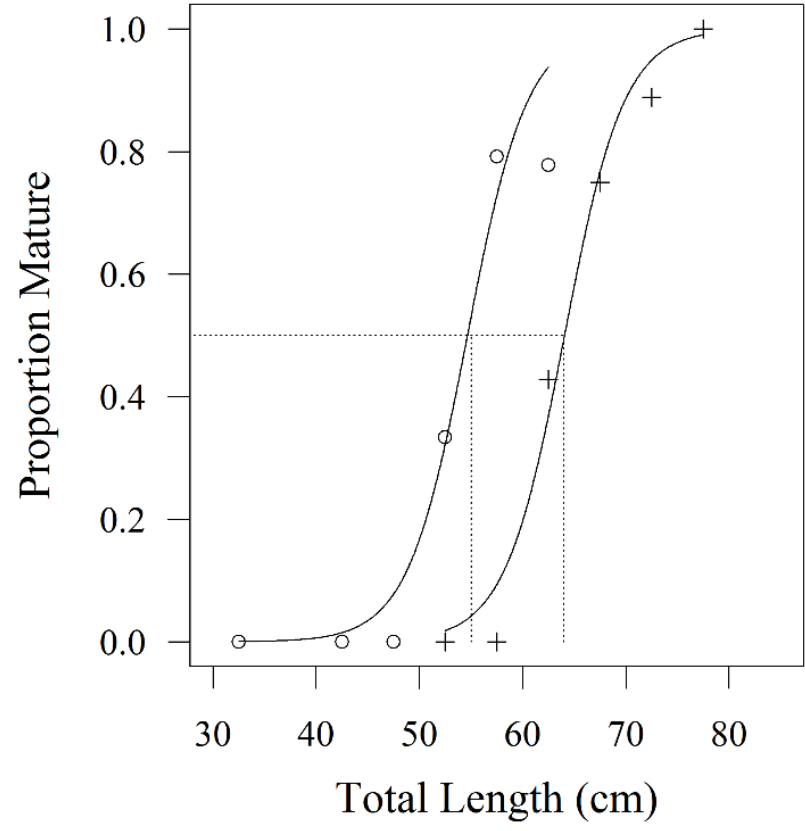

Figure 4.5 Logistic model fitted total length $\left(L_{\mathrm{T}}\right)$-maturity ogive for Oxynotus bruniensis, with the observed proportion of mature (०) males and (+) females in each size interval. $\vdots$, $L_{\mathrm{T}}$ at $50 \%$ mature $\left(L_{50}\right)$ for males $\left(L_{50}=54.7 \mathrm{~cm}\right)$ and females $\left(L_{50}=64 \mathrm{~cm}\right)$. 
In the male sample, $35 \%(n=14)$ were classified in early maturity stages (immature or maturing) and $65 \%(n=26)$ were mature. The $L_{\mathrm{T}}$ of the smallest mature individual was $54.9 \mathrm{~cm}$, while the largest immature was $64.8 \mathrm{~cm}$. There was no significant difference between the left and right inner clasper length ( $t$-test, d.f. $=20, P>$ $0 \cdot 05)$, or the left and right testis mass ( $t$-test, d.f. $=15, P>0 \cdot 05)$. Gonad mass ranged from 0.05 to $4.79 \mathrm{~g}$ in immature males and from 4.27 to $28.6 \mathrm{~g}$ in mature males. All males over $56 L_{\mathrm{T}} \mathrm{cm}$ had fully calcified claspers and sperm in the seminal vesicles. Both $I_{\mathrm{G}}$ and $L_{\mathrm{C}}$ increased abruptly at $c .55 \mathrm{~cm} L_{\mathrm{T}}$ (Fig. 4.6), which coincided with the mean size-at-maturity estimate from macroscopic staging of $54 \cdot 7 \mathrm{~cm} L_{\mathrm{T}}(95 \%$ C.I. $=52 \cdot 2$ $-57.6 \mathrm{~cm})($ Fig. 4.5$)$. The mean size-at-maturity occurred at $84 \%$ of the maximum observed $L_{\mathrm{T}}$, and represented $81 \%$ of the male sample.

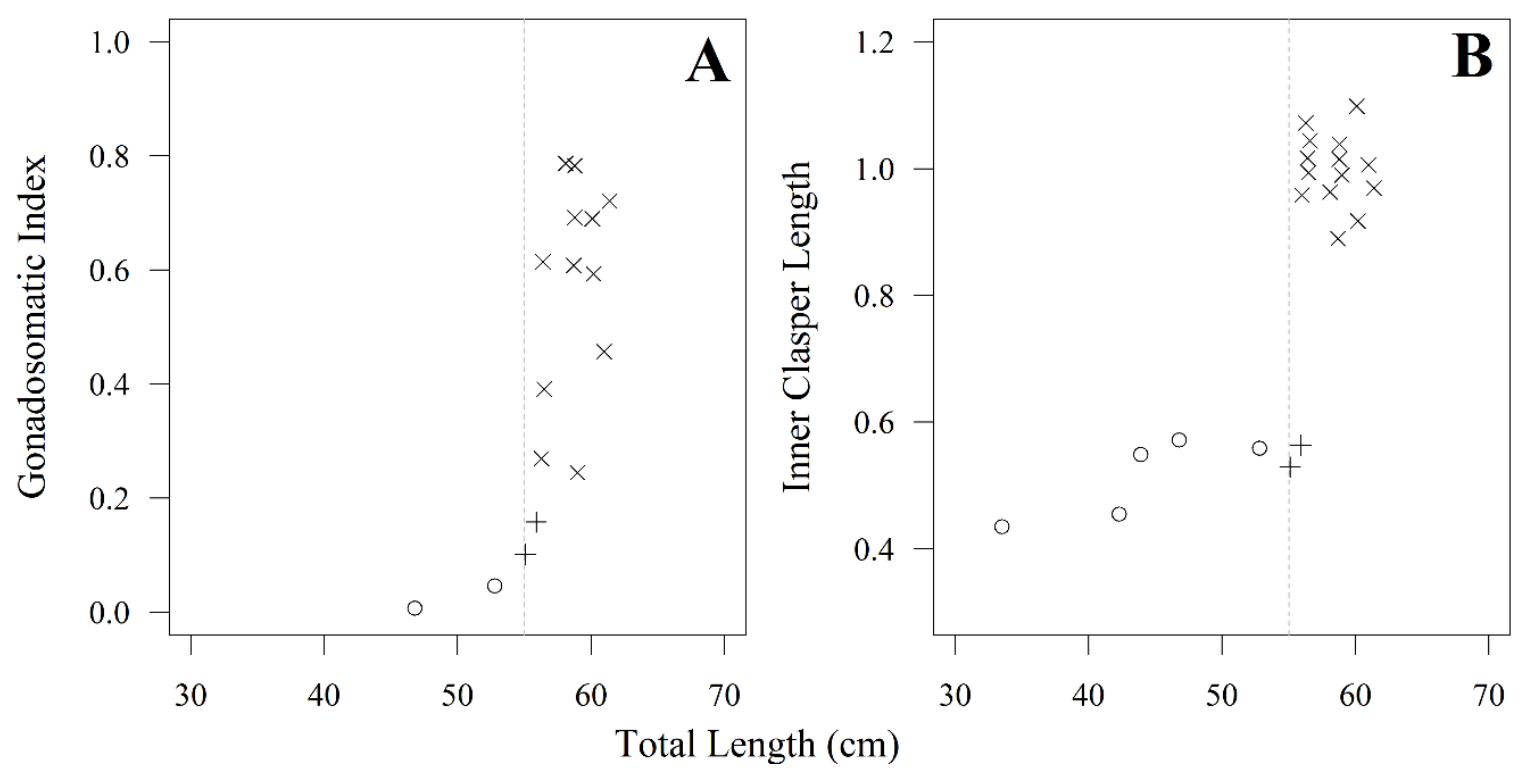

Figure 4.6 Relationship between total length $\left(L_{\mathrm{T}}\right)$ and $(\mathrm{A})$ gonadosomatic index $\left(I_{\mathrm{G}}\right)$, and (B) proportional inner clasper length $\left(L_{\mathrm{C}}\right)$, for Oxynotus bruniensis based on macroscopic maturity stages: $\circ$, juvenile; + , maturing; $\times$, mature). $\vdots$, estimated $L_{\mathrm{T}}$ at $50 \%$ mature $\left(L_{50}=\right.$ $54 \cdot 7 \mathrm{~cm})$. 


\subsubsection{Female reproduction}

In females, $40 \%(n=18)$ were classified as immature/maturing and 60\% $(n=27)$ were mature. The smallest mature female was $61.9 \mathrm{~cm} L_{\mathrm{T}}$ and the largest immature female was $71.5 \mathrm{~cm} L_{\mathrm{T}}$. Both ovaries were functional in all mature specimens. Gonad mass ranged from 1.31 to $13 \cdot 10 \mathrm{~g}$ for immature females, and from $10 \cdot 28$ to $596 \mathrm{~g}$ for mature females, accounting for up to $12.6 \%$ of $M_{\mathrm{T}}$. The $I_{\mathrm{G}}$ increased abruptly at around 64-65 $\mathrm{cm} L_{\mathrm{T}}$ [Fig 7(A)], which correlated with the estimated size-at-maturity from macroscopic staging of $64.0 \mathrm{~cm} L_{\mathrm{T}}(95 \%$ C.I. $=62 \cdot 4-65.8 \mathrm{~cm})($ Fig. 4.5$)$. Size-atmaturity was at $84 \%$ of the maximum observed $L_{\mathrm{T}}$ and represented $61 \%$ of the female sample. A marked increase in the uterus and oviducal gland width [Fig. 4.7(B)] was also found at around $64 \mathrm{~cm} L_{\mathrm{T}}$ [Fig. 4.7(C)]. There was no significant difference between left and right oviducal gland width $(t$-test, d.f. $=20, P>0.05)$ or uterus width $(t$-test, d.f. $=$ $29, P>0 \cdot 05)$.

Ovarian fecundity varied from eight to 17 , with a mean \pm S.D. of $11 \cdot 1 \pm 3 \cdot 3(n=$ 10). The diameter of the largest follicle measured $2 \cdot 46-51 \cdot 22 \mathrm{~mm}$ (mean \pm S.D. $=27 \cdot 48$ $\pm 17 \cdot 08)$ [Fig. 4.7(D)]. Three females $\left(65 \cdot 0,67.5\right.$ and $\left.71 \cdot 2 \mathrm{~cm} L_{\mathrm{T}}\right)$ with embryos were found, having eight embryos each. The $65.0 \mathrm{~cm} L_{\mathrm{T}}$ female had embryos ranging in size from 21.0 to $23.4 \mathrm{~cm} L_{\mathrm{T}}($ mean $=22.6 \mathrm{~cm})$ and 62.0 to $88.0 \mathrm{~g}($ mean $=80.0 \mathrm{~g})$, with no external yolk. Embryos of the $67.5 \mathrm{~cm} L_{\mathrm{T}}$ female ranged between 20.4 and 21.4 $\mathrm{cm} L_{\mathrm{T}}($ mean $=20.9 \mathrm{~cm})$ and $114 \cdot 3-130.3 \mathrm{~g}($ mean $=123.3 \mathrm{~g})$, with external yolk sac retained. The largest gravid female $\left(71.2 \mathrm{~cm} L_{\mathrm{T}}\right)$ had near-term embryos. Embryo size ranged from 24.3 to $27.0 \mathrm{~cm} L_{\mathrm{T}}($ mean $=25.9 \mathrm{~cm})$ and 113.5 to $141.0 \mathrm{~g}($ mean $=128.9$

g). The external yolk sac of all pups in this litter was nearly consumed, with several nearing the closing of the umbilical scar. Based on these observations, size-at-birth was estimated at $25-27 \mathrm{~cm} L_{\mathrm{T}}$. In each case, four embryos were present in each uterus. Ovaries in gravid females lacked any follicles and appeared to be atrophied. The sex ratio of pups was 1:1 overall, but was variable in individual litters. Eight females were classified as post-partum, and considered reproductively inactive due to atrophied ovaries. 

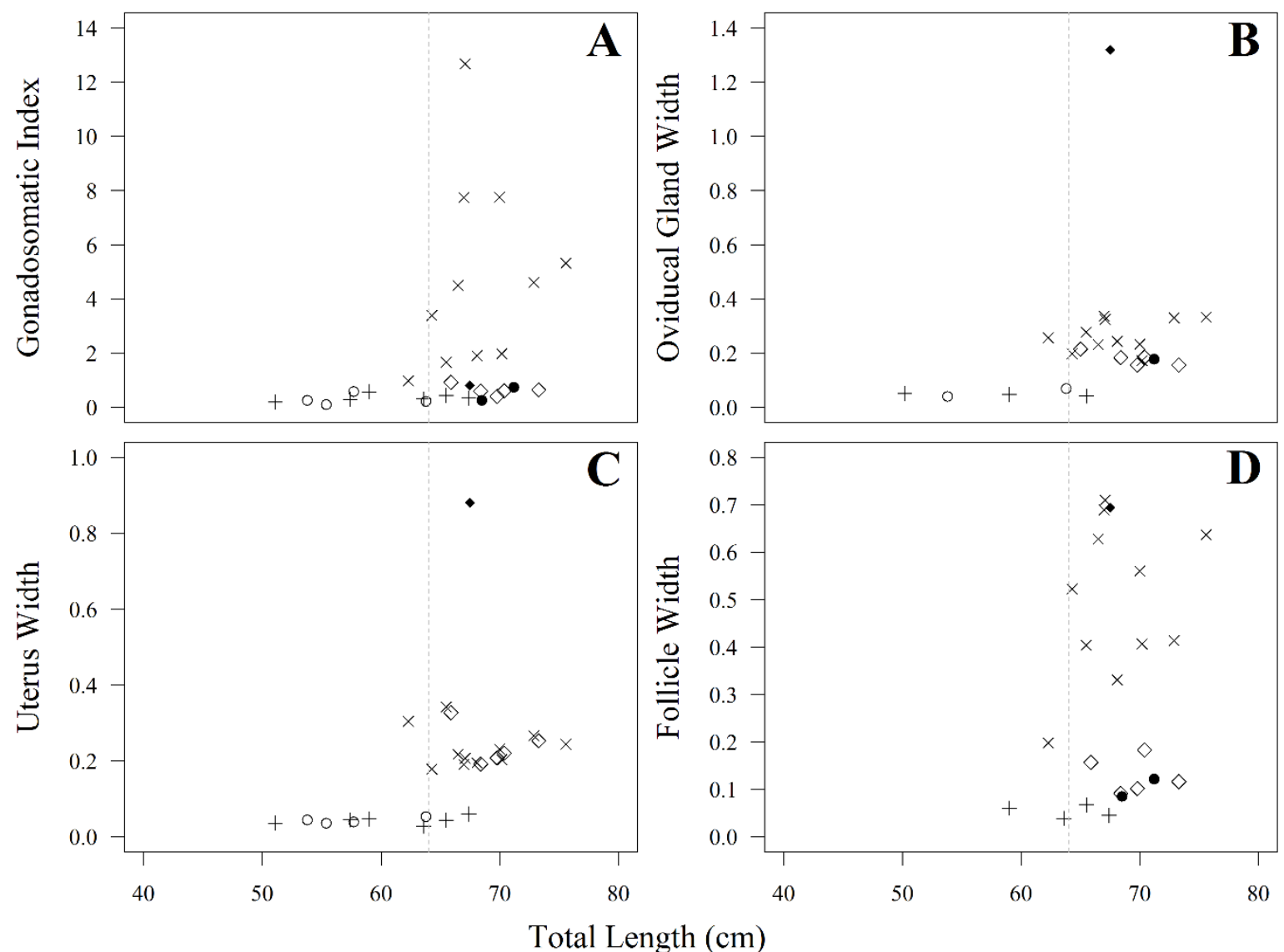

Figure 4.7 Relationship between total length $\left(L_{\mathrm{T}}\right)$ and $(\mathrm{A})$ gonadosomatic index $\left(I_{\mathrm{G}}\right),(\mathrm{B})$ proportional width of oviducal gland, (C) uterus and (D) diameter of largest ovarian follicle for female Oxynotus bruniensis, based on macroscopic maturity stages: ○, juvenile; +, maturing; $\times$, mature; $\bullet$, gravid I; $\bullet$, gravid II; $\diamond$, post-partum. $\vdots$, estimated $L_{\mathrm{T}}$ at $50 \%$ mature $\left(L_{50}=64 \cdot 0 \mathrm{~cm}\right)$. 


\subsubsection{Diet analysis}

Of the 53 stomachs, four were empty, 26 contained only fluid and 23 had prey items retained by the sieve. Of those containing prey, unidentifiable white and yellow material consistent with chondrichthyan egg yolk (vitellus) was found in every stomach. Fourteen stomachs contained only vitellus from unidentified species. Four stomachs contained vitellus and fragments of egg case capsules from unidentified species [Fig. 4.8(A)], and five others contained vitellus and embryos. Of the latter, two of the embryos were visually identified as the longnose chimaera Harriotta raleighana Goode \& Bean 1895 [Fig. 4.8(B)], and the remaining three embryos required further analysis for identification. From the 21 stomach samples where none of the prey could be visually identified, 15 yielded DNA that could be amplified and sequenced. These 15 samples included six from Chatham Rise in January, four from the west coast of the South Island in June and five from east coast of the North Island in March and June.
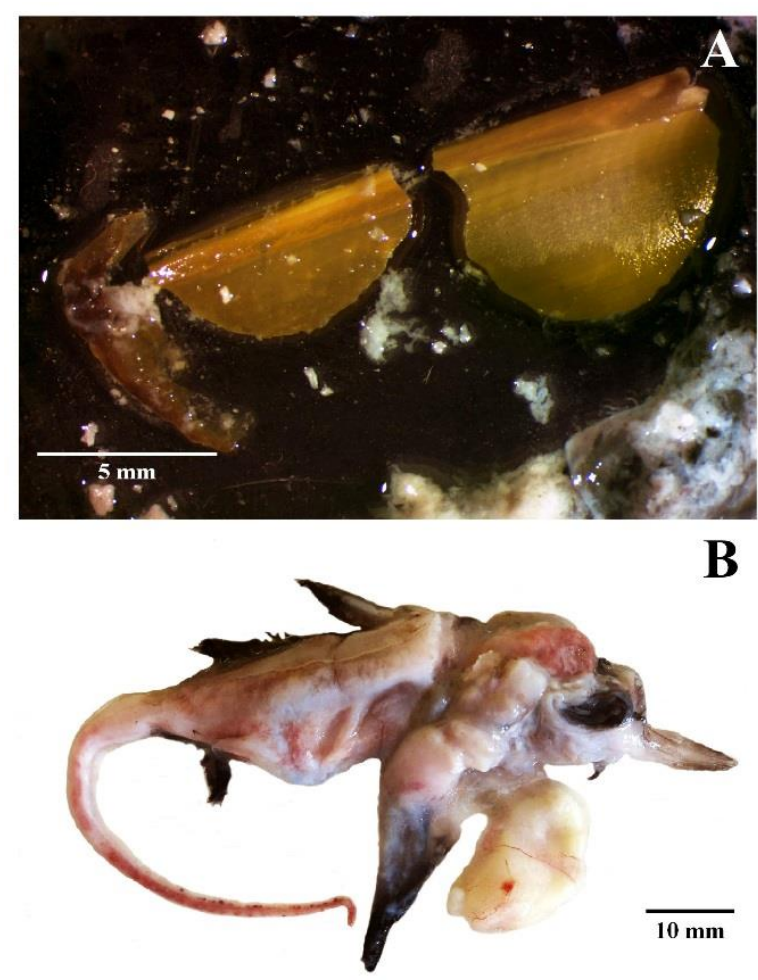

Figure 4.8 Examples of stomach contents from Oxynotus bruniensis including (A) egg case fragments and (B) Harriotta raleighana embryo. 
Fragments of cox 1 and nadh2 genes were successfully sequenced in all 15 samples. The analysis involved 128 sequences of 21 species for $\operatorname{cox} 1$ and 25 sequences of 10 species for nadh2. The aligned data contained 652 base pairs (bp) and $1054 \mathrm{bp}$ for cox 1 and nadh2, respectively. The species and haplotypes used for comparison analysis are listed in Appendix V. Alignment of sequences of cox1 showed 340 identical sites and 223 variable sites with a ML estimate of transition/transversion bias (R) value 9.64. The alignment of nadh2 sequences showed 486 conserved and 485 variable sites with a ML estimate of transition/transversion bias (R) value 4.67. Bootstrap analysis consensus trees obtained from ML and NJ (Figs 4.9 and 4.10) have sufficient discriminatory power to identify species from content samples and the taxa studies. There was no haplotype sharing or overlapping. The genetic distance between samples was from 0.00 to 2.23 in cox 1 and 0.00 to 1.23 in nadh2, due the inclusion of Oxynotus species as outgroup. Only one prey type was identified per sample.

Low genetic distances for $\operatorname{cox} 1$ and nadh2 genes were observed between $O$. bruniensis and sequences from two stomach content samples, suggesting potential contamination from host tissue during dissections. Sequences from 13 stomach content samples, however, were clustered across two holocephalan clades. Two samples were grouped inside the Rhinochimaera pacifica (Mitsukuri 1895) clade, and the haplotype of Chimaera carophila Kemper, Ebert, Naylor \& Didier 2014 was shared among 11 samples. The phylogenetic trees inferred from the haplotypes detected may suggest that both cox 1 and nadh2 genes unambiguously differentiated all stomach content samples in three species, the predator (O. bruniensis) and two prey (C. carophila and R. pacifica). 


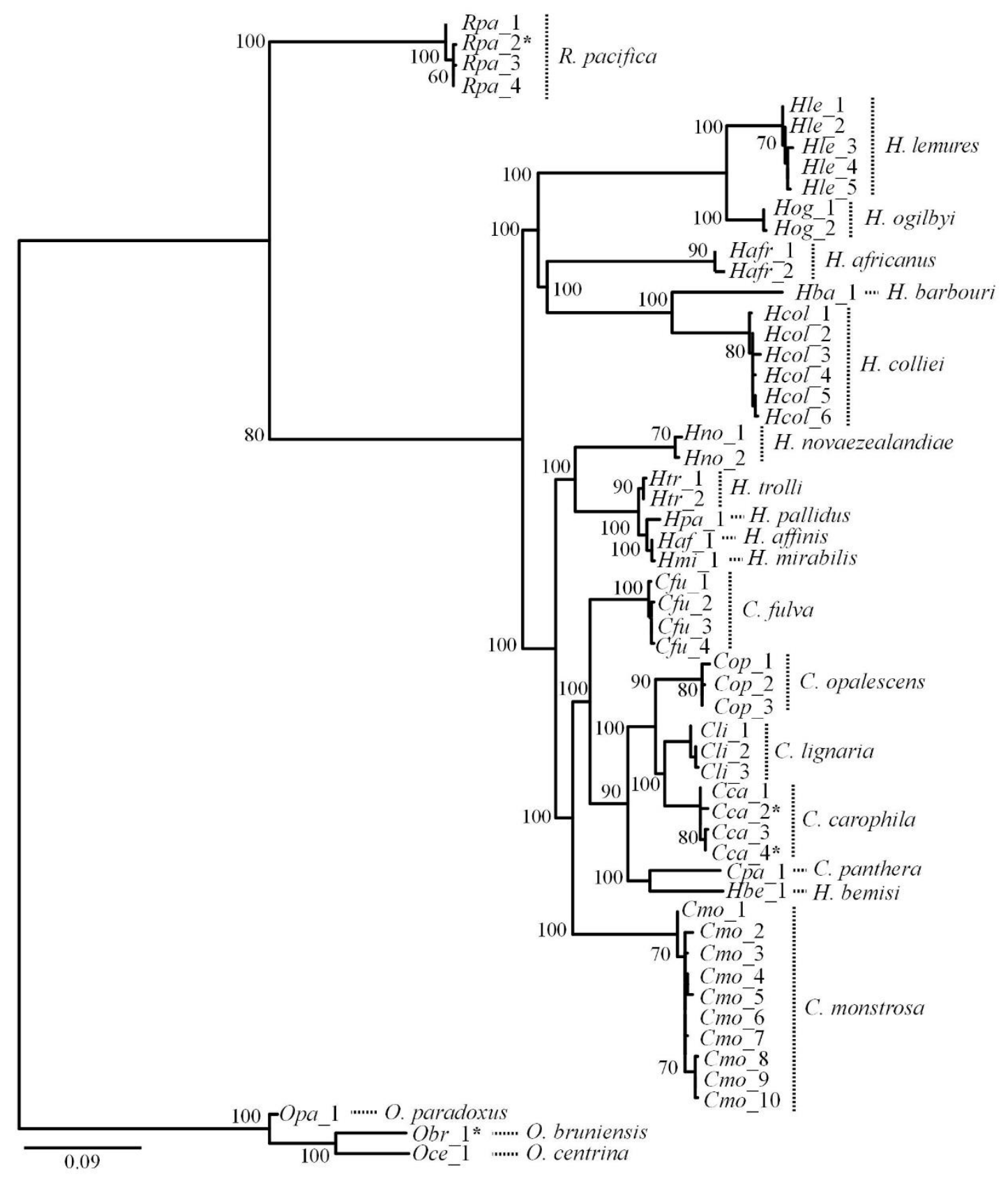

Figure 4.9 Neighbour-joining distance (NJ) and maximum-likelihood (ML) tree of cytochrome oxidase subunit 1 ( coxl) haplotypes found from stomach contents (*) and 18 holocephalan species (R., Rhinochimaera spp.; H., Hydrolagus spp.; C., Chimaera spp.), including the Oxynotidae clade (O., Oxynotus spp.) as the out group, based on the K2P model. Estimation of the nodes stability is expressed as a percentage (1000 bootstrap replicates). 


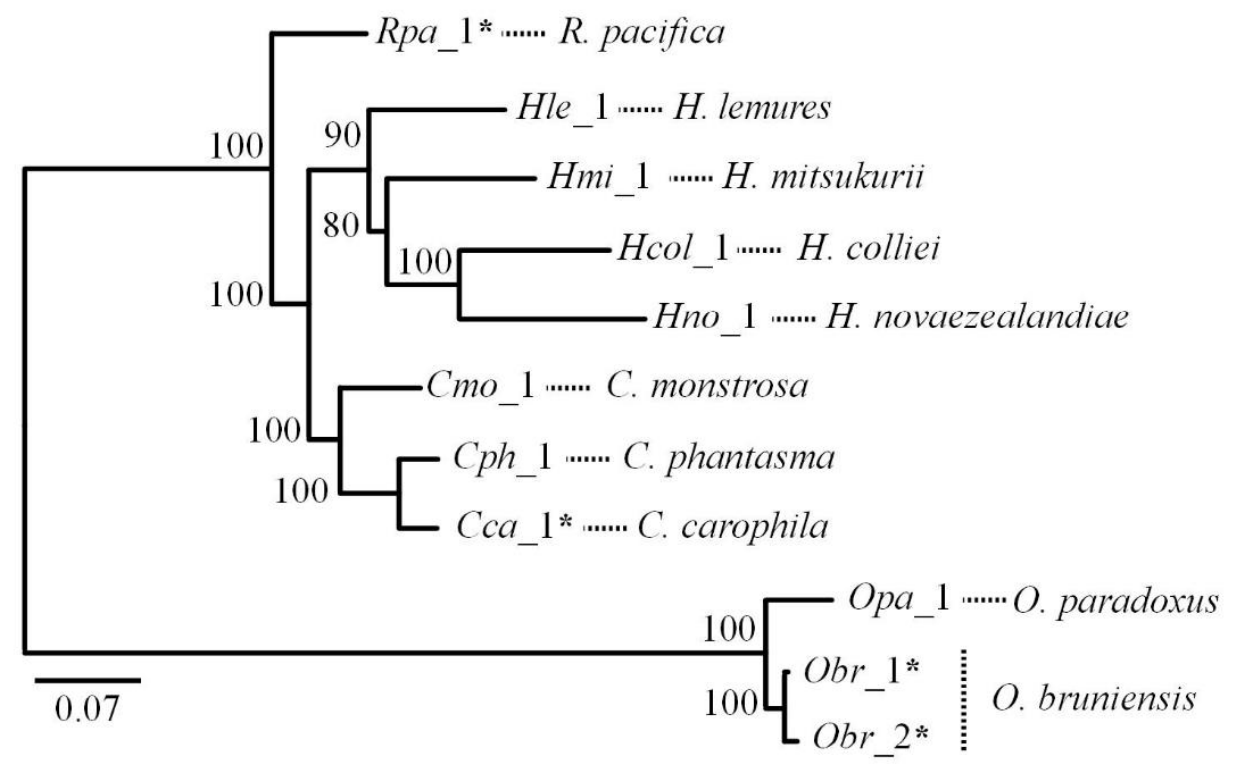

Figure 4.10 Neighbour-joining distance (NJ) and maximum-likelihood (ML) tree of nicotinamide adenine dinucleotide subunit 2 (nadh2) haplotypes found from stomach contents (*) and eight holocephalan species (R., Rhinochimaera spp. H., Hydrolagus spp.; C., Chimaera spp.), including the Oxynotidae clade (O., Oxynotus spp.) as the out group, based on the $\mathrm{K} 2 \mathrm{P}$ model. Estimation of the nodes stability is expressed as a percentage (1000 bootstrap replicates). 


\subsection{Discussion}

Oxynotus bruniensis exhibits reproductive characteristics similar to other lecithotrophic viviparous elasmobranchs. Females grow larger and attain sexual maturity at a greater size than males. Both sexes in this study reached sexual maturity at $84 \%$ of the maximum observed $L_{\mathrm{T}}$, suggesting that $O$. bruniensis may undergo little somatic growth after maturity as reported in many chondrichthyans (Cortés, 2000). These characteristics are commonly observed in cartilaginous fishes, particularly deep-sea species with relatively low productivity (slow growth, high longevity and low fecundity) (Graham \& Daley, 2011; Irvine et al., 2012). While no attempts have been made to age any members of the genus Oxynotus, external bandings have been noticed on the spine of the first dorsal fin in $O$. bruniensis, which might be used for ageing this species in future studies (Irvine et al., 2006).

\subsubsection{Reproductive characteristics}

Reproductive characteristics suggested $O$. bruniensis is likely to have relatively low reproductive output. Ovarian and uterine fecundity were estimated to be lower than $O$. centrina, which was estimated to have an ovarian fecundity of nine to 22 , and a uterine fecundity of 10 to 12 embryos (Capapé et al., 1999). The rarity of gravid females in the present study reduces confidence in overall fecundity estimations, although such low fecundity is not uncommon among deep-sea species (Simpfendorfer \& Kyne, 2009). Large maternal size is probably a requirement for increased reproductive maternal investment (Springer, 1967; Cortés, 2000). Size-at-birth was estimated at 25-27 cm $L_{\mathrm{T}}$, which accounted for up to $35 \%$ of the maternal $L_{\mathrm{T}}$. This large size-at-birth most likely reflects a trade-off between size-at-birth and reduced fecundity (Smith \& Fretwell, 1974).

The presence of atrophied ovaries in gravid females, and minimal follicle development and distended uteri in post-partum females, provided some evidence that $O$. bruniensis may engage in a non-continuous reproductive cycle, in which there are periods of inactive follicle development between pregnancies (Pethybridge et al., 2011b; Irvine et $a l ., 2012$ ). This reproductive behaviour is typical of deep-sea chondrichthyans (Kyne \& Simpfendorfer, 2010; Rigby \& Simpfendorfer, 2015), and was also observed in $O$. centrina (Capapé et al., 1999). Species with a non-continuous reproductive strategy are thought to have shorter gestation periods than those with continual cycles of follicle 
development (Pethybridge et al., 2011b). Oxynotus centrina was suggested to have a gestation period of up to 1 year (Capapé et al., 1999), which seems to be a reasonable hypothesis for $O$. bruniensis on account of their similar biology. The small sample size and lack of seasonal available information, however, impedes further conclusions.

There was some indication of sex-based segregation by depth in $O$. bruniensis. Mature males predominately occupied shallower water, while mature females were found in deeper water. Segregation by sex is common in many elasmobranchs (Wetherbee, 1996; Clarke et al., 2001), and other studies have shown that males favour shallower depths, and females deeper water (Yano \& Tanaka, 1988). Most individuals observed in this study, of both sexes, were greater in length than the estimated size-atmaturity. Although this demersal, thick-bodied, and presumably slow-swimming species would not be expected to easily avoid bottom trawls, there were few records of small individuals $\left(<50 \mathrm{~cm} L_{\mathrm{T}}\right)$. One free-swimming male of $25 \cdot 1 \mathrm{~cm} L_{\mathrm{T}}$ with an open umbilical scar $0.03 \mathrm{~cm}$ was reported by a scientific observer from a trawl near the Auckland Islands (M.P. Francis, NIWA, pers. comm.), and one small individual of $33.0 \mathrm{~cm} L_{\mathrm{T}}$ was recorded in this study on Chatham Rise, suggesting O. bruniensis may pup in multiple locations around New Zealand. Immature individuals have been previously reported at depths deeper than adults (Moura et al., 2014), however, in the case of $O$. bruniensis, any small individuals would still occur within the range of commercial and research trawls, and should have been detected. The presence of young $O$. centrina specimens in coastal waters suggests inshore mating and pupping areas (Capapé et al., 1999). Oxynotus bruniensis may engage in a similar strategy, and the identification of potential inshore pupping and nursery grounds merits further investigation.

\subsubsection{Oxynotus bruniensis diet}

The examination of stomach contents of $O$. bruniensis suggested a diet based primarily on the eggs and embryos of chimaeras (holocephalans). Previously, on the basis of its small ventral mouth, $O$. bruniensis was thought to have a similar diet to other demersal chondrichthyans, consisting of small benthic invertebrates and fishes (Dunn et al., 2013). Other members of the Oxynotidae are considered to be suction feeders, specializing in worm-like prey, and $O$. centrina has been reported to feed primarily on polychaetes, sipunculids, crustaceans and teleosts (Capapé, 2008). More recently, Guallart et al. (2015) suggested that $O$. centrina has a more specialized trophic 
preference, after captive specimens were found to prey exclusively on the contents of elasmobranch egg cases. Previous studies on Oxynotus have reported mostly empty stomachs (Megalofonou \& Damalas, 2004; Capapé, 2008; Dragičević et al., 2009; Dunn et al., 2013). The present study found that stomachs were often full of water, and could rupture easily upon dissection, which could explain the previous lack of evidence of vitellus in stomach contents. The use of molecular markers, however, has been demonstrated as a useful tool for estimating the potential identity of otherwise unidentifiable prey from stomach contents (Dunn et al., 2010b).

Predation on cartilaginous fish egg cases is considered widespread among marine animals (Cox et al., 1999), and identified predators have included teleosts (Long, 1996; Powter \& Gladstone, 2008), marine mammals (Bor \& Santos, 2003), gastropods (Lucifora \& García, 2004; Hoff, 2009) and other elasmobranchs (Powter \& Gladstone, 2008; Bustamante et al., 2013). The remains of embryonic small-spotted catshark Scyliorhinus canicula were reported from the stomach contents of $O$. centrina in the Mediterranean Sea (Barrull \& Mate, 2001). Predation on egg cases of the chimaera Callorhinchus milii Bory de Saint-Vincent 1823 have also been reported based upon observations of bore holes on the dorsal surface of the capsules, but predators have yet to be identified (Bell, 2012). Egg case predation has been suggested as a major factor influencing abundance of some elasmobranch populations (Cox et al., 1999). It seems unlikely that predation on egg cases by $O$. bruniensis would have a significant effect on populations of the Pacific spookfish Rhinochimaera pacifica (Mitsukuri 1895) or the longnose spookfish Harriotta raleighana Goode \& Bean 1895, as both are considered to have large populations within New Zealand (Ford et al., 2015), and also have geographical distributions well beyond New Zealand waters (Last \& Stevens, 2009). Predation of egg cases of C. carophila, however, may impact local populations, as this recently described species is thought to be endemic to New Zealand waters, is expected to have a low reproductive potential (Kemper et al., 2014), and is assumed to have a relatively small population (Ford et al., 2015).

There are several examples of chondrichthyans playing a large role in the diet of other chondrichthyans (Cortés, 1999). Very few of these have come from the deep sea, and the predators are generally large-sized species (Navarro et al., 2014). Specialist diets have been identified amongst elasmobranchs, although the degree of specialization can vary with species, competition, and fluctuations in spatial and temporal prey availability (Munroe et al., 2014). While the diet sample size in the present study was small, 
specimens were collected over 3 years, throughout the New Zealand EEZ, and throughout the year, reducing the potential for sampling bias. Within this specialized feeding behaviour, there may be a preference for certain chimaera species. Rather surprisingly, the two most common chimaera species in New Zealand, the pale ghost shark Hydrolagus bemisi Didier 2002 and the dark ghost shark Hydrolagus novaezealandiae (Fowler 1911), were not detected in any of the stomach samples, despite having substantially overlapping depth ranges with $O$. bruniensis (400-1100 m for $H$. bemisi; 32-800 m for $H$. novaezealandiae, Roberts et al., 2015). An embryonic unidentified skate, however, was also previously found in the stomach contents of an O. bruniensis (C.A.J. Duffy, Department of Conservation, pers. comm.), suggesting additional oviparous chondrichthyans not identified in this study may also be preyed upon. Areas of breeding importance for oviparous chondrichthyans, including $H$. bemisi and $H$. novaezealandiae, have yet to be identified in New Zealand. Without this information, reasons for the absence of additional prey species in $O$. bruniensis is speculative. Future studies assessing the importance of the ecological relationship between $O$. bruniensis and oviparous chondrichthyans should be considered.

The described exclusivity of $O$. bruniensis foraging on egg cases of select chondrichthyan species may increase its vulnerability to human impacts. Not only may $O$. bruniensis be particularly vulnerable to excess fishing mortality (Dulvy et al., 2014), but the prey species may be similarly vulnerable, and there may be damage to egg cases and egg-laying habitat by bottom trawling and exploratory mineral mining (Clark \& Rowden, 2009). Whilst $O$. bruniensis is widespread in New Zealand waters, most records are reported from Chatham Rise, an area which is relatively heavily fished (MPI, 2014). Other areas where $O$. bruniensis have been reported are sampled infrequently, and although the species is known from areas with restricted fishing efforts (e.g. one specimen has been recorded on the summit of the Rumble V Submarine Volcano, southern Kermadec Ridge; Roberts et al., 2015), it has been estimated that up to $60 \%$ of the distribution range of $O$. bruniensis overlaps with commercial fisheries for the majority of the year (Ford et al., 2015). There is no clear trend in relative biomass estimates for $O$. bruniensis on Chatham Rise (O'Driscoll et al., 2011), but considering its low reproductive output (e.g. low fecundity and large size-at-maturity), specialized diet, and spatial and depth distribution overlap with fisheries, it seems likely that $O$. bruniensis is at higher risk from the impact of fishing than currently estimated (Ford et al., 2015). 


\section{CHAPTER V}

Reproductive biology of two deep-sea chimaeras, longnose spookfish (Harriotta raleighana), and Pacific spookfish (Rhinochimaera pacifica) 


\subsection{Abstract}

The family Rhinochimaeridae, the long-nose chimaeroids, consists of eight species across three genera, two of which occur in New Zealand waters. Very little is known about the biology of the rhinochimaerids. Longnose spookfish, (Harriotta raleighana, Goode and Bean 1895), and Pacific spookfish, (Rhinochimaera pacifica, Mitsukuri 1895), were collected from research trawl surveys and from commercial vessels around New Zealand at depths between 400 and $1300 \mathrm{~m}$. A total of $300 \mathrm{H}$. raleighana were caught which varied in length from 18.7 to $90.4 \mathrm{~cm}$ chimaera length $(L c)$, and 168 R. pacifica at lengths of 20.9 to $139.9 \mathrm{~cm} \mathrm{Lc}$. External assessment of male claspers and female gonad mass and oviducal gland width were the best indicators for maturity. Both species matured at a large proportion of their maximum length. Length-at-maturity was estimated at $62.8 \mathrm{~cm} \mathrm{Lc}$ and $75.8 \mathrm{~cm} \mathrm{Lc}$ for male and female $H$. raleighana respectively, and $105.3 \mathrm{~cm} \mathrm{Lc}$ and $125.0 \mathrm{~cm} L \mathrm{c}$ for male and female $R$. pacifica. Fecundity was low and estimated to be up to 27 eggs for H. raleighana, and 31 eggs for R. pacifica. Sperm storage was confirmed in females of both species. Sexual dimorphism in snout length was found in H. raleighana, where male relative snout size increased at sexual maturity, suggesting the snout is a secondary sexual characteristic. This study contributes to a better understanding of the life histories of $H$. raleighana and $R$. pacifica and their vulnerability to exploitation as fisheries bycatch. 


\subsection{Introduction}

Chimaeroids, also known as ratfish or ghost sharks, form the subclass Holocephali, and along with Elasmobranchii (sharks and rays), make up the class Chondrichthyes (Didier, 1995). The holocephalan family Rhinochimaeridae is globally distributed, consisting of eight species across three genera, and are distinguished from other chimaeroids by having long tapered snouts. The longnose spookfish (Harriotta raleighana, Goode \& Bean 1895) is the only chimaeroid with a presumed worldwide distribution (Didier et al., 2012). It is relatively common across the continental shelf and ocean floor of the Northern Atlantic, as well as the Northwest and Southwest Pacific (Last \& Stevens 2009). The Pacific spookfish (Rhinochimaera pacifica, Mitsukuri 1895) has a patchy known distribution on the continental shelf of the Pacific and Indian Oceans (Didier \& Nakaya, 1999), with reports across the Japanese Archipelago to the South China Sea (Shao \& Hwang, 1997; Shinohara et al., 2009), Australia (Last \& Stevens, 2009), New Zealand (McMillian et al., 2011), and Peru (Chirichigno 1974). Around New Zealand, both species are widely distributed on the continental shelf at depths from 400 to 1300 m (Inada \& Garrick, 1979; McMillian et al., 2011), with $R$. pacifica typically found deeper than $H$. raleighana (Dunn et al., 2010a). Elsewhere, H. raleighana has been recorded at depths up to $2600 \mathrm{~m}$ (Priede et al., 2006).

Harriotta raleighana and $R$. pacifica are listed as Least Concern by the International Union for the Conservation of Nature (IUCN), but very little is known about their biology. This is largely due to a lack of targeted sampling and taxonomic confusion (Kyne \& Simpfendorfer, 2007; Holt et al., 2013). Chimaeroids that have been studied have expressed K-selected characteristics similar to deep-sea elasmobranchs (relatively slow growth, high longevity, late maturation, low fecundity, see Barnett et al., 2012; King \& McPhie, 2013). These factors, along with their tendency to aggregate (Quinn et al., 1980; Barnett et al., 2012) and distribution overlap with fishing effort (Bagley et al., 2013), make chimaeroids susceptible to overfishing.

Harriotta raleighana has been reported to grow to a length of $120 \mathrm{~cm}$, although it has never been recorded larger than $90 \mathrm{~cm}$ chimaera length $\left(L_{\mathrm{C}}\right)$ in New Zealand, and $R$. pacific to $165 \mathrm{~cm}$ (McMillian et al., 2011). Both species are oviparous, and it is likely that individual embryos contained in an egg case are deposited onto the ocean floor as exhibited by other chimaeroids (Dean, 1906; Didier et al., 2012). Gestation for chimaeroids may last up to 8 months (Barnett et al., 2009), and one report estimated size- 
at-birth for H. raleighana to be between 10 and $13 \mathrm{~cm}$ (Cox \& Francis, 1999). Based on preserved specimens, size-at-maturity for $H$. raleighana has previously been estimated between 25 and $30 \mathrm{~cm}$ body length $\left(L_{\mathrm{BD}}\right.$, dorsal edge of gill opening to origin of upper caudal fin) for males and $35 \mathrm{~cm} L_{\mathrm{BD}}$ for females, and at $50 \mathrm{~cm} L_{\mathrm{BD}}$ for R. pacifica (D.D. Didier, Millersville University, pers. comm.).

There are no biomass trends available for H. raleighana and $R$. pacifica (O’Driscoll et al., 2011; Bagley et al., 2013). Nevertheless, there are many documented instances of declining trends in abundance of many deep-sea taxa as a result of fishing (Heymans et al., 2010; Norse et al., 2012), and chimaeroid populations could be negatively affected with expanding deep-sea fisheries (Simpfendorfer \& Kyne, 2009). No target fisheries for H. raleighana or R. pacifica exist in New Zealand, but both are caught as bycatch in deep-sea trawl fisheries (Jacob et al., 1998; Allain et al., 2003; MPI, 2015). Harriotta raleighana is caught regularly in New Zealand waters, making it one of the most common bycatch species that is not managed by the fisheries Quota Management System (QMS). An estimated catch of $82 \mathrm{t}$ was recorded between the 2008 09 to 2012-13 fishing years (Ford et al., 2015), making it one of the largest catches of deep-sea chondrichthyans fishes in New Zealand. In contrast, $R$. pacifica is only occasionally caught as bycatch, with less than $5 \mathrm{t}$ of estimated catch between the 200809 to 2012-13 fishing years (Ford et al., 2015). It is also occasionally recorded by the Ocean Trawl Fishery of Australia at midslope depths of 760-1290 m (Pethybridge et al., 2012).

The purpose of this research was to quantify reproductive parameters to investigate the reproductive biology of $H$. raleighana and $R$. pacifica, and determine if these species possess the same biological characteristics that make other deep-sea chondrichthyan vulnerable to overfishing. To add in this comparison, we also review other studies that focused on holocephalan reproductive biology, expanding previous work by Kyne \& Simpfendorfer (2007). 


\subsection{Methods}

\subsubsection{Study area and specimen collection}

Harriotta raleighana and Rhinochimaera pacifica were collected by RV Tangaroa in 2014 and 2016 from the continental shelf and slope of the Chatham Rise (January surveys) and sub-Antarctic waters (December surveys) of New Zealand. Trawl surveys were stratified-random with a primary objective to provide relative abundance indices of important commercial middle-depth fishes (see O'Driscoll et al., 2011; Bagley et al., 2013). Sampling strata were defined by location and depth, and fishing occurred on trawlable habitats at depths of 400-1300 m. A full-wing bottom trawl with a $60 \mathrm{~mm}$ codend mesh was towed at each station for 3 nautical miles $(5.6 \mathrm{~km})$, at a speed of 3.5 knots $(6 \cdot 5 \mathrm{~km} / \mathrm{h})$. A total of 370 tows were conducted by the surveys. All fish caught were frozen whole at sea and brought back to the laboratory for analyses. An additional 5 samples of $R$. pacifica were obtained from within the New Zealand Exclusive Economic Zone (EEZ) by on-board scientific observers of the Ministry for Primary Industry (MPI) during commercial fishing operations in 2015.

\subsubsection{Biological data}

In all specimens, total mass $\left(M_{\mathrm{T}}\right)$, chimaera length $(L c$; tip of the snout to the posterior edge of the supracaudal fin, excluding the caudal filament), snout to vent length $\left(L_{\mathrm{SV}}\right)$, preorbital length $\left(L_{\mathrm{PB}}\right)$; tip of the snout to anterior edge of the eye) (Fig. 5.1), and inner clasper length $\left(L_{\mathrm{CL}}\right)$ were recorded following Garrick \& Inada (1975) and Francis (2006a). Sex was determined externally by the presence (males) or absence (females) of claspers. The mass of the liver and gonads (ovaries in females, testes in males) were recorded, and the oviducal gland, uterus (in females) and testes (in males) were measured for maximum length and width. The degree of calcification of claspers and development of the epididymis were also noted, as defined in Table 5.1. The number of vitellogenic follicles (> $10 \mathrm{~mm}$ diameter) and the largest ovarian follicle diameter were recorded for each ovary. Ovarian fecundity was determined by the number of follicles yellow in colour and greater than $10 \mathrm{~mm}$ diameter in mature females. Lengths were measured to the nearest $\mathrm{mm}$ and all mass to the nearest $0 \cdot 1 \mathrm{~g}$. One adult female $R$. pacifica was missing a substantial part of the tail, and was removed from the data analysis. 


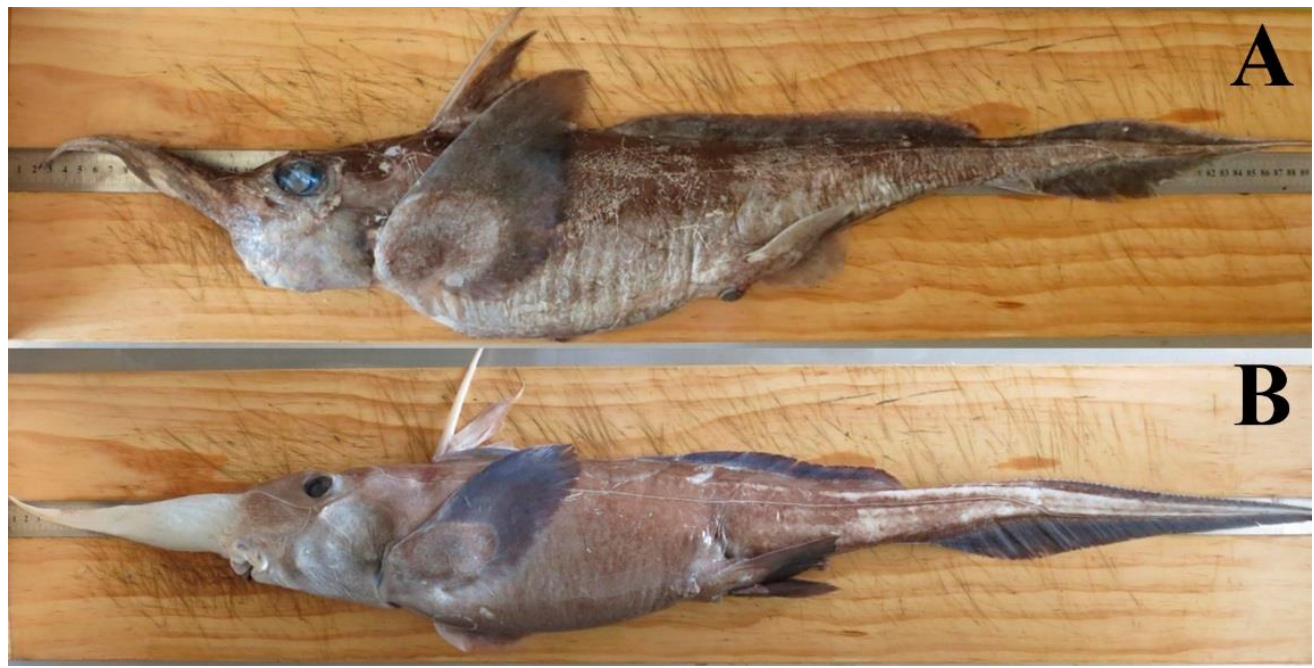

Figure 5.1 (A) Adult female Harriotta raleighana $\left(89 \mathrm{~cm} L_{\mathrm{C}}\right)$ and (B) adult male Rhinochimaera pacifica $\left(106 \mathrm{~cm} L_{\mathrm{C}}\right)$.

For each specimen, the maturity stage was defined by macroscopic evaluation of reproductive organ development. Maturity was classified into one of six maturity stages based on New Zealand research survey protocol by M.P. Francis \& W.S. Lyon (NIWA, pers. comm.) with adaptations following Stehmann (2002), Barnett et al. (2009), and ICES (2010) (Table 5.1). As both right and left sides of the reproductive system are functional in males and females (Wourms, 1977; Malagrino et al., 1981), left and right measurements were plotted against each other to test for differences. In males, inner clasper length was standardized by chimaera length $\left(L_{\mathrm{CL}} / L_{\mathrm{C}}\right)$. Measurements of testes length and seminal vesicle width in males, and oviducal gland width and uterus width in females, were similarly standardized by chimaera length. The presence or absence of a sperm plug in the female accessory gland was recorded. Gonadosomatic index $\left(I_{\mathrm{G}}\right)$ was calculated as the total gonad mass/total body mass x 100 . The onset of maturity was assumed to be represented by an abrupt increase in these statistics. Pairedsample $t$-tests were used to evaluate differences between measurements of the right and left reproductive tracts of males and females (Zar, 1996).

Size-at-maturity $\left(L_{50}\right)$ was calculated for males and females separately by fitting a logistic model to the proportion of mature individuals at length using non-linear least squares. Maturity data (immature 0 , mature 1) were aggregated by $5 \mathrm{~cm} L \mathrm{c}(H$. raleighana) and $10 \mathrm{~cm} \mathrm{Lc} \mathrm{(R.} \mathrm{pacifica)} \mathrm{size} \mathrm{class} \mathrm{intervals} \mathrm{for} \mathrm{each} \mathrm{sex.} \mathrm{The} \mathrm{logistic}$ 
function had the form $Y_{a}=\left[1+19^{\left(L_{50}-a\right) / L} \text { to95 }\right]^{-1}$, where $Y_{a}$ is the proportion of mature individuals in size class $a$, and $L_{50}$ and $L_{\text {to95 }}$ are parameters. All statistical analyses were carried out using R (R Core Team, 2012). 
Table 5.1. Macroscopic criteria used to determine maturity in male and female chimaeroids.

\begin{tabular}{|c|c|c|c|c|}
\hline Maturity & Stage & Trait & Male & Female \\
\hline \multirow[t]{6}{*}{ Immature } & \multirow[t]{6}{*}{1} & \multirow[t]{3}{*}{ External } & Claspers: flexible, non-calcified and shorter than pelvic fins & \multirow[t]{3}{*}{ Paired oviducal openings: not visible } \\
\hline & & & Frontal tenaculum: not erupted & \\
\hline & & & Pre-pelvic tenacula: no development & \\
\hline & & \multirow[t]{3}{*}{ Internal } & Testes: thin and soft; no development & $\begin{array}{l}\text { Ovaries: small, whitish; indistinguishable ovarian follicles }(<10 \\
\mathrm{mm})\end{array}$ \\
\hline & & & \multirow[t]{2}{*}{ Epididymis: thread-like and straight } & Oviducal gland: no differentiation from uterus \\
\hline & & & & Uteri: thread-like and narrow \\
\hline \multirow[t]{6}{*}{ Maturing } & \multirow[t]{6}{*}{2} & \multirow[t]{3}{*}{ External } & $\begin{array}{l}\text { Claspers: flexible, partially calcified; extend to } 1 / 2 \text { length of } \\
\text { pelvic fin, not yet perforated at their free ends }\end{array}$ & \multirow[t]{3}{*}{ Paired oviducal openings: small and visible } \\
\hline & & & $\begin{array}{l}\text { Frontal tenaculum: erupted, but small, smooth with } \\
\text { uncalcified hooks }\end{array}$ & \\
\hline & & & Pre-pelvic tenacula: erupted, but no calcified hooks & \\
\hline & & \multirow[t]{3}{*}{ Internal } & Testes: thin, round and soft & $\begin{array}{l}\text { Ovaries: follicles of different stages of development; whitish to } \\
\text { cream-coloured }(>10 \mathrm{~mm})\end{array}$ \\
\hline & & & Epididymis: developing, beginning to coil & $\begin{array}{l}\text { Oviducal gland: developing and differentiating from uterus, but } \\
\text { no visible contrasting tissue zones }\end{array}$ \\
\hline & & & Seminal vesicle: no sperm present & Uteri: enlarging, partly enlarged posteriorly \\
\hline \multirow[t]{6}{*}{ Mature } & \multirow[t]{6}{*}{3} & \multirow[t]{3}{*}{ External } & $\begin{array}{l}\text { Claspers: rigid, fully calcified; extended to or past the tip of } \\
\text { pelvic fin; perforated, bulbous free ends }\end{array}$ & Paired oviducal openings: large and swollen \\
\hline & & & Frontal tenaculum: fully developed with calcified hooks & Spermatophore may be present \\
\hline & & & Pre-pelvic tenacula: hard and protruding with calcified hooks & \\
\hline & & \multirow[t]{3}{*}{ Internal } & Testes: large, firm and well-developed & $\begin{array}{l}\text { Ovaries: presence of oocytes of different sizes with large, } \\
\text { vascularize oocytes yellow in colour }\end{array}$ \\
\hline & & & Epididymis: tightly coiled, filled with sperm & $\begin{array}{l}\text { Oviducal gland: fully developed; bulbous in shape with } \\
\text { contrasting tissue zones }\end{array}$ \\
\hline & & & Seminal vesicles: enlarged, sperm present & Uteri: fully developed \\
\hline
\end{tabular}




\subsubsection{Light microscopy and transmission electron microscopy}

The presence of sperm storage in females was evaluated following the descriptions of Pratt \& Tanaka (1994) and Smith et al. (2004). Oviducal glands were removed and fixed in $10 \%$ neutral buffered formalin. Samples were cut on the coronal plane and placed in biopsy cassettes. Tissues were dehydrated through a series of ascending alcohol concentrations (70-100\%) and infiltrated with paraffin wax in a Tissue Tek VIP 5 Processor before being embedded into wax blocks. Sections of embedded tissue were sliced $4 \mu \mathrm{m}$ thick with a Leica microtome, mounted onto glass slides, and stained in Harris's haematoxylin and eosin (H\&E). Samples were photographed on a Leica DM LB light microscope with a Canon EOS 70D camera attachment.

Areas of interest selected from prepared slides were marked, and the corresponding wax block had the marked area removed with a $2 \mathrm{~mm}$ biopsy punch. To prepare the wax biopsy for transmission electron microscopy (TEM), the sample was placed in xylol overnight to dewax, and then rehydrated using $100 \%$ and $50 \%$ alcohol and a sodium cacodylate buffer.

The biopsy was processed through a secondary fixation procedure in an aqueous solution of osmium tetroxide and dehydrated in a series of increasing alcohol concentrations (15-100\%). Sections were placed into propylene oxide (transition fluid), followed by a 50/50 mix of propylene oxide and resin to aid resin infiltration, then transferred to fresh resin where they were set into resin blocks and placed in a $60^{\circ} \mathrm{C}$ oven overnight to harden. Resin blocks were sectioned $1 \mu \mathrm{m}$ thick on a LKB ultrotome and stained in toluidine blue to be used as survey slides to ascertain the area of interest. After confirmation of area of interest, sections $80-90 \mathrm{~nm}$ were cut, mounted onto grids, and stained with uranyl acetate followed by Reynold's lead citrate. Images were viewed on a Phillip's CM100 Electron microscope and captured at a series of magnifications (3400X, 5800X, 9700X, 13,500X, and 17,500X) using the Morada soft imaging system. 


\subsection{Results}

\subsubsection{Harriotta raleighana}

A total of 300 specimens of Harriotta raleighana (180 females, 120 males) were examined. Females ranged from 18.7 to $90.4 \mathrm{~cm} L \mathrm{c}($ median $=70.0 \mathrm{~cm} L \mathrm{c})$ and males 27.4 to $79.6 \mathrm{~cm} L \mathrm{c}$ (median $=65 \cdot 1 \mathrm{~cm} L \mathrm{c}$ ). Total mass for females and males varied from 14 to $2189 \mathrm{~g}$ and 58 to $1123 \mathrm{~g}$, respectively. Females were more numerous in larger length classes (> $60 \mathrm{~cm} L \mathrm{c}$ ) and reached a greater size than males (Fig. 5.2). The length (cm) to mass ( $\mathrm{g}$ ) relationship of $H$. raleighana was described by $W=1.06 \times 10^{-3}(\mathrm{Lc})^{3.228}$. There was a strong correlation between $L_{\mathrm{SV}}$ on $L \mathrm{c}\left(L_{\mathrm{SV}}=0.521 L \mathrm{c}+1.462, R^{2}=0.97\right)$.

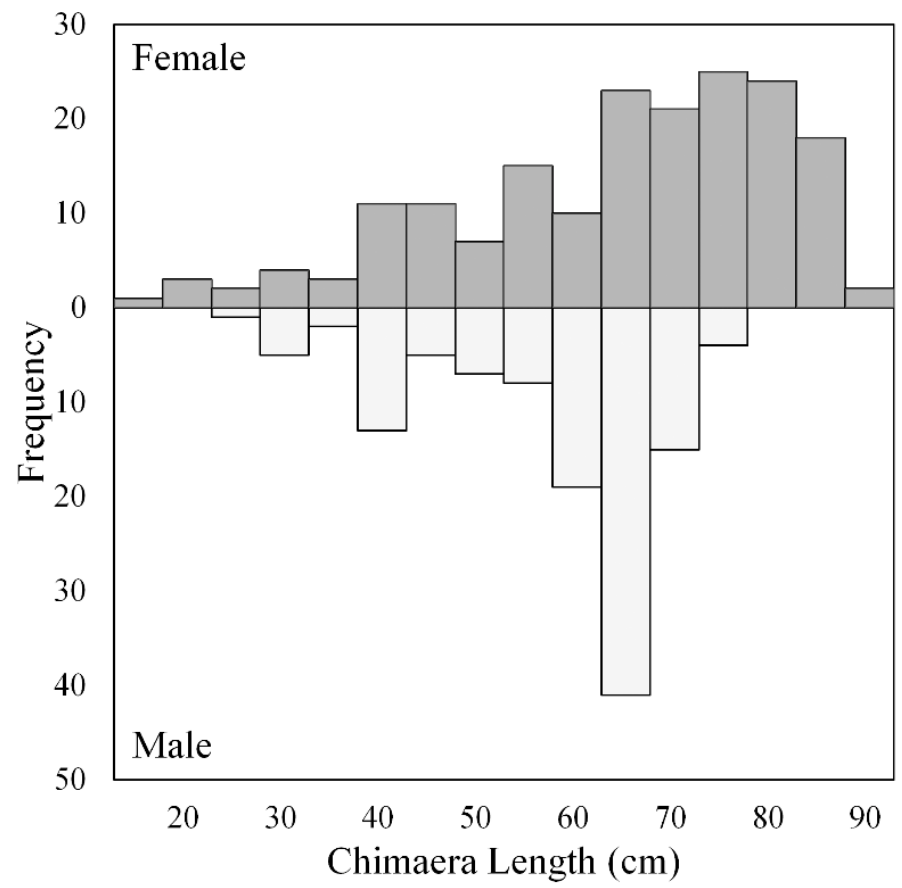

Figure 5.2 Chimaera length $\left(L_{C}\right)$-frequency distribution for $(\square)$ female and ( $\square$ ) male Harriotta raleighana $(n=300)$. 


\subsubsection{Size-at-maturity}

Based on the macroscopic assessments, size at $50 \%$ maturity $\left(L_{50}\right)$ was $75.8 \mathrm{~cm} L \mathrm{c}$ $(95 \%$ C.I. $=75 \cdot 1-76.6 \mathrm{~cm} \mathrm{Lc})$ for females and $62 \cdot 8 \mathrm{~cm} \mathrm{Lc}(95 \%$ C.I. $=62 \cdot 8-62.9 \mathrm{~cm} \mathrm{Lc})$ for males (Fig. 5.3). Maturity took place at 0.84 and 0.79 of the maximum observed length for females and males respectively.

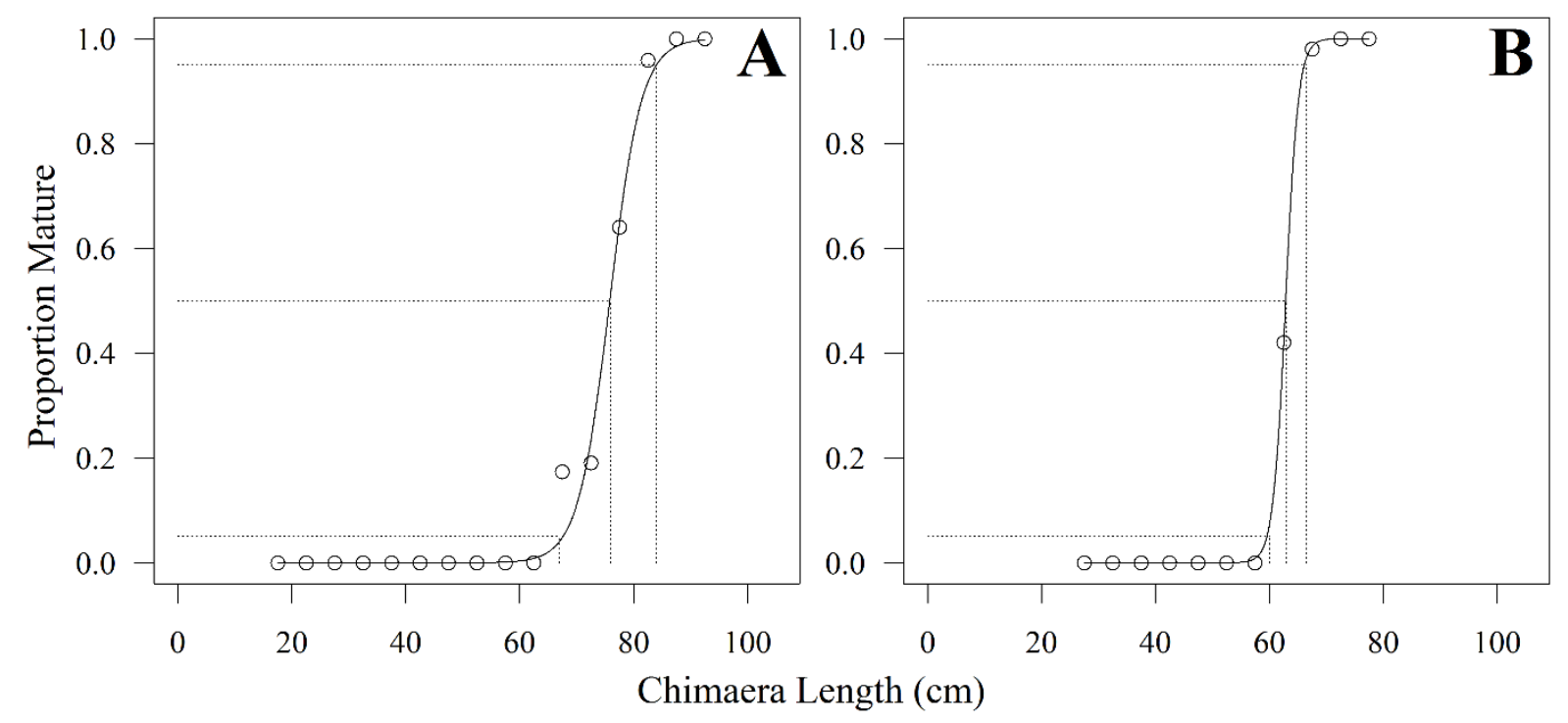

Figure 5.3 Logistic model fitted chimaera length $(L c)$-maturity ogive for Harriotta raleighana, with the observed proportion of mature (A) females and (B) males in each size interval. $\vdots, L c$ at $5 \%, 50 \%\left[\left(L_{50}\right)\right.$ for females $\left.\left(L_{50}=75.8 \mathrm{~cm}\right)\right]$ and males $\left(L_{50}=\right.$ $62 \cdot 8 \mathrm{~cm})]$, and $95 \%$ maturity determined from fitted logistic curves. 
Approximately $63 \%(n=113)$ of the female $H$. raleighana were macroscopically classified as immature or maturing and $37 \%(n=67)$ as mature. The smallest mature specimen was $66 \cdot 2 \mathrm{~cm} L \mathrm{c}$ and the largest immature was $84 \cdot 3 \mathrm{~cm} L \mathrm{c}$. The mean size of an adult was $81.3 \mathrm{~cm} L \mathrm{c}$. There was no difference between left and right oviducal gland width $(t$-test, d.f. $=174, P>0.05)$ or uterus width $(t$-test, d.f. $=178, P>0 \cdot 05)$. An abrupt increase in the gonadosomatic index $\left(I_{\mathrm{G}}\right)$ [Fig. 5.4(A)], as well as oviducal gland width [Fig. 5.4(B)], corresponded well with the onset of female maturity as evaluated macroscopically. Uterus width also increased with the onset of macroscopic maturity [Fig. 5.4(C)], but the change was not as pronounced. Ovarian follicle width ranged from 0.8 to $3.9 \mathrm{~cm}$, but did not appear to change with increasing chimaera length [Fig. 5.4(D)]. Maximum ovarian fecundity ranged from 15 in the left ovary to 16 in the right, with a combined fecundity of up to 27 . A total of $10.4 \%(n=7)$ of adult females were found with an egg capsule developing in each uterus. Half $(n=36)$ of the adult females had a large, gelatinous mass in the accessory genital gland. 

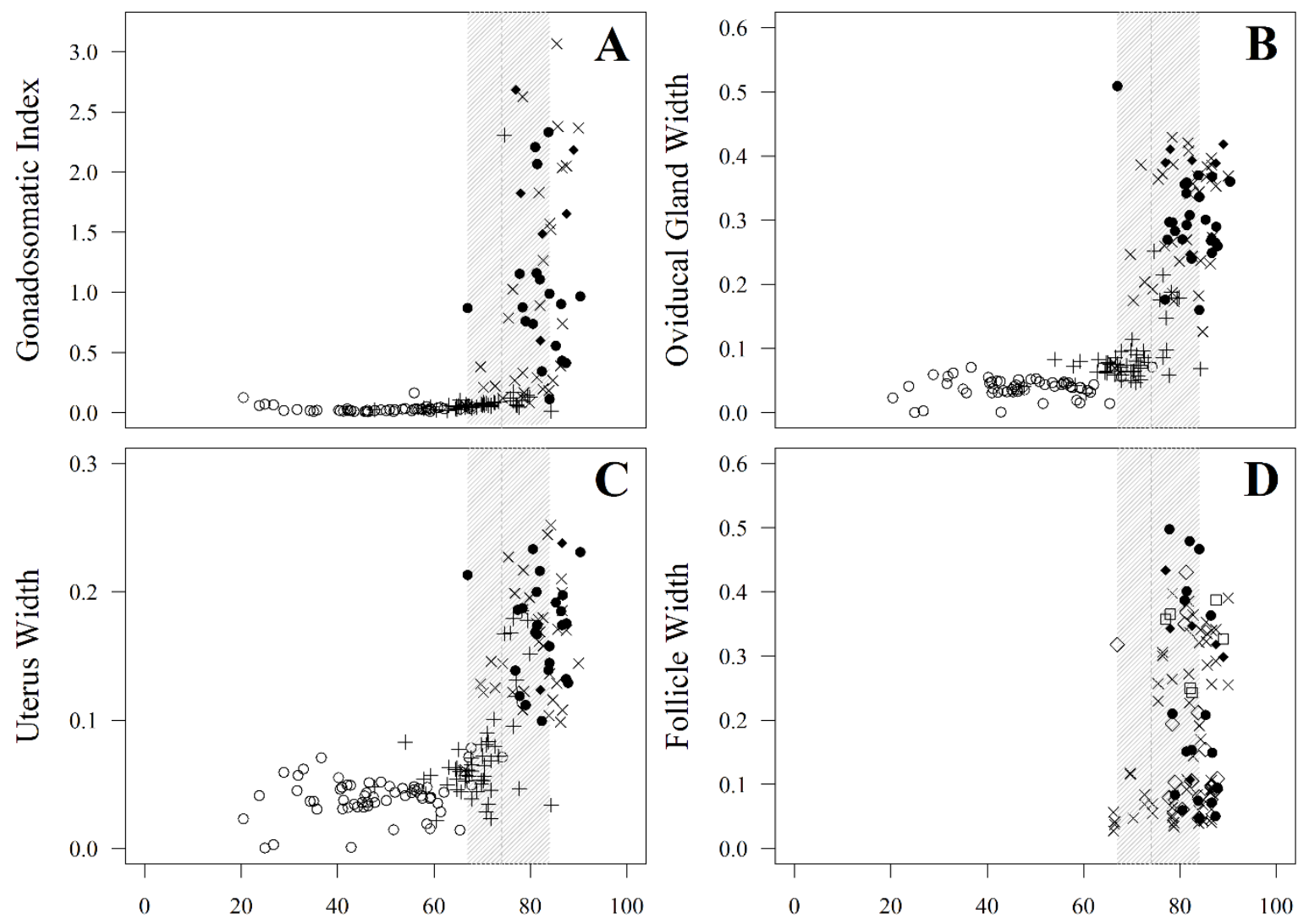

Chimaera Length $(\mathrm{cm})$

Figure 5.4 Relationship between chimaera length $(L c)$ and $(A)$ gonadosomatic index $\left(I_{\mathrm{G}}\right)$, (B) proportional width of oviducal gland, (C) uterus and (D) diameter of largest ovarian follicle for female Harriotta raleighana, based on macroscopic maturity stages: $\circ$, juvenile; +, maturing; $\times$, mature; $\bullet$, gravid; $\bullet$, post-laying. $\vdots$, estimated $L_{\mathrm{T}}$ at $50 \%$ mature $\left(L_{50}=75 \cdot 8 \mathrm{~cm}\right)$ with $5-95 \%$ mature as grey bar. 
In males, 44\% ( $n=53)$ of specimens were classified as immature or maturing and $56 \%(n=67)$ as mature. The smallest mature specimen was $60 \cdot 2 \mathrm{~cm} L \mathrm{c}$ and the largest immature was $65.3 \mathrm{~cm} L \mathrm{c}$. The mean size of an adult was $68.6 \mathrm{~cm} L \mathrm{c}$. There was no difference between left and right inner clasper length $(t$-test, d.f. $=119, P>0.05)$ or seminal vesicle width $(t$-test, d.f. $=115, P>0 \cdot 05)$, but there was a difference in testes length $(t$-test, d.f. $=115, P<0.05)$. An abrupt change in the gonadosomatic index $\left(I_{\mathrm{G}}\right)$, observed at approximately $62 \mathrm{~cm} \mathrm{Lc} \mathrm{[Fig.} \mathrm{5.5(A)],} \mathrm{corresponded} \mathrm{well} \mathrm{with} \mathrm{an} \mathrm{abrupt}$ increase in standardized inner clasper length [Fig. 5.5(B)], and testes length [Fig. 5.5(C)]. An increase in seminal vesicle width was also noted slightly larger, at approximately $66 \mathrm{~cm} \mathrm{Lc} \mathrm{[Fig.} \mathrm{5.5(D)].} \mathrm{Clasper} \mathrm{calcification} \mathrm{was} \mathrm{observed} \mathrm{as} \mathrm{the} \mathrm{last} \mathrm{macroscopic} \mathrm{change}$ to take place during maturation. 

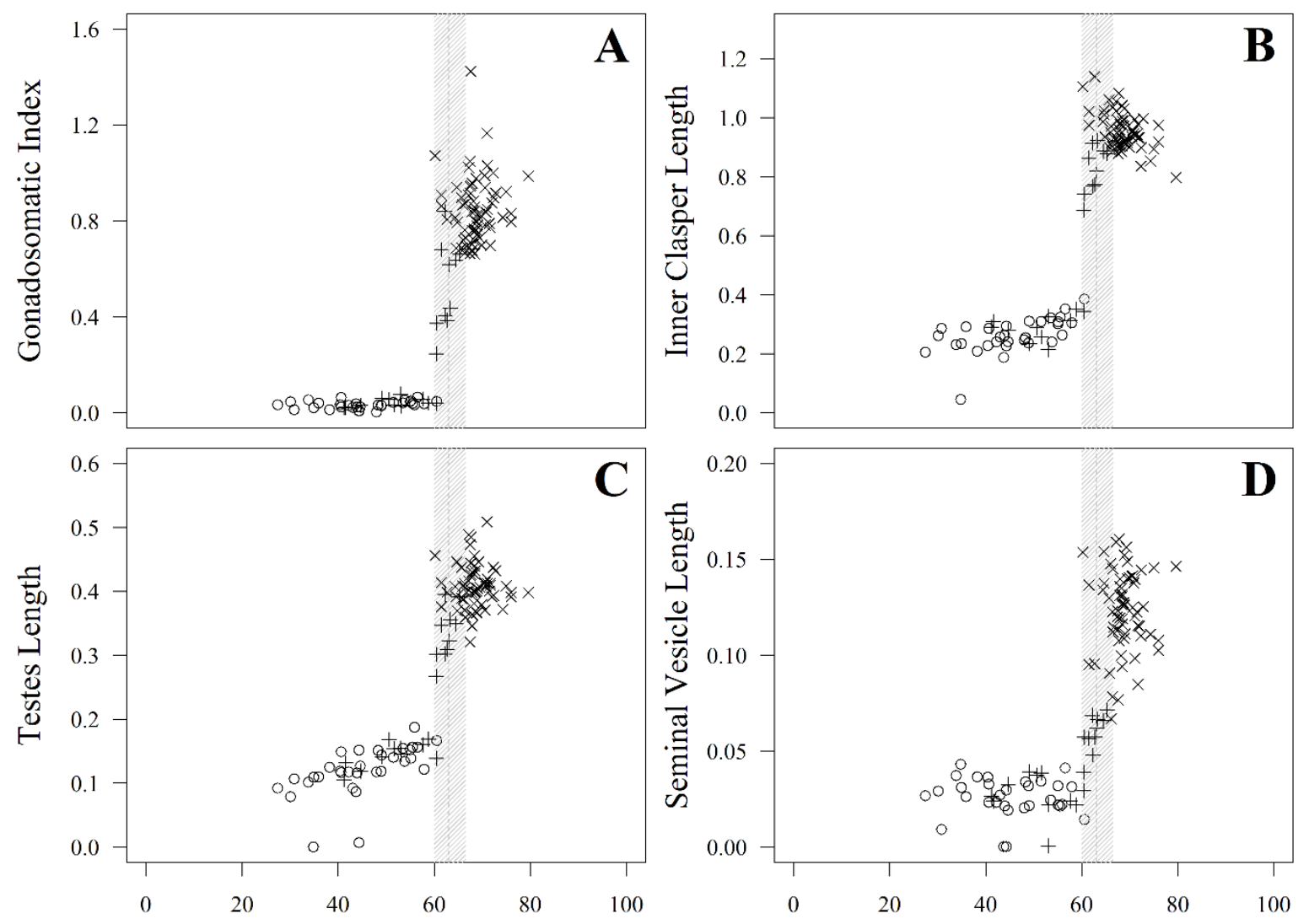

Chimaera Length $(\mathrm{cm})$

Figure 5.5 Relationship between chimaera length $(L c)$ and $(\mathrm{A})$ gonadosomatic index $\left(I_{\mathrm{G}}\right)$, (B) proportional inner clasper length $\left(L_{\mathrm{CL}}\right),(\mathrm{C})$ testes length, and (D) seminal vesicle width for male Harriotta raleighana based on macroscopic maturity stages: ○, juvenile; + , maturing; $\times$, mature $)$ !, estimated $L_{\mathrm{T}}$ at $50 \%$ mature $\left(L_{50}=62.8 \mathrm{~cm}\right)$ with $5-95 \%$ mature as grey bar. 
A total of 168 specimens of Rhinochimaera pacifica (104 males, 64 females) were examined. Females ranged from 20.9 to $139.9 \mathrm{~cm} L \mathrm{c}($ median $=105.85 \mathrm{~cm} \mathrm{Lc})$ and males 50.8 to $123.3 \mathrm{~cm} L \mathrm{c}($ median $=107.7 \mathrm{~cm} L \mathrm{c})$. Total mass for females and males varied from 17 to $6268 \mathrm{~g}$ and 136 to $3182 \mathrm{~g}$ respectively. Females reached a greater size than males, with a relatively uniform length frequency distribution, while males had a mode at $110-120 \mathrm{~cm}$ Lc (Fig. 5.6). The length (cm) to mass (g) relationship of $R$. pacifica was described by $W=5.993 \times 10^{-5}(L \mathrm{c})^{3.717}$. There was a strong correlation between $L_{\mathrm{SV}}$ on $L \mathrm{c}$ $\left(L_{\mathrm{SV}}=0.501 L \mathrm{c}+1 \cdot 831, R^{2}=0 \cdot 92\right)$.

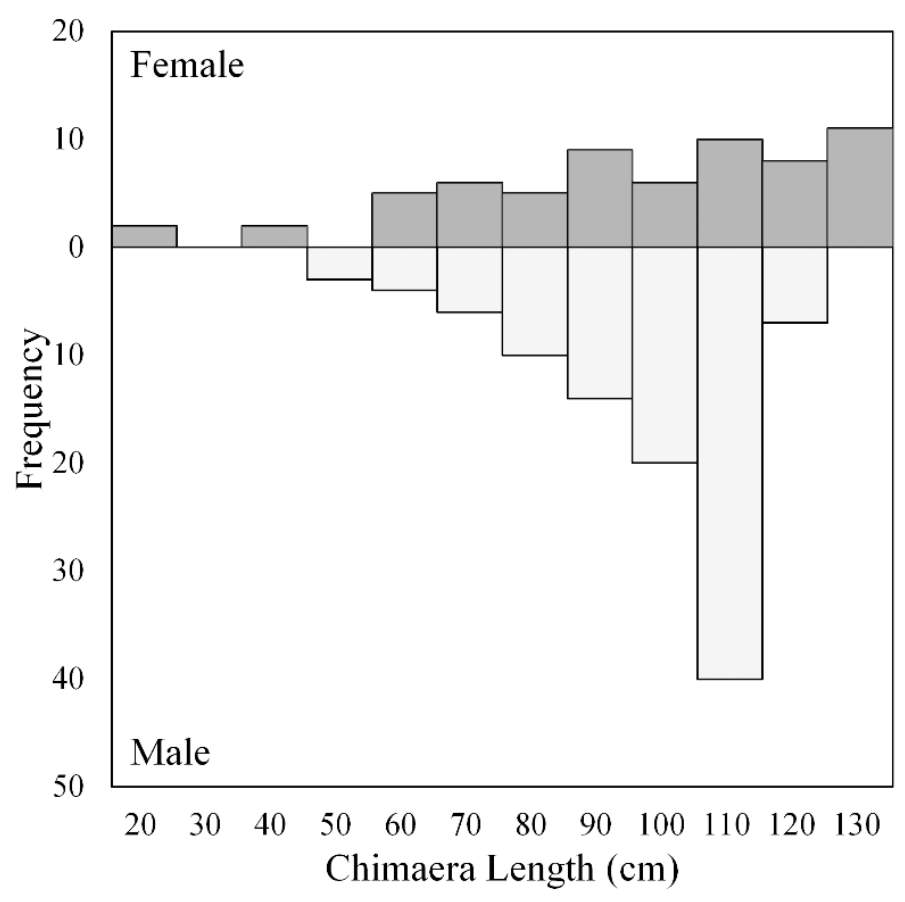

Figure 5.6 Chimaera length $\left(L_{C}\right)$-frequency distribution for $(\square)$ female and ( $\square$ ) male Rhinochimaera pacifica $(n=168)$. 


\subsubsection{Size-at-maturity}

Macroscopic size at $50 \%$ maturity $\left(L_{50}\right)$ was $125 \cdot 0 \mathrm{~cm} \mathrm{Lc}(95 \%$ C.I. $=123 \cdot 6-$ $126 \cdot 4 \mathrm{~cm} \mathrm{Lc})$ for females and $105 \cdot 3 \mathrm{~cm} \mathrm{Lc}(95 \%$ C.I. $=104 \cdot 7-105.6 \mathrm{~cm} \mathrm{Lc})$ for males (Fig. 5.7). Maturity took place at 0.90 and 0.85 of the maximum observed length for females and males respectively.

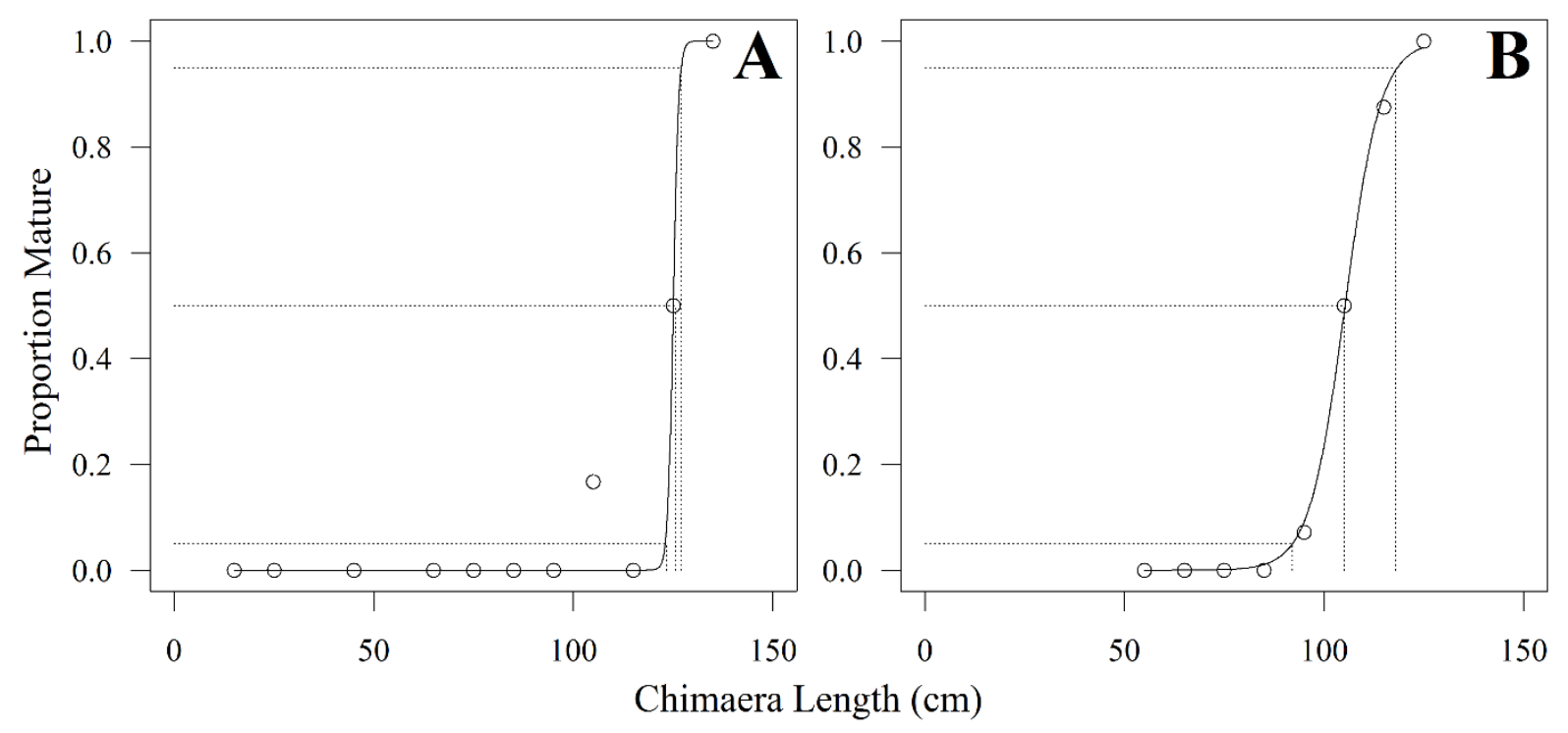

Figure 5.7 Logistic model fitted chimaera length $(L c)$-maturity ogive for Rhinochimaera pacifica, with the observed proportion of mature (A) females and (B) males in each size interval. $\vdots, L \mathrm{c}$ at $5 \%, 50 \%\left[\left(L_{50}\right)\right.$ for females $\left(L_{50}=125.0 \mathrm{~cm}\right)$ and males $\left(L_{50}=\right.$ $105 \cdot 3 \mathrm{~cm})]$, and $95 \%$ maturity determined from fitted logistic curves.

\subsubsection{Female reproductive characteristics}

Approximately $75 \%(n=41)$ of the females were classified as immature or maturing and $25 \%(n=16)$ as mature. The smallest mature specimen measured $108 \cdot 3 \mathrm{~cm}$ $L c$ and the largest immature was $129.5 \mathrm{~cm} L \mathrm{c}$. The mean size of an adult female was $132 \cdot 1 \mathrm{~cm} \mathrm{Lc}$. While data on mature female $R$. pacifica were limited, there were distinct separations between immature and mature measurements of gonad mass $\left(I_{\mathrm{G}}\right)$ [Fig. 5.8(A)], oviducal gland width [Fig. 5.8(B)], and uterus width [Fig. 5.8(D)]. There was no difference between left and right oviducal gland width ( $t$-test, d.f. $=34, P>0 \cdot 05$ ), but the right uterus was significantly larger than the right $(t$-test, d.f. $=60, P<0 \cdot 05)$. Ovarian 
follicle width ranged from 2.0 to $4.4 \mathrm{~cm}$ and did not appear to change with increasing chimaera length [Fig. 5.8(D)]. Ovarian fecundity ranged from 16 in the left ovary to 19 in the right, with a combined maximum fecundity of up to 31 . One gravid females was sampled with one egg capsule in each uterus, and two adult females were found with a gelatinous mass in the accessory genital gland.
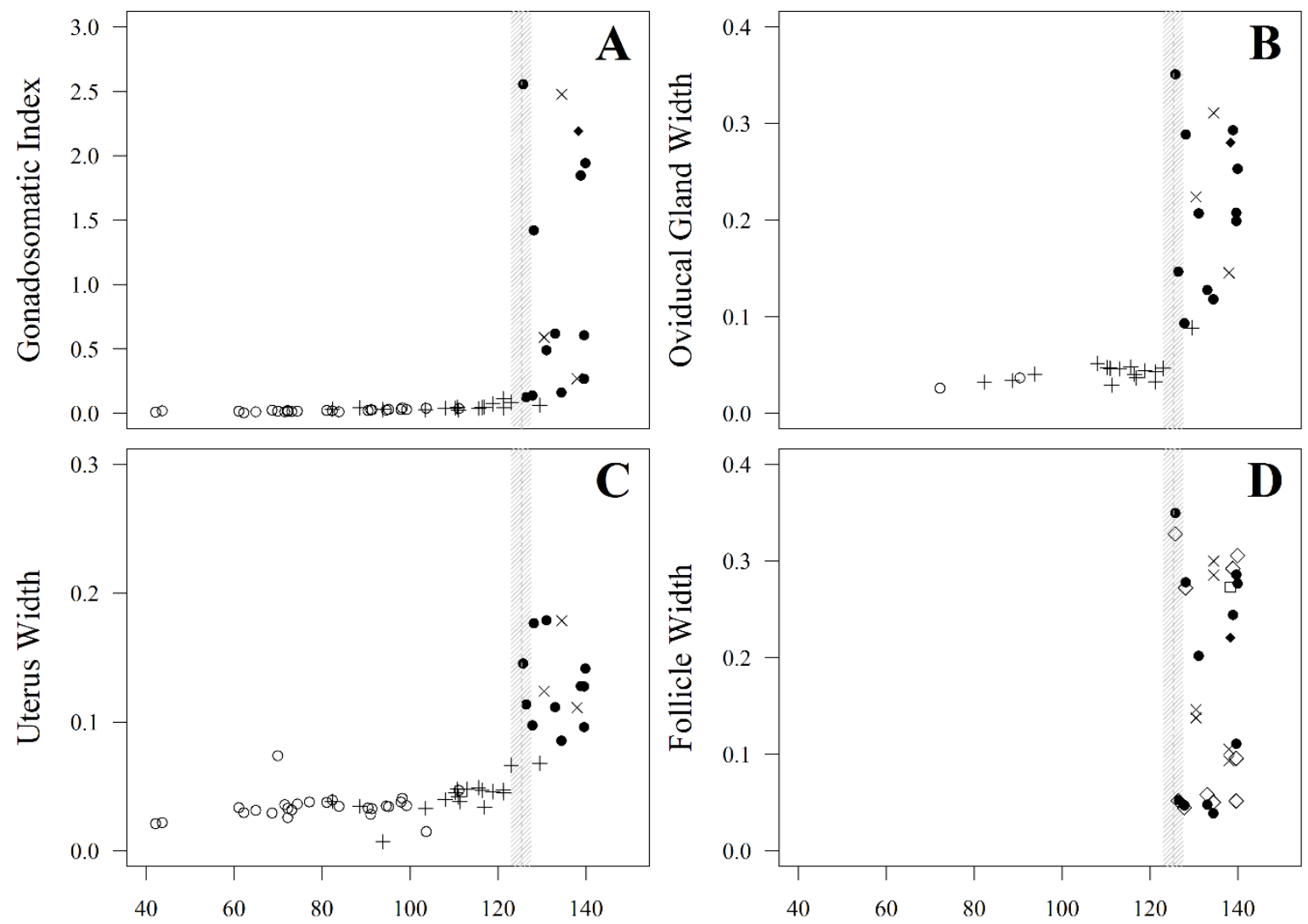

Chimaera Length $(\mathrm{cm})$

Figure 5.8 Relationship between chimaera length $(L c)$ and $(\mathrm{A})$ gonadosomatic index $\left(I_{\mathrm{G}}\right)$, (B) proportional width of oviducal gland, (C) uterus and (D) diameter of largest ovarian follicle for female Rhinochimaera pacifica, based on macroscopic maturity stages: $\odot$, juvenile; +, maturing; $\times$, mature; $\bullet$, gravid; $\bullet$, post-laying. !, estimated $L_{\mathrm{T}}$ at $50 \%$ mature $\left(L_{50}=125 \cdot 0 \mathrm{~cm}\right)$ with $5-95 \%$ mature as grey bar. 


\subsubsection{Male reproductive characteristics}

In males, approximately half were immature or maturing $(n=50)$ and half adult $(n=54)$. The smallest mature specimen was $99.7 \mathrm{~cm} \mathrm{Lc}$ and the largest immature $116.6 \mathrm{~cm} L \mathrm{c}$. The mean size of an adult was $114.1 \mathrm{~cm} L \mathrm{c}$. A significant difference was found between left and right measurements of inner clasper length ( $t$-test, d.f. $=102, P<$ $0 \cdot 05)$ and testes length $(t$-test, d.f. $=100, P<0.05)$, with the right reproductive tract found to be slightly larger. No difference in measurements was found in seminal vesicle mass ( $t$-test, d.f. $=101, P>0 \cdot 05)$. An abrupt change in standardized inner clasper length was observed at $105 \mathrm{~cm} \mathrm{Lc} \mathrm{[Fig.} \mathrm{5.9(B)],} \mathrm{which} \mathrm{coincided} \mathrm{with} \mathrm{macroscopic} \mathrm{length-at-}$ maturity. Results were similar for testes length [Fig. 5.9(C)], and gonadosomatic index $\left(I_{\mathrm{G}}\right)[$ Fig. 5.9(A)]. An increase in seminal vesicle width was also noted around macroscopic length-at-maturity [Fig. 5.9(D)]. One individual was found to be fully mature internally, but was not developed externally (non-calcified tenaculum and claspers but calcified pre-pelvic tenacula with hooks). Clasper calcification was observed as the last macroscopic change to take place during maturation. 

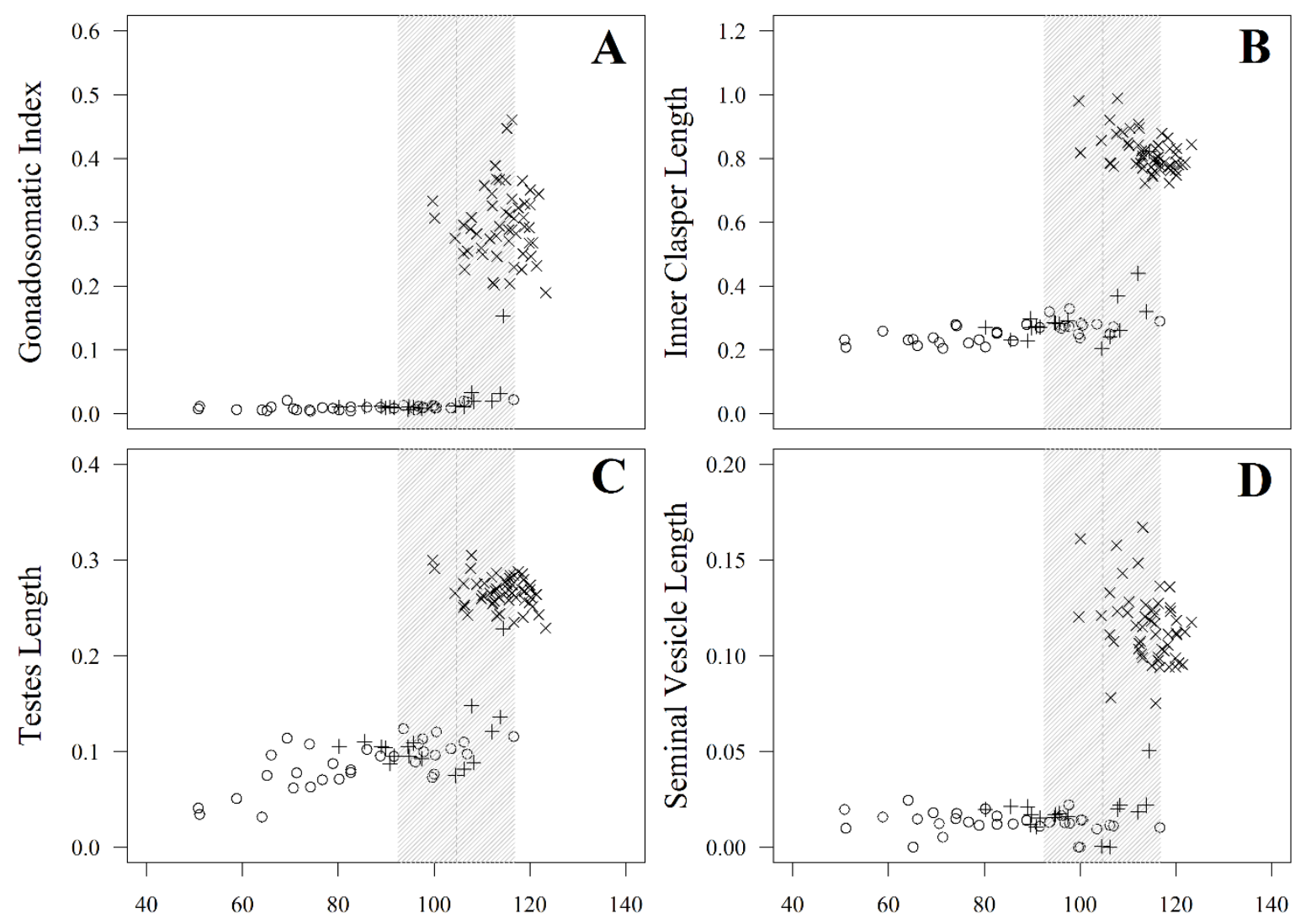

Chimaera Length $(\mathrm{cm})$

Figure 5.9 Relationship between chimaera length $(L c)$ and $(\mathrm{A})$ gonadosomatic index $\left(I_{\mathrm{G}}\right)$, (B) proportional inner clasper length $\left(L_{\mathrm{CL}}\right),(\mathrm{C})$ testes length, and (D) seminal vesicle width for male Rhinochimaera pacifica based on macroscopic maturity stages: $\bigcirc$, juvenile; +, maturing; $\times$, mature). $\vdots$, estimated $L_{\mathrm{T}}$ at $50 \%$ mature $\left(L_{50}=105.3 \mathrm{~cm}\right)$ with 5 $95 \%$ mature as grey bar. 


\subsubsection{Sperm storage}

Sperm storage tubules (SSTs) and sperm bundles were identified in the terminal zone of the oviducal gland of two mature H. raleighana and two mature $R$. pacifica [Fig. 5.10(A)]. SSTs were identified in both active and resting stages. Suspected sperm bundles using light microscopy were confirmed with transmission electron microscopy (TEM)

[Fig. 5.10(B)].

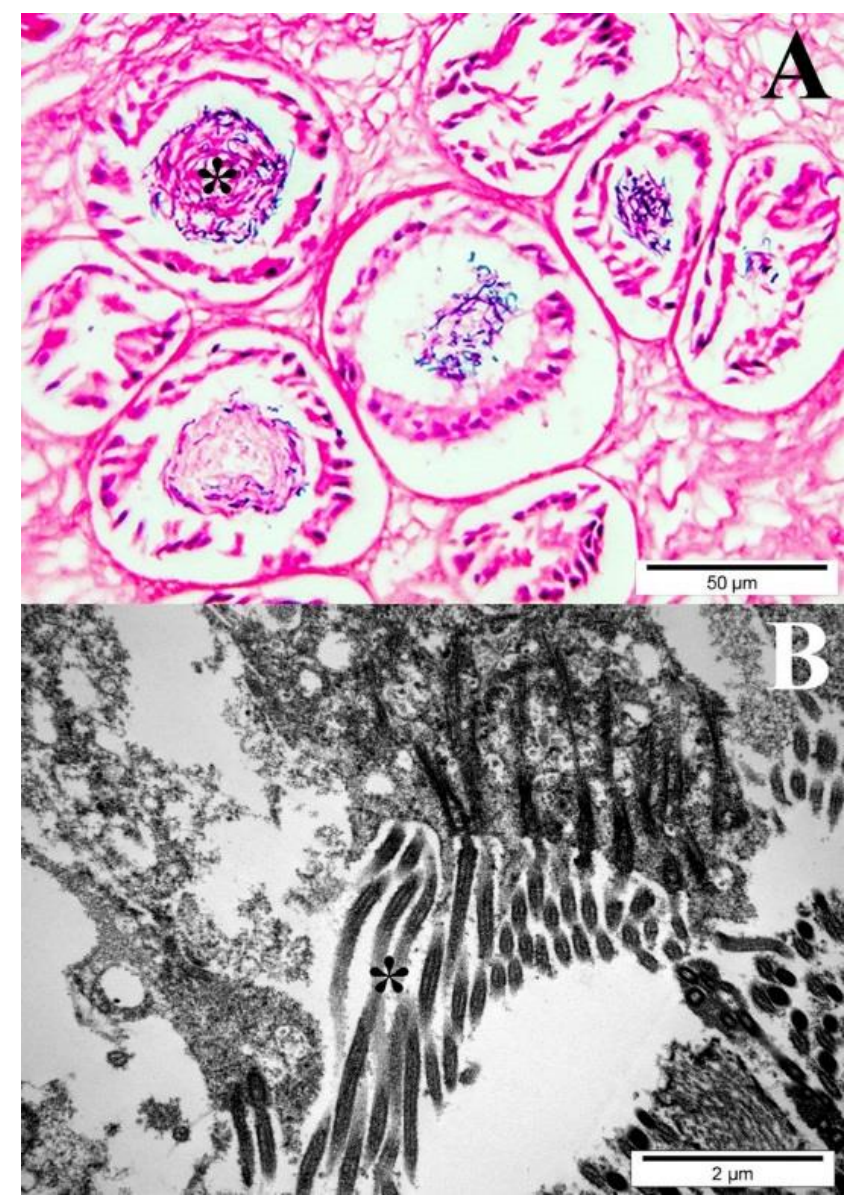

Figure 5.10 (A) Light microscopy image of sperm storage tubules from terminal zone of the oviducal gland of Harriotta raleighana (* indicates sperm bundle), and (B) transmission electron microscopy of a sperm bundles $(*)$. 


\subsubsection{Preorbital snout length}

The ratio of preorbital length $\left(L_{\mathrm{PB}}\right)$ to chimaera length $(L \mathrm{c})$ decreased with increasing $L c$ for both species (Fig. 5.11). In H. raleighana, at approximately $60 \mathrm{~cm} \mathrm{Lc}$, there was a divergence between male and female $L_{\mathrm{PB}}$, with the ratio of $L_{\mathrm{PB}}$ increasing in males [Fig. 5.11(A)]. This sexual dimorphism in $L_{\mathrm{PB}}$ was observed in $H$. raleighana, but not in $R$. pacifica [Fig. 5.10(B)].

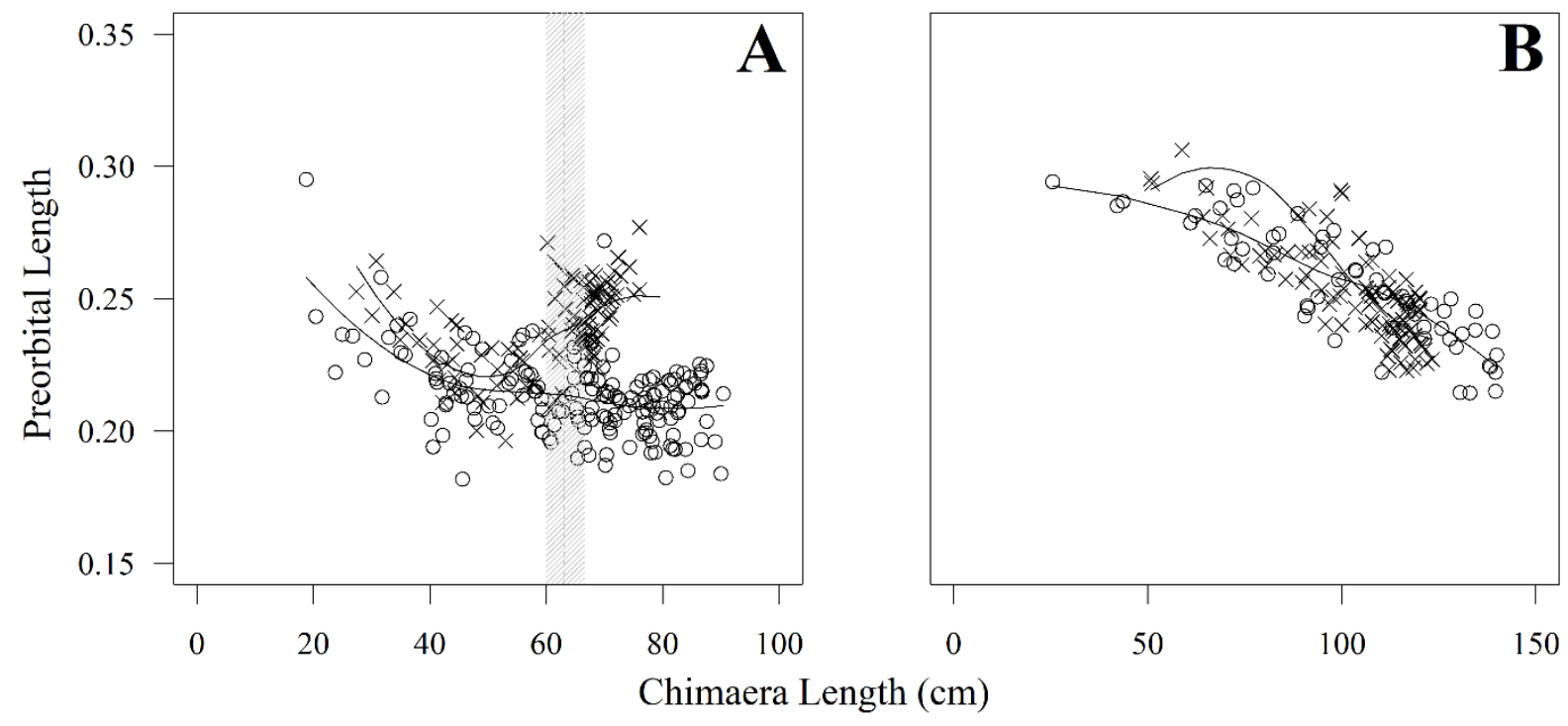

Figure 5.11 Preorbital length $\left(L_{\mathrm{PB}}\right)$ as a function of chimaera length $(L \mathrm{c})$ for $(\mathrm{A})$ Harriotta raleighana and (B) Rhinochimaera pacifica ( $\circ$, female; $\times$, male). The vertical dashed line and bar indicates the macroscopic $L_{50}$ (line) and 95\% C.I. (bar) for male $H$. raleighana. A LOESS (moving average) smoother is plotted for male and female samples (solid line). 


\subsection{Discussion}

We believe that this study is the first to examine the reproductive strategies of Harriotta raleighana and Rhinochimaera pacifica, and of any member of the family Rhinochimaeridae. Females of both species attained a larger overall size than males and reached maturity at a larger size. These characteristics are commonly observed across chondrichthyans, and are most likely a requirement for significant reproductive maternal investment (Springer, 1967; Hoenig \& Gruber, 1990). In R. pacifica, maturity in both sexes was reached at a large proportion of the maximum observed length, which may indicate the full length range of $R$. pacifica was not sampled, or that there is little somatic growth after maturity. Ageing data are not available for $H$. raleighana or $R$. pacifica. In Hydrolagus colliei, maturity was not reached for up to 14 years, and with a life span of around 20 years, this suggests a very late maturity given longevity (King \& McPhie, 2013). Late maturity might also occur in $H$. raleighana and $R$. pacifica. While information on bycatch mortality is not available for either species, maturing relatively late in their life cycles may increase their vulnerability to fishing efforts.

\subsubsection{Sperm storage}

Both $H$. raleighana and $R$. pacifica appear to be capable of storing sperm, a trait presumed to be common amongst chimaeroids (Didier et al., 2012). Until now, it has only been confirmed in the elephantfish, Callorhinchus milii (Smith et al., 2004). Over half of the mature $H$. raleighana females were also found with a gelatinous mass in the accessory gland, which may act as a sperm plug (Smith et al., 2004). Females of all maturity stages were observed during this sampling period, suggesting this species engages in an asynchronous breeding cycle. The frequency of sperm plugs may suggest that at least half of the mature female population is capable of breeding at any time. In contrast, only two (<13\%) of the mature $R$. pacifica were found with a sperm plug, suggesting a much smaller proportion of the females of $R$. pacifica had mated. However, since these samples were only taken at one time of the year, monthly samples are encouraged to measure any temporal differences in population structure and evaluate the possibility of breeding cycles. 


\subsubsection{Secondary sexual characteristics}

The relative increase in male $H$. raleighana snout length was at the approximate size of first sexual maturity, suggesting the snout as a secondary sexual characteristic. Chimaeroids are sexually dimorphic, and males possess several obvious secondary sexual characteristics used for mating purposes, including the frontal tenaculum, paired prepelvic tenaculum, and paired pelvic claspers (Didier et al., 2012). Secondary sexual characteristics may be a consequence of male competition or female selection (Fitzpatrick et al., 2012). Male competition may arise when reproductively active females are low in numbers (Lucifora et al., 2002) or through sexual segregation (Wearmouth \& Sims, 2008). The latter of these is a common observation in elasmobranchs (e.g. Klimley, 1987; Mucientes et al., 2009), and has been more recently identified in holocephalans (Holt et al., 2013). Sperm storage, through cryptic female choice, may also increase male competition (Fitzpatrick et al., 2012). Garrick \& Inada (1975) first noted sexual dimorphism in snout size of $H$. raleighana, but lacked a representative sample size, and no further conclusions were made. While no detailed research has investigated the use of $H$. raleighana snout, it has been assumed that it is used as a sensory probe to locate prey in the sediment (Mauchline \& Gordon, 1983). This may indeed be the case for $R$. pacifica, where no difference in snout size between the sexes was observed in this study. Previous investigation of the diet of $H$. raleighana did not find any diet composition difference between the sexes (Dunn et al., 2010a). In elasmobranchs, dental sexual dimorphism has been confirmed in several species, with the onset of change in male tooth morphology occurring around the time of maturity (Duffy, 2007; Last et al., 2008). Given the potential for sexual segregation and female sperm storage, we speculate that the snout of $H$. raleighana may prove to be used in male competition, but if, and how, this might occur is unknown at this time.

\subsubsection{Sampling}

A lack of neonate individuals in the sample may be accounted for by escapement through the net meshes, but the capture of other species of small size $(<40 \mathrm{~cm})$ (Stevens et al., 2015) suggests this is not likely. Alternatively, if $H$. raleighana and $R$. pacifica engage in congregative nursery behaviour, as exhibited by other oviparous 
chondrichthyans (Etnoyer \& Warrenchuk, 2007), these species may be egg laying in areas not sampled by the surveys. It seems likely that juvenile $H$. raleighana and $R$. pacifica may have nursery grounds distinct from adult areas (Holt et al., 2013), and possibly in deeper waters (Quinn et al., 1980; Dunn et al., 2010a). Chimaeroids deposit egg capsules on the ocean floor, including rocky habitat, where they persist for the remainder of the gestation period (Dean, 1906; Didier et al., 2012). One R. pacifica of $20.9 \mathrm{~cm} L_{\mathrm{C}}$ with an umbilical scar was captured at $42.48{ }^{\circ} \mathrm{S} 177.12{ }^{\circ} \mathrm{W}$ at a depth of approximately $930 \mathrm{~m}$, while another with a length of $25.5 \mathrm{~cm} L_{\mathrm{C}}$ was found further south at $46.35{ }^{\circ} \mathrm{S} 170.44{ }^{\circ} \mathrm{E}$ at a depth of $907 \mathrm{~m}$. Egg capsules of $\mathrm{H}$. raleighana have been previously collected at approximately $685 \mathrm{~m}$ and for $R$. pacifica at 548 to $1077 \mathrm{~m}$ (Dean, 1906). If these depths are correct for New Zealand, then egg capsules should be found within the depth range of the trawl survey. However, if ideal spawning grounds for chimaeroids include hard substrate, then $H$. raleighana and $R$. pacifica may be spawning in areas inaccessible to trawl surveys. Alternatively, these fish may be burying their eggs in the substrate (Dean, 1906), and could be missed by trawls altogether.

Large females, particularly $R$. pacifica, were rare in the sample. A rarity of reproductively active females has been reported for other New Zealand chondrichthyans (Wetherbee, 1996; Finucci et al., 2016a), and the North Atlantic (Holt et al., 2013; Moura et al., 2014). Some chimaeroids segregate by sex and size (Quinn et al., 1980; Holt et al., 2013), which may be a trade-off for minimizing intraspecific competition and sexual conflict (Wearmouth \& Sims, 2008). Female chimaeroids have been found at greater depths than their male counterparts (Holt et al., 2013). Additionally, chimaeroids transition through diel (Quinn et al., 1980) and seasonal movements (Mathews, 1975), and it is possible that these females inhabit depths beyond the survey range or move out of the survey regions. Large female $R$. pacifica (up to $165.3 \mathrm{~cm} L_{\mathrm{C}}$ ) have been recorded previously within the area of study (McMillian et al., 2011), although the largest animal of this study was $139.9 \mathrm{~cm} L_{\mathrm{C}}$. The largest $H$. raleighana recorded in New Zealand waters was $99.3 \mathrm{~cm} L_{\mathrm{C}}$, which is less than the maximum known length of $120 \mathrm{~cm} L_{\mathrm{C}}$ (McMillian et al., 2011). Whether larger fish are elsewhere, or have succumbed to fishing mortality, is unknown. 


\subsubsection{Review of chimaeroid reproductive biology}

There is general acceptance of the use of macroscopic maturity indicators in chimaeroid reproductive studies. Inner clasper length was the prevalent macroscopic observation used to assess male maturity in holocephalans (Table 5.2). As clasper calcification was observed as the last macroscopic change to take place during maturation, we agree with others (e.g. Barnett et al., 2009) that measurement of inner clasper length is sufficient to use for maturity staging males. Tenaculum eruption and development may also be used in conjunction with clasper length (Barnett et al., 2009). Since the transition between immature and mature male specimens is clear-cut, and rapid in terms of fish size, including a maturing stage (Stage 2 in Table 5.1) adds little information, and seems redundant.

No known external measurements for female maturity exist. Previous studies have used follicle development to assess maturation. However, the use of this measurement alone may make distinguishing between resting or regenerating females and maturing individuals difficult (Bubley et al., 2013). Histology (Moura et al., 2004) and skeletal muscle concentration of steroid hormones (Barnett et al., 2009) have proven to be useful tools to support macroscopic observations of size-at-maturity. The overlap between maturity stages in measurements of $I_{\mathrm{G}}$, oviducal gland width, and uterus width in this study demonstrates there is no single measurement that provides a clear-cut indicator of maturity for females. It is recommended that multiple measurements might be made for better assessing female maturity, with gonad mass and oviducal gland width showing promise based on this study and previous results (e.g. Barnett et al., 2009; King \& McPhie, 2013).

An inconsistency in morphometric measurements for holocephalans has been noted, making it difficult to review records of size-at-maturity (Table 5.2). Inconsistent measurements of fish length were previously discussed by Francis (2006a), who recommended chimaera length $\left(L_{\mathrm{C}}\right)$ as an acceptable measurement for total length of Chimaeriformes (fork length for the family Callorhinchidae). While chimaeroids are subject to losing portions of the caudal filament, $L_{\mathrm{C}}$ was found to be a robust measurement to represent chimaeroid length in this study, as only one individual needed to be removed due to tail loss. Body length $\left(L_{\mathrm{BD}}\right)$ can be found in earlier reports (see Inada \& Garrick, 1979), which is only inclusive of the gill opening to upper caudal origin. In the case of Rhinochimaeridae, this measurement can misrepresent the whole animal 
size, where snout length contributes to a large proportion of the total length size of a specimen. Elsewhere, precaudal length $\left(L_{\mathrm{PC}}\right)$ is used in Canada (King \& McPhie, 2013) and Scotland (Neat et al., 2010), and snout-to-vent length $\left(L_{\mathrm{SV}}\right)$ in Japan (Malagrino et al., 1977; Malagrino et al., 1981) and the US (Barnett et al., 2009). Multiple length measurements, or the inclusion of conversion factors, are becoming more frequent in recent publications (Table 5.2). It is therefore recommended that a combination of $L_{\mathrm{C}}$ and $L_{\mathrm{PC}}$ or $L_{\mathrm{SV}}$ are recorded so conversions can be made (Francis, 2006a).

Methods for estimating fecundity in chimaeroids also vary. These have included observations of captive animals (Barnett et al., 2009), or by averaging the mean number of fully developed follicles from wild specimens (Moura et al., 2004). Respectively, these studies concluded with an estimated annual fecundity of 19.5 to 28.9 in Hydrolagus colliei, and a lower fecundity estimate of 6.46 in rabbitfish Chimaera monstrosa L. 1758. In Hydrolagus bemisi and Hydrolagus novaezealandiae, fecundity was estimated at 15 or greater, based on the number of large follicles counted in each individual (McCutcheon, 1980). Horn (1997) postulated $H$. bemisi and H. novaezealandiae may produce 50 eggs per year, presuming these animals have a six month spawning season and produce two eggs on a weekly basis, based on observations of $H$. colliei by Dean (1906). The differences in fecundity estimates may therefore be method, as well as species-specific. We found up to 27 follicles (>10 mm and yellow in colour) in $H$. raleighana and 31 in $R$. pacifica, although it is unknown how many of those follicles would contribute to parturition. Sampling throughout the year is required to determine annual, and lifetime, fecundity (Kyne \& Simpfendorfer, 2007). Nevertheless, fecundity in Chimaeriformes seems to be higher than in other chondrichthyan classes (Barnett et al., 2009), which, for other species, has been estimated as low as 12 for a lifetime fecundity (Kyne \& Simpfendorfer, 2007). A higher fecundity, combined with sperm storage, and enhanced embryonic growth from relatively stable environmental conditions of the deep-sea (Hoff, 2010; Treude et al., 2011), may contribute to a higher reproductive capacity than other chondrichthyans. In part, this may explain why large catches of chimaeroids are still caught in New Zealand (MPI, 2015), with no indication of reduced biomass (Bagley et al., 2013).

This is the first reporting of the reproductive biology of $H$. raleighana and $R$. pacifica, two poorly known deep-sea chimaeroids caught as bycatch in New Zealand deep-sea trawl fisheries. In this study, we have shown that these species are capable of storing sperm, and based on the presence of sexual dimorphism in snout size, we propose 
the snout of $H$. raleighana is a secondary sexual characteristic. Both species matured at a large proportion of their maximum length, suggesting both $H$. raleighana and $R$. pacifica are late maturing fish. In addition, we have reviewed all known literature on holocephalan reproduction to assist with recommendations for future research in this field. Reproductive parameters, such as size-at-maturity and fecundity, contribute to the ecological understanding of a relatively unknown group of deep-sea fish, and have implications for fisheries management of these species. 
Table 5.2. Published and unpublished studies on holocephalan reproduction by species, location, depth, sample size by sex (M: male; F: female), length measurement (SL: standard length; TL: total length; FL: fork length; CL: chimaera length; PCL: precaudal length; BDL: body length; SVL: snout to vent length), length range by sex, measurements to assess maturity by sex (Cl: clasper length; G: gonad mass; OV: oviducal gland width; U: uterus width; H: histology; S: steroid hormone), length-at-maturity $\left(L_{50}\right), L_{50}$ as proportion of maximum length, age at maturity $\left(A_{50}\right)$, and ovarian fecundity. $*$ refers to those studies that have included more than one length measurement.

\begin{tabular}{|c|c|c|c|c|c|c|c|c|c|c|c|c|}
\hline Family & Species & Location & $\begin{array}{l}\text { Depth } \\
\text { range } \\
\text { of } \\
\text { study } \\
\text { (m) }\end{array}$ & $n$ & Length & $\begin{array}{l}\text { Length range } \\
(\mathrm{cm})\end{array}$ & $\begin{array}{l}\text { Maturity } \\
\text { method }\end{array}$ & $L_{50}$ & $\begin{array}{l}L_{50} \text { as \% } \\
\text { max } \\
\text { length }\end{array}$ & $A_{50}$ & $\begin{array}{l}\text { Ovarian } \\
\text { fecundity }\end{array}$ & Source \\
\hline \multirow[t]{12}{*}{ Callorhinchidae } & \multirow{2}{*}{$\begin{array}{l}\text { Callorhinchus } \\
\text { callorhynchus }\end{array}$} & \multirow[t]{2}{*}{ Chile } & \multirow[t]{2}{*}{$>30$} & M: 209 & \multirow[t]{2}{*}{ SL } & M: 20-62 & $\mathrm{M}: \mathrm{Cl}$ & M: 43.7 & M: 0.70 & M: 4-5 & \multirow[t]{2}{*}{-} & \multirow{2}{*}{$\begin{array}{l}\text { Alarcon et al. } \\
\text { (2011) }\end{array}$} \\
\hline & & & & F: 193 & & $\mathrm{~F}: 21-70$ & F: G & F: 50.2 & $F: 0.72$ & F: $6-7$ & & \\
\hline & \multirow{4}{*}{$\begin{array}{l}\text { Callorhinchus } \\
\text { capensis }\end{array}$} & \multirow[t]{2}{*}{ Argentina } & \multirow[t]{2}{*}{$20-130$} & \multirow[t]{2}{*}{123} & \multirow[t]{2}{*}{ SL } & M: 26-55 & $\mathrm{M}: \mathrm{Cl}$ & M: 40 & M: 0.73 & \multirow[t]{2}{*}{-} & \multirow[t]{2}{*}{ - } & \multirow{2}{*}{$\begin{array}{l}\text { Di Giacomo } \\
\text { \& Perier } \\
\text { (1994) }\end{array}$} \\
\hline & & & & & & F: up to 71 & $\mathrm{~F}: \mathrm{G}$ & F: 48 & F: 0.68 & & & \\
\hline & & South & \multirow[t]{2}{*}{ - } & \multirow[t]{2}{*}{173} & \multirow[t]{2}{*}{$\mathrm{TL}^{*}$} & M: 36.1-74 & $\mathrm{M}: \mathrm{Cl}$ & M: 59.3 & M: 0.78 & \multirow[t]{2}{*}{ - } & \multirow{2}{*}{$\begin{array}{l}5.3+/- \\
3.79\end{array}$} & \multirow{2}{*}{$\begin{array}{l}\text { Nibam } \\
(2010)\end{array}$} \\
\hline & & Africa & & & & F: $40.3-85.2$ & F: U, G & $F: 75.4$ & $F: 0.88$ & & & \\
\hline & \multirow{6}{*}{$\begin{array}{l}\text { Callorhinchus } \\
\text { milli }\end{array}$} & South & \multirow[t]{2}{*}{$<40$} & \multirow[t]{2}{*}{578} & \multirow[t]{2}{*}{ FL* } & M: up to 55 & $\mathrm{M}: \mathrm{Cl}, \mathrm{G}$ & M: 43.5 & M: 0.79 & M: 3.3 & \multirow[t]{2}{*}{22} & \multirow{2}{*}{$\begin{array}{l}\text { Freer \& } \\
\text { Griffiths } \\
\text { (1993a \& b) }\end{array}$} \\
\hline & & Africa & & & & F: up to $\sim 58$ & $\mathrm{~F}: \mathrm{OV}, \mathrm{G}$ & F: 49.6 & F: 0.86 & $\mathrm{~F}: 4.2$ & & \\
\hline & & \multirow{2}{*}{$\begin{array}{l}\text { New } \\
\text { Zealand }\end{array}$} & \multirow[t]{2}{*}{$<50$} & \multirow[t]{2}{*}{$1000+$} & \multirow[t]{2}{*}{ FL } & M: up to 76 & $\mathrm{M}: \mathrm{Cl}$ & M: $51 / 52$ & M:0.68 & M:2.9-4.5 & \multirow[t]{2}{*}{ - } & \multirow{2}{*}{$\begin{array}{l}\text { Gorman } \\
(1963) ; \\
\text { Francis } \\
(1997)\end{array}$} \\
\hline & & & & & & F: up to 97 & $\mathrm{~F}: \mathrm{G}$ & $\mathrm{F}: 71$ & F: 0.73 & F: 4.6-6 & & \\
\hline & & Australia & - & M: 467 & FL & - & $\mathrm{M}: \mathrm{Cl}, \mathrm{G}$ & M: 53.9 & M: 0.61 & M: 4/10 & $20-25$ & Bell (2012) \\
\hline & & & & F: 858 & & & $\mathrm{~F}: \mathrm{OV}, \mathrm{U}, \mathrm{G}$ & F: 58.7 & F: 0.58 & F: $5 / 10$ & & \\
\hline Rhinochimaeridae & Harriotta & New & $400-$ & M: 120 & CL & M: 27.4-79.6 & $\mathrm{M}: \mathrm{Cl}$ & M: 62.8 & M: 0.78 & - & 27 & this study \\
\hline & raleighana & Zealand & 1300 & F: 168 & & F: 18.7-90.4 & $\mathrm{F}: \mathrm{OV}, \mathrm{G}$ & F: 75.9 & F: 0.84 & & & \\
\hline & Rhinochimaera & Australia & $500-$ & M: 88 & PCL & - & $\mathrm{M}: \mathrm{Cl}, \mathrm{G}$ & M: 66.8 & M: 0.9 & M: 16 & - & Bell (2012) \\
\hline & pacifica & & 1300 & F: 106 & & & $\mathrm{~F}: \mathrm{OV}, \mathrm{U}, \mathrm{G}$ & F: 84.8 & F: 0.9 & $\mathrm{~F}: 21$ & & \\
\hline & & New & $400-$ & M: 103 & $\mathrm{CL}$ & M: 50.8-123.3 & $\mathrm{M}: \mathrm{Cl}$ & M: 104.7 & M: 0.85 & - & 31 & this study \\
\hline & & Zealand & 1300 & F: 51 & & F: 20.9-139.9 & $\mathrm{F}: \mathrm{OV}, \mathrm{G}$ & $\mathrm{F}: 125.3$ & $F: 0.9$ & & & \\
\hline
\end{tabular}




\begin{tabular}{|c|c|c|c|c|c|c|c|c|c|c|c|c|}
\hline Family & Species & Location & $\begin{array}{l}\text { Depth } \\
\text { range } \\
\text { of } \\
\text { study } \\
\text { (m) }\end{array}$ & $n$ & Length & $\begin{array}{l}\text { Length range } \\
(\mathrm{cm})\end{array}$ & $\begin{array}{l}\text { Maturity } \\
\text { method }\end{array}$ & $L_{50}$ & $\begin{array}{l}L_{50} \text { as \% } \\
\text { max } \\
\text { length }\end{array}$ & $A_{50}$ & $\begin{array}{l}\text { Ovarian } \\
\text { fecundity }\end{array}$ & Source \\
\hline \multirow[t]{28}{*}{ Chimaeridae } & \multirow{2}{*}{$\begin{array}{l}\text { Hydrolagus } \\
\text { bemisi }\end{array}$} & \multirow{2}{*}{$\begin{array}{l}\text { New } \\
\text { Zealand }\end{array}$} & \multirow{2}{*}{$\begin{array}{l}496- \\
801\end{array}$} & M: 48 & \multirow[t]{2}{*}{ BDL* } & M: $18.5-62.7$ & $\mathrm{M}: \mathrm{Cl}$ & M: 44-45 & M: 0.72 & \multirow[t]{2}{*}{ - } & \multirow[t]{2}{*}{$15+$} & \multirow{2}{*}{$\begin{array}{l}\text { McCutcheon } \\
\text { (1980) }\end{array}$} \\
\hline & & & & F: 37 & & F: $16.8-59.8$ & F: G & F: $42-45$ & F: 0.75 & & & \\
\hline & \multirow{4}{*}{$\begin{array}{l}\text { Hydrolagus } \\
\text { colliei }\end{array}$} & \multirow[t]{2}{*}{ Canada } & \multirow[t]{2}{*}{$15-660$} & M: 124 & \multirow[t]{2}{*}{ PCL* } & \multirow[t]{2}{*}{$7.4-49.5$} & $\mathrm{M}: \mathrm{Cl}, \mathrm{G}$ & M: 30.2 & M: 0.75 & M: 12 & \multirow[t]{2}{*}{-} & King \& \\
\hline & & & & F: 151 & & & F: OV, G & F: 39.3 & F: 0.79 & F: 14 & & $\begin{array}{l}\text { McPhie } \\
\text { (2013) }\end{array}$ \\
\hline & & \multirow{2}{*}{$\begin{array}{l}\text { United } \\
\text { States }\end{array}$} & \multirow[t]{2}{*}{ - } & M: 48 & \multirow[t]{2}{*}{ SVL* } & M: 4.6-20.8 & $\mathrm{M}: \mathrm{Cl}$ & M: 15.72 & M: 0.76 & \multirow[t]{2}{*}{ - } & \multirow[t]{2}{*}{$19.5-28.9$} & \multirow{2}{*}{$\begin{array}{l}\text { Barnett et al. } \\
(2009)\end{array}$} \\
\hline & & & & F: 37 & & F: 4.1-28.3 & $\mathrm{F}: \mathrm{OV}, \mathrm{G}, \mathrm{S}$ & F: 20.28 & F: 0.72 & & & \\
\hline & \multirow{2}{*}{$\begin{array}{l}\text { Hydrolagus } \\
\text { barbouri }\end{array}$} & \multirow[t]{2}{*}{ Japan } & \multirow{2}{*}{$\begin{array}{l}250- \\
900\end{array}$} & 1613 & \multirow[t]{2}{*}{ PCL } & M: up to 60 & M: Cl, G & M: 48 & M: 0.8 & \multirow[t]{2}{*}{-} & \multirow[t]{2}{*}{-} & \multirow{2}{*}{$\begin{array}{l}\text { Kokuho et al. } \\
\text { (2003) }\end{array}$} \\
\hline & & & & & & F: up to 68 & F: OV, G & F: 55 & F: 0.81 & & & \\
\hline & \multirow{4}{*}{$\begin{array}{l}\text { Hydrolagus } \\
\text { homonycteris }\end{array}$} & Australia & - & M: 54 & PCL & - & $\mathrm{M}: \mathrm{Cl}, \mathrm{G}$ & M: 59.6 & M: 0.71 & - & - & Bell (2012) \\
\hline & & & & $\mathrm{F}: 23$ & & & $\mathrm{~F}: \mathrm{OV}, \mathrm{U}, \mathrm{G}$ & F: 67.7 & $F: 0.81$ & & & \\
\hline & & New & $400-$ & M: 51 & $\mathrm{CL}$ & M: 65.1-96.3 & $\mathrm{M}: \mathrm{Cl}$ & M: 78.5 & M: 0.82 & - & - & Finucci \\
\hline & & Zealand & 1300 & F: 17 & & F: 50.6-99.8 & F: OV, G & F: 82.8 & F: 0.82 & & & (unpublished) \\
\hline & Hydrolagus & Australia & - & M: 19 & PCL & - & $\mathrm{M}: \mathrm{Cl}, \mathrm{G}$ & M: 52.5 & M: 0.77 & - & - & Bell (2012) \\
\hline & lemures & & & $F: 54$ & & & F:OV,U,G & F: 57.7 & F: 0.68 & & & \\
\hline & Hydrolagus & New & $290-$ & M: 47 & BDL* & M: 15.3-46.1 & $\mathrm{M}: \mathrm{Cl}$ & M:34-35 & M: 0.76 & - & $15+$ & McCutcheon \\
\hline & novaezealandiae & Zealand & 464 & F: 63 & & F: 24.5-55.1 & F: G & $F: 35-38$ & F: 0.69 & & & (1980) \\
\hline & Hydrolagus & Australia & - & M: 144 & PCL & - & $\mathrm{M}: \mathrm{Cl}, \mathrm{G}$ & M: 55.6 & M: 0.82 & M: 16 & - & Bell (2012) \\
\hline & ogilbyi & & & F: 200 & & & $\mathrm{~F}: \mathrm{OV}, \mathrm{U}, \mathrm{G}$ & F: 65.5 & F: 0.81 & F:28(18) & & \\
\hline & Chimaera & New & $400-$ & M: 163 & CL & M: 28.7-103.1 & $\mathrm{M}: \mathrm{Cl}$ & M: 74.9 & M: 0.79 & - & 31 & Finucci \\
\hline & carophila & Zealand & 1300 & F: 58 & & F: 43.3-103.9 & $\mathrm{F}: \mathrm{OV}, \mathrm{G}$ & $F: 82.5$ & F: 0.73 & & & (unpublished) \\
\hline & Chimaera & Australia & $500-$ & M: 88 & PCL & - & $\mathrm{M}: \mathrm{Cl}, \mathrm{G}$ & M: 50.8 & M: 0.65 & M: 13 & - & Bell (2012) \\
\hline & fulva & & 1300 & F: 97 & & & $\mathrm{~F}: \mathrm{OV}, \mathrm{U}, \mathrm{G}$ & F: 62.8 & F: 0.75 & F: 18 & & \\
\hline & Chimaera & Australia & $500-$ & M: 65 & PCL & - & $\mathrm{M}: \mathrm{Cl}, \mathrm{G}$ & M: 65.9 & M: 0.66 & M: 17 & - & Bell (2012) \\
\hline & lignaria & & 1300 & F: 45 & & & $\mathrm{~F}: \mathrm{OV}, \mathrm{U}, \mathrm{G}$ & F: 88.6 & F: 0.77 & $\mathrm{~F}: 33(27)$ & & \\
\hline & Chimaera & Portugal & $400-$ & 305 & PCL & M: 9.1-50.3 & $\mathrm{M}: \mathrm{Cl}$ & M: 40.2 & M: 0.80 & - & $6.46+/-$ & Moura et al. \\
\hline & monstrosa & & 850 & & & F: $8.1-57.1$ & $\mathrm{~F}: \mathrm{OV}, \mathrm{G}, \mathrm{H}$ & $F: 45.9$ & F: 0.80 & & 2.32 & (2004) \\
\hline & Chimaera & Japan & $100-$ & M: 164 & SVL & M: up to $\sim 70$ & $\mathrm{M}: \mathrm{Cl}, \mathrm{G}$ & M: 51 & M: 0.73 & - & - & Malagrino $e t$ \\
\hline & phantasma & & 600 & F: 244 & & F: up to $\sim 90$ & F: G & $\mathrm{F}: 63$ & F: 0.7 & & & $\begin{array}{l}\text { al. } \\
(1977 ; 1981)\end{array}$ \\
\hline
\end{tabular}




\section{CHAPTER VI}

First observations of the biology of two rarely observed deep-sea chimaeras, Chimaera carophila and Hydrolagus homonycteris 


\subsection{Abstract}

Chimaera carophila and Hydrolagus homonycteris are two deep-sea chimaerids rarely caught in the waters off New Zealand. Little is known of their biology or ecology. Chimaera carophila $(n=183)$ and H. homonycteris $(n=79)$ were collected from research trawl catches and commercial fishery catches around New Zealand at depths between 400 and $1300 \mathrm{~m}$, between 2014 and 2016. Additional preserved specimens of both species ( $n$ $=58$ ) from museum collections were analysed for size, sex, and maturity. External assessment of male claspers, and a combination of internal assessments of female gonad weight and oviducal gland width, were used to determine maturity. For both species, length at first maturity was $0 \cdot 70-0 \cdot 82$ of their maximum observed length, with females maturing at a larger size. Length-at-maturity for C. carophila (length range: 28·7-103.9 cm chimaera length, $L c)$ was estimated at $72.5 \mathrm{~cm} L c$ for males $(n=163)$ and $82.5 L \mathrm{c}$ for females ( $n=58)$. In $H$. homonycteris, length-at-maturity (length range: $78 \cdot 6-99 \cdot 8 \mathrm{~cm} \mathrm{Lc}$ ) was estimated at $79 \cdot 1 \mathrm{~cm} L \mathrm{c}$ for males $(n=51)$ and $80 \cdot 1 \mathrm{~cm} L \mathrm{c}$ for females $(n=17)$. Ovarian fecundity was up to 31 for $C$. carophila, and sperm storage was confirmed in the oviducal gland of this species. Both species predated on benthic invertebrates. Some $C$. carophila and $H$. homonycteris inhabit depths beyond most current fisheries, but both species appear to be relatively rare and have reproductive parameters characteristic of low productivity, which may make these species vulnerable to population decline if mortality was to increase in the future. 


\subsection{Introduction}

The chimaerids (order Chimaeriformes) are a relatively small group of mainly deep-sea chondrichthyan fishes. The order is currently comprised of 49 recognized species across three distinct families, the Callorhinchidae (plow-nose chimaeras), Rhinochimaeridae (long-nose chimaeras), and Chimaeridae (short-nose chimaeras) (Didier et al., 2012; Weigmann, 2016). New species continue to be described (e.g. Luchetti et al., 2011; Angulo et al., 2014; Kemper et al., 2014), and since 2002, half of these new species have come from the southern Pacific Ocean around New Zealand and Australia (Last \& Stevens, 2009; Didier et al., 2012). The most speciose family, the Chimaeridae (38 species), also referred to as ratfish or ghost sharks, includes two genera: Chimaera (16 species) and Hydrolagus (22 species) (Weigmann, 2016). As an order, chimaerids are widespread geographically, but individual species are known to have rather restricted ranges, and most are described as deep-sea (Didier et al., 2012). At this time, chimaerids are thought to be at a relatively low risk of extinction compared to other chondrichthyan families, however, biological information on chimaerids is sparse and many species remain data deficient (Dulvy et al., 2014). Many deep-sea fishes are known for their low productivity (Morato et al., 2006a; Rigby \& Simpfendorfer, 2015), and as fisheries continue to expand into deeper waters (Watson \& Morato, 2013), chimaerids could become increasingly threatened from fishing-induced mortality (Simpfendorfer \& Kyne, 2009).

Chimaera carophila Kemper, Ebert, Naylor \& Didier, 2014 and Hydrolagus homonycteris Didier, 2008 are two deep-sea chimaerids that are occasionally caught in the waters off New Zealand. The former was previously thought to be Chimaera macrospina Didier, Last \& White, 2008 (or a conspecific) (McMillan et al., 2011), until it was described in 2014 as an species endemic to New Zealand waters (Kemper et al., 2014). Chimaera carophila, the brown chimaera, is known from central and southern New Zealand waters and has been caught at depths between 846 and $1350 \mathrm{~m}$ (Roberts et al., 2015). Its geographic distribution, uniform pale-brown coloration, and a combination of morphological characters separate $C$. carophila from its congeners (Kemper et al., 2014), whereas Hydrolagus homonycteris, the black ghost shark, is distinguishable by its purplish-black fins and deciduous, patchy black skin (Didier, 2008). Hydrolagus homonycteris is thought to be widespread around New Zealand, and has also been caught off southeast Australia, from southern New South Wales to Victoria and Tasmania, 
between depths of 866 to 1447 m (Didier, 2008; Roberts et al., 2015). In addition, research surveys have collected $H$. homonycteris from seamounts and slopes of the northern Tasman and southern Coral Seas, at depths between 1051 to $1345 \mathrm{~m}$, suggesting these locations as a potential trans-Tasman link between the slope waters of New Zealand and southeast Australia (Zintzen et al., 2011).

There are no age or reproductive data, abundance indices, or population structure information for either C. carophila or H. homonycteris, and few data on their biology, or the impacts of fishing. The International Union for the Conservation of Nature (IUCN) Red List of Threated Species currently lists H. homonycteris as Least Concern (Theiss et al., 2015) while C. carophila has yet to be evaluated. Both C. carophila and $H$. homonycteris have been measured to lengths of about $105 \mathrm{~cm}$ chimaera length $\left(L_{\mathrm{C}}\right.$, tip of the snout to the posterior edge of the supracaudal fin, excluding the caudal filament) (McMillan et al., 2011). Like all chimaerids, they are oviparous (Last \& Stevens, 2009). Population sizes in New Zealand waters have been classified as relatively small for $C$. carophila and moderate for H. homonycteris based on the scale of catches (Ford et al., 2015). Estimated catches of both species in New Zealand are low (less than 5 tonnes of each species reported for the five year period 2008-09 to 2012-13; Ford et al., 2015), where they are caught in both deep-sea trawl and longline fisheries (Francis, 2015). The potential impact of fishing has been classified as moderate for C. carophila and low for $H$. homonycteris, largely due to relatively little fishing taking place beyond $800 \mathrm{~m}$ depth (Ford et al., 2015). Outside of New Zealand waters, H. homonycteris was once caught commonly as bycatch in the orange roughy Hoplostethus atlanticus target fishery on the South Tasman Rise (Last \& Stevens 2009), but this fishery closed in 2007 (Patterson et al., 2016). Hydrolagus homonycteris has not been reported as bycatch in the New Zealand orange roughy fishery (Ballara, 2015).

The objective of this study was to establish some basic life history parameters of C. carophila and H. homonycteris, which are essential for better estimation of the risk the face from fisheries, and for successful fisheries and conservation management. In addition, this study aims to determine if these species possess similar biological characteristics to other deep-sea chondrichthyan species (Simpfendorfer \& Kyne, 2009; Rigby \& Simpfendorfer, 2015). 


\subsection{Methods}

\subsubsection{Specimen collection and biological data}

Chimaera carophila and H. homonycteris were collected by RV Tangaroa in January of 2014 and 2016 from the continental shelf and slope of Chatham Rise off New Zealand. Trawl surveys were stratified-random, with a primary objective to provide relative abundance indices of important commercial fishes (see O'Driscoll et al., 2011). Sampling strata were defined by location and depth, and fishing occurred on trawlable habitats at depths of 200-1300 m. A full-wing bottom trawl with a $60 \mathrm{~mm}$ codend mesh was towed at each station for 3 nautical miles $(5 \cdot 6 \mathrm{~km})$, at a speed of 3.5 knots $(6 \cdot 5$ $\mathrm{km} / \mathrm{h}$ ). All fish caught were frozen whole at sea and brought back to the laboratory for analyses. Additional samples of $C$. carophila were obtained from within the New Zealand Exclusive Economic Zone (EEZ) by on-board scientific observers of the Ministry for Primary Industries (MPI) during commercial trawl fishing operations in 2015.

In all specimens, total mass $\left(M_{\mathrm{T}}\right)$, chimaera length ( $L \mathrm{c}$ - tip of the snout to the posterior edge of the supracaudal fin, excluding the caudal filament) and snout to vent length $\left(L_{\mathrm{SV}}\right)$ were recorded following Francis (2006a) (Fig. 6.1). Sex was determined externally by the presence (males) or absence (females) of claspers. The mass of the liver and gonads was recorded, and the oviducal gland, uterus (in females) and testes (in males) were measured for maximum length and width. The number of vitellogenic follicles (> 10 mm diameter, M.P. Francis \& W.S. Lyon, NIWA, pers. comm.) and the largest ovarian follicle diameter were recorded for each ovary. Ovarian fecundity was determined by the number of follicles yellow in colour and greater than $10 \mathrm{~mm}$ diameter in mature females. Lengths were measured to the nearest $\mathrm{mm}$ and all mass to the nearest $0 \cdot 1$ gram. 


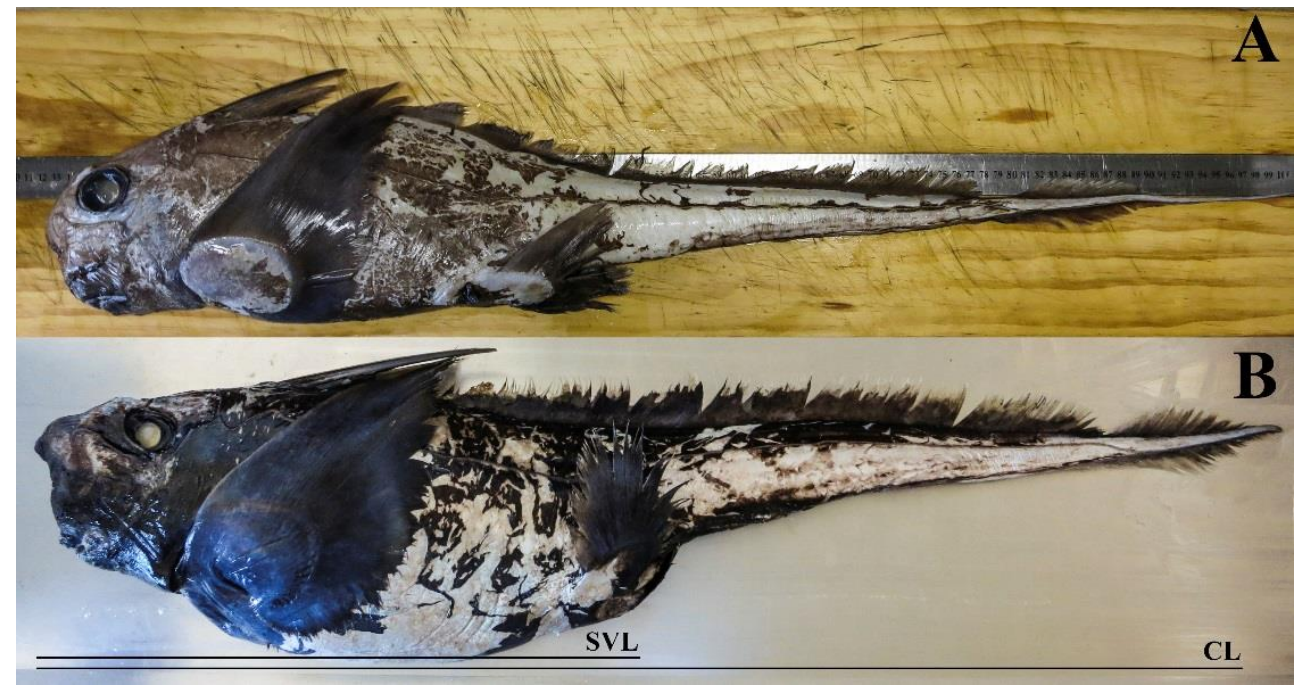

Figure 6.1 Adult (A) male Chimaera carophila $(78 \mathrm{~cm} \mathrm{Lc)} \mathrm{and} \mathrm{(B)} \mathrm{female} \mathrm{Hydrolagus}$ homonycteris $(86 \mathrm{~cm} \mathrm{Lc).} \mathrm{Patchy} \mathrm{colouration} \mathrm{of} \mathrm{both} \mathrm{specimens} \mathrm{due} \mathrm{to} \mathrm{skin} \mathrm{damage} \mathrm{from}$ trawl.

Maturity stage for each specimen was defined by macroscopic evaluation of reproductive organ development. Maturity was classified into one of six maturity stages based on New Zealand research survey protocol by M.P. Francis \& W.S. Lyon (NIWA, pers. comm.) with adaptations to stage descriptions following Stehmann (2002), Walker (2005), Barnett et al. (2009) and ICES (2010) (Table 5.1). In males, inner clasper length $\left(L_{\mathrm{CL}}\right)$ was measured, and the degree of calcification of claspers and development of the epididymis were also noted, as defined in Table 5.1. As both right and left sides of the reproductive system are functional in males and females (Wourms, 1977; Malagrino et al., 1981), left and right measurements were made and compared. In males, inner clasper length was standardized by chimaera length $\left(L_{\mathrm{CL}} / L \mathrm{c}\right)$. Measurements of testes length and seminal vesicle width in males, and oviducal gland width and uterus width in females, were similarly standardized by chimaera length. Gonado-somatic index $\left(I_{\mathrm{G}}\right)$ was calculated as the gonad mass divided by $M_{\mathrm{T}}$. The onset of maturity was assumed to be represented by an abrupt increase in these parameters. To test if the ratio of males to females varied significantly from 1:1, a $\chi^{2}$ goodness of fit (Sokal \& Rohlf, 1987) was used, while ANCOVA was used to compare relationships between $L c$ and $M_{\mathrm{T}}$ and $L c$ and $L_{\mathrm{SV}}$ between sexes. Paired-sample $t$-tests were used to evaluate differences between measurements of the right and left reproductive tracts of males and females (Zar, 1996), with significance for all tests accepted at $P<0 \cdot 05$. 
Preserved specimens deposited at the Museum of New Zealand Te Papa Tongarewa (NMNZ) were also examined for length and reproductive parameters. Specimens of both species were collected across the New Zealand EEZ between 1981 and 2010. An additional six specimens of $H$. homonycteris were recorded from the Challenger Plateau, Lord Howe Rise, and Norfolk Ridge outside of the New Zealand EEZ.

Commonly used preservation methodology including fixative (formalin) and preservative (ethyl alcohol) have been found to shrink fish proportionally along multidimensional planes (Gaston et al., 2013). Previously studies on the effects of preservation on elasmobranch and teleost body size have found a decrease in total body length from $2 \%$ (Francis, 2006b) to nearly 6\% (Jones \& Geen, 1977; Jawad, 2003; Ebert, 2005; Gaston et $a l ., 2013)$. To address shrinkage, an increase of $4 \%$ was added to external measurements of these specimens.

Historical information on catch and biological data (length, mass, sex, and maturity assessment) of $C$. carophila and $H$. homonycteris caught during previous survey trawls were also accessed from the New Zealand Ministry for Primary Industries research trawl database. Bottom trawl catches dating back to 1982, and made across the New Zealand EEZ between $38.69^{\circ}-44.63^{\circ} \mathrm{S}$ and $176 \cdot 30^{\circ} \mathrm{E}-173.94^{\circ} \mathrm{W}$, provided 772 and 413 catch records of $C$. carophila and H. homonycteris, respectively. Of these, $163 C$. carophila and $68 \mathrm{H}$. homonycteris were also sampled for biological data, of which none were sampled for maturity stage before 2010 .

Size-at-maturity $\left(L_{50}\right)$ was calculated for males and females separately by fitting a logistic model to the proportion of mature individuals at length using non-linear least squares. Maturity data (immature 0 , mature 1) were aggregated by $5 \mathrm{~cm} L \mathrm{c}$ size class intervals for each sex. The logistic function had the form $Y_{a}=\left[1+19^{\left(L_{50}-a\right) / L} \text { to95 }\right]^{-1}$, where $Y_{a}$ is the proportion of mature individuals in size class $a$, and $L_{50}$ and $L_{\text {to95 }}$ are parameters. Confidence intervals (95\% C.I.) for $L_{50}$ were calculated using a non-parametric bootstrap (1000 iterations) (Efron, 1981). All statistical analyses were carried out using R (R Core Team, 2012).

\subsubsection{Sperm storage}

The presence of sperm storage in females was evaluated following the descriptions and methods of Pratt \& Tanaka (1994), Smith et al. (2004), and Finucci et al. (2016b). Oviducal glands of mature specimens were removed and fixed in $10 \%$ neutral 
buffered formalin. Samples were cut on the coronal plane and placed in biopsy cassettes. Tissues were dehydrated through a series of ascending alcohol concentrations $(70-$ $100 \%$ ) and infiltrated with paraffin wax in a Tissue Tek VIP 5 Processor before being embedded into wax blocks. Sections of embedded tissue were sliced $4 \mu \mathrm{m}$ thick with a Leica microtome, mounted onto glass slides, and stained in Harris's haematoxylin and eosin (H\&E). Samples were photographed on a Leica DM LB light microscope with a Canon EOS 70D camera attachment.

\subsubsection{Diet}

Digestive tracts were removed and dissected. Contents of digestive tracts were rinsed with water into a petri dish. Prey items were then identified to the lowest possible taxon under a stereo microscope. For each prey taxon, the number of individual prey was estimated, and wet weight recorded to the nearest $0.01 \mathrm{~g}$ after removal of surface water by blotting paper. A fragmented prey count was based on the number of eyes, heads, mouth parts, tails or telsons, paired otoliths or other anatomical parts traceable to a single specimen. For the diet analysis, prey items were aggregated into taxonomic categories. The contribution of different prey items to the diet was determined by the frequency of occurrence $(F)$, mass $(W)$, and numerical importance $(N)$ (Hyslop, 1980). Unidentifiable prey, abiotic items, as well as parasites found in the digestive tract, were excluded from calculations for $W$ and $N$. 


\subsection{Results}

\subsubsection{Chimaera carophila distribution}

Combining all available information, Chimaera carophila had been caught more frequently and over a wider area than Hydrolagus homonycteris (Fig. 6.2). Most catch records of $C$. carophila were from Chatham Rise and Hikurangi Trench [Fig. 6.2(A)]. The northernmost record from within the New Zealand EEZ was from the western tip of the North Island $\left(34.5^{\circ} \mathrm{S}, 171 \cdot 7^{\circ} \mathrm{E}\right)$ at a depth of $1051 \mathrm{~m}$, while the most southern record was documented on Campbell Plateau $\left(54 \cdot 2^{\circ} \mathrm{S}, 167 \cdot 6^{\circ} \mathrm{E}\right)$ at $987 \cdot 5 \mathrm{~m}$. The largest catch record was 44 individuals, although most fish were caught individually (62\%) and $93 \%$ of catches were five or fewer fish. Depth of captures ranged from 375 to $1525 \mathrm{~m}$, with fish most frequently captured between 1000 and $1200 \mathrm{~m}($ mean $=1091 \mathrm{~m}$, S.E. $=5 \cdot 0)$ [Fig. 3(A)]. The proportion of survey trawls that caught $C$. carophila declined at depths greater than $1100 \mathrm{~m}$. Juvenile specimens of both sexes were more frequently caught with increasing depth, and there was overlap in depth distribution of mature males and females [Fig. 6.3(C)].

\subsubsection{Hydrolagus homonycteris distribution}

Most catch records for $H$. homonycteris were from eastern Chatham Rise and Hikurangi Trench, with additional records along the Challenger Plateau and Norfolk Ridge. Few catch records existed south of Chatham Rise, with two occurrences documented on the northwest Campbell Plateau (southernmost capture at $48 \cdot 2^{\circ} \mathrm{S}$, $165 \cdot 2^{\circ} \mathrm{E}$ at $1055 \mathrm{~m}$ ) [Fig. 6.2(B)]. The northernmost record from within the New Zealand EEZ was also from the western tip of the North Island $\left(35^{\circ} \mathrm{S}, 171 \cdot 8^{\circ} \mathrm{E}\right)$ at a depth of 1102 m. Most specimens were caught as individuals (62\%), and all but one catch (97\%) were five or fewer fish (one catch of eight). Depth of captures ranged from 612 to $1517 \mathrm{~m}$, with fish most frequently captured between depths of 1100 to $1200 \mathrm{~m}$ (mean $=1227 \mathrm{~m}$, S.E. $=$ 6.9) [Fig. 6.3(B)]. There were very few records of $H$. homonycteris in depths shallower than $1000 \mathrm{~m}(n=20)$, despite high sampling effort. Only males of lengths $<65 \mathrm{~cm} \mathrm{Lc}$ were captured at depths shallower than $1000 \mathrm{~m}$, and females $<70 \mathrm{~cm} \mathrm{Lc}$ caught at depths greater than $1300 \mathrm{~m}$. Mature males were most frequently captured, and there was overlap in catch depth distribution of sex and size classes at depths $>1100 \mathrm{~m}$ [Fig. 6.3(D)]. 


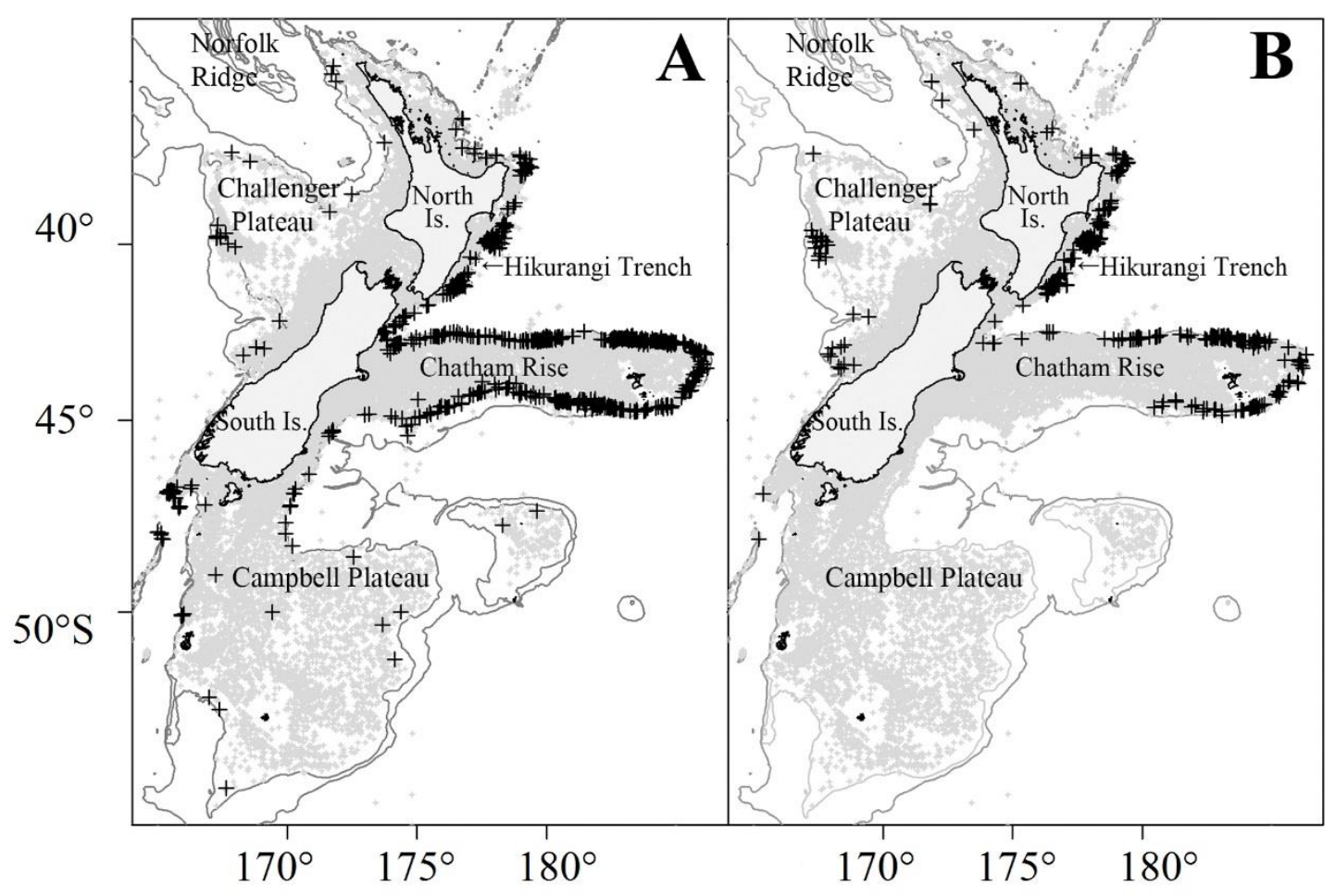

Figure 6.2 Distribution of deep-sea trawl-survey stations $(\square, n=23245)$ and catch-records of (A) Chimaera carophila $(+, n=781)$ and (B) Hydrolagus homonycteris $(+, n=415)$ within New Zealand waters. 

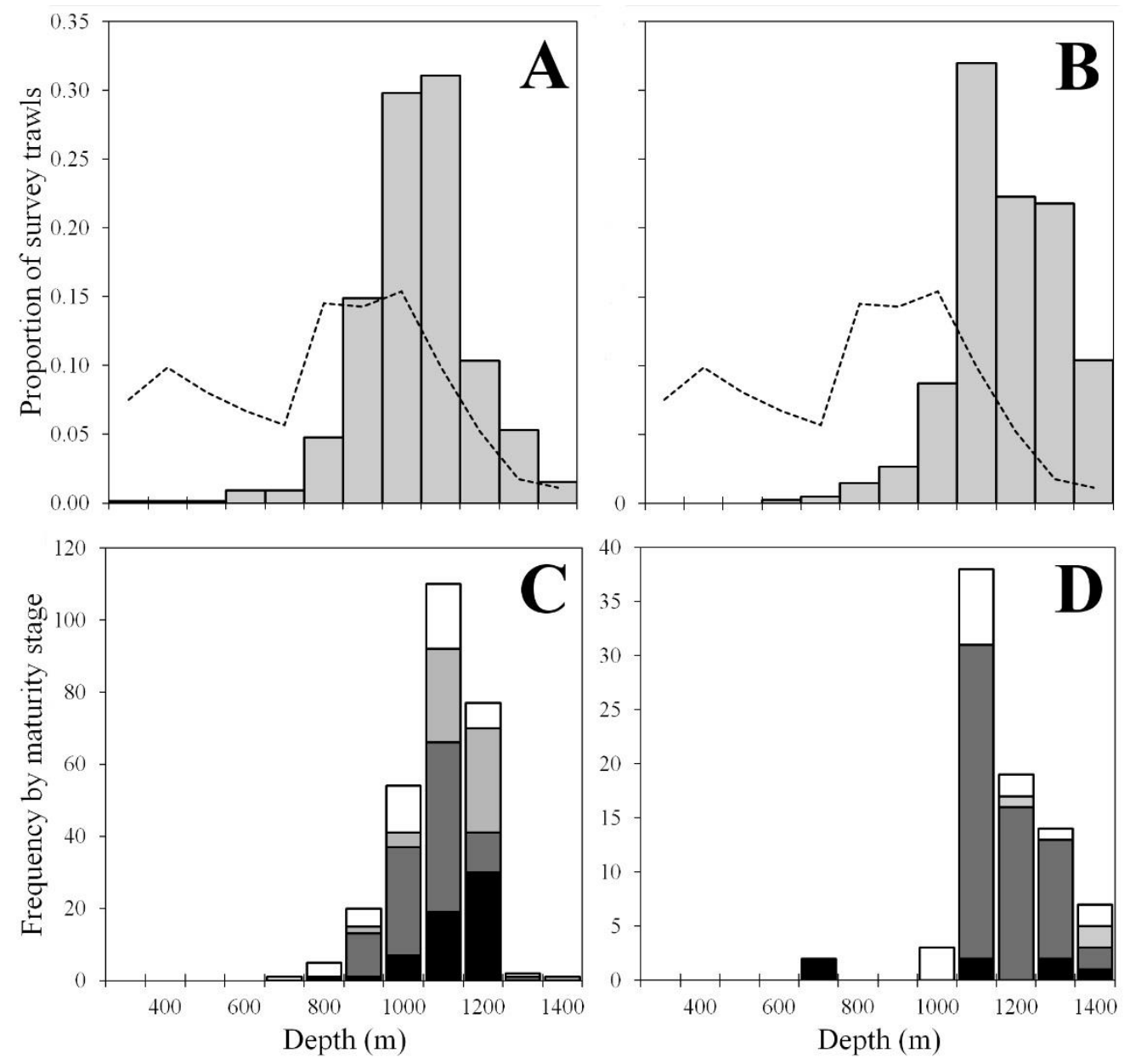

Figure 6.3 Proportion of survey trawls conducted by depth class $(\cdots, n=23245)$, and proportion of survey trawls that captured (A) Chimaera carophila and (B) Hydrolagus homonycteris by depth class ( $\square, n=781$ and $n=415$ ); and frequency of trawl survey captures of (C) Chimaera carophila and (D) Hydrolagus homonycteris by sex and maturity stage: $\square$, immature male; $\square$, mature male; $\square$, immature female; $\square$, mature female. 


\subsubsection{Chimaera carophila specimen size range and length-mass relationship}

To describe species biology, a total of 45 specimens of Chimaera carophila were collected and dissected between 2014 and 2016: 14 (7 males, 7 females) were obtained from survey trawls on Chatham Rise, and 6 (4 males, 2 females) were obtained from the MPI observer program. The additional 25 samples (length range: 22.0-83.0 cm Lc; 16 male, 9 female) were examined from NMNZ museum collections. Biological data extracted for 163 specimens from historical survey trawl catches included 105 males and 58 females, giving a total data set of 208 individuals (132 males, 76 females). Males ranged from $28 \cdot 7-103 \cdot 1 \mathrm{~cm} L \mathrm{c}($ median $=79 \cdot 7 \mathrm{~cm} L \mathrm{c})$ and females from $43.3-103.9 \mathrm{~cm}$ $L \mathrm{c}($ median $=82 \cdot 1 \mathrm{~cm} \mathrm{Lc})$ [Fig. 6.4(A)]. $M_{\mathrm{T}}$ varied from 300 to $4805 \mathrm{~g}$ and 430 to $4829 \mathrm{~g}$ in males and females, respectively. Males and females reached similar lengths, although females were more abundant in the largest size classes. Overall, males were more abundant than females in the sample $\left(1 \cdot 7: 1, \chi^{2}=9 \cdot 6\right.$, d.f. $\left.=1, P<0 \cdot 05\right)$. The relationship between $L c$ and $M_{\mathrm{T}}$ by sex was described by $W=0 \cdot 16 \times L c^{2.18}\left(n=113, R^{2}=0 \cdot 85\right)$ for males and $W=0.04 \times L c^{2.52}\left(n=60, R^{2}=0.93\right)$ for females. Significant differences between the sexes were found in the relationship between $L \mathrm{c}$ and $M_{\mathrm{T}}\left(\mathrm{ANCOVA}, F_{1,175}=\right.$ $30 \cdot 2, P<0 \cdot 05)$. No significant difference was found between the sexes in the $L \mathrm{c}$ to $L_{\mathrm{SV}}$ relationship (ANCOVA, $F_{1,41}=0 \cdot 05, P>0 \cdot 05$ ), which was described by $L_{\mathrm{SV}}=0.34 L \mathrm{c}-$ $4 \cdot 87\left(R^{2}=0 \cdot 73\right)$. 
For Hydrolagus homonycteris, 11 (10 males, 1 female) were obtained from the survey trawls on Chatham Rise. Thirty three samples (length range: 22.0-93.5 cm Lc; 19 male, 14 female) were examined from museum collections. Biological data for 68 specimens measured from historical survey trawls included 51 males and 17 females, for a total data set of 112 individuals (80 males, 32 females). Length ranged from $65 \cdot 1-96 \cdot 3$ $\mathrm{cm} L \mathrm{c}($ median $=87 \mathrm{~cm} L \mathrm{c})$ in males and $50.6-99.8 \mathrm{~cm} L \mathrm{c}($ median $=91.8 \mathrm{~cm} L \mathrm{c})$ in females [Fig. 6.4(B)]. Additional specimens caught by research trawl surveys were measured at up to $110.0 \mathrm{~cm} L \mathrm{c}$, but sex for these individuals was not recorded. $M_{\mathrm{T}}$ ranged from 1320-3330 $\mathrm{g}$ and 652-4310 $\mathrm{g}$ for males and females, respectively. Males and females reached similar lengths, although females were more abundant in the largest size classes. Overall, males were much more abundant than females in the sample $\left(2 \cdot 5: 1, \chi^{2}=13 \cdot 1\right.$, d.f. $=1, P<0.05)$. The relationship between $L \mathrm{c}$ and $M_{\mathrm{T}}$ was described by $W=0.98 \times L \mathrm{c}$ $1.78\left(n=61, R^{2}=0.77\right)$ for males and $W=0.11 \times L c^{2.29}\left(n=18, R^{2}=0.93\right)$ for females. Significant differences between the sexes were found in the relationship between $L c$ and $M_{\mathrm{T}}\left(\mathrm{ANCOVA}, F_{1,73}=8 \cdot 85, P<0 \cdot 05\right)$. No significant difference was found between the sexes in the $L c$ to $L_{\mathrm{SV}}$ relationship (ANCOVA, $F_{1,39}=2 \cdot 76, P>0 \cdot 05$ ), which was described by $L_{\mathrm{SV}}=0 \cdot 38 L \mathrm{c}-2 \cdot 15\left(R^{2}=0 \cdot 86\right)$. 

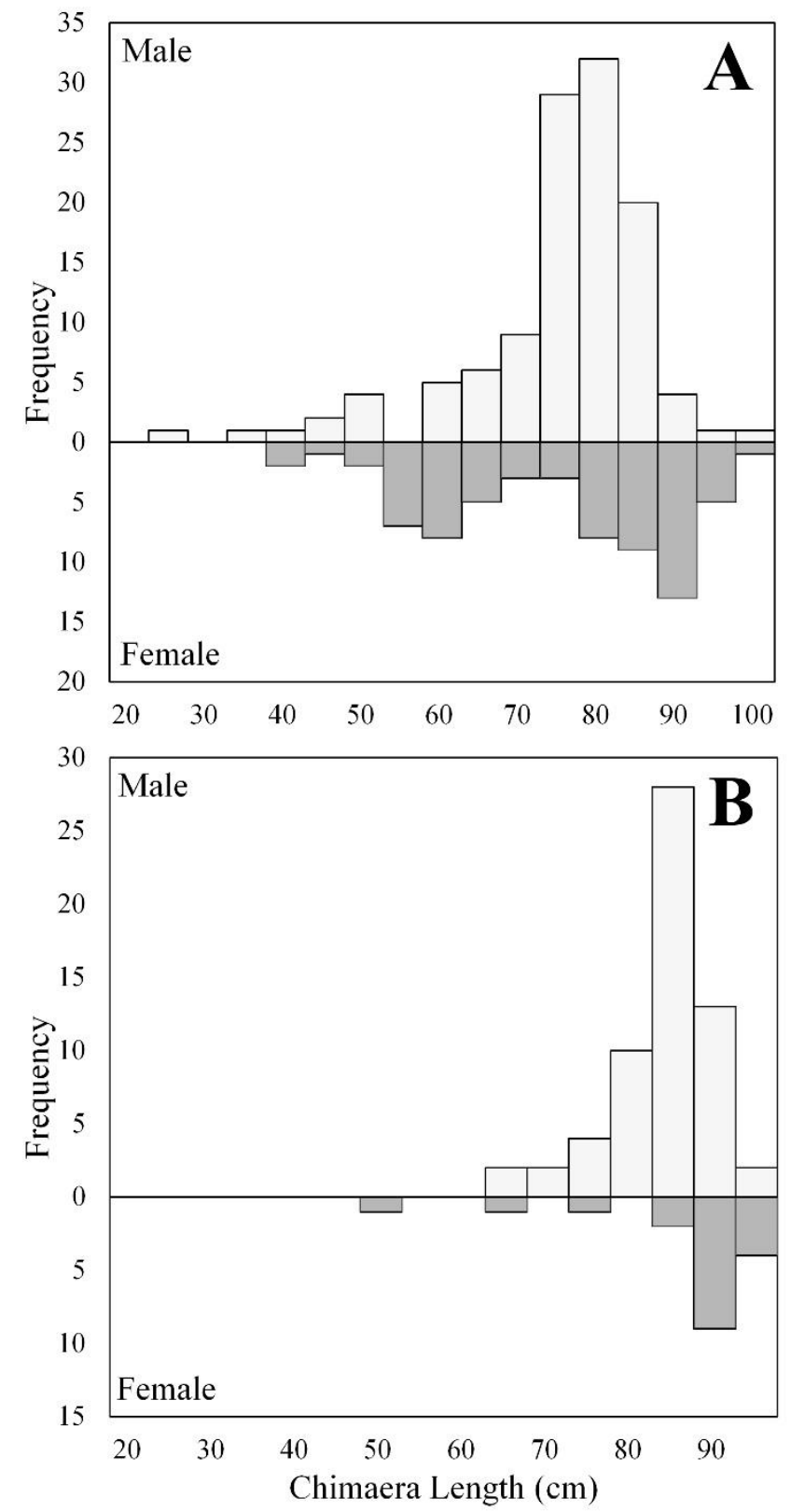

Figure 6.4 Chimaera length $(L c)$-frequency distributions by sex $[(\square)$ male and ( $\square)$ female] for trawl survey specimens of (A) Chimaera carophila $(n=183)$ and (B) Hydrolagus homonycteris $(n=79)$. 


\subsubsection{Size-at-maturity}

Based on the macroscopic assessments of dissected individuals and museum specimens, in Chimaera carophila, the size-at-maturity parameter $\left(L_{50}\right)$ was estimated at $72 \cdot 5 \mathrm{~cm} \mathrm{Lc}(95 \%$ C.I. $=67 \cdot 7-75 \cdot 1 \mathrm{~cm})$ for males and $82 \cdot 5 \mathrm{~cm} \mathrm{Lc}(95 \%$ C.I. $=77 \cdot 3-93 \cdot 5$ $\mathrm{cm}$ ) for females [Fig. 6.5(A)]. Maturity took place at 0.70 and 0.80 of the maximum observed Lc for males and females, respectively. Size-at-maturity for Hydrolagus homonycteris was $79 \cdot 1 \mathrm{~cm} \mathrm{Lc}(95 \%$ C.I. $=76 \cdot 0-81 \cdot 1 \mathrm{~cm})$ for males and $80 \cdot 1 \mathrm{~cm} \mathrm{Lc}(95 \%$ C.I. $=74 \cdot 6-87 \cdot 3 \mathrm{~cm}$ ) for females [Fig. 6.5(B)] and was equivalent to $0 \cdot 82$ and $0 \cdot 80$ of the maximum observed $L c$ for males and females, respectively.

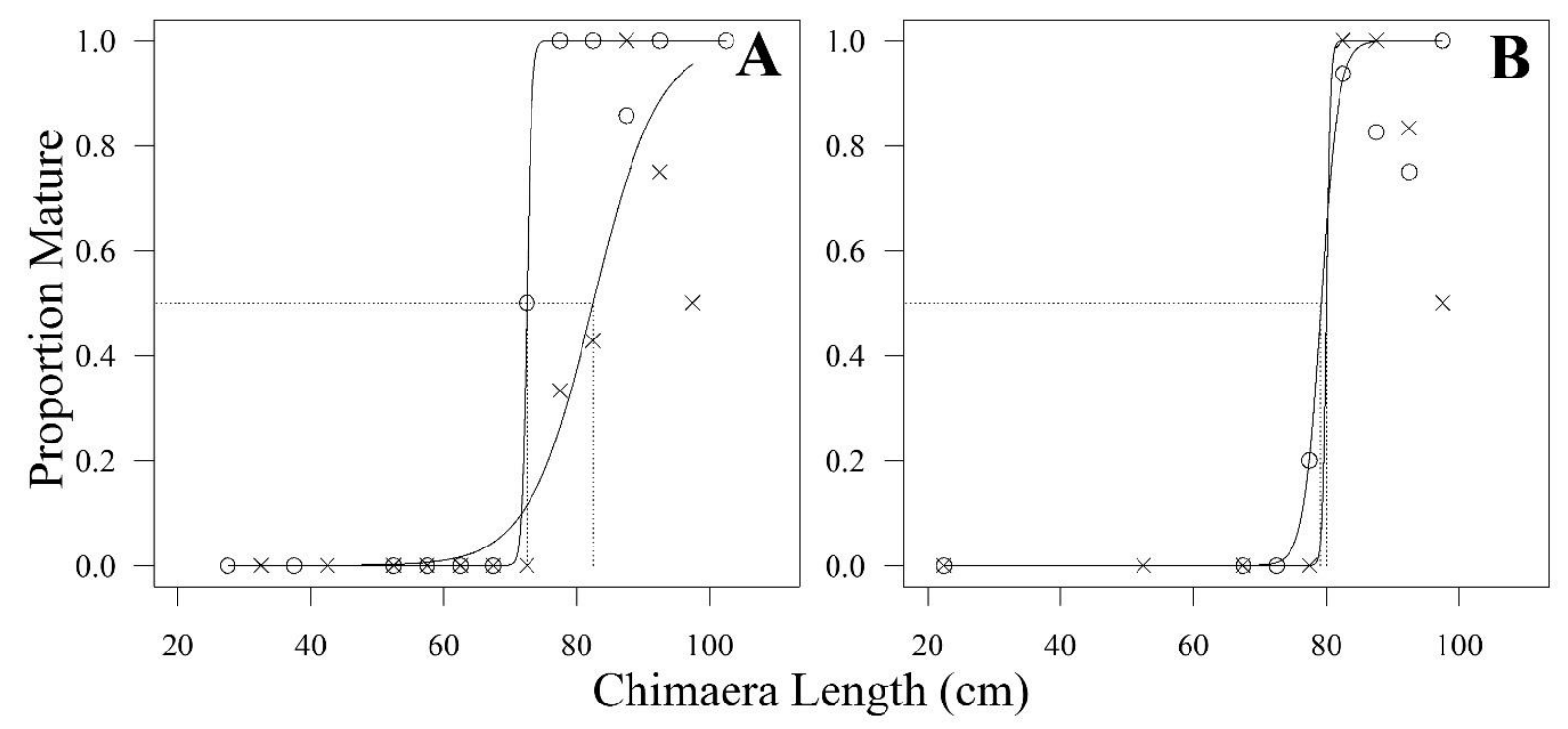

Figure 6.5 Logistic model fitted total length $(L c)$-maturity ogive for (A) Chimaera carophila and (B) Hydrolagus homonycteris, with the observed proportion of mature (०) males and $(\times)$ females in each size interval. $\vdots, L c$ at $50 \%\left(L_{50}\right)$ determined from fitted logistic curves. 


\subsubsection{Chimaera carophila reproductive characteristics}

Of the Chimaera carophila dissected, 22\% $(n=6)$ of the males were classified in early maturity stages (immature or maturing) and 78\% $(n=21)$ were mature. The smallest mature male individual was $75.4 \mathrm{~cm} L \mathrm{c}$, while the largest immature was $85.0 \mathrm{~cm} L \mathrm{c}$. There was no significant difference between the left and right $L_{\mathrm{CL}}(t$-test, d.f. $=26, P>$ $0 \cdot 05)$ or the left and right testis mass ( $t$-test, d.f. $=10, P>0 \cdot 05)$. Gonad mass ranged from 0.01 to $0.03 \mathrm{~g}$ in immature males and from 12.41 to $28.03 \mathrm{~g}$ in mature males. An abrupt increase in standardized $L_{\mathrm{CL}}(n=37)$, observed at approximately $73.0 \mathrm{~cm} L \mathrm{c}$, corresponded well with the onset of male maturity as evaluated by macroscopic staging $\left(L_{50}\right)$ [Fig. 6.6(A)].

Half $(n=9)$ of the female $C$. carophila were macroscopically classified as immature or maturing, and half $(n=9)$ as mature. The length of the smallest mature female specimen was $80.7 \mathrm{~cm} L \mathrm{c}$ and the largest immature was $99.0 \mathrm{~cm} L \mathrm{c}$. There was no difference between left and right oviducal gland width ( $t$-test, d.f. $=6, P>0.05$ ) or uterus width ( $t$-test, d.f. $=8, P>0 \cdot 05)$. An abrupt increase in oviducal gland width $(n=13)$ [Fig. 6.7(A)], as well as uterus gland width $(n=16)$ [Fig. 6.7(B)], corresponded with the onset of female maturity as evaluated macroscopically $\left(L_{50}\right)$. Three mature females were found with ovarian fecundity counts of up to 31 mature follicles (14 in left, 17 in right).

Diameter of the largest follicle measured was $0 \cdot 12-4 \cdot 67 \mathrm{~cm}($ mean \pm S.D. $=1 \cdot 67 \pm 1 \cdot 81)$. No gravid females were observed. Sperm storage tubules (SSTs) and sperm bundles were identified in the terminal zone of the oviducal gland of two mature individuals (Fig. 6.8).

\subsubsection{Hydrolagus homonycteris reproductive characteristics}

Of the H. homonycteris dissected, 24\% $(n=7)$ of the males were classified in early maturity stages (immature or maturing) and 76\% $(n=22)$ were mature. The smallest mature male specimen was $79.8 \mathrm{~cm} L \mathrm{c}$ and the largest immature was $92.3 \mathrm{~cm} L \mathrm{c}$. There was no significant difference between the left and right $L_{\mathrm{CL}}(t$-test, d.f. $=26, P>0.05)$ or the left and right testis mass ( $t$-test, d.f. $=7, P>0.05$ ). Gonads weighed up to $11.49 \mathrm{~g}$ in mature males. An abrupt increase in standardized $L_{\mathrm{CL}}(n=46)$, observed at approximately $78.0 \mathrm{~cm} L \mathrm{c}$, corresponded well with the onset of male maturity as evaluated macroscopically $\left(L_{50}\right)$ [Fig. 6.6(B)]. 
Among female $H$. homonycteris, $20 \%(n=3)$ were classified as immature or maturing and $80 \%(n=12)$ as mature. The length of the smallest mature female specimen was $86.1 \mathrm{~cm} L \mathrm{c}$ and the largest immature was $96.5 \mathrm{~cm} L \mathrm{c}$. There was no difference between left and right oviducal gland width $(t$-test, d.f. $=6, P>0.05)$ or uterus width $(t$ test, d.f. $=6, P>0 \cdot 05)$. An abrupt increase in oviducal gland width $(n=14)$ [Fig. 6.7(C)], as well as uterus gland width $(n=14)$ [Fig. 7(D)], corresponded with the onset of female maturity as evaluated macroscopically $\left(L_{50}\right)$. Only one mature female provided an ovarian fecundity count of 11 fully developed follicles (4 in left, 7 in right). The oviducal glands of this female were not available to examine for sperm storage. One preserved specimen had a developing egg capsule present in the left uterus.
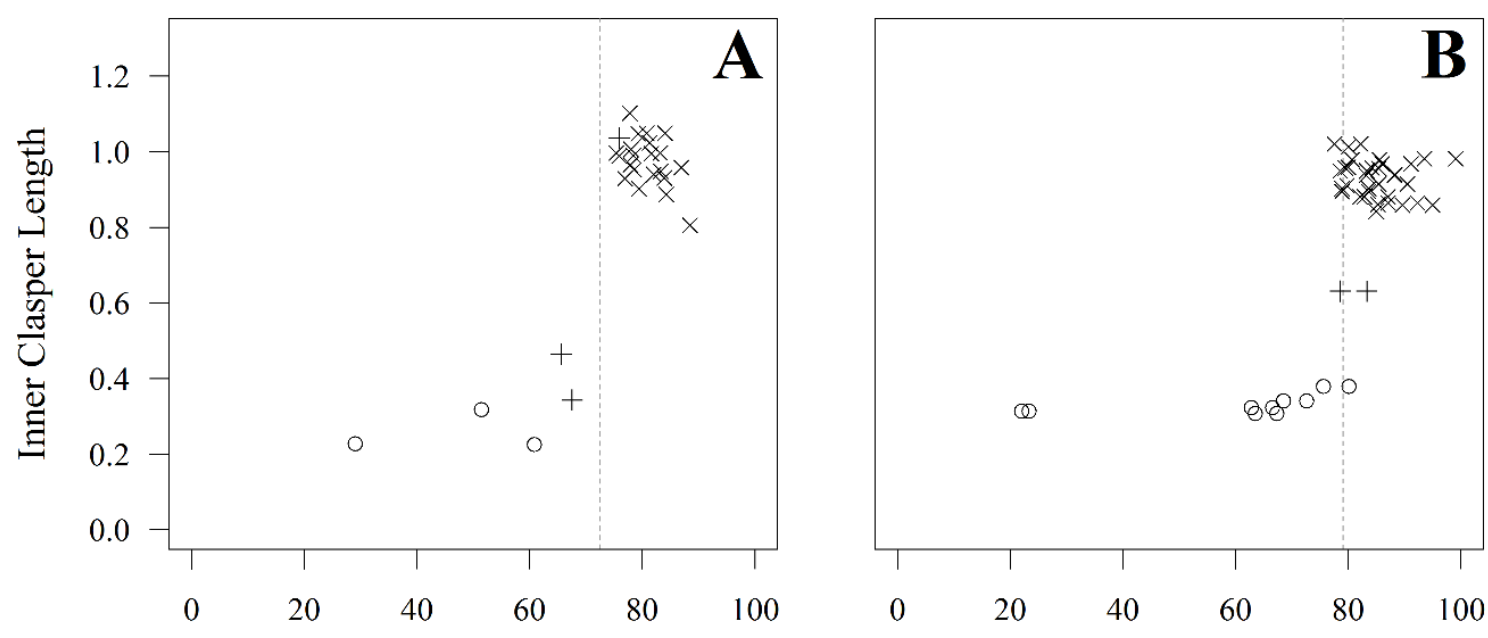

Chimaera Length $(\mathrm{cm})$

Figure 6.6 Relationship between chimaera length $(L c)$ and proportional inner clasper length $\left(L_{\mathrm{CL}}\right)$, for (A) Chimaera carophila and (B) Hydrolagus homonycteris, based on macroscopic maturity stages: $\odot$, juvenile; +, maturing; $\times$, mature). mature $\left(L_{50}=72 \cdot 5 \mathrm{~cm} \mathrm{[a]} \mathrm{and} 79 \cdot 1 \mathrm{~cm} \mathrm{[b]).}\right.$ 


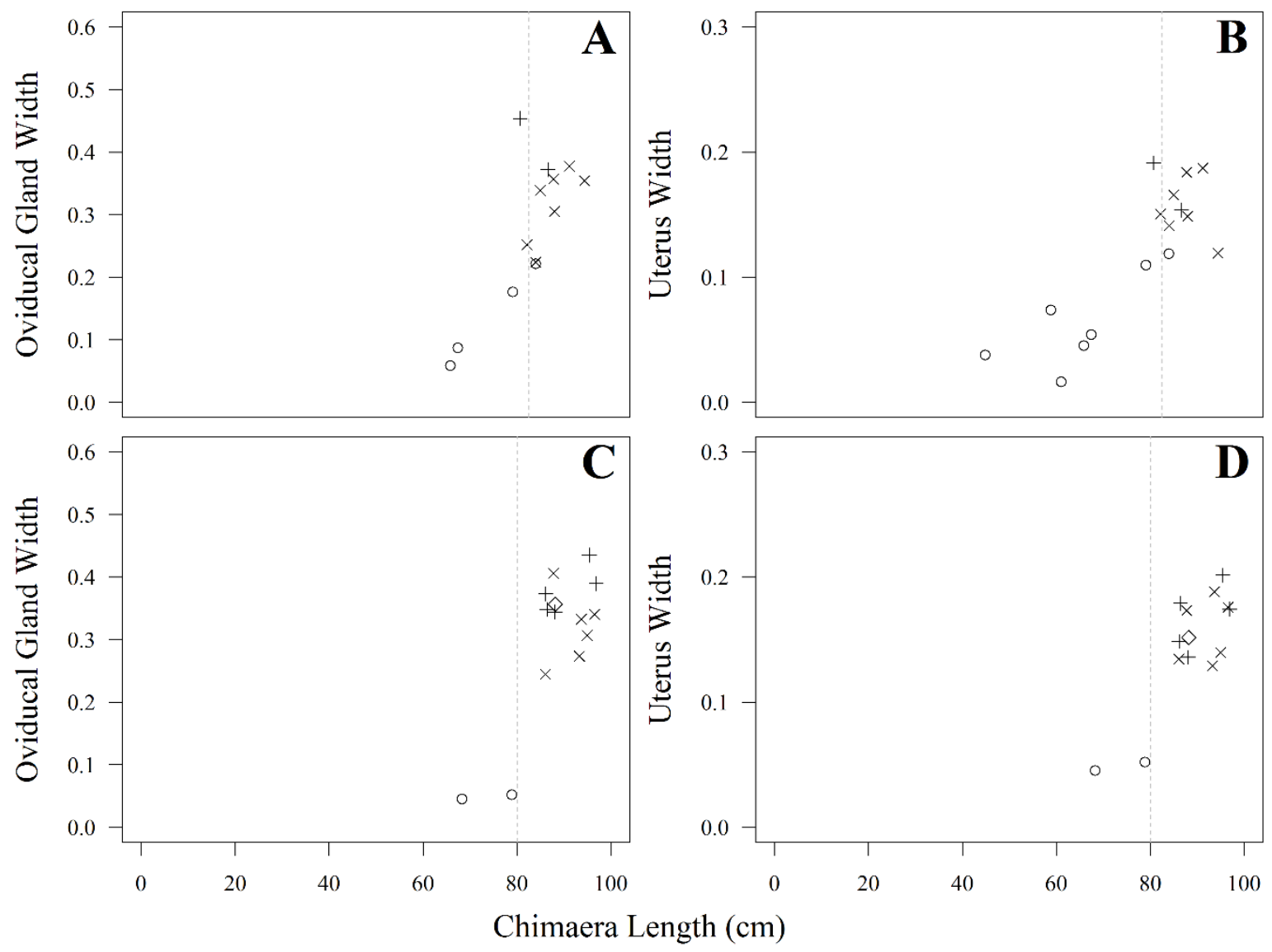

Figure 6.7 Relationship between chimaera length $(L c)$ and $(A)$ proportional width of oviducal gland, (B) uterus for female Chimaera carophila, and relationship between chimaera length $(L c)$ and $(C)$ proportional width of oviducal gland, $(D)$ uterus for female Hydrolagus homonycteris based on macroscopic maturity stages: $\odot$, juvenile; +, maturing; $\times$, mature; $\diamond$ gravid I; $\bullet$, gravid II; $\diamond$, post-partum. $\vdots$, estimated $L c$ at $50 \%$ mature $\left(L_{50}=\right.$ $82 \cdot 7 \mathrm{~cm} L \mathrm{c}$ and $80 \cdot 1 \mathrm{~cm} L \mathrm{c})$. 


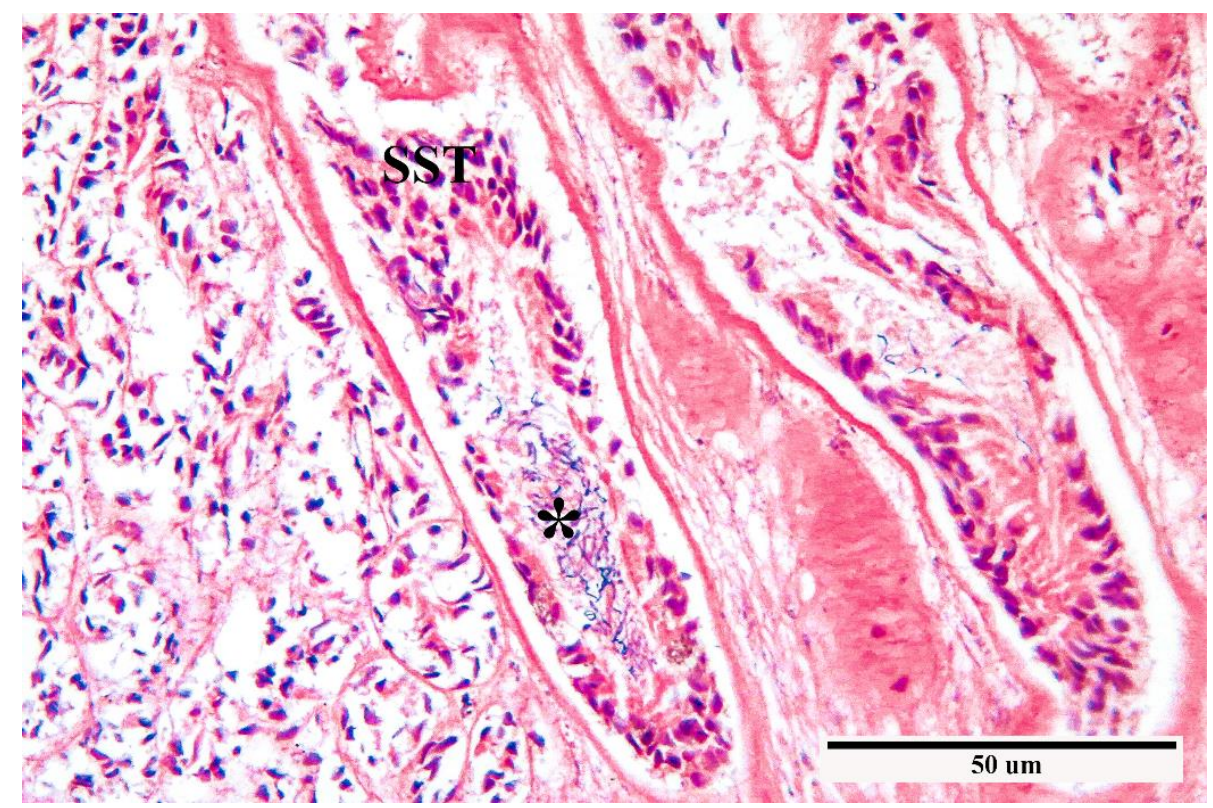

Figure 6.8 Light microscopy image of sperm storage tubules (SST) from terminal zone of the oviducal gland of Chimaera carophila (* indicates sperm bundle).

\subsubsection{Diet}

The digestive tracts of 35\% $(n=7)$ Chimaera carophila and $27 \%(n=3)$ Hydrolagus homonycteris contained visually identifiable prey items (Table 6.1). Small benthic invertebrates, including gastropods, ophiuroids, barnacles, and polychaetes, were prey in both species. Fish, cephalopods, and a number of crustaceans were only found in the digestive tracts of Chimaera carophila. 
Table 6.1. Digestive tract contents composition from Chimaera carophila and

Hydrolagus homonycteris by absolute number $(N)$, frequency of occurrence $(F)$, and weight $(W)$ for prey grouped at taxonomic level.

\begin{tabular}{|c|c|c|c|c|c|c|c|}
\hline & & & caro & & H. & omonyct & teris \\
\hline & & $\mathrm{N}$ & $\mathrm{F}$ & W & $\mathrm{N}$ & $\mathrm{F}$ & $\mathrm{W}$ \\
\hline Salpida & unidentified salp & 2 & 1 & 15.5 & & & \\
\hline & Soestia zonaria & & & & 1 & 1 & 0.44 \\
\hline Polychaetes & unidentified polychaete & 2 & 2 & 0.13 & 1 & 1 & 0.04 \\
\hline Eunicida & & 5 & 1 & 9.55 & & & \\
\hline Crustacea & & & & & & & \\
\hline Cirripedia & unidentified barnacle & 1 & 1 & 0.05 & 1 & 1 & 0.01 \\
\hline Pedunculata & unidentified stalked barnacle & 1 & 1 & 0.01 & & & \\
\hline Euphausiacea & unidentified euphausid & 1 & 1 & 0.02 & & & \\
\hline Euphausiidae & Nematoscelis megalops & 1 & 1 & 0.02 & & & \\
\hline Anomura & & & & & & & \\
\hline Paguroidea & unidentified hermit crab & 2 & 2 & 0.06 & & & \\
\hline Parapaguridae & Parapagurus latimanus & 2 & 1 & 0.06 & & & \\
\hline Brachyura & unidentified crab & 2 & 2 & 0.07 & & & \\
\hline Mollusca & & & & & & & \\
\hline Bivalvia & unidentified bivalve & 2 & 1 & 0.01 & & & \\
\hline Solemyidae & Acharax clarificatus & 1 & 1 & 2.01 & & & \\
\hline Gastropoda & unidentified gastropod & 3 & 5 & 0.21 & & & \\
\hline Muricidae & Enixotrophon sp. & 3 & 1 & 2.36 & & & \\
\hline Nassariidae & Nassarius ephamillus & 6 & 3 & 0.45 & 1 & 1 & 0.04 \\
\hline Raphitomidae & Pleurotomella sp. & 1 & 1 & 0.01 & & & \\
\hline Scaphandridae & Scaphander otagoensis & 2 & 2 & 0.29 & & & \\
\hline Solariellidae & Archiminolia meridiana & & & & 2 & 1 & 0.23 \\
\hline & Zetela variabilis & 2 & 1 & 0.04 & & & \\
\hline Pteropoda & unidentied pteropod & 1 & 1 & 0.06 & & & \\
\hline Cephalopoda & & & & & & & \\
\hline Teuthoidea & unidentified squid & 1 & 5 & 0.3 & & & \\
\hline Octopodidae & Amphitretus thielei & 1 & 1 & 0.01 & & & \\
\hline Osteichthyes & unidentified fish & 1 & 1 & 0.02 & & & \\
\hline Anguilliformes & unidentified eel & 1 & 1 & 2.57 & & & \\
\hline Echinodermata & & & & & & & \\
\hline Echinoidea & unidentifed sea urchin & 1 & 1 & 0.01 & & & \\
\hline Asteroidea & unidentified sea star & 1 & 3 & 1.46 & & & \\
\hline $\begin{array}{l}\text { Ophiuroidea } \\
\text { Other }\end{array}$ & unidentifed brittle star & 4 & 4 & 3.5 & 1 & 1 & 0.05 \\
\hline Cestoda & unidentified tapeworm & 5 & 1 & 2.87 & 6 & 3 & 3.89 \\
\hline small stone & & 1 & 1 & 0.01 & 1 & 1 & 0.02 \\
\hline Sum & & $n=$ & $n=$ & 9.32 & $n=$ & $n=7$ & 0.81 \\
\hline & & 5 & 21 & $\mathrm{~g}$ & 3 & & $\mathrm{~g}$ \\
\hline $\begin{array}{l}\text { Chimaeras sampled } \\
n\end{array}$ & & & 7 & & & 3 & \\
\hline Length range (cm) & & & $61.0-$ & & & $30.1-88.3$ & \\
\hline Male : Female & & & 2.5: & & & $2: 01$ & \\
\hline Depth (m) & & & 1064- & & & $064-127$ & \\
\hline
\end{tabular}




\subsection{Discussion}

Chimaera carophila and Hydrolagus homonycteris appear to have similar biological characteristics to that of other chimaeroids, and more generally, to deep-sea chondrichthyans. Female $C$. carophila matured at a larger size than males, which has been observed in a wide range of elasmobranchs (Cortés, 2000) and holocephalans (e.g. Francis, 1997; Finucci et al., 2016a). Male and female H. homonycteris reached maturity at a similar size, which may indicate the population was not representatively sampled. Maturity in both species was reached at a large proportion of the maximum observed length. There was very good correspondence between macroscopic $L_{50}$, male $L_{\mathrm{CL}}$, and female oviducal gland and uterus width, suggesting using the macroscopic key to determine length-at-maturity was accurate for the sample. Ovarian fecundity counts were restricted to a few individuals and over a limited temporal span, but the counts of 31 and 11 mature follicles in C. carophila and H. homonycteris, respectively, were comparable in number to the deep-sea chimaeroids Harriotta raleighana Goode \& Bean, 1895 and Rhinochimaera pacifica (Mitsukuri, 1895) (Finucci et al., 2016a).

Female $C$. carophila were found to be capable of storing sperm, making it the first confirmation of sperm storage in the family Chimaeridae. This trait has been presumed to be common amongst chimaeroids (Didier et al., 2012), and has now been confirmed in all three families of the order Chimaeriformes: Callorhinchidae (elephantfish Callorhinchus milii Bory de Saint-Vincent, 1823, Smith et al., 2004); Rhinochimaeridae (H. raleighana and $R$. pacifica, Finucci et al., 2016a). Sperm storage could be particularly advantageous for deep-sea species, increasing reproductive efficiency by allowing fertilization to take place at the optimal time in an environment where food resources may be limited (Moura et al., 2011), or where mating may be difficult due to infrequent encounters or sexual spatial segregation (Birkhead \& Møller, 1993). Hydrolagus homonycteris may also store sperm, but further sampling of female individuals will be required to confirm this hypothesis.

\subsubsection{Biological sampling}

Large, reproductively active females (e.g. showing vitellogenic activity or egg capsules) were rarely observed in either species in this study. The males and females of both $C$. carophila and $H$. homonycteris were found to reach a similar length in the 
samples, although females were heavier. The absence of larger females could be because (a) they have suffered additional mortality, perhaps from fishing, or (b) they were not captured, because they could either avoid the net, or were elsewhere. Chimaera carophila have not been recorded any larger than observed in this study, but unsexed $H$. homonycteris has been measured up to $110 \mathrm{~cm} L_{\mathrm{C}}$ by research trawl surveys (maximum length in this study was $99.8 \mathrm{~cm} L_{\mathrm{T}}$ ). A rarity of reproductive females has been reported for other New Zealand chondrichthyans (Wetherbee, 1996; Finucci et al., 2016b), and in the North Atlantic (Holt et al., 2013; Moura et al., 2014). Chimaeroids may segregate by sex and size (Quinn et al., 1980), which may be a trade-off for minimizing intraspecific competition and sexual conflict (Wearmouth \& Sims, 2008). Female chimaeroids have been found at greater depths than their male counterparts (Holt et al., 2013). Diel (Quinn et al., 1980) or seasonal movements (Mathews, 1975) are also possible. Although greater samples may increase the maximum size observed, the available information tends to support the hypothesis of large females being unavailable because they inhabit depths beyond the survey range, or move out of the surveyed regions (perhaps onto rougher or deeper areas where research trawls do not operate, and perhaps for egg-laying).

The few juveniles that were caught, were caught more frequently at greater depths, but sampling was insufficient to establish segregation by size. Segregation of juveniles from adults has been documented in other chimaeroids (Dunn et al., 2010a; Holt et al., 2013), and has been attributed to predator avoidance in elasmobranchs (Heupel \& Simpfendorfer, 2005; Guttridge et al., 2011). Many species of elasmobranch utilize nursery grounds, defined as areas where neonates and small juveniles remain after pupping or hatching, which are presumed to be optimal for somatic growth and low mortality rates (Heupel et al., 2007). If C. carophila and H. homonycteris are engaging in congregative nursery behaviour, as exhibited by other oviparous chondrichthyans (Etnoyer \& Warrenchuk, 2007; Hoff, 2016), these species may have breeding grounds in areas not sampled by the surveys, perhaps on rougher grounds less accessible to trawls.

Nursery areas for chimaeroids have yet to be identified, although the discovery of dense aggregations of juveniles and egg cases in the troughs of large sand waves on the U.S. West Coast outer continental shelf have been suspected as nursery areas for Hydrolagus colliei (Barnett et al., 2012). Large clusters of egg cases of other oviparous chondrichthyans have been located near cold seeps (Truede et al., 2011), cold-water coral reef habitat (Henry et al., 2013), and various discreet locations of the outer and middle shelf and upper slope of canyons (Hoff, 2016). However, if the females burying their eggs 
in the substrate (Dean, 1906), they could be missed by trawls and visual surveys altogether. Continued surveying over a wider range of area is necessary to identify potential breeding areas, nursery grounds, and areas of importance for specific life cycles of these species.

\subsubsection{Diet}

The diets of $C$. carophila and H. homonycteris both predominately consisted of benthic invertebrates. The presence of pelagic prey such as salps, pteropods, squids, and the pelagic octopus Amphitretus thielei, indicates these species are likely to opportunistically scavenge pelagic food fall, as suspected in other chimaerids (Dunn et al., 2010a). Substantial catch of several chimaerids, including pale ghost shark Hydrolagus bemisi Didier 2002, dark ghost shark Hydrolagus novaezealandiae (Fowler, 1911), and giant chimaera Chimaera lignaria Didier 2002, as well as $C$. carophila and $H$. homonycteris, have been recorded from bottom longline fisheries in New Zealand waters (Francis, 2015), indicating that more generally, chimaerids do engage in benthic foraging and scavenging. The indicative diets from this study are similar to those previously described for other chimaeroids caught on Chatham Rise, suggesting any niche separation is likely to occur more through environmental preferences (e.g. depth) than dietary specialisation (Dunn et al., 2010a).

The identification of diet in chimaerids is practically difficult, as these fish crush their prey with tooth plates, resulting in a large proportion of fragmented prey items which cannot always be identified to a species level. The presence of unidentifiable material makes separating stomach contents into prey categories for quantification ambiguous, leaving frequency of mass $(W)$ and frequency of importance $(N)$ as less reliable measurements. Instead, frequency of occurrence ( $F$; presence/absence) seems the best measure for a robust characterisation of diet composition for these species (Baker $e t$ al., 2014).

\subsubsection{Rare, uncommon, or under-sampled?}

Annual trawl surveys (biennial as of 2014) have been carried out on Chatham Rise since 1992, creating the most comprehensive time series of marine species observations within the New Zealand EEZ (O'Driscoll et al., 2011). Most observations of C. carophila 
and $H$. homonycteris have been recorded from Chatham Rise. Despite extensive sampling, both $C$. carophila and $H$. homonycteris have been infrequently encountered across Chatham Rise, recorded in $4.12 \%$ and $0.66 \%$ of 4541 bottom research trawls at depths from 300 to $1400+\mathrm{m}$ from 1978 to 2013 (Table 6.3), and they have most often been caught as individual fish. Compared to other chimaeroids (H. bemisi, $H$. novaezealandiae, $H$. raleighana, $R$. pacifica) caught in this region, $C$. carophila and $H$. homonycteris are rarely encountered (Table 6.3). The apparent rarity of these species, however, could be due to lack of sampling at their preferred habitat and/or depth range. Chimaeroids have been recorded at some of the deepest depth ranges of any chondrichthyan; in the northeast Atlantic, H. raleighana was found at $2600 \mathrm{~m}$ (Priede et al., 2006). Both C. carophila and H. homonycteris were caught most frequently at depths beyond $1000 \mathrm{~m}$, particularly $H$. homonycteris, and are known to occur at depths beyond 1400 m (Roberts et al., 2015). In New Zealand, most deep-sea trawling occurs at depths less than $800 \mathrm{~m}$, with a maximum commercial fishing depth is considered to be around $1500 \mathrm{~m}$ due to logistical constraints on the deployment of commercial fishing nets (and depth distribution of the target species) (Black \& Tilney, 2015).

Outside of Chatham Rise, C. carophila appears to have a widespread distribution across the New Zealand EEZ, whilst the distribution of H. homonycteris may be restricted to more temperate waters, including off Australia (Didier, 2008). Population genetics would be useful to establish if individuals within New Zealand, and for H. homonycteris, between New Zealand and Australia, belong to the same population. If these species were to be found to be part of widespread populations, this would suggest a lesser risk to exploitation despite their apparent rarity. Given their apparent scavenging nature, it could be useful to sample the catches from bottom longline fisheries as well, which operate at depths of 200-800 m when targeting ling (Genypterus blacodes) (Anderson, 2014), and occasionally fish deeper (SPRFMO, 2008).

Chimaera carophila and $H$. homonycteris have been most frequently caught by research trawls at depths below the range of most deep-sea commercial fishing activity at this time, and it therefore seems unlikely that these species are under immediate threat from population decline as a result of fishing. Nonetheless, C. carophila and $H$. homonycteris appear to be characterised by low productivity life histories similar to other deep-sea chondrichthyans (Simpfendorfer \& Kyne, 2009). Sustained collection of data for these species, particularly new information on age, and thus longevity, mortality rates, and growth, is required to accurately assess their productivity. In addition, continued 
monitoring of the deep-sea environment, including the footprint of deep-sea fisheries, the potential new impact of deep-sea seabed mining (Leduc et al., 2015), and the mortality and habitat modifications that these cause, is needed to fully evaluate impacts of human activity on these species. 
Table 6.2. Research bottom trawl catch records of chimaera species on Chatham Rise from 1978 to 2013 by depth (m).

\begin{tabular}{|c|c|c|c|c|c|c|c|c|c|c|c|c|c|c|}
\hline \multirow[t]{2}{*}{ Species } & \multicolumn{13}{|c|}{ Depth (m) } & \multirow[b]{2}{*}{$\begin{array}{c}\% \\
\text { occurrence } \\
\text { in all } \\
\text { trawls }\end{array}$} \\
\hline & $\begin{array}{c}300- \\
399\end{array}$ & $\begin{array}{l}400- \\
499\end{array}$ & $\begin{array}{c}500- \\
599\end{array}$ & $\begin{array}{c}600- \\
699\end{array}$ & $\begin{array}{l}700- \\
799\end{array}$ & $\begin{array}{c}800- \\
899\end{array}$ & $\begin{array}{c}900- \\
999\end{array}$ & $\begin{array}{c}1000- \\
1099\end{array}$ & $\begin{array}{l}1100- \\
1199\end{array}$ & $\begin{array}{l}1200- \\
1299\end{array}$ & $\begin{array}{c}1300- \\
1399\end{array}$ & $>1400$ & $\begin{array}{l}\text { Total } \\
\text { catch } \\
\text { records }\end{array}$ & \\
\hline Chimaera panthera & 0 & 0 & 1 & 2 & 3 & 0 & 0 & 0 & 0 & 0 & 0 & 1 & 7 & 0.15 \\
\hline Hydrolagus trolli & 0 & 1 & 0 & 1 & 0 & 0 & 3 & 1 & 4 & 8 & 7 & 3 & 28 & 0.62 \\
\hline Hydrolagus homonycteris & 0 & 0 & 0 & 0 & 0 & 2 & 0 & 4 & 14 & 7 & 3 & 0 & 30 & 0.66 \\
\hline Chimaera lignaria & 0 & 2 & 3 & 9 & 13 & 18 & 54 & 33 & 10 & 0 & 0 & 0 & 142 & 3.13 \\
\hline Chimaera carophila & 0 & 0 & 0 & 3 & 2 & 13 & 46 & 80 & 36 & 5 & 2 & 0 & 187 & 4.12 \\
\hline Rhinochimaera pacifica & 0 & 2 & 3 & 14 & 57 & 148 & 223 & 93 & 23 & 3 & 0 & 0 & 566 & 12.46 \\
\hline Hydrolagus novaezealandiae & 654 & 484 & 50 & 4 & 4 & 3 & 2 & 1 & 0 & 0 & 1 & 0 & 1203 & 26.49 \\
\hline Harriotta raleighana & 24 & 367 & 295 & 229 & 192 & 273 & 226 & 53 & 10 & 2 & 0 & 0 & 1671 & 36.80 \\
\hline Hydrolagus bemisi & 126 & 740 & 469 & 279 & 215 & 313 & 207 & 38 & 8 & 2 & 0 & 0 & 2397 & 52.79 \\
\hline Total no. of trawls & 701 & 879 & 492 & 294 & 261 & 504 & 669 & 411 & 233 & 60 & 29 & 8 & 4541 & \\
\hline
\end{tabular}


Chapter VII

General Discussion 
As inshore fisheries become overexploited, the expansion and commercial importance of deep-sea fisheries have increased on a global scale (Watson \& Morato, 2013; Clark et al., 2016). Many deep-sea fisheries have proven to be unsustainable, as deep-sea species are generally characterised with life history traits which result in low productivity (slow growth, high longevity, late age of maturity, and low fecundity) (García et al., 2008; Simpfendorfer \& Kyne, 2009). Low biological productivity, coupled with particular patterns of habitat use (e.g. aggregations near topographic features such as seamounts), can contribute to high vulnerability to overfishing (Koslow et al., 2000; Simpfendorfer \& Kyne, 2009; Clark et al., 2016). Deep-sea chondrichthyans, those occurring below $200 \mathrm{~m}$, and representing nearly half of the known species of sharks, rays, and chimaeras (Kyne \& Simpfendorfer, 2007), are no exception. Their inaccessibility to most fisheries has allowed for these species to be generally considered less threatened than shallow water relatives (Dulvy et al., 2014). However, some deep-sea chondrichthyans have already shown significant population declines, which in turn has resulted in the implementation of zero allowable landed catch for species in certain regions (Graham et al., 2001; ICES, 2009; Neat et al., 2015). Regardless of their high risk status (e.g. Ford et al., 2015), research efforts are often prioritized for high value commercial species (Mace et al., 2013), and as a result, many deep-sea chondrichthyans are listed as Data Deficient on the International Union for the Conservation of Nature (IUCN) Red List of Threatened Species (Dulvy et al., 2014).

Work on deep-sea chondrichthyans often comes with considerable costs and challenges. The rarity and inaccessibility of these fish makes obtaining sufficient sample sizes for life history studies difficult, research surveys are expensive, and the logistics of working with live animals (e.g. tagging) in the deep-sea still face many technical restrictions that have yet to be addressed. Despite these challenges, the proportion of literature devoted to deep-sea taxa has been increasing in recent years, in part due to a recent increase in the number of new taxonomic descriptions of deep-sea species (Cotton \& Grubbs, 2015). Between 2000 and 2014, 230 species of chondrichthyans were described, with $65 \%$ of them recognized as deep-sea taxa (Pollerspöck, 2014). However, life history data on deep-sea species is still limited, and only available for just under 7\% of taxa (Rigby \& Simpfendorfer, 2015).

The research in this thesis had added to the knowledge of deep-sea chondrichthyan ecology through the collection of new information on several species for which data were previously unavailable. Data has included length-weight relationships 
for twenty species, many of which have yet to be reported; reproductive parameters (e.g. size-at-maturity, fecundity, and sperm storage) for five species; diet for three species; and characteristics of aggregations and social associations for common and uncommon bycatch species on Chatham Rise. This information is critical for informed management decisions (e.g. input into ecological risk assessments) and determining conservation status (e.g. New Zealand Threat Classification System, IUCN Red List of Threatened Species).

\subsection{Implications for management}

Both targeted fishing and incidental bycatch has depleted deep-sea chondrichthyan populations in Australia (Graham et al., 2001; Graham \& Daley, 2011) and the European Union (ICES, 2009). This has resulted in management arrangements such as the implementation of zero catch limits for some species (Villasante et al., 2012), and scientific recommendations to limit fishing beyond $600 \mathrm{~m}$ altogether (Clarke et al., 2015). Over the course of 1998-2013, despite management action to reduce the effects of fishing in the Rockall Trough, current data suggests minimal recovery of deep-sea chondrichthyan populations, possibly hindered by the generalized low productivity of deep-sea species (Neat et al., 2015). Some deep-sea trawling bans employed to protect important commercial fish stocks (e.g. orange roughy in the Tasman Sea, Patterson et al., 2016), may also indirectly provide some refuge for deep-sea chondrichthyans. With minimal fishing at depths $>800 \mathrm{~m}$ in New Zealand, many deep-sea chondrichthyans with depth ranges beyond $800 \mathrm{~m}$, including Chimaera carophila and Hydrolagus homonycteris, are considered less threatened than their shallow water relatives at this time. The mean depth of catches for most species in Chapter III, however, was $<800 \mathrm{~m}$, meaning that their core depth ranges overlap with commercial fishing activities (Table 3.2). New industries, such as deep-sea mining, should also be taken into consideration as they will most likely impact the New Zealand deep-sea environment and benthicassociated species (Leduc et al., 2015). Chimaeroids may be at particular risk, given their need for egg-laying grounds, which have yet to be identified, and their diet that consists primarily of benthic macrofauna (Chapter V, VI).

At this time, spatial use of the water column or depth preference of different life history stages for many deep-sea species is not well known and thus, cannot be taken into account for risk and conservation assessments. Vertical migration patterns have been recognized in several deep-sea elasmobranchs, migrating to shallow depths at night and 
remaining in deeper waters during the day (Nelson et al., 1997; Daley et al., 2015; Nakamura et al., 2015; Shipley et al., 2016). These migrations may reduce species vulnerability to bottom trawl fishing (Dunn et al., 2013), or alternatively, may increase vulnerability to fishing mortality from multiple types of fishing gear (Speed et al., 2010). In addition, there is virtually nothing known about stock structure or migration patterns for these species, both of which are crucial pieces of information for stock assessments. The spatial, and possibly depth, influence of size- and sex-segregated groups described in Chapter III will have important implications for differential mortality of sexes and/or size classes. Suspected male or female dominated seamount aggregations of Etmopterus granulosus, in particular, further highlights this species' high risk to fishing and the need for continual monitoring.

Data from this thesis will provide biological and ecological context to better assess species vulnerability to population depletion as a result of fishing. Accurate lengthweight relationships (LWRs), which can be applied to stock assessment models and fisheries management applications, are required to accurately assess biomass trends which may otherwise be under- or overestimated with the use of currently available online parameter estimates (Chapter II). Species-specific biological parameters provides data required for national and international threat classification schemes. In the case of Chimaera carophila, data from Chapter VI will be useful to assess this species for the IUCN Red List for the first time, and for other species, data will be used to reassess their status in the future. For Oxynotus bruniensis, where its vulnerability to fishing has been previously disputed (Ford et al., 2015), data from Chapter IV provides further evidence that beyond its distributional overlap with fishing effort, the low productivity of $O$. bruniensis strengthens the need to classify this species as high risk from overfishing. In addition, its selective diet highlights a need to incorporate consideration for ecological specialisation into future risk assessment criteria, as this factor will most likely increase the species' susceptibility to population decline.

\subsection{Continued data collection}

As of 2014, of the 37 chimaeroids to have been assessed on the IUCN Red List, 22 were categorized as Data Deficient (Dulvy et al., 2014). Since the release of that synthesis paper, many of those statuses have been upgraded to Least Concern on the grounds of their deep-sea distributions being beyond most current fishing effort (e.g. 
Dagit \& Kyne, 2015; Dagit et al., 2016). However, many of these species are still data poor; even the most basic of biological data, such as length-weight relationships (Chapter II), are unreported, and their low vulnerability risk is inferred in the absence of data. In New Zealand alone, nothing is known of life histories, population size or structure for most chimaeroids (e.g. Duffy, 2003; Compagno \& Dagit, 2015; Dagit \& Walls, 2015), despite consistent, and sometimes, considerable catch records for some of these species. For example, a substantial number of giant chimaera, Chimaera lignaria, are caught in deep-sea research and commercial trawls around both Australia and New Zealand, including a number of records from the Bounty Islands (Ford et al., 2015). Nothing further is known about its biology or population size and structure (Dagit, 2015). Despite their frequent occurrence in catches, there is still much to learn about the two ghost sharks, Hydrolagus bemisi and Hydrolagus novaezealandiae, species that are managed under the Quota Management System (QMS); growth has yet to be validated, spawning and nursery grounds have yet to be identified, and few data exist on their fecundity, reproductive seasonality, movement and behaviour (MacGibbon \& Fu, 2013). This is not unexpected given that between 2000 and 2010, less than 50 individual $H$. bemisi, and not a single $H$. novaezealandiae, have been examined by the Ministry of Primary Industries (MPI) Observer Program for gonad development (MacGibbon \& Fu, 2013; MacGibbon, 2016). Even less is known about most of the deep-sea batoids, which are considered some of the most threatened species amongst the chondrichthyans (Dulvy et al., 2014). At this time, research surveys are generally the only means of data collection for many species, and additional measurements beyond the standard length, mass, and sex measurements (O'Driscoll et al., 2011) are needed to adequately describe species biology. These should include genetic sampling (e.g. for examination of population connectivity), growth and ageing, longevity, and basic evaluation of stomach contents, all of which would be invaluable data for filling in biological knowledge gaps. To accurately assess annual (or lifetime) reproductive output, measurements including maturation stage, fecundity estimates (ovarian and uterine fecundity counts) throughout the year, and evaluation of the proportion of the population contributing to recruitment, should also be routinely collected. Previously collected data from these surveys should also be fully utilized, and, as highlighted in Chapter II, may be useful in providing inferences about important life history stages, such as length-at-maturity.7.3 Future research 


\subsubsection{Ageing}

Novel results from this thesis, including the selective diet of Oxynotus bruniensis and the snout of Harriotta raleighana as a secondary sexual characteristic, have shown that basic biological studies can reveal interesting aspects for a greater understanding of chondrichthyan ecology. All species examined here were shown to have biological characteristics similar to that of other deep-sea chondrichthyans with low productivity, including low fecundity and sexual maturation at a large proportion of their overall size. At this time, it is unclear if these species are maturing late in life as this would require age estimates. Age estimates are the basis of many key biological parameters, such as longevity, mortality, and productivity, which are essential to manage chondrichthyan populations and assess their ability to withstand fisheries exploitation (Cailliet et al., 2006; Goldman et al., 2012). Studies have indicated high longevity and low growth rates for deep-sea chondrichthyans (see Kyne \& Simpfendorfer, 2010), although many deepsea species have yet to be aged, and few studies have been validated (Cailliet $e t a l$., 2015). Until recently, band pair counts of dorsal fin spines were the traditional age reading approach for age estimation of squalid sharks (e.g. Clarke et al., 2002). Not only can this method be both time and cost consuming, but band pair counts also require practice and experience to achieve accurate results, can be highly subjective, and many deep-sea sharks do not have dorsal fin spines, or have reduced band resolution which complicates age interpretation (see Cailliet et al., 2015).

Band counts in the dorsal fin spines of chimaeroids, such as the rabbitfish Chimaera monstrosa (L. 1758), have estimated age ranges of up to 30 years, and maturity between 11 and 13 years, indicating a long-lived, late maturing species (Calis et al., 2006). However, this technique, as well as other ageing methods such as measuring weight and diameter of eye lens, and band counts of vertebrae, have been found to be unreliable or not validated (Francis \& Ó Maolagáin, 2000; Barnett et al., 2009). Thus, any ageing of the study species were excluded from this thesis. The need for alternative ageing approaches to address these limitations has resulted in experimental techniques, including counting tritor ridges on the vomerine tooth plates of chimaeroids (King \& McPhie., 2013), as well as validation approaches such as near infrared spectroscopy (NIRS) (Rigby et al., 2015), and vertebral (Christiansen et al., 2016) and eye lens (Nielsen et al., 2016) bomb radiocarbon dating. Other emerging methods include computed tomography (CT) scanning to detect growth bands in vertebrae (or other hard 
parts) (Geraghty et al., 2012; Moe \& Cotton, 2016). Further research for alternative ageing methods is essential, particularly for the deep-sea chimaeroids, and investigations for methods of ageing are currently underway for $O$. bruniensis (Finucci, unpublished data) and several other deep-sea chondrichthyans mentioned in this thesis (M.P. Francis, NIWA, pers. comm.).

\subsubsection{Identification of regions of importance for life history stages}

Most deep-sea chimaeroids not managed by the QMS have a presence outside of New Zealand and at depths beyond most fishing, suggesting that there is probably no immediate threat from fisheries, regardless of their presumed low productivity. However, the absence of particular size classes, particularly large females and small juveniles, from the areas sampled warrants further investigation to assess whether these individuals have refuge areas from fishing. Areas of importance to these species (e.g. mating grounds, egg laying or pupping grounds, nursery grounds) have yet to be identified. On a global scale, there are very few confirmed records of chondrichthyan egg-laying grounds, but published accounts are growing (see ICES, 2015; Henry et al., 2016). While it is unknown if all oviparous chondrichthyans use specific sites for egg-laying, the lack of accounts may be due to egg cases being overlooked rather than a lack of egg-laying habitat or areas not yet surveyed (Henry \& Roberts, 2016). Seamounts can support diverse and abundant fish communities (Morato \& Clarke, 2007), including commercially valuable species (Clark, 1999; Clark \& Dunn, 2012), and have been recognized as areas of importance (e.g. mating grounds, cleaning stations) for elasmobranchs (Oliver $e t$ $a l ., 2011)$. Identifying regions of importance for various life history stages is essential for the spatial and temporal management of such deep-sea features before they are impacted by human activities, such as bottom trawling or deep-sea mining (Clark \& Dunn, 2012; Leduc et al., 2015).

Technologies such as autonomous underwater vehicles (AUV), remotely operated vehicles (ROV), and baited remote underwater video (BRUV) are becoming readily available for ocean exploration, offering novel opportunity to observe deep-sea chondrichthyans in situ. They can also be used in areas where trawls cannot sample, and as a result, have allowed for sampling of new species (Quaranta et al., 2006), as well as revealing egg-laying grounds of several deep-sea elasmobranchs (Henry et al., 2014; Henry et al., 2016). A database of BRUV footage collected by the Museum of New 
Zealand Te Papa Tongarewa (NMNZ) already exists, but has yet to be analysed for chondrichthyan ecology (V. Zintzen, Department of Conservation, pers. comm.). Moored underwater video deployed on a Chatham Rise seamount also captured several deep-sea elasmobranchs (O’Driscoll et al., 2012). These archives potentially offer a wealth of information on chondrichthyan density, distribution, foraging behaviour, and habitat use, and should be utilised to their full potential, while also offering guidance for future research in this discipline.

It is also recommended that increased collaboration with the fishing industry and the Ministry for Primary Industries Observer Program be encouraged, as it can also allow for greater sampling coverage beyond the spatial and temporal range of research surveys. Research surveys generally, and deliberately, sample certain regions at the same time of year with the same gear type (Chatham Rise survey in January, for example) (O'Driscoll et al., 2011). Alternative sampling methods, such as longlining, may catch individuals that may otherwise evade trawl capture (Clarke, 2005). Centroscymnus owstonii, for example, was almost always recorded from commercial surface longlining between 2008 and 2013, while most commercial catches of $C$. lignaria were documented from bottom longlining (Francis, 2015). A previous study on Etmopterus granulosus across Chatham Rise found few reproductively active females and none of these contained embryos, despite extensive spatial survey coverage over a number years (Wetherbee, 1999). In contrast, E. granulosus samples collected by local fishers from the Wellington region during the course of this thesis included many reproductively active females, with several gravid specimens (B. Finucci, pers. obs.). Etmopterus granulosus was ranked as relatively high risk from the recent qualitative risk assessment for New Zealand chondrichthyans, which described data for this species as "exist but poor" on the basis of a lack of reproductive frequency information or reliable abundance indices (Ford et al., 2015). Collaboration with industry offers opportunity to collect valuable data for species that would otherwise remain data poor when relying solely on research surveys.

\subsubsection{Taxonomy}

Finally, there is still a need for accurate taxonomic work. While taxonomy is often overlooked, it remains the vital foundation for all other biological studies. It assists researchers with the identification of their research subjects, and allows managers to distinguish which species are caught, and evaluate species range, which is essential to 
implement practical management measures (Simpfendorfer et al., 2011). Accurate taxonomy is vital for revealing cryptic species and resolving species complex issues, which, in recent years, has employed molecular markers to assist in distinguishing between closely related species (see White et al., 2015). Multiple sister species have been identified within species thought to be widespread, which can reveal reduced, and even biogeographically isolated, ranges (White \& Last, 2012). The eventual recognition of the endemic ghost sharks, Hydrolagus bemisi and Hydrolagus novaezealandiae, as two species in 2002 signified that they were to be managed separately (MPI, 2014), rather than as one as was previously the case, allowing for more accurate catch records and species management (Didier, 2002). Other New Zealand species where catch records are known or suspected to include multiple species include E. granulosus, the catsharks (Apristurus spp.), and skates of the genus Brochiraja (Ford et al. 2015). Without resolving these fundamental identification issues, and confirming species identification, species vulnerability to exploitation may be underestimated (Simpfendorfer et al. 2011).

A recent collation of all known chondrichthyans presented a list of 1188 extant sharks, rays, and chimaeroids, across 61 families and 199 genera (Weigmann, 2016). Since the previous review ten years earlier by Compagno (2005), 217 new species were described, with many species being synonymized or resurrected. Valid species are still being regularly described from museum collections, during research cruises, and from local fish markets (Weigmann, 2016). Currently, there are 117 valid chondrichthyan fishes recognized from New Zealand waters (Roberts et al., 2015), with additional specimens yet to be described. However, there are still many unresolved issues over species identification (particularly within the chimaeroids), family phylogenetic placement, and population structure between New Zealand and neighbouring regions (e.g. Australia). Even species mentioned in previous chapters have been subject to taxonomic changes during the course of this thesis. Etmopterus baxteri was found to be a junior synonym of $E$. granulosus, a lanternshark now confirmed to be widespread across the Southern Hemisphere (Straube et al., 2015). Chimaera carophila, previously reported as Chimaera sp. C (McMillan et al., 2011), was thought to be C. macrospina or a conspecific, until it was formally described as a new species endemic to New Zealand waters (Kemper et al., 2014). The genus Proscymnodon, which included P. plunketi, was placed into synonymy with Scymnodon by White et al. (2015), and molecular data has further indicated that $P$. plunketi is most likely a junior synonym of $S$. macracanthus (Weigmann, 2016). Several chimaeroid specimens collected for dissections during this 
thesis could not be confidently identified to the species level, and are currently undergoing molecular analysis (B. Finucci, unpublished data). With a large ongoing investigation of chondrichthyan phylogenetics conducted by the Chondrichthyan Tree of Life project (CTOL, see https://sharksrays.org/), a broader understanding of New Zealand chondrichthyan diversity and biogeography through further identification of valid species and corrections among phylogenies are expected within New Zealand and globally.

Research presented here has provided novel life history information on the biology and ecology of data deficient deep-sea chimaeroids and squaloids, some of which are endemic to New Zealand, while others are more globally distributed. The costs and constraints affliated with deep-sea research highlights the need for increased collaborative efforts across disciplines and institutes, while fully exploiting datasets that may be readily available, but underutilized. There are many data-poor chondrichthyans from New Zealand which have not been examined in this thesis, including more cryptic chimaeroids, such as leopard chimaera Chimaera panthera Didier, 1998, catsharks (Apristurus spp.), and batoids. Future research should be directed to these species, which are considered rare, or threatened by fishing (Ford et al., 2015). 


\section{References}

Akaike, H. (1987). Factor analysis and AIC. Psychometrika 52, 317-332. DOI: 10.1007/BF02294359.

Akhilesh, K. V. (2014). Fishery and biology of deep-sea chondrichthyans off the southwest coast of India. Faculty of Marine Sciences, Cochin University of Science and Technology.

Alarcón, C., Cubillos, L.A. \& Acuña, E. (2011). Length-based growth, maturity and natural mortality of the cockfish Callorhinchus callorhynchus (Linnaeus, 1758) off Coquimbo, Chile. Environmental Biology of Fishes 92, 65-78. DOI: 10.1007/s10641-011-9816-0.

Albo-Puigserver, M., Navarro, J., Coll, M., Aguzzi, J., Cardona, L. \& Sáez-Liante, R. (2015). Feeding ecology and trophic position of three sympatric demersal chondrichthyans in the northwestern Mediterranean. Marine Ecology Progress Series 524, 255-268. DOI: 10.3354/meps11188.

Allain, V., Biseau, A. \& Kergoat, B. (2003). Preliminary estimates of French deepwater fishery discards in the Northeast Atlantic Ocean. Fisheries Research 60, 185-192. DOI: 10.1016/S0165-7836(02)00031-0.

Alonso, M.K., Crespo, E.A., García, N.A., Pedraza, S.N., Mariotti, P.A. \& Mora, N.J. (2002). Fishery and ontogenetic driven changes in the diet of the spiny dogfish, Squalus acanthias, in Patagonian waters, Argentina. Environmental Biology of Fishes 63, 193-202. DOI: 10.1023/A:1014229432375.

Anderson, O.F. (2014). Fish and invertebrate bycatch and discards in New Zealand ling longline fisheries from 1992-93 until 2011-12. New Zealand Aquatic Environment and Biodiversity Report No. 138. Wellington: Ministry for Primary Industries.

Angulo, A., Lopez, M.I., Bussing, W.A. \& Murase, A. (2014). Records of chimaeroid fishes (Holocephali: Chimaeriformes) from the Pacific coast of Costa Rica, with the description of a new species of Chimaera (Chimaeridae) from the eastern Pacific Ocean. Zootaxa, 3861, 554-574. DOI: 10.11646/zootaxa.3861.6.3.

Annala, J.H. \& Sullivan, K.J. (1997). Report from the Fishery Assessment Plenary, May 1997: stock assessments and yield estimates. Unpublished report held in NIWA library, Wellington.

Apostolidis, C., Bal, G., Froese, R., Kopra, J., Kuikka, S., Leach, A., Levontin, P., Mäntyniemi, S., Maoileidigh, N.O., Mumford, J., et al. (2015). Best practices for the provision of prior information for Bayesian stock assessment. ICES Cooperative Research Report 328. Copenhagen: ICES.

Armansin, N.C., Lee, K.A., Huveneers, C. \& Harcourt, R.G. (2016). Integrating social network analysis and fine-scale positioning to characterize the associations of a benthic shark. Animal Behaviour 115, 245-258. DOI: 10.1016/j.anbehav.2016.02.014. 
Araújo, M.S., Bolnick, D.I. \& Layman, C.A. (2011). The ecological causes of individual specialisation. Ecology Letters 14, 948-958. DOI: 10.1111/j.1461-0248.2011.01662.x.

Bagley, N.W. \& O'Driscoll, R.L. (2012). Trawl survey of hoki, hake, and ling in the Southland and Sub-Antarctic areas, November-December 2009 (TAN0911). New Zealand Fisheries Assessment Report, 5.

Bagley, N.W., Ballara, S.L., O’Driscoll, R.L., Fu, D. \& Lyon, W. (2013). A review of hoki and middle depth summer trawl surveys of the Sub-Antarctic, November December 19911993 and 2000-2009. New Zealand Fisheries Assessment Report No. 41. Wellington: Ministry for Primary Industries.

Ballara, S.L. (2015). Fish and invertebrate bycatch in New Zealand deepwater fisheries from 1990-91 until 2012-13. New Zealand Aquatic Environment and Biodiversity Report No. 158. Wellington: Ministry for Primary Industries.

Baker, R. \& Buckland, A., Sheaves, M. (2014). Fish gut content analysis: robust measures of diet composition. Fish and Fisheries 15, 170-177. DOI: 10.1111/faf.12026.

Baremore, I.E. \& Passerotti, M.S. (2013). Reproduction of the blacktip shark in the Gulf of Mexico. Marine and Coastal Fisheries 5, 127-138. DOI: 10.1080/19425120.2012.758204.

Barnett, L.A.K., Earley, R., Ebert, D.A. \& Cailliet, G.M. (2009). Maturity, fecundity, and reproductive cycle of the spotted ratfish, Hydrolagus colliei. Marine Biology 156, 301-316. DOI: $10.1007 / \mathrm{s} 00227-008-1084-\mathrm{y}$.

Barnett, L.A.K., Ebert, D.A. \& Cailliet, G.M. (2009). Assessment of the dorsal fin spine for chimaeroid (Holocephali: Chimaeriformes) age estimation. Journal of Fish Biology 75, 1258-1270. DOI: 10.1111/j.1095-8649.2009.02362.x.

Barnett, L.A.K., Ebert, D.A. \& Cailliet, G.M. (2012). Evidence of stability in a chondrichthyan population: case study of the spotted ratfish Hydrolagus colliei (Chondrichthyes: Chimaeridae). Journal of Fish Biology 80, 1765-1788. DOI: $10.1111 /$ j.1095-8649.2011.03216.x.

Barría, C., Navarro, J., Coll, M., Fernandez-Arcaya, U. \& Sáez-Liante, R. (2015). Morphological parameters of abundant and threatened chondrichthyans of the northwestern Mediterranean Sea. Journal of Applied Ichthyology 31, 114-119. DOI: 10.1111/jai.12499.

Barrull, J. \& Mate, I. (2001). First confirmed record of angular roughshark Oxynotus centrina (Linnaeus, 1758) predation on shark egg case of small-spotted catshark Scyliorhinus canicula (Linnaeus, 1758) in Mediterranean waters. Annales Series Historia Naturalis 11, 23-28.

Beentjes, M.P., Bull, B., Hurst, R.J. \& Bagley, N.W. (2002). Demersal fish assemblages along the continental shelf and upper slope of the east coast of the South Island, New Zealand. New Zealand Journal of Marine and Freshwater Research 36, 197-223. DOI: 10.1080/00288330.2002.9517080. 
Bell, J. D. (2012). Reproduction and ageing of Australian holocephalans and white-fin swell shark. Ph.D. Thesis. Melbourne: Deakin University.

Belleggia, M., Figueroa, D.E., Sánchez, F. \& Bremec, C. (2012). Long-term changes in the spiny dogfish (Squalus acanthias) trophic role in the southwestern Atlantic. Hydrobiologia 684, 57-67. DOI: 10.1007/s10750-011-0967-y.

Birkhead, T.R. \& Møller, A.P. (1993). Sexual selection and the temporal separation of reproductive events: sperm storage data from reptiles, birds and mammals. Biological Journal of the Linnean Society, 50, 295-311. DOI: 10.1111/j.1095-8312.1993.tb00933.x.

Black, J. \& Tilney, R. (2015). Monitoring New Zealand's trawl footprint for deepwater fisheries: 1989-1990 to 2010-2011. New Zealand Aquatic Environment and Biodiversity Report No. 142. Wellington: Ministry for Primary Industries.

Blackwell, R.G. (2010). Distribution and abundance of deepwater sharks in New Zealand waters, 2000-01 to 2005-06. New Zealand Aquatic Environment and Biodiversity Report No. 57. Wellington: Ministry of Fisheries.

Bor, P.H.F. \& Santos, M.B. (2003). Findings of elasmobranch eggs in the stomachs of sperm whales and other marine organisms. Journal of the Marine Biological Association of the UK 83, 1351-1353. DOI: 10.1017/S0025315403008804.

Bornatowski, H., Braga, R.R. \& Vitule, J.R.S. (2013). Shark mislabeling threatens biodiversity. Science 340, 923-923. DOI: 10.1126/science.340.6135.923-a.

Braccini, J.M., Gillanders, B.M. \& Walker, T.I. (2006). Hierarchical approach to the assessment of fishing effects on non-target chondrichthyans: case study of Squalus megalops in southeastern Australia. Canadian Journal of Fisheries and Aquatic Sciences 63, 2456-2466. DOI: 10.1139/f06-141.

Braccini, J.M., Hamlett, W.C., Gillanders, B.M. \& Walker, T.I. (2007). Embryo development and maternal-embryo nutritional relationships of piked spurdog (Squalus megalops). Marine Biology 150, 727-737. DOI: 10.1007/s00227-006-0393-2.

Bradbury, J. (1981). The evolution of leks. In Natural selection and social behavior: recent research and new theory (Alexander, R.D. \& Tinkle, D.W., eds.), pp. 138-169. Chiron Press

Bubley, W.J., Sulikowski, J.A., Koester, D.M. \& Tsang, P.C. (2013). Using a multiparameter approach to reassess maturity of spiny dogfish, Squalus acanthias, following increased fishing pressure in the Western North Atlantic. Fisheries Research 147, 202-212. DOI: 10.1016/j.fishres.2013.06.004.

Bustamante, C., Kyne, P.M. \& Bennett, M.B. (2013). Comparative morphology of the egg cases of Asymbolus analis, Asymbolus rubiginosus and Figaro boardmani (Carcharhiniformes: Scyliorhinidae) from southern Queensland, Australia. Journal of Fish Biology 83, 133-143. DOI: 10.1111/jfb.12155. 
Cailliet, G.M. \& Goldmann, K.J. (2004). Age determination and validation in chondrichthyan fishes. In Biology of Sharks and Their Relatives (Carrier, J. C., Musick, J. A. \& Heithaus, M.R., eds.), pp. 399-447. Boca Raton, FL: CRC Press. DOI: 10.1201/9780203491317.pt3.

Cailliet, G.M., Smith, W., Mollet, H. \& Goldman, K. (2006). Age and growth studies of chondrichthyan fishes: the need for consistency in terminology, verification, validation, and growth function fitting. Environmental Biology of Fishes 77, 211-228. DOI: 10.1007/s10641-006-9105-5.

Cailliet, G.M. (2015). Perspectives on elasmobranch life-history studies: a focus on age validation and relevance to fishery management. Journal of Fish Biology 87, 1271-1292. DOI: $10.1111 / \mathrm{jfb} .12829$.

Calder, W. A. (1984). Size, function, and life history. New York: Dover Publications, Inc.

Calis, E., Jackson, E.H., Nolan, C.P. \& Jeal, F. (2005). Preliminary age and growth estimates of the rabbitfish, Chimaera monstrosa, with implications for future resource management. Journal of Northwest Atlantic Fishery Science 35, 15-26.

Capapé, C. (2008). Diet of the angular rough shark Oxynotus centrina (Chondrichthyes: Oxynotidae) off the Languedocian coast (southern France, northwestern Mediterranean). Vie et Milieu 58, 57-61.

Capapé, C., Seck, A.A. \& Quignard, J.P. (1999). Observations on the reproductive biology of the angular rough shark, Oxynotus centrina (Oxynotidae). Cybium, 23, 259-271.

Carrier, J.C., Pratt Jr, H.L. \& Martin, L.K. (1994). Group reproductive behaviors in freeliving nurse sharks, Ginglymostoma cirratum. Copeia, 646-656. DOI: 10.2307/1447180.

Chirichigno, F. (1974). Registro de dos especies de Peces Tucanes (Familia: Rhinochimaeridae) en las aguas marinas del Perú. IMARPE.

Christiansen, H.M., Campana, S.E., Fisk, A.T., Cliff, G., Wintner, S.P., Dudley, S.F., Kerr, L.A. \& Hussey, N.E. (2016). Using bomb radiocarbon to estimate age and growth of the white shark, Carcharodon carcharias, from the southwestern Indian Ocean. Marine Biology 163, 1-13. DOI: 10.1007/s00227-016-2916-9.

Clark, M.R. (1999). Fisheries for orange roughy (Hoplostethus atlanticus) on seamounts in New Zealand. Oceanol Acta 22, 593-602. DOI: 10.1016/S0399-1784(00)88950-1.

Clark, I.N., Major, P.J. \& Mollett, N. (1988). Development and implementation of New Zealand's ITQ management system. Marine Resource Economics 5, 325-349.

Clark, M.R., Anderson, O.F., Francis, R.C. \& Tracey, D.M. (2000). The effects of commercial exploitation on orange roughy (Hoplostethus atlanticus) from the continental slope of the Chatham Rise, New Zealand, from 1979 to 1997. Fisheries Research 45, 217238. DOI: 10.1016/S0165-7836(99)00121-6. 
Clark, M.R. \& Rowden, A.A. (2009). Effect of deepwater trawling on the macroinvertebrate assemblages of seamounts on the Chatham Rise, New Zealand. Deep Sea Research Part I: Oceanographic Research Papers 56, 1540-1554. DOI: 10.1016/j.dsr.2009.04.015.

Clark, M.R. \& Dunn, M.R. (2012). Spatial management of deep-sea seamount fisheries: balancing sustainable exploitation and habitat conservation. Environmental Conservation 39, 204-214. DOI: 10.1017/S0376892912000021.

Clark, M.R., Althaus, F., Schlacher, T.A., Williams, A., Bowden, D A. \& Rowden, A.A. (2016). The impacts of deep-sea fisheries on benthic communities: a review. ICES Journal of Marine Science 73(suppl 1), i51-i69. DOI: 10.1093/icesjms/fsv123.

Clarke, M.W., Connolly, P.L. \& Bracken, J.J. (2001). Aspects of reproduction of the deep water sharks Centroscymnus coelolepis and Centrophorus squamosus from west of Ireland and Scotland. Journal of the Marine Biological Association of the UK 81, 1019-1029. DOI: $10.1017 / \mathrm{S} 0025315401005008$.

Clarke, M.W., Connolly, P.L. \& Bracken, J.J. (2002). Age estimation of the exploited deepwater shark Centrophorus squamosus from the continental slopes of the Rockall Trough and Porcupine Bank. Journal of Fish Biology 60, 501-514. DOI: 10.1111/j.10958649.2002.tb01679.x.

Clarke, M.W., Borges, L. \& Officer, R.A. (2005). Comparisons of trawl and longline catches of deepwater elasmobranchs west and north of Ireland. Journal of Northwest Atlantic Fishery Science 35, 429-442. DOI: 10.2960/J.v35.m516.

Clarke, J., Milligan, R.J., Bailey, D.M. \& Neat, F. C. (2015). A scientific basis for regulating deep-sea fishing by depth. Current Biology 25, 2425-2429. DOI: 10.1016/j.cub.2015.07.070.

Coelho, R. \& Erzini, K. (2010). Depth distribution of the velvet belly, Etmopterus spinax, in relation to growth and reproductive cycle: The case study of a deep-water lantern shark with a wide-ranging critical habitat. Marine Biology Research 6, 381-389. DOI: 10.1080/17451000802644706.

Compagno, L.J.V. (1984). FAO Species catalogue, vol 4, part 1 and 2: sharks of the world: an annotated and illustrated catalogue of shark species known to date. FAO fisheries synopsis, 125.

Compagno, L.J.V. \& Dagit, D.D. (2015). Hydrolagus trolli. The IUCN Red List of Threatened Species 2015, e.T60197A70709551. Available at http://www.iucnredlist.org (Accessed 23 September 2016). DOI: 10.2305/IUCN.UK.20154.RLTS.T60197A70709551.en.

Cortés, E. (1999). Standardized diet compositions and trophic levels of sharks. ICES Journal of Marine Science: Journal $d u$ Conseil 56, 707-717. DOI: 10.1006/jmsc.1999.0489. 
Cortés, E. (2000). Life history patterns and correlations in sharks. Reviews in Fisheries Science 8, 299-344. DOI: 10.1080/10408340308951115.

Cortés, E. (2007). Chondrichthyan demographic modelling: an essay on its use, abuse and future. Marine and Freshwater Research 58, 4-6. DOI: 10.1071/MF06191.

Cortés, E., Arocha, F., Beerkircher, L., Carvalho, F., Domingo, A., Heupel, M., Holtzhausen, H., Santos, M.N., Ribera, M. \& Simpfendorfer, C. (2010). Ecological risk assessment of pelagic sharks caught in Atlantic pelagic longline fisheries. Aquatic Living Resources 23, 25-34. DOI: 10.1051/alr/2009044.

Cortés, E., Domingo, A., Miller, P., Forselledo, R., Mas, F., Arocha, F., Campana, S., Coelho, R., Da Silva, C., Hazin, F.H.V., et al. (2015). Expanded ecological risk assessment of pelagic sharks caught in Atlantic pelagic longline fisheries. Collect Vol Sci Pap ICCAT 71, 2637-2688

Cotton, C.F. \& Grubbs, R.D. (2015). Biology of deep-water chondrichthyans: Introduction. Deep Sea Research Part II: Topical Studies in Oceanography 115: 1-10. DOI: 10.1016/j.dsr2.2015.02.030.

Coull, K.A., Jermyn, A.S., Newton, A.W., Henderson, G.I. \& Hall, W.B. (1989). Lengthweight relationships for 88 species of fish encountered in the North East Atlantic. Scottish Fisheries Research Report Number 43. Aberdeen: Department of Agriculture and Fisheries for Scotland (DAFS).

Cox, G., \& Francis, M. (1997). Sharks and rays of New Zealand. Canterbury Univ. Press, Univ. of Canterbury.

Cox, D.L., Walker, P. \& Koob, T.J. (1999). Predation on eggs of the thorny skate. Transactions of the American Fisheries Society 128, 380-384. DOI: 10.1577/15488659(1999)128<0380:POEOTT>2.0.CO;2.

Croft, D.P., James, R. \& Krause, J. (2008). Exploring animal social networks. Princeton, New Jersey: Princeton University Press.

Dean, B. (1906). Chimeroid Fishes and Their Development. The Wilkens-Sheiry Printing Co., Washington.

Dagit, D.D. (2015). Chimaera lignaria. The IUCN Red List of Threatened Species 2015, e.T60185A68617238. Available at http://www.iucnredlist.org (Accessed 23 September 2016). DOI: 10.2305/IUCN.UK.2015-4.RLTS.T60185A68617238.en.

Dagit, D.D. \& Kyne, P.M. (2015). Rhinochimaera pacifica. The IUCN Red List of Threatened Species 2015, e.T60146A68642001. Available at http://www.iucnredlist.org (Accessed $23 \quad$ September 2016). DOI: 10.2305/IUCN.UK.20154.RLTS.T60146A68642001.en.

Dagit, D.D. \& Walls, R.H.L. (2015). Harriotta haeckeli. The IUCN Red List of Threatened Species 2015, e. T60139A70709321. Available at http://www.iucnredlist.org (Accessed 23 September 2016). DOI: 10.2305/IUCN.UK.2015-4.RLTS.T60139A70709321.en 
Dagit, D.D., Walls, R.H.L. \& Buscher, E. (2016). Harriotta raleighana. The IUCN Red List of Threatened Species 2015, e. T60140A3088899. Available at http://www.iucnredlist.org (Accessed 23 September 2016). DOI: 10.2305/IUCN.UK.20161.RLTS.T60140A3088899.en.

Daley, R.K., Stevens, J.D. \& Graham, K.J. (2002). Catch analysis and productivity of the deepwater dogfish resource in southern Australia. FRDC Final Report, 1998/108. Canberra: Fisheries Research and Development Corporation.

Daley, R.K., Williams, A., Green, M., Barker, B. \& Brodie, P. (2015). Can marine reserves conserve vulnerable sharks in the deep sea? A case study of Centrophorus zeehaani (Centrophoridae), examined with acoustic telemetry. Deep Sea Research Part II: Topical Studies in Oceanography 115, 127-136. DOI: 10.1016/j.dsr2.2014.05.017.

Daly-Engel, T.S., Grubbs, R.D., Feldheim, K.A., Bowen, B.W. \& Toonen, R.J. (2010). Is multiple mating beneficial or unavoidable? Low multiple paternity and genetic diversity in the shortspine spurdog Squalus mitsukurii. Marine Ecology Progress Series 403, 255-267. DOI: $10.3354 /$ meps08417.

DeAngelis, D.L. \& Huston, M.A. (1987). Effects of growth rates in models of size distribution formation in plants and animals. Ecological Modelling 36, 119-137. DOI: 10.1016/0304-3800(87)90062-7.

De Roos, A.M., Persson, L. \& McCauley, E. (2003). The influence of size-dependent lifehistory traits on the structure and dynamics of populations and communities. Ecology Letters 6, 473-487. DOI: 10.1046/j.1461-0248.2003.00458.x.

Didier, D.A. (2002). Two new species of chimaeroid fishes from the southwestern Pacific Ocean (Holocephali, Chimaeridae). Ichthyological Research 49, 299-306. DOI: $10.1007 / \mathrm{s} 102280200045$.

Didier, D.A. (2008). Two new species of the genus Hydrolagus Gill (Holocephali: Chimaeridae) from Australia. In Descriptions of new Australian chondrichthyans (Last, P.R., White, W.T., Pogonoski, J.J. eds). pp 349-356. CSIRO Marine and Atmospheric Research Paper.

Didier, D.A. \& Nakaya, K. (1999). Redescription of Rhinochimaera pacifica (Mitsukuri) and first record of $R$. africana Compagno, Stehmann \& Ebert from Japan (Chimaeriformes: Rhinochimaeridae). Ichthyology Research 46, 139-152. DOI: 10.1007/BF02675432.

Didier, D.A. \& Rosenberger, L.J. (2002). The spotted ratfish, Hydrolagus colliei: notes on its biology with a redescription of the species (Holocephali: Chimaeridae). California Fish and Game 88, 112-125.

Didier, D.A., Kemper, J.M. \& Ebert, D.A. (2012). Phylogeny, biology, and classification of extant holocephalans. In Biology of Sharks and Their Relatives, $2^{\text {nd }}$ edn. (Carrier, J. C., Musick, J. A. \& Heithaus, M.R., eds.), pp. 97-124. New York, NY: CRC Press.

Di Giacomo, E.E. \& Perier, M.R. (1994). Reproductive biology of cockfish, Callorhinchus 
callorhynchus (Holocephali: Callorhynchidae), in Patagonian waters. Fisheries Bulletin 92, 531-539.

Domeier, M.L. \& Nasby-Lucas, N. (2013). Two-year migration of adult female white sharks (Carcharodon carcharias) reveals widely separated nursery areas and conservation concerns. Animal Bioteleometry 1. DOI: 10.1186/2050-3385-1-2.

Dragičević, B., Dulčić, J. \& Capapé, C. (2009). Capture of a rare shark, Oxynotus centrina (Chondrichthyes: Oxynotidae) in the eastern Adriatic Sea. Journal of Applied Ichthyology 25, 56-59. DOI: 10.1111/j.1439-0426.2009.01265.x.

Drazen, J.C. \& Seibel, B.A. (2007). Depth-related trends in metabolism of benthic and benthopelagic deep-sea fishes. Limnology and Oceanography 52, 2306-2316. DOI: 10.4319/lo.2007.52.5.2306.

Drazen, J.C. \& Haedrich, R.L. (2012). A continuum of life histories in deep-sea demersal fishes. Deep Sea Research Part I 61, 34-42. DOI: 10.1016/j.dsr.2011.11.002.

Driggers, W., Carlson, J., Cullum, B., Dean, J. \& Oakley, D. (2004). Age and growth of the blacknose shark, Carcharhinus acronotus, in the western North Atlantic Ocean with comments on regional variation in growth rates. Environmental Biology of Fishes 71, 171178. DOI: $10.1007 / \mathrm{s} 10641-004-0105-\mathrm{z}$.

Duffy, C.A.J. (2003). Chimaera panthera. The IUCN Red List of Threatened Species 2015, e. T41793A10548093. Available at http://www.iucnredlist.org (Accessed 23 September 2016). DOI: 10.2305/IUCN.UK.2003.RLTS.T41793A10548093.en.

Dulvy, N.K., Sadovy, Y. \& Reynolds, J.D. (2003). Extinction vulnerability in marine populations. Fish and Fisheries 4, 25-64. DOI: 10.1046/j.1467-2979.2003.00105.x.

Dulvy, N.K., Baum, J.K., Clarke, S., Compagno, L.J., Cortes, E., Domingo, A., Fordham, S., Fowler, S., Francis, M.P., Gibson, C., et al. (2008). You can swim but you can't hide: the global status and conservation of oceanic pelagic sharks and rays. Aquatic Conservation: Marine and Freshwater Ecosystems 18, 459-482. DOI: 10.1002/aqc.975.

Dulvy, N.K., Fowler, S.L., Musick, J.A., Cavanagh, R.D., Kyne, P.M., Harrison, L.R., Carlson, J.K., Davidson, L.N.K., Fordham, S.V., Francis, M.P., et al. (2014). Extinction risk and conservation of the world's sharks and rays. eLife 3, e00590. DOI: 10.7554/eLife.00590.

Dunn, M.R. \& Forman, J.S. (2011). Hypotheses of spatial stock structure in orange roughy Hoplostethus atlanticus inferred from diet, feeding, condition, and reproductive activity. PloS one, 6(11), e26704. DOI: 10.1371/journal.pone.0026704.

Dunn, M.R., Rickard, G.J., Sutton, P.J.H. \& Doonan, I.J. (2009). Nursery grounds of the orange roughy around New Zealand. ICES Journal of Marine Science: Journal du Conseil fsp093. DOI: 10.1093/icesjms/fsp093.

Dunn, M.R., Griggs, L., Forman, J. \& Horn, P. (2010a). Feeding habits and niche separation among the deep-sea chimaeroid fishes Harriotta raleighana, Hydrolagus bemisi and 
Hydrolagus novaezealandiae. Marine Ecology Progress Series 407, 209-225. DOI: $10.3354 /$ meps 08580 .

Dunn, M.R., Szabo, A., McVeagh, M.S. \& Smith, P.J. (2010b). The diet of deepwater sharks and the benefits of using DNA identification of prey. Deep Sea Research Part I: Oceanographic Research Papers 57, 923-930. DOI: 10.1016/j.dsr.2010.02.006.

Dunn, M.R., Stevens, D.W., Forman, J.S. \& Connell, A. (2013). Trophic interactions and distribution of some squaliforme sharks, including new diet descriptions for Deania calcea and Squalus acanthias. PLoS ONE 8, e59938. DOI: 10.1371/journal.pone.0059938.

Ebenman, B. (1988). Dynamics of age- and size-structured populations; intraspecific competition. In Size-structured populations: ecology and evolution (Ebenman, B., Persson, L., eds.), pp. 127-139. Berlin: Springer.

Ebert, D.A. (2005). Reproductive biology of skates, Bathyraja (Ishiyama), along the eastern Bering Sea continental slope. Journal of Fish Biology 66, 618-649. DOI: $10.1111 /$ j.0022-1112.2005.00628.x.

Economakis, A.E. \& Lobel, P.S. (1998). Aggregation behavior of the grey reef shark, Carcharhinus amblyrhynchos, at Johnston Atoll, Central Pacific Ocean. Environmental Biology of Fishes 51, 129-139. DOI: 10.1023/A:1007416813214.

Efron, B. (1981). Nonparametric estimates of standard error: the jackknife, the bootstrap and other methods. Biometrika 68, 589-599. DOI: 10.1093/biomet/68.3.589.

Eschmeyer, W.N., Fricke, R. \& van der Laan, R. (eds.) (2016). Catalog of Fishes. http://researcharchive.calacademy.org/research/ichthyology/catalog/fishcatmain.asp.

Version 2016.

Espinoza, M., Farrugia, T.J., Webber, D.M., Smith, F. \& Lowe, C.G. (2011). Testing a new acoustic telemetry technique to quantify long-term, fine-scale movements of aquatic animals. Fisheries Research 108, 364-371. DOI: 10.1016/j.fishres.2011.01.011.

Etnoyer, P. \& Warrenchuk, J. (2007). A catshark nursery in a deep gorgonian field in the Mississippi Canyon, Gulf of Mexico. Bulletin of Marine Science 81, 553-559.

Farré, M., Tuset, V.M., Cartes, J.E., Massutí, E. \& Lombarte, A. (2016). Depth-related trends in morphological and functional diversity of demersal fish assemblages in the western Mediterranean Sea. Progress in Oceanography 147, 22-37. DOI: 10.1016/j.pocean.2016.07.006.

Feldheim, K.A., Gruber, S.H. \& Ashley, M.V. (2002). The breeding biology of lemon sharks at a tropical nursery lagoon. Proceedings of the Royal Society of London Series B 269, 1655-1661. DOI: 10.1098/rspb.2002.2051.

Finucci, B., Bustamante, C., Jones, E.G. \& Dunn, M.R. (2016a) Reproductive biology and feeding habits of the prickly dogfish Oxynotus bruniensis. Journal of Fish Biology 89, 2326-2344. DOI: 10.1111/jfb.13116. 
Finucci, B., Dunn, M.R., Jones, E.G. \& Anderson, J. (2016b). Reproductive biology of the two deep-sea chimaerids, longnose spookfish (Harriotta raleighana) and Pacific spookfish (Rhinochimaera pacifica). Deep Sea Research Part I: Oceanographic Research Papers. DOI: 10.1016.j.dsr2016.11.008.

Finucci, B., Stevens, D.W., Jones, E.G. and Dunn, M.R. (2017). Some observations on the biology of two rarely seen deep-sea chimaerids, Chimaera carophila and Hydrolagus homonycteris. Journal of Fish Biology 90, 2020-2040. DOI: 10.1111/jfb.13284.

Fitzpatrick, J.L., Kempster, R.M., Daly-Engel, T.S., Collin, S.P. \& Evans, J.P. (2012). Assessing the potential for post-copulatory sexual selection in elasmobranchs. Journal of Fish Biology 80, 1141-1158. DOI: 10.1111/j.1095-8649.2012.03256.x.

Ford, E. (1921). A contribution to our knowledge of the life-histories of the dogfishes landed at Plymouth. Journal of Marine Biological Association of the United Kingdom 12, 468-505.

Ford, R.B., Galland, A., Clark, M.R., Crozier, P., Duffy, C.A.J., Dunn, M., Francis, M.P. \& Wells, R. (2015). Qualitative (Level 1) Risk Assessment of the impact of commercial fishing on New Zealand Chondrichthyans. New Zealand Aquatic Environment and Biodiversity Report No. 157. Wellington: Ministry for Primary Industries.

Francis, M.P. (1979). Checklist of the marine fishes of Kaikoura, New Zealand. Mauri ora 7, 63-71.

Francis, M.P. (1997). Spatial and temporal variation in the growth rate of elephantfish (Callorhinchus milii). New Zealand Journal of Marine and Freshwater Research 31, 9-23. DOI: 10.1080/00288330.1997.9516741.

Francis, M.P. (1998). New Zealand shark fisheries: development, size and management. Marine and Freshwater Research 49, 579-591. DOI: 10.1071/MF97076.

Francis, M.P. (2003). Oxynotus bruniensis. The IUCN Red List of Threatened Species 2003, e.T41840A10578144. Available at http://www.iucnredlist.org (Accessed 25 March 2016). DOI: 10.2305/IUCN.UK.2003.RLTS.T41840A10578144.en.

Francis, M.P. (2006a). Morphometric minefields - towards a measurement standard for chondrichthyan fishes. Environmental Biology of Fishes 77, 407-421. DOI: 10.1007/s10641-006-9109-1.

Francis, M.P. (2006b). Distribution and biology of the New Zealand endemic catshark, Halaelurus dawsoni. Environmental Biology of Fishes 75, 295-306. DOI: 10.1007/s10641006-0026-0.

Francis, M.P. (2015). Geographic distribution of commercial catches of cartilaginous fishes in New Zealand waters, 2008-13. New Zealand Aquatic Environment and Biodiversity Report No. 156. Wellington: Ministry for Primary Industries. 
Francis, M.P. \& Ó Maolagáin, C. (2000). Age and growth of ghost sharks. Final Research Report for Ministry of Fisheries Project GSH1999/01 Objective 1. (Unpublished report held by Ministry for Primary Industries, Wellington).

Francis, M.P. \& Stevens, J.D. (2000). Reproduction, embryonic development, and growth of the porbeagle shark, Lamna nasus, in the southwest Pacific Ocean. Fishery Bulletin 98, 41-63.

Francis, M.P., Hurst, R.J., McArdle, B.H., Bagley, N.W. \& Anderson, O.F. (2002). New Zealand demersal fish assemblages. Environmental Biology of Fishes 65, 215-234. DOI: 10.1023/A:1020046713411.

Francis, M.P. \& Lyon, W.S. (2012). Review of research and monitoring studies on New Zealand sharks, skates, rays and chimaeras, 2008-2012. New Zealand Aquatic Environment and Biodiversity Report No. 102. Wellington: Ministry for Primary Industries.

Francis, M.P. \& Lyon, W.S. (2013). Review of anthropogenic impacts other than fishing on cartilaginous fishes. New Zealand Aquatic Environment and Biodiversity Report No. 107. Wellington: Ministry for Primary Industries.

Francis, M.P., Duffy, C.A.J. \& Lyon, W. (2015). Spatial and temporal habitat use by white sharks (Carcharodon carcharias) at an aggregation site in southern New Zealand. Marine and Freshwater Research 66, 900-918. DOI: 10.1071/MF14186.

Freer, D.W.L. \& Griffiths, C.L. (1993a). The fishery for, and general biology of, the St Joseph Callorhinchus capensis (Dumeril) off the south-western Cape, South Africa. South African Journal of Marine Science 13, 63-74. DOI: 10.2989/025776193784287428.

Freer, D.W.L. \& Griffiths, C.L. (1993b). Estimation of age and growth in the St Joseph Callorhinchus capensis (Dumeril). South African Journal of Marine Science 13, 75-81. DOI: $10.2989 / 025776193784287194$.

Frisk, M.G., Miller, T.J. \& Fogarty, M.J. (2001). Estimation and analysis of biological parameters in elasmobranch fishes: a comparative life history study. Canadian Journal of Fisheries and Aquatic Sciences 58, 969-981. DOI: 10.1139/f01-051.

Froese, R. (2006). Cube law, condition factor and weight-length relationships: history, meta-analysis and recommendations. Journal of Applied Ichthyology 22, 241-253. DOI: 10.1111/j.1439-0426.2006.00805.x.

Froese, R., Thorson, J.T. \& Reyes, R.B. (2014). A Bayesian approach for estimating lengthweight relationships in fishes. Journal of Applied Ichthyology 30, 78-85. DOI: 10.1111/jai.12299.

Fulton, T.W. (1904). The rate of growth of fishes. Twenty-second Annual Report, Part III. Fisheries Board of Scotland, Edinburgh.

Gage, J.D. \& Tyler, P.A. (1991). Deep-sea biology: a natural history of organisms at the deep-sea floor. United Kingdom: Cambridge University Press. 
Gallagher, A.J., Kyne, P.M. \& Hammerschlag, N. (2012). Ecological risk assessment and its application to elasmobranch conservation and management. Journal of Fish Biology 80, 1727-1748. DOI: 10.1111/j.1095-8649.2012.03235.x.

Galland, A. R. (2015). Demographics of Etmopterus lucifer (Lucifer dogfish). M.Sc. Thesis. New Zealand: Victoria University of Wellington.

García, V.B., Lucifora, L.O. \& Myers, R.A. (2008). The importance of habitat and life history to extinction risk in sharks, skates, rays and chimaeras. Proceedings of the Royal Society of London Series B 1630, 83-89. DOI: 10.1098/rspb.2007.1295.

Garman, S. (1901). Genera and families of the chimaeroids. Proceedings of the New England Zoological Club 2, 75-77.

Garrick, J.A.F. (1959). Studies on New Zealand Elasmobranchii-Part IX. Scymnodon plunketi (Waite, 1910), an abundant deep-water shark of New Zealand waters. Transactions of the Royal Society of New Zealand 87, 271-282.

Garrick, J.A.F. (1960). Studies on New Zealand Elasmobranchii. Part XII. The species of Squalus from New Zealand and Australia; and a general account and key to the New Zealand Squaloidea. Transactions of the Royal Society of New Zealand 88, 519-557.

Garrick, J.A.F. (1971). Harriotta raleighana, a long-nosed chimaera (Family Rhinochimaeridae), in New Zealand waters. Transactions of the Royal Society of New Zealand 1, 203-213.

Garrick, J.A.F. \& Inada, T. (1975). Dimensions of long-nosed Chimaera Harriotta Raleighana from New Zealand. New Zealand Journal of Marine and Freshwater Research 9, 159-167.

García de La Rosa, S.B., \& Sánchez, M.F. (1997). Alimentación de Squalus acanthias y predación sobre Merluccius hubbsi en el Mar Argentino entre $34^{\circ} 47^{\prime}-47^{\circ}$ S. Revista de Investigación y Desarrollo Pesquero 11, 119-133.

Gaston, K.A., Jacquemin, S.J. \& Lauer, T.E. (2013). The influence of preservation on fish morphology in museum collections based on two species of the genus Lepomis (Actinopterygii: Perciformes: Centrarchidae). Acta Ichthyologica et Piscatoria 43, 219227.

Geer, L. Y., Marchler-Bauer, A., Geer, R. C., Han, L., He, J., He, S., Liu, C., Shi, W. \& Bryant, S.H. (2010). The NCBI BioSystems database. Nucleic Acids Research 38, D492D496. DOI: 10.1093/nar/gkp858.

Geraghty, P.T., Jones, A.S., Stewart, J. \& Macbeth, W.G. (2012). Micro-computed tomography: an alternative method for shark ageing. Journal of Fish Biology 80, 12921299. DOI: $10.1111 / \mathrm{j} .1095-8649.2011 .03188 . x$.

Gerritsen, H.D. \& McGrath, D. (2007). Significant differences in the length-weight relationships of neighbouring stocks can result in biased biomass estimates: examples of 
haddock (Melanogrammus aeglefinus, L.) and whiting (Merlangius merlangus, L.). Fisheries Research 85, 106-111. DOI: 10.1016/j.fishres.2007.01.004.

Girard, M. \& Du Buit, M. H. (1999). Reproductive biology of two deep-water sharks from the British Isles, Centroscymnus coelolepis and Centrophorus squamosus (Chondrichthyes: Squalidae). Journal of the Marine Biological Association of the UK 79, 923-931.

Gordon, D.P. (2013). Life on the Edge: Parachnoidea (Ctenostomata) and Barentsia (Kamptozoa) on Bathymodiolin Mussels from an active submarine volcano in the Kermadec Volcanic Arc. In: Bryozoan Studies 2010 (Ernst, A., Schäfer, P. \& Scholz, J., eds.), pp. 75-89. Berlin: Springer.

Goldman, K.J., Cailliet, G.M., Andrews, A.H. \& Natanson, L.J. (2012). Assessing the age and growth of chondrichthyan fishes. In Biology of Sharks and Their Relatives, $2^{\text {nd }}$ edn. (Carrier, J. C., Musick, J. A. \& Heithaus, M.R., eds.), pp. 423-451. New York, NY: CRC Press.

Gorman, T.B. (1963). Biological and economic aspects of the elephant fish, Callorhynchus milii Bory, in Pegasus Bay and the Canterbury bight. New Zealand Marine Department Fisheries Technical Report 8.

Graham, K.J., Andrew, N.L. \& Hodgson, K.E. (2001). Changes in relative abundance of sharks and rays on Australian South East Fishery trawl grounds after twenty years of fishing. Marine and Freshwater Research 52, 549-561. DOI: 10.1071/MF99174.

Graham, K.J. \& Daley, R.K. (2011). Distribution, reproduction and population structure of three gulper sharks (Centrophorus, Centrophoridae) in south-east Australian waters. Marine and Freshwater Research 62, 583-595. DOI: 10.1071/MF10158.

Grinnell, J., Packer, C., \& Pusey, A.E. (1995). Cooperation in male lions: kinship, reciprocity or mutualism? Animal Behaviour. 49, 95-105. DOI: 10.1016/00033472(95)80157-X.

Gruber, S.H., Nelson, D.R. \& Morrissey, J.F. (1988). Patterns of activity and space utilization of lemon sharks, Negaprion brevirostris, in a shallow Bahamian lagoon. Bulletin of Marine Science. 43, 61-76.

Guallart, J., García-Salinas, P., Ahuir-Baraja, A.E., Guimerans, M., Ellis, J.R. \& Roche, M. (2015). Angular roughshark Oxynotus centrina (Squaliformes: Oxynotidae) in captivity feeding exclusively on elasmobranch eggs: an overlooked feeding niche or a matter of individual taste? Journal of Fish Biology 87, 1072-1079. DOI: 10.1111/jfb.12761.

Guindon, S. \& Gascuel, O. (2003). A simple, fast, and accurate algorithm to estimate large phylogenies by maximum likelihood. Systematic Biology 52, 696-704. DOI: 10.1080/10635150390235520.

Guttridge, T.L., Gruber, S.H., Gledhill, K.S., Croft, D.P., Sims, D.W. \& Krause, J. (2009). Social preferences of juvenile lemon sharks, Negaprion brevirostris. Animal Behaviour 78, 543-548. DOI: 10.1016/j.anbehav.2009.06.009. 
Guttridge, T.L., Gruber, S.H., Franks, B.R., Kessel, S.T., Gledhill, K.S., Uphill, J., Krause, J. \& Sims, D.W. (2011). Deep danger: intra-specific predation risk influences habitat use and aggregation formation of juvenile lemon sharks Negaprion brevirostris. Marine Ecology Progress Series 445, 279-291. DOI: 10.3354/meps09423.

Guttridge, T.L., Gruber, S.H., Franks, B.R., Kessel, S.T., Gledhill, K.S., Uphill, J., Krause, J. \& Sims, D.W. (2012). Deep danger: intra-specific predation risk influences habitat use and aggregation formation of juvenile lemon sharks Negaprion brevirostris. Marine Ecology Progress Series 445, 279-291. DOI: 10.3354/meps09423.

Haedrich, R.L. \& Rowe, G.T. (1977). Megafaunal biomass in the deep sea. Nature 269, 141-142. DOI: 10.1038/269141a0.

Hallett, C.S. \& Daley, R.K. (2010). Feeding ecology of the southern lanternshark (Etmopterus baxteri) and the brown lanternshark (E. unicolor) off southeastern Australia. ICES Journal of Marine Science: Journal du Conseil fsq143. DOI: 10.1093/icesjms/fsq143.

Hanchet, S. (1988). Reproductive biology of Squalus acanthias from the east coast, South Island, New Zealand. New Zealand Journal of Marine and Freshwater Research 22, 537549.

Heath, R. A. (1985). A review of the physical oceanography of the seas around New Zealand-1982._New Zealand Journal of Marine and Freshwater Research 19, 79-124. DOI: $10.1080 / 00288330.1985 .9516077$.

Henry, L.A., Navas, J.M., Hennige, S.J., Wicks, L.C., Vad, J. \& Roberts, J.M. (2013). Cold-water coral reef habitats benefit recreationally valuable sharks. Biological Conservation 161, 67-70. DOI: 10.1016/j.biocon.2013.03.002.

Henry, L.A., Vad, J., Findlay, H.S., Murillo, J., Milligan, R., \& Roberts, J.M. (2014). Environmental variability and biodiversity of megabenthos on the Hebrides Terrace Seamount (Northeast Atlantic). Scientific Reports 4, 55-89. DOI: 10.1038/srep05589.

Henry, L.A., Roberts, J.M. (2016). Global biodiversity of cold-water coral reefs. In Marine Animal Forests: the Ecology of Benthic Biodiversity Hotspots (Rossi, S., Bramanti, L., Gori, A. \& Orejas, C., eds). New York, NY: Springer.

Henry, L.A., Stehmann, M.F.W., De Clippele, L., Findlay, H.S., Golding, N. \& Roberts, J.M. (2016). Seamount egg-laying grounds of the deep-water skate Bathyraja richardsoni. Journal of Fish Biology 89, 1473-1481. DOI: 10.1111/jfb.13041.

Hensor, E., Couzin, I.D., James, R. \& Krause, J. (2005). Modelling density-dependent fish shoal distributions in the laboratory and field. Oikos 110, 344-352. DOI: 10.1111/j.00301299.2005.13513.x.

Heupel, M.R. \& Simpfendorfer, C.A. (2005). Quantitative analysis of aggregation behavior in juvenile blacktip sharks. Marine Biology 147, 1239-1249. DOI: 10.1007/s00227-0050004-7. 
Heupel, M.R., Carlson, J.K. \& Simpfendorfer, C.A. (2007). Shark nursery areas: concepts, definition, characterization and assumptions. Marine Ecology Progress Series 337, 287297. DOI: 10.3354/meps337287.

Heupel, M.R. \& Simpfendorfer, C.A. (2010). Science or slaughter: need for lethal sampling of sharks. Conservation Biology 24, 1212-1218. DOI: 10.1111/j.1523-1739.2010.01491.x.

Heymans, J.J., Howell, K.L., Ayers, M., Burrows, M.T., Gordon, J.D.M., Jones, E.G. \& Neat, F. (2010). Do we have enough information to apply the ecosystem approach to management of deep-sea fisheries? An example from the West of Scotland. ICES Journal of Marine Science: Journal du Conseil 68, 265-280. DOI: 10.1093/icesjms/fsq065.

Hile, R. (1936). Age and growth of the cisco Leucichthys artedi (Le Sueur), in the lakes of the north-eastern highlands, Wisconsin. Bulletin of the U.S. Bureau of Fisheries 48, 211317.

Hobday, A.J., Dowdney, J., Bulman, C., Sporcic, M., Fuller, M. \& Ling, S. (2007). Ecological risk assessment for the effects of fishing: methodology. Australian Fisheries Management Authority Report R04/1072. Canberra: Bureau of Rural Sciences

Hobday, A.J., Smith, A.D.M., Stobutzki, I.C., Bulman, C., Daley, R., Dambacher, J.M., Deng, R.A., Dowdney, J., Fuller, M., Furlani, D., et al. (2011). Ecological risk assessment for the effects of fishing. Fisheries Research 108, 372-384. DOI: 10.1016/j.fishres.2011.01.013.

Hoff, G.R. (2008). A nursery site of the Alaska skate (Bathyraja parmifera) in the eastern Bering Sea. Fishery Bulletin 106, 233-244.

Hoff, G.R. (2009). Skate Bathyraja spp. egg predation in the eastern Bering Sea. Journal of Fish Biology 74, 250-269. DOI: 10.1111/j.1095-8649.2008.02137.x.

Hoff, G.R. (2010). Identification of skate nursery habitat in the eastern Bering Sea. Marine Ecology Progress Series 403, 243-254. DOI: 10.1111/jfb.12939.

Hoff, G.R. (2016). Identification of multiple nursery habitats of skates in the eastern Bering Sea. Journal of Fish Biology 88, 1746-1757. DOI: 10.1111/jfb.12939.

Höglund, J. \& Alatalo, R.V. (1995). Leks. New Jersey: Princeton University Press.

Holt, R.E., Foggo, A., Neat, F.C. \& Howell, K.L. (2013). Distribution patterns and sexual segregation in chimaeras: implications for conservation and management. ICES Journal of Marine Science: Journal du Conseil. DOI: 10.1093/icesjms/fst058.

Horn, P.L. (1997). Biology and commercial landings and a stock assessment of ghost sharks (Hydrolagus spp.) in New Zealand waters. New Zealand Fisheries Assessment Research Document $97 / 3$.

Huber, D.R., Dean, M.N. \& Summers, A.P. (2008). Hard prey, soft jaws and the ontogeny of feeding mechanics in the spotted ratfish Hydrolagus colliei. Journal of the Royal Society Interface 5, 941-953. DOI: 10.1098/rsif.2007.1325. 
Hurst, R.J., Bagley, N., Chatterton, T., Hanchet, S., Schofield, K. \& Vignaux, M. (1992). Standardisation of hoki/middle depth time series trawl surveys. MAF Fisheries Greta Point Internal Report 194.

Hussey, N E., Wintner, S.P., Dudley, S.F., Cliff, G., Cocks, D.T. \& MacNeil, M.A. (2010). Maternal investment and size-specific reproductive output in carcharhinid sharks. Journal of Animal Ecology 79, 184-193. DOI: 10.1111/j.1365-2656.2009.01623.x.

Hyslop, E.J. (1980). Stomach contents analysis: review of methods and their applications. Journal of Fish Biology 17, 411-429. DOI: 10.1111/j.1095-8649.1980.tb02775.x.

ICCAT (2008). Report of the 2007 inter-sessional meeting of the sub-committee on ecosystems. SCRS 2007/010 Collect Vol Sci Pap ICCAT 62, 1671-1720.

ICES (2009). Report of the Joint Meeting between ICES Working Group on Elasmobranch Fishes (WGEF) and ICCAT Shark Subgroup. ICES CM 2009/ACOM, 16.

ICES (2010). Report of the Workshop on Sexual Maturity Staging of Elasmobranchs (WKMSEL), 11-15 October 2010, Valetta, Malta, ICES Document CM 2010/ACOM 2010, 48.

ICES (2015). Report of the ICES/NAFO Joint Working Group on Deep-water Ecology (WGDEC). ICES Document CM 2015/ACOM, 27.

Iitembu, J.A. \& Richoux, N.B. (2015). Trophic relationships of hake (Merluccius capensis and $M$. paradoxus) and sharks (Centrophorus squamosus, Deania calcea and D. profundorum) in the Northern (Namibia) Benguela Current region. African Zoology 50, 273-279.

Inada, T. \& Garrick, J.A.F. (1979). Rhinochimaera pacifica, a long-snouted chimaera (Rhinochimaeridae), in New Zealand waters. Japan Journal of Ichthyology 25, 235-243.

Irvine, S.B., Stevens, J.D. \& Laurenson, L.J. (2006). Surface bands on deepwater squalid dorsal-fin spines: an alternative method for ageing Centroselachus crepidater. Canadian Journal of Fisheries and Aquatic Sciences 63, 617-627. DOI: 10.1139/f05-237.

Irvine, S.B., Daley, R.K., Graham, K.J. \& Stevens, J.D. (2012). Biological vulnerability of two exploited sharks of the genus Deania (Centrophoridae). Journal of Fish Biology 80, 1181-1206. DOI: 10.1111/j.1095-8649.2012.03262.x.

Ismen, A., Cigdem Yigin, C., Altinagac, U. \& Ayaz, A. (2009). Length-weight relationships for ten shark species from Saros Bay (North Aegean Sea). Journal of Applied Ichthyology 25, 109-112. DOI: 10.1111/j.1439-0426.2009.01263.x.

Jacob, W., McClatchie, S., Probert, P.K. \& Hurst, R.J. (1998). Demersal fish assemblages off southern New Zealand in relation to depth and temperature. Deep Sea Research Part I: Oceanographic Research Papers 45, 2119-2155. DOI: 10.1016/S0967-0637(98)00051-X. 
Jacoby, D.M., Busawon, D.S. \& Sims, D.W. (2010). Sex and social networking: the influence of male presence on social structure of female shark groups. Behavioural Ecology arq061. DOI: $10.1093 /$ beheco/arq061.

Jacoby, D.M.P., Croft, D.P. \& Sims, D.W. (2012). Social behaviour in sharks and rays: analysis, patterns and implications for conservation. Fish and Fisheries 13, 399-417. DOI: 10.1111/j.1467-2979.2011.00436.x.

Jawad, L.A. (2003). The effect of formalin, alcohol and freezing on some body proportions of Alepes djeddaba (Pisces: Carangidae) collected from the Red Sea coast of Yemen. Revista de Biología Marina y Oceanografía 38, 77-80.

Jobling, M. (1997). Temperature and growth: modulation of growth rate via temperature change. In Seminar Series - Society for Experimental Biology, pp. 225-254. United Kingdom: Cambridge University Press.

Jobling, M. (2002). Environmental factors and rates of development and growth. Handbook of fish biology and fisheries 1, 97-122.

Jones, B.C. \& Geen, G.H. (1977). Morphometric changes in an elasmobranch (Squalus acanthias) after preservation. Canadian Journal of Zoology 55, 1060-1062. DOI: 10.1139/z77-135.

Johnson, A.G. (1967). Biology of the ratfish, Hydrolagus colliei (Lay and Bennett). P.h.D Thesis. Oregon: Oregon State University.

Karachle, P. K. \& Stergiou, K.I. (2012). Morphometrics and allometry in fishes. In Morphometrics (Wahl, C., ed.), pp. 65-86. InTech.

Kemper, J.M., Ebert, D.A., Naylor, G.J.P. \& Didier, D.A. (2014). Chimaera carophila (Chondrichthyes: Chimaeriformes: Chimaeridae), a new species of chimaera from New Zealand. Bulletin of Marine Science 91, 63-81. DOI: 10.5343/bms.2014.1042.

Kimber, J.A., Sims, D.W., Bellamy, P.H. \& Gill, A.B. (2009). Male-female interactions affect foraging behaviour within groups of small-spotted catshark, Scyliorhinus canicula. Animal Behaviour 77, 1435-1440. DOI: 10.1016/j.anbehav.2009.02.014.

Kimura, M. (1980). A simple method for estimating evolutionary rates of base substitutions through comparative studies of nucleotide sequences. Journal of Molecular Evolution 16,111-120. DOI: 10.1007/BF01731581.

King, K.J. \& Clark, M.R. (1987). Sharks from the upper continental slope - are they of value. Catch 14, 3-6.

King, J.R. \& McPhie, R.P. (2013). Preliminary age, growth and maturity estimates of spotted ratfish (Hydrolagus colliei) in British Columbia. Deep Sea Research Part II: Topical Studies in Oceanography 115, 55-63. DOI:10.1016/j.dsr2.2013.11.002.

Kirby, D.S. \& Molony, B. (2006). An ecological risk assessment for species caught in WCPO longline and purse seine fisheries. WCPFC SC2 EB WP-1. Manila: WCPFC. 
Klimley, P.A. (1987). The determinants of sexual segregation in the scalloped hammerhead shark, Sphyrna lewini. Environmental Biology of Fishes. 18, 27-40. DOI: 10.1007/BF00002325.

Kokuho, T., Nakaya, K. \& Kitagawa, D. (2003). Distribution and reproductive biology of the nine-spot ratfish Hydrolagus barbouri (Holocephali; Chimaeridae). Memoirs of the Graduate School of Fisheries Sciences, Hokkaido University 50, 63-87.

Koslow, J., Boehlert, G.W., Gordon, J.D.M., Haedrich, R.L., Lorance, P. \& Parin, N. (2000). Continental slope and deep-sea fisheries: implications for a fragile ecosystem. ICES Journal of Marine Science 57, 548-557. DOI: 10.1006/jmsc.2000.0722.

Krause, J. \& Ruxton, G.D. (2002). Living in groups. New York, NY: Oxford University Press.

Kulbicki, M., Guillemot, N., \& Amand, M. (2005). A general approach to length-weight relationships for New Caledonian lagoon fishes. Cybium 29, 235-252.

Kyne, P.M. \& Simpfendorfer, C.A. (2007). A collation and summarization of available data on deepwater Chondrichthyans: biodiversity, life history and fisheries. IUCN Shark Specialist Group.

Kyne, P.M. \& Simpfendorfer, C.A. (2010). Deepwater chondrichthyans. In Sharks and Their Relatives II. Biodiversity, Adaptive Physiology, and Conservation (Carrier, J. C., Musick, J. A., Heithaus, M. R., eds.), pp. 37-113. Boca Raton, FL: CRC Press.

Last, P.R. \& Stevens, J.D. (2009). Sharks and Rays of Australia, Second Ed. Hobart: CSIRO Marine and Atmospheric Research.

Last, P.R., Séret, B. \& Naylor, G.J. (2016). A new species of guitarfish, Rhinobatos borneensis sp. nov. with a redefinition of the family-level classification in the order Rhinopristiformes (Chondrichthyes: Batoidea). Zootaxa 4117, 451-475. DOI: 10.11646/zootaxa.4117.4.1.

Le Cren, E.D. (1951) The length-weight relationship and seasonal cycle in gonad weight and condition in the perch (Perca fluviatilis). Journal of Animal Ecology 20, 201-219.

Leduc, D., Rowden, A.A., Torres, J.G., Nodder, S.D. \& Pallentin, A. (2015). Distribution of macro-infaunal communities on Chatham Rise, Southwest Pacific: Implications for management of seabed mining. Deep Sea Research I: Oceanographic Research Papers 99: 105-118. DOI: 10.1016/j.dsr.2015.01.006.

Le Gros Clark, W.E. \& Medawar, P.B. (1945). Essays on growth and form presented to D'Arcy Wentworth Thompson. In Essays on growth and form presented to D'Arcy Wentworth Thompson. Clarendon Press.

Librado, P. \& Rozas, J. (2009). DnaSP v5: a software for comprehensive analysis of DNA polymorphism data. Bioinformatics 25, 1451-1452. DOI: 10.1093/bioinformatics/btp187. 
Long, D.J. (1996). First confirmed record of teleost predation on a shark egg case. California Fish and Game 82, 103-104.

Lowe, C.G., Wetherbee, B.M., Crow, G.L. \& Tester, A.L. (1996). Ontogenetic dietary shifts and feeding behavior of the tiger shark, Galeocerdo cuvier, in Hawaiian waters. Environmental Biology of Fishes 47, 203-211. DOI: 10.1007/BF00005044.

Luchetti, E.A., Iglésias, S.P. \& Sellos, D.Y. (2011). Chimaera opalescens $n$. sp., a new chimaeroid (Chondrichthyes: Holocephali) from the north-eastern Atlantic Ocean. Journal of Fish Biology 79, 399-417. DOI: 10.1111/j.1095-8649.2011.03027.x.

Lucifora, L.O., Valero, J.L. \& García, V.B. (1999). Length at maturity of the greeneye spurdog shark, Squalus mitsukurii (Elasmobranchii: Squalidae), from the SW Atlantic, with comparisons with other regions. Marine and Freshwater Research 50, 629-632. DOI: http://dx.doi.org/10.1071/MF98167.

Lucifora, L.O., Menni, R.C. \& Escalante, A.H. (2002). Reproductive ecology and abundance of the sand tiger shark, Carcharias taurus, from the southwestern Atlantic ICES Journal of Marine Science: Journal $d u$ Conseil 59, 553-561. DOI: $10.1006 / j m s c .2002 .1183$.

Lucifora, L.O. \& García, V.B. (2004). Gastropod predation on egg cases of skates (Chondrichthyes, Rajidae) in the southwestern Atlantic: quantification and life history implications. Marine Biology 145, 917-922. DOI: 10.1007/s00227-004-1377-8.

Mace, P.M., Sullivan, K.J. \& Cryer, M. (2013). The evolution of New Zealand's fisheries science and management systems under ITQs. ICES Journal of Marine Science: Journal du Conseil fst159. DOI: 10.1093/icesjms/fst 159 .

MacGibbon, D.J. (2016). Fishery characterisation and standardised CPUE analyses for dark ghost shark, Hydrolagus novaezealandiae (Fowler, 1911) (Chimaeridae), 1989-90 to 2010-11. New Zealand Fisheries Assessment Report 9, 162. Wellington: Ministry for Primary Industries.

MacGibbon, D.J. \& Fu, D. (2013). Fishery characterisation and standardised CPUE analyses for pale ghost shark, Hydrolagus bemisi (Didier, 2002) (Chimaeridae), 1989-90 to 2009-10. New Zealand Fisheries Assessment Report 33, 120. Wellington: Ministry for Primary Industries.

MacPherson, E. (1979). Estudio sobre las relaciones tróficas en peces bentónicos de la costa catalana. Ph.D. Thesis. Spain: Universitat de Barcelona.

Macpherson, E. (1980). Regime alimentaire de Galeus melastomus Raønesque, 1810, Etmopterus spinax (L., 1758) et Scymnorhinus licha (Bonnaterre, 1788) en Mediterranee occidentale. Vie et Milieu 30, 139-148.

Magurran, A.E. \& Seghers, B.H. (1994). A cost of sexual harassment in the guppy, Poecilia reticulata. Proceedings of the Royal Society of London Series B 258, 89-92. DOI: 10.1098/rspb.1994.0147. 
Malagrino, G., Takemura, A. \& Mizue, K. (1977). Studies on Holocephali-I On the morphology and ecology of Chimaera phantasma, and male reproductive organs. 長崎大 学水産学部研究報告, 42, 11-19.

Malagrino, G., Takemura, A. \& Mizue, K. (1981). Studies on Holocephali-II On the Reproduction of Chimaera phantasma JORDAN et SNYDER Caught in the Coastal Waters of Nagasaki. 長崎大学水産学部研究報告 51, 1-7.

Matallanas, J. (1982). Feeding habits of Scymnorhinus licha in Catalan waters. Journal of Fish Biology 20, 155-163. DOI: 10.1111/j.1095-8649.1982.tb03916.x.

Mathews, C.P. (1975). Note on the ecology of the ratfish, Hydrolagus collei, in the Gulf of California. California Fish and Game 61, 47-53.

Mauchline, J. \& Gordon, J.D.M. (1983). Diets of the sharks and chimaeroids of the Rockall Trough, Northeastern Atlantic Ocean. Marine Biology 75, 269-278. DOI: 10.1007/BF00406012.

McClatchie, S., Millar, R.B., Webster, F., Lester, P. ., Hurst, R. \& Bagley, N. (1997). Demersal fish community diversity off New Zealand: Is it related to depth, latitude and regional surface phytoplankton? Deep Sea Research Part I: Oceanographic Research Papers 44, 647-667. DOI: 10.1016/S0967-0637(96)00096-9.

McCutcheon, F.I.A. (1980). Comparative Study of Some Aspects of the Biology of Two New Zealand Ghost Sharks (Elasmobranchii: Holocephalis: Chimaeridae: Hydrolagus Novaezelandiae (Fowler, 1911) and Hydrolagus Sp. A (Sp. Nov.?). PhD dissertation. Wellington: Victoria University of Wellington.

McLean, D.L., Green, M., Harvey, E.S., Williams, A., Daley, R. \& Graham, K.J. (2015). Comparison of baited longlines and baited underwater cameras for assessing the composition of continental slope deepwater fish assemblages off southeast Australia. Deep Sea Research Part I: Oceanographic Research Papers 98, 10-20. DOI: 10.1016/j.dsr.2014.11.013.

McMillan, P.J., Francis, M.P., James, G.D., Paul, L.J., Marriott, P.J., Mackay, E., Wood, B.A., Griggs, L.H., Sui, H. \& Wei, F. (2011). New Zealand fishes. Volume 1: A field guide to common species caught by bottom and midwater fishing. New Zealand Aquatic Environment and Biodiversity Report No. 68. Wellington: Ministry of Fisheries.

Megalofonou, P. \& Damalas, D. (2004). Morphological and biological characteristics of a gravid angular rough shark (Oxynotus centrina) and its embryos from the eastern Mediterranean Sea. Cybium 28, 105-110.

Messing, C.G., Stanley, K., Reed, J.K. \& Gilmore, R.G. (2013). The first in situ habitat observations and images of the Caribbean roughshark, Oxynotus caribbaeus Cervigón, 1961 (Squaliformes: Oxynotidae). Proceedings of the Biological Society of Washington 126, 234-239. DOI: 10.2988/0006-324X-126.3.234.

Miller, S.A., Dykes, D.D. \& Polesky, H.F.R.N. (1988). A simple salting out procedure for extracting DNA from human nucleated cells. Nucleic acids research, 16, 1215. 
Ministry of Fisheries (2008). New Zealand National Plan of Action for the Conservation and Management of Sharks. Wellington: Ministry of Fisheries.

Mitani, J.C. \& Watts, D.P. (1999). Demographic influences on the hunting behavior of chimpanzees. American Journal of Physical Anthropology 109, 439-454. DOI: 10.1002/(SICI)1096-8644(199908)109:4<439::AID-AJPA2>3.0.CO;2-3.

Moe, B. \& Cotton, C. (2016). Estimating length-at-age for the little gulper shark Centrophorus cf. uyato in the Gulf of Mexico: an evaluation of alternative aging techniques. Joint Meeting of Ichthyologists and Herpetologists, 2016 July 6-10. New Orleans, LA.

Moore, D.M., Neat, F.C. \& McCarthy, I.D. (2013). Population biology and ageing of the deep water sharks Galeus melastomus, Centroselachus crepidater and Apristurus aphyodes from the Rockall Trough, north-east Atlantic. Journal of Marine Biological Association of the United Kingdom 93, 1941-1950. DOI: 10.1017/S0025315413000374.

Morato, T., Afonso, P., Lourinho, P., Barreiros, J.P., Santos, R.S. \& Nash, R.D.M. (2001). Length-weight relationships for 21 coastal fish species of the Azores, north-eastern Atlantic. Fisheries Research 50, 297-302. DOI: 10.1016/S0165-7836(00)00215-0.

Morato, T., Cheung, W.W.L. \& Pitcher, T.J. (2006a). Vulnerability of seamount fish to fishing: fuzzy analysis of life history attributes. Journal of Fish Biology 68, 209-221. DOI: 10.1007/BF00406012.

Morato, T., Watson, R., Pitcher, T.J. \& Pauly, D. (2006b). Fishing down the deep. Fish and Fisheries 7, 24-34. DOI: 10.1111/j.1467-2979.2006.00205.x.

Morato, T. \& Clark, M.R. (2007) Seamount fishes: ecology and life histories. In: Seamounts: Ecology, Fisheries, and Conservation (Pitcher, T.J., Morato, T., Hart, P.J.B., Clark, M.R., Haggan, N.\& Santos, R.S., eds.) pp. 170-188. Oxford, UK: Blackwell Publishing.

Morson, J.M. \& Morrissey, J.F. (2007). Morphological variation in the electric organ of the little skate (Leucoraja erinacea) and its possible role in communication during courtship. Environmental Biology of Fishes 80, 267-275. DOI: 10.1007/s10641-007-9221$\mathrm{x}$.

Motta, P.J. \& Wilga, C.D. (2001). Advances in the study of feeding behaviors, mechanisms, and mechanics of sharks. In The behavior and sensory biology of elasmobranch fishes: an anthology in memory of Donald Richard Nelson, pp. 131-156. Netherlands: Springer.

Moura, T., Figuieredo, I., Machado, P.B. \& Gordo, L.S. (2004). Growth pattern and reproductive strategy of the holocephalan Chimeara monstrosa along the Portuguese continental shelf. Journal of Marine Biological Association of the United Kingdom 84, 801-804. DOI: 10.1017/S002531540400997Xh.

Moura, T., Serra-Pereira, B., Gordo, L.S. \& Figueiredo, I. (2011). Sperm storage in males and females of the deepwater shark Portuguese dogfish with notes on oviducal gland 
microscopic organization. Journal of Zoology 283, 210-219. DOI: 10.1111/j.14697998.2010.00775.x.

Moura, T., Jones, E., Clarke, M.W., Cotton, C.F., Crozier, P., Daley, R.K., Diez, G., Dobby, H., Dyb, J.E., Fossen, I. et al. (2014). Large-scale distribution of three deep-water squaloid sharks: Integrating data on sex, maturity and environment. Fisheries Research 157, 47-61. DOI: 10.1016/j.fishres.2014.03.019.

Mourier, J., Vercelloni, J. \& Planes, S. (2012). Evidence of social communities in a spatially structured network of a free-ranging shark species. Animal Behaviour 83, 389401. DOI: 10.1016/j.anbehav.2011.11.008.

MPI (2013). National Plan of Action for the Conservation and Management of Sharks (NPOA-Sharks) 2013. Ministry for Wellington: Ministry for Primary Industries.

MPI (2014). Fisheries Assessment Plenary, May 2014: stock assessments and stock status. Fisheries Science Group. Wellington: Ministry for Primary Industries.

MPI (2015). Annual Review Report for Deepwater Fisheries for 2013/14. MPI Technical Paper No: 2015/07. Wellington: Ministry for Primary Industries.

MPI (2016a). NZ Fisheries InfoSite. http://fs.fish.govt.nz/, Version 2016.

MPI (2016b) Annual Review Report for Deepwater Fisheries for 2014/15. MPI Technical Paper 2016/09. Wellington: Ministry for Primary Industries.

Mucientes, G.R., Queiroz, N., Sousa, L.L., Tarroso, P. \& Sims, D.W. (2009). Sexual segregation of pelagic sharks and the potential threat from fisheries. Biology Letters $\mathbf{5}, 156-$ 159. DOI: $10.1098 / \mathrm{rsbl} .2008 .0761$.

Munroe, S.E.M., Simpfendorfer, C.A. \& Heupel, M.R. (2014). Defining shark ecological specialisation: concepts, context, and examples. Reviews in Fish Biology and Fisheries 24, 317-331. DOI: 10.1007/s11160-013-9333-7.

Musick, J.A., Burgess, G., Cailliet, G., Camhi, M. \& Fordham, S. (2000). Management of sharks and their relatives (Elasmobranchii)._Fisheries_25, 9-13. DOI: 10.1577/15488446(2000)025<0009:MOSATR>2.0.CO;2.

Nakamura, I., Meyer, C.G.,\& Sato, K. (2015). Unexpected Positive Buoyancy in Deep Sea Sharks, Hexanchus griseus, and Echinorhinus cookei. PloS one 10, e0127667. DOI: 10.1371/journal.pone.0127667.

Navarro, J., López, L., Coll, M., Barría, C. \& Sáez-Liante, R. (2014). Short-and long-term importance of small sharks in the diet of the rare deep-sea shark Dalatias licha. Marine biology 161, 1697-1707. DOI: 10.1007/s00227-014-2454-2.

Naylor, G.J.P., Ryburn, J.A., Fedrigo, O. \& López, J.A. (2005). Phylogenetic relationships among the major lineages of modern elasmobranchs. In Reproductive biology and phylogeny of Chondrichthyes: sharks, batoids and chimaeras (Hamlett, W. C., ed.), pp. 125. Enfield, NH: Science Publishers, Inc. 
Neat, F., Kynoch, R., Drewery, J. \& Burns, F. (2010). Deepwater Trawl Survey Manual. Marine Scotland Science Report 03/10. Aberdeen: Marine Scotland Science.

Neat, F.C. \& Campbell, N. (2013). Proliferation of elongate fishes in the deep sea. Journal of Fish Biology 83, 1576-1591. DOI: 10.1111/jfb.12266.

Neat, F.C., Burns, F., Jones, E. \& Blasdale, T. (2015). The diversity, distribution and status of deep-water elasmobranchs in the Rockall Trough, north-east Atlantic Ocean. Journal of Fish Biology 87, 1469-1488. DOI: 10.1111/jfb.12822.

Neer, J.A., \& Thompson, B.A. (2005). Life history of the cownose ray, Rhinoptera bonasus, in the northern Gulf of Mexico, with comments on geographic variability in life history traits. Environmental Biology of Fishes 73, 321-331. DOI: 10.1007/s10641-0052136-5.

Nei, M. \& Kumar, S. (2000). Molecular Evolution and Phylogenetics. New York: Oxford University Press.

Neiva, J., Coelho, R. \& Erzini, K. (2006). Feeding habits of the velvet belly lanternshark Etmopterus spinax (Chondrichthyes: Etmopteridae) off the Algarve, southern Portugal. Journal of Marine Biological Association of the United Kingdom 86, 835-841. DOI: $10.1017 / \mathrm{S} 0025315406013762$.

Nelson, D.R., McKibben, J.N., Strong Jr, W.R., Lowe, C.G., Sisneros, J.A., Schroeder, D. M. \& Lavenberg, R.J. (1997). An acoustic tracking of a megamouth shark, Megachasma pelagios: a crepuscular vertical migrator.Environmental Biology of Fishes 49, 389-399. DOI: 10.1023/A:1007369619576.

Nibam, A.H. (2011). Reproductive biology and diet of the St. Joseph (Callorhinchus capensis) in South Africa. M.Sc. Thesis. South Africa: University of Cape Town.

Nielsen, J., Hedeholm, R.B., Heinemeier, J., Bushnell, P.G., Christiansen, J.S., Olsen, J., Ramsey, C.B., Brill, R.W., Simon, M., Steffensen, K.F., et al. (2016). Eye lens radiocarbon reveals centuries of longevity in the Greenland shark (Somniosus microcephalus). Science 353, 702-704. DOI: 10.1126/science.aaf1703.

Niimi, A.J. \& Beamish, F.W.H. (1974). Bioenergetics and growth of largemouth bass (Micropterus salmoides) in relation to body weight and temperature. Canadian Journal of Zoology 52, 447-456. DOI: 10.1139/z74-056.

Nordeide, J.T. \& Folstad, I. (2000). Is cod lekking or a promiscuous group spawner? Fish and Fisheries 1, 90-93. DOI: 10.1046/j.1467-2979.2000.00005.x.

Nosal, A.P., Cartamil, D.C., Long, J.W., Lührmann, M., Wegner, N.C. \& Graham, J.B. (2013). Demography and movement patterns of leopard sharks (Triakis semifasciata) aggregating near the head of a submarine canyon along the open coast of southern California, USA. Environmental Biology of Fishes 96, 865-878. DOI: 10.1007/s10641012-0083-5. 
Norse, E.A., Brooke, S., Cheung, W.W.L., Clark, M.R., Ekeland, I., Froese, R., Gjerde, K.M., Haedrich, R.L., Heppell, S.S. \& Morato, T. (2012). Sustainability of deep-sea fisheries. Marine Policy 36, 307-320. doi:10.1016/j.marpol.2011.06.008.

O'Driscoll, R.L. \& Bagley, N.W. (2004). Trawl survey of middle depth species in the Southland and Sub-Antarctic areas, November-December 2003 (TAN0317). New Zealand Fisheries Assessment Report 2004/49. Wellington: Ministry of Fisheries.

O’Driscoll, R.L., MacGibbon, D., Fu, S., Lyon, W. \& Stevens, D.W. (2011). A review of hoki and middle-depth trawl surveys of the Chatham Rise, January 1992-2010. New Zealand Fisheries Assessment Report No. 47. Wellington: Ministry of Fisheries.

O'Driscoll, R.L., de Joux, P., Nelson, R., Macaulay, G.J., Dunford, A.J., Marriott, P.M., Stewart, C. \& Miller, B.S. (2012). Species identification in seamount fish aggregations using moored underwater video. ICES Journal of Marine Science: Journal du Conseil, 69 , 648-659. DOI: 10.1093/icesjms/fss010.

Ohlberger, J., Otero, J., Edeline, E., Winfield, I.J., Stenseth, N.C. \& Vøllestad, L.A. (2013). Biotic and abiotic effects on cohort size distributions in fish. Oikos 122, 835-844. DOI: 10.1111/j.1600-0706.2012.19858.

Olden, J.D., Hogan, Z.S. \& Zanden, M. (2007). Small fish, big fish, red fish, blue fish: sizebiased extinction risk of the world's freshwater and marine fishes. Global Ecology and Biogeography 16, 694-701. DOI: 10.1111/j.1466-8238.2007.00337.x.

Oliver, S.P., Hussey, N.E., Turner, J.R. \& Beckett, A.J. (2011). Oceanic sharks clean at coastal seamount. PLoS ONE 6, e1475. DOI: 10.1371/journal.pone.0014755.

Pardo, S.A., Kindsvater, H.K., Cuevas-Zimbrón, E., Sosa-Nishizaki, O., Pérez-Jiménez, J.C. \& Dulvy, N. K. (2016). Devil in the details: growth, productivity, and extinction risk of a data-sparse devil ray. bioRxiv, 043885. DOI: 10.1038/srep33745.

Parker, S.J. \& Francis, M.P. (2012). Productivity of two species of deepwater sharks, Deania calcea and Centrophorus squamosus in New Zealand. New Zealand Aquatic Environment and Biodiversity Report 103. Wellington: Ministry for Primary Industries.

Patterson, H., Noriega, R., Georgeson, L., Stobutzki, I. \& Curtotti, R. (2016). Fishery status reports 2016. Australian Bureau of Agricultural and Resource Economics and Sciences, Canberra: Bureau of Rural Sciences.

Pauly, D. (1984). Fish population dynamics in tropical waters: a manual for use with programmable calculators. International Center for Living Aquatic Resources Management, Studies and Reviews No. 8. Manila: WorldFish.

Peacor, S.D. \& Pfister, C.A. (2006). Experimental and model analyses of the effects of competition on individual size variation in wood frog (Rana sylvatica) tadpoles. Journal of Animal Ecology 75, 990-999. DOI: 10.1111/j.1365-2656.2006.01119.x.

Peters, R.H. (1986). The ecological implications of body size (Vol. 2). New York: Cambridge University Press. 
Pethybridge, H., Daley, R.K., \& Nichols, P.D. (2011a). Diet of demersal sharks and chimaeras inferred by fatty acid profiles and stomach content analysis. Journal of Experimental Marine Biology and Ecology 409, 290-299. DOI: 10.1016/j.jembe.2011.09.009.

Pethybridge, H., Daley, R., Virtue, P. \& Nichols, P.D. (2011b). Lipid (energy) reserves, utilisation and provisioning during oocyte maturation and early embryonic development of deepwater chondrichthyans. Marine biology 158, 2741-2754. DOI: 10.1007/s00227-0111773-9.

Pethybridge, H., Butler, E.C.V., Cossa, D., Daley, R. \& Boudou, A. (2012). Trophic structure and biomagnification of mercury in an assemblage of deepwater chondrichthyans from southeastern Australia. Marine Ecology Progress Series 451, 163-174. DOI: 10.3354/meps09593.

Pitcher, T. J. \& Partridge, B. L. (1979). Fish school density and volume.Marine Biology 54, 383-394. DOI: 10.1007/BF00395444.

Pollerspöck, J. (2014). www.shark-references.com. World Wide Web Electronic Publication, Version 2014.

Ponte, S. (2012). The Marine Stewardship Council (MSC) and the making of a market for 'sustainable fish'. Journal of Agrarian Change 12, 300-315. DOI: 10.1111/j.14710366.2011.00345.x.

Powter, D.M. \& Gladstone, W. (2008). Embryonic mortality and predation on egg capsules of the Port Jackson shark Heterodontus portusjacksoni (Meyer). Journal of Fish Biology 72, 573-584. DOI: 10.1111/j.1095-8649.2007.01721.x.

Pratt Jr, H.L. \& Tanaka, S. (1994). Sperm storage in male elasmobranchs: a description and survey. Journal of Morphology 219, 297-308. DOI: 10.1002/jmor.1052190309.

Pratt Jr, H.L. \& Carrier, J.C. (2001). A review of elasmobranch reproductive behavior with a case study on the nurse shark, Ginglymostoma cirratum. Environmental Biology of Fishes 60, 157-188. DOI: 10.1007/s10641-012-0083-5.

Preciado, I., Cartes, J.E., Serrano, A., Velasco, F., Olaso, I., Sánchez, F. \& Frutos, I. (2009). Resource utilization by deep-sea sharks at the Le Danois Bank, Cantabrian Sea, north-east Atlantic Ocean. Journal of Fish Biology 75, 1331-1355. DOI: 10.1111/j.10958649.2009.02367.x.

Priede, I.G., Froese, R., Bailey, D.M., Bergstad, O.A., Collins, M.A., Dyb, J.E., Henriques, C., Jones, E.G. \& King, N. (2006). The absence of sharks from abyssal regions of the world's oceans. Proceedings of the Royal Society of London Series B 273, 1435-1441. DOI: $10.1098 / \mathrm{rspb} .2005 .3461$.

Quaranta, K.L., Didier, D.A., Long, D.J., Ebert, D.A. (2006). A new species of chimaeroid, Hydrolagus alphus sp. nov. (Chimaeriformes: Chimaeridae) from the Galapagos Islands. Zootaxa 1377, 33-45. 
Quinn, T.P., Miller, B.S. \& Wingert, R.C. (1980). Depth distribution and seasonal and diel movements of ratfish, Hydrolagus colliei, in Puget Sound, Washington. Fisheries Bulletin 78, 816-821.

R Core Team. (2012). R: A language and environment for statistical computing. Vienna, Austria: R Foundation for Statistical Computing.

Ranta, E., Peuhkuri, N., \& Laurila, A. (1994). A theoretical exploration of antipredatory and foraging factors promoting phenotype-assorted fish schools._Ecoscience 1, 99-106. DOI: $10.1080 / 11956860.1994 .11682233$.

Ratnasingham, S. \& Hebert, P.D. (2007). The barcode of life data system (http://www.barcodinglife.org). Molecular Ecology Notes 7, 355-364. DOI: 10.1111/j.1471-8286.2007.01678.x.

Reynolds, J.D., Dulvy, N.K., Goodwin, N.B. \& Hutchings, J.A. (2005). Biology of extinction risk in marine fishes. Proceedings of the Royal Society of London B: Biological Sciences, 272, 2337-2344. DOI: 10.1098/rspb.2005.3281.

Ricker, W.E. (1958). Handbook of computations for biological statistics of fish populations. Bulletins of the Fisheries Research Board of Canada 119, 1-300.

Ricker, W.E. (1979). Growth rates and models. Fish Physiology 8, 677-743.

Rigby, C., \& Simpfendorfer, C.A. (2015). Patterns in life history traits of deep-water chondrichthyans. Deep Sea Research Part II: Topical Studies in Oceanography 115, 3040. DOI: 10.1016/J.DSR2.2013.09.004.

Roberts, C.D., Stewart, A.L. \& Struthers, C.D. (2015). The Fishes of New Zealand. Wellington: Te Papa Press.

Rodríguez-Cabello, C., \& Sánchez, F. (2014). Is Centrophorus squamosus a highly migratory deep-water shark? Deep Sea Research Part I: Oceanographic Research Papers 92, 1-10. DOI: 10.1016/j.dsr.2014.06.005.

Rodríguez-Cabello, C., González-Pola, C., \& Sánchez, F. (2016). Migration and diving behavior of Centrophorus squamosus in the NE Atlantic. Combining electronic tagging and Argo hydrography to infer deep ocean trajectories. Deep Sea Research Part I: Oceanographic Research Papers 115, 48-62. DOI: 10.1016/j.dsr2016.05.009.

Roff, D. A. (1983). An allocation model of growth and reproduction in fish. Canadian Journal of Fisheries and Aquatic Sciences 40, 1395-1404. DOI: 10.1139/f83-161.

Rubenstein, D.I. (1981). Individual variation and competition in the Everglades pygmy sunfish. The Journal of Animal Ecology 50, 337-350. DOI: 10.2307/4059.

Schluessel, V. (2015). Who would have thought that 'Jaws' also has brains? Cognitive functions in elasmobranchs. Animal Cognition 18, 19-37. DOI: 10.1007/s10071-014-0762$\mathrm{Z}$. 
Shao, K.T. \& Hwang, D.F. (1997). Rhinochimaera pacifica (Chimaeriformes, Rhinochimaeridae), the first rhinochimaerid recorded from Taiwan. Acta Zoological Taiwanica 8, 97-102.

Shelton, W.L., Davies, W.D., King, T.A. \& Timmons, T.J. (1979). Variation in the growth of the initial year class of largemouth bass in West Point Reservoir, Alabama and Georgia. Transactions of the American Fisheries Society 108, 142-149. DOI: 10.1577/1548-8659(1979)108<142:VITGOT>2.0.CO;2.

Shinohara, G., Narimatsu, Y., Hattori, T., Ito, M., Takata, Y. \& Matsuura, K. (2009). Annotated checklist of deep-sea fishes from the Pacific coast off Tohoku District, Japan. National Museum of Nature and Science Monographs 39, 683-735.

Shipley, O.N., Howey, L.A., Tolentino, E.R., Jordan, L.K., \& Brooks, E.J. (2016). Novel techniques and insights into the deployment of pop-up satellite archival tags on a smallbodied deep-water chondrichthyan. Deep Sea Research Part I: Oceanographic Research Papers. DOI: 10.1016/j.dsr.2016.11.005.

Simmons, G., Bremner, G., Whittaker, H., Clarke, P., Teh, L., Zylich, K., Zeller, D., Pauly, D., Stringer, C., Torkington, B. \& Haworth, N. (2016). Reconstruction of marine fisheries catches for New Zealand (1950-2010). Institute for the Oceans and Fisheries Working Paper 2015 - 87. Vancouver: University of British Columbia.

Simpfendorfer, C.A. \& Kyne, P.M. (2009). Limited potential to recover from overfishing raises concerns for deep-sea sharks, rays and chimaeras. Environmental Conservation 36, 97-103. DOI: 10.1017/S0376892909990191.

Simpfendorfer, C.A., Heupel, M.R., White, W.T. \& Dulvy, N.K. (2011). The importance of research and public opinion to conservation management of sharks and rays: a synthesis. Marine and Freshwater Research 62, 518-527. DOI: 10.1071/MF11086.

Simpfendorfer, C.A. \& Wetherbee, B. (2015). Shark and ray life history. Marine and Freshwater Research 66: i-ii.

Sims, D.W. (2005). Differences in habitat selection and reproductive strategies of male and female sharks. In Sexual segregation in vertebrates: Ecology of the two sexes (Ruckstuhl, K. \& Neuhaus, P., eds.), pp. 127-147. New York, NY: Cambridge University Press.

Sims, D.W., Wearmouth, V.J., Southall, E.J., Hill, J.M., Moore, P., Rawlinson, K., Hutchinson, N., Budd, G.C., Righton, D., Metcalfe, J.D., et al. (2006). Hunt warm, rest cool: bioenergetic strategy underlying diel vertical migration of a benthic shark. Journal of Animal Ecology 75, 176-190. DOI: 10.1111/j.1365-2656.2005.01033.x.

Smith, C.C., \& Fretwell, S.D. (1974). The optimal balance between size and number of offspring. American Naturalist 108, 499-506.

Smith, R.M., Day, R.W., Walker, T.I., Hamlett, W.C. (2004). Microscopic organisation of the oviducal gland of the holocephalan elephant fish, Callorhynchus milii. Marine and 
Freshwater Research 55, 155-164. DOI: 10.1071/MF01078.

Smith, K.R., Scarpaci, C., Louden, B.M. \& Otway, N.M. (2015). Behaviour of aggregated grey nurse sharks Carcharias taurus off eastern Australia: similarities and differences among life-history stages and sites. Endangered Species Research 27, 69-85. DOI: 10.3354/esr00652.

Speed, C.W., Meekan, M.G., Field, I.C., McMahon, C.R., Stevens, J.D., McGregor, F., Huveneers, C., Berger, Y. \& Bradshaw, C.J. (2011). Spatial and temporal movement patterns of a multi-species coastal reef shark aggregation. Marine Ecology Progress Series 429, 261-275. DOI: 10.3354/meps09080.

Speed, C.W., Meekan, M.G., Field, I.C., McMahon, C.R., \& Bradshaw, C.J. (2012). Heatseeking sharks: Support for behavioural thermo-regulation in reef sharks. Marine Ecology Progress Series 463, 231. DOI: 10.3354/meps09864.

SPRFMO. (2008). Bottom fishing activities by New Zealand vessels fishing in the high seas in the SPRFMO area during 2008 and 2009. Document SP-7-SWG-DW-01. Wellington: SPRFMO.

Sokal, R.R. \& Rohlf, F.J. (1987). Introduction to Biostatistics, 2nd edn. New York, NY: W. H. Freeman Press.

Springer, S. (1967). Social organization of shark populations. In Sharks, skates and rays (Gilbert, P. W., Mathewson, R. F., Rall, D. P., eds.), pp. 149-174. Baltimore: John Hopkins Press.

Stehmann, M.F.W. (2002). Proposal of a maturity stages scale for oviparous and viviparous cartilaginous fishes (Pisces, Chondrichthyes). Archive of Fishery and Marine Research 50, $23-48$.

Stergiou, K.I. \& Fourtouni, H. (1991). Food habits, ontogenetic diet shift and selectivity in Zeus faber Linnaeus, 1758. Journal of Fish Biology 39, 589-603. DOI: 10.1111/j.10958649.1991.tb04389.x.

Stevens, J.D., Bonfil, R., Dulvy, N.K., \& Walker, P.A. (2000). The effects of fishing on sharks, rays, and chimaeras (Chondrichthyans), and the implications for marine ecosystems. ICES Journal of Marine Science: Journal du Conseil 57, 476-494. DOI: 10.1006/JMSC.2000.0724.

Stevens, D.W., O’Driscoll, R.L., Ladroit, Y., Ballara, S.L., MacGibbon, D.J. \& Horn, P.L. (2015). Trawl survey of hoki and middle-depth species on the Chatham Rise, January 2014 (TAN1401). New Zealand Fisheries Assessment Report 2015/19. Wellington: Ministry for Primary Industries.

Stevenson, M.L. \& Hanchet, S.M. (1999). Trawl survey design and data analysis procedures

for inshore fisheries research. NIWA Technical Report. Wellington: Ministry of Fisheries. 
Stobutzki, I.C., Miller, M.J., Heales, D.S. \& Brewer, D.T. (2002). Sustainability of elasmobranchs caught as bycatch in a tropical prawn (shrimp) trawl fishery. Fisheries Bulletin 100, 800-821.

Straube, N., Leslie, R.W., Clerkin, P.J., Ebert, D.A., Rochel, E., Corrigan, S., Li, C. \& Naylor, G.J. (2015). On the occurrence of the Southern Lanternshark, Etmopterus granulosus, off South Africa, with comments on the validity of E. compagnoi. Deep Sea Research Part II: Topical Studies in Oceanography 115 11-17. DOI: 10.1016/j.dsr2.2014/04.004.

Taborsky, M. (2009). Reproductive skew in cooperative fish groups: virtue and limitations of alternative modeling approaches. In Reproductive Skew in Vertebrates: Proximate and Ultimate Causes, pp. 265-304. New York, NY: Cambridge University Press.

Theiss, S.M., Huveneers, C. \& Ebert, D.A. (2015). Hydrolagus homonycteris. The IUCN Red List of Threatened Species 2015 e.T195433A68629582. Available at http://www.iucnredlist.org (Accessed 23 September 2016). DOI: 10.2305/IUCN.UK.20154.RLTS.T195433A68629582.en.

Thorson, J.T., Clarke, M.E., Stewart, I.J. \& Punt, A.E. (2012). The implications of spatially varying catchability on bottom trawl surveys of fish abundance: a proposed solution involving underwater vehicles. Canadian Journal of Fisheries and Aquatic Sciences 70, 294-306. DOI: 10.1139/cjfas-2012-0330.

Tozer, H. \& Didier, D.D. (2004). Husbandry of spotted ratfish, Hydrolagus colliei. The Elasmobranch Husbandry Manual: Captive Care of Sharks, Rays and their Relatives, 487.

Tracey, D.M., Bull, B., Clark, M.R. \& Mackay, K.A. (2004). Fish species composition on seamounts and adjacent slope in New Zealand waters. New Zealand Journal of Marine and Freshwater Research 38, 163-182. DOI: 10.1080/00288330.2004.9517226.

Tracey, D.M., Clark, M.R., Anderson, O.F. \& Kim, S.W. (2012). Deep-sea fish distribution varies between seamounts: results from a seamount complex off New Zealand. PloS one 7, e36897. DOI:10.1371/journal.pone.0036897.

Treude, T., Kiel, S., Linke, P., Peckmann, J. \& Goedert, J.L. (2011). Elasmobranch egg capsules associated with modern and ancient cold seeps: a nursery for marine deep-water predators. Marine Ecology Progress Series 437, 175-181. DOI: 10.3354/meps09305.

Underwood, R. (1981). Companion preference in an eland herd. African Journal of Ecology 19, 341-354.

Veríssimo, A., Grubbs, D., McDowell, J., Musick, J. \& Portnoy, D. (2010). Frequency of multiple paternity in the spiny dogfish Squalus acanthias in the Western North Atlantic. Journal of Heredity esq084. DOI: 10.1093/jhered/esq084.

Villasante, S., Morato, T., Rodriguez-Gonzalez, D., Antelo, M., Österblom, H., Watling, L., Nouvian, C., Gianni, M. \& Macho, G. (2012). Sustainability of deep-sea fish species under the European Union Common Fisheries Policy. Ocean \& Coastal Management 70, 31-37. DOI: 10.1016/j.ocecoaman.2012.07.033. 
Walker, T.I. (2005). Reproduction in fisheries science, In Reproductive biology and phylogeny of chondrichthyes. (Hamlett W.C., ed), pp. 97-124. New Hampshire: Science Publishers Inc.

Ward, R.D., Zemlak, T. S., Innes, B.H., Last, P.R. \& Hebert, P.D.N. (2005). DNA barcoding Australia's fish species. Philosophical Transactions of the Royal Society B 360, 1847-1851. DOI: 10.1098/rstb.2005.1716.

Watson, R.A. \& Morato, T. (2013). Fishing down the deep: Accounting for within-species changes in depth of fishing. Fisheries Research 140, 63-65. DOI: 10.1016/j.fishres.2012.12.004.

Wearmouth, V.J. \& Sims, D.W. (2008). Sexual segregation in marine fish, reptiles, birds and mammals: behaviour patterns, mechanisms and conservation implications. Advances in Marine Biology 54, 107-170. DOI: 10.1016/S0065-2881(08)00002-3.

Weigmann, S. (2016). Annotated checklist of the living sharks, batoids and chimaeras (Chondrichthyes) of the world, with a focus on biogeographical diversity. Journal of Fish Biology 88, 837-1037. DOI: 10.1111/jfb.12874.

Werner, E.E. \& Gilliam, J.F. (1984). The ontogenetic niche and species interactions in sizestructured populations. Annual review of Ecology and Systematics 15, 393-425.

Wetherbee, B.M. (1996). Distribution and reproduction of the southern lantern shark from New Zealand. Journal of Fish Biology, 49: 1186-1196. DOI: 10.1111/j.10958649.1996.tb01788.x.

Wetherbee, B.M. (2000). Assemblage of deep-sea sharks on Chatham Rise, New Zealand. Fishery Bulletin 98, 189-198.

White, W.T. \& Last, P.R. (2012). A review of the taxonomy of chondrichthyan fishes: a modern perspective. Journal of Fish Biology 80, 901-917. DOI: 10.1111/j.10958649.2011.03192.x.

White, W.T., Vaz, D.F., Ho, H.C., Ebert, D.A., de Carvalho, M.R., Corrigan, S., Rochel, E., de Carvalho, M., Tanaka, S. \& Naylor, G.J. (2015). Redescription of Scymnodon ichiharai Yano and Tanaka 1984 (Squaliformes: Somniosidae) from the western North Pacific, with comments on the definition of somniosid genera. Ichthyology Research 62 , 213-229. DOI: 10.1007/s10228-014-0430-y.

Whitehead, H. (2008). Analyzing animal societies: quantitative methods for vertebrate social analysis. Chicago: University of Chicago Press.

Whitney, N.M., Pratt, H.L. \& Carrier, J.C. (2004). Group courtship, mating behaviour and siphon sac function in the whitetip reef shark, Triaenodon obesus. Animal Behaviour 68, 1435-1442. DOI: 10.1016/j.anbehav.2004.02.018. 
Wickman, P. \& Jansson, P. (1997). An estimate of female mate searching costs in the lekking butterfly Coenonympha pamphilus. Behavioral Ecology and Scoiobiology 40, 321328. DOI: 10.1023/A:1011069914753.

Wiedenmann, J., Wilen, J., Levin, P., Plummer, M. \& Mangel, M. (2016). A framework for exploring the role of bioeconomics on observed fishing patterns and ecosystem dynamics. Coastal Management 44, 529-546. DOI: 10.1080/08920753.2016.1208886.

Williams, R., Okey, T.A., Wallace, S. \& Gallucci, V.F. (2010). Shark aggregation in coastal waters of British Columbia. Marine Ecology Progress Series 414, 249-256. DOI: 10.3354/meps08718.

Wilson, S.G, Taylor, J.G. \& Pearce, A.F. (2001). The seasonal aggregation of whale sharks at Ningaloo Reef, Western Australia: currents, migrations and the El Nino/Southern Oscillation. Environmental Biology of Fishes 61, 1-11. DOI: 10.1023/A:1011069914753.

Wilson, A.D.M., Croft, D.P. \& Krause, J. (2014). Social networks in elasmobranchs and teleost fishes. Fish and Fisheries 15, 676-689. DOI: 10.1111/faf.12046.

Wootton, R.J. (1990). Ecology of teleost fishes. Netherlands: Springer.

Wourms, J.P. (1977) Reproduction and development in chondrichthyan fishes. American Zoology 17, 379-410. DOI:10.1093/icb/17.2.379.

Yano, K. \& Tanaka, S. (1988). Size at maturity, reproduction cycle, fecundity, and depth segregation of the deep sea squaloid sharks Centroscymnus owstoni and Centroscymnus coelolepis in Suruga Bay, Japan. Nippon Suisan Gakkaishi 54, 167-174. DOI: http://doi.org/10.2331/suisan.54.167.

Yano, K. (1995). Reproductive biology of the black dogfish, Centroscyllium fabricii, collected from waters off western Greenland. Journal of the Marine Biological Association of the United Kingdom 75, 285-310. DOI: 10.1017/S002531540001818X.

Yano, K. \& Matsuura, K. (2002). A review of the genus Oxynotus (Squaliformes, Oxynotidae). Bulletin of the National Science Museum, Tokyo, Series A 28, 109-117.

Yano, K., Matsuura, K., \& Tsukada, O. (2002). Redescription of the rare squaloid shark Oxynotus japonicus from Suruga Bay and the Enshu-nada Sea, Japan. Species Diversity 7, 363-369.

Yano, K. (2004). Oxynotus japonicus. The IUCN Red List of Threatened Species 2004, e.T44206A10870794. Available at http://www.iucnredlist.org (Accessed 25 March 2016). DOI: 10.2305/IUCN.UK.2004.RLTS.T44206A10870794.en.

Zar, J. H. (1996). Biostatistical Analysis, Third edition. New Jersey: Prentice Hall.

Zintzen, V., Roberts, C.D., Clark, M.R., Williams, A., Althaus, F. \& Last, P.R. (2011). Composition, distribution and regional affinities of the deepwater ichthyofauna of the Lord Howe Rise and Norfolk Ridge, south-west Pacific Ocean. Deep Sea Research Part II: Topical Studies in Oceanography 58, 933-947. DOI: 10.1016/j.dsr2.2010.10.049. 


\section{Appendix I.}

Length-weight relationships (LWR, $W=a L^{b}$ ) (left panel) and log-transformed LWR [log $(W)=\log (a)+b \times \log (L)]$ (right panel) for for Harriotta haeckeli (HHA), Harriotta raleighana $(\mathrm{LCH})$, Chimaera carophila $(\mathrm{CHP})$, Chimaera lignaria $(\mathrm{CHG})$, Hydrolagus bemisi (GSP), Hydrolagus homonycteris (HYB), Hydrolagus novaezealandiae (GSH), Squalus acanthias (SPD), Centrophorus squamosus (CSQ), Deania calcea (SND), Etmopterus granulosus (ETB), Etmopterus lucifer (ETL), Centroscymnus coelolepis (CYL), Centroscymnus owstoni (CYO), Centroscymnus crepidater (CYP), Scymnodon plunketi (PLS), and Dalatias licha (BSH). 

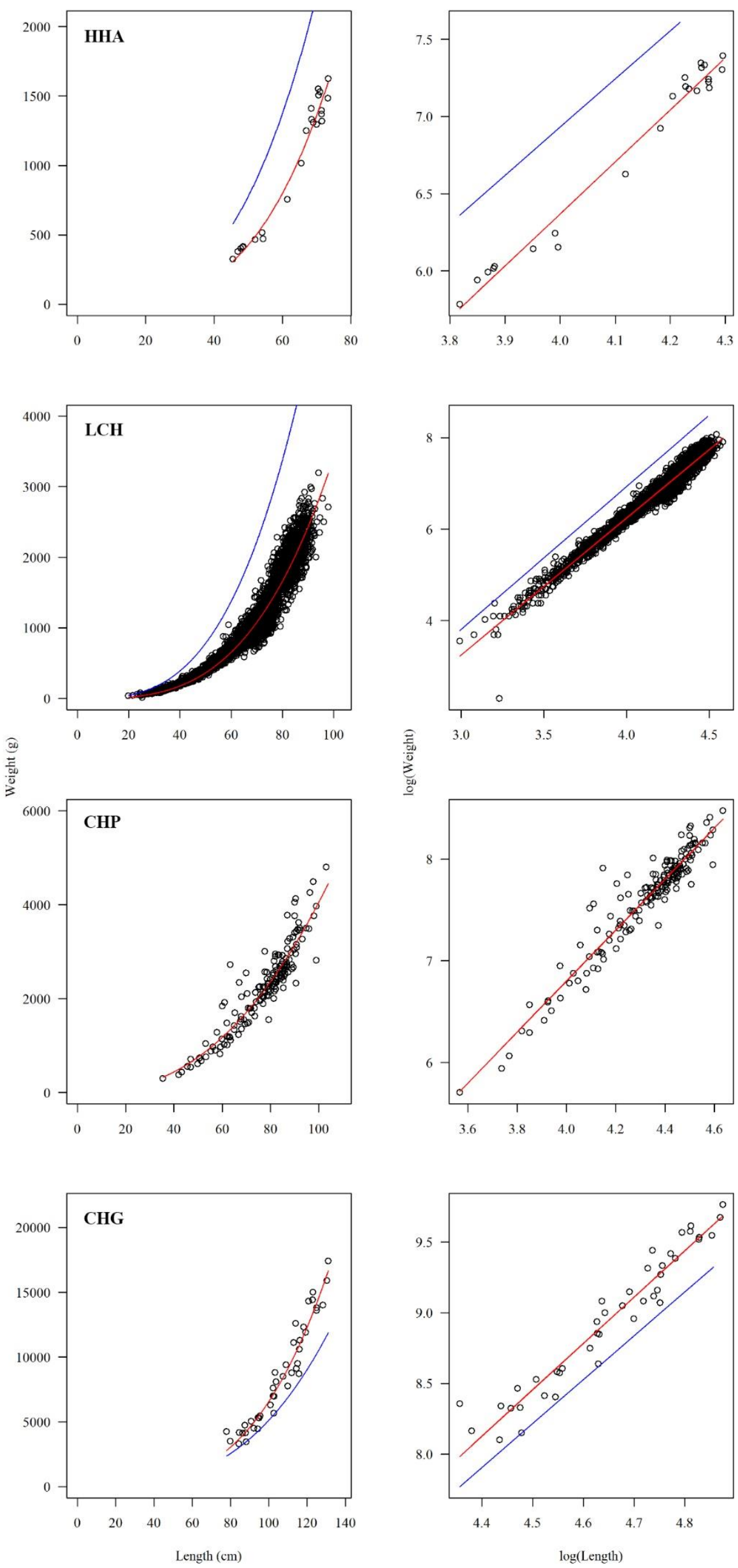

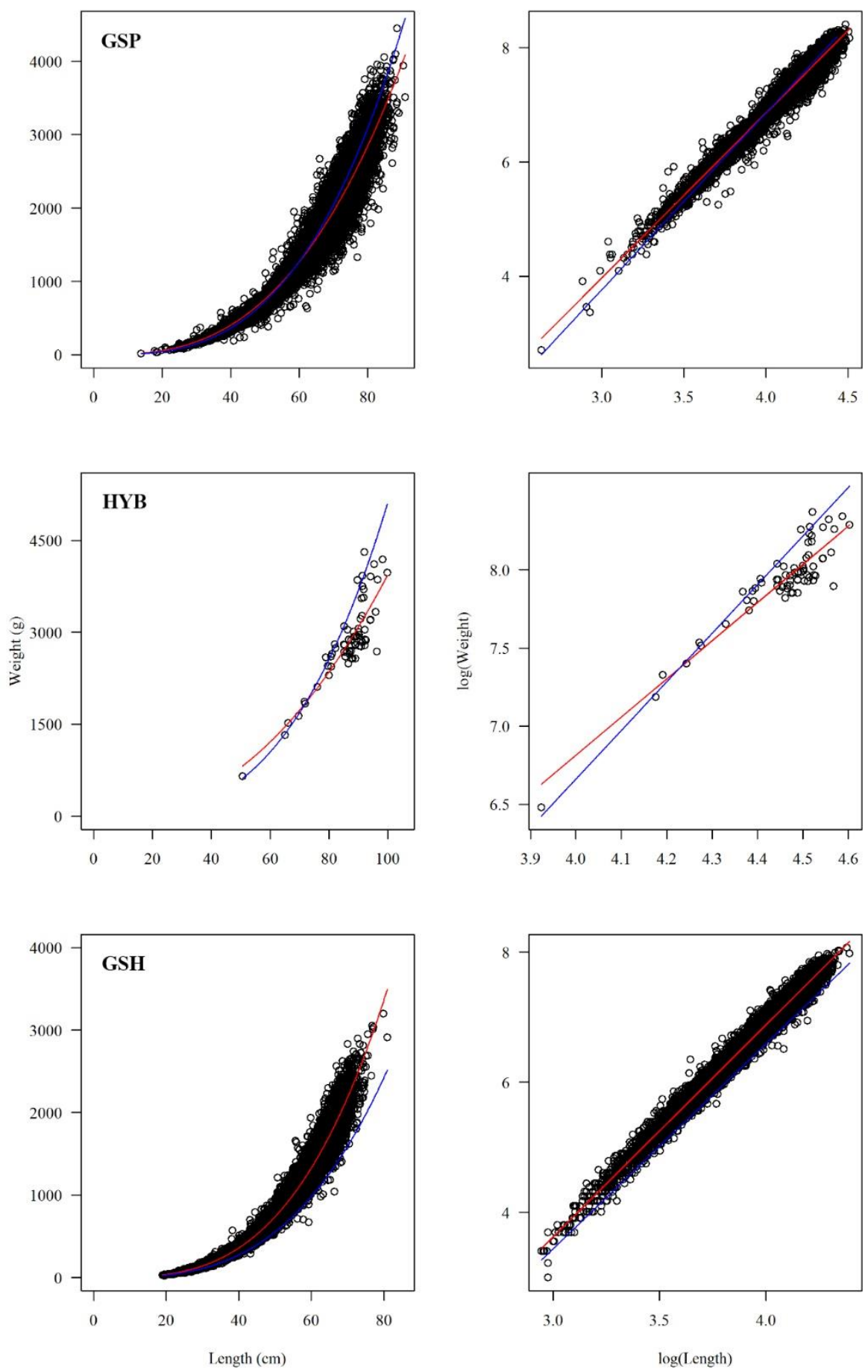

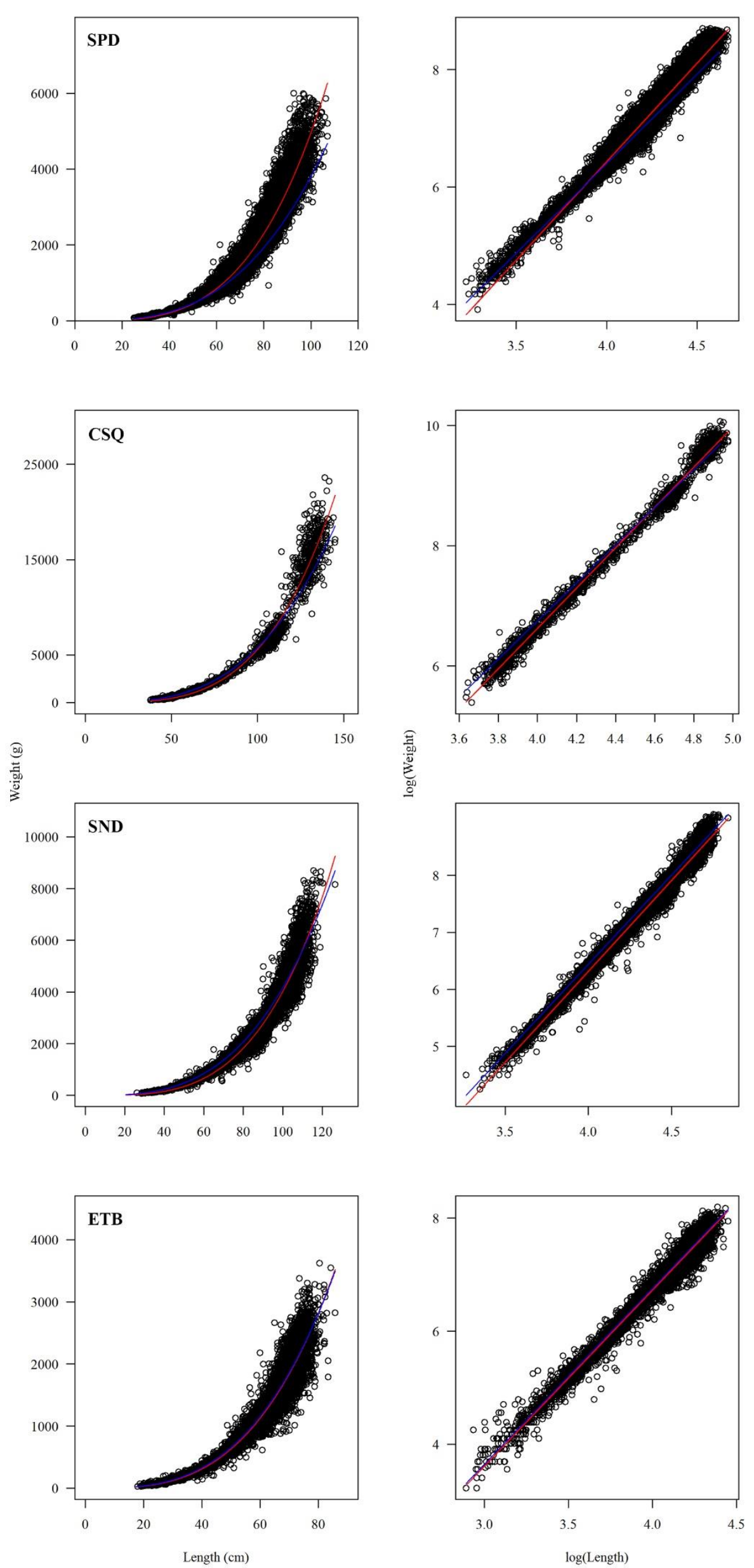

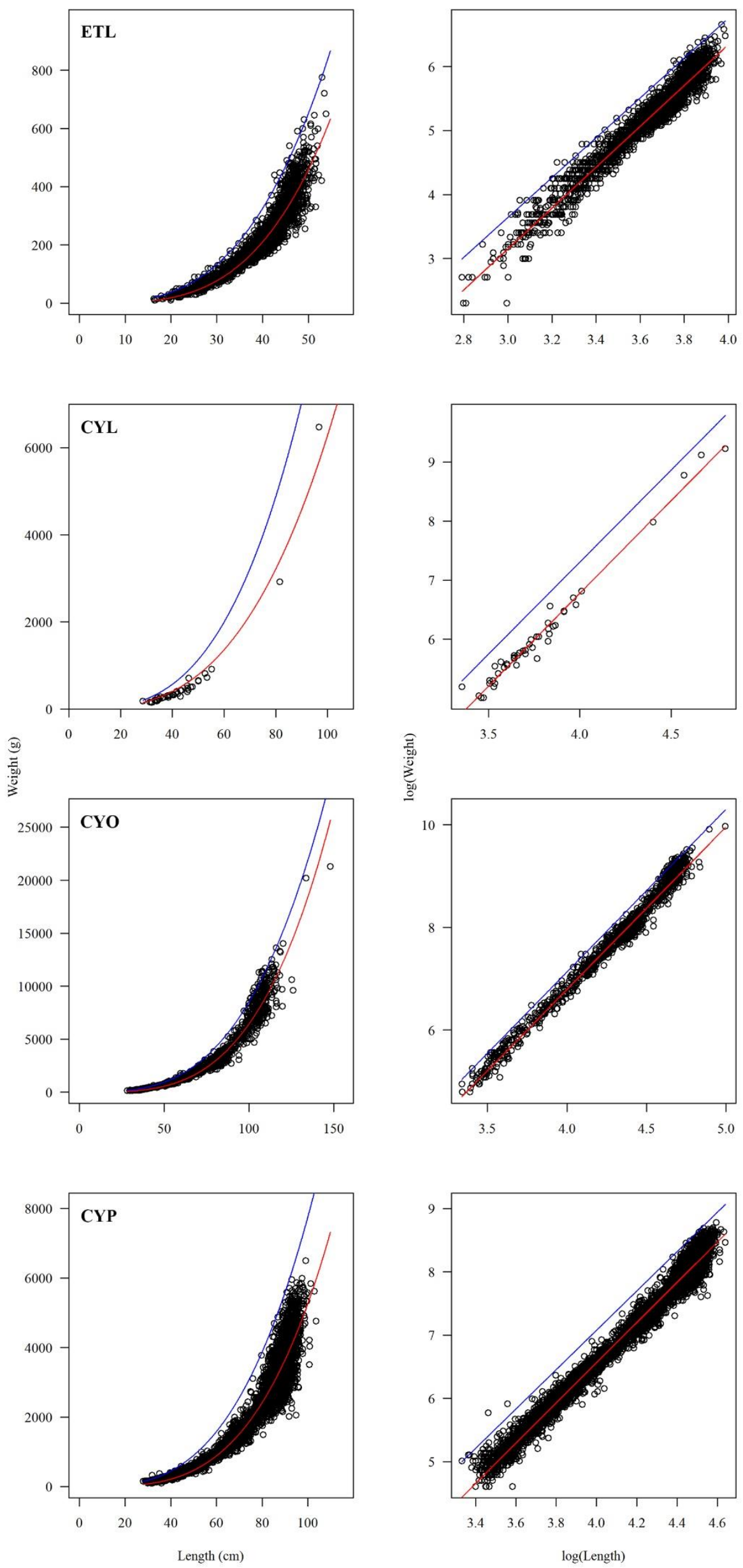

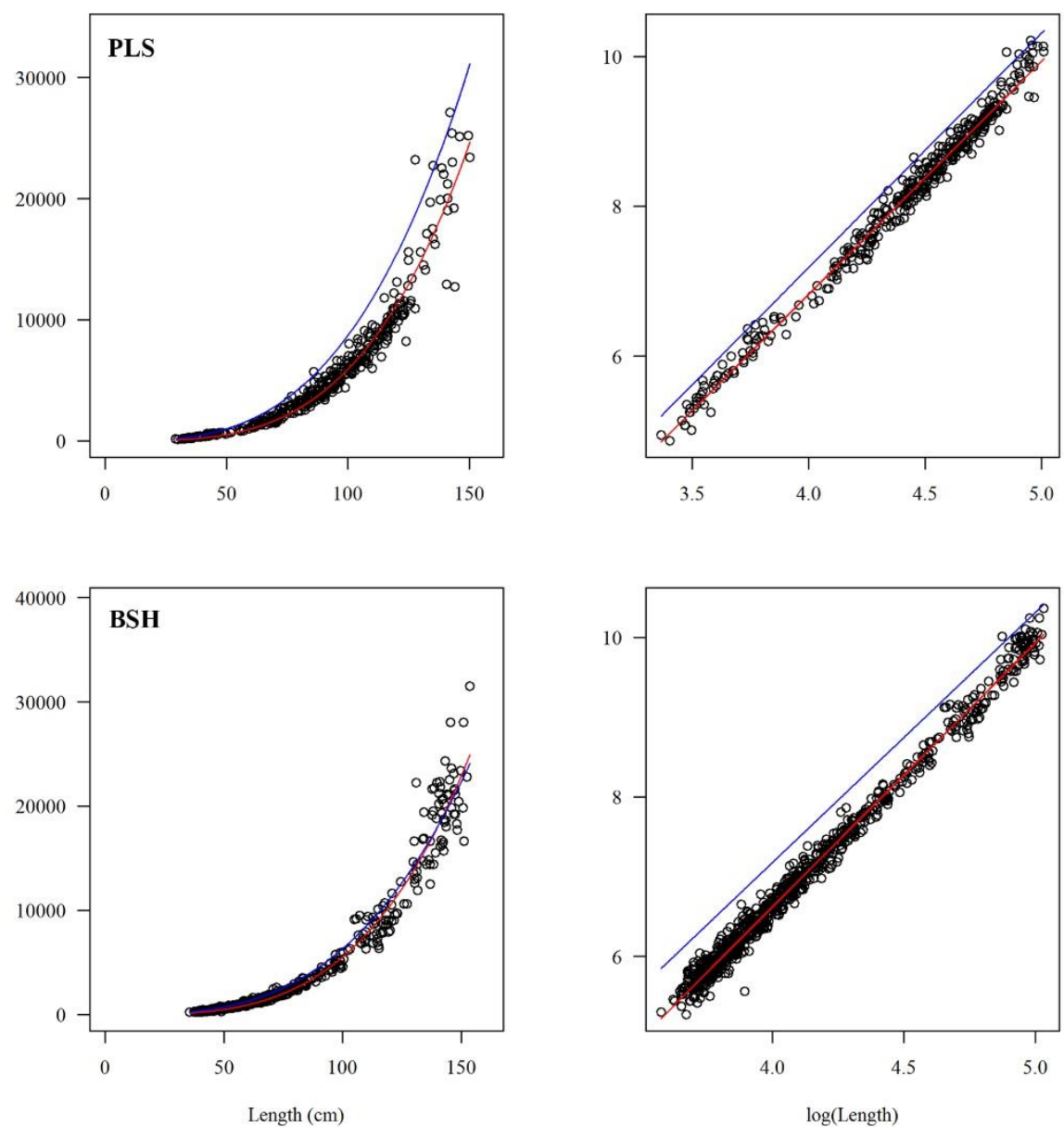


\section{Appendix II.}

For each species, estimates of the variance $(\alpha)$ and mean $(\beta)$ inflection points in logspace by the best fitting model, as determined by AIC. Standard error (S.E.), t-value (T), confidence intervals (CI1, CI2), and significance (p-value) also reported. 


\begin{tabular}{lrrrrrr}
\hline \multicolumn{2}{l}{ Harriotta raleighana, male } & & & & & \\
\hline Parameter & Value & SE & T & CI1 & CI2 & p-value \\
\hline $\boldsymbol{\alpha}_{\mathbf{0}}$ & -4.60 & 0.03 & -138.38 & -4.66 & -4.53 & $<0.001$ \\
$\boldsymbol{\alpha}_{1}$ & 4.33 & 0.01 & 582.42 & 4.32 & 4.35 & $<0.001$ \\
$\boldsymbol{\alpha}_{2}$ & 1.29 & 0.17 & 7.64 & 0.96 & 1.62 & $<0.001$ \\
$\boldsymbol{\alpha}_{3}$ & -4.61 & & & & & \\
\hline $\boldsymbol{\beta}_{0}$ & -4.79 & 0.06 & -74.75 & -4.92 & -4.67 & $<0.001$ \\
$\boldsymbol{\beta}_{1}$ & 2.76 & 0.02 & 178.52 & 2.73 & 2.79 & $<0.001$ \\
$\boldsymbol{\beta}_{2}$ & 3.69 & 0.02 & 158.49 & 3.65 & 3.74 & $<0.001$ \\
$\boldsymbol{\beta}_{3}$ & 0.31 & 0.07 & 4.66 & 0.18 & 0.44 & $<0.001$ \\
\hline
\end{tabular}

\begin{tabular}{lrrrrrr}
\hline \multicolumn{2}{l}{ Harriotta raleighana, female } & & & & & \\
\hline Parameter & Value & SE & T & CI1 & CI2 & p-value \\
\hline $\boldsymbol{\alpha}_{\mathbf{0}}$ & -3.51 & 0.26 & -13.51 & -4.02 & -3.00 & $<0.001$ \\
$\boldsymbol{\alpha}_{1}$ & 3.58 & 0.02 & 200.44 & 3.54 & 3.61 & $<0.001$ \\
$\boldsymbol{\alpha}_{2}$ & -0.92 & 0.26 & -3.51 & -1.43 & -0.41 & $<0.001$ \\
$\boldsymbol{\alpha}_{3}$ & -4.61 & & & & & \\
\hline $\boldsymbol{\beta}_{0}$ & -5.99 & 0.06 & -103.24 & -6.10 & -5.87 & $<0.001$ \\
$\boldsymbol{\beta}_{1}$ & 3.07 & 0.01 & 227.25 & 3.04 & 3.09 & $<0.001$ \\
$\boldsymbol{\beta}_{2}$ & 3.89 & 0.03 & 137.71 & 3.83 & 3.95 & $<0.001$ \\
$\boldsymbol{\beta}_{3}$ & -0.13 & 0.05 & -2.33 & -0.23 & -0.02 & 0.02 \\
\hline
\end{tabular}

\begin{tabular}{lcrrrrr}
\hline \multicolumn{2}{l}{ Rhinochimaera pacifica, male } & \multicolumn{2}{l}{} \\
\hline Parameter & Value & SE & T & CI1 & CI2 & p-value \\
\hline $\boldsymbol{\alpha}_{0}$ & -4.36 & 0.05 & -83.95 & -4.46 & -4.26 & $<0.001$ \\
$\boldsymbol{\alpha}_{1}$ & & & & & & \\
$\boldsymbol{\alpha}_{2}$ & & & & & & \\
$\boldsymbol{\alpha}_{3}$ & & & & & & \\
\hline $\boldsymbol{\beta}_{0}$ & -0.59 & 0.52 & -1.14 & -1.60 & 0.42 & 0.25 \\
$\boldsymbol{\beta}_{1}$ & 1.79 & 0.11 & 16.64 & 1.58 & 2.01 & $<0.001$ \\
$\boldsymbol{\beta}_{2}$ & 4.74 & 0.01 & 943.99 & 4.73 & 4.75 & $<0.001$ \\
$\boldsymbol{\beta}_{3}$ & 1.33 & 0.12 & 11.27 & 1.10 & 1.56 & $<0.001$ \\
\hline
\end{tabular}

\begin{tabular}{|c|c|c|c|c|c|c|}
\hline \multicolumn{7}{|c|}{ Rhinochimaera pacifica, female } \\
\hline Parameter & Value & SE & $\mathbf{T}$ & CI1 & CI2 & p-value \\
\hline$\alpha_{0}$ & -3.74 & 0.09 & -43.35 & -3.91 & -3.57 & $<0.001$ \\
\hline$\alpha_{1}$ & 4.91 & 0.01 & 547.12 & 4.89 & 4.93 & $<0.001$ \\
\hline $\boldsymbol{\alpha}_{2}$ & -0.90 & 0.14 & -6.57 & -1.17 & -0.63 & $<0.001$ \\
\hline $\boldsymbol{\alpha}_{3}$ & -4.61 & & & & & \\
\hline $\boldsymbol{\beta}_{0}$ & -7.26 & 0.11 & -64.94 & -7.48 & -7.04 & $<0.001$ \\
\hline$\beta_{1}$ & 3.20 & 0.02 & 138.65 & 3.16 & 3.25 & $<0.001$ \\
\hline \multicolumn{7}{|l|}{$\beta_{2}$} \\
\hline$\beta_{3}$ & & & & & & \\
\hline
\end{tabular}




\begin{tabular}{|c|c|c|c|c|c|c|}
\hline \multicolumn{7}{|c|}{ Chimaera carophila, male } \\
\hline Parameter & Value & SE & $\mathbf{T}$ & CI1 & CI2 & p-value \\
\hline$\alpha_{0}$ & -2.69 & 0.34 & -7.82 & -3.38 & -2.01 & $<0.001$ \\
\hline $\boldsymbol{\alpha}_{1}$ & 4.26 & 0.01 & 397.72 & 4.24 & 4.28 & $<0.001$ \\
\hline $\boldsymbol{\alpha}_{2}$ & -2.08 & 0.38 & -5.46 & -2.84 & -1.33 & $<0.001$ \\
\hline$\alpha_{3}$ & -4.61 & & & & & \\
\hline $\boldsymbol{\beta}_{0}$ & -2.17 & 7.09 & -0.31 & -16.24 & 11.89 & 0.76 \\
\hline$\beta_{1}$ & 2.26 & 0.11 & 21.12 & 2.05 & 2.48 & $<0.001$ \\
\hline $\boldsymbol{\beta}_{2}$ & 5.08 & & & & & \\
\hline$\beta_{3}$ & 0.03 & & & & & \\
\hline
\end{tabular}

\begin{tabular}{lrrrrrr}
\hline \multicolumn{6}{l}{ Chimaera carophila, female } & \multicolumn{2}{l}{} \\
\hline Parameter & Value & SE & T & CI1 & CI2 & p-value \\
\hline $\boldsymbol{\alpha}_{\mathbf{0}}$ & -3.38 & 0.33 & -10.22 & -4.05 & -2.72 & $<0.001$ \\
$\boldsymbol{\alpha}_{\mathbf{1}}$ & 4.26 & 0.03 & 155.39 & 4.20 & 4.31 & $<0.001$ \\
$\boldsymbol{\alpha}_{2}$ & -1.16 & 0.43 & -2.72 & -2.02 & -0.30 & 0.01 \\
$\boldsymbol{\alpha}_{3}$ & -4.61 & & & & & \\
\hline $\boldsymbol{\beta}_{\mathbf{0}}$ & -3.77 & 0.45 & -8.30 & -4.68 & -2.86 & $<0.001$ \\
$\boldsymbol{\beta}_{\mathbf{1}}$ & 2.64 & 0.10 & 25.72 & 2.44 & 2.85 & $<0.001$ \\
$\boldsymbol{\beta}_{2}$ & & & & & & \\
$\boldsymbol{\beta}_{3}$ & & & & & & \\
\hline
\end{tabular}

\begin{tabular}{|c|c|c|c|c|c|c|}
\hline \multicolumn{7}{|c|}{ Hydrolagus bemisi, male } \\
\hline Parameter & Value & SE & $\mathbf{T}$ & CI1 & CI2 & p-value \\
\hline$\alpha_{0}$ & -4.58 & 0.01 & -364.43 & -4.61 & -4.56 & $<0.001$ \\
\hline \multicolumn{7}{|l|}{$\boldsymbol{\alpha}_{1}$} \\
\hline \multicolumn{7}{|l|}{$\alpha_{2}$} \\
\hline \multicolumn{7}{|l|}{$\boldsymbol{\alpha}_{3}$} \\
\hline$\beta_{0}$ & -2.21 & 0.24 & -9.23 & -2.67 & -1.74 & $<0.001$ \\
\hline$\beta_{1}$ & 2.30 & 0.06 & 41.29 & 2.20 & 2.41 & $<0.001$ \\
\hline$\beta_{2}$ & 4.26 & 0.00 & 1226.62 & 4.25 & 4.26 & $<0.001$ \\
\hline$\beta_{3}$ & 0.61 & 0.06 & 10.64 & 0.49 & 0.72 & $<0.001$ \\
\hline
\end{tabular}

\begin{tabular}{lcrrrrr}
\hline \multicolumn{2}{l}{ Hydrolagus bemisi, female } & & & & & \\
\hline Parameter & Value & SE & T & CI1 & CI2 & p-value \\
\hline $\boldsymbol{\alpha}_{0}$ & -4.57 & 0.01 & -364.26 & -4.59 & -4.54 & $<0.001$ \\
$\boldsymbol{\alpha}_{1}$ & & & & & & \\
$\boldsymbol{\alpha}_{2}$ & & & & & & \\
$\boldsymbol{\alpha}_{3}$ & & & & & & \\
\hline $\boldsymbol{\beta}_{0}$ & -4.06 & 0.09 & -47.27 & -4.23 & -3.89 & $<0.001$ \\
$\boldsymbol{\beta}_{1}$ & 2.74 & 0.02 & 137.26 & 2.70 & 2.78 & $<0.001$ \\
$\boldsymbol{\beta}_{2}$ & 4.23 & 0.01 & 493.15 & 4.21 & 4.25 & $<0.001$ \\
$\boldsymbol{\beta}_{3}$ & 0.18 & 0.02 & 7.84 & 0.13 & 0.22 & $<0.001$ \\
\hline
\end{tabular}




\begin{tabular}{lcrrrrr}
\hline \multicolumn{2}{l}{ Hydrolagus homonycteris, male } & & & & & \\
\hline Parameter & Value & SE & T & CI1 & CI2 & p-value \\
\hline $\boldsymbol{\alpha}_{0}$ & -5.71 & 0.20 & -28.84 & -6.11 & -5.31 & $<0.001$ \\
$\boldsymbol{\alpha}_{1}$ & & & & & & \\
$\boldsymbol{\alpha}_{2}$ & & & & & & \\
$\boldsymbol{\alpha}_{3}$ & 4.33 & 0.99 & 4.38 & 2.34 & 6.32 & $<0.001$ \\
\hline $\boldsymbol{\beta}_{0}$ & 0.81 & 0.22 & 3.66 & 0.36 & 1.25 & $<0.001$ \\
$\boldsymbol{\beta}_{1}$ & 4.41 & 0.01 & 635.33 & 4.39 & 4.42 & $<0.001$ \\
$\boldsymbol{\beta}_{2}$ & 2.16 & 0.36 & 6.06 & 1.44 & 2.87 & $<0.001$ \\
$\boldsymbol{\beta}_{3}$ & & & & & & \\
\hline
\end{tabular}

\begin{tabular}{lrrrrrr}
\hline \multicolumn{2}{l}{ Hydrolagus homonycteris, female } & & & & \\
\hline Parameter & Value & SE & T & CI1 & CI2 & p-value \\
\hline $\boldsymbol{\alpha}_{\mathbf{0}}$ & -5.07 & 0.34 & -14.78 & -5.82 & -4.32 & $<0.001$ \\
$\boldsymbol{\alpha}_{\mathbf{1}}$ & & & & & & \\
$\boldsymbol{\alpha}_{\mathbf{2}}$ & & & & & & \\
$\boldsymbol{\alpha}_{3}$ & 2.52 & 3.05 & 0.82 & -4.14 & 9.18 & 0.43 \\
\hline $\boldsymbol{\beta}_{0}$ & 1.26 & 0.67 & 1.87 & -0.21 & 2.73 & 0.09 \\
$\boldsymbol{\beta}_{1}$ & 4.45 & 0.06 & 78.31 & 4.33 & 4.58 & $<0.001$ \\
$\boldsymbol{\beta}_{\mathbf{2}}$ & 1.85 & 0.72 & 2.57 & 0.28 & 3.41 & 0.02 \\
$\boldsymbol{\beta}_{3}$ & & & & & & \\
\hline
\end{tabular}

\begin{tabular}{|c|c|c|c|c|c|c|}
\hline \multicolumn{7}{|c|}{ Hydrolagus novaezealandiae, male } \\
\hline Parameter & Value & $\mathbf{S E}$ & $\mathbf{T}$ & CI1 & CI2 & p-value \\
\hline$\alpha_{0}$ & -4.23 & 0.05 & -91.58 & -4.32 & -4.14 & $<0.001$ \\
\hline$\alpha_{1}$ & 3.70 & 0.01 & 544.66 & 3.69 & 3.71 & $<0.001$ \\
\hline$\alpha_{2}$ & -0.66 & 0.05 & -13.56 & -0.75 & -0.56 & $<0.001$ \\
\hline$\alpha_{3}$ & -4.61 & & & & & \\
\hline $\boldsymbol{\beta}_{0}$ & -3.74 & 0.12 & -31.82 & -3.97 & -3.51 & $<0.001$ \\
\hline$\beta_{1}$ & 2.66 & 0.03 & 91.75 & 2.60 & 2.72 & $<0.001$ \\
\hline$\beta_{2}$ & 4.01 & 0.00 & 1405.81 & 4.00 & 4.01 & $<0.001$ \\
\hline$\beta_{3}$ & 0.66 & 0.03 & 20.92 & 0.60 & 0.72 & $<0.001$ \\
\hline
\end{tabular}

\begin{tabular}{|c|c|c|c|c|c|c|}
\hline \multicolumn{7}{|c|}{ Hydrolagus novaezealandiae, female } \\
\hline Parameter & Value & SE & $\mathbf{T}$ & CI1 & CI2 & p-value \\
\hline $\boldsymbol{\alpha}_{0}$ & -4.26 & 0.07 & -58.07 & -4.40 & -4.12 & $<0.001$ \\
\hline \multirow{2}{*}{$\begin{array}{l}\alpha_{1} \\
\alpha_{2}\end{array}$} & 3.58 & 0.03 & 141.68 & 3.53 & 3.63 & $<0.001$ \\
\hline & -0.52 & 0.07 & -7.10 & -0.67 & -0.38 & $<0.001$ \\
\hline$\alpha_{3}$ & -4.61 & & & & & \\
\hline$\beta_{0}$ & -6.22 & 0.02 & -353.03 & -6.25 & -6.18 & $<0.001$ \\
\hline$\beta_{1}$ & 3.27 & 0.00 & 745.53 & 3.27 & 3.28 & $<0.001$ \\
\hline$\beta_{2}$ & & & & & & \\
\hline$\beta_{3}$ & & & & & & \\
\hline
\end{tabular}




\begin{tabular}{lrrrrrr}
\hline \multicolumn{2}{l}{ Squalus acanthias, male } & & & & & \\
\hline Parameter & Value & SE & T & CI1 & CI2 & p-value \\
\hline $\boldsymbol{\alpha}_{0}$ & -4.36 & 0.01 & -347.01 & -4.38 & -4.34 & $<0.001$ \\
$\boldsymbol{\alpha}_{1}$ & 4.46 & 0.01 & 299.18 & 4.43 & 4.49 & $<0.001$ \\
$\boldsymbol{\alpha}_{2}$ & 2.10 & 0.70 & 3.00 & 0.73 & 3.47 & $<0.001$ \\
$\boldsymbol{\alpha}_{3}$ & -4.61 & & & & & \\
\hline $\boldsymbol{\beta}_{0}$ & -5.85 & 0.04 & -133.91 & -5.94 & -5.77 & $<0.001$ \\
$\boldsymbol{\beta}_{1}$ & 3.06 & 0.01 & 292.34 & 3.04 & 3.09 & $<0.001$ \\
$\boldsymbol{\beta}_{2}$ & 3.85 & 0.02 & 192.26 & 3.81 & 3.89 & $<0.001$ \\
$\boldsymbol{\beta}_{3}$ & -0.11 & 0.03 & -3.73 & -0.17 & -0.05 & $<0.001$ \\
\hline
\end{tabular}

\begin{tabular}{lrrrrrr}
\hline \multicolumn{2}{l}{ Squalus acanthias, female } & & & & & \\
\hline Parameter & Value & SE & T & CI1 & CI2 & p-value \\
\hline $\boldsymbol{\alpha}_{\mathbf{0}}$ & -4.25 & 0.01 & -422.65 & -4.27 & -4.23 & $<0.001$ \\
$\boldsymbol{\alpha}_{\mathbf{1}}$ & & & & & & \\
$\boldsymbol{\alpha}_{\mathbf{2}}$ & & & & & & \\
$\boldsymbol{\alpha}_{3}$ & -6.96 & 0.02 & -303.71 & -7.01 & -6.92 & $<0.001$ \\
\hline $\boldsymbol{\beta}_{0}$ & 3.36 & 0.01 & 629.60 & 3.35 & 3.37 & $<0.001$ \\
$\boldsymbol{\beta}_{1}$ & 3.84 & 0.02 & 234.66 & 3.81 & 3.87 & $<0.001$ \\
$\boldsymbol{\beta}_{2}$ & -0.44 & 0.03 & -14.03 & -0.50 & -0.38 & $<0.001$ \\
$\boldsymbol{\beta}_{3}$ & & & & & & \\
\hline
\end{tabular}

\begin{tabular}{lrrrrrr}
\hline \multicolumn{2}{l}{ Centrophorus squamosus, male } & & & & & \\
\hline Parameter & Value & SE & T & CI1 & CI2 & p-value \\
\hline $\boldsymbol{\alpha}_{\mathbf{0}}$ & -4.42 & 0.06 & -77.52 & -4.54 & -4.31 & $<0.001$ \\
$\boldsymbol{\alpha}_{\mathbf{1}}$ & & & & & & \\
$\boldsymbol{\alpha}_{\mathbf{2}}$ & & & & & & \\
$\boldsymbol{\alpha}_{3}$ & -6.34 & 0.06 & -110.98 & -6.45 & -6.23 & $<0.001$ \\
\hline $\boldsymbol{\beta}_{0}$ & 3.24 & 0.01 & 248.61 & 3.21 & 3.27 & $<0.001$ \\
$\boldsymbol{\beta}_{1}$ & 3.80 & 0.02 & 209.22 & 3.77 & 3.84 & $<0.001$ \\
$\boldsymbol{\beta}_{2}$ & -1.02 & 0.35 & -2.89 & -1.72 & -0.33 & $<0.001$ \\
$\boldsymbol{\beta}_{3}$ & & & & & & \\
\hline
\end{tabular}

\begin{tabular}{lrrrrrr}
\hline \multicolumn{2}{l}{ Centrophorus squamosus, female } & & & & & \\
\hline Parameter & Value & SE & T & CI1 & CI2 & p-value \\
\hline $\boldsymbol{\alpha}_{0}$ & -4.40 & 0.06 & -71.60 & -4.52 & -4.28 & $<0.001$ \\
$\boldsymbol{\alpha}_{1}$ & 4.63 & 0.03 & 161.54 & 4.58 & 4.69 & $<0.001$ \\
$\boldsymbol{\alpha}_{2}$ & 0.46 & 0.09 & 5.11 & 0.28 & 0.64 & $<0.001$ \\
$\boldsymbol{\alpha}_{3}$ & -4.61 & & & & & \\
\hline $\boldsymbol{\beta}_{0}$ & -7.99 & 0.14 & -55.18 & -8.28 & -7.71 & $<0.001$ \\
$\boldsymbol{\beta}_{1}$ & 3.61 & 0.03 & 118.50 & 3.55 & 3.67 & $<0.001$ \\
$\boldsymbol{\beta}_{2}$ & 4.45 & 0.02 & 241.17 & 4.41 & 4.49 & $<0.001$ \\
$\boldsymbol{\beta}_{3}$ & -0.39 & 0.05 & -8.28 & -0.48 & -0.30 & $<0.001$ \\
\hline
\end{tabular}




\begin{tabular}{lrrrrrr}
\hline \multicolumn{2}{l}{ Deania calcea, male } & & & & & \\
\hline Parameter & Value & SE & T & CI1 & CI2 & p-value \\
\hline $\boldsymbol{\alpha}_{0}$ & -4.84 & 0.02 & -286.54 & -4.87 & -4.80 & $<0.001$ \\
$\boldsymbol{\alpha}_{1}$ & & & & & & \\
$\boldsymbol{\alpha}_{2}$ & & & & & & \\
$\boldsymbol{\alpha}_{3}$ & & & & & & \\
\hline $\boldsymbol{\beta}_{0}$ & -3.43 & 0.20 & -17.07 & -3.82 & -3.03 & $<0.001$ \\
$\boldsymbol{\beta}_{1}$ & 2.50 & 0.05 & 55.45 & 2.41 & 2.59 & $<0.001$ \\
$\boldsymbol{\beta}_{2}$ & 4.38 & 0.01 & 494.06 & 4.37 & 4.40 & $<0.001$ \\
$\boldsymbol{\beta}_{3}$ & 0.64 & 0.04 & 14.74 & 0.56 & 0.73 & $<0.001$ \\
\hline
\end{tabular}

\begin{tabular}{lrrrrrr}
\hline \multicolumn{2}{l}{ Deania calcea, female } & & & & & \\
\hline Parameter & Value & SE & T & CI1 & CI2 & p-value \\
\hline $\boldsymbol{\alpha}_{\mathbf{0}}$ & -4.70 & 0.02 & -243.99 & -4.74 & -4.67 & $<0.001$ \\
$\boldsymbol{\alpha}_{\mathbf{1}}$ & 4.60 & 0.00 & 924.57 & 4.59 & 4.61 & $<0.001$ \\
$\boldsymbol{\alpha}_{2}$ & 0.86 & 0.04 & 20.21 & 0.78 & 0.95 & $<0.001$ \\
$\boldsymbol{\alpha}_{3}$ & -4.61 & & & & & \\
\hline $\boldsymbol{\beta}_{\mathbf{0}}$ & -6.65 & 0.02 & -336.32 & -6.69 & -6.61 & $<0.001$ \\
$\boldsymbol{\beta}_{1}$ & 3.24 & 0.00 & 710.47 & 3.23 & 3.25 & $<0.001$ \\
$\boldsymbol{\beta}_{2}$ & 3.75 & & & & & \\
$\boldsymbol{\beta}_{3}$ & -0.50 & & & & & \\
\hline
\end{tabular}

\begin{tabular}{lrrrrrr}
\hline \multicolumn{2}{l}{ Etmopterus granulosus, male } & & & & & \\
\hline Parameter & Value & SE & T & CI1 & CI2 & p-value \\
\hline $\boldsymbol{\alpha}_{\mathbf{0}}$ & -3.86 & 0.05 & -72.28 & -3.96 & -3.75 & $<0.001$ \\
$\boldsymbol{\alpha}_{1}$ & 3.79 & 0.01 & 418.07 & 3.77 & 3.81 & $<0.001$ \\
$\boldsymbol{\alpha}_{2}$ & -0.75 & 0.06 & -12.95 & -0.86 & -0.63 & $<0.001$ \\
$\boldsymbol{\alpha}_{3}$ & -4.61 & & & & & \\
\hline $\boldsymbol{\beta}_{0}$ & -5.30 & 0.04 & -150.16 & -5.37 & -5.23 & $<0.001$ \\
$\boldsymbol{\beta}_{1}$ & 3.00 & 0.01 & 343.95 & 2.99 & 3.02 & $<0.001$ \\
$\boldsymbol{\beta}_{2}$ & & & & & & \\
$\boldsymbol{\beta}_{3}$ & & & & & & \\
\hline
\end{tabular}

\begin{tabular}{lrrrrrr}
\hline \multicolumn{2}{l}{ Etmopterus granulosus, female } & \multicolumn{7}{l}{} \\
\hline Parameter & Value & SE & T & CI1 & CI2 & p-value \\
\hline $\boldsymbol{\alpha}_{\mathbf{0}}$ & -4.17 & 0.02 & -240.85 & -4.20 & -4.14 & $<0.001$ \\
$\boldsymbol{\alpha}_{\mathbf{1}}$ & 4.34 & 0.01 & 794.43 & 4.33 & 4.35 & $<0.001$ \\
$\boldsymbol{\alpha}_{\mathbf{2}}$ & 1.05 & 0.20 & 5.32 & 0.66 & 1.44 & $<0.001$ \\
$\boldsymbol{\alpha}_{3}$ & -4.61 & & & & & \\
\hline $\boldsymbol{\beta}_{\mathbf{0}}$ & -6.01 & 0.04 & -170.37 & -6.08 & -5.94 & $<0.001$ \\
$\boldsymbol{\beta}_{1}$ & 3.19 & 0.01 & 374.89 & 3.17 & 3.20 & $<0.001$ \\
$\boldsymbol{\beta}_{2}$ & 3.60 & 0.03 & 131.87 & 3.54 & 3.65 & $<0.001$ \\
$\boldsymbol{\beta}_{3}$ & -0.27 & 0.04 & -7.69 & -0.34 & -0.20 & $<0.001$ \\
\hline
\end{tabular}




\begin{tabular}{lrrrrrr}
\hline \multicolumn{2}{l}{ Etmopterus lucifer, male } & & & & & \\
\hline Parameter & Value & SE & T & CI1 & CI2 & p-value \\
\hline $\boldsymbol{\alpha}_{0}$ & -4.47 & 0.03 & -142.27 & -4.53 & -4.41 & $<0.001$ \\
$\boldsymbol{\alpha}_{1}$ & & & & & & \\
$\mathbf{\alpha}_{2}$ & & & & & & \\
$\boldsymbol{\alpha}_{3}$ & & & & & & \\
\hline $\boldsymbol{\beta}_{0}$ & -4.01 & 0.20 & -19.59 & -4.41 & -3.61 & $<0.001$ \\
$\boldsymbol{\beta}_{1}$ & 2.52 & 0.06 & 45.59 & 2.42 & 2.63 & $<0.001$ \\
$\boldsymbol{\beta}_{2}$ & 3.61 & 0.01 & 452.28 & 3.60 & 3.63 & $<0.001$ \\
$\boldsymbol{\beta}_{3}$ & 0.65 & 0.07 & 9.33 & 0.52 & 0.79 & $<0.001$ \\
\hline
\end{tabular}

\begin{tabular}{lrrrrrr}
\hline \multicolumn{2}{l}{ Etmopterus lucifer, female } & & & & & \\
\hline Parameter & Value & SE & T & CI1 & CI2 & p-value \\
\hline $\boldsymbol{\alpha}_{\mathbf{0}}$ & -2.91 & 0.14 & -21.43 & -3.18 & -2.64 & $<0.001$ \\
$\boldsymbol{\alpha}_{\mathbf{1}}$ & 3.33 & 0.03 & 116.42 & 3.27 & 3.38 & $<0.001$ \\
$\boldsymbol{\alpha}_{\mathbf{2}}$ & -1.05 & 0.13 & -7.81 & -1.32 & -0.79 & $<0.001$ \\
$\boldsymbol{\alpha}_{\mathbf{3}}$ & -4.61 & & & & & \\
\hline $\boldsymbol{\beta}_{\mathbf{0}}$ & -5.29 & & & & & \\
$\boldsymbol{\beta}_{1}$ & 2.94 & 0.02 & 153.90 & 2.90 & 2.97 & $<0.001$ \\
$\boldsymbol{\beta}_{\mathbf{2}}$ & 4.08 & & & & & \\
$\boldsymbol{\beta}_{\mathbf{3}}$ & 0.36 & 0.00 & 224.21 & 0.36 & 0.36 & $<0.001$ \\
\hline
\end{tabular}

\begin{tabular}{lrrrrrr}
\hline \multicolumn{2}{l}{ Centroscymnus owstoni, male } & & & & & \\
\hline Parameter & Value & SE & T & CI1 & CI2 & p-value \\
\hline $\boldsymbol{\alpha}_{\mathbf{0}}$ & -4.74 & 0.05 & -99.46 & -4.84 & -4.65 & $<0.001$ \\
$\boldsymbol{\alpha}_{\mathbf{1}}$ & 4.48 & 0.01 & 543.40 & 4.46 & 4.50 & $<0.001$ \\
$\boldsymbol{\alpha}_{\mathbf{2}}$ & 1.78 & 0.37 & 4.86 & 1.06 & 2.49 & $<0.001$ \\
$\boldsymbol{\alpha}_{3}$ & -4.61 & & & & & \\
\hline $\boldsymbol{\beta}_{\mathbf{0}}$ & -5.12 & 0.15 & -33.47 & -5.42 & -4.82 & $<0.001$ \\
$\boldsymbol{\beta}_{\mathbf{1}}$ & 2.98 & 0.04 & 85.04 & 2.91 & 3.05 & $<0.001$ \\
$\boldsymbol{\beta}_{\mathbf{2}}$ & 4.03 & 0.03 & 156.16 & 3.98 & 4.08 & $<0.001$ \\
$\boldsymbol{\beta}_{\mathbf{3}}$ & 0.10 & 0.07 & 1.41 & -0.04 & 0.23 & 0.16 \\
\hline
\end{tabular}

\begin{tabular}{lrrrrrr}
\hline \multicolumn{2}{l}{ Centroscymnus owstoni, female } & & & & & \\
\hline Parameter & Value & SE & T & CI1 & CI2 & p-value \\
\hline $\boldsymbol{\alpha}_{0}$ & -4.47 & 0.07 & -65.58 & -4.60 & -4.34 & $<0.001$ \\
$\boldsymbol{\alpha}_{1}$ & 4.59 & 0.01 & 336.10 & 4.56 & 4.62 & $<0.001$ \\
$\boldsymbol{\alpha}_{\mathbf{2}}$ & 0.71 & 0.11 & 6.47 & 0.49 & 0.92 & $<0.001$ \\
$\boldsymbol{\alpha}_{3}$ & -4.61 & & & & & \\
\hline $\boldsymbol{\beta}_{0}$ & -7.66 & 0.24 & -32.24 & -8.13 & -7.20 & $<0.001$ \\
$\boldsymbol{\beta}_{1}$ & 3.57 & 0.05 & 68.61 & 3.47 & 3.67 & $<0.001$ \\
$\boldsymbol{\beta}_{2}$ & 4.41 & 0.02 & 246.09 & 4.37 & 4.44 & $<0.001$ \\
$\boldsymbol{\beta}_{3}$ & -0.48 & 0.06 & -7.88 & -0.60 & -0.36 & $<0.001$ \\
\hline
\end{tabular}




\begin{tabular}{lrrrrrr}
\hline \multicolumn{2}{l}{ Centroselachus crepidater, male } & & & & & \\
\hline Parameter & Value & SE & T & CI1 & CI2 & p-value \\
\hline $\boldsymbol{\alpha}_{0}$ & -4.38 & 0.07 & -65.40 & -4.52 & -4.25 & $<0.001$ \\
$\boldsymbol{\alpha}_{1}$ & & & & & & \\
$\boldsymbol{\alpha}_{2}$ & & & & & & \\
$\boldsymbol{\alpha}_{3}$ & & & & & & \\
\hline $\boldsymbol{\beta}_{0}$ & -8.69 & 0.42 & -20.81 & -9.51 & -7.87 & $<0.001$ \\
$\boldsymbol{\beta}_{1}$ & 3.79 & 0.09 & 41.59 & 3.61 & 3.97 & $<0.001$ \\
$\boldsymbol{\beta}_{2}$ & 4.45 & 0.02 & 215.65 & 4.41 & 4.49 & $<0.001$ \\
$\boldsymbol{\beta}_{3}$ & -0.74 & 0.10 & -7.64 & -0.93 & -0.55 & $<0.001$ \\
\hline
\end{tabular}

\begin{tabular}{lrrrrrr}
\hline \multicolumn{2}{l}{ Centroselachus crepidater, female } & & & & & \\
\hline Parameter & Value & SE & T & CI1 & CI2 & p-value \\
\hline $\boldsymbol{\alpha}_{\mathbf{0}}$ & -4.64 & 0.05 & -98.57 & -4.73 & -4.55 & $<0.001$ \\
$\boldsymbol{\alpha}_{\mathbf{1}}$ & 4.46 & 0.01 & 625.46 & 4.45 & 4.48 & $<0.001$ \\
$\boldsymbol{\alpha}_{\mathbf{2}}$ & 1.11 & 0.12 & 9.52 & 0.88 & 1.33 & $<0.001$ \\
$\boldsymbol{\alpha}_{\mathbf{3}}$ & -4.61 & & & & & \\
\hline $\boldsymbol{\beta}_{\mathbf{0}}$ & -6.89 & 0.25 & -27.45 & -7.38 & -6.39 & $<0.001$ \\
$\boldsymbol{\beta}_{\mathbf{1}}$ & 3.39 & 0.06 & 59.97 & 3.27 & 3.50 & $<0.001$ \\
$\boldsymbol{\beta}_{\mathbf{2}}$ & 4.40 & 0.01 & 443.31 & 4.38 & 4.42 & $<0.001$ \\
$\boldsymbol{\beta}_{\mathbf{3}}$ & -0.36 & 0.06 & -6.02 & -0.47 & -0.24 & $<0.001$ \\
\hline
\end{tabular}

\begin{tabular}{lrrrrrr}
\hline \multicolumn{2}{l}{ Centroselachus crepidater, male 2 } & & & & & \\
\hline Parameter & Value & SE & T & CI1 & CI2 & p-value \\
\hline $\boldsymbol{\alpha}_{\mathbf{0}}$ & -4.08 & 0.17 & -23.40 & -4.43 & -3.74 & $<0.001$ \\
$\boldsymbol{\alpha}_{\mathbf{1}}$ & 4.11 & 0.02 & 270.99 & 4.08 & 4.14 & $<0.001$ \\
$\boldsymbol{\alpha}_{\mathbf{2}}$ & -0.74 & 0.19 & -3.80 & -1.12 & -0.36 & $<0.001$ \\
$\boldsymbol{\alpha}_{\mathbf{3}}$ & -4.61 & & & & & \\
\hline $\boldsymbol{\beta}_{\mathbf{0}}$ & -5.04 & 0.15 & -33.04 & -5.34 & -4.74 & $<0.001$ \\
$\boldsymbol{\beta}_{\mathbf{1}}$ & 2.97 & 0.04 & 84.12 & 2.90 & 3.03 & $<0.001$ \\
$\boldsymbol{\beta}_{\mathbf{2}}$ & 3.72 & 0.02 & 208.21 & 3.68 & 3.75 & $<0.001$ \\
$\boldsymbol{\beta}_{\mathbf{3}}$ & 0.57 & 0.19 & 3.06 & 0.20 & 0.94 & $<0.001$ \\
\hline
\end{tabular}

\begin{tabular}{lrrrrrr}
\hline \multicolumn{2}{l}{ Centroselachus crepidater, female 2} & & & & \\
\hline Parameter & Value & SE & T & CI1 & CI2 & p-value \\
\hline $\boldsymbol{\alpha}_{\mathbf{0}}$ & -4.63 & 0.05 & -102.37 & -4.72 & -4.54 & $<0.001$ \\
$\boldsymbol{\alpha}_{\mathbf{1}}$ & & & & & & \\
$\boldsymbol{\alpha}_{\mathbf{2}}$ & & & & & & \\
$\boldsymbol{\alpha}_{3}$ & -3.18 & 0.42 & -7.50 & -4.01 & -2.35 & $<0.001$ \\
\hline $\boldsymbol{\beta}_{0}$ & 2.54 & 0.10 & 26.34 & 2.35 & 2.73 & $<0.001$ \\
$\boldsymbol{\beta}_{1}$ & 4.31 & 0.02 & 229.46 & 4.28 & 4.35 & $<0.001$ \\
$\boldsymbol{\beta}_{2}$ & 0.54 & 0.10 & 5.29 & 0.34 & 0.73 & $<0.001$ \\
$\boldsymbol{\beta}_{3}$ & & & & & & \\
\hline
\end{tabular}




\begin{tabular}{lrrrrrr}
\hline \multicolumn{2}{l}{ Scymnodon } & plunketi, male & & & & \\
\hline Parameter & Value & SE & T & CI1 & CI2 & p-value \\
\hline $\boldsymbol{\alpha}_{0}$ & -4.07 & 0.21 & -19.02 & -4.50 & -3.65 & $<0.001$ \\
$\boldsymbol{\alpha}_{1}$ & 4.32 & 0.04 & 110.73 & 4.24 & 4.39 & $<0.001$ \\
$\boldsymbol{\alpha}_{2}$ & -0.58 & 0.25 & -2.29 & -1.08 & -0.08 & 0.02 \\
$\boldsymbol{\alpha}_{3}$ & -4.61 & & & & & \\
\hline $\boldsymbol{\beta}_{0}$ & -5.38 & 0.12 & -43.59 & -5.62 & -5.14 & $<0.001$ \\
$\boldsymbol{\beta}_{1}$ & 3.05 & 0.03 & 111.94 & 3.00 & 3.11 & $<0.001$ \\
$\boldsymbol{\beta}_{2}$ & & & & & & \\
$\boldsymbol{\beta}_{3}$ & & & & & & \\
\hline
\end{tabular}

\begin{tabular}{lrrrrrr}
\hline \multicolumn{2}{l}{ Scymnodon plunketi, female } & & & & & \\
\hline Parameter & Value & SE & T & CI1 & CI2 & p-value \\
\hline $\boldsymbol{\alpha}_{\mathbf{0}}$ & -4.10 & 0.10 & -42.55 & -4.28 & -3.91 & $<0.001$ \\
$\boldsymbol{\alpha}_{\mathbf{1}}$ & 4.83 & 0.02 & 272.95 & 4.79 & 4.86 & $<0.001$ \\
$\boldsymbol{\alpha}_{\mathbf{2}}$ & 0.99 & 0.31 & 3.17 & 0.38 & 1.61 & $<0.001$ \\
$\boldsymbol{\alpha}_{\mathbf{3}}$ & -4.61 & & & & & \\
\hline $\boldsymbol{\beta}_{\mathbf{0}}$ & -6.37 & 0.21 & -29.63 & -6.79 & -5.94 & $<0.001$ \\
$\boldsymbol{\beta}_{1}$ & 3.28 & 0.05 & 68.49 & 3.18 & 3.37 & $<0.001$ \\
$\boldsymbol{\beta}_{\mathbf{2}}$ & 4.12 & 0.04 & 95.95 & 4.04 & 4.21 & $<0.001$ \\
$\boldsymbol{\beta}_{\mathbf{3}}$ & -0.36 & 0.09 & -3.93 & -0.55 & -0.18 & $<0.001$ \\
\hline
\end{tabular}

\begin{tabular}{lrrrrrr}
\hline \multicolumn{2}{l}{ Oxynotus bruniensis, male } & & & & & \\
\hline Parameter & Value & SE & T & CI1 & CI2 & p-value \\
\hline $\boldsymbol{\alpha}_{0}$ & -4.24 & 0.27 & -15.58 & -4.80 & -3.68 & $<0.001$ \\
$\boldsymbol{\alpha}_{1}$ & & & & & & \\
$\boldsymbol{\alpha}_{2}$ & & & & & & \\
$\boldsymbol{\alpha}_{3}$ & & & & & & \\
\hline $\boldsymbol{\beta}_{0}$ & -4.00 & 0.93 & -4.28 & -5.93 & -2.07 & $<0.001$ \\
$\boldsymbol{\beta}_{1}$ & 2.86 & 0.23 & 12.37 & 2.38 & 3.34 & $<0.001$ \\
$\boldsymbol{\beta}_{2}$ & & & & & & \\
$\boldsymbol{\beta}_{3}$ & & & & & & \\
\hline
\end{tabular}

\begin{tabular}{lrrrrrr}
\hline \multicolumn{2}{l}{ Oxynotus bruniensis, female } & & & & & \\
\hline Parameter & Value & SE & T & CI1 & CI2 & p-value \\
\hline $\boldsymbol{\alpha}_{0}$ & -3.65 & 0.25 & -14.60 & -4.16 & -3.14 & $<0.001$ \\
$\boldsymbol{\alpha}_{1}$ & & & & & & \\
$\boldsymbol{\alpha}_{2}$ & & & & & & \\
$\boldsymbol{\alpha}_{3}$ & & & & & \\
\hline $\boldsymbol{\beta}_{0}$ & -6.50 & 1.30 & -5.02 & -9.15 & -3.85 & $<0.001$ \\
$\boldsymbol{\beta}_{1}$ & 3.50 & 0.31 & 11.23 & 2.86 & 4.14 & $<0.001$ \\
$\boldsymbol{\beta}_{2}$ & & & & & & \\
$\boldsymbol{\beta}_{3}$ & & & & & & \\
\hline
\end{tabular}




\begin{tabular}{lrrrrrr}
\hline \multicolumn{2}{l}{ Dalatias licha, male } & & & & & \\
\hline Parameter & Value & SE & T & CI1 & CI2 & p-value \\
\hline $\boldsymbol{\alpha}_{0}$ & -4.24 & 0.08 & -55.56 & -4.39 & -4.09 & $<0.001$ \\
$\boldsymbol{\alpha}_{\mathbf{1}}$ & & & & & & \\
$\boldsymbol{\alpha}_{\mathbf{2}}$ & & & & & & \\
$\boldsymbol{\alpha}_{\mathbf{3}}$ & -6.36 & 0.09 & -72.59 & -6.53 & -6.19 & $<0.001$ \\
\hline $\boldsymbol{\beta}_{0}$ & 3.24 & 0.02 & 151.53 & 3.20 & 3.29 & $<0.001$ \\
$\boldsymbol{\beta}_{1}$ & 3.74 & 0.02 & 198.06 & 3.70 & 3.78 & $<0.001$ \\
$\boldsymbol{\beta}_{2}$ & -0.97 & 0.52 & -1.85 & -2.00 & 0.06 & 0.07 \\
$\boldsymbol{\beta}_{3}$ & & & & & & \\
\hline
\end{tabular}

\begin{tabular}{lrrrrrr}
\hline \multicolumn{2}{l}{ Dalatias licha, female } & \multicolumn{7}{l}{} \\
\hline Parameter & Value & SE & T & CI1 & CI2 & p-value \\
\hline $\boldsymbol{\alpha}_{\mathbf{0}}$ & -4.49 & 0.07 & -65.61 & -4.63 & -4.36 & $<0.001$ \\
$\boldsymbol{\alpha}_{\mathbf{1}}$ & 4.61 & 0.03 & 148.15 & 4.55 & 4.67 & $<0.001$ \\
$\boldsymbol{\alpha}_{\mathbf{2}}$ & 0.89 & 0.18 & 4.89 & 0.53 & 1.25 & $<0.001$ \\
$\boldsymbol{\alpha}_{\mathbf{3}}$ & -4.61 & & & & & \\
\hline $\boldsymbol{\beta}_{\mathbf{0}}$ & -6.68 & 0.07 & -94.29 & -6.81 & -6.54 & $<0.001$ \\
$\boldsymbol{\beta}_{\mathbf{1}}$ & 3.32 & 0.02 & 192.66 & 3.29 & 3.36 & $<0.001$ \\
$\boldsymbol{\beta}_{\mathbf{2}}$ & 3.77 & 0.01 & 321.72 & 3.75 & 3.79 & $<0.001$ \\
$\boldsymbol{\beta}_{3}$ & -0.55 & 0.24 & -2.28 & -1.03 & -0.08 & 0.02 \\
\hline
\end{tabular}




\section{Appendix III.}

Linear and best fitting model for each species [Harriotta raleighana (LCH),

Rhinochimaera pacifica (RCH), Chimaera carophila (CHP), Hydrolagus bemisi (GSP), Hydrolagus homonycteris (HYB), Hydrolagus novaezealandiae (GSH), Squalus acanthias (SPD), Centrophorus squamosus (CSQ), Deania calcea (SND), Etmopterus granulosus (ETB), Etmopterus lucifer (ETL), Centroscymnus owstoni (CYO), Centroselachus crepidater (CYP), truncated Centroselachus crepidater (CPT), Scymnodon plunketi (PLS), Oxynotus bruniensis (PDG), and Dalatias licha (BSH)] and by sex (male, M; female, F). Top left panel: linear model with constant variance; top right panel: residuals for the best fitting model from a linear model with constant variance (horizontal lines show the mean with \pm 1 standard deviations); middle two panels: same data summarised by boxplots; bottom left panel: the same as top left, with residuals from the linear model, but with the mean and \pm 1 standard deviation superimposed; bottom right: fitted model plotted on top of the original data on the original linear scale. :, inflection point of the variance shelf $(\alpha)$; |, inflection point of the broken stick $(\beta)$. 

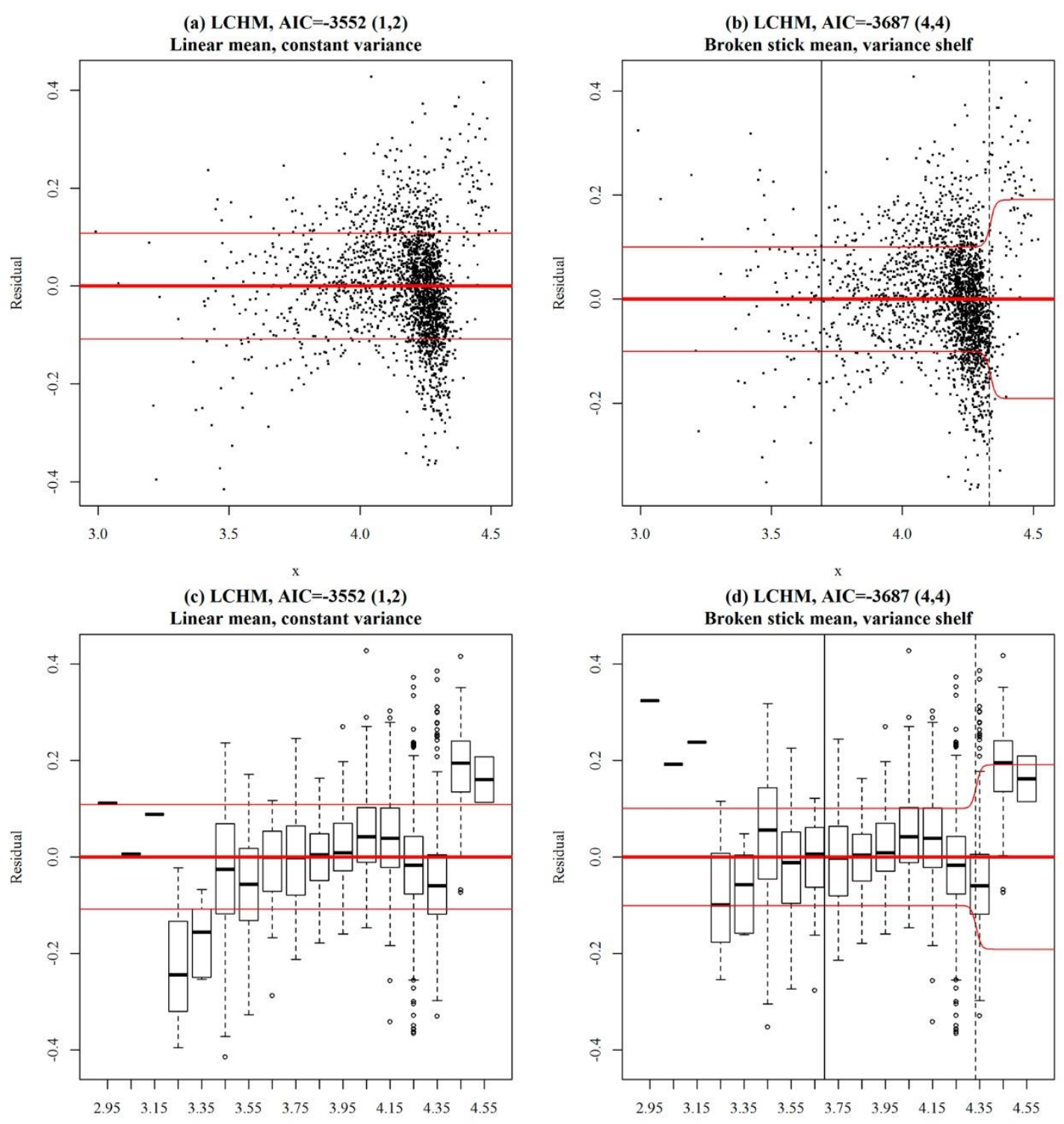

(e) LCHM, AIC $=-3687(4,4)$
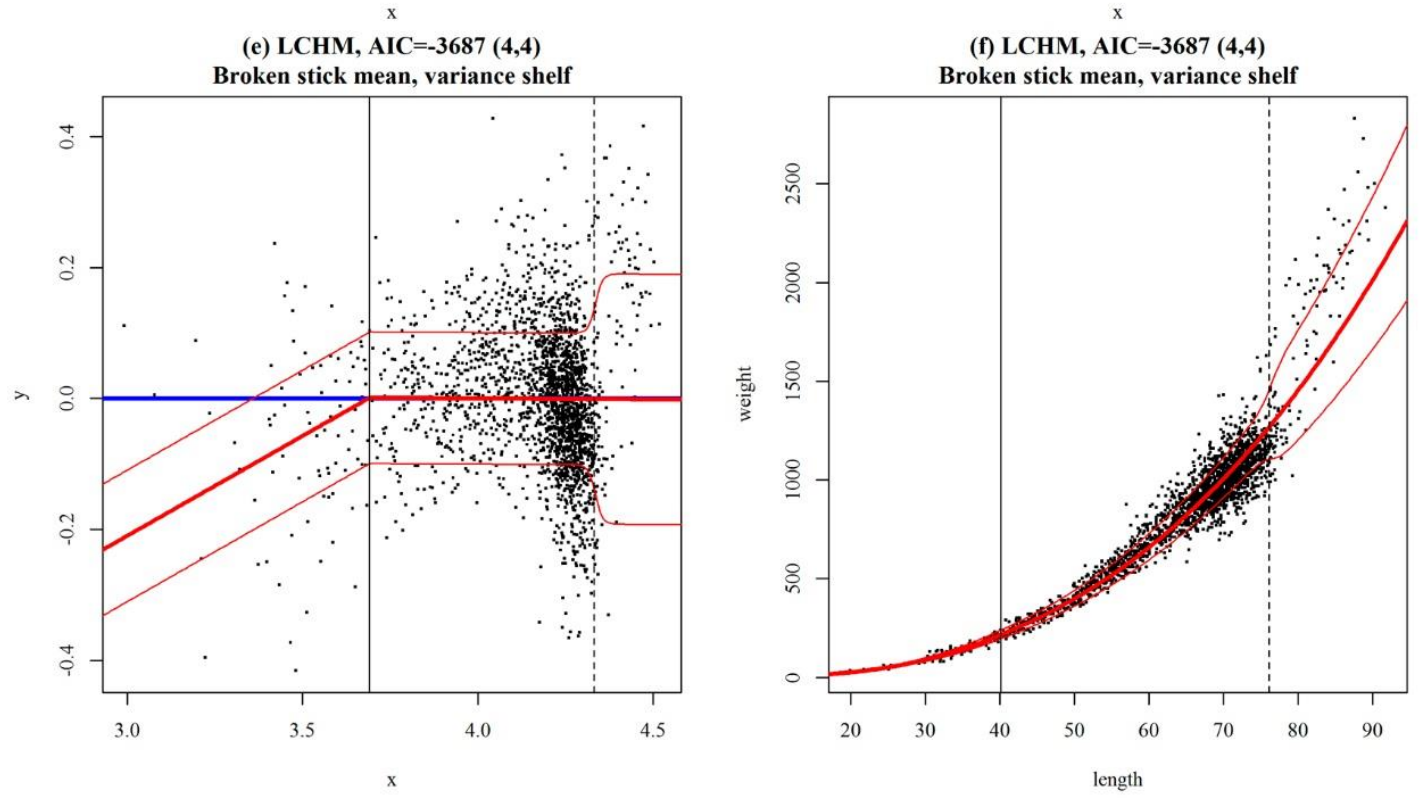

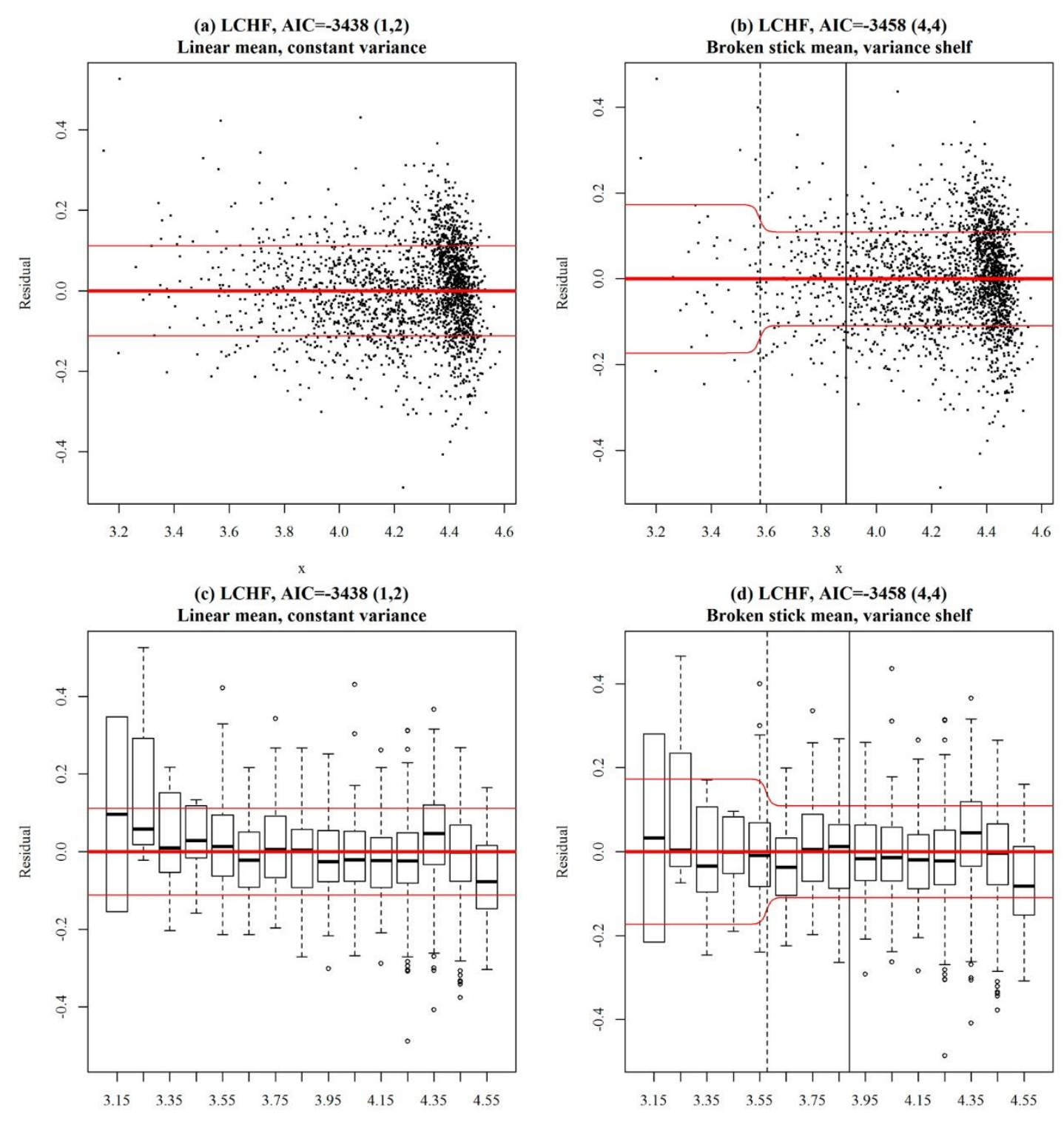

(e) LCHF, AIC=-3458 $(4,4)$
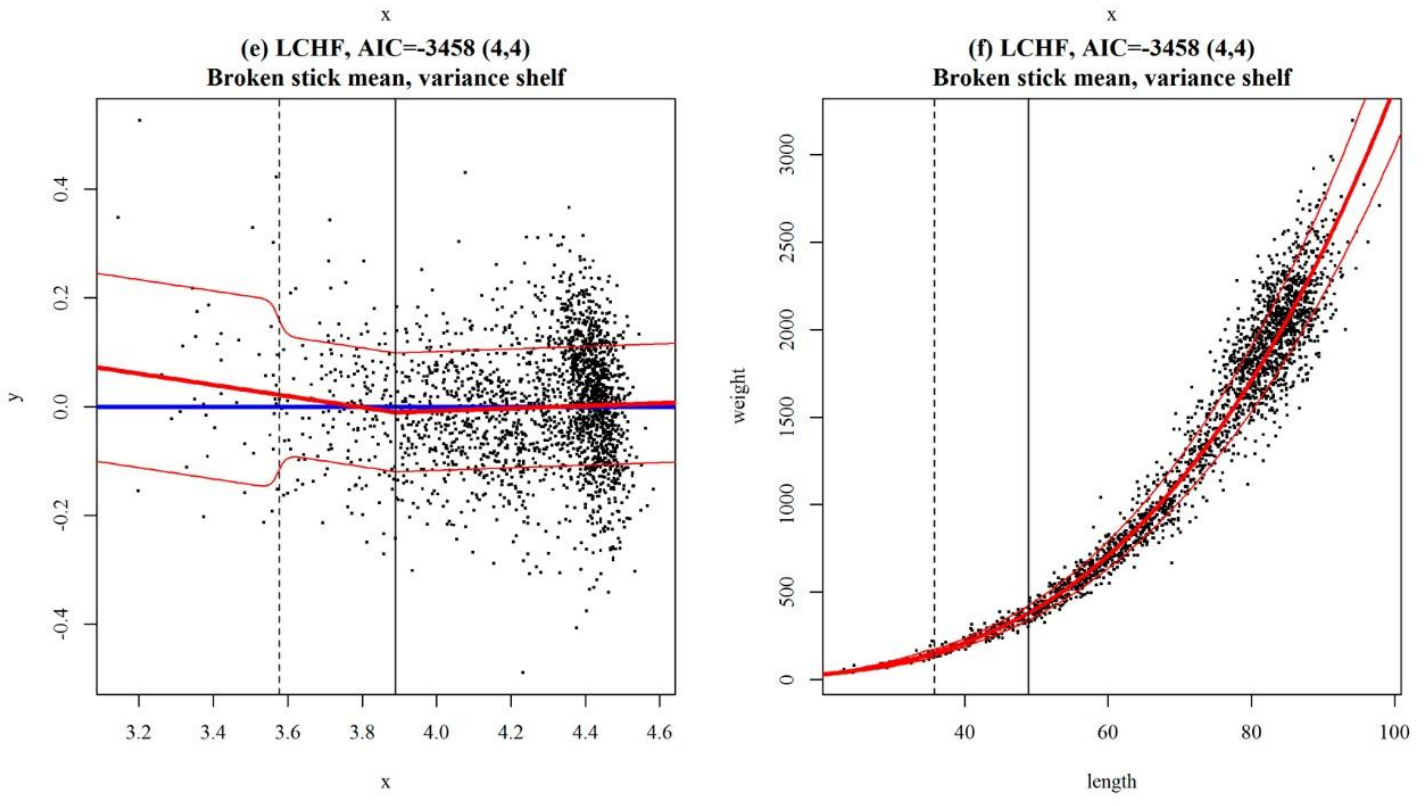
(a) RCHM, AIC =-999 $(1,2)$

Linear mean, constant variance

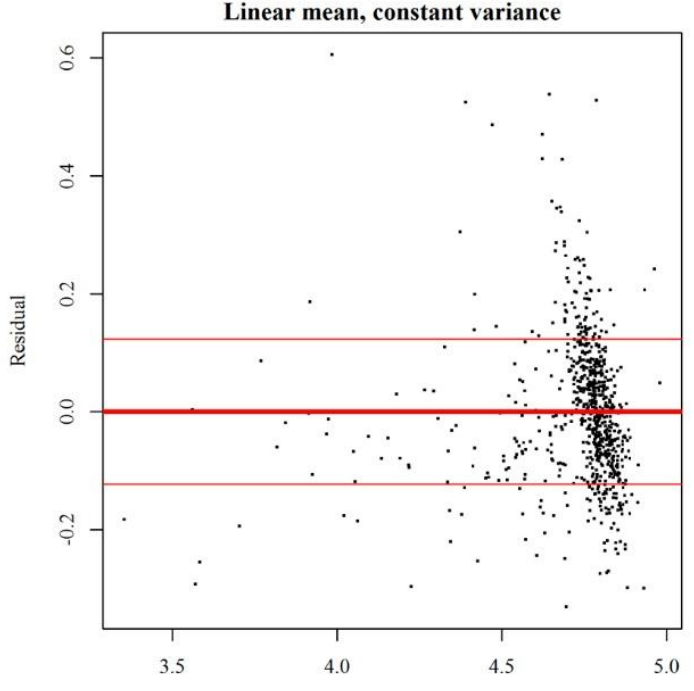

(c) RCHM, AIC=-999 $(1,2)$

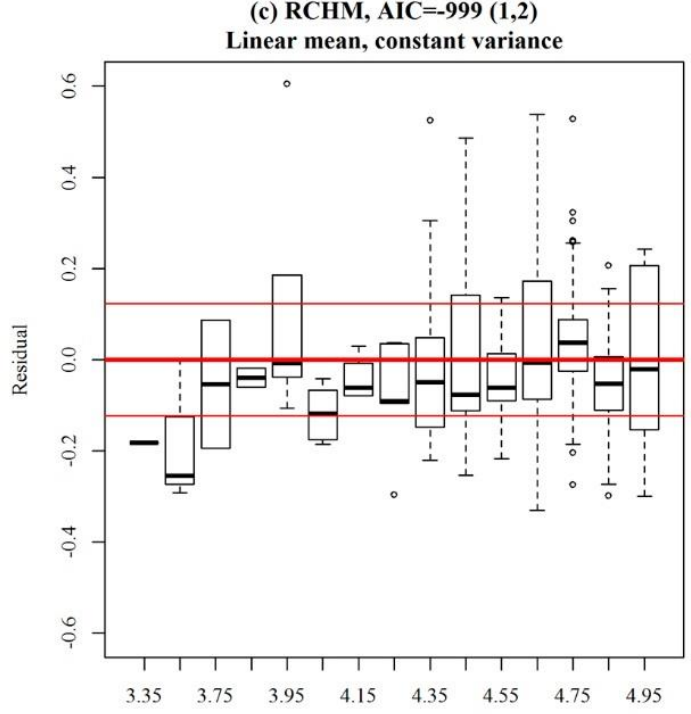

(e) RCHM, AIC=-1119 $(1,4)$

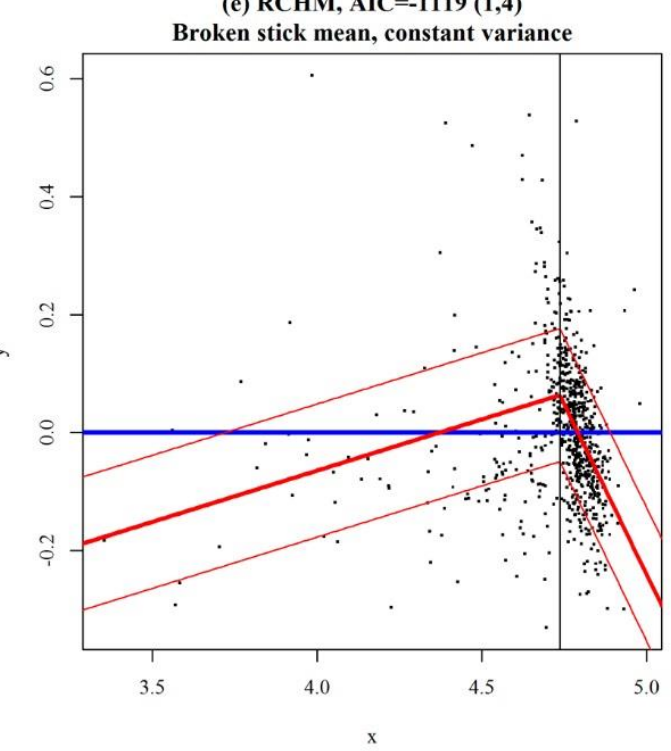

(b) RCHM, AIC=-1119 $(1,4)$

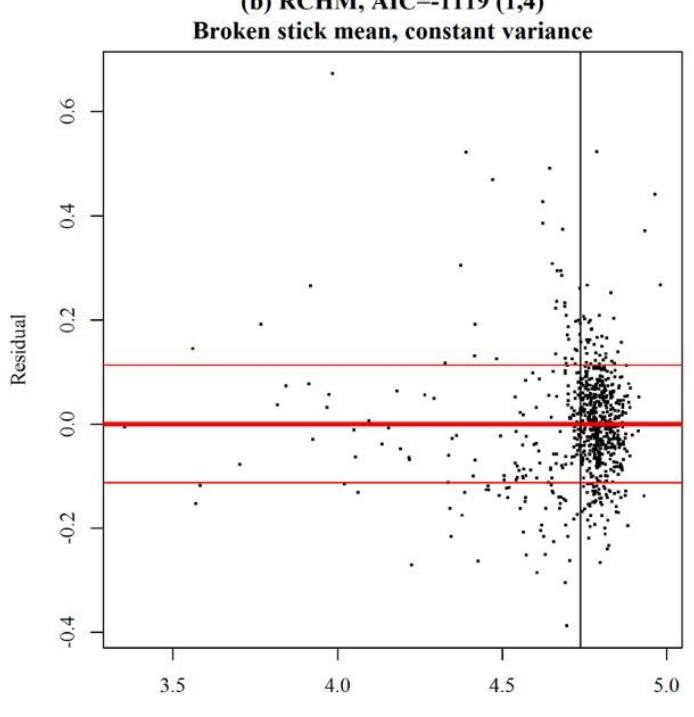

(d) RCHM, AIC=-1119 $(1,4)$

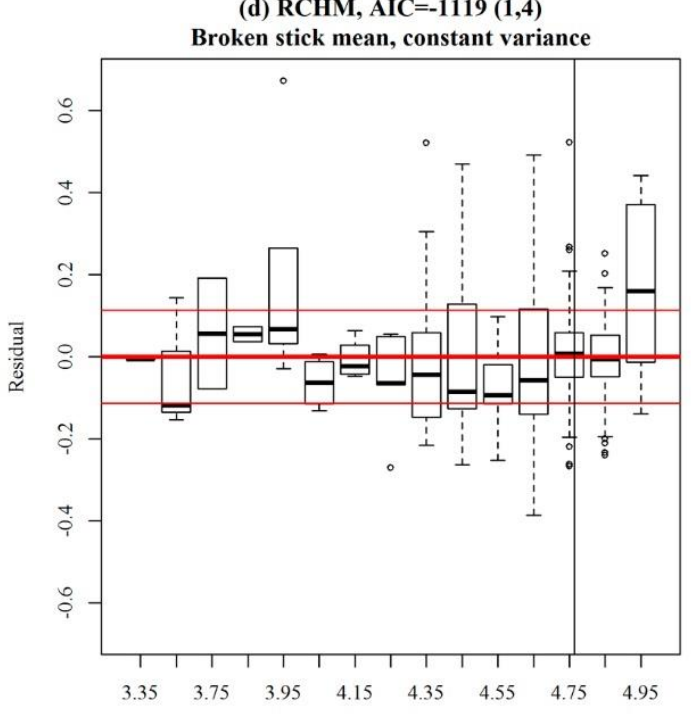

$\mathrm{x}$

(f) RCHM, AIC=-1119 $(1,4)$

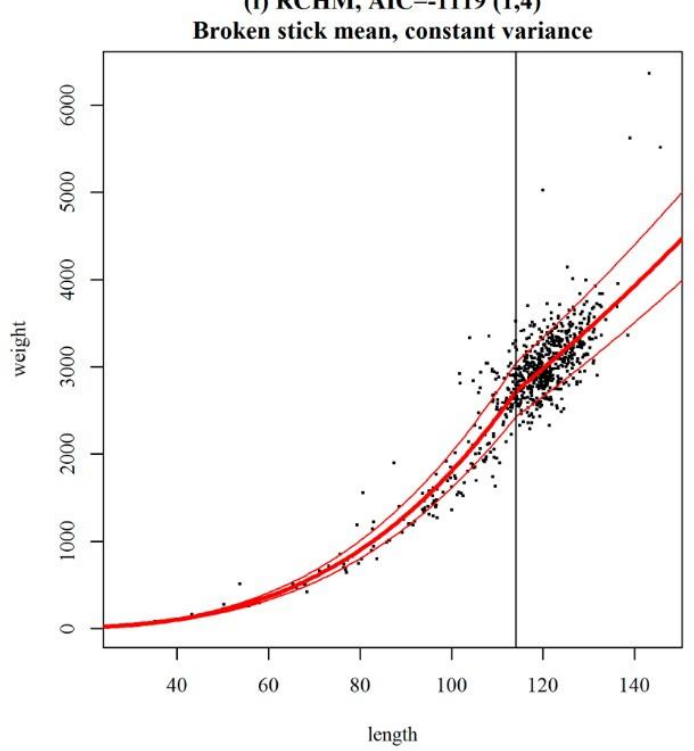


(a) $\mathrm{RCHF}, \mathrm{AIC}=-629(1,2)$

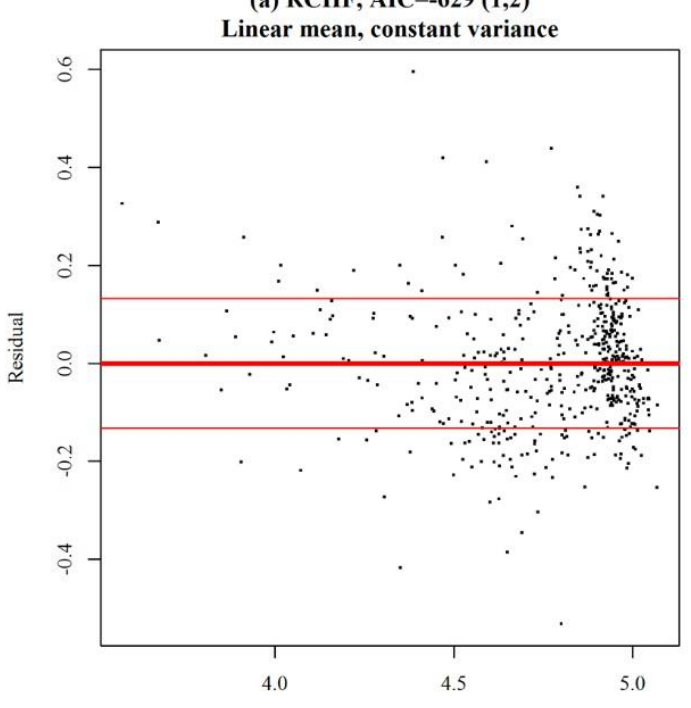

(c) RCHF, AIC =-629 $(1,2)$

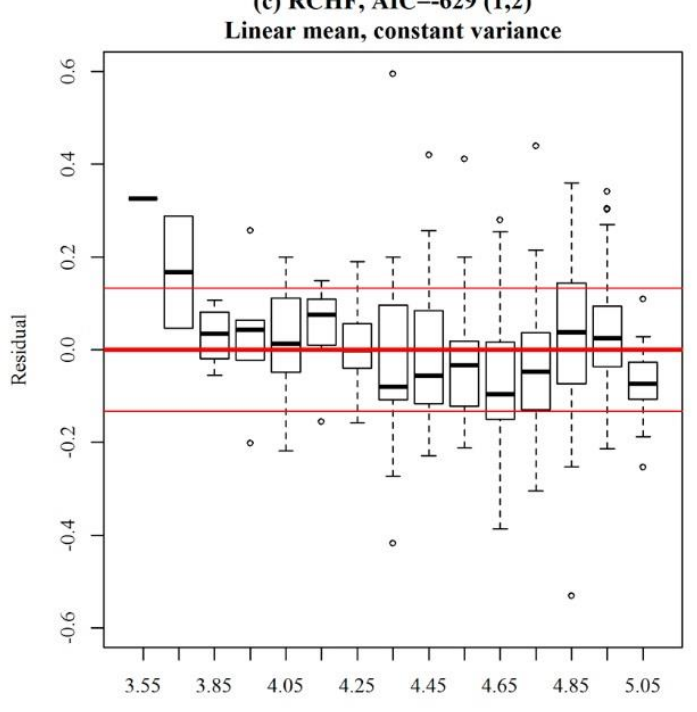

(e) RCHF, AIC $=-667(4,2)$

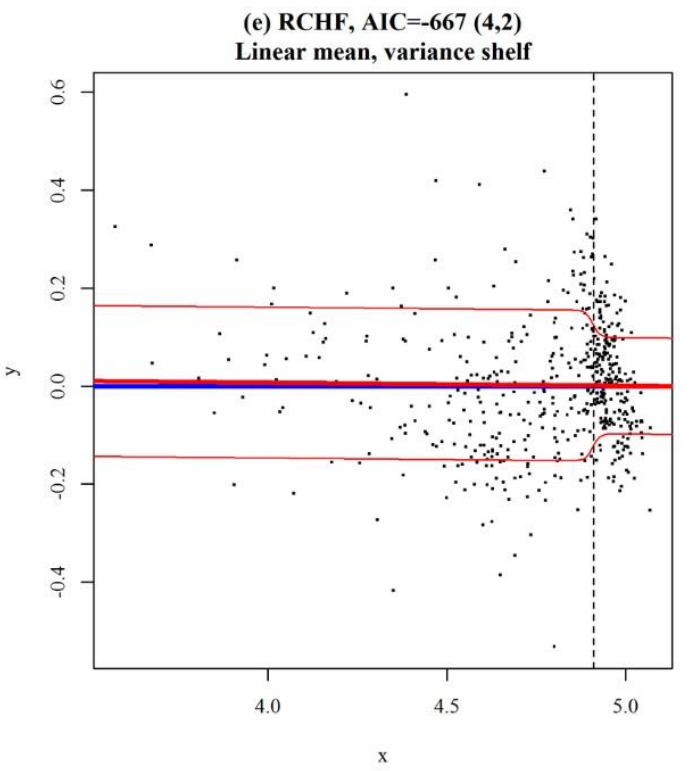

(b) RCHF, AIC =-667 (4,2)

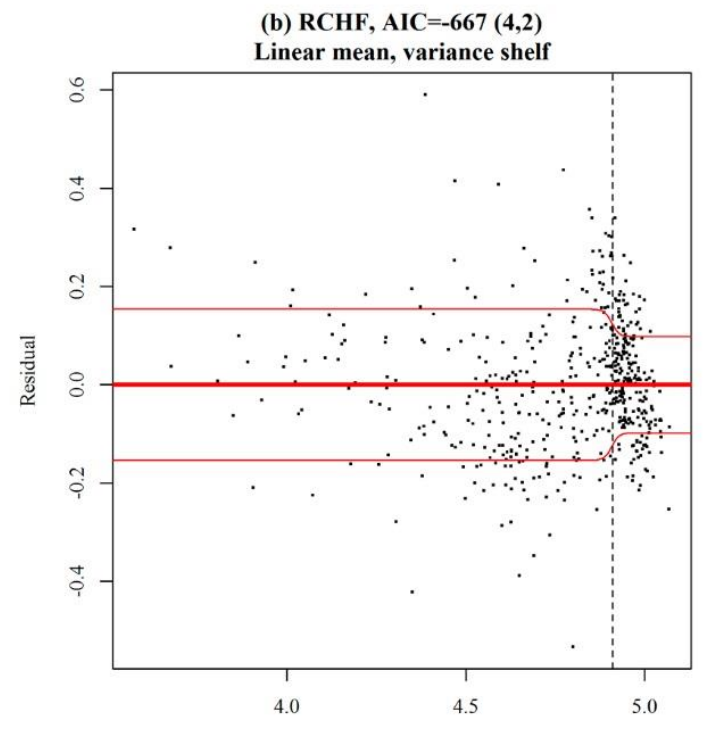

(d) RCHF, AIC $=-667(4,2)$

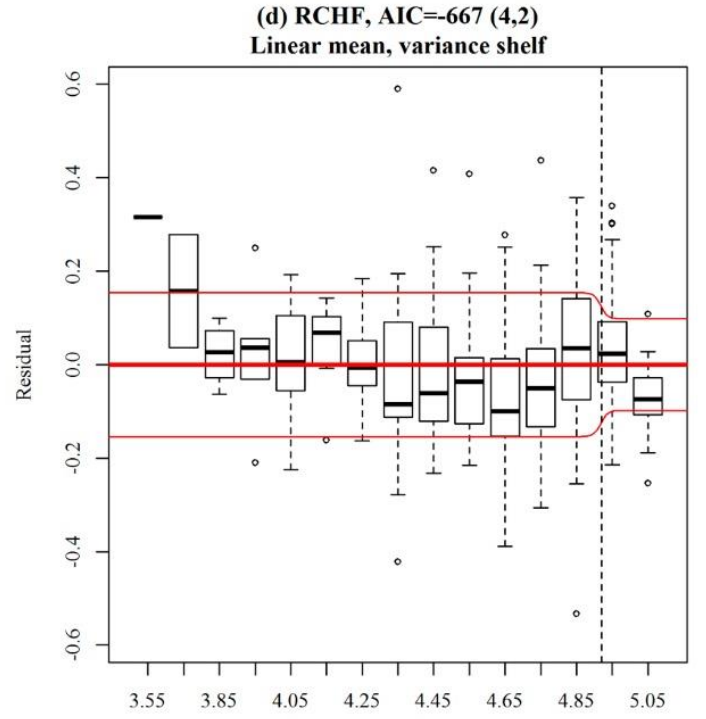

(f) RCHF, AIC =-667 $(4,2)$

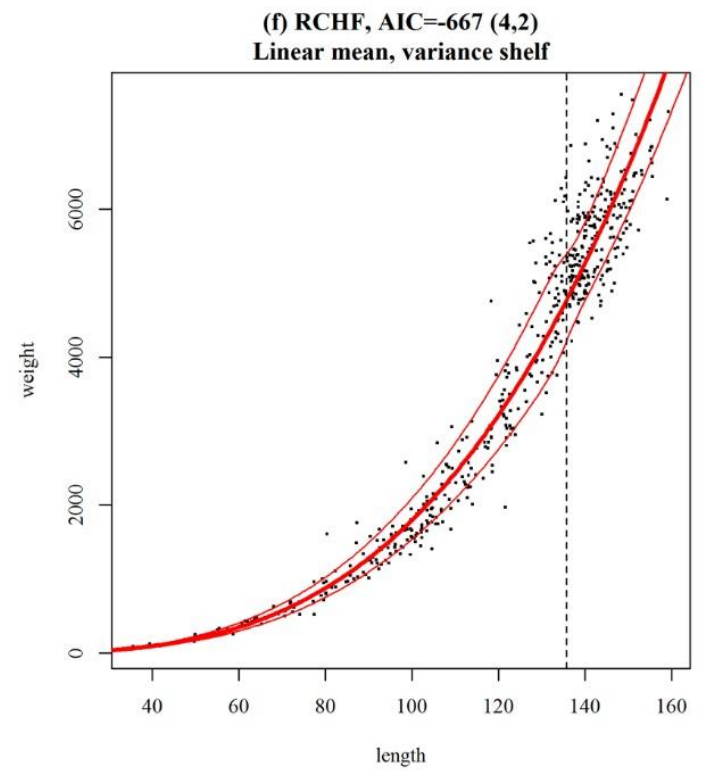


(a) CHPM, AIC=-111 $(1,2)$

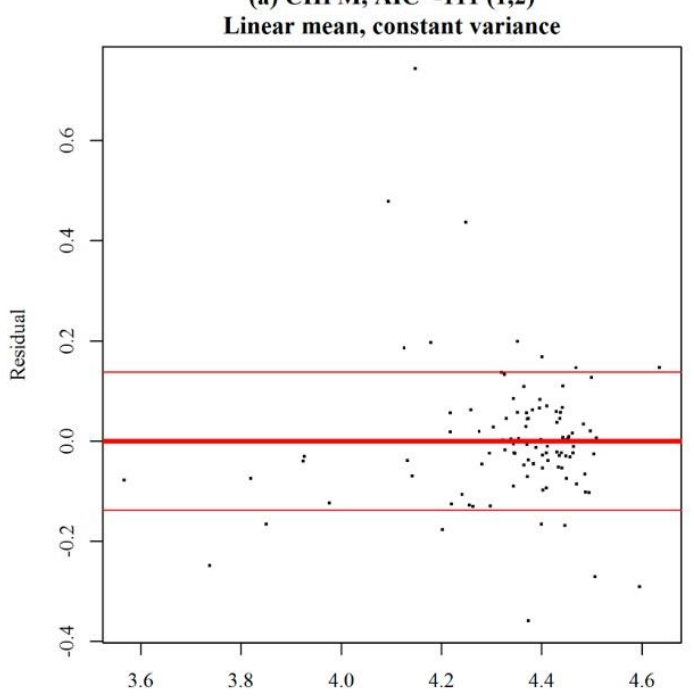

(c) CHPM, AIC=-111 $(1,2)$

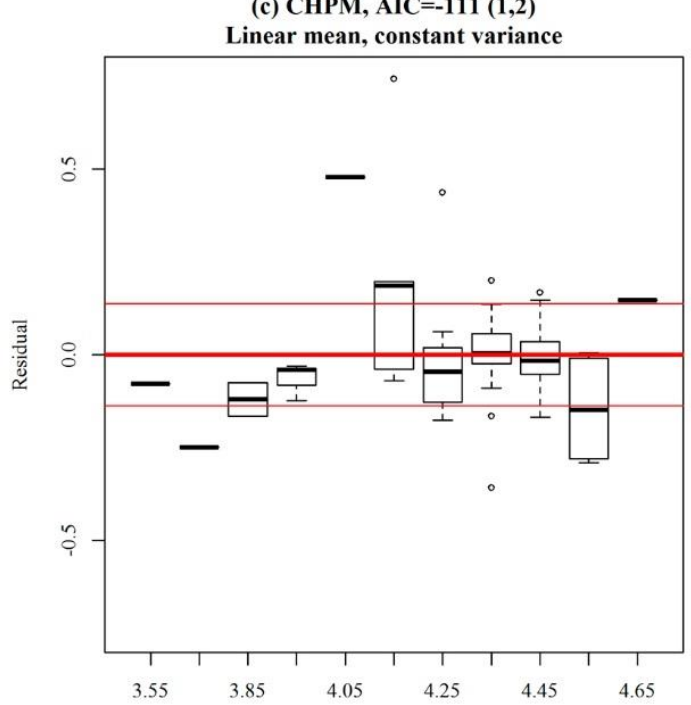

(e) CHPM, AIC =-145 $(4,4)$

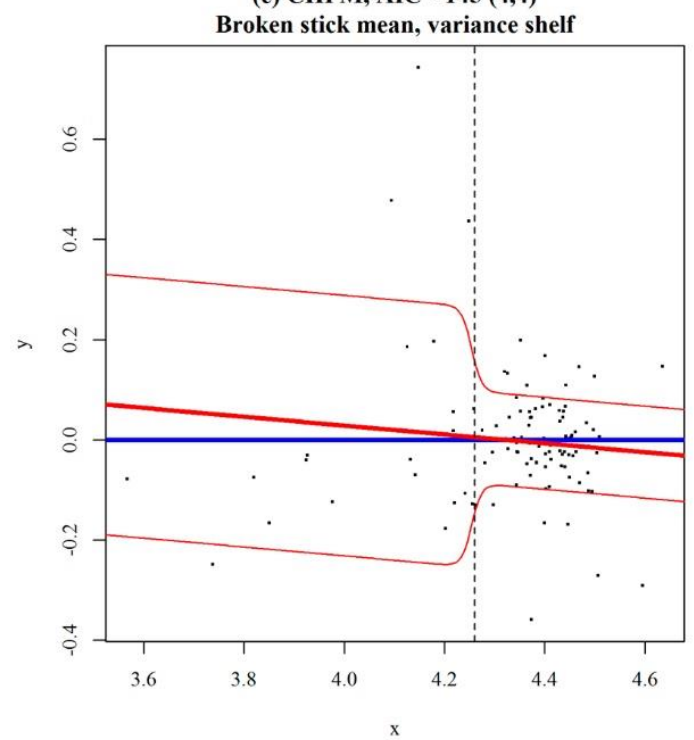

(b) CHPM, AIC=-145 $(4,4)$

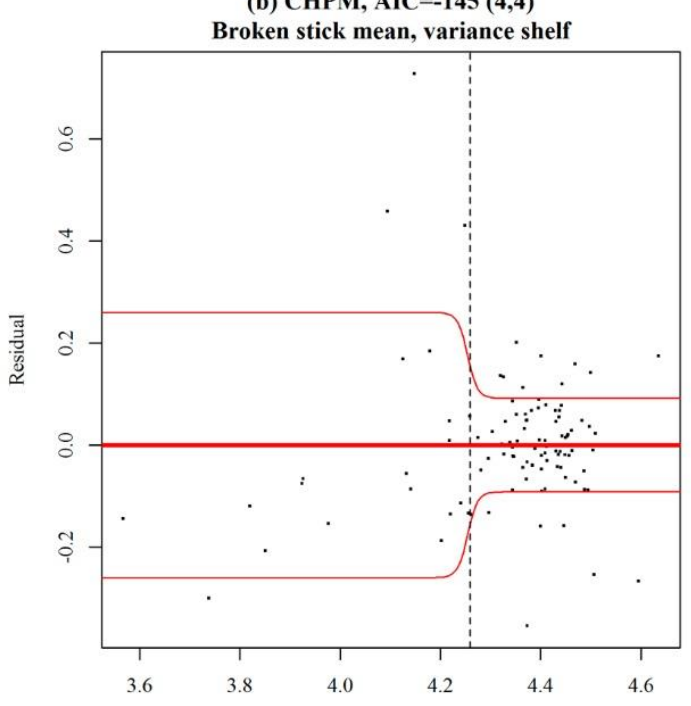

(d) CHPM, AIC=-145 $(4,4)$

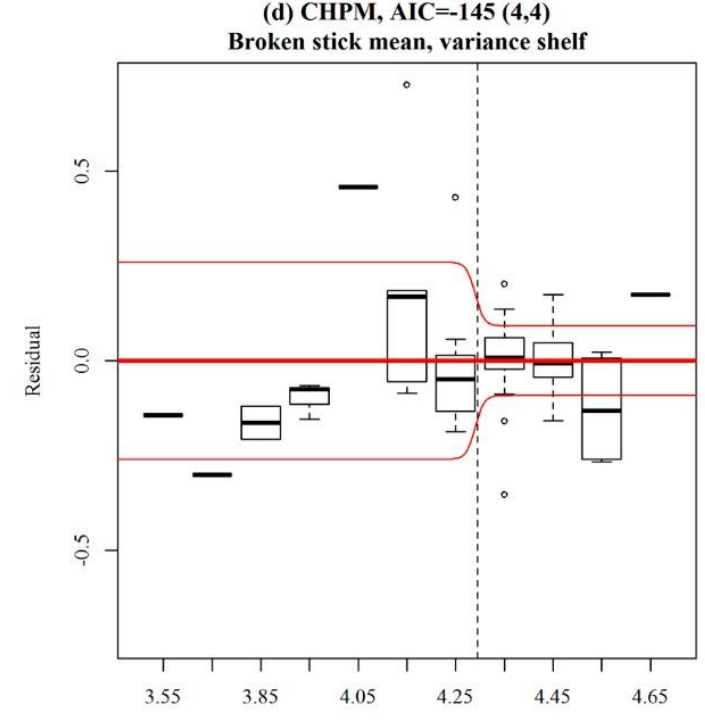

(f) CHPM, AIC $=-145(4,4)$

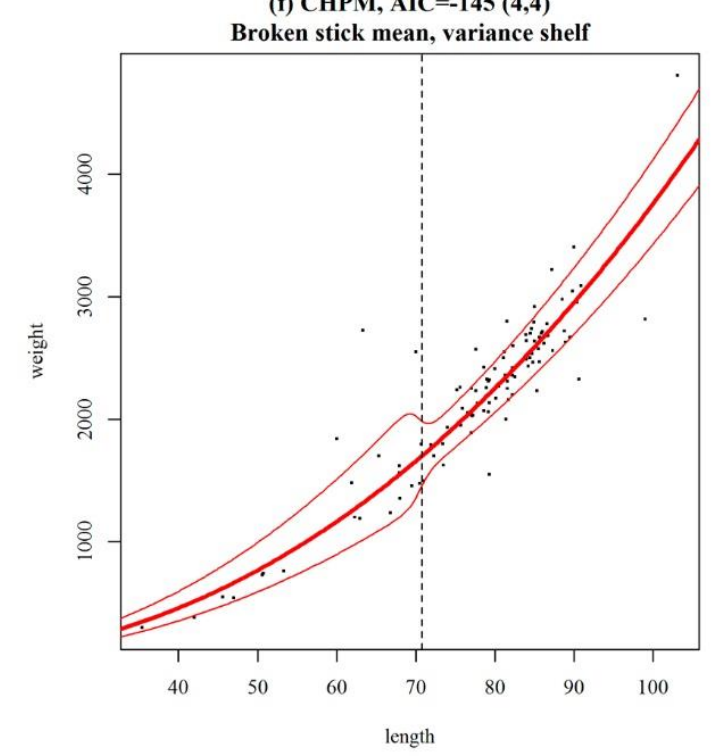


(a) $\mathrm{CHPF}, \mathrm{AIC}=-56(1,2)$

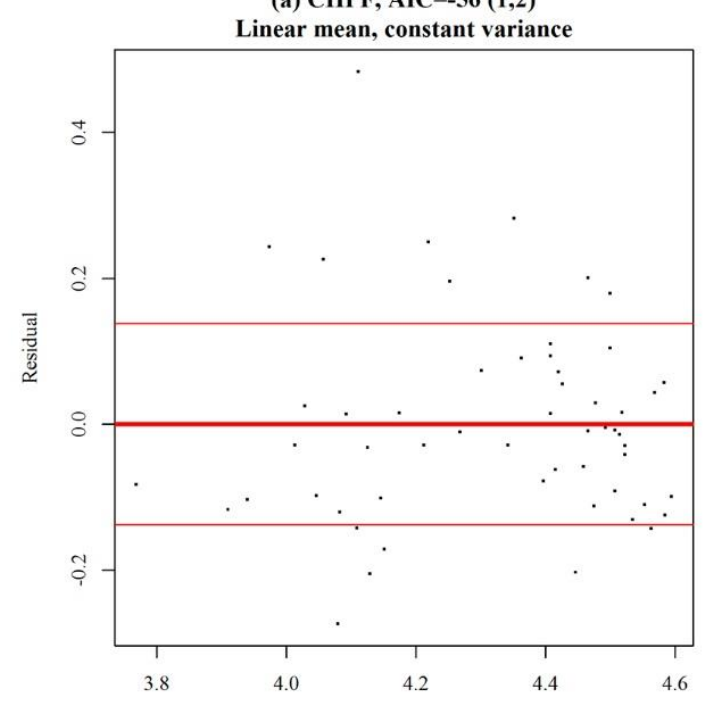

(e) CHPF, AIC $=-60(4,2)$

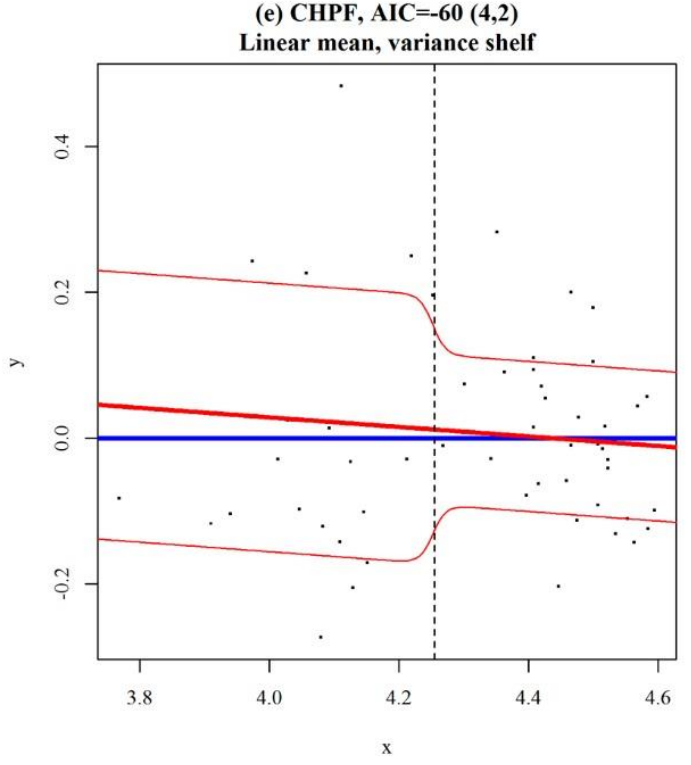

(b) CHPF, AIC =-60 (4,2)

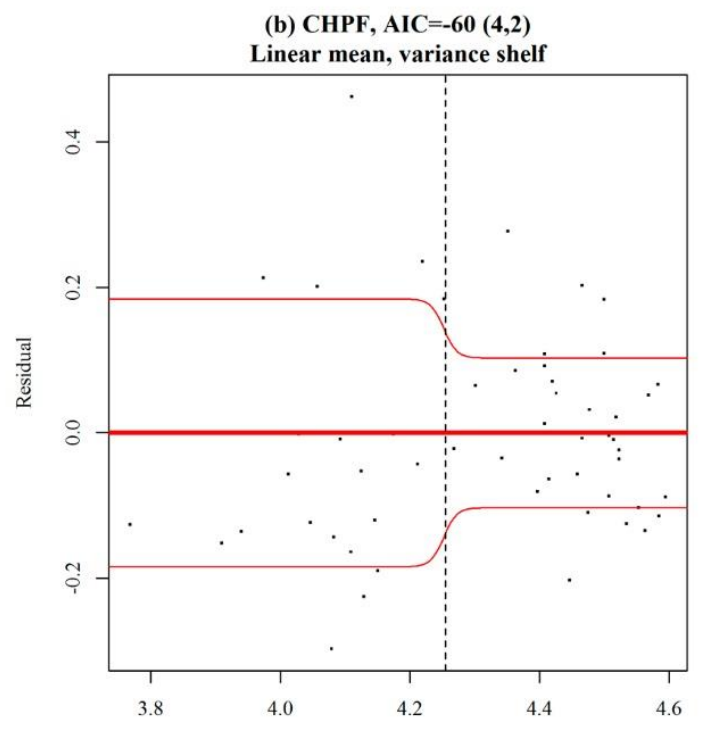

(f) CHPF, AIC $=-60(4,2)$

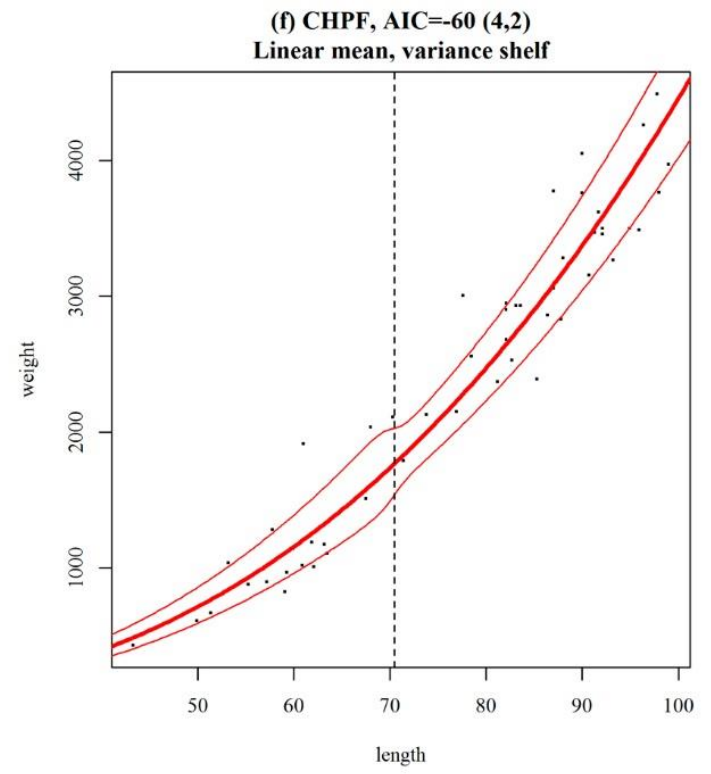



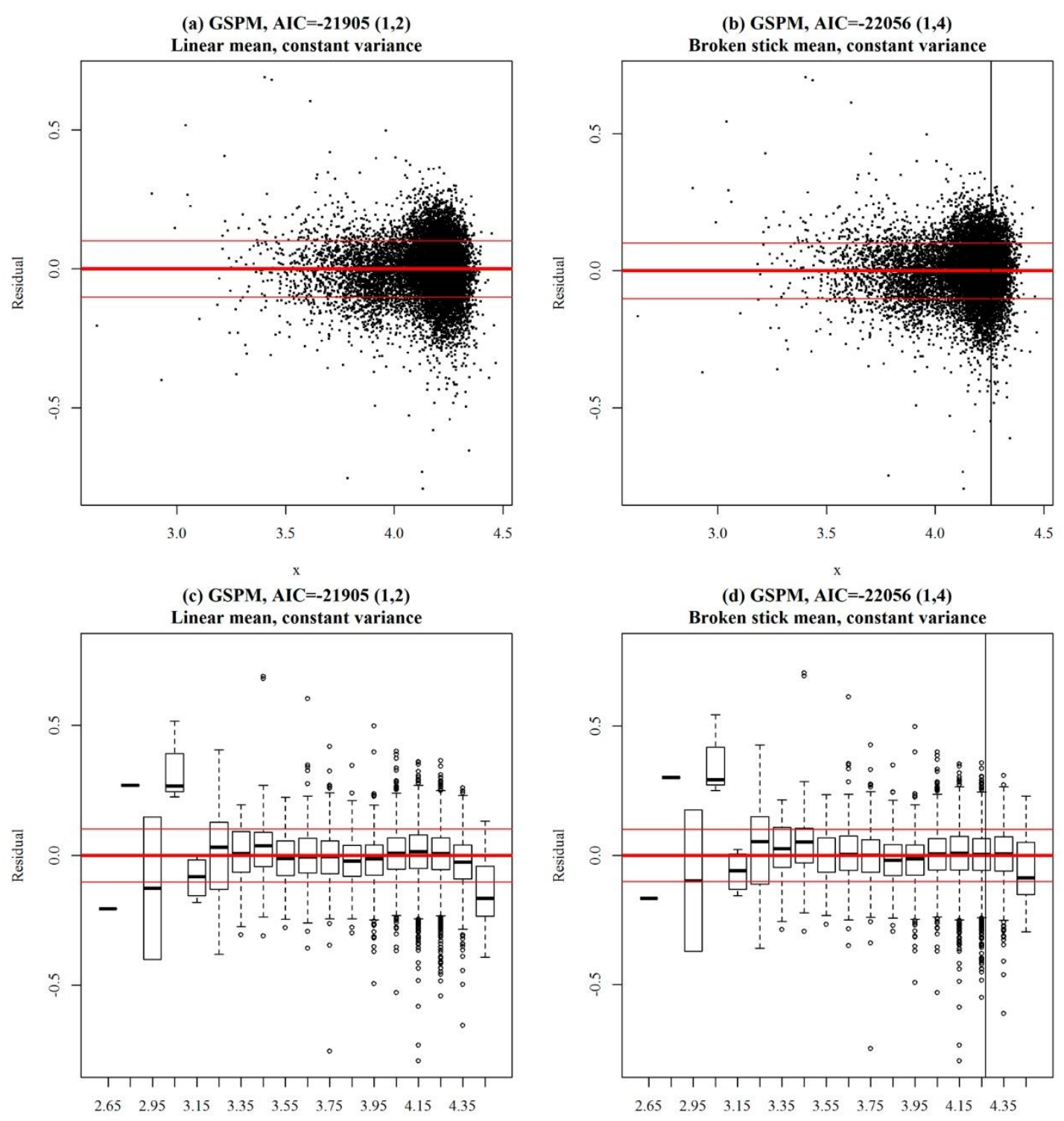

(e) GSPM, AIC=-22056 (1,4)
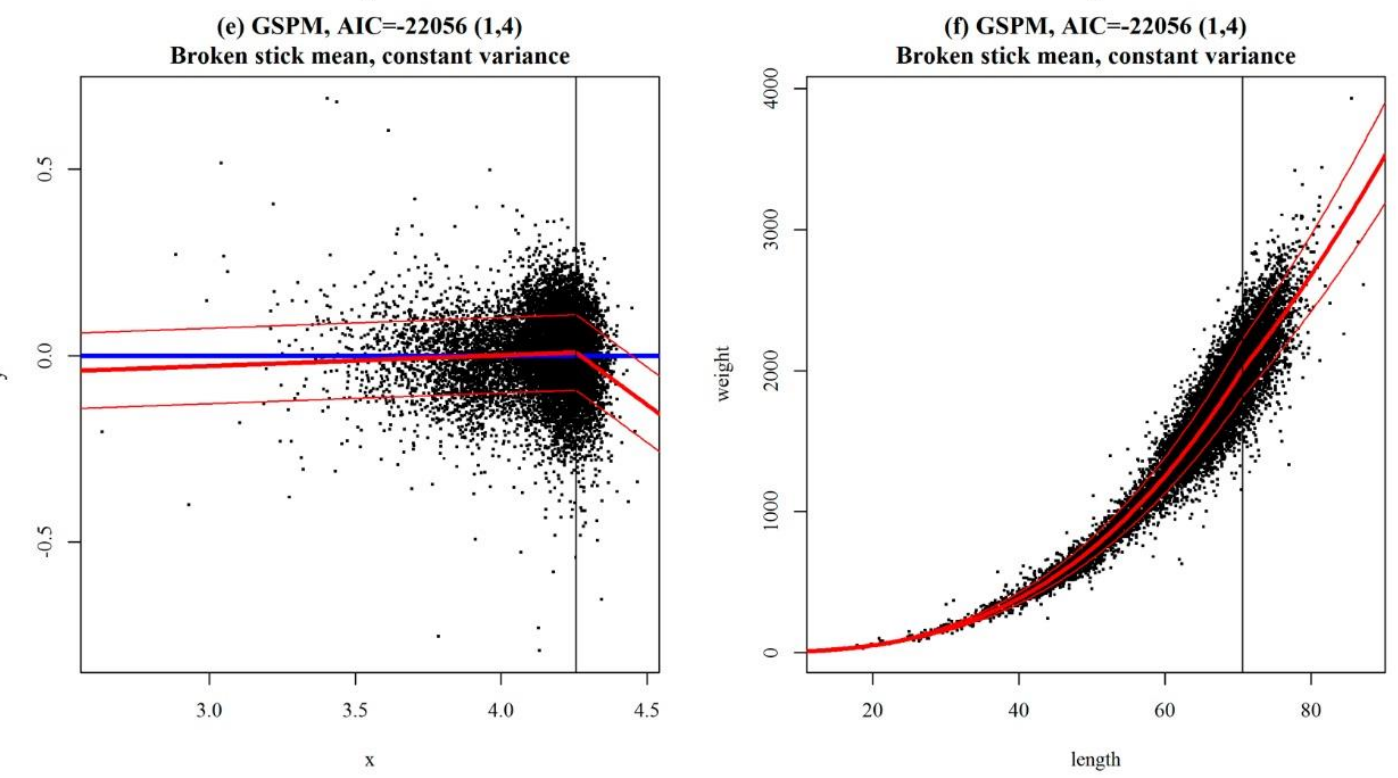

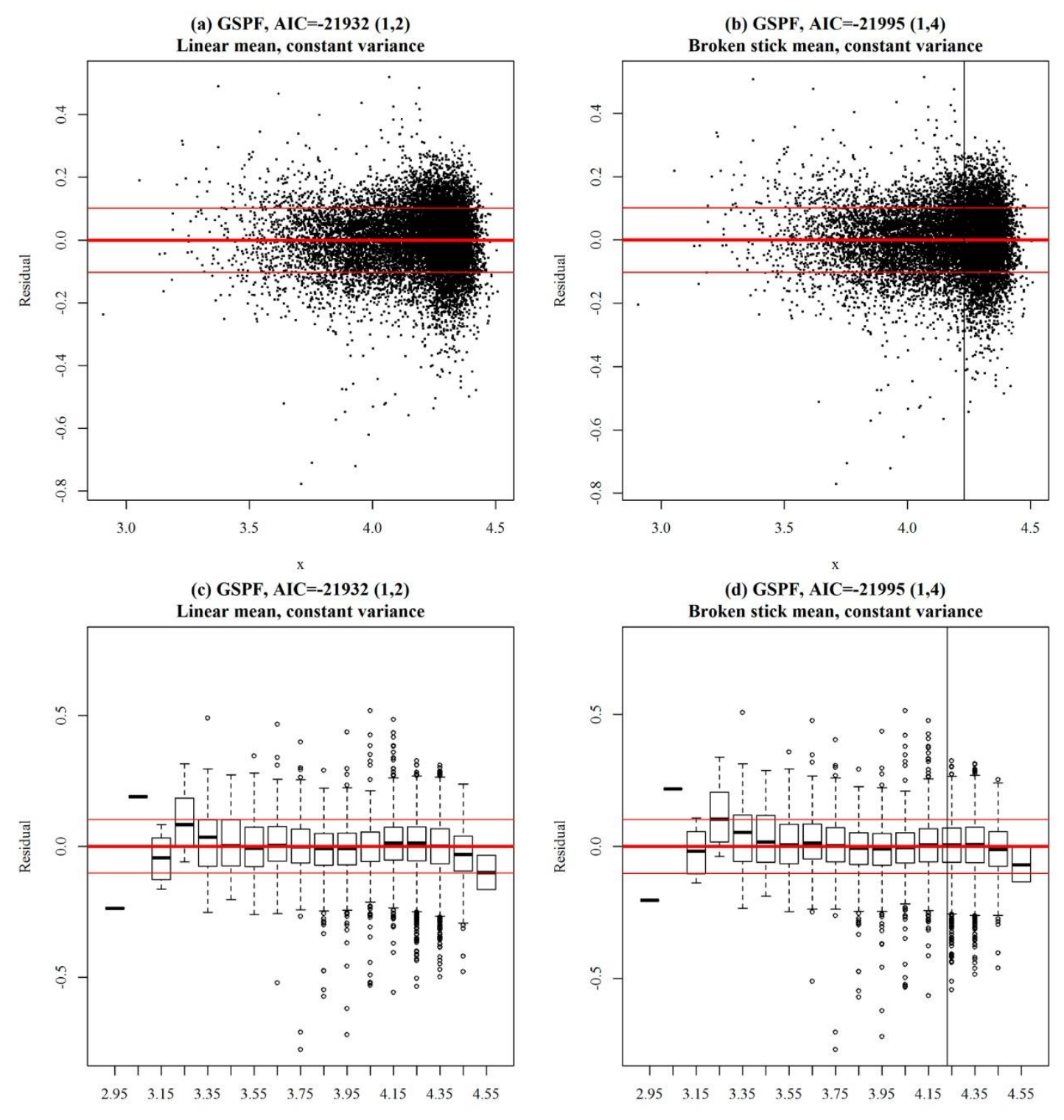

(e) GSPF, AIC=-21995 $(1,4)$
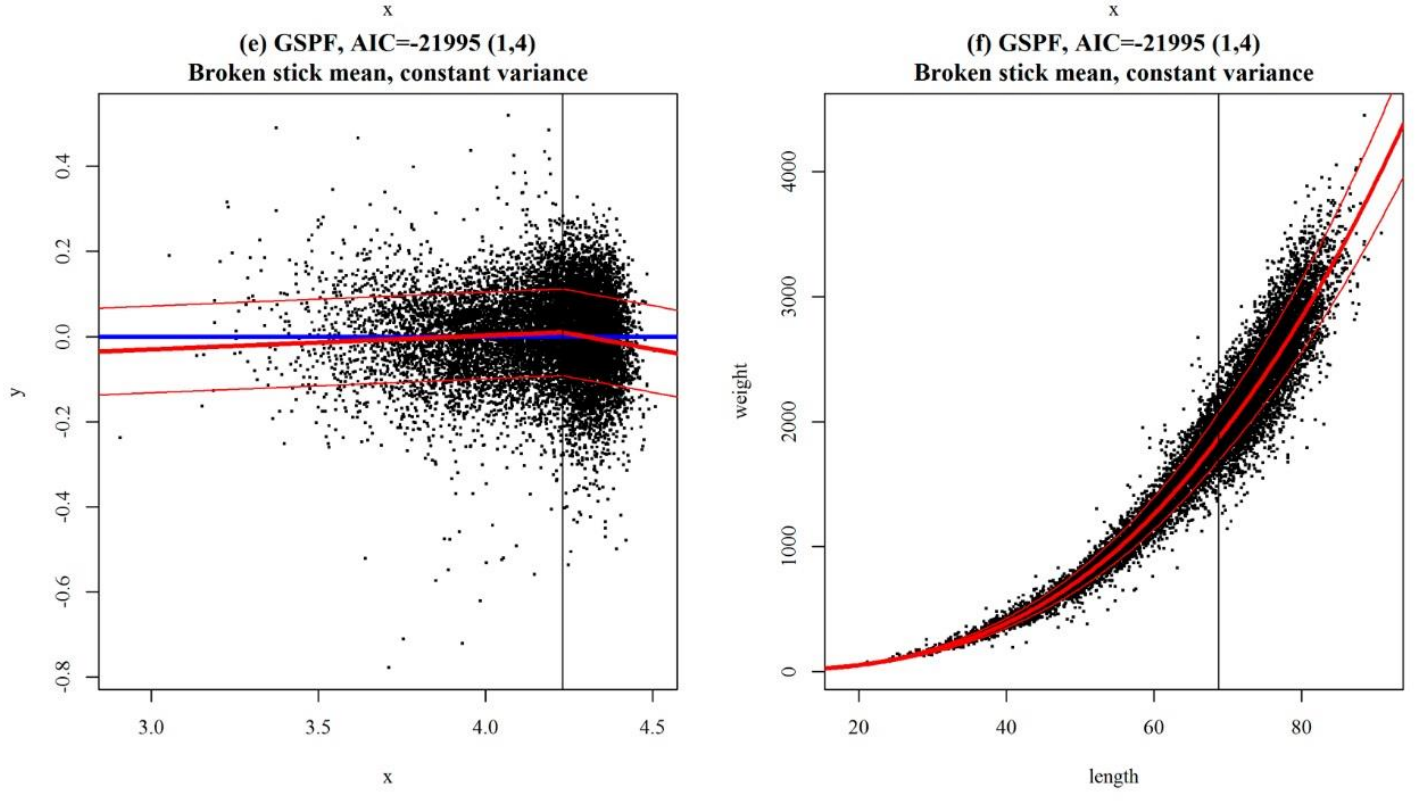
(a) HYBM, AIC=-114 (1,2)

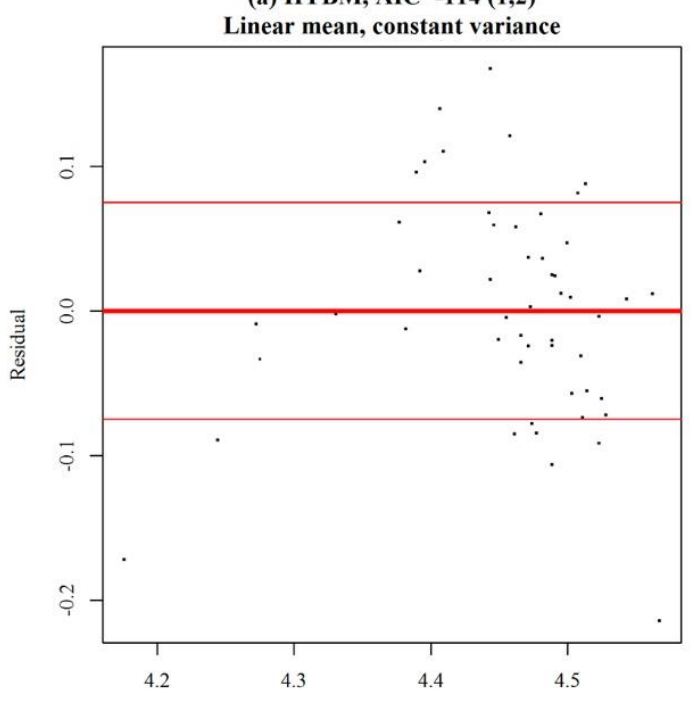

(e) HYBM, AIC=-137 $(1,4)$

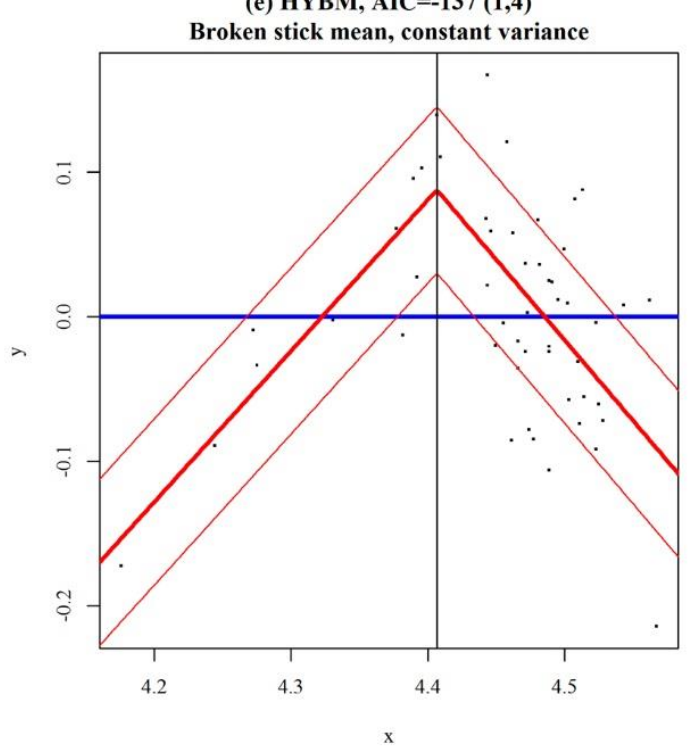

(b) HYBM, AIC=-137 $(1,4)$

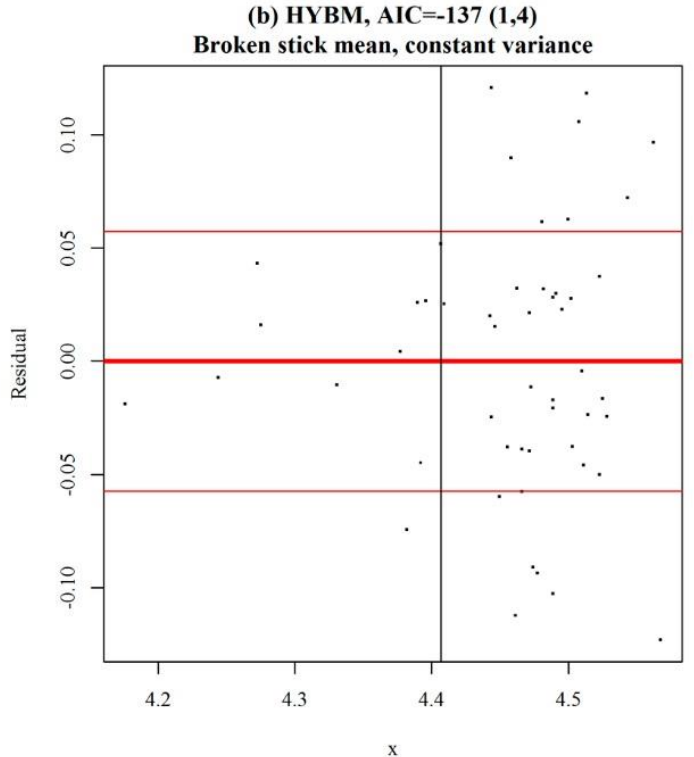

(f) HYBM, AIC=-137 (1,4)

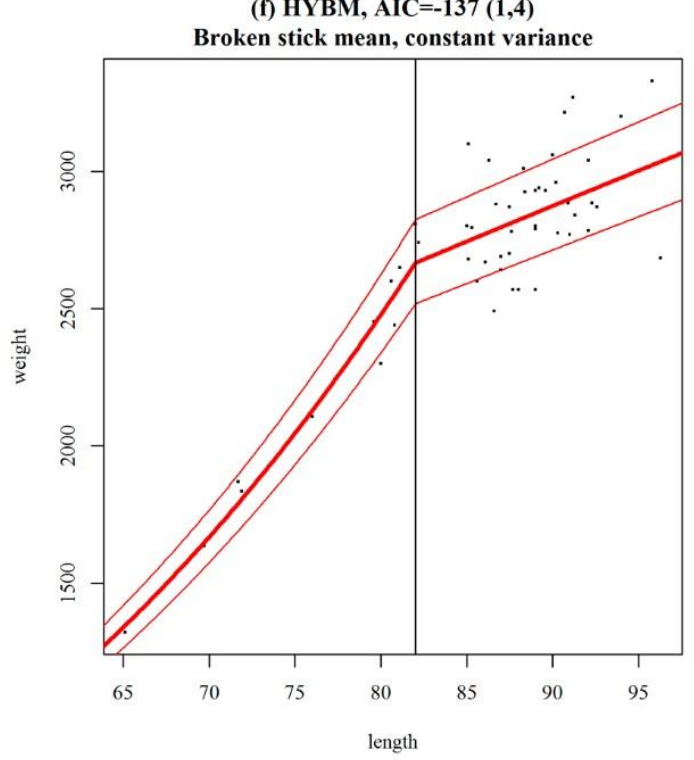


(a) HYBF, AIC $=-26(1,2)$

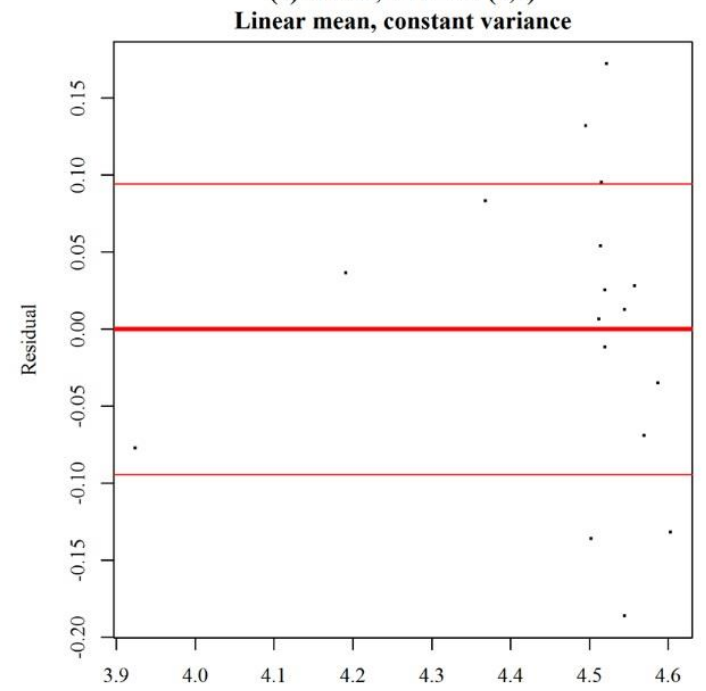

(e) HYBF, AIC=-28 $(1,4)$

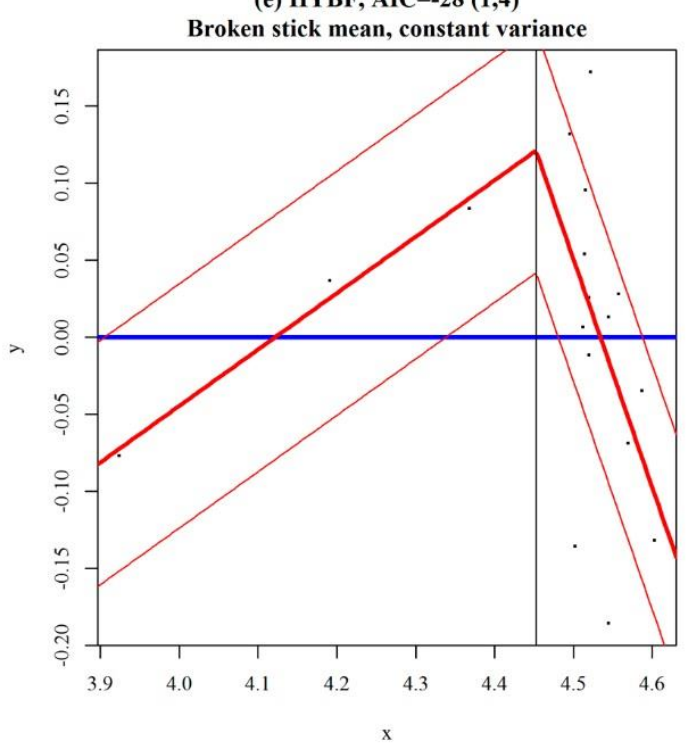

(b) HYBF, AIC=-28 $(1,4)$

Broken stick mean, constant variance

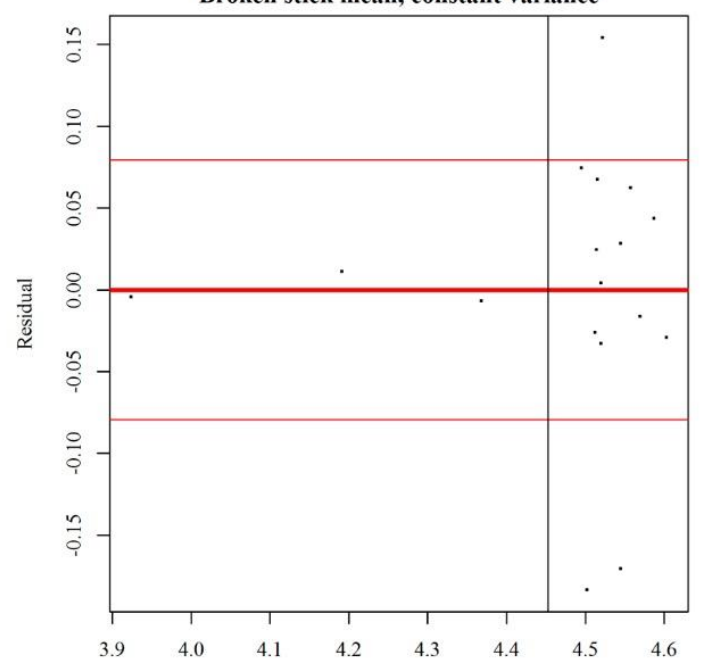

(f) HYBF, AIC=-28 $(1,4)$

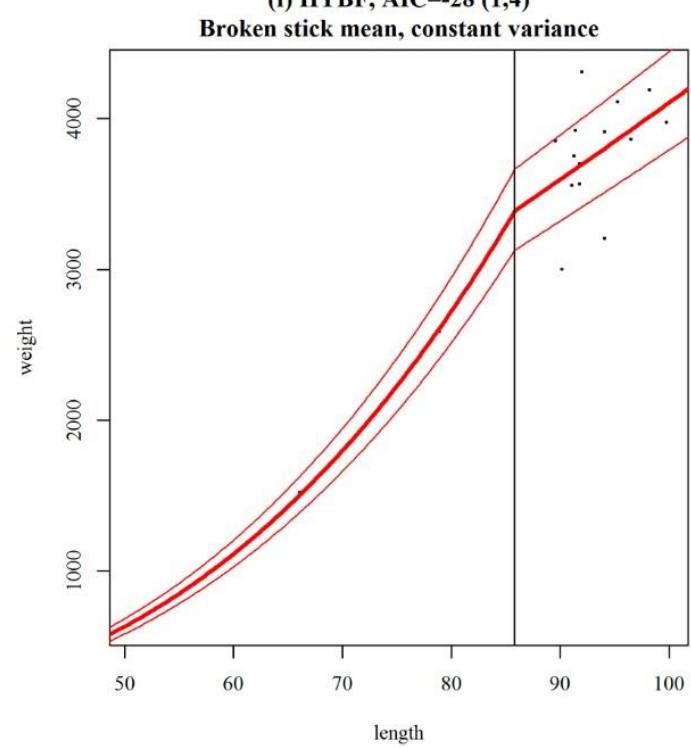



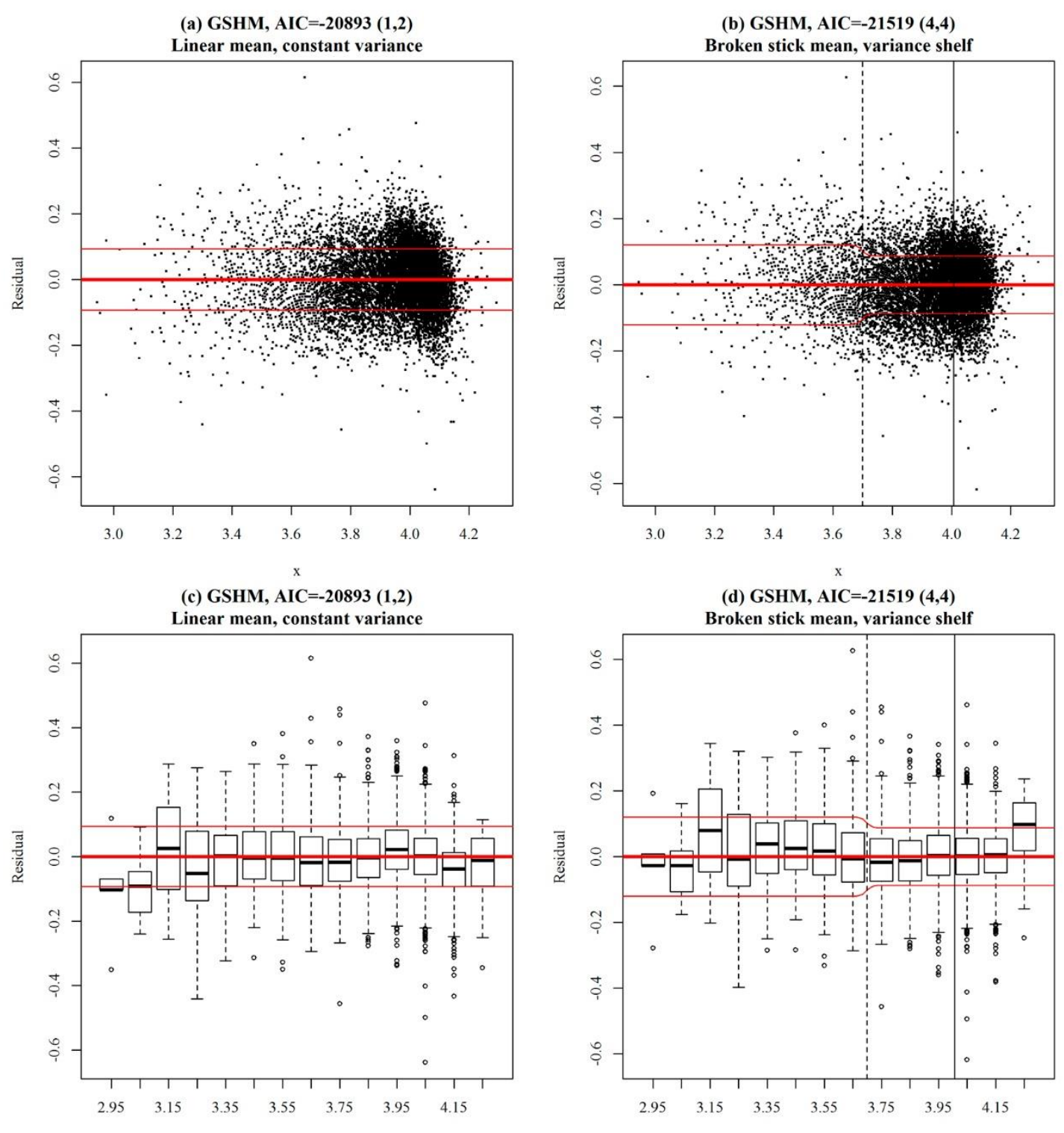

(e) GSHM, AIC=-21519 $(4,4)$
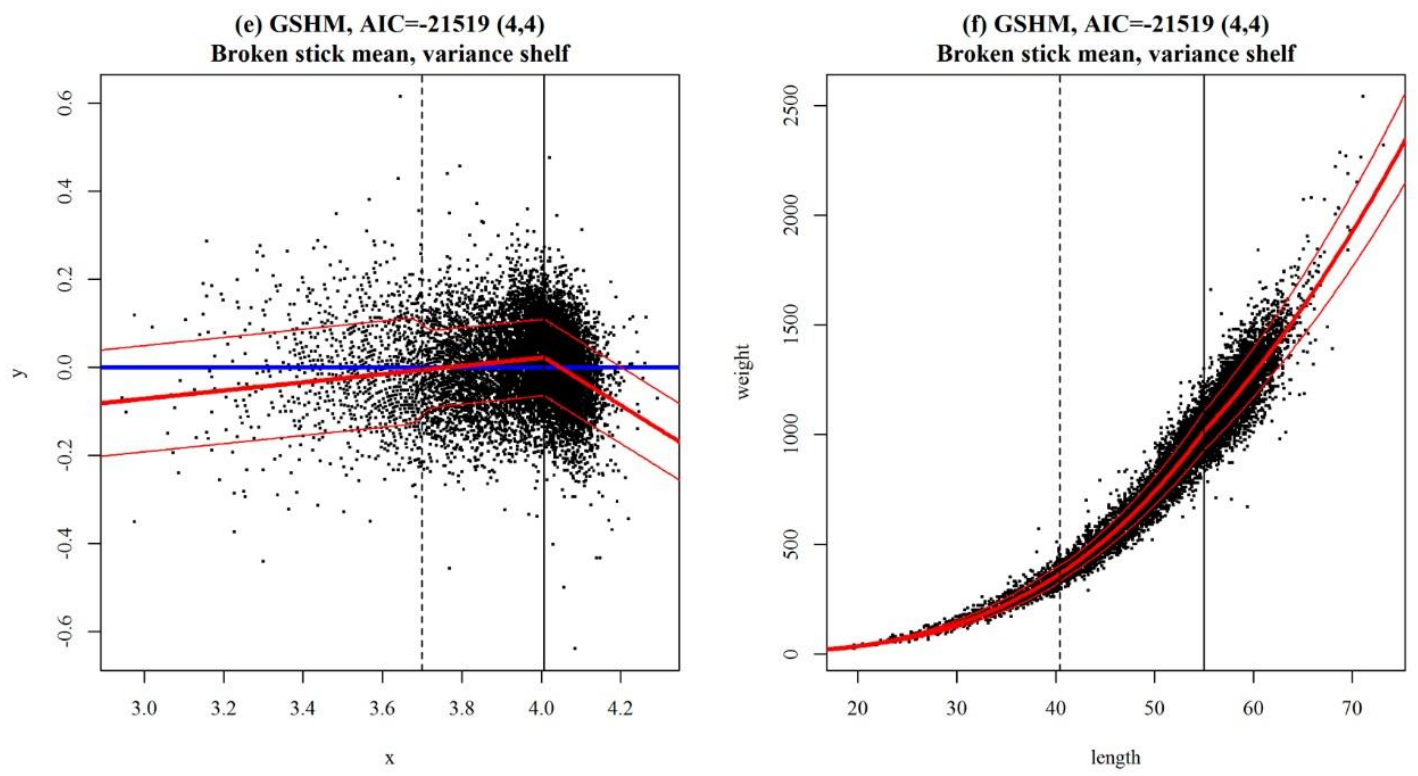

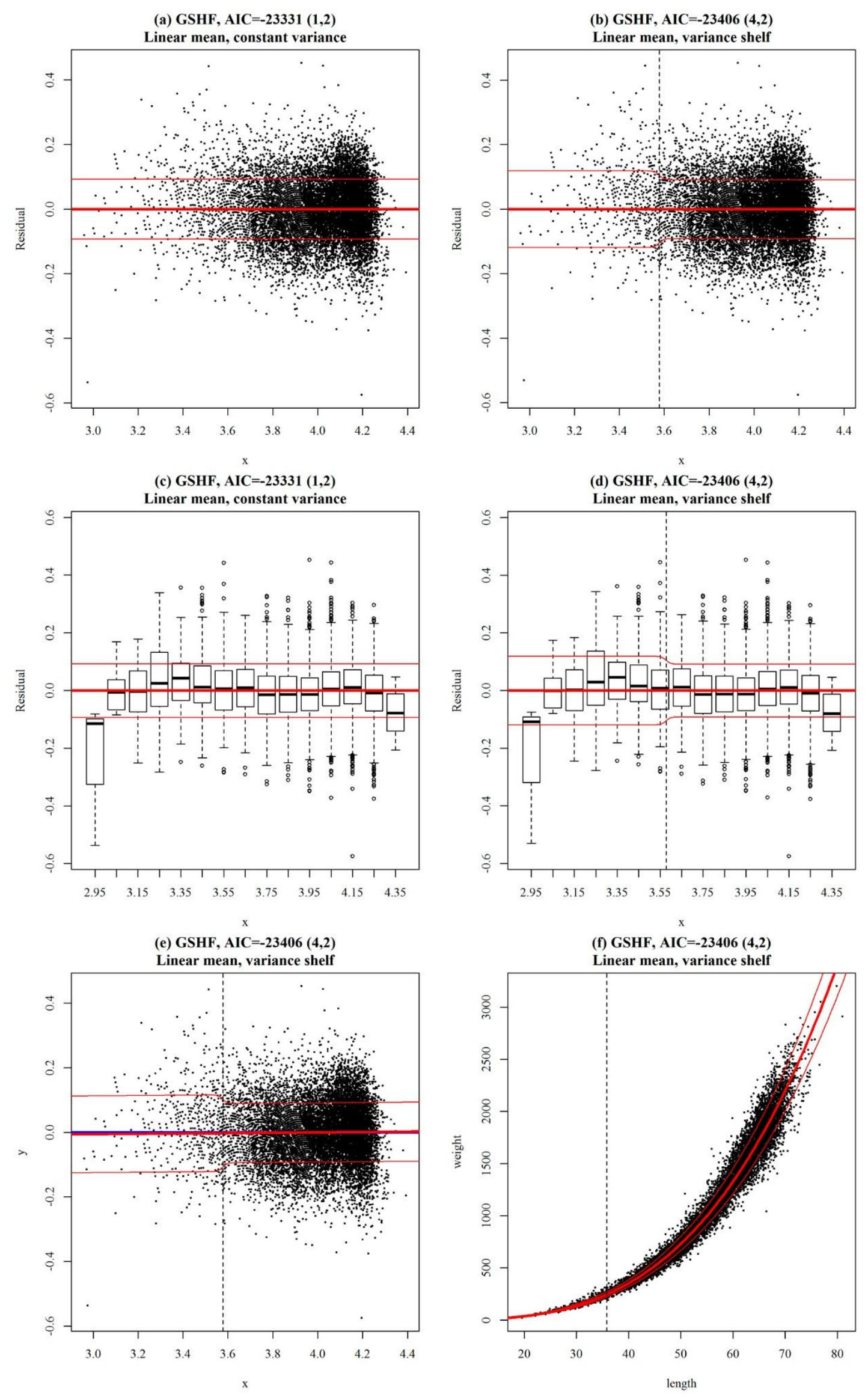

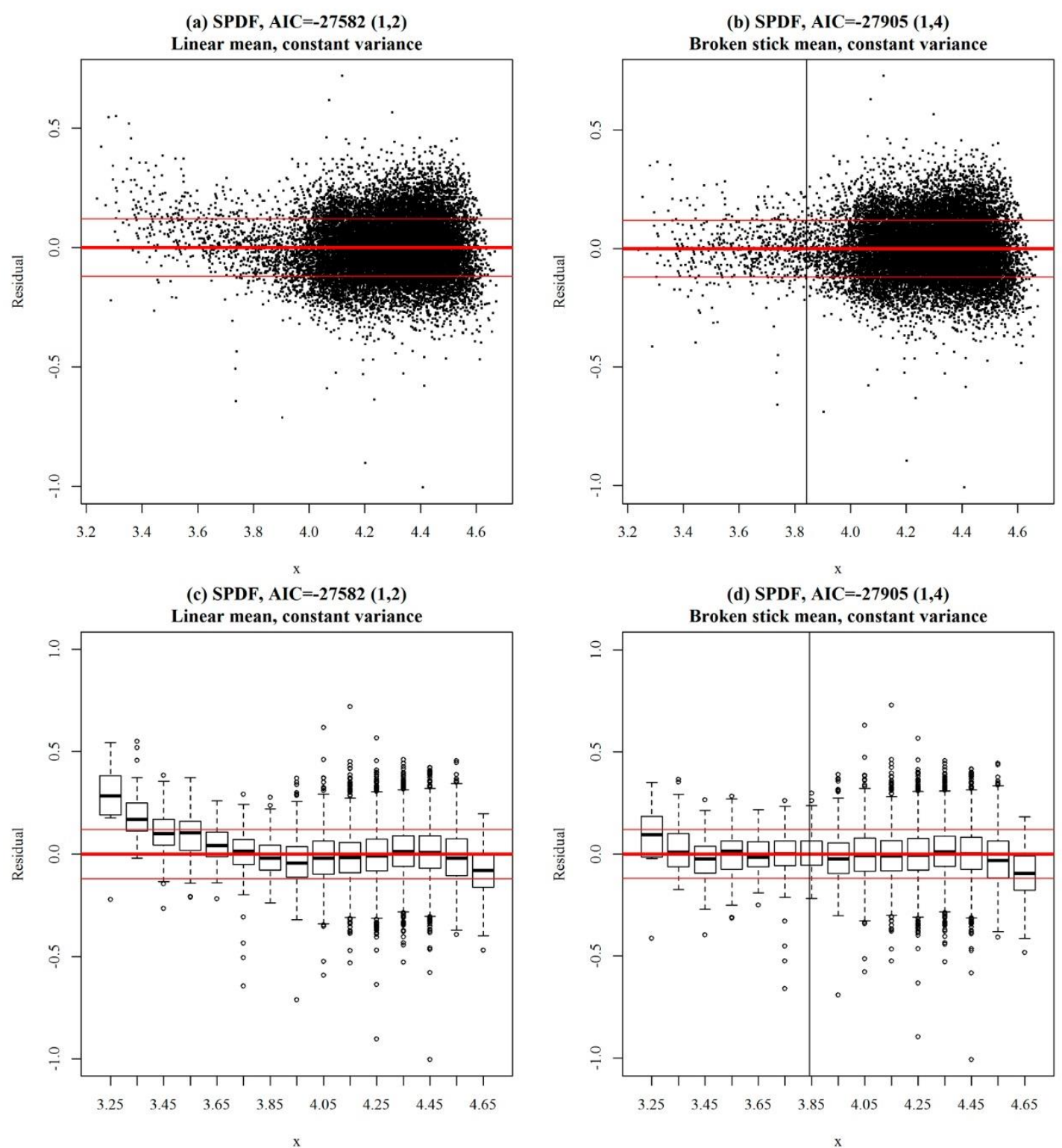

(e) SPDF, AIC=-27905 $(1,4)$
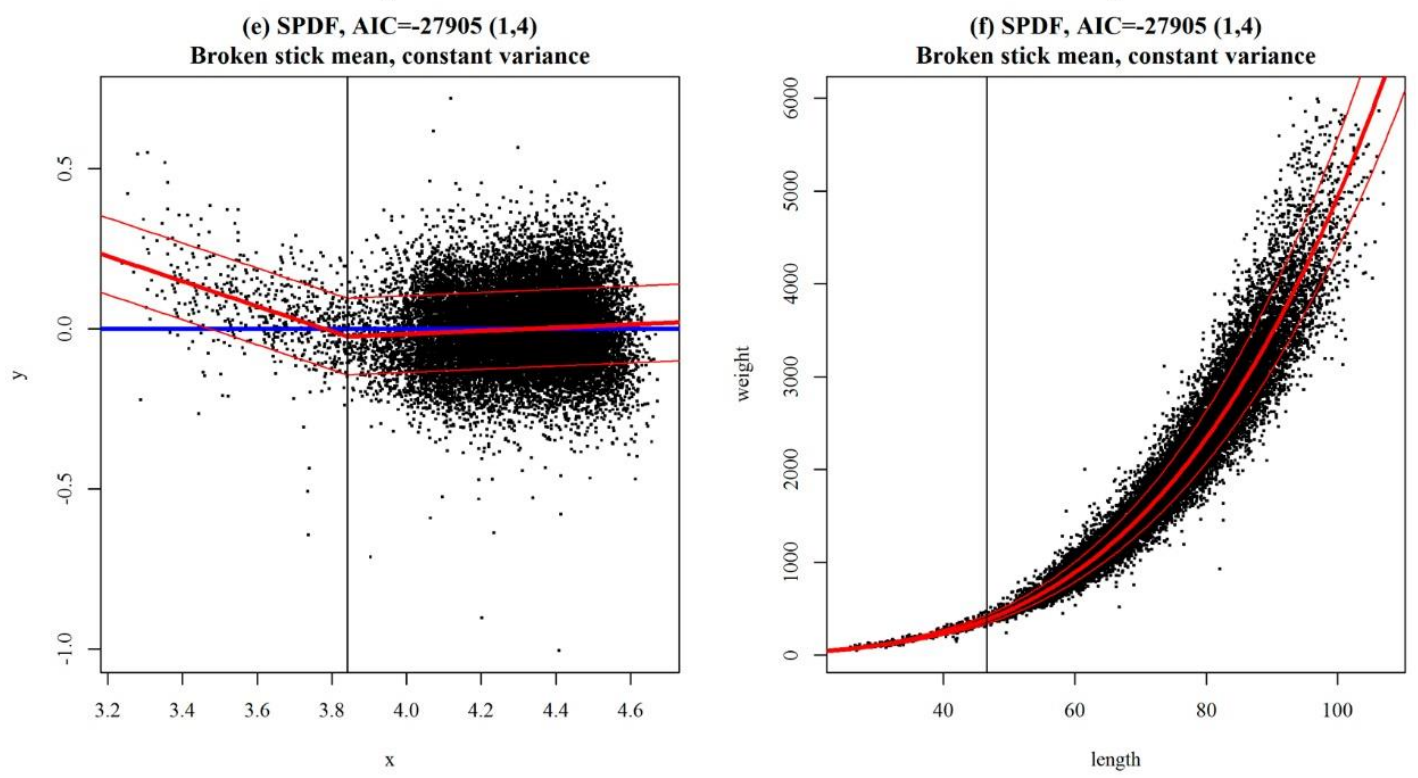
(a) CSQM, AIC $=-957(1,2)$

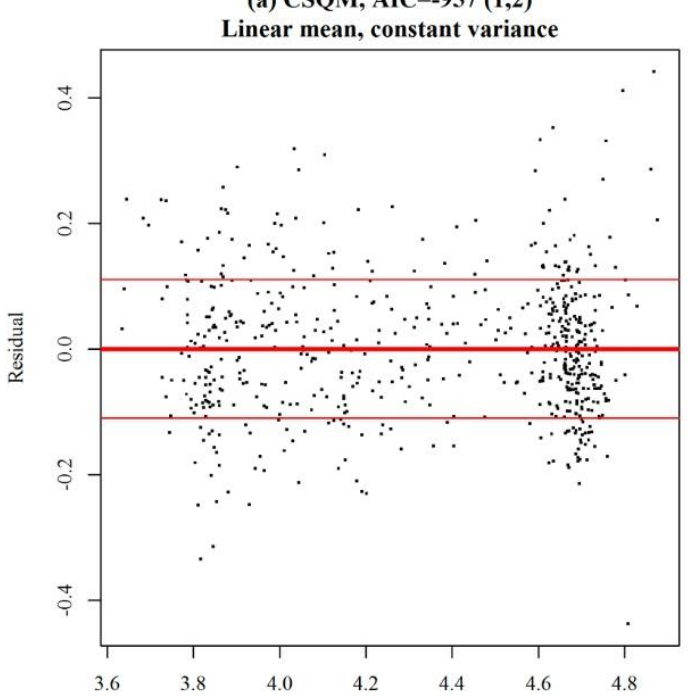

(c) CSQM, AIC $=-957(1,2)$

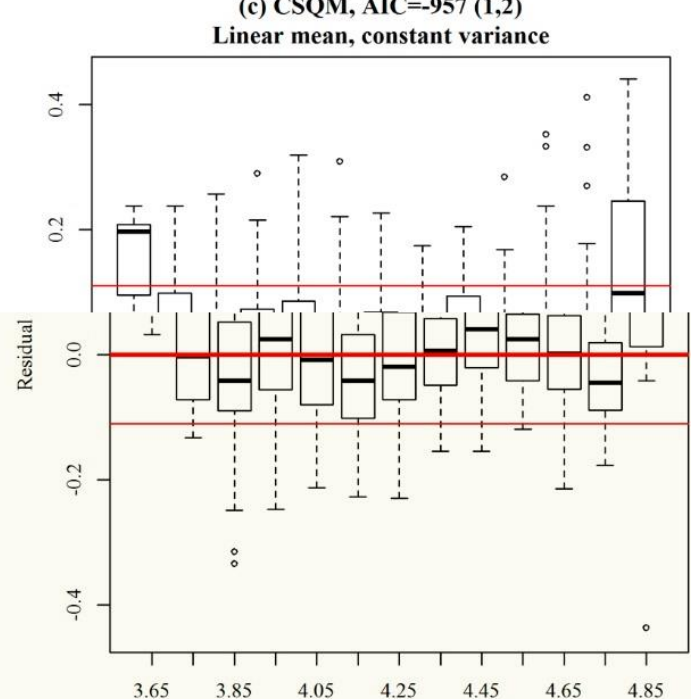

(e) CSOM, AIC=-964 $(1,4)$

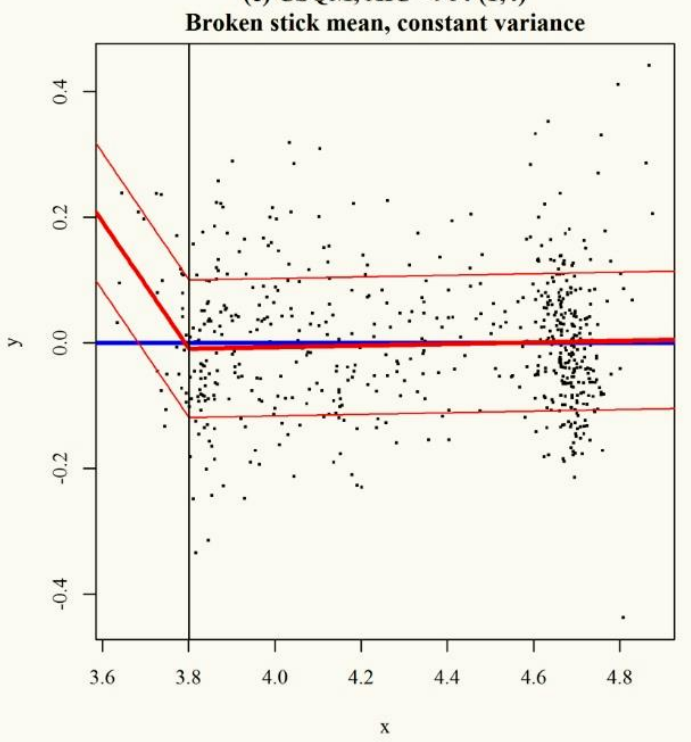

(b) CSQM, AIC=-964 $(1,4)$

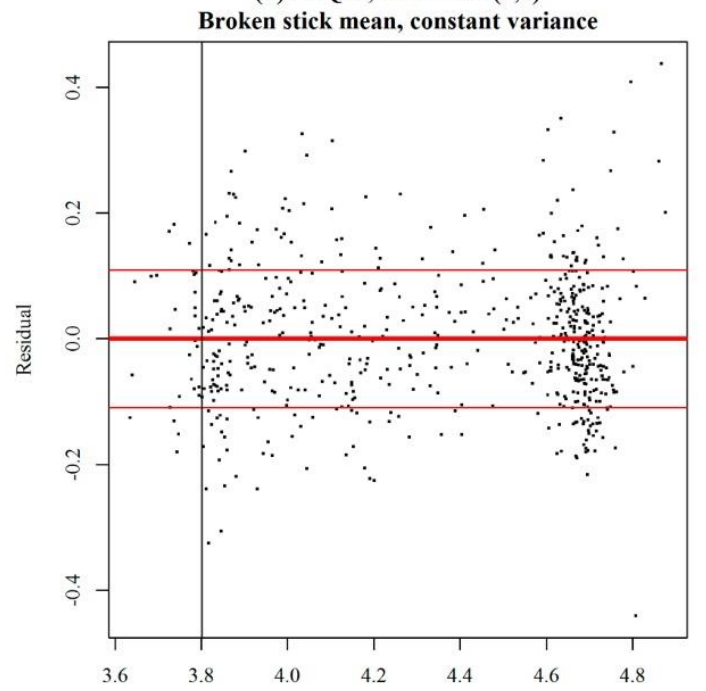

(d) CSQM, AIC=-964 (1,4)

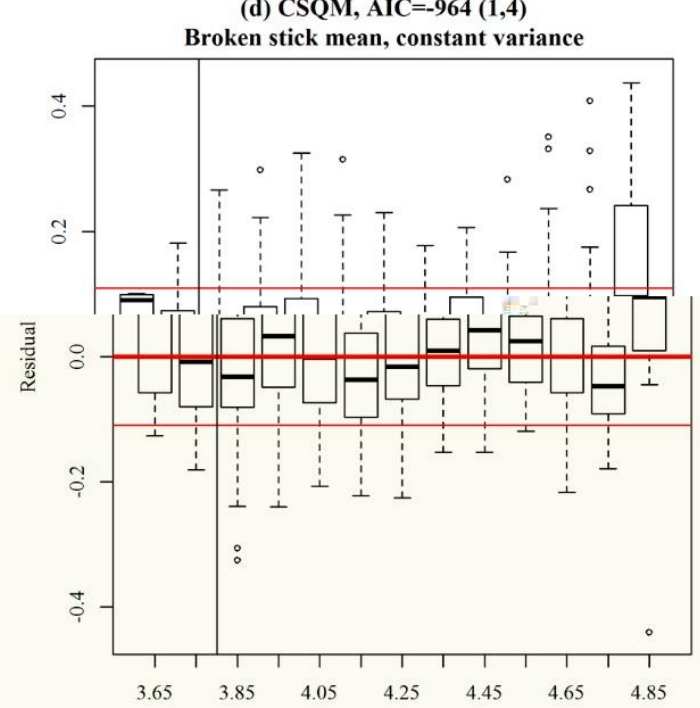

$\mathrm{x}$

(f) CSQM, AIC=-964 (1,4)

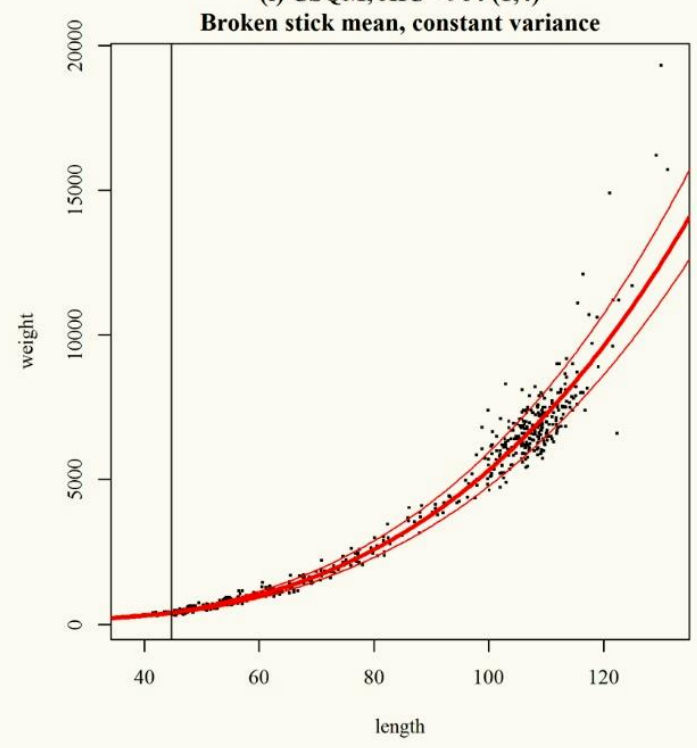



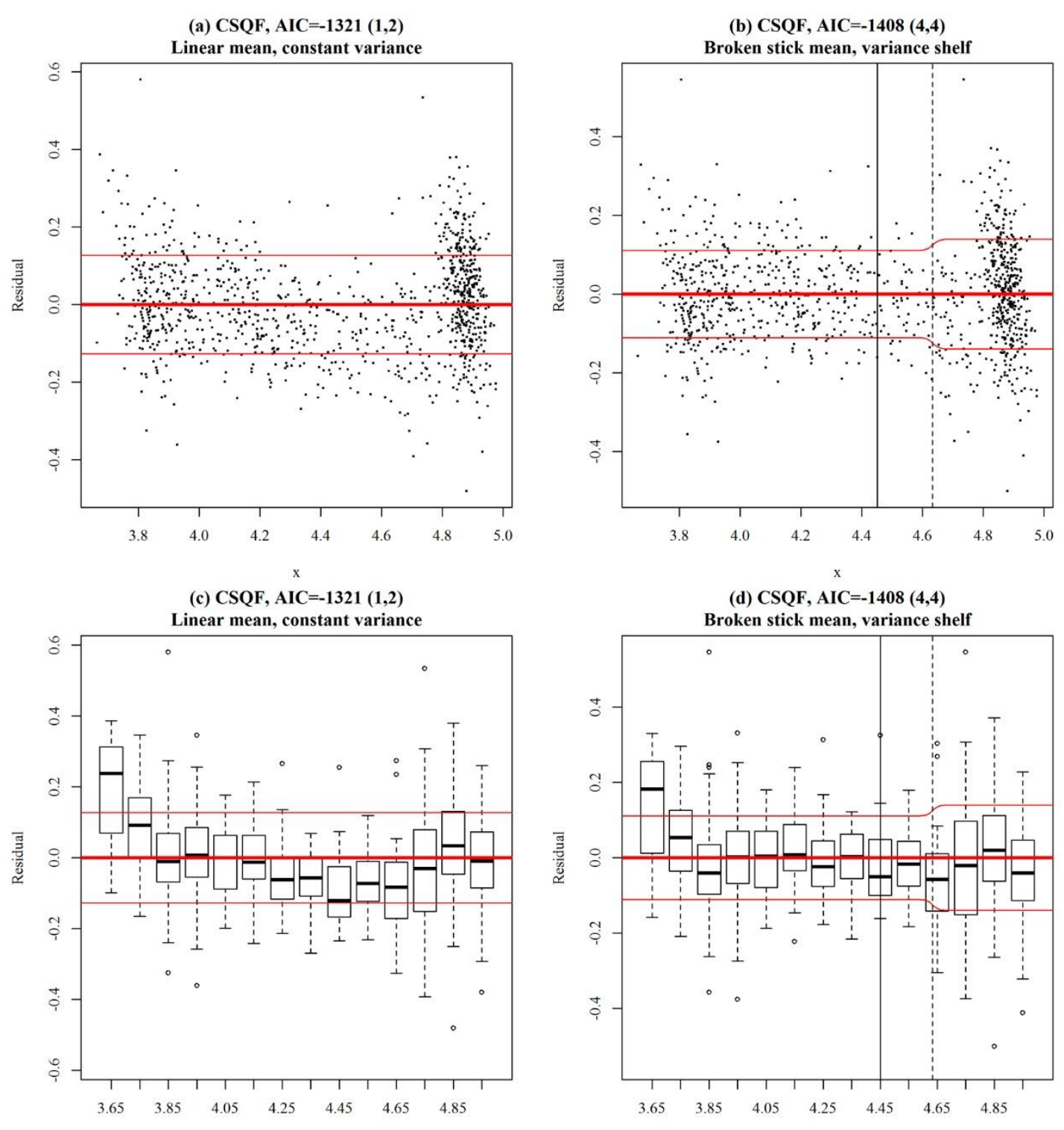

(e) CSQF, AIC=-1408 (4,4)
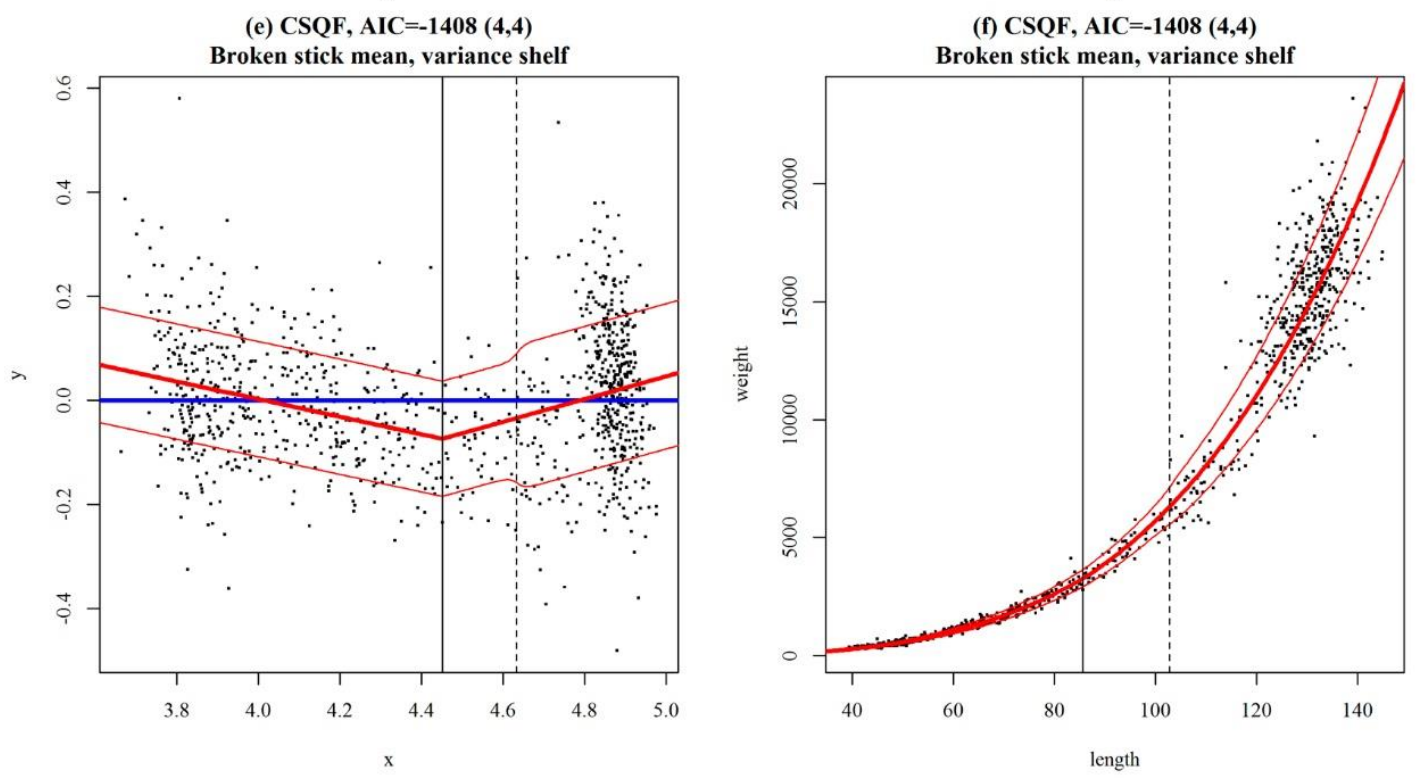
(a) SNDF, AIC=-12271 (1,2)

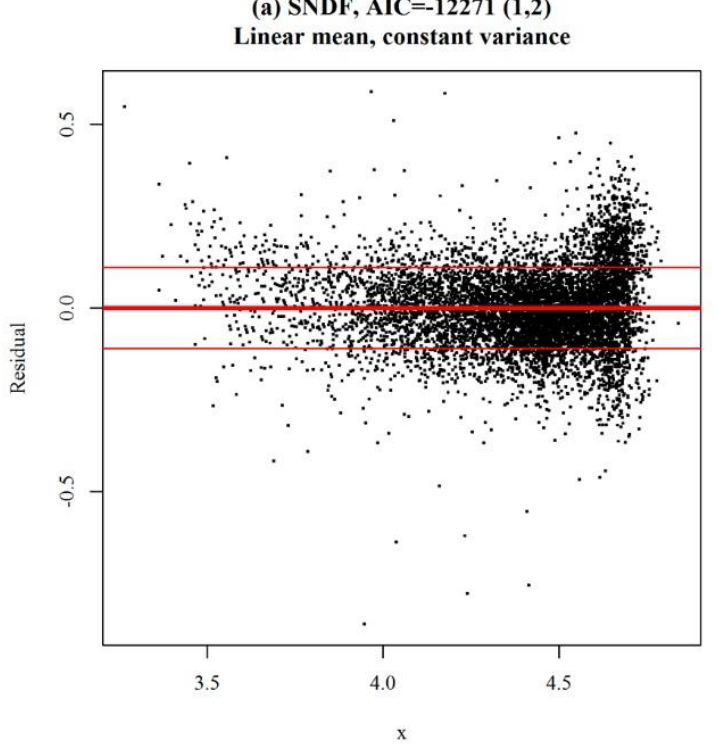

(c) SNDF, AIC=-12271 $(1,2)$

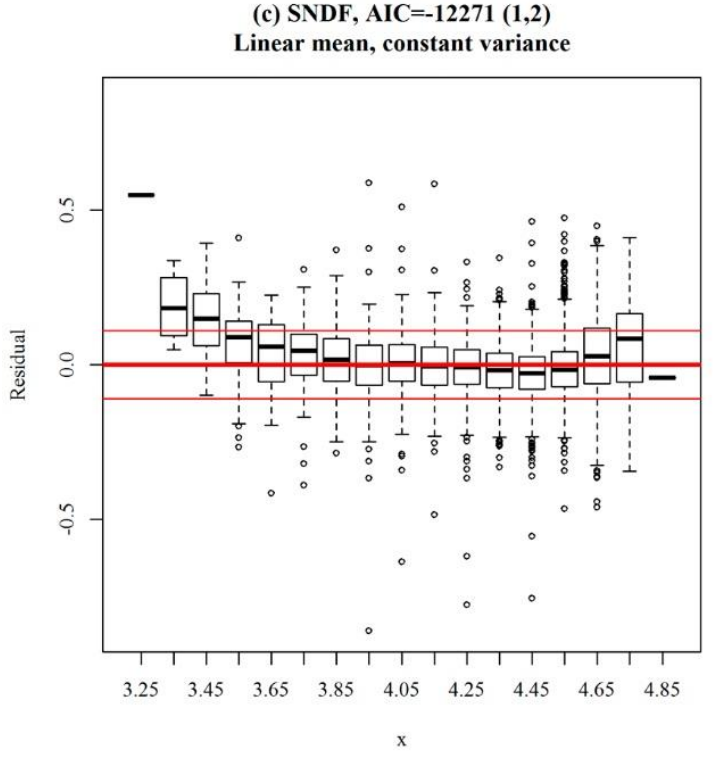

(e) SNDF, AIC=-12915 $(4,4)$

Broken stick mean, variance shelf

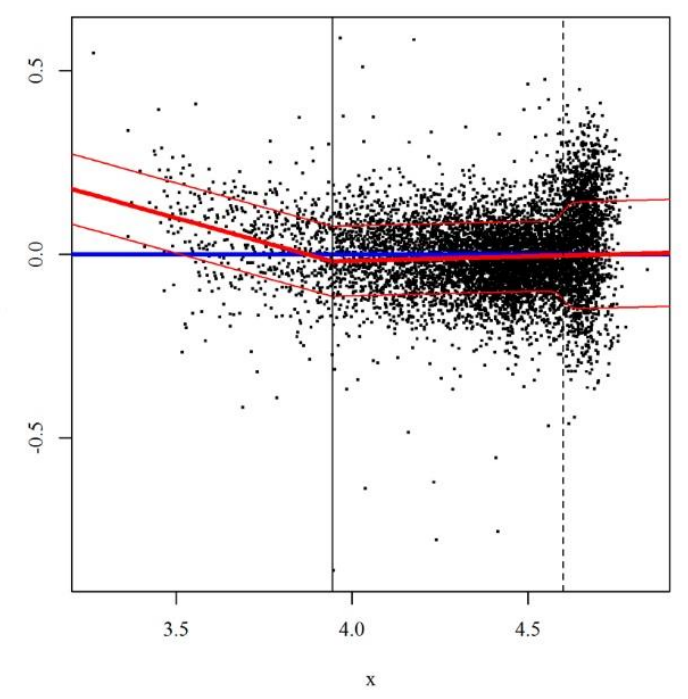

(b) SNDF, AIC=-12915 (4,4)

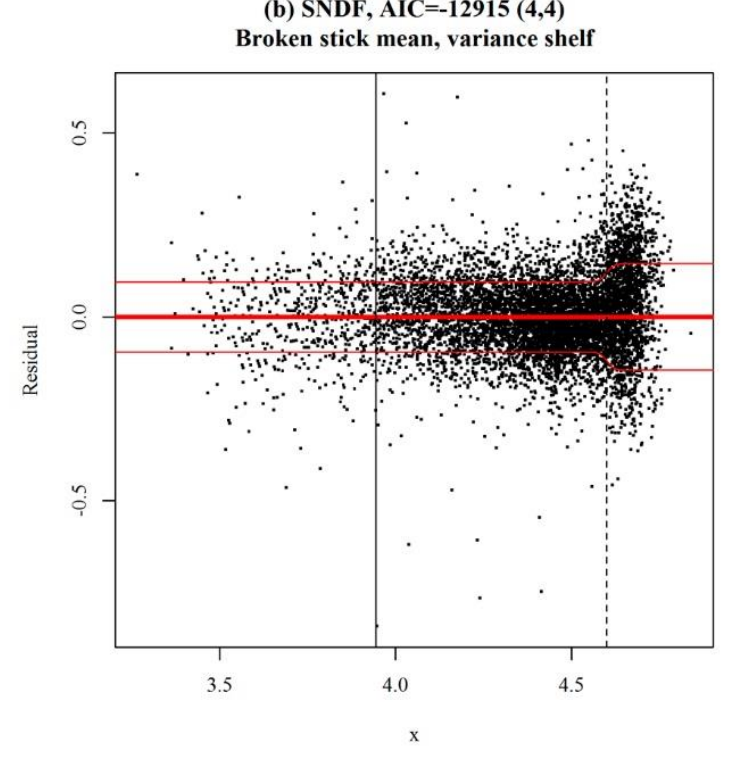

(d) SNDF, AIC=-12915 (4,4)

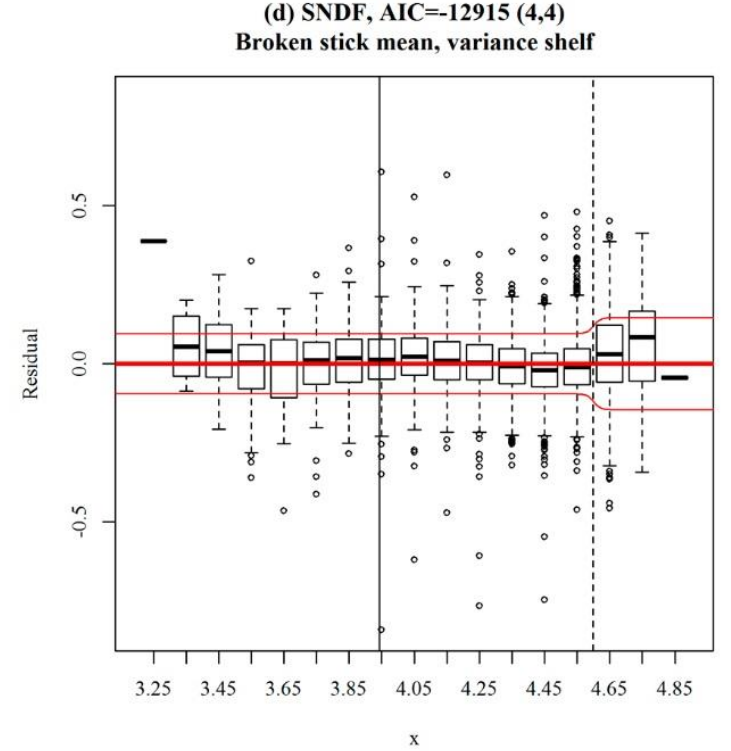

(f) SNDF, AIC=-12915 $(4,4)$

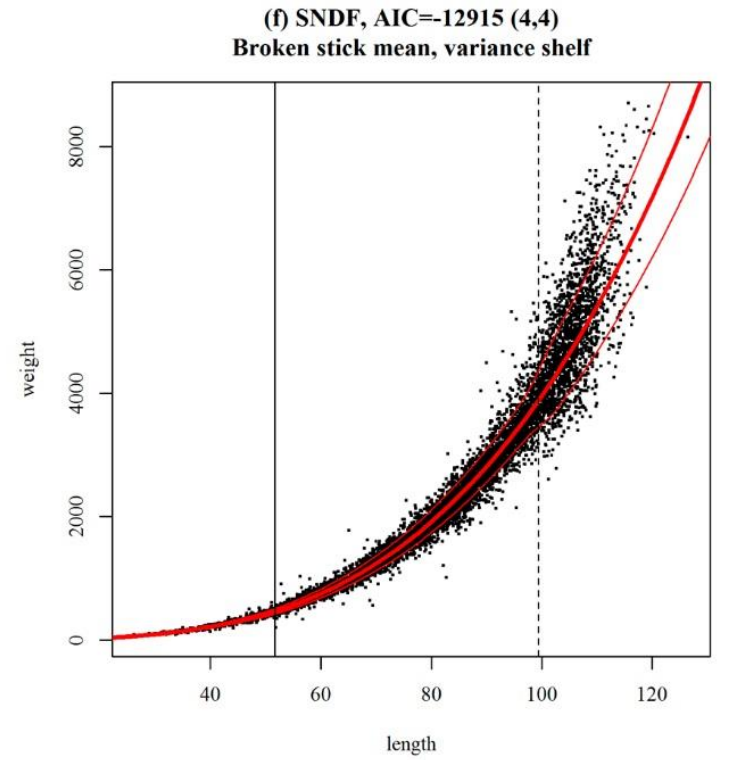



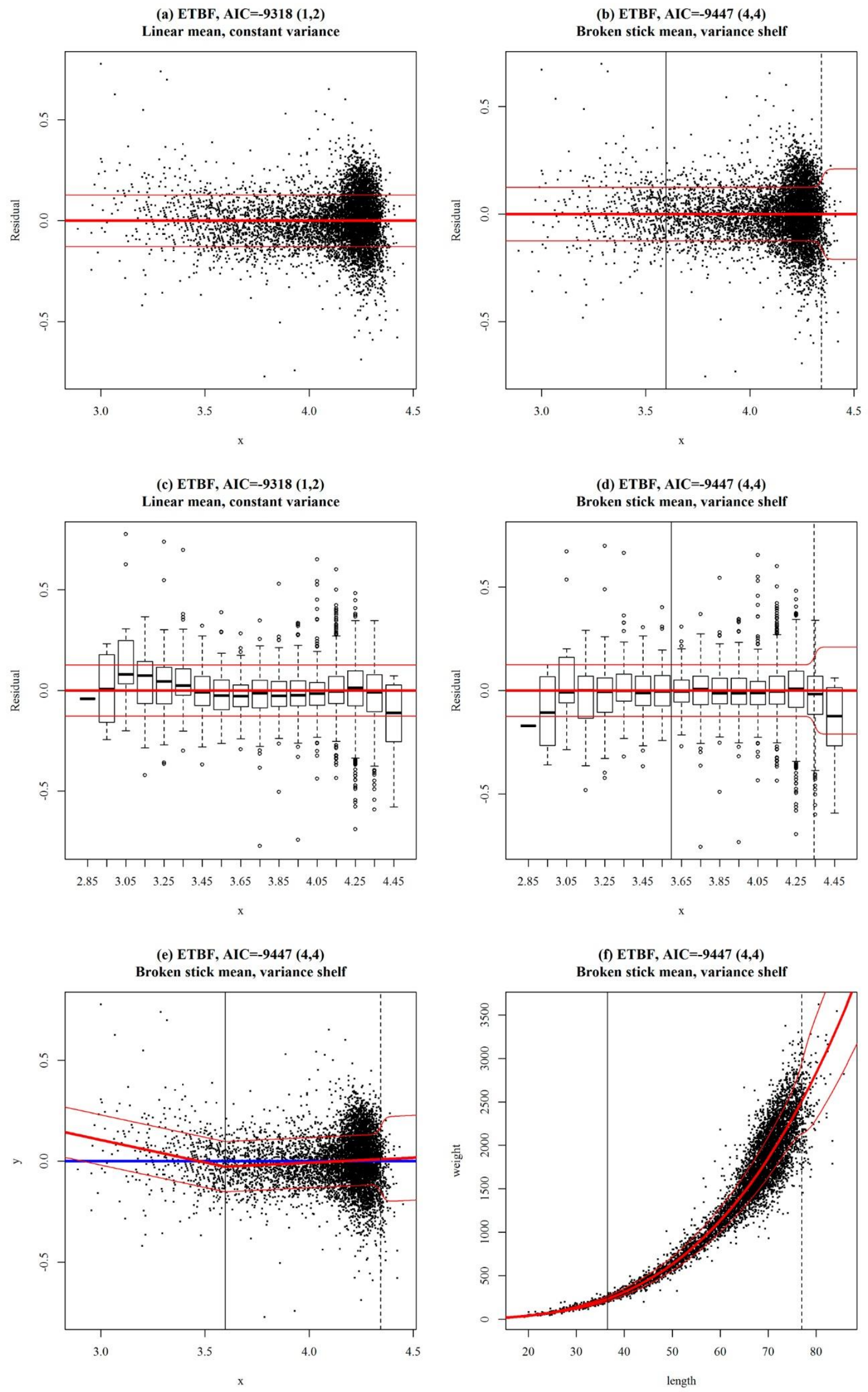
(a) ETLM, AIC=-3214 (1,2)

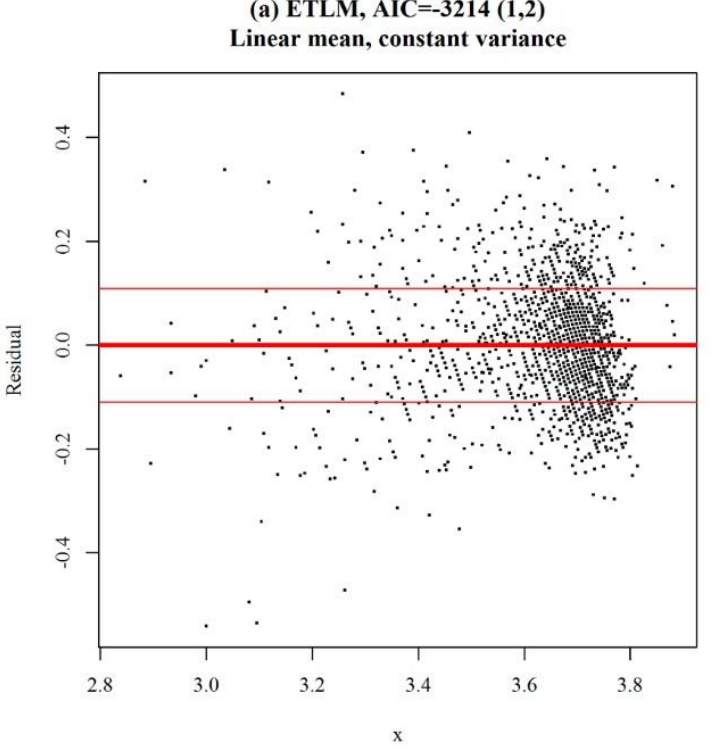

(c) ETLM, AIC=-3214 $(1,2)$

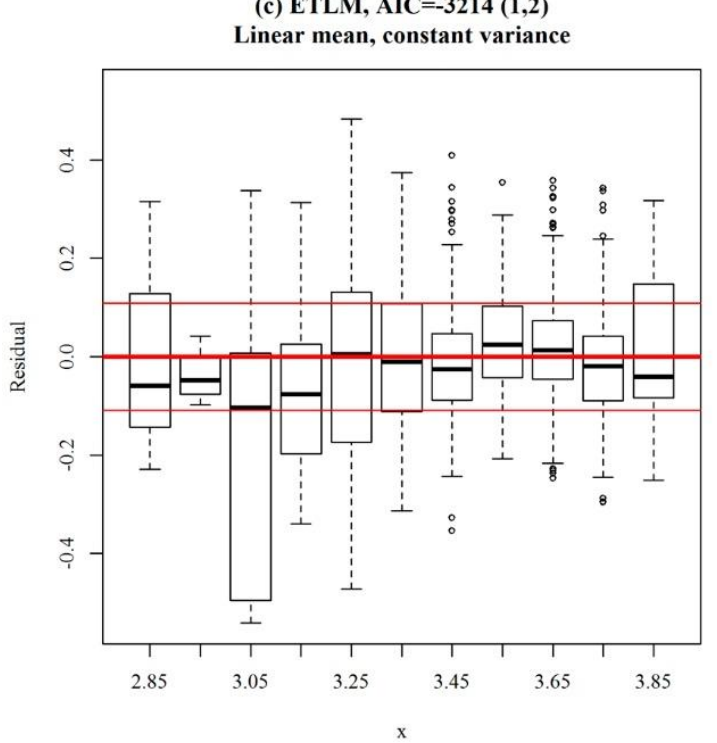

(e) ETLM, AIC=-3297 $(1,4)$

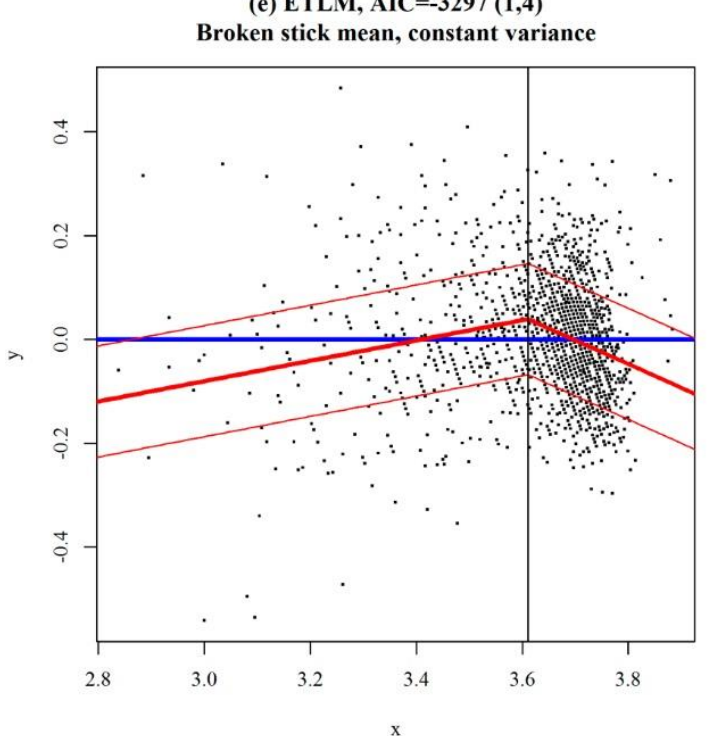

(b) ETLM, AIC=-3297 $(1,4)$

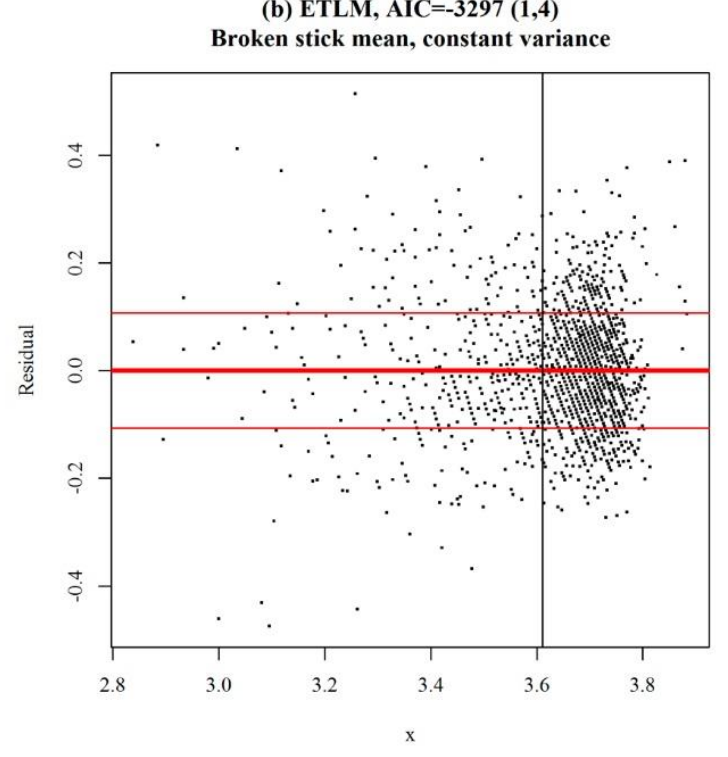

(d) ETLM, AIC=-3297 $(1,4)$

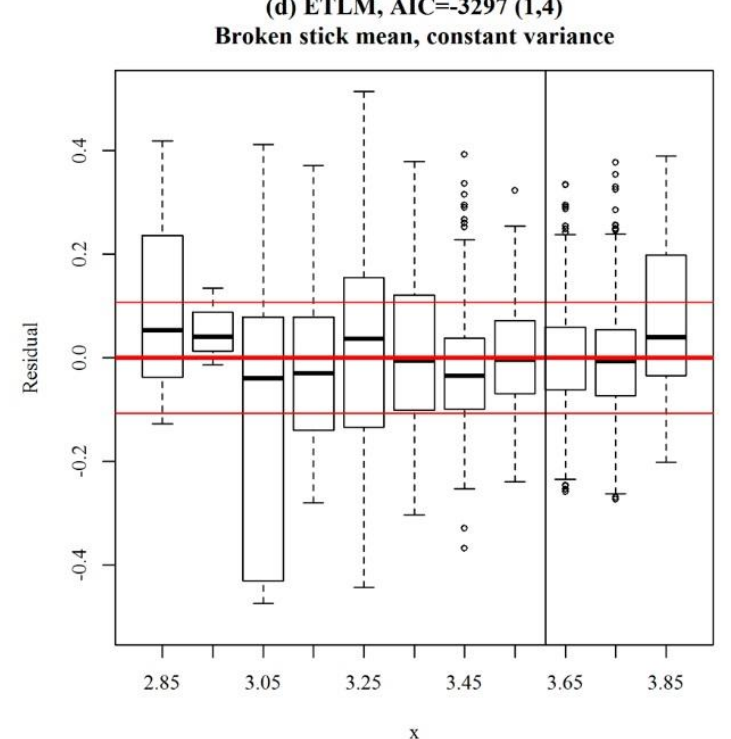

(f) ETLM, AIC=-3297 (1,4)

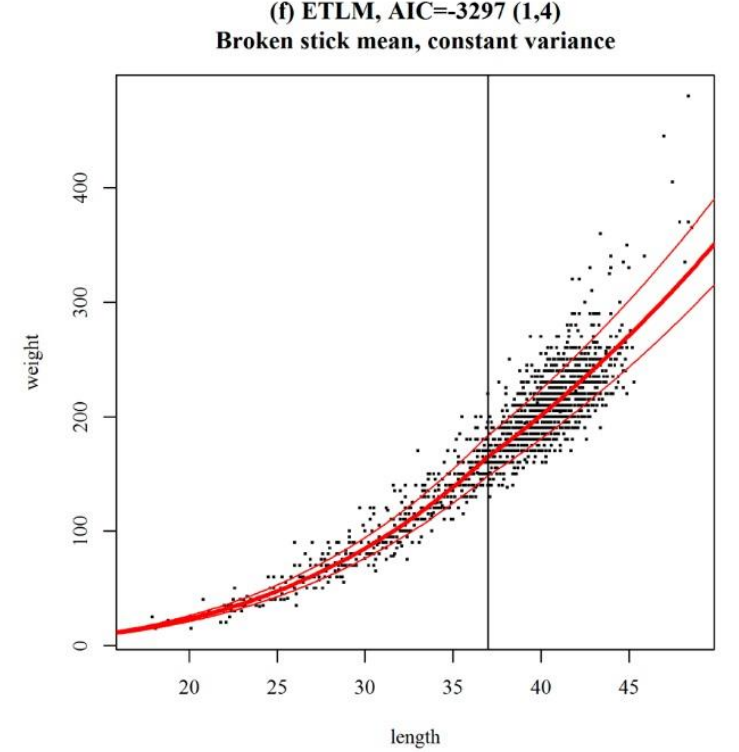



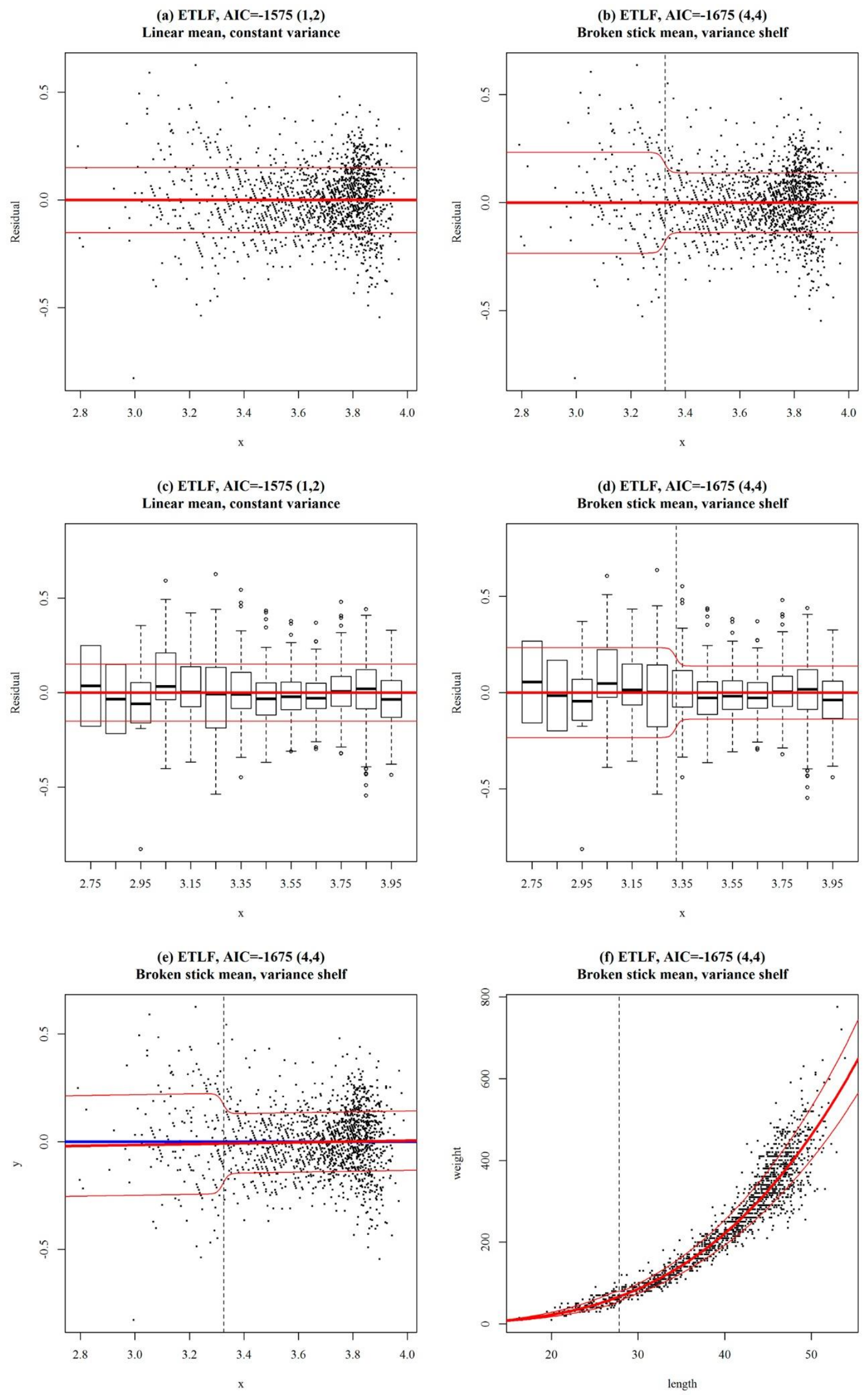
(a) CYOM, AIC=-1746 (1,2)

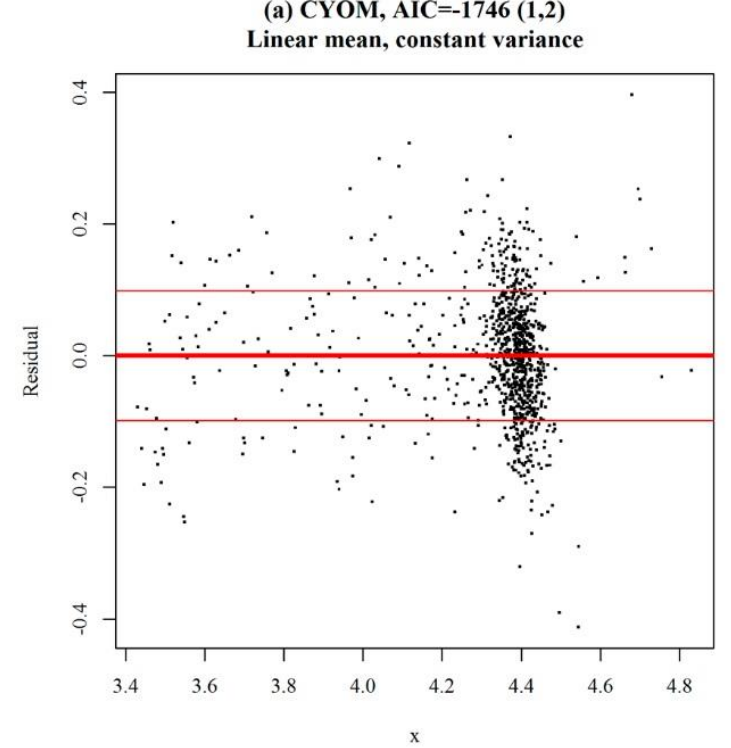

(c) CYOM, AIC=-1746 (1,2)

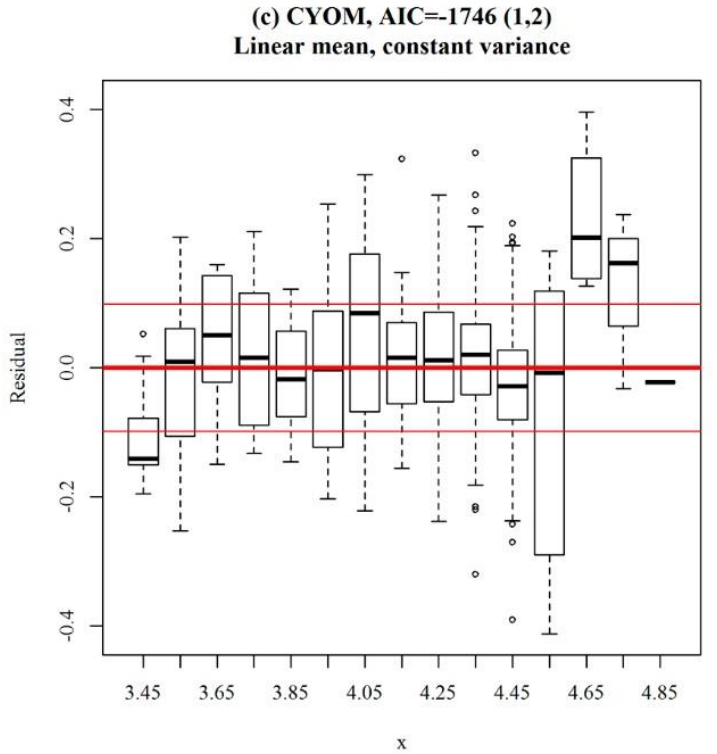

(e) CYOM, AIC=-1798 $(4,4)$

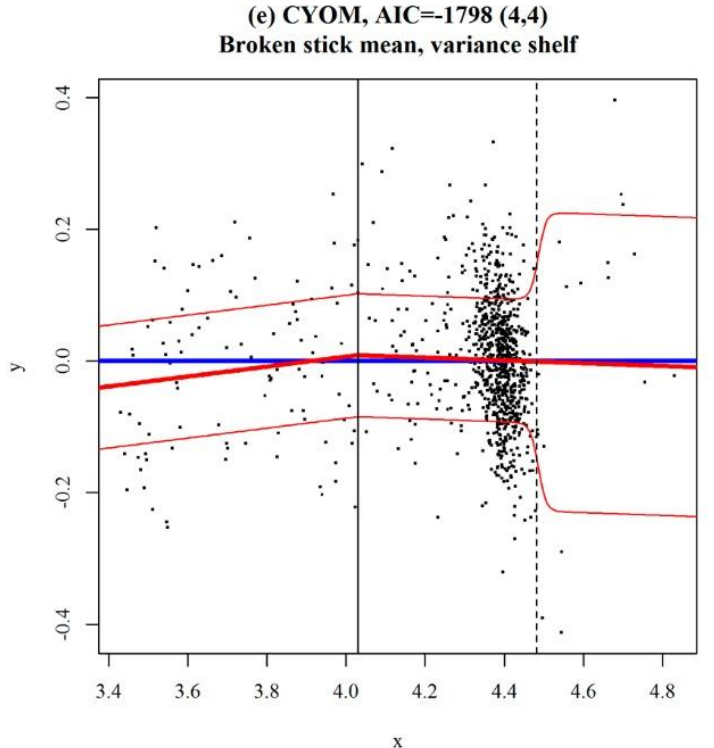

(b) CYOM, AIC=-1798 (4,4)

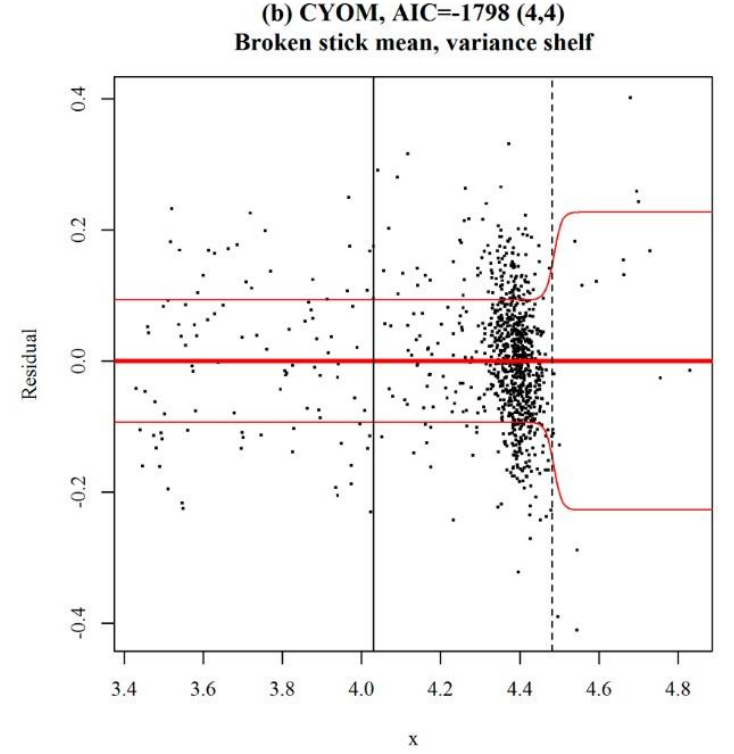

(d) CYOM, AIC=-1798 (4,4)
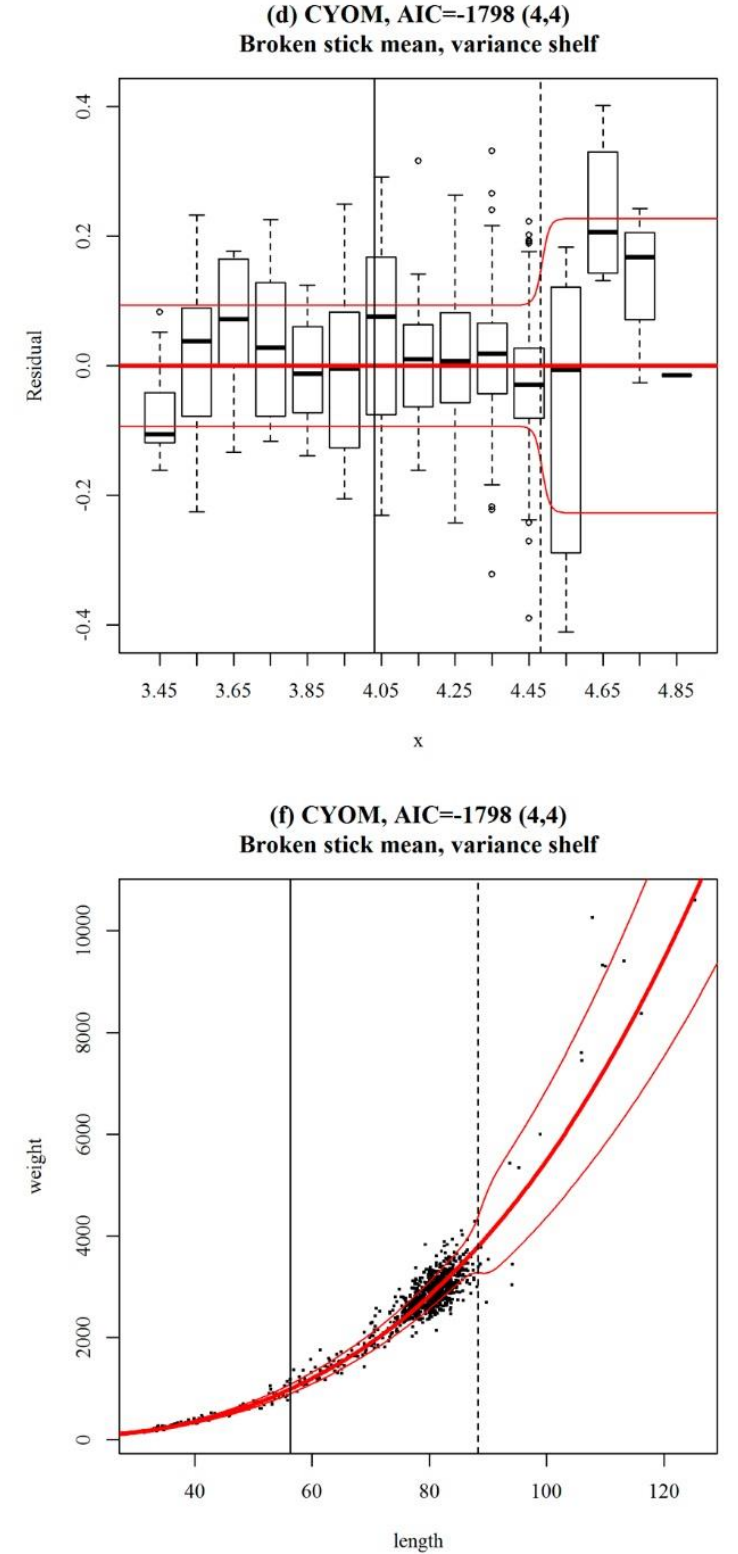
(a) CYPM, AIC $=-597(1,2)$

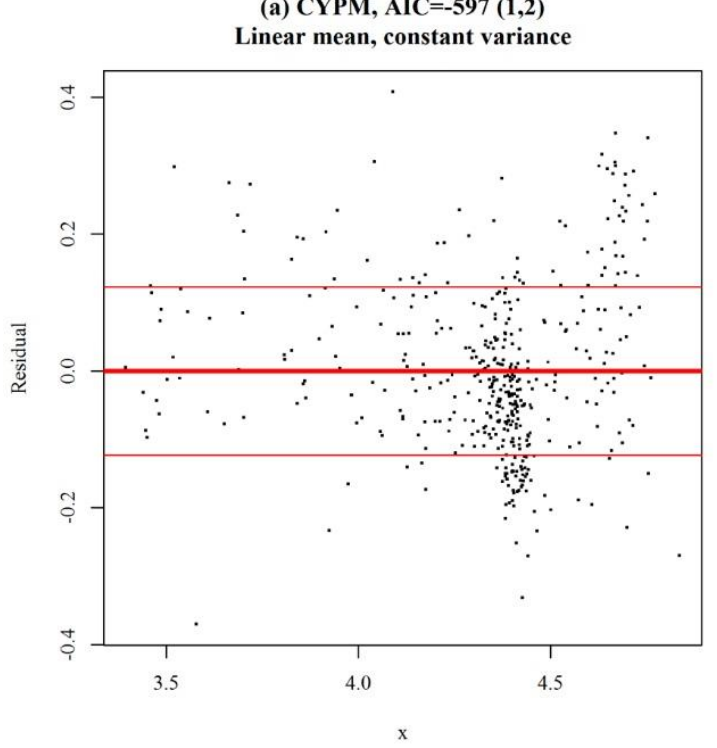

(c) CYPM, AIC=-597 (1,2)

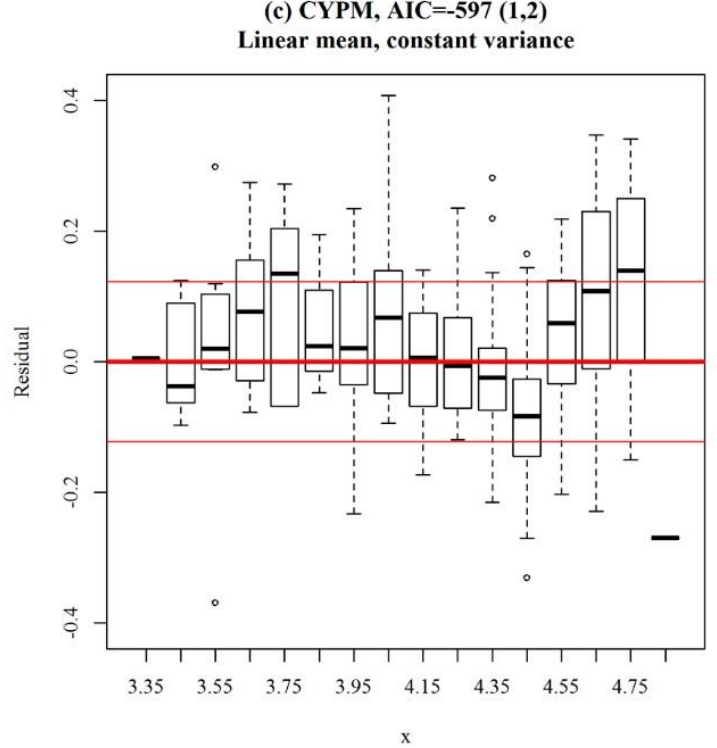

(e) CYPM, AIC $=-678(1,4)$

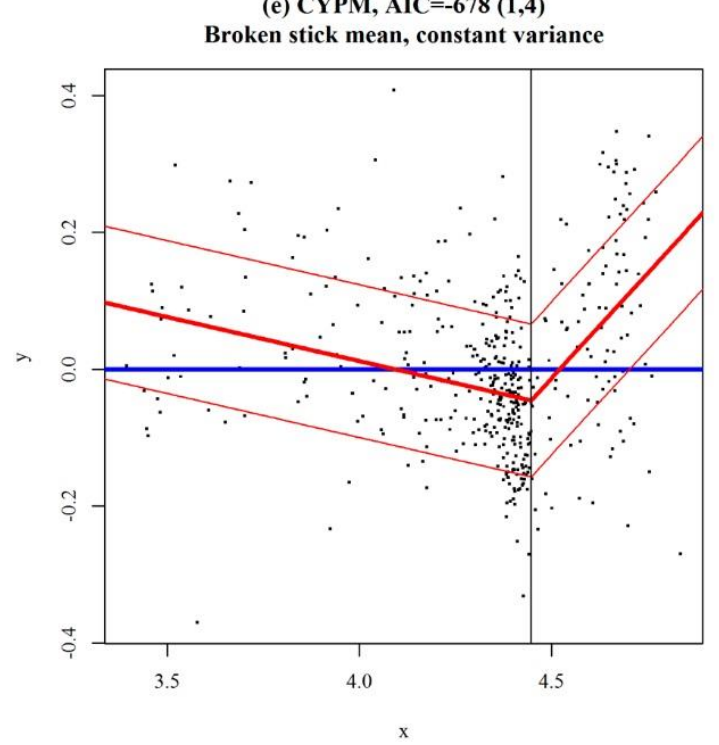

(b) CYPM, AIC=-678 $(1,4)$

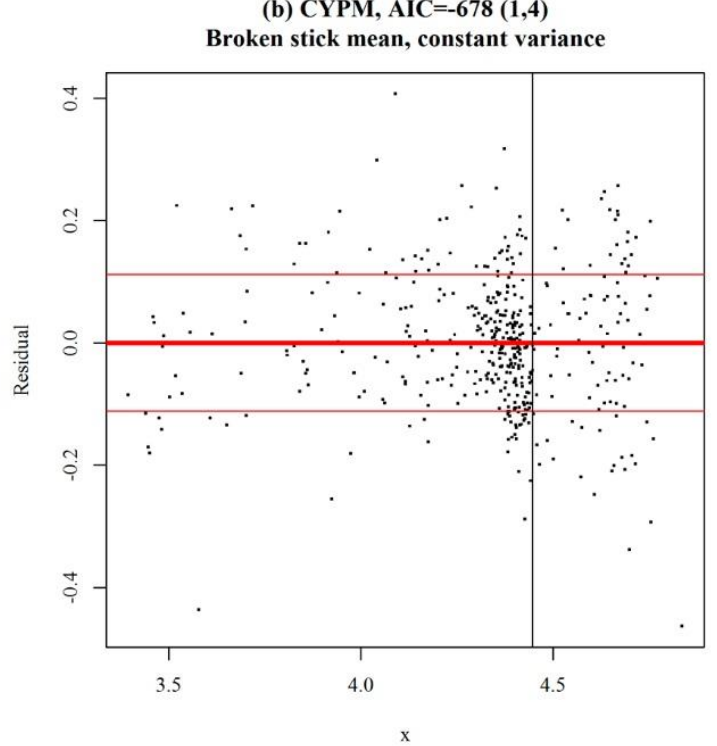

(d) CYPM, AIC $=-678(1,4)$

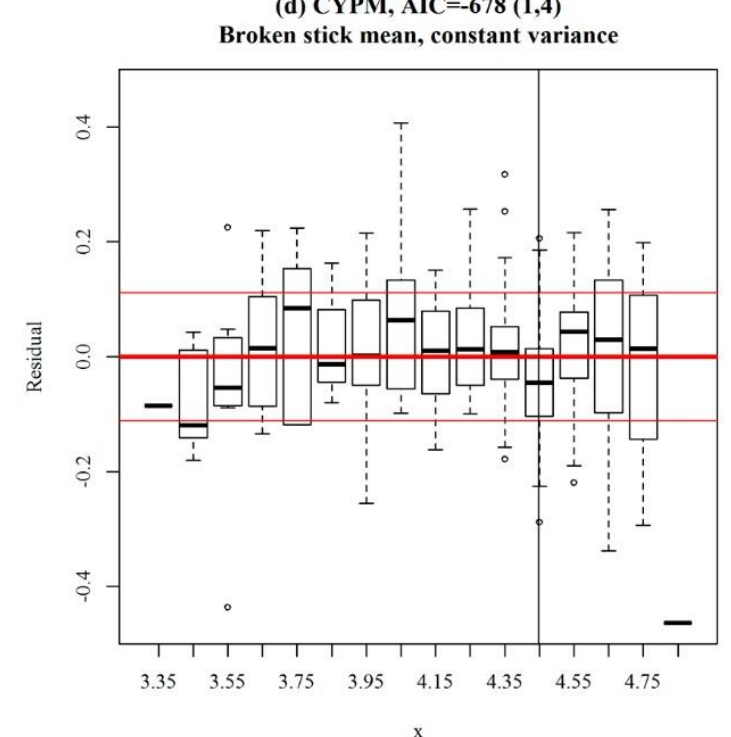

(f) CYPM, AIC=-678 $(1,4)$

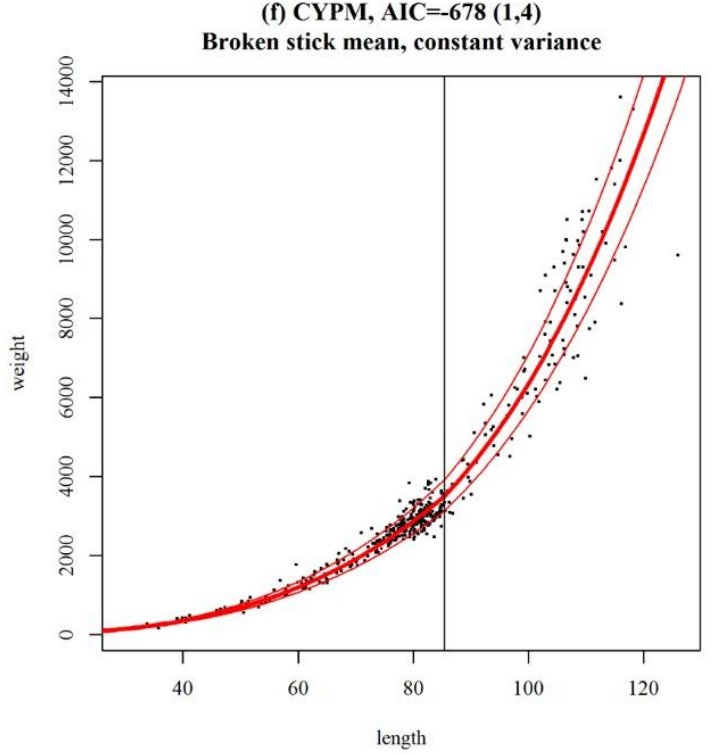


(a) CYPF, AIC=-1589 $(1,2)$

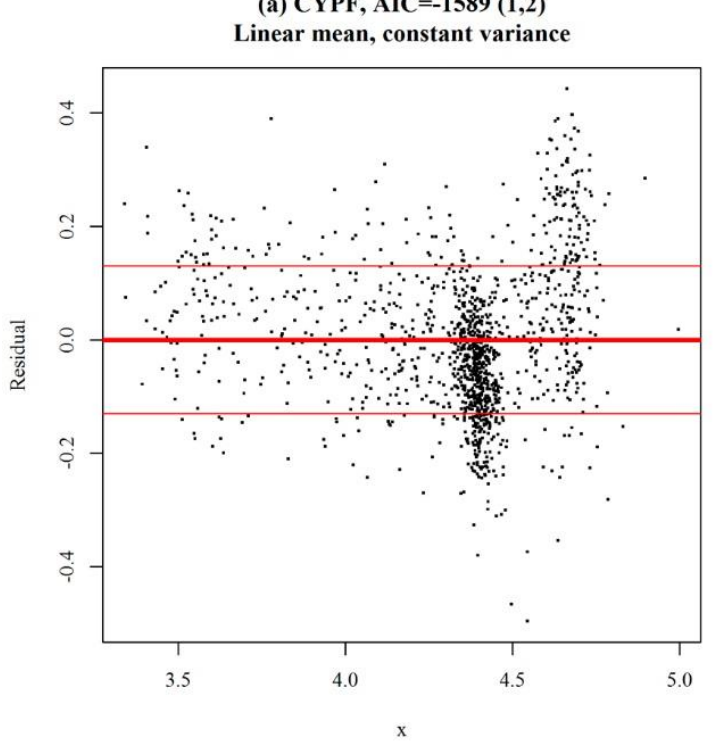

(c) CYPF, AIC=-1589 (1,2)

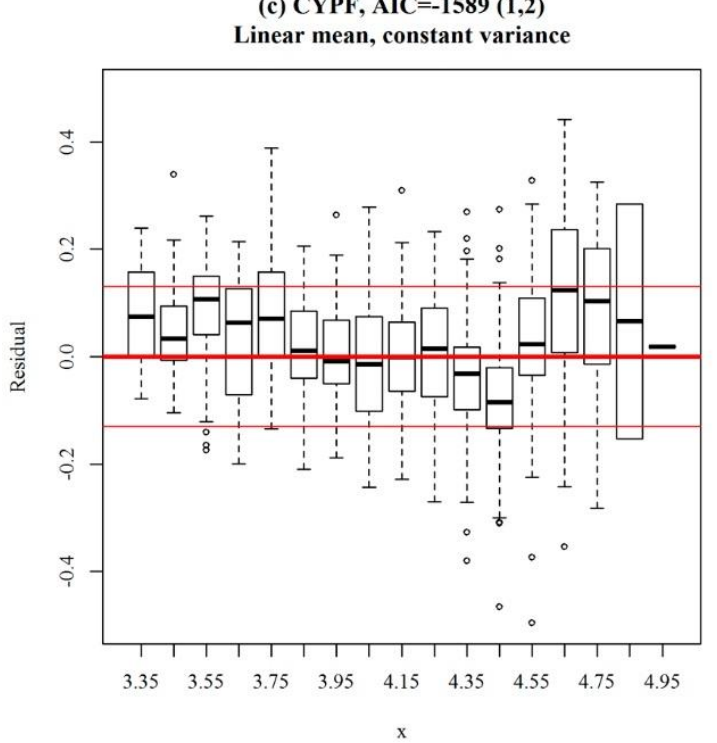

(e) CYPF, AIC=-1922 $(4,4)$

Broken stick mean, variance shelf

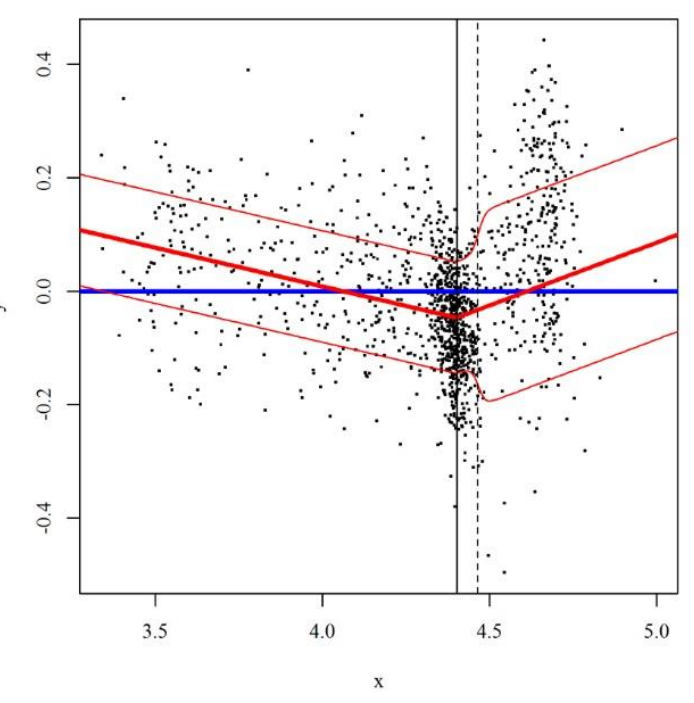

(b) CYPF, AIC=-1922 (4,4)

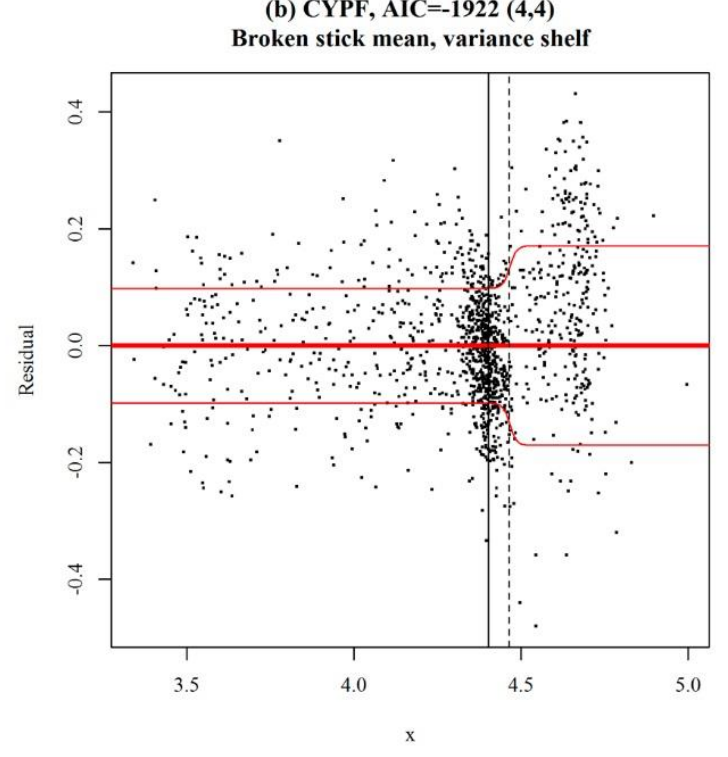

(d) CYPF, AIC=-1922 (4,4)

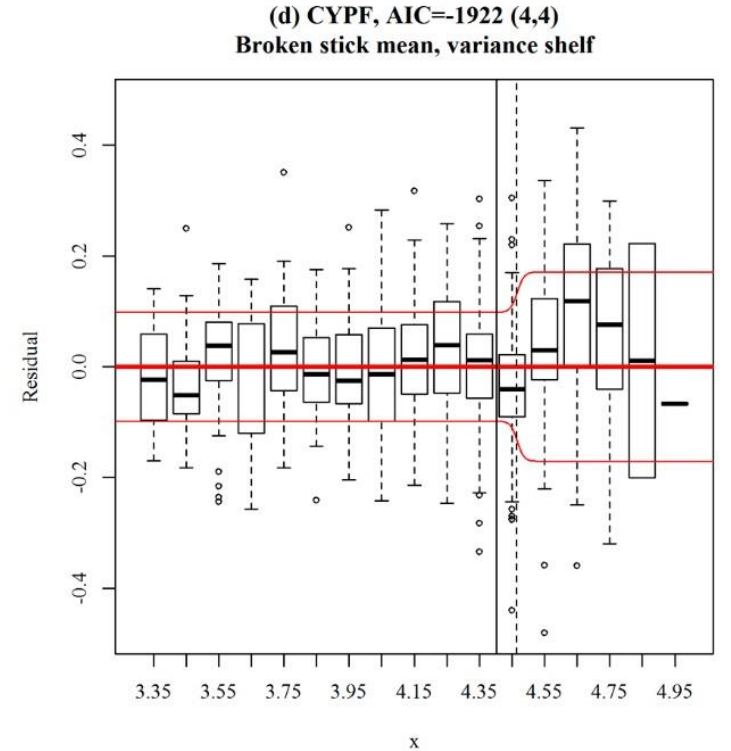

(f) CYPF, AIC=-1922 $(4,4)$

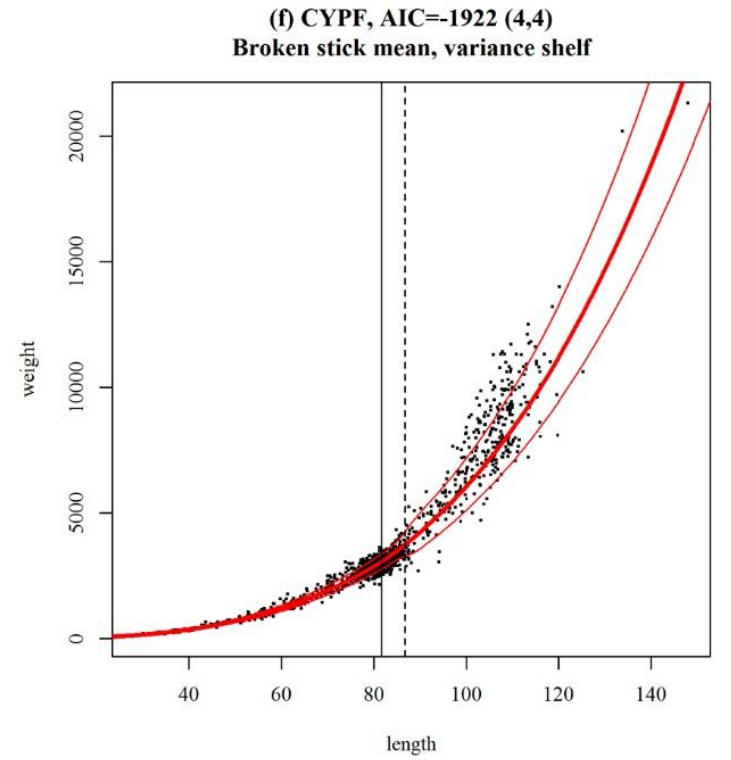


(a) CPTM, AIC $=-615(1,2)$

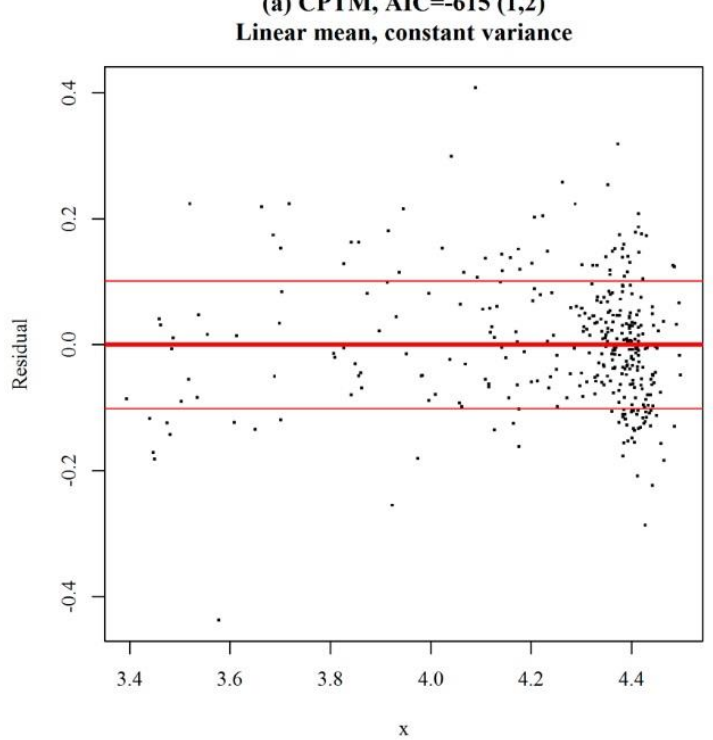

(c) CPTM, AIC $=-615(1,2)$

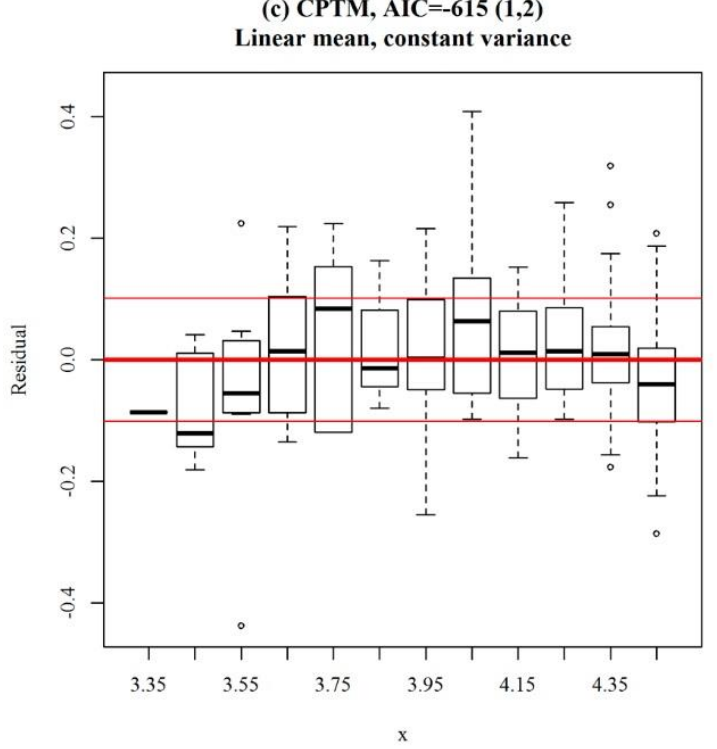

(e) CPTM, AIC=-641 $(4,4)$

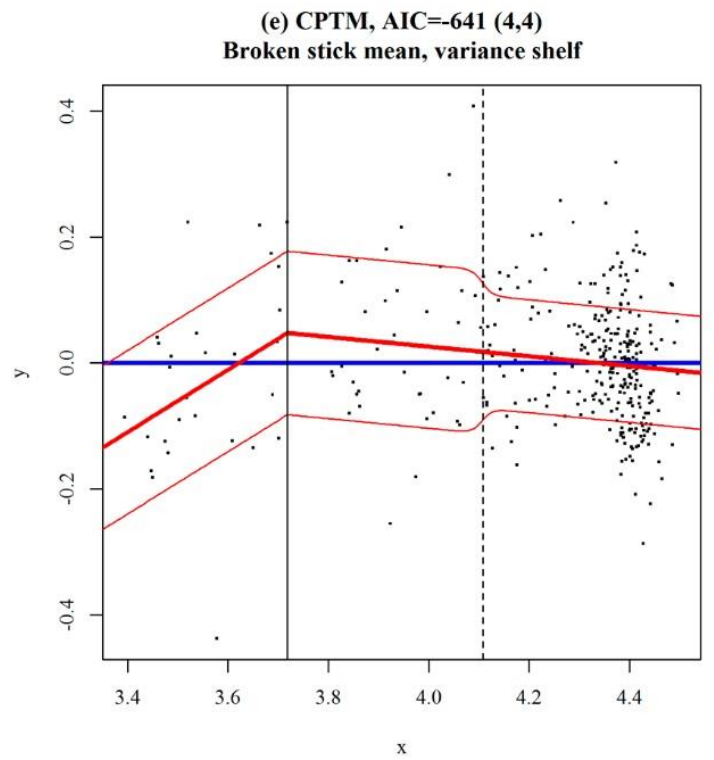

(b) CPTM, AIC =-641 $(4,4)$

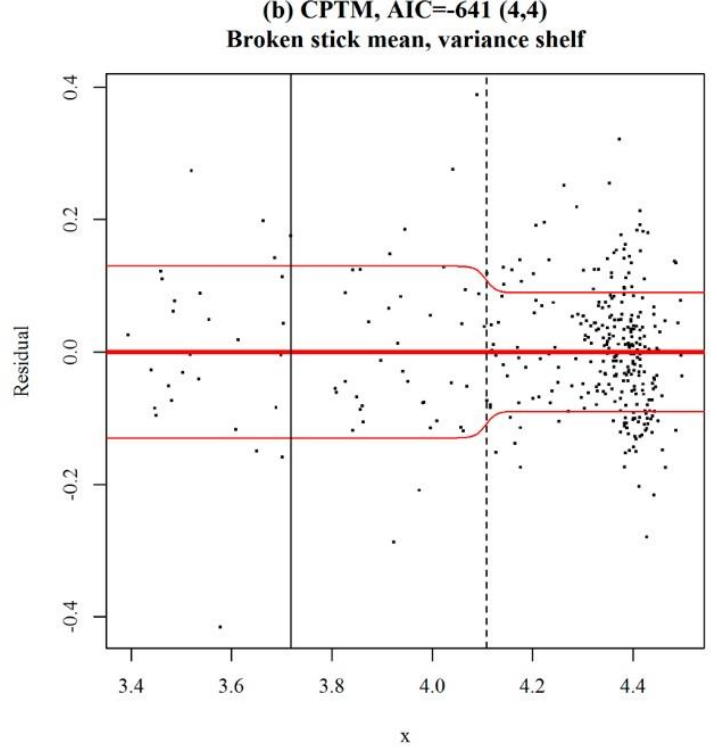

(d) CPTM, AIC $=-641(4,4)$
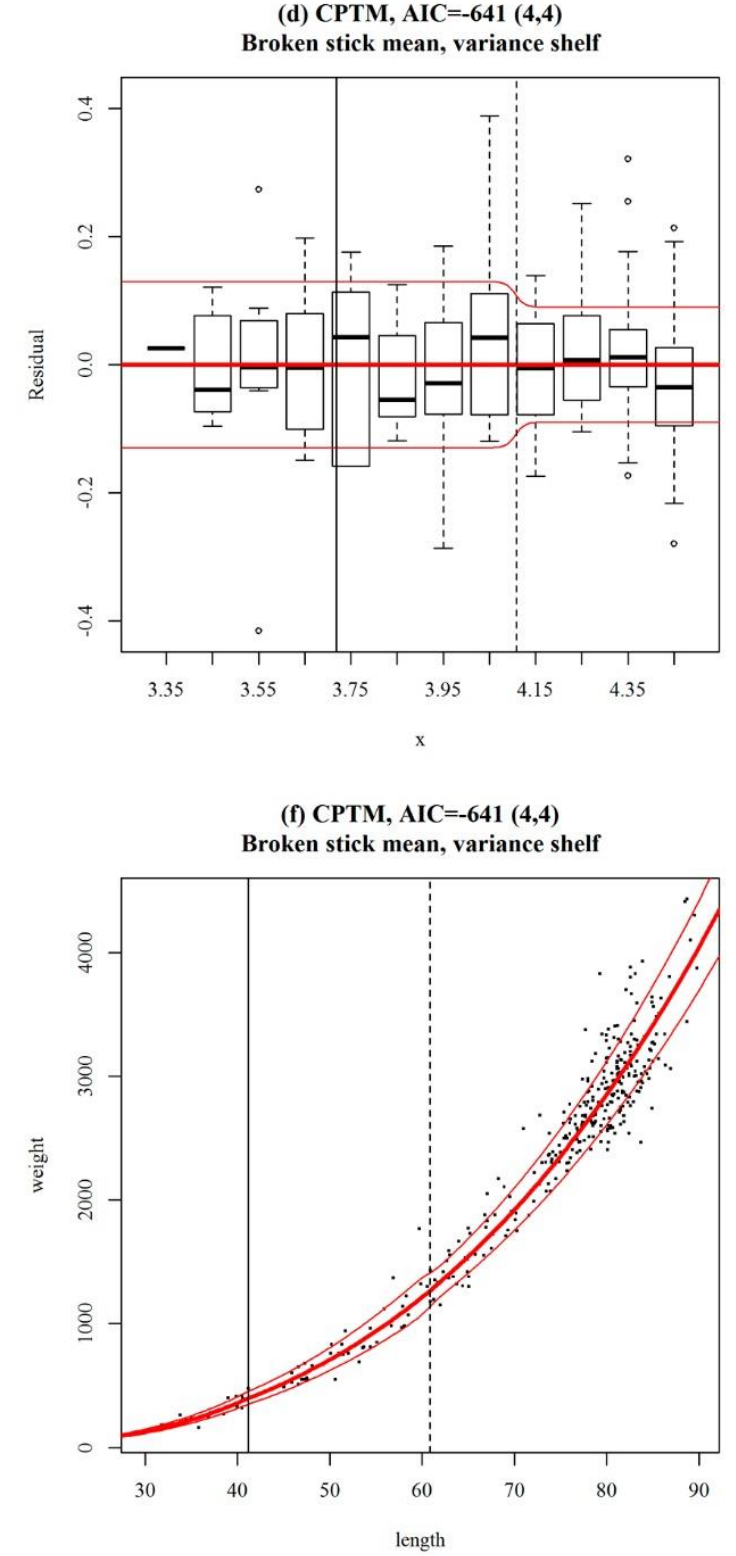
(a) CPTF, AIC=-1711 (1,2)

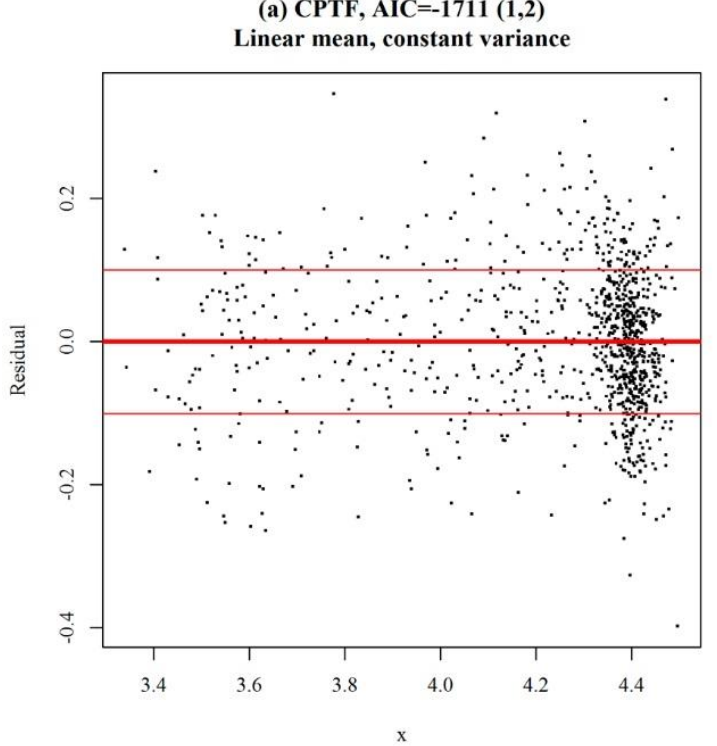

(c) CPTF, AIC=-1711 (1,2)

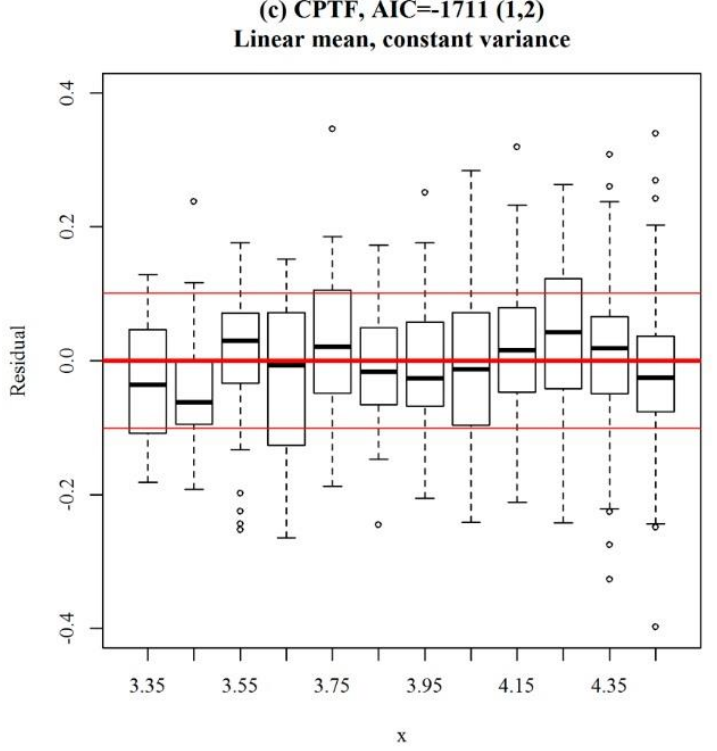

(e) CPTF, AIC=-1742 $(1,4)$

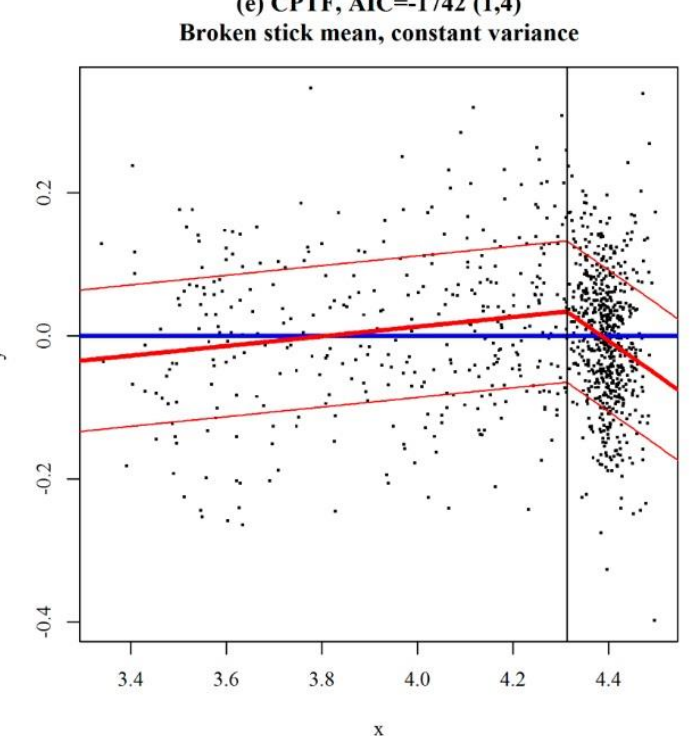

(b) CPTF, AIC=-1742 (1,4)

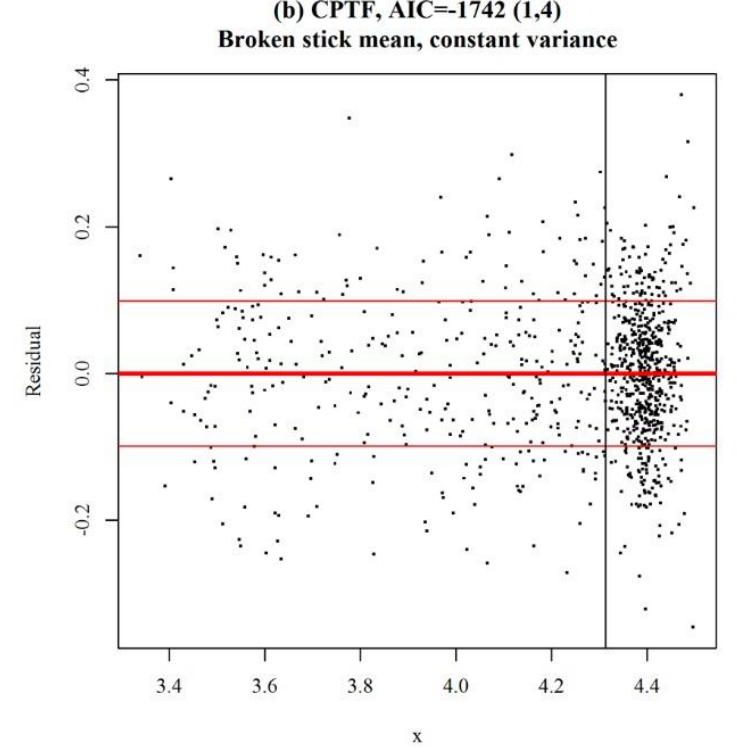

(d) CPTF, AIC=-1742 $(1,4)$

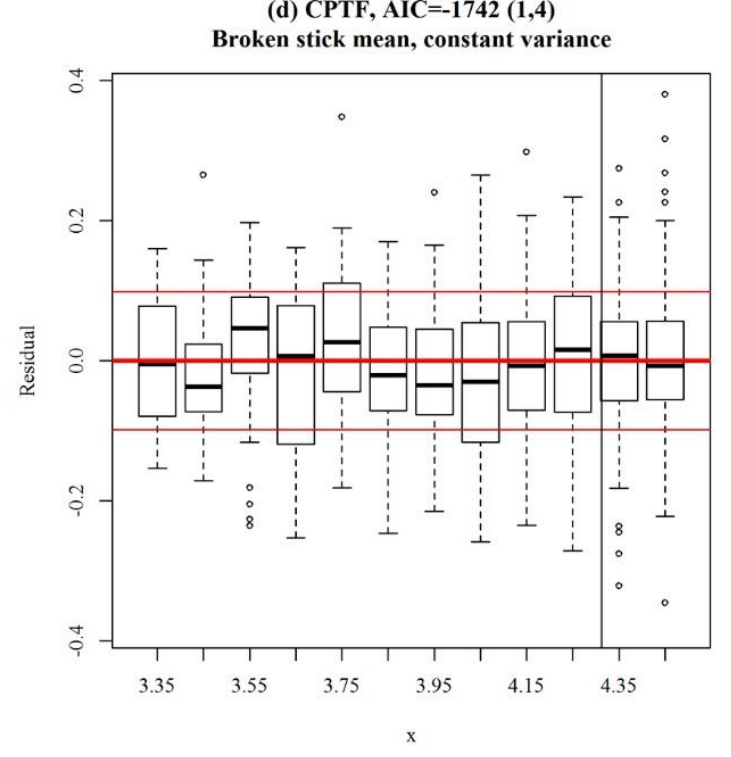

(f) CPTF, AIC=-1742 $(1,4)$

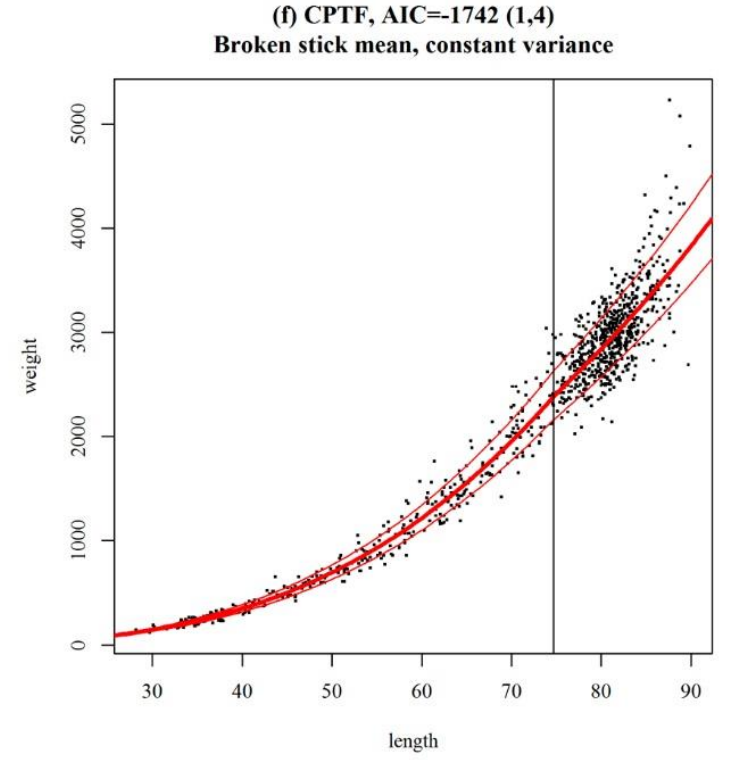


(a) PLSM, AIC =-243 (1,2)

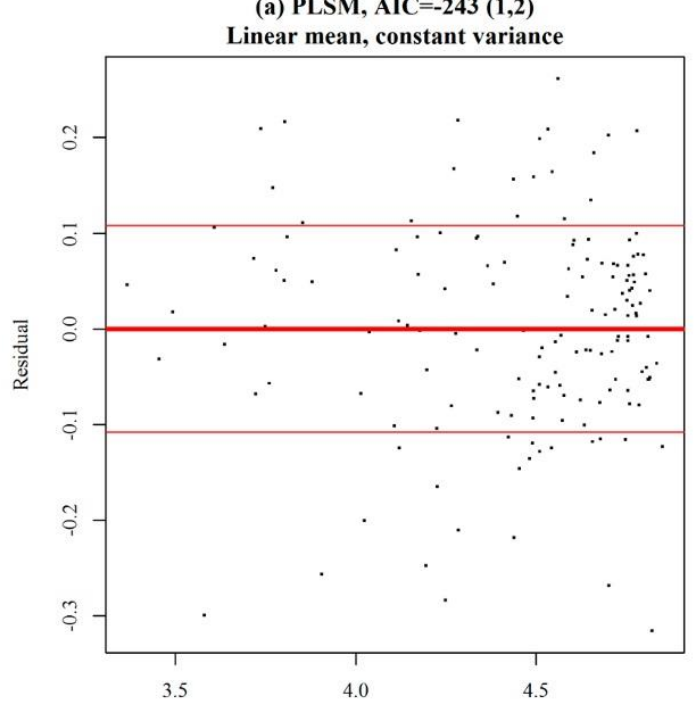

$x$

(c) PLSM, AIC=-243 (1,2)

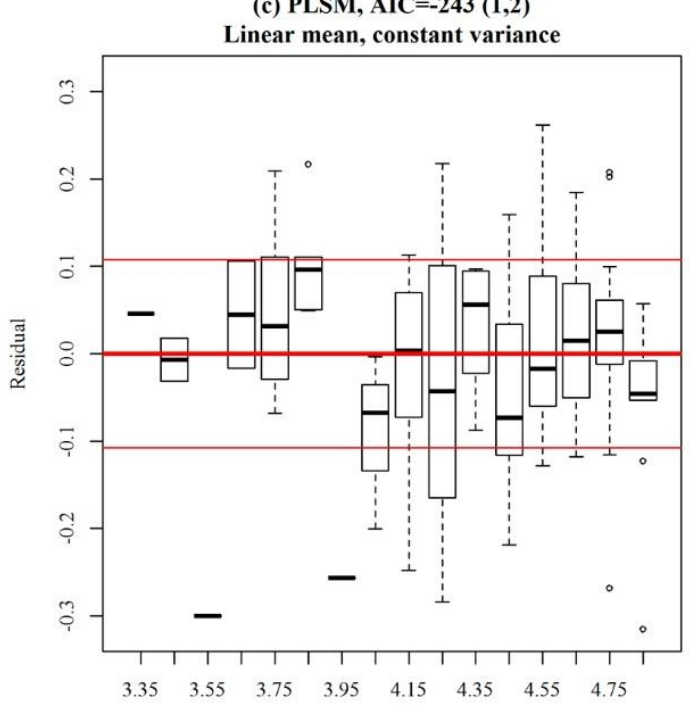

(e) PLSM, AIC=-244 (4,2)

Linear mean, variance shelf

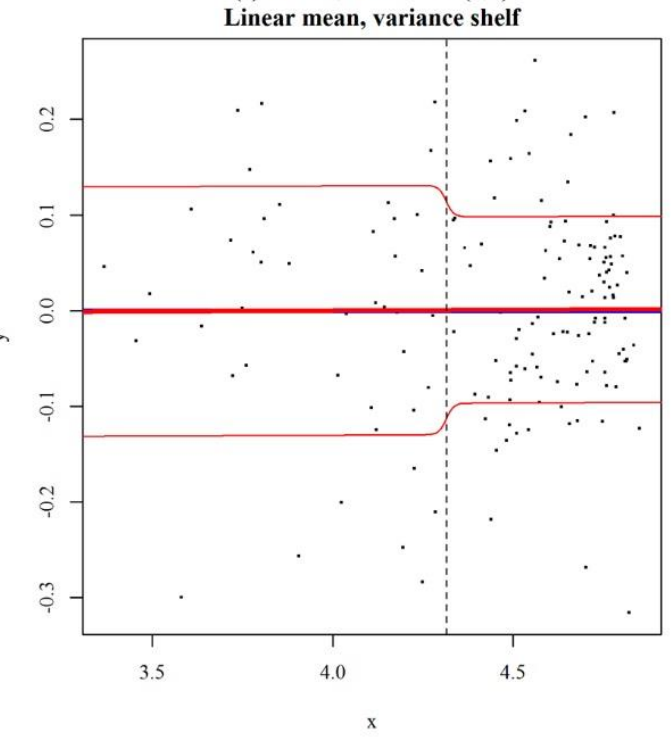

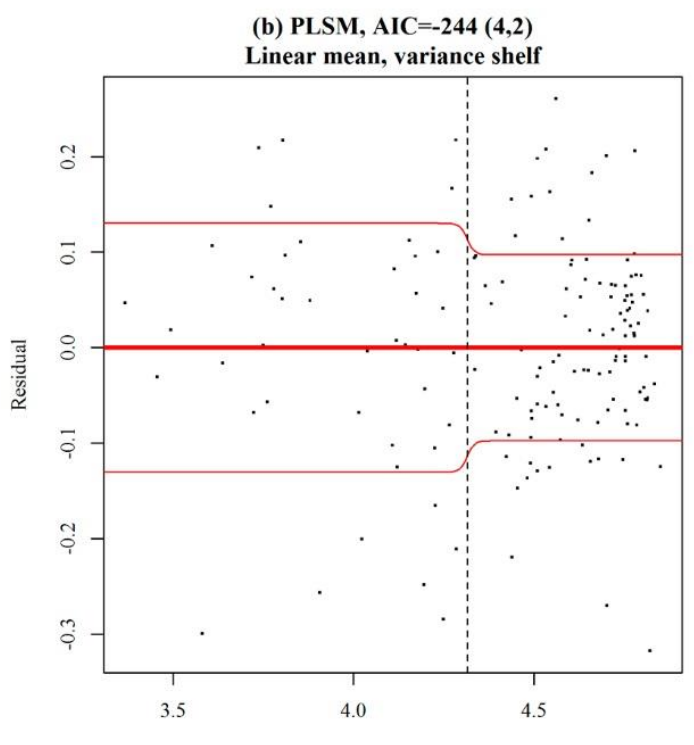

(d) PLSM, AIC=-244 (4,2)

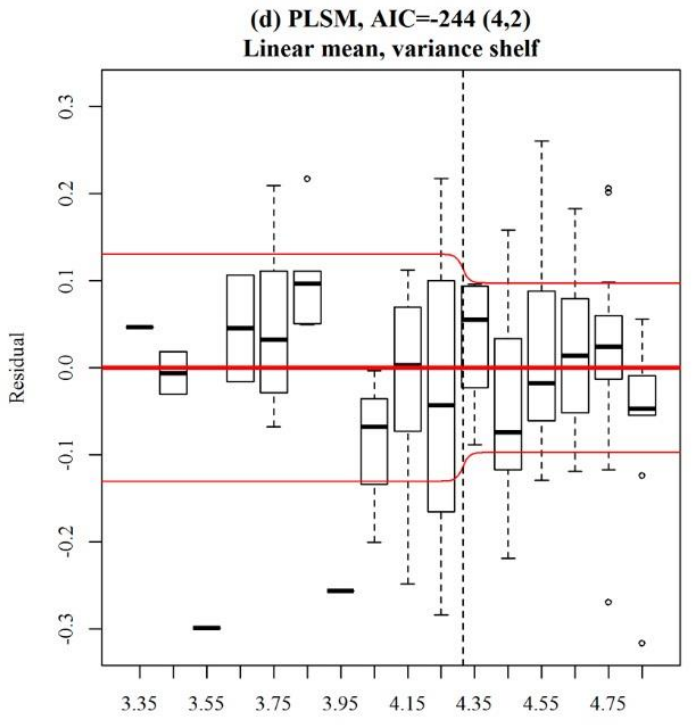

$x$

(f) PLSM, AIC=-244 (4,2)

Linear mean, variance shelf

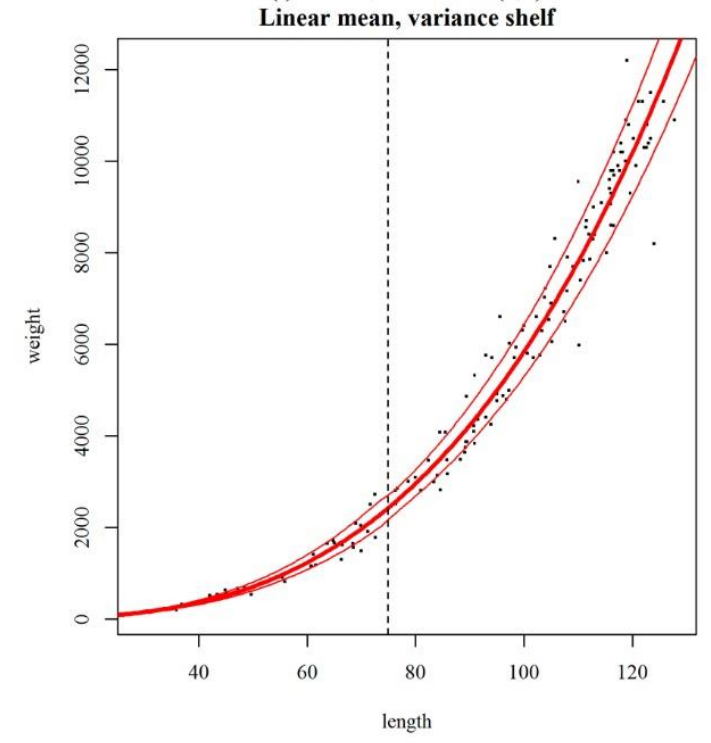


(a) PLSF, AIC=-243 (1,2)

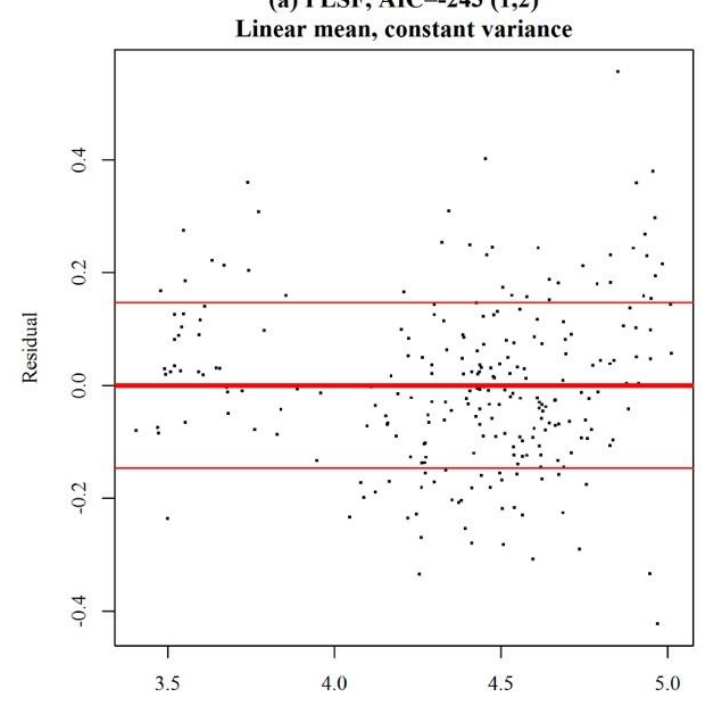

(c) PLSF, AIC=-243 (1,2)

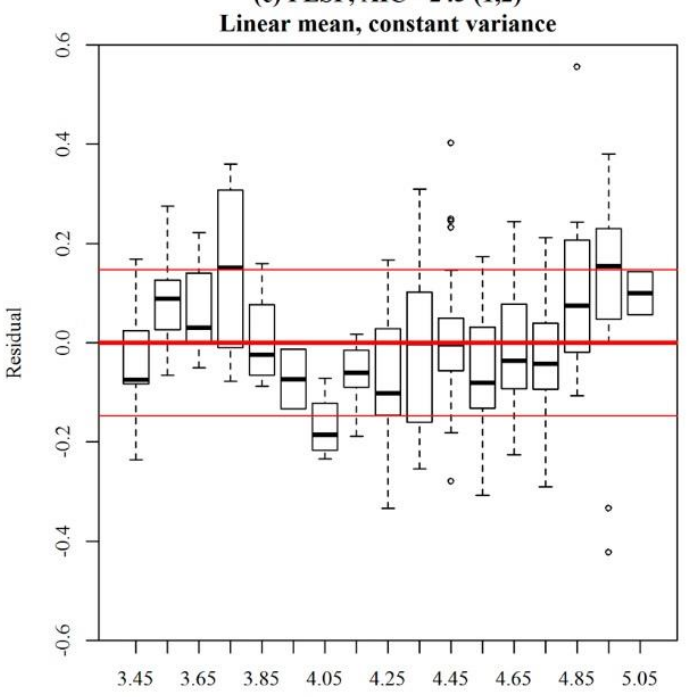

(e) PLSF, AIC=-271 (4,4)

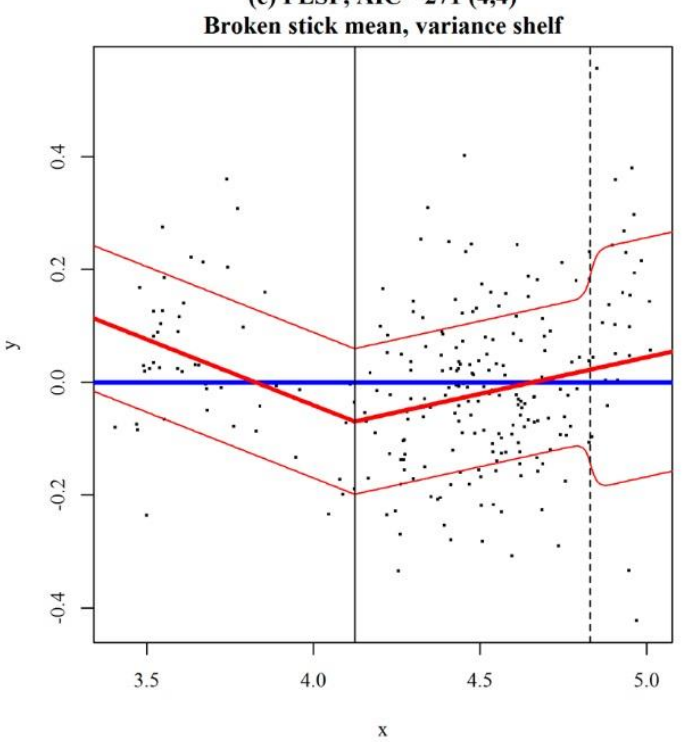

(b) PLSF, AIC=-271 $(4,4)$

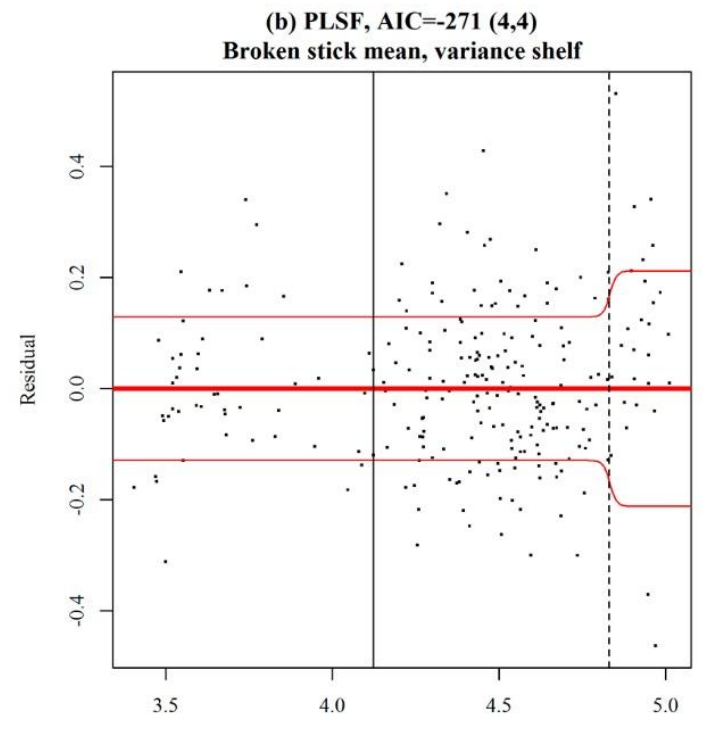

(d) PLSF, AIC=-271 $(4,4)$

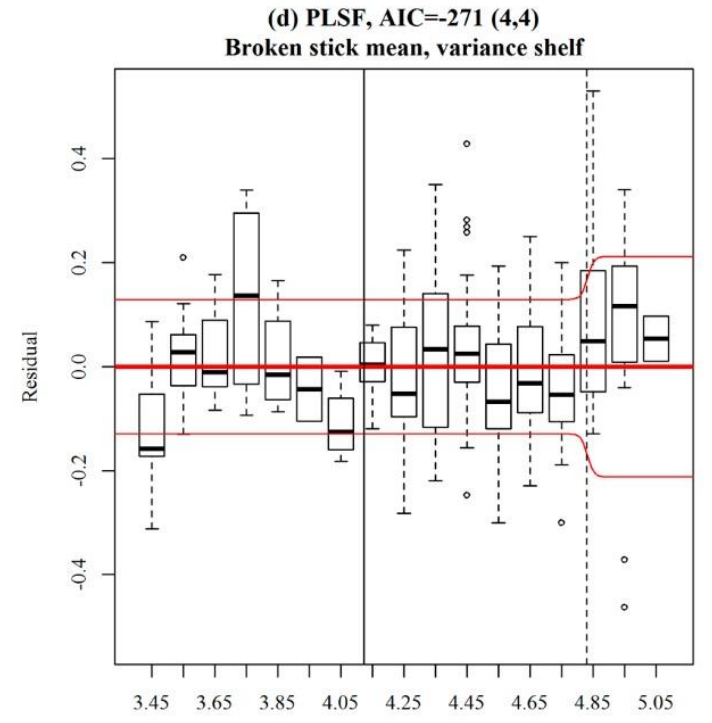

(f) PLSF, AIC=-271 (4,4)

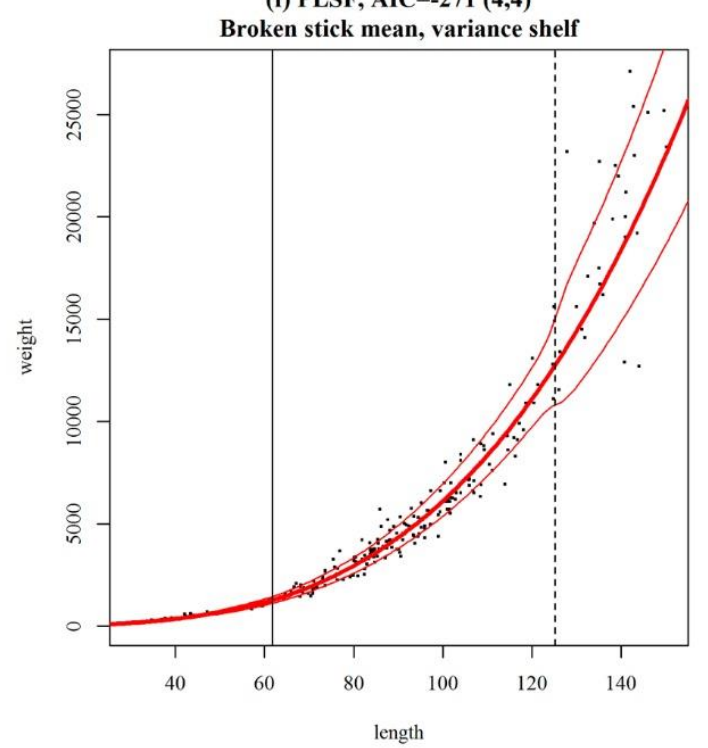


(a) PDGM, AIC=-32 $(1,2)$

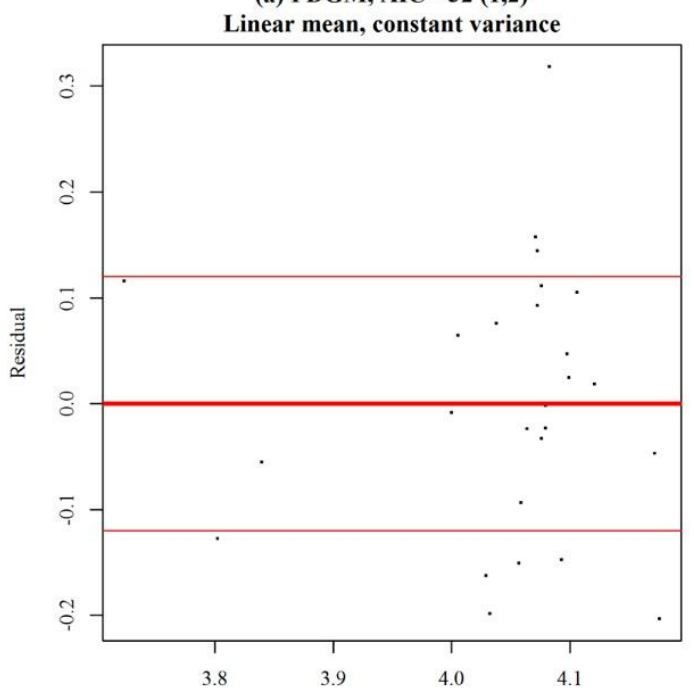

(e) PDGM, AIC=-32 $(1,2)$

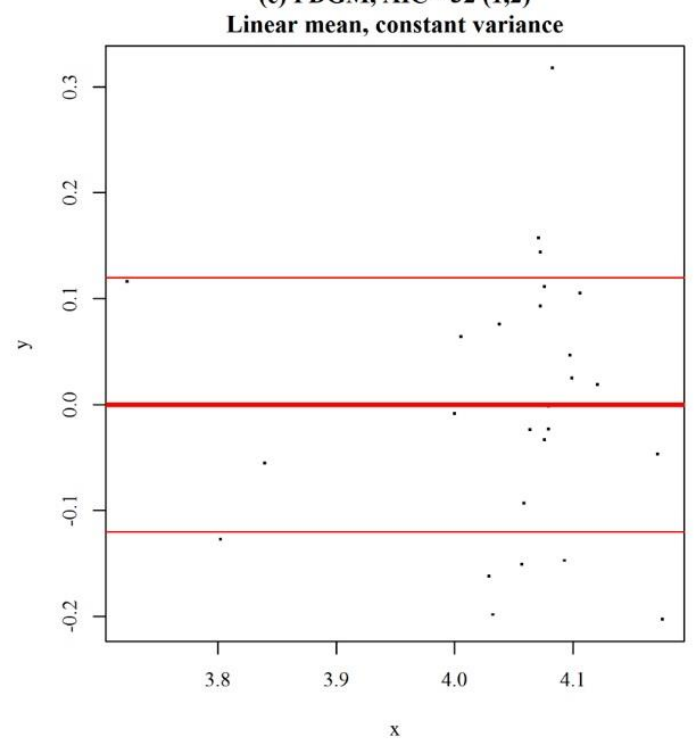

(b) PDGM, AIC =-32 $(1,2)$

Linear mean, constant variance

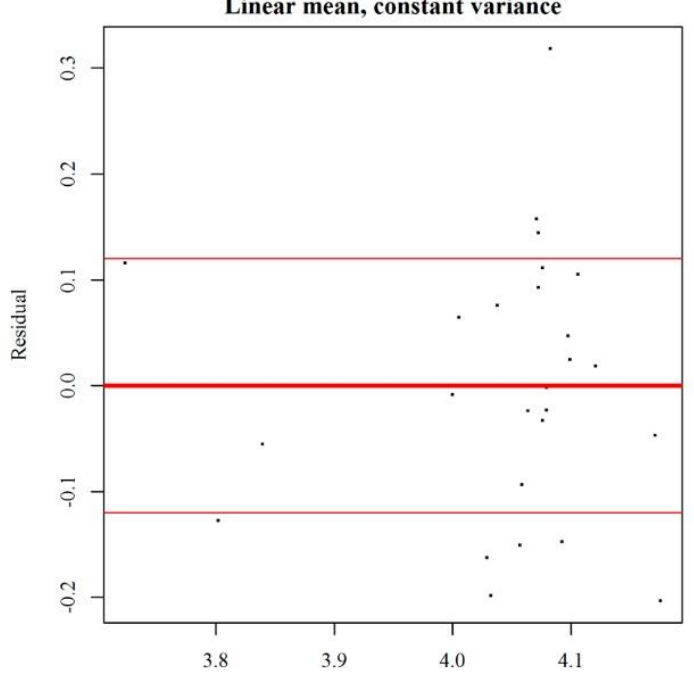

$x$

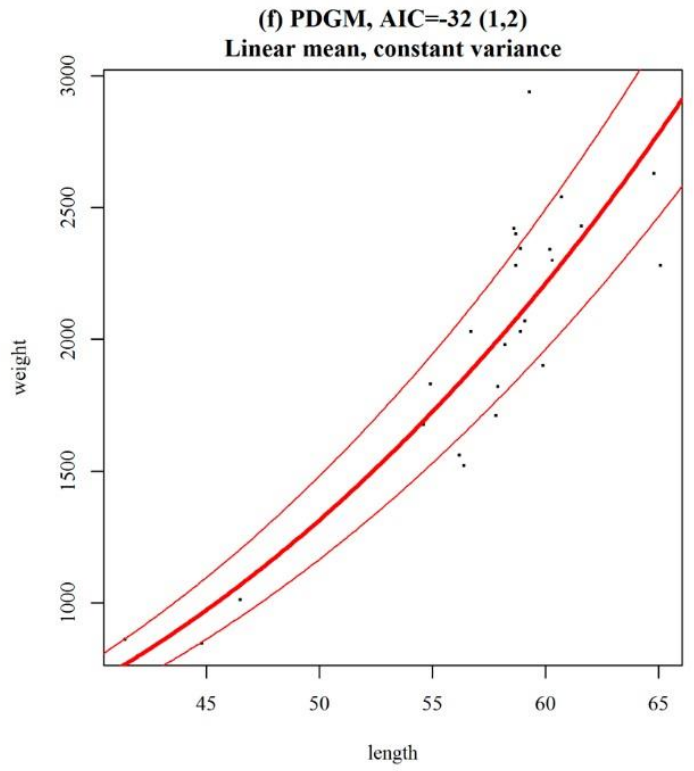


(a) PDGF, AIC=-20 (1,2)

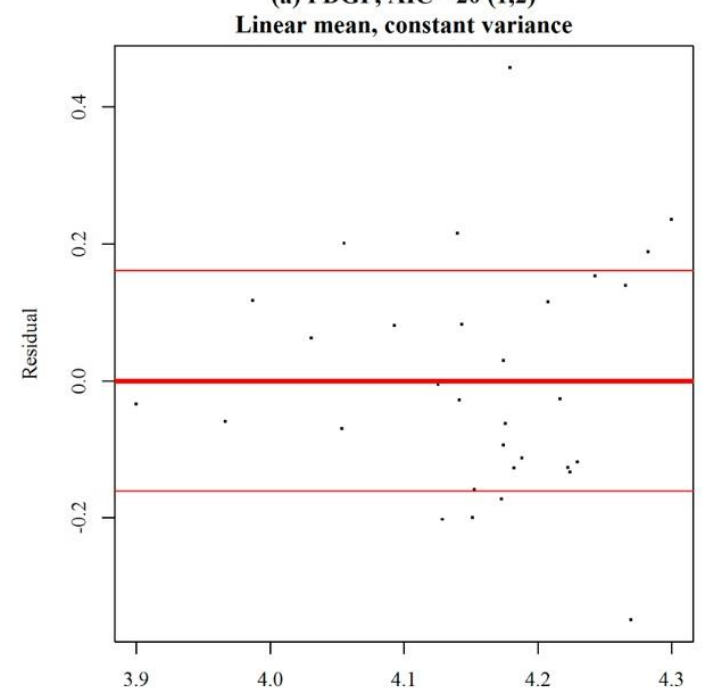

(e) PDGF, AIC =-20 (1,2)

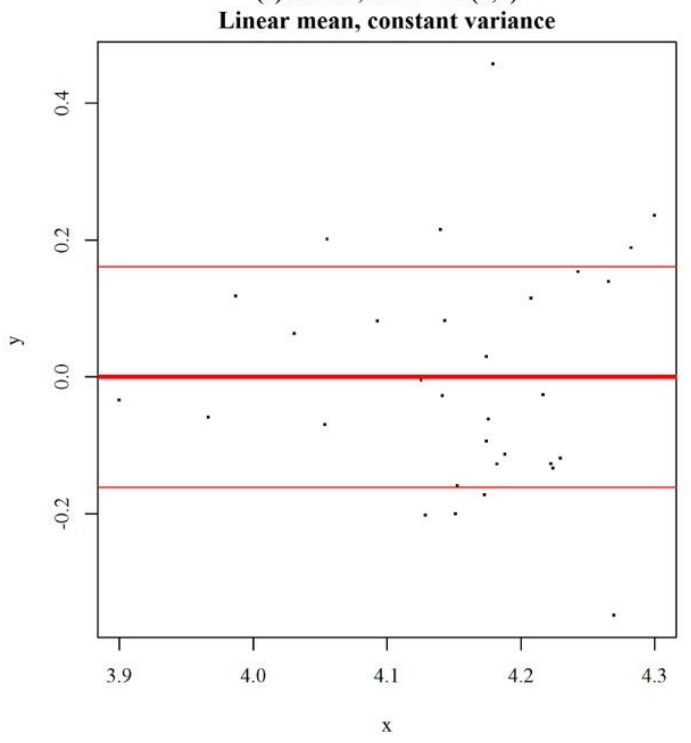

(b) PDGF, AIC=-20 (1,2)

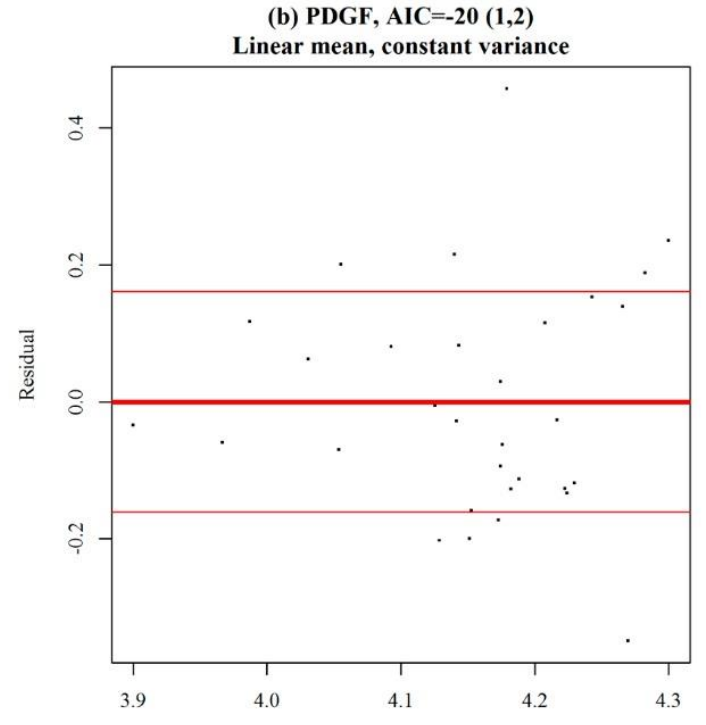

(f) PDGF, AIC $=-20(1,2)$

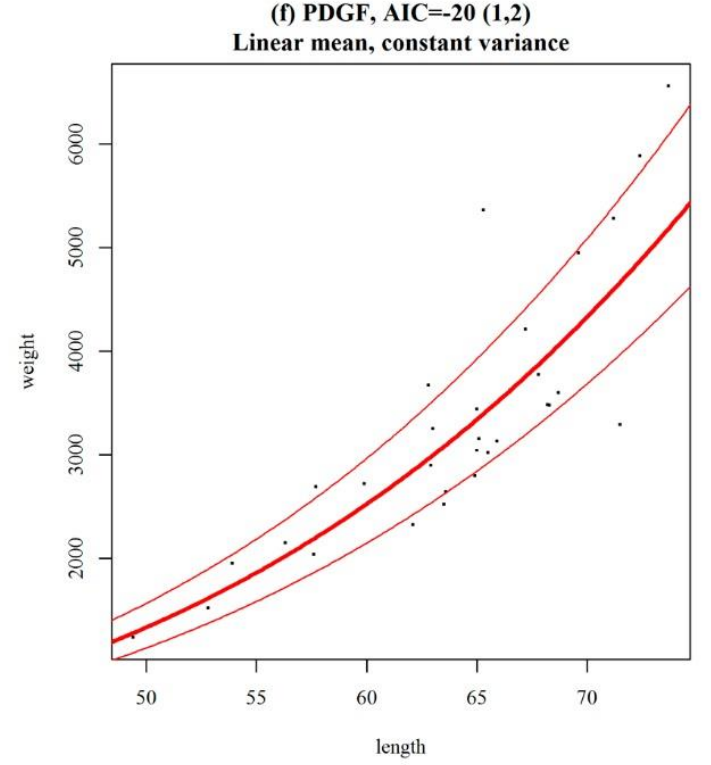


(a) BSHM, AIC $=-471(1,2)$

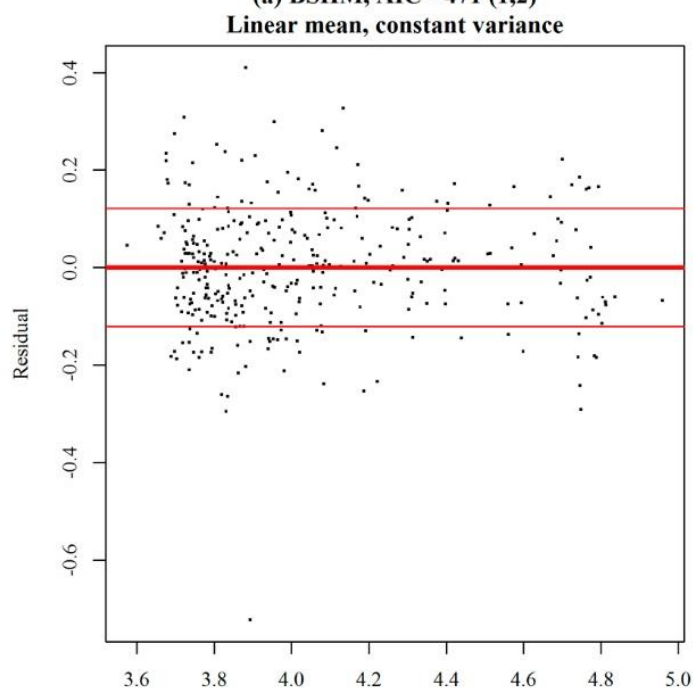

(c) BSHM, AIC $=-471(1,2)$

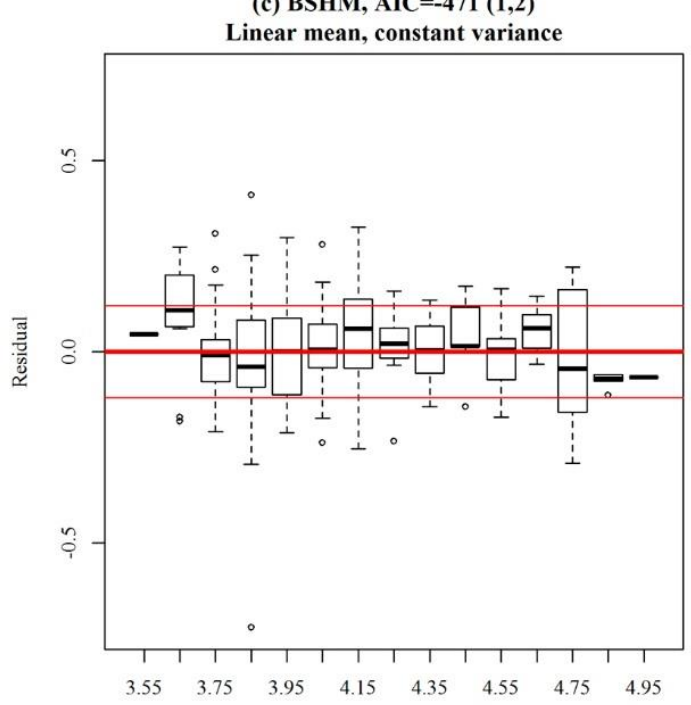

(e) BSHM, AIC=-471 $(1,4)$

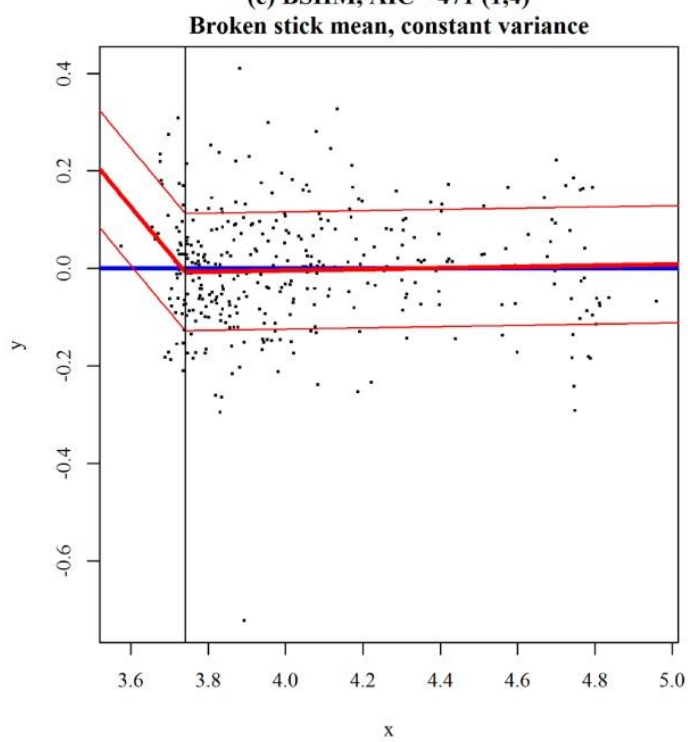

(b) BSHM, AIC=-471 $(1,4)$

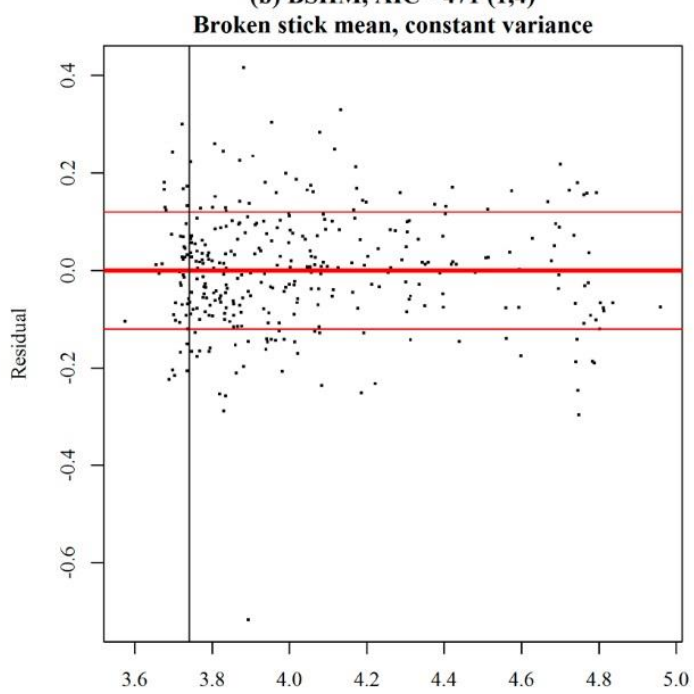

(d) BSHM, AIC=-471 $(1,4)$

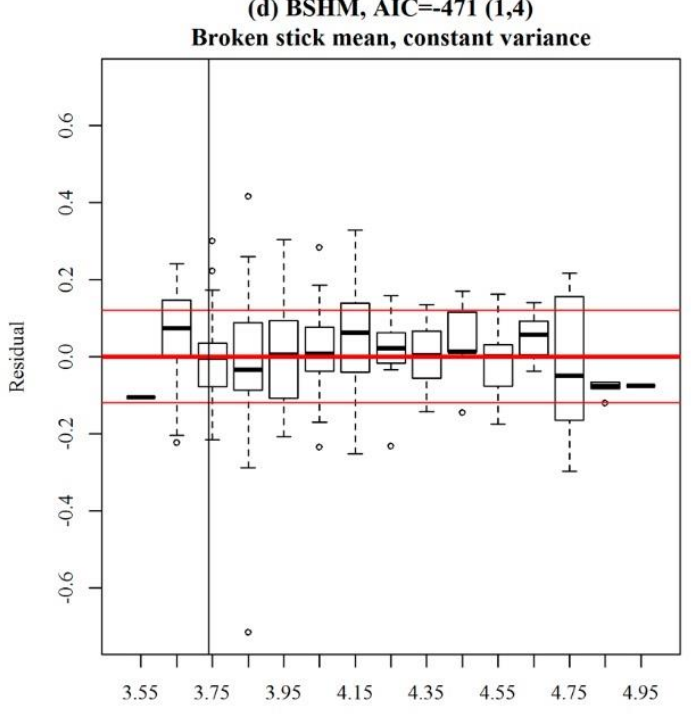

(f) BSHM, AIC=-471 $(1,4)$

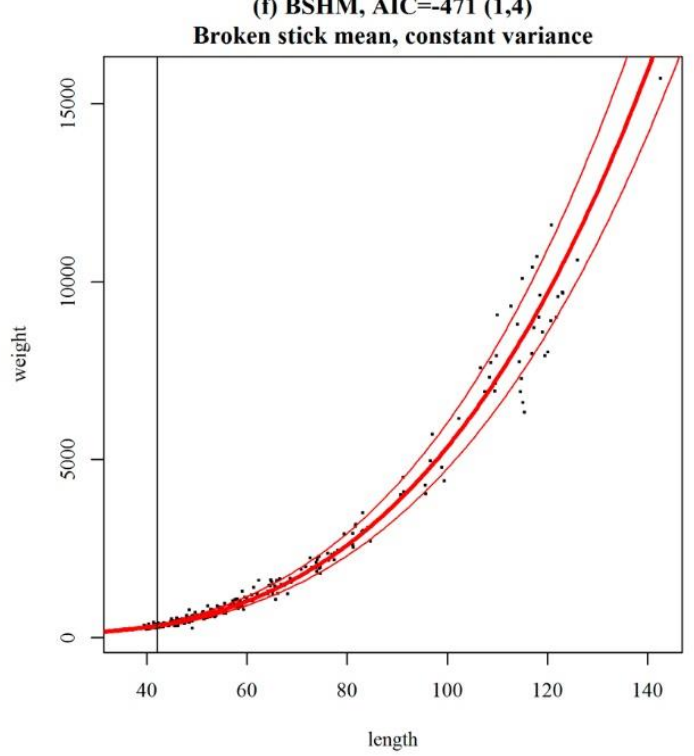


(a) BSHF, AIC=-753 (1,2)

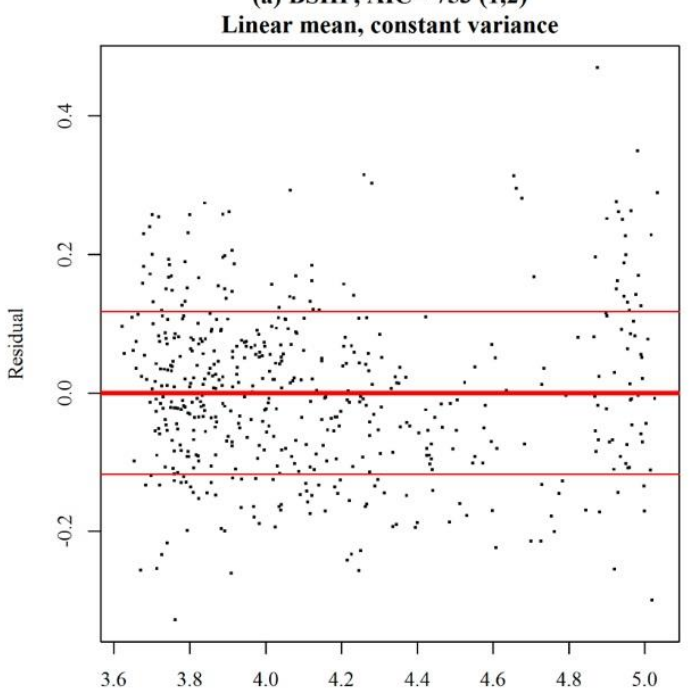

(c) BSHF, AIC =-753 (1,2)

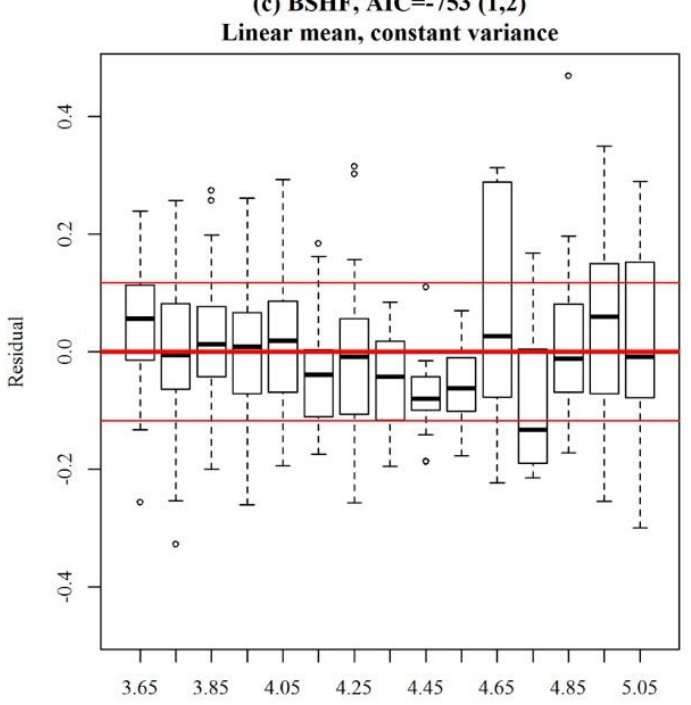

(e) BSHF, AIC=-782 $(4,4)$

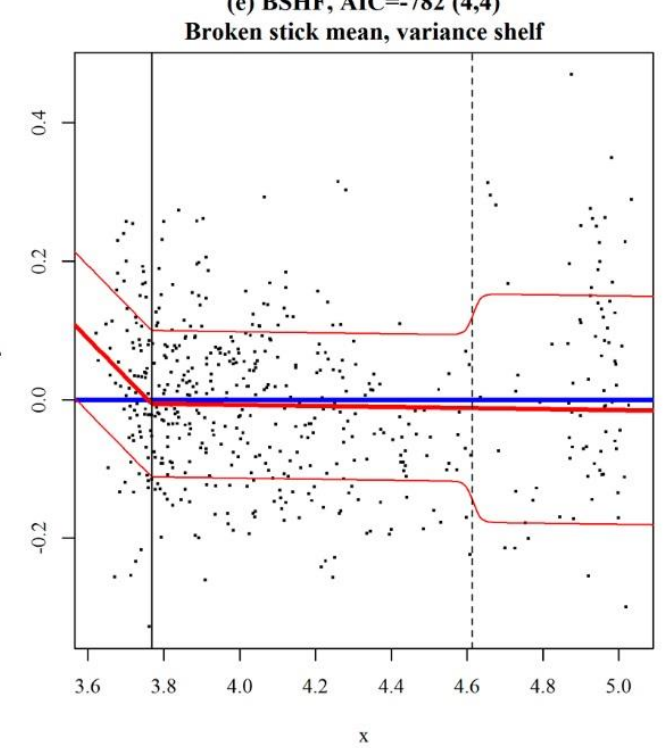

(b) BSHF, AIC=-782 (4,4)

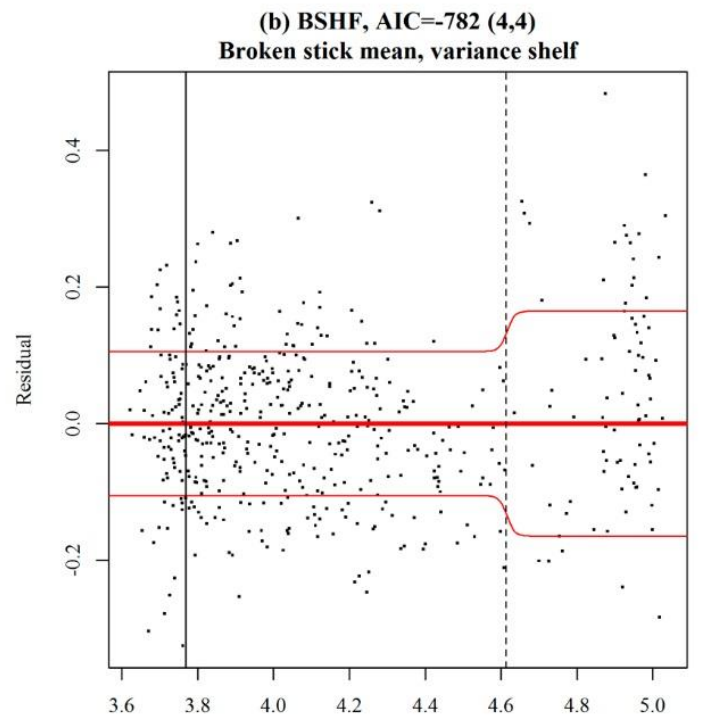

(d) BSHF, AIC=-782 $(4,4)$

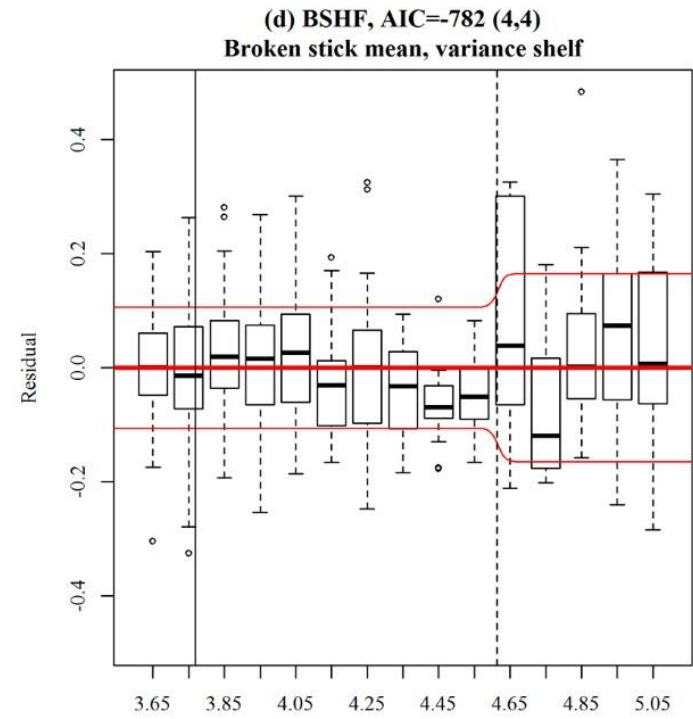

(f) BSHF, AIC =-782 (4,4)

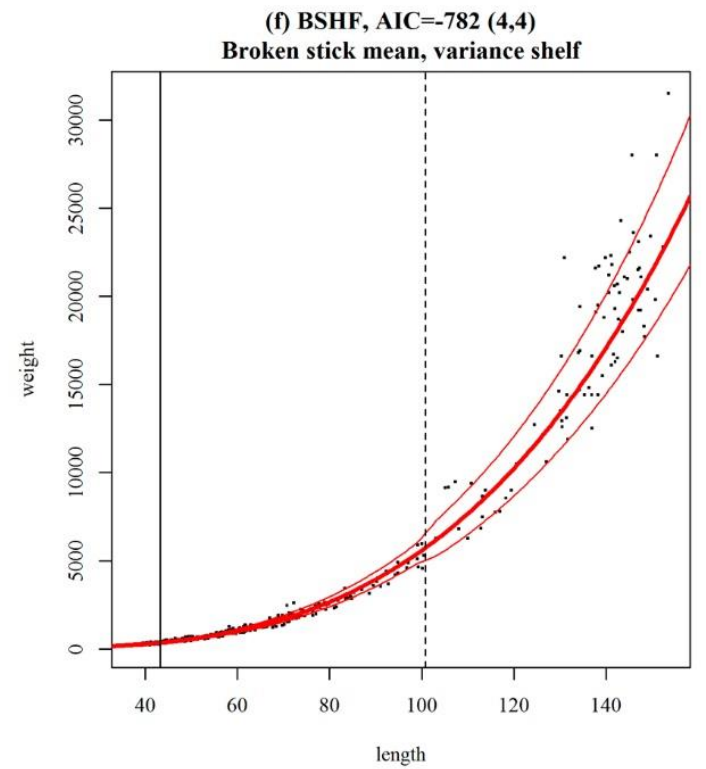




\section{Appendix IV.}

By species, frequency of individual catch sizes $(n)$ by weight $(\mathrm{kg})$; mass of individuals $(\mathrm{kg})$; number of individuals; and density (number of individuals per kilometre), as used in analyses, for Harriotta raleighana (LCH), Rhinochimaera pacifica (RCH), Chimaera carophila (CHP), Hydrolagus bemisi (GSP), Hydrolagus homonycteris (HYB), Hydrolagus novaezealandiae (GSH), Squalus acanthias (SPD), Centrophorus squamosus (CSQ), Deania calcea (SND), Etmopterus granulosus (ETB), Etmopterus lucifer (ETL), Centroscymnus owstoni (CYO), Centroselachus crepidater (CYP), Scymnodon plunketi (PLS), Oxynotus bruniensis (PDG), and Dalatias licha (BSH). 

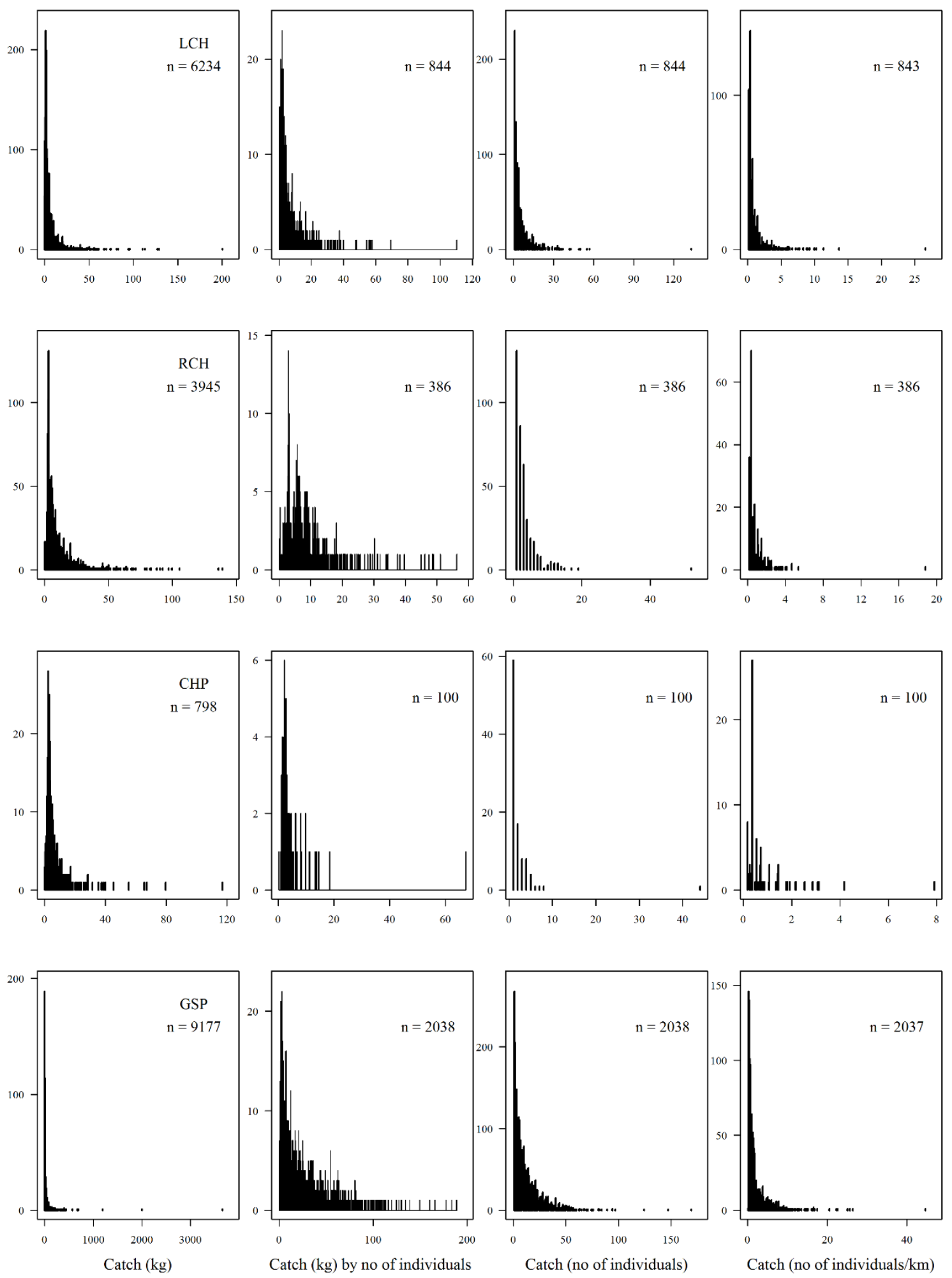

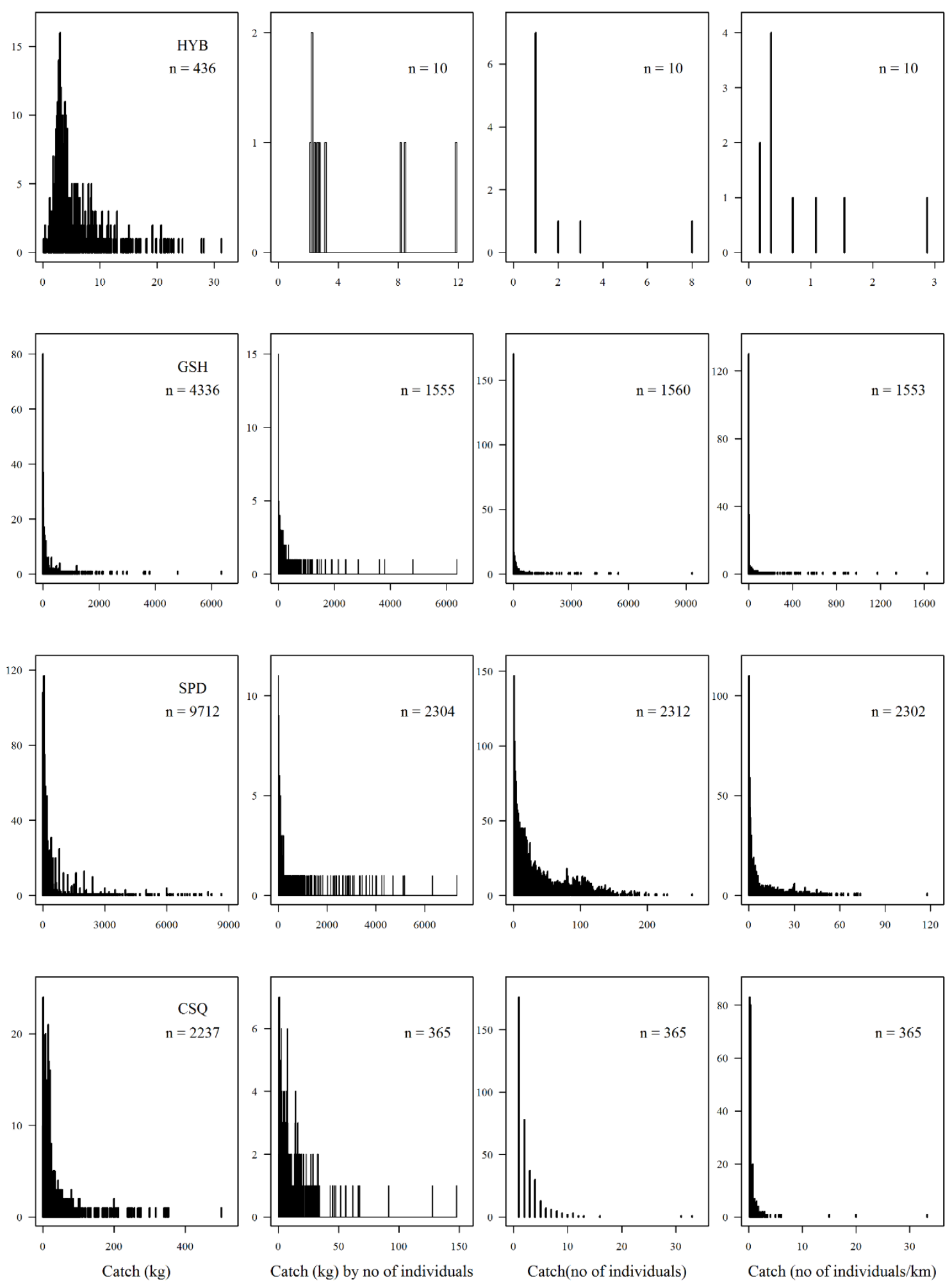

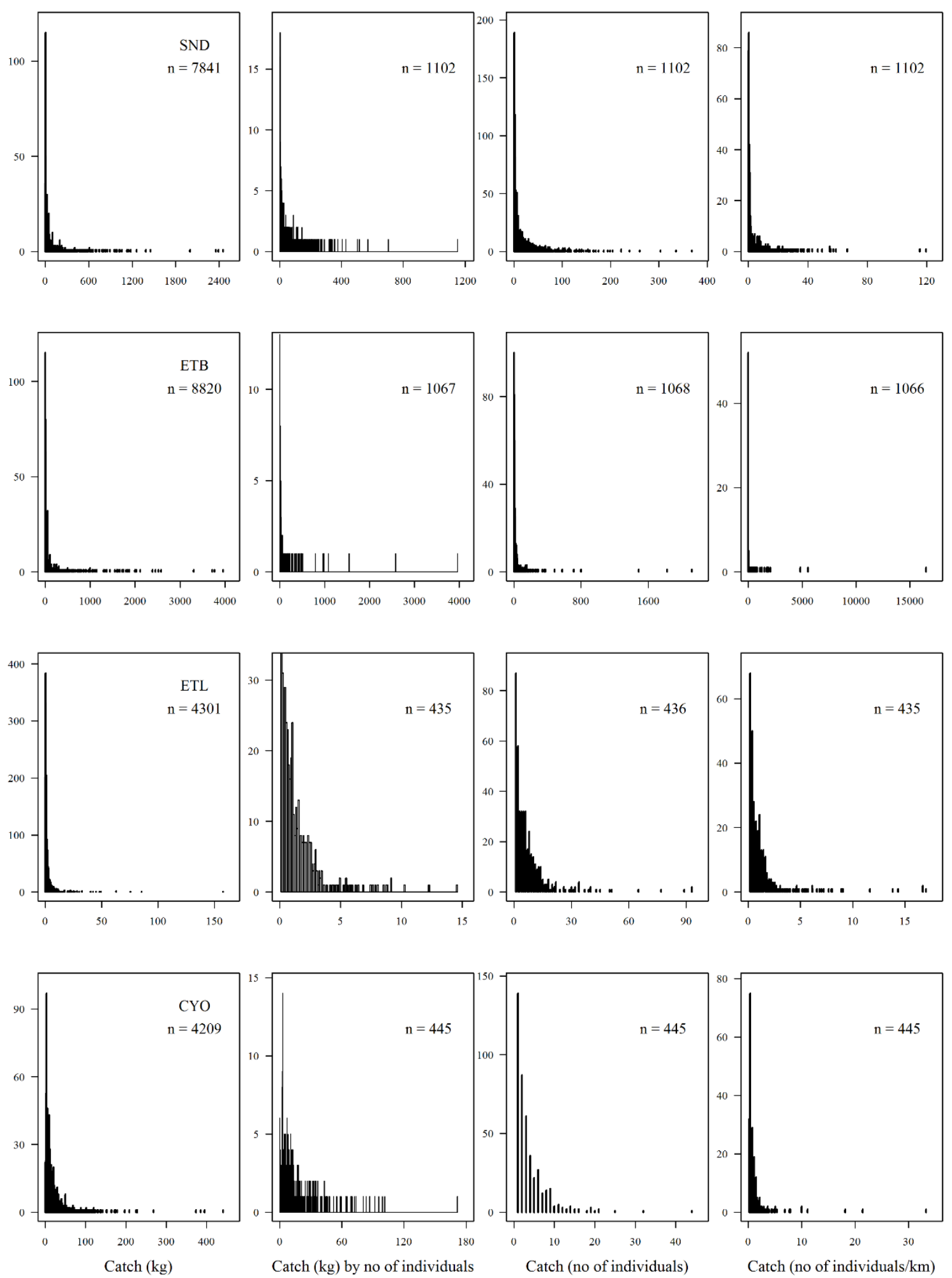

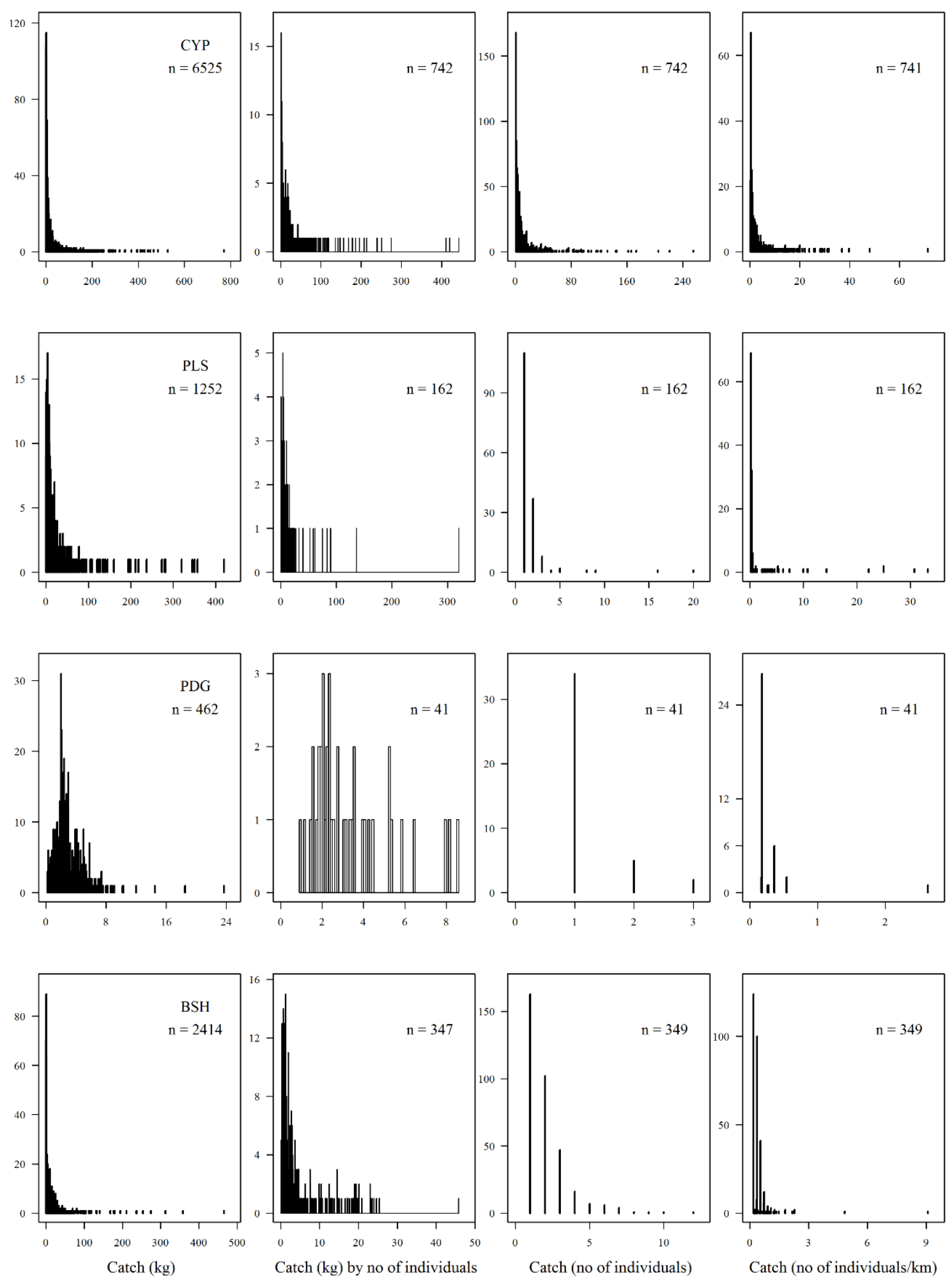


\section{Appendix V.}

List of species and haplotypes used for comparison analysis, including accessions numbers for BOLD and GenBank databases. Novel sequences are indicated in bold. $n a=$ represents sequence not available for the species.

\begin{tabular}{|c|c|c|c|}
\hline Family & Species & $\operatorname{cox} 1$ gene & nadh2 gene \\
\hline \multirow[t]{3}{*}{ Oxynotidae } & Oxynotus bruniensis (Ogilby 1893) & Obr_1 (KX002063, KX002064) & $\begin{array}{l}\text { Obr_1 (JQ518965, KX002045), Obr_2 } \\
\text { (KX002046) }\end{array}$ \\
\hline & Oxynotus centrina (Linnaeus 1758) & Oce_1 (FMS051-10, ELAME112-09, ELAME113-09) & $n a$ \\
\hline & Oxynotus paradoxus Frade 1929 & Opa_1 (ANGBF417-12) & Opa_1 (JQ518966) \\
\hline \multirow[t]{5}{*}{ Chimaeridae } & Chimaera carophila Kemper, Ebert, Naylor \& Didier 2014 & 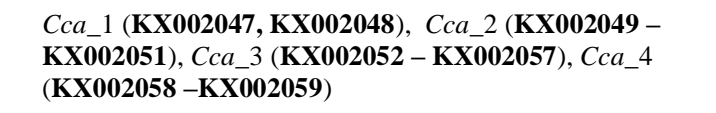 & $\begin{array}{l}\text { Cca_1 (GN12992, GN12993, GN12994, } \\
\text { KX002035 - KX002042) }\end{array}$ \\
\hline & Chimaera fulva Didier, Last \& White 2008 & $\begin{array}{l}\text { Cfu_1 (CYTC3536-12, CYTC4919-12), Cfu_2 (FOAF579- } \\
\text { 07), Cfu_3 (FOA274-04, FOAF580-07, FOAF583-07), } \\
\text { Cfu_4 (FOA280-04; FOA281-04) }\end{array}$ & na \\
\hline & Chimaera lignaria Didier 2002 & $\begin{array}{l}\text { Cli_1 (FOA279-04), Cli_2 (FOA282-04, FOAF586-07), } \\
\text { Cli_3 (FOAF585-07) }\end{array}$ & na \\
\hline & Chimaera monstrosa Linnaeus 1758 & $\begin{array}{l}\text { Cmo_1 (ANGBF588-12, EFBC007-09), Cmo_2 (FCFPS022- } \\
\text { 06), Cmo_3 (FCFPS020-06), Cmo_4 (ANGBF586-12, } \\
\text { ANGBF11765-15, EFBC005-09), Cmo_5 (GBGC0231-06, } \\
\text { GBGC1531-06), Cmo_6 (ANGBF587-12, ANGBF11122-15, } \\
\text { EFBC006-09, FCFPI004-06, FCFPS019-06, FCFPS021-06), } \\
\text { Cmo_7 (ANGBF589-12, EFBC008-09), Cmo_8 (BIM389-14, } \\
\text { ELAME549-09), Cmo_9 (ELAME547-09, ELAME551-09, } \\
\text { ELAME576-09, ELAME577-09, ELAME578-09, } \\
\text { ELAME601-09, ELAME602-09, ELAME603-09, } \\
\text { ELAME604-09, ELAME605-09), Cmo_10 (ELAME548-09) }\end{array}$ & Cmo_1 (AJ310140) \\
\hline & Chimaera opalescens Luchetti, Iglésias \& Sellos 2011 & $\begin{array}{l}\text { Cop_1(ANGBF582-12, EFBC001-09), Cop_2 (ANGBF414- } \\
\text { 12, ANGBF584-12, EFBC003-09), Cop_3 (ANGBF583-12, } \\
\text { ANGBF585-12, EFBC002-09, EFBC004-09) }\end{array}$ & $n a$ \\
\hline
\end{tabular}




\begin{tabular}{|c|c|c|c|}
\hline Family & Species & cox 1 gene & nadh2 gene \\
\hline & Chimaera panthera Didier 1998 & Cpa_1 (FOAD658-05) & $n a$ \\
\hline & Chimaera phantasma Jordan \& Snyder 1900 & $n a$ & Cph_1 (JQ518718) \\
\hline & Hydrolagus affinis (de Brito Capello 1868) & Haf_1 (GLF111-14, GLF186-14) & $n a$ \\
\hline & Hydrolagus africanus (Gilchrist 1922) & $\begin{array}{l}\text { Hafr_1 (ANGBF10936-15, ANGBF10937-15, } \\
\text { ANGBF10938-15, ANGBF10939-15), Hafr_2 (DSFSF300- } \\
\text { 09, DSFSF301-09) }\end{array}$ & $n a$ \\
\hline & Hydrolagus barbouri (Garman 1908) & Hba_1 (ABFJ147-06) & $n a$ \\
\hline & Hydrolagus bemisi Didier 2002 & Hbe_1 (FOA295-04) & \\
\hline & Hydrolagus colliei (Lay \& Bennett 1839) & $\begin{array}{l}\text { Hco_1 (MFC025-08), Hco_2 (FMV005-08, FMV041-08, } \\
\text { TZFPB175-05, TZFPB185-05, TZFPB186-05, TZFPB187-05, } \\
\text { TZFPB484-06, TZFPB485-06, TZFPB487-06, TZFPB488-06, } \\
\text { TZFPB721-06, TZFPB775-06), Hco_3 (TZFPB184-05), } \\
\text { Hco_4 (FMV040-08), Hco_5 (TZFPB486-06), Hco_6 } \\
\text { (TZFPB75-06) }\end{array}$ & Hco_1 (JQ518720) \\
\hline & Hydrolagus lemures (Whitley 1939) & $\begin{array}{l}\text { Hle_1 (FOA292-04), Hle_2 (FOA291-04), Hle_3 (FOAF587- } \\
\text { 07), Hle_4 (FOA288-04, FOA290-04, FOAF590-07), Hle_5 } \\
\text { (FOA289-04) }\end{array}$ & Hle_1 (HM147139) \\
\hline & Hydrolagus mitsukurii (Jordan \& Snyder 1904) & $n a$ & Hmi_1 (KF927900) \\
\hline & Hydrolagus novaezealandiae (Fowler 1911) & Hno_1 (FOA293-04), Hno_2 (FOA294-04) & Hno_1 (JQ518721) \\
\hline & Hydrolagus ogilbyi (Waite 1898) & $\begin{array}{l}\text { Hog_1(CYTC3538-12, CYTC4920-12, FOA296-04, FOA297- } \\
\text { 04, FOA298-04, FOAF588-07, FOAF589-07, FOAF591-07), } \\
\text { Hog_2 (FOA299-04) }\end{array}$ & $n a$ \\
\hline & Hydrolagus pallidus Hardy \& Stehmann 1990 & Hpa_1 (GLF234-14) & $n a$ \\
\hline & Hydrolagus trolli Didier \& Séret 2002 & $\begin{array}{l}\text { Htr_1 (FOA283-04, FOA285-04, FOA286-04, FOA287-04), } \\
\text { Htr_2 (FOA284-04) }\end{array}$ & $n a$ \\
\hline Rhinochimaeridae & Rhinochimaera pacifica (Mitsukuri 1895) & $\begin{array}{l}\text { Rpa_1 (CYTC4922-12), Rpa_2, (CYTC3541-12), Rpa_3 } \\
\text { (KX002060, KX002061),Rpa_4 (KX002062) }\end{array}$ & $\begin{array}{l}\text { Rpa_1 (HM147141, KX002043, } \\
\text { KX002044) }\end{array}$ \\
\hline
\end{tabular}


